\title{
INEL BNCT Research Program Publications 1993
}

Published May 1994

\section{Idaho National Engineering Laboratory EG\&G Idaho, Inc. Idaho Falls, Idaho 83415}

Prepared for the

U.S. Department of Energy Office of Energy Research Under DOE Idaho Operations Office Contract DE-AC07-76ID01570 


\section{,}




\section{DISCLAIMER}

This report was prepared as an account of work sponsored by an agency of the United States Government. Neither the United States Government nor any agency thereof, nor any of their employees, make any warranty, express or implied, or assumes any legal liability or responsibility for the accuracy, completeness, or usefulness of any information, apparatus, product, or process disclosed, or represents that its use would not infringe privately owned rights. Reference herein to any specific commercial product, process, or service by trade name, trademark, manufacturer, or otherwise does not necessarily constitute or imply its endorsement, recommendation, or favoring by the United States Government or any agency thereof. The views and opinions of authors expressed herein do not necessarily state or reflect those of the United States Government or any agency thereof. 


\section{DISCLAIMER}

Portions of this document may be illegible in electronic image products. Images are produced from the best available original document. 


\begin{abstract}
This document is a collection of the published reports describing research supporting the Idaho National Engineering Laboratory Boron Neutron Capture Therapy Research Program for calendar year 1993. Contributions from the principal investigators are included, covering chemistry (pituitary tumor studies, boron drug development including liposomes, lipoproteins, and carboranylalanine derivatives), pharmacology (murine screenings, toxicity testing, ICP-AES analysis of biological samples), physics (radiation dosimetry software, neutron beam and filter design, neutron beam measurement dosimetry), and radiation biology (tissue and efficacy studies of small and large animal models). These reports have previously appeared in the book: Advances in Neutron Capture Therapy, edited by A. H. Soloway, R. F. Barth, D. E. Carpenter, Plenum Press, 1993. Reports have also appeared in three journals: Angewandte Chemie, Strahlentherapie und Onkologie, and Nuclear Science and Engineering.
\end{abstract}

The Govermment reserves for itself and others acting on its behalf a royalty free, nonexclusive, irrevocable, world-wide license for govermental purposes to publish, distribute, translate, duplicate, exhibit, and perform any such data copyrighted by the contractor. 



\section{CONTENTS}

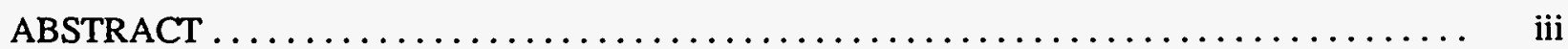

INTRODUCTION $\ldots \ldots \ldots \ldots \ldots \ldots \ldots \ldots \ldots \ldots \ldots \ldots \ldots \ldots \ldots \ldots \ldots \ldots \ldots \ldots \ldots$

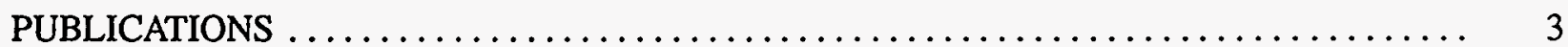

Angewandte Chemie, 32, 950-984, (1993)

The Role of Chemistry in the Development of Boron Neutron Capture Therapy of Cancer . . 5 M. F. Hawthorne

Strahlentherapie und Onkologie, 169, 48-56, (1993)

Large Animal Normal Tissue Tolerance Using an Epithermal Neutron Beam and

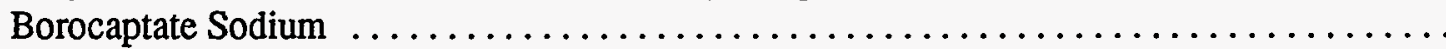

P. R. Gavin, R. Huiskamp, F. J. Wheeler, S. L. Kraft, C. E. DeHaan

Nuclear Science and Engineering, 113, 314-323, (1993)

Creating and Using a Type of Free-Form Geometry in Monte Carlo Particle Transport ..... D. E. Wessol and F. J. Wheeler

Advances in Neutron Capture Therapy, edited by A. H. Soloway, R. F. Barth, and D. E. Carpenter, Plenum Press, (1993)

Nuclear Characterization of the HFR Petten BNCT Facility

P. Watkins, Y. Harker, C. Amaro, W. Voorbraak, F. Stecher-Rasmussen, H. Verhagen,

C. Perks, H. Delafield, G. Constantine, and R. Moss

Physics Parameters for an Epithermal Neutron Beam at the Georgia Institute

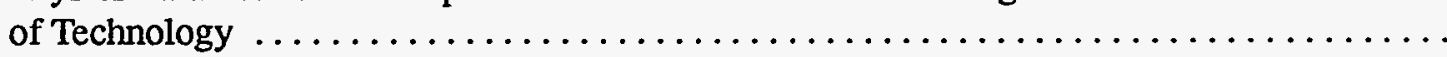

D. Nigg, G. Storr; and F. Wheeler

PLENARY TALK: Analytical Dosimetry and Treatment Planning for BNCT .......... D. Nigg

Post Treatment Dose Distribution Evaluation for a Recent NCT Patient $G$. Storr and $F$. Wheeler

Enantioselective Synthesis of an Unnatural Amino Acid, L-Carboranylalanine P. Radel and S. Kahl

Carbonyl Nonahydrodecaborate(1-): A Versatile Synthon for Functionalized Borane Anions . . . . . . . . . . . . . . . . . . . . . . . . . . . K. Shelly, M. F. Hawthorne, and C. Knobler

PLENARY PRESENTATION: Boron Delivery to Tumors Mediated by Macromolecules

and Vesicles

M.F. Hawthorne 
Liposomal Delivery of Boron to Murine Tumors for Boron Neutron Capture Therapy .....

D. Feakes, K. Shelly, M. F. Hawthorne, P. Schmidt, C. Elstad, G. Meadows, and W. Bauer

Low Density Lipoprotein Reconstitutions with Alkyl and Aryl Carboranes

S. Kahl, D. Pate, and L. Wainschel

A Rapid Method for the Direct Analysis of Boron in Whole Blood by Atomic

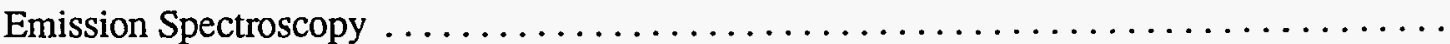

W. Bauer, P. Micca, and B. White

Quantification of Boron in Biological Tissue by Secondary Ion Mass Spectrometry .......

V. Chia, R. Bleiler; D. Sams, C. Jones, R. Odom, W. Bauer, A. Gianotto, C. Swartz, and

$J$. Weidner

Determination of Strongly Protein-Bound Borocaptate Species by High Performance Liquid Chromatography with Online Inductively Coupled Plasma Atomic Emission

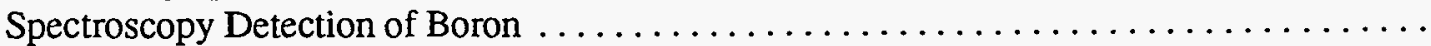

N. Hotz and W. Bauer

NMR Studies of the Interaction of Borocaptate Sodium with Serum Albumin

P. Tang, M. Schweizer, W. Bauer, and K. Bradshaw

Purity and Quality Determinations of Borocaptate Sodium

A. Gianotto and W. Bauer

PLENARY PRESENTATION: Large Animal Studies on the Use of BNCT for the

Treatment of Brain Tumors . . . . . . . . . . . . . . . . . . . . . . . . . .

P. Gavin, C. DeHaan, M. Moore, J. Weidner, D. Swartz, S. Kraft, C. Atkinson,

C. Amaro, W. Bauer, and A. Siefert

Cardiovascular Toxicities Associated with Intravenous Administration of

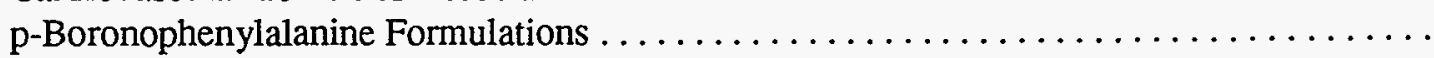

T. LaHann, C. Sills, G. Hematillake, T. Dymock, and G. Daniell

Dose Effect Comparisons between HFR and BMRR Irradiated Dogs with Respect to

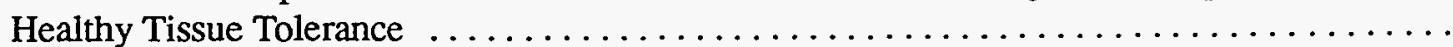

R. Huiskamp, P. Gavin, F. Wheeler; A. Siefert, and K. Philipp

Pharmacokinetics of Borocaptate Sodium in Canine Head Determined by ${ }^{11} \mathrm{~B}$ Magnetic

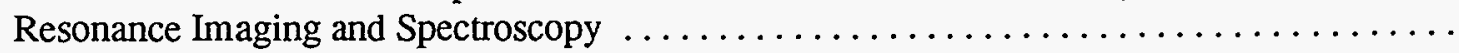

K. Bradshaw, M. Schweizer, G. Glover, and J. Hadley

Bioavailability of Intravenous Formulations of p-Boronophenylalanine in Dog and Rat ....

T. LaHann, D. Lu, C. Sills, G. Daniell, S. Kraft, P. Gavin, and W. Bauer

Computer Modeling the Boron Compound Factor in Normal Brain Tissue

P. Gavin, R. Huiskamp, F. Wheeler, and M. Griebenow

Computer Modeling the Boron Compound Factor in Brain Tumors

P. Gavin, R. Huiskamp, F. Wheeler; and M. Griebenow 
Determination of Fast-Neutron Dose Distributions in the Canine Central Nervous System . . 225 D. Nigg, J. Moran, and F. Wheeler

Dose Optimization for Boron Neutron Capture Therapy of Spontaneous Canine

Brain Tumors .

C. Atkinson and F. Wheeler

In Vivo and In Vitro Uptake of Boronated Compounds by B16-BL6 Murine Melanoma .....

C. Elstad, K. Meinkoth, B. Mathison, G. Meadow', D. Kinder, M. F. Hawthorne, K. Shelly, D. Feakes, and W. Bauer

The Use of Boron Neutron Capture for the Treatment of Pituitary Tumors

B. Albertson, M. Millan, S. Binney, G. Willeke, S. Martsolf, J. Johnson, and D. Loriaux 


\section{INEL BNCT Research \\ Program Publications \\ 1993}

\section{INTRODUCTION}

This compilation of technical publications is intended as a companion document for the Idaho National Engineering Laboratory (INEL) Boron Neutron Capture Therapy (BNCT) Research Program Annual Report for 1993, EGG-2738, published in May 1994.

Overall, a total of 28 papers were published by the INEL BNCT Research Program participants in calendar year 1993 . These 28 papers included 3 plenary talks, 5 articles on boron chemistry/ compound development, 5 articles on reactor/ radiation physics, 11 articles on analytical chemistry/pharmacology, and 4 articles on radiation biology. The breadth of the integrated program at the INEL is reflected in both the number of these reports and the topics covered. In addition, the high level of collaboration, which is absolutely essential for technological development and implementation of BNCT, is displayed in these reports.

The reports represent some earlier presentations, notably the Proceedings of the Fifth International Symposium on Neutron Capture Therapy for Cancer, held in September 1992 in Columbus, Ohio, and published in Advances in
Neutron Capture Therapy, edited by Albert H. Soloway, Rolf F. Barth and David E. Carpenter, Plenum Press, 1993.

The contents herein represent the combined efforts of numerous research groups, both onsite at the INEL and at numerous university groups throughout the country. Contributions of these groups will help lead to clinical usage of BNCT.

For more information about the INEL BNCT Research Program, contact:

J. R. Venhuizen

Program Manager

National Center for BNCT

Measurement and Development

phone: (208) 526-9421

FAX: (208) 526-0528

e-mail: jrv@inel.gov

\section{R. J. Wiersema}

Director

National Center for BNCT

Measurement and Development

phone: (208) 526-9264

FAX: (208) 526-0528

e-mail: rw9@inel.gov 



\section{PUBLICATIONS}

Of the 28 papers that follow, all but the first three appear in Advances in Neutron Capture Therapy, edited by Albert $\mathrm{H}$. Soloway, Rolf F.
Barth, and David E. Carpenter (Plenum Press, 1993). The first three papers appear in separate journals. 



\section{The Role of Chemistry in the Development of Boron Neutron Capture Therapy of Cancer}

M. F. Hawthorne

This article is a reprint from Angewandte Chemie, 32, 950-984, (1993). 



\title{
The Role of Chemistry in the Development of Boron Neutron Capture Therapy of Cancer
}

\author{
By M. Frederick Hawthorne* \\ Dedicated to Professor Heinrich Nöth on the occasion of his 65 th birthday.
}

A therapeutic method that selectively destroys malignant cells in the presence of normal cells is a highly valued goal of oncologists and the possible salvation of cancer patients afflicted with some incurable forms of the disease. Selective cell destruction is, in principle, possible with a binary therapeutic strategy based upon the neutron capture reaction observed with the ${ }^{10} \mathrm{~B}$ nucleus and a neutron of low kinetic energy (thermal neutron). This nuclear fission reaction produces both ${ }^{4} \mathrm{He}$ and ${ }^{7} \mathrm{Li}^{+}$nuclei along with about $2.4 \mathrm{MeV}$ of kinetic energy and weak $\gamma$-radiation. Since the energetic and cytotoxic product ions travel only about one cell diameter in tissue one may specify the cell type to be destroyed by placing innocent ${ }^{10} \mathrm{~B}$ nuclei on or within only the doomed cells. This article describes the current status of chemical research aimed at the eventual adoption of this therapeutic method (boron neutron capture therapy or BNCT). The multidisciplinary nature of this research effort involves chemistry, biology, nuclear physics, medicine, and related specialties. Methods devised for bringing ${ }^{10} \mathrm{~B}$ nuclei to tumor cells in therapeutic amounts are correlated with the structure of a generalized cell and the various cellular compartments available for boron localization. The synthesis methods employed for the creation of boron-containing biomolecules and drugs are presented along with representative data concerning their efficacy in tumor localization. The outlook for BNCT is especially bright at this time because of rapid developments in the fields of bioorganometallic chemistry, microbiology, immunology, and nuclear science, to name but a few. Very effective boron delivery vehicles have been demonstrated, and through the interaction of chemistry and biology these species are undergoing further improvement and evaluation of their suitability for BNCT.

\section{Introduction}

What would result if it were possible to selectively attach a nontoxic species $\mathrm{A}$ to cancer cells and, upon command, destroy only these malignant cells by converting their cellbound $A$ to a deadly cytotoxin through its activation with a second nontoxic reagent $B$ ? One would have created a binary method for cancer therapy characterized by greater selectivity, efficacy, and safety than the unitary, single agent, radiation and chemotherapy protocols commonly in use today. Most importantly, tumors now beyond any hope of longterm control could be destroyed. The key to this proposed binary therapy is the requirement that the innocent $A$ and $B$ species have a great propensity for mutual reaction and that the extremely energetic products of this reaction, $X$ and $Y$, are confined to an extremely small volume by their very brief lifetimes. However, while $\mathrm{X}$ and $\mathrm{Y}$ exist they must function

$$
\begin{aligned}
& A+B \longrightarrow[A B] \longrightarrow X+Y \text { (short-lived cytotoxins) } \\
& B+\infty l l \# \\
& A+\infty e l l \longrightarrow A \cdot c e l l \text { (interactions specific to cancer } \infty \text { ells) } \\
& A \cdot c e l l+B \longrightarrow[A B \cdot c e l l] \longrightarrow[(X+Y) \cdot c e l l] \text { (cell deatb) }
\end{aligned}
$$

Scheme 1. A hypothetical binary cytotoxic reaction of two innocent reagents $A$ and $B$ to form the cell-killing reaction products $X$ and $Y$. In BNCT, $A$ represents a ${ }^{10} B$ nucleus selectively emplaced in or on a malignant cell. $B$ is a slow neutron. and $X$ and $Y$ represent high-energy ${ }^{4} \mathrm{He}^{2+}$ and ${ }^{7} \mathrm{Li}^{3+}$ ions.

["] Prof. Dr. M. F. Hawthorne Department of Chemistry and Biochemistry University of Calffornia, Los Angeles 405 Hilgard Avenue, Los Angeles, CA 90024 (USA) as efficient killers of cells. This binary process is described by the equations of Scheme 1, and an example of such a process, boron neutron capture therapy (BNCT), forms the subject of this article.

\section{Boron Neutron Capture and Therapy}

\subsection{Historical Account of the Conceptualization of Boron Neutron Capture Theory}

The rampant development of quantum mechanics, nuclear physics, and associated chemistry that occurred in the 1930s led James Chadwick ${ }^{[1]}$ to the discovery of the neutron in 1932. Studies of the interactions of neutrons with a variety of materials uncovered the phenomenon of neutron scattering by elastic collisions ${ }^{[2]}$ with atomic nuclei, especially the ubiquitous proton of the $\mathrm{H}$ atom. Capture of slow (or thermal) neutrons by certain nuclei was observed by Fermi, ${ }^{[3]}$ and the disintegration of other specific nuclei by interaction with these thermal neutrons was observed by others, as well. ${ }^{[4]}$ By 1935 a great mass of such experimental information had been collected; it was then clear that the ability of an atomic nucleus to capture a neutron was related not to the mass of the target nucleus, but to the actual structure of that nucleus. The concept of a characteristic effective cross-sectional area of a nucleus, expressed as units of $10^{-24} \mathrm{~cm}^{2}$ (known as barn units), was introduced with this early work. The effective nuclear cross-section of boron for neutron capture was known to be exceptionally large, while boron's neighbors in the periodic table, nitrogen and carbon, exhib- 
ited nuclear cross-sections that were comparatively quite small. An important 1935 paper by Taylor ${ }^{[3]}$ described the capture of thermal neutrons by ${ }^{10} \mathrm{~B}$ nuclei followed by the production of ${ }^{4} \mathrm{He}^{2+}$ ( $\alpha$-particles) and ${ }^{7} \mathrm{Li}^{3+}$. About $2 \mathrm{MeV}$ of kinetic energy is distributed between these two heavy ion products. The translational range of the product ions was particularly short: ${ }^{[5]}$ about $7.6 \mu \mathrm{m}$ in photographic gelatin and $1.1 \mathrm{~cm}$ in air. Consequently, the lithium nucleus and the $\alpha$-particle were short-lived, energetic products capable of doing immense local damage to organic materials through ionization processes. Thus, by 1936 the stage was set for the suggestion of the concept of boron neutron capture therapy (BNCT).

Gordon L. Locher of the Bartol Research Foundation of the Franklin Institute in Philadelphia, Pennsylvania, pointed out ${ }^{[6]}$ in 1936 the potential medical applications of neutrons and boron neutron capture (BNC). His concept invoked the simple neutron capture reaction by boron for the basis of a binary therapeutic method as defined in Scheme 1. Accordingly, Locher's analogues of species $A$ and $B$ were the ${ }^{10} \mathrm{~B}$ nucleus contained in a compound that specifically localizes in tumor and a thermal neutron, respectively. The energetic cytotoxic reaction products $X$ and $Y$ correspond to the $\alpha$ particle and the lithium nucleus. The beauty of this proposal is the fact that no radioactive materials are involved and that the therapeutic process may be modulated by controlling the supply of neutrons to the tumor site.

The two necessary components of the boron neutron capture (BNC) process, a controllable source of low-energy neutrons with a usefully high flux and suitable boron compounds for concentration in tumors, were unknown in 1936, and Locher's concept was to remain prophetic until nuclear reactors were available to support an initial experimental test with thermal neutrons. This initial event did not occur until 1954 when Sweet, Farr et al. ${ }^{[7]}$ attacked human brain tumors (glioblastoma multiforme) using ${ }^{10} \mathrm{~B}$-enriched borate as the ${ }^{10} \mathrm{~B}$ target species in terminal patients at the Brookhaven National Laboratory (BNL) in cooperation with the Massachusetts General Hospital (MGH). These initial experiments will be discussed in Section 3.1. In these first examples,
BNCT was applied to the problem of killing malignant glioma cells that remained at the tumor site following normal surgical procedures.

As expected from its potential medical applications, neutron capture therapy embraces a wide spectrum of scientific disciplines. The research literature pertinent to BNC and BNCT abounds with studies derived from such highly specialized fields as cell biology, nuclear science and engineering, radiation oncology, pathology, pharmacology, immunology, and others, in addition to chemistry. The present article is, however, restricted to topics at the interface of chemistry with these other disciplines, and it is written from the viewpoint of a chemist.

\subsection{Rudimentary Aspects of BNCT}

The correct description of the boron neutron capture reaction obtained with thermal neutrons $(293 \mathrm{~K} \cong 0.025 \mathrm{eV})$ is given in Scheme 2. The ${ }^{11} \mathrm{~B}$ nucleus is incapable of undergo-

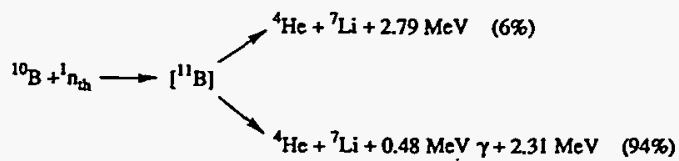

Scheme 2. The two parallel nuclear fission reactions that occur upon capture of a slow (thermal) neutron, $\mathrm{n}_{1,}$, by a ${ }^{30} \mathrm{~B}$ nucleus.

ing a $\mathrm{BNC}$ reaction, whereas the effective nuclear cross-section of ${ }^{10} \mathrm{~B}$ is 3837 barns. Table 1 presents the accepted nuclear cross-sections of the elements having unusually high values, as well as those elements of physiological importance commonly found in tissue. The ${ }^{157} \mathrm{Gd}$ nucleus has been considered as a target for neutron capture therapy due to its very high nuclear cross-section. However, the products of neutron capture are photons and Auger electrons of low energy. If ${ }^{157} \mathrm{Gd}$ can be placed in contact with DNA, double strand breaking may occur upon neutron capture. ${ }^{\left[{ }^{[}\right]}$

Two nuclides abundant in tissue (Table $1 \mathrm{~A}$ ) stand out as participants in important neutron capture side-reactions

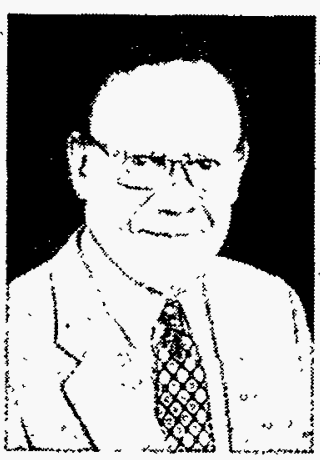

M. Frederick Hawthorne was born in 1928 in the state of Kansas. He entered the Missouri School of Mines and Metallurgy as a chemical engineering student in 1944. In 1947 he transfered to Pomona College and obtained his B.A. degree in 1949. In 1953 he completed his Ph.D. under Donald J. Cram at the University of California at Los Angeles (UCLA). After a year of postdoctoral research with George S. Hammond at Iowa State University, he accepted a position with the Rohm and Haas Co. in Huntsville, Alabama, in 1954, where he began his investigations in boron chemistry two years later. He was appointed Professor at the University of California, Riverside, in 1962. In 1969 he took up his present position as Professor of Chemistry (Inorganic) at UCLA. From that year he has been Editor-in-Chief of Inorganic Chemistry. Among his numerous awards are the American Chemical Society Award in Inorganic Chemistry (1973) and the Distinguished Service in the Advancement of Inorganic Chemistry Award (1988). He is a member of the U.S. National Academy of Sciences and the American Academy of Arts and Sciences. He was awarded an Honorary Ph.D. from Uppsala University in 1992 and is currently an Alexander von Humboldt Senior Scientist associated with Professor Nöth in Münich. He is a member of the Executive Committee of the International Society for Neutron Capture Therapy. His major research themes are centered about the use of boron clusters as building blocks in host-guest and supramolecular complexes, in homogeneous catalysis, as components of nanomechanical assemblies, and in the chemistry of BNCT biodelivery systems. 
Table 1. Neutron capture cross-sections measured with thermal neutrons and natural isotopic abundances of physiologically important elements $(A)$ and elements that display the greatest cross-sections, including ${ }^{10} \mathrm{~B}$ (B) [a]

\begin{tabular}{|c|c|c|c|}
\hline \multicolumn{2}{|c|}{ Nuclide } & Nuclear cross-section [Barn] & $\begin{array}{l}\text { Natural abundance [\%] } \\
99.8\end{array}$ \\
\hline (A) & $\begin{array}{l}{ }^{13} \mathrm{H} \\
{ }^{12} \mathrm{C} \\
{ }^{14} \mathrm{~N} \\
{ }^{23} \mathrm{O} \\
{ }^{31} \mathrm{Na} \\
{ }^{32} \mathrm{~S} \\
{ }^{35} \mathrm{Cl}\end{array}$ & $\begin{array}{l}0.33 \\
3.4 \times 10^{-3} \\
1.8 \\
1.8 \times 10^{-4} \\
0.43 \\
0.18 \\
0.53 \\
43\end{array}$ & $\begin{array}{r}99.8 \\
98.9 \\
99.6 \\
99.8 \\
100.0 \\
100.0 \\
95.0 \\
76.0\end{array}$ \\
\hline (B) & $\begin{array}{l}{ }^{3} \mathrm{He} \\
{ }^{6} \mathrm{Li} \\
{ }^{10} \mathrm{~B} \\
{ }^{113} \mathrm{Cd} \\
{ }^{149} \mathrm{Sm} \\
{ }^{155} \mathrm{Gd} \\
{ }^{857} \mathrm{Gd} \\
{ }^{235 \mathrm{U}}\end{array}$ & $\begin{array}{l}5.3 \times 10^{3} \\
9.4 \times 10^{2} \\
3.8 \times 10^{3} \\
2.0 \times 10^{4} \\
4.2 \times 10^{4} \\
6.1 \times 10^{4} \\
2.6 \times 10^{5} \\
5.8 \times 10^{2}\end{array}$ & $\begin{array}{l}1.4 \times 10^{-4} \\
7.5 \\
19.8 \\
12.2 \\
13.9 \\
14.8 \\
15.7 \\
7.2 \times 10^{-1}\end{array}$ \\
\hline
\end{tabular}

[a] Data taken from G. Friedlander, J. W. Kennedy, E. S. Macias, J. M. Miller, Nuclear and Radlochemistry, 3rd ed., John Wiley, New York, 1981.

that occur during BNCT and thus contribute important doses of background radiation to the subject. These two neutron capture reactions play a role, not because of enhanced nuclear cross-sections of the target nuclei, but because of their very high concentrations in tissue. The neutron capture reactions ${ }^{[96]}$ of these nuclides, ${ }^{1} \mathrm{H}$ and ${ }^{14} \mathrm{~N}$, are detailed in Scheme 3. The passage of a neutron through hydro-

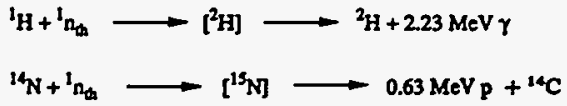

Scheme 3. Neutron capture reactions involving ${ }^{1} \mathrm{H}$ and ${ }^{14} \mathrm{~N}$. Capture by ${ }^{1} \mathrm{H}$ releases high-energy $\gamma$-photons and a deuteron, whereas capture by ${ }^{14} \mathrm{~N}$ yields an energetic proton and ${ }^{14} \mathrm{C}$.

gen-rich media such as tissue results in the slowing and scattering of these neutrons by collisions with nuclei of the $\mathrm{H}$ atoms. Occasionally, a slowly moving neutron will be captured by such a proton to produce a deuteron accompanied by characteristic $\boldsymbol{\gamma}$-radiation, which contributes to the total radiation dose. In another competing capture reaction, the nitrogen atoms available in tissue may capture a lowenergy neutron and generate ${ }^{14} \mathrm{C}$ and a proton whose kinetic energy is $0.63 \mathrm{MeV}$. The kinetic energy imparted to the ${ }^{7} \mathrm{Li}^{3+}$ and ${ }^{4} \mathrm{He}^{2+}$ ions derived from the BNC reaction and that similarly associated with the proton and $\gamma$-photons produced as shown in Scheme 3 is transferred to the surrounding media. Since all of these energetic nuclear reaction products with the exception of the $\gamma$-photons, are heavy particles, this kinetic energy transfer is rapid and takes place along a very short path length. The rate of linear energy transfer (LET) of these particles is characteristically high; the immense energy of these reactions is therefore dissipated in a very small volume. As an example, the ${ }^{7} \mathrm{Li}^{3+}$ and ${ }^{4} \mathrm{He}^{2+}$ ions generated in the BNC reaction leave ionization tracks about $0.01 \mathrm{~mm}$ long or the equivalent of approximately one cell diameter. Thus, the high LET characteristic of particles produced by nuclear reactions that occur within tissue are especially lethal to affected cells because of the high density of deposited energy. Ideally, those cells that carry large numbers of ${ }^{10} \mathrm{~B}$ nuclei are subject to destruction by $\mathrm{BNC}$, while neighboring cells that are free of ${ }^{10} \mathrm{~B}$ would be spared save for the contribution of the background ${ }^{1} \mathrm{H}(\mathrm{n}, \gamma){ }^{2} \mathrm{H}$ and ${ }^{14} \mathrm{~N}(\mathrm{n}, \mathrm{p}){ }^{14} \mathrm{C}$ reactions. In order to achieve this desired effect in BNCT, ${ }^{10} \mathrm{~B}$ delivery should be as selective as possible to tumor tissue and should avoid normal tissue that will be subject to neutron irradiation. In addition, the actual concentration of ${ }^{10} \mathrm{~B}$ in tumor must be sufficiently high to offer a localized binary therapeutic effect well above the background radiation dose delivered by the neutron capture processes of Scheme 3. The minimum ${ }^{10} \mathrm{~B}$ concentration necessary for effective $\mathrm{BNC}$ has been repeatedly calculated and is generally accepted to be between 10 and $30 \mu \mathrm{g}{ }^{10} \mathrm{~B}$ per $\mathrm{g}$ tumor depending upon the precise location of the ${ }^{10} \mathrm{~B}$ with respect to vital components of the tumor cell structure. As the position of the ${ }^{10} \mathrm{~B}$ nuclei is changed from the external cell wall to the cytoplasm to the nucleus of the cell, the required concentration of ${ }^{10} \mathrm{~B}$ for effective BNCT decreases, as expected. Thus, if the ${ }^{10} \mathrm{~B}$ is bound to the cell wall, $30 \mathrm{ppm}$ or greater concentrations might be required, while if ${ }^{10} \mathrm{~B}$ is localized within the nucleus of the tumor cell, a concentration of only $10 \mathrm{ppm}$ or less might be required. Obviously, an additional factor is the steady state concentration of thermal neutrons in the targeted volume of tissue, since very low neutron intensities require proportionally longer irradiation times to produce the required number of BNC events for effective therapy. The quantification of the effective lethality of product particles or $\gamma$-radiation from neutron capture reactions is expressed in terms of their relative biological effectiveness (RBE) relative to $\gamma$-radiation. The RBE of particulate species or photons is expressed as the ratio of the energy dose of $\gamma$-radiation to the energy delivered by the particles or photons in question that accomplishes equivalent cell-killing or physiological damage to living tissue. While the precise values of RBE characteristic of commonly encountered particles and photons depend somewhat upon the conditions of the measurement, the heavy ${ }^{7} \mathrm{Li}^{3+}$ and ${ }^{4} \mathrm{He}^{2+}$ ions that arise from BNC have the commonly accepted (conservative) value of about 2.5 when taken together, (per neutron captured), although much higher values have been suggested. Similarly the products of the ${ }^{14} \mathrm{~N}(\mathrm{n}, \mathrm{p}){ }^{14} \mathrm{C}$ reaction are assigned an RBE of 2.7 . Thermal $(0.025 \mathrm{eV})$ neutrons themselves are relatively harmiess (negligible $\mathrm{RBE}$ ). The ${ }^{1} \mathrm{H}(\mathrm{n}, \gamma)^{2} \mathrm{H}$ reaction mainly contributes the energy of its characteristic $\gamma$-radiation (RBE of approximately 1.0) (Scheme 3). In practice, the total effective radiation dose delivered to a volume of tissue containing a fixed concentration of homogeneously distributed ${ }^{10} \mathrm{~B}$ would be the sum of the energies delivered by each of the independent ${ }^{1} \mathrm{H}(\mathrm{n}, \gamma)^{2} \mathrm{H},{ }^{14} \mathrm{~N}(\mathrm{n}, \mathrm{p}){ }^{14} \mathrm{C}$, and ${ }^{10} \mathrm{~B}(\mathrm{n}, \mathrm{a})^{7} \mathrm{Li}$ reactions after multiplication by their respective $\mathrm{RBE}$ values. The ${ }^{1} \mathrm{H}$ and ${ }^{14} \mathrm{~N}$ capture reactions obviously provide a radiation background upon which is superimposed the ${ }^{10} \mathrm{~B}$ concentration-dependent (binary) BNC reaction.

The discussion thus far has centered upon the use of thermal neutrons as the applied neutron source, since their energy is sufficiently low for immediate nuclear capture to occur. However, the scattering of thermal neutrons as they encounter hydrogen atoms in tissue very seriously diminishes their availability at tumor sites located below the tissue surface. 
Such deeply buried tumors can be addressed with BNCT by applying more energetic neutrons found in the $0.5 \mathrm{eV}$ $1.0 \mathrm{keV}$ region of the epithermal ${ }^{[10]}$ range. Neutrons of this type are not sufficiently energetic to cause destructive ionization in tissue through hydrogen-recoil processes, but they are effectively slowed by continual encounters with $\mathrm{H}$ atoms in the media. These collisions are accompanied by the generation of heat, which is not destructive to tissue. However, as these epithermal neutrons pass through tissue, they will lose sufficient kinetic energy to become thermal neutrons and subject to capture by ${ }^{10} \mathrm{~B},{ }^{14} \mathrm{~N}$, and ${ }^{1} \mathrm{H}$ nuclei. Thus, while directly applied thermal neutrons will give the maximum dose due to capture processes at the point of entry into tissue (zero depth), epithermal neutrons are not sufficiently slowed for effective capture until they have penetrated some distance into tissue. ${ }^{[10]}$ This is the desired condition for noninvasive BNCT of deeply buried tumors.

Fast neutrons $(E>10 \mathrm{keV})$ are capable, through collision, of causing the ionization of hydrogen atoms from hydrogencontaining substrates, and in so doing, they create a severe type of radiation damage (hydrogen-recoil reaction). ${ }^{[1]}$ This process is so biologically disruptive that an RBE as large as 5 has been suggested for fast neutrons. ${ }^{[12 b]}$ Fast neutrons are encountered as impurities in epithermal neutron beams, but they may be reduced in population by neutron beam moderation devices ${ }^{[13 b]}$ including specific band-pass filters. Whenever fast neutrons are present in a neutron beam used in BNCT, the effective radiation dose supplied by hydrogen recoil must be added to the total background dose supplied by the ${ }^{1} \mathrm{H}(\mathrm{n}, \gamma)^{2} \mathrm{H} \gamma$-photons and the ${ }^{14} \mathrm{~N}(\mathrm{n}, \mathrm{p})^{14} \mathrm{C}$ protons.

\subsection{Neutron Sources for BNCT}

\subsubsection{Nuclear Reactors}

As noted in Section 2.1, BNCT was conceptualized before a viable source of neutrons had been discovered. This restriction was lifted following the initial development of nuclear reactor technology in the 1940s, and Farr and Sweet could conduct the first clinical trials of BNCT with a thermal neutron beam in 1954. ${ }^{[7 \mathrm{el}}$ Epithermal beams have replaced ther$\mathrm{mal}$ beams as the neutron source of choice for the reasons presented in Section 2.2 .

It is currently believed that a dose (fluence) of $5 \times 10^{12}$ epithermal neutrons per $\mathrm{cm}^{2}$ will be required ${ }^{[14]}$ for successful BNCT if every epithermal neutron is converted to a thermal neutron. To minimize the duration of patient exposure to the neutron source, reactors not less powerful than one megawatt would be required to achieve a clinically useful flux ${ }^{[14]}$ of at least $10^{9}$ neutrons per $\mathrm{cm}^{2}$ per s. In addition, these epithermal neutron beams must be relatively free of both extraneous $\gamma$-photons and fast neutrons $(E>10 \mathrm{keV})$ and collimated to the greatest extent possible. Potentially, these therapeutic flux requirements could be met, in the United States alone, by approximately thirty-five nuclear reactors ${ }^{[15 b]}$ which could be modified for BNCT. Among the reactors of this type that have already been specifically dedicated for medical research are the Brookhaven Medical Research Reactor, the MIT (Massachusetts Institute of
Technology) Research Reactor, the Georgia Institute of Technology Research Reactor, and the European Collaboration Reactor located at Petten, the Netherlands. The Power Burst Nuclear Reactor Facility located at the Idaho National Engineering Laboratory also has a reactor potentially capable of producing an intense therapeutic neutron beam if it were to be modified for BNCT research and therapy. The potential of epithermal neutron beams for the noninvasive therapy of internal tumors has yet to be fully realized; research efforts now underway promise the availability of such $1-1000 \mathrm{eV}$ neutron beams with intensities of greater than $10^{9}$ neutrons per $\mathrm{cm}^{2}$ per $\mathrm{s}$. The many interdependent variables that come into play in any actual BNCT experiment are the subject of extensive studies carried out by radiation physicists, radiation biologists, and nuclear reactor specialists, to name but a few. The interested reader is referred to the extensive work on computational modeling of BNCT radiation effects coupled with experimental verification exemplified by the pioneering work of Fairchild et al. ${ }^{[16 b]}$ at the Brookhaven National Laboratory, the work of Wheeler and Nigg et al. ${ }^{[17]}$ at the Idaho National Engineering Laboratory, and Harling et al. ${ }^{[18]}$ at MIT.

The fact remains that nuclear reactors are the only sources of thermal and epithermal neutrons available for research and clinical trials today. The limited number of potentially available reactors capable of clinical applications and the immobility of these reactors would limit the general usefulness of BNCT even if efficacious modalities for particular tumor types were developed. Consequently, the search for epithermal neutron beam sources other than nuclear reactors has begun. ${ }^{[19,20]}$

\subsubsection{Neutron Sources Other Than Nuclear Reactors}

The development of BNCT as a generally available therapy depends upon securing a useful, safe, and relatively inexpensive epithermal neutron beam source that can be widely distributed in hospitals and medical centers located in populated urban areas. Research directed to this end is currently in progress. ${ }^{[19-21]}$

Collision of low energy protons, produced in an accelerator, with a lithuim target will produce epithermal neutrons. To secure epithermal neutrons for BNCT in this manner, proton currents would necessarily be high, and lithium target cooling problems would be severe. In addition, the high energy neutrons produced would require thermal moderation with materials such as $\mathrm{D}_{2} \mathrm{O}$. In an alternative procedure, the bombardment of heavy elements with high energy protons provides an array of neutrons whose energy distribution is generally richer in high energy neutrons than that observed in nuclear fission. These very energetic neutrons may be brought into the desired epithermal region through the use of moderating materials.

The synthetic nuclide, californium-252, is the only practical spontaneous neutron-emitter. ${ }^{[14,22]}$ This isotope has a half-life of 2.65 years. The neutron spectrum produced by ${ }^{252} \mathrm{Cf}$ decay is similar to that produced by a one megawatt nuclear reactor. Since it isotropically emits $2.3 \times$ $10^{6}$ neutrons per sec per $\mu \mathrm{g}$, large (gram) quantities of this nuclide would be required to obtain the desired neutron 
beam strength following thermal moderation and collimation, etc. The continual synthesis of large quantities of ${ }^{252} \mathrm{Cf}$ to fulfill the world's need of epithermal neutrons for BNCT would be a technically formidable and very expensive task, and, at the same time, would create a new set of international problems in nuclear safety and security.

The available sources of epithermal neutrons for BNCT based on the proton accelerator are now well understood in principle, and remaining problems are in the realm of engineering. Hopefully, these obstacles can be overcome, so that safe, economically viable neutron beams can become established in a variety of clinical settings.

\subsection{Modeling BNCT for Clinical Applications}

The interaction of the components of a specific neutron beam with the ${ }^{10} \mathrm{~B}$ nuclei present in tumor, the normal tissues near the tumor, and the blood raise questions that must be answered before a complete BNCT system can be employed in actual therapy experiments. These questions concern the spatial distribution and effective dose (physical dose in rads $\times \mathrm{RBE}=$ effective dose in rads or cGys) of each of the component radiation processes that came into play during the experiment and procedural matters such as choosing between a single-dose therapy mode versus the delivery of the required radiation dose in increments ${ }^{[10]}$ (fractionation) with intervening time alotted for repair of radiation damage arising from low LET processes, principally $\gamma$-photon interactions. Since epithermal neutron beams are most desired because of their deeper tissue penetration, one must be able to quantify the effective dose contributed by the processes of Scheme 3 ( $\gamma$-dose and nitrogen capture), fast neutron beam contaminant (H-recoil dose), and the ${ }^{10} \mathrm{~B}(\mathrm{n}, \alpha)^{7} \mathrm{Li}$ capture reactions in both tumor $\left({ }^{10} \mathrm{~B}\right.$ tumor capture) and normal tissues $\left({ }^{10} \mathrm{~B}\right.$ tissue capture) as a function of depth and radial distance from the hypothetical neutron beam centerline. ${ }^{[10,23]}$ Precision is required in these calculations to avoid the delivery of a total effective dose to normal tissue that surpasses its ability to survive. The total effective dose that can be safely delivered to innocent tissues during tumor therapy limits the total effective dose that may be given to tumor. Blood and the vascularity associated with its distribution within the radiation volume present additional problems, which are now under scrutiny. As a general premise, one wishes to minimize the ${ }^{10} \mathrm{~B}$ content of blood during therapy, and a high ${ }^{10} \mathrm{~B}$ (tumor) $/{ }^{10} \mathrm{~B}$ (blood) concentration ratio is desired. However, in certain circumstances a tumor/blood concentration ratio for ${ }^{10} \mathrm{~B}$ of unity is acceptable. ${ }^{[24]}$ It is beyond the scope of this article to develop the reasons for, and the consequences of, these observations.

In order to approach the generalized problem of quantifying the effective radiation doses delivered to each point in the irradiated volume comprising normal tissues and tumor, use has been made of very refined microdosimetry measurements within phantom tissues during actual neutron beam irradiation. As an example, brain tumors (gliomas) have been popular subjects for study and consequently phantom human (and dog) heads have been constructed from acrylic polymers and provided with instruments for the determination of the effective doses from each of the various radiation sources encountered during neutron beam irradiation. ${ }^{[25]}$ Due to the unique nature of each neutron beam employed, this procedure must be repeated for each beam in order to thoroughly characterize the flux and characteristics of its neutrons as well as the dose rate arising from indigenous $\gamma$-photon and fast neutron beam contaminants. After the complete characterization of the neutron beam and its interaction with tissue through the phantom procedure, one then employs these data using Monte Carlo methods for the description of neutron transport. ${ }^{[26]}$ The resulting calculation of the therapeutic performance of defined concentrations of ${ }^{10} \mathrm{~B}$ placed at various points within the phantom simulates the selective accretion of ${ }^{10} \mathrm{~B}$ by tumor. ${ }^{[27]}$ The concentration of ${ }^{10} \mathrm{~B}$ simultaneously delivered to normal tissues within the radiation volume is usually assumed to be a definite fraction of the tumor ${ }^{10} \mathrm{~B}$ concentration and to be homogenously distributed. Thus, a boron compound or boronated delivery system that provided a tumor/normal tissue selectivity factor of 10 would be assumed to be homogeneously distributed in normal tissue with a concentration of $2.5 \mu \mathrm{g}$ ${ }^{10} \mathrm{~B}$ per $\mathrm{g}$ tissue if the simultaneous tumor concentration were $25 \mu \mathrm{g}{ }^{10} \mathrm{~B}$ per $\mathrm{g}$. The performance of the therapeutic system is time-dependent, since each boron delivery system will have a characteristic biological half-life, and the concentration of ${ }^{10} \mathrm{~B}$ in tumor, tissue, and blood will decrease with time.

Figure 1 is a graphical representation of the results of a typical BNCT simulation calculation supported by microdosimetry data obtained with a phantom human head. ${ }^{[28]}$ The epithermal beam employed here is typical of those obtained from large multimegawatt reactors. Fast neutrons are present in the beam giving a significant $\mathrm{H}$-recoil dose, while the beam is relatively free of significant $\gamma$-photon contamination. The beam port is $10 \mathrm{~cm}$ square. The hypothetical boron delivery system described above is used in the calculations; the concentration of ${ }^{10} \mathrm{~B}$ in tumor and in tissue are 25 and $2.5 \mu \mathrm{g}{ }^{10} \mathrm{~B}$ per $\mathrm{g}$, respectively. The boron is assumed to be homogeneously distributed within both tumor and normal tissue. Blood is not treated as a separate tissue, but it is included in the normal tissue category. Recently, sophisticated treatments of expected damage rendered to the epithelial cells of the cranial capillaries due to the presence of ${ }^{10} \mathrm{~B}$ in blood have been advanced. ${ }^{[24]}$ The interesting result obtained is that sufficiently small capillaries with diameters similar to the $0.05 \mathrm{~mm}$ length of the ${ }^{10} \mathrm{~B}$ fission fragment track are spared, because the effective ${ }^{10} \mathrm{~B}$ concentration within them is reduced by their geometry and the simultaneous presence of the blood-brain barrier, which prevents the migration of the ${ }^{10} \mathrm{~B}$-containing species to positions behind the capillary endothelial cells. This effect can be incorporated into estimations of effective dose ( $\mathrm{rads} \times \mathrm{RBE}$ ) to normal tissue by using a reduced value for the RBE of the ${ }^{10} \mathrm{~B}(\mathrm{n}, \alpha)^{7} \mathrm{Li}$ reaction in normal brain tissue that is 0.33 rather than 2.5. The higher value of the RBE was used in the construction of Figure 1, which represents the worst possible case for glioma therapy, but fairly approximates the results to be expected in the therapy of tumors located outside the cranium.

Curve $\mathrm{C}$ in Figure 1 (total effective dose rate to tumor) clearly shows a maximum at a depth of $2 \mathrm{~cm}$. Normal tissue would also receive a maximum total effective dose rate near 


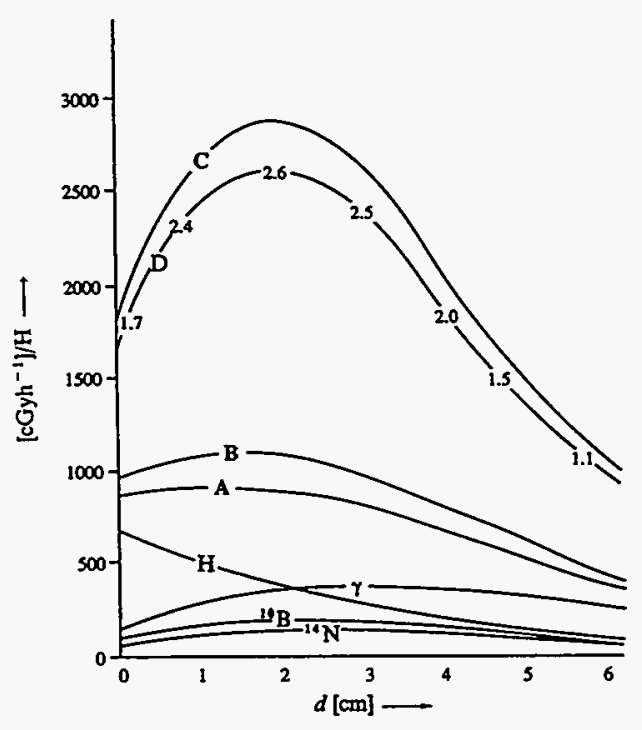

Fig. 1. Graphical display of the relative effective dose rate $I$ of component energy imputs as a function of depth in tissue $d$, viewed on the centerline of a hypothetical epithermal neutron beam that impinges upon a human head. RBE values employed in these calculations were as follows: ${ }^{10} \mathrm{~B}(\mathrm{n}, \alpha)^{7} \mathrm{Li} 2.5$, ${ }^{14} \mathrm{~N}(\mathrm{n}, \mathrm{p}){ }^{14} \mathrm{C} 2.7, \mathrm{H}$ (H-recoil from fast neutron-beam impurity) 5.0, and $\gamma$ photons contributed by ${ }^{1} \mathrm{H}(\mathrm{n}, \gamma)^{2} \mathrm{H},{ }^{10} \mathrm{~B}(\mathrm{n}, \alpha)^{1} \mathrm{~L} \mathrm{i}$, and beam impurity photons i.0. The curves labeled ${ }^{14} \mathrm{~N}, \gamma$, and $\mathrm{H}$ refer to the contributions of the individual processes to the background radiation dose rate as a function of depth in tissue. The curve labeled ${ }^{10} \mathrm{~B}$ is the contribution from $2.5 \dot{\mu} \mathrm{g}$ of ${ }^{10} \mathrm{~B}$ per gram of tissue homogeneously distributed throughout normal tissue. Curve $A$ is the sum of curves ${ }^{14} \mathrm{~N}, \gamma$, and $\mathrm{H}$, while in curve $\mathrm{B}$ the dose rate provided by ${ }^{10} \mathrm{~B}$ in normal tissue (Curve ${ }^{10} \mathrm{~B}$ ) is added. Curve $\mathrm{C}$ is the sum of the dose rate provided by $25 \mu \mathrm{g}$ of ${ }^{10} \mathrm{~B}$ per $\mathrm{g}$ of tissue homogeneously distributed in tumor and the background dose rate of curve $A$. The therapeutic gain (curve D) at any depth is obtained by dividing the dose rate of curve $C$ by the maximum of curve $B$ $\left(1100 \mathrm{cGyh}^{-t}\right)$. See the discussion for details.

$2 \mathrm{~cm}$ in depth; thus, the total effective dose rate of approximately $1100 \mathrm{cGy} \mathrm{h}^{-1}$ in normal tissue would deliver the maximum allowable total effective dose for normal brain of about $1300 \mathrm{cGy}$ (or $13 \mathrm{~Gy}$ ) in approximately 71 minutes if the ${ }^{10} \mathrm{~B}$ concentration remained unchanged throughout this irradiation period. Regardless of the tumor depth, the 71 minute irradiation period and the appropriate total effective dose rate for tumor (Curve $\mathrm{C}$ ) would determine the total effective dose that could be given to the tumor site. For example, this dose is equal to $2200 \mathrm{cGy} \times 71 / 60 \approx 2600 \mathrm{cGy}$ at a tumor depth of $4 \mathrm{~cm}$, while the corresponding value at $2 \mathrm{~cm}$ is 3500 cGy.

The efficacy attained in a particular BNCT experiment is defined as the dimensionless parameter known as therapeutic gain ${ }^{[27 c]}$ and defined as the total effective dose rate available to tumor at a specified depth (Curve $C$ ) divided by the maximum total effective dose rate delivered to normal tissue (Curve B). Thus the therapeutic gain at a depth of $2 \mathrm{~cm}$ is $2900 \mathrm{cGy} \mathrm{h}^{-1} / 1100 \mathrm{cGy} \mathrm{h}^{-1} \approx 2.6$. At a tumor depth of $4 \mathrm{~cm}$ this value is $2200 / 1100 \approx 2.0$. In practice, therapeutic gain values less than 1.2, that is, at a depth greater than $5.5 \mathrm{~cm}$ in the example of Figure 1 (see Curve D), would probably not be considered to be useful. Since effective dose rates and exposure times must be computed prior to therapy, knowledge of the tumor's position must be accurately determined along with the concentration of ${ }^{10} \mathrm{~B}$ in both tumor and nor- mal tissue. These data, when combined with the biological half-life of the ${ }^{10} \mathrm{~B}$ delivery system, permit dosimetric calculations to be made to determine the length of the neutron radiation period. The safety and convenience of the patient as well as the effect of ${ }^{10} \mathrm{~B}$ loss from the tumor with time suggests that the beam exposure time must be minimized. Thus large reactors are required to provide the necessary optimized flux of greater than $10^{9}$ epithermal neutrons per $\mathrm{cm}^{2}$ per s. Calculations show that a single irradiation of $5 \times 10^{12}$ thermalized ${ }^{[14]}$ epithermal neutrons per $\mathrm{cm}^{2}$ would provide the desired therapeutic dose.

Thermal neutron beams have not been treated in this article, since it is apparent that the analog of Figure 1 would contain total effective dose vs depth curves that have their maxima at the surface and decay rapidly with depth in tissue. While thermal neutron beams comparable in flux to the epithermal beam of Figure 1 provide therapeutic gain values greater than 2 at the surface, this parameter rapidly decreases to the useful limit of 1.2 at about $2 \mathrm{~cm}$ depth. These observations clearly support the need for epithermal neutron beams of high flux derived from a large nuclear reactor or an as yet unavailable proton accelerator discussed above (Section 2.3.2).

It is important at this point to compare the radiation damage mechanism of the ${ }^{10} \mathrm{~B}(\mathrm{n}, \alpha)^{7} \mathrm{Li}$ process with that produced by the more conventional radiation source, $\gamma$-photons. ${ }^{[29]}$ Conventional $\gamma$-photon doses of therapeutic significance result from an average of tens of thousands of $\gamma$-photon-initiated ionization tracks per cell, and stochastic principles do not apply since the $\gamma$-photons are presented to the tumor target cells in a homogeneous manner. On the other hand, BNC provides a therapeutic $10 \mathrm{~Gy}$ dose of about 25 high LET events per cell. Damage resulting from nonlethal doses of $\gamma$-photons is reparable with time, whereas the extreme damage per BNC event is not reparable, since heavy $\alpha$ and ${ }^{7} \mathrm{Li}$ fission products with a high LET are involved. The average number of BNC events per cell during a fixed exposure period will vary with the depth of the cell and the spatial distribution and concentration of ${ }^{10} \mathrm{~B}$ nuclei within cells..$^{\text {[30] }}$ Since the ranges of the $\alpha$ and ${ }^{7} \mathrm{Li}$ fission products are short in tissue and comparable to one cell diameter, the response of individual cells will vary with intra- and intercellular distribution of ${ }^{10} \mathrm{~B}$ and target cell geometry. Consequently, stochastic effects are of great importance in BNCT. If ${ }^{10} \mathrm{~B}$ can be placed in or near a vital cellular component such as the nucleus, the number of $\mathrm{BNC}$ events required for cellular death is greatly reduced. ${ }^{10} \mathrm{~B}$ that resides in the intercellular volume or on external cell walls is much less effective. Thus, the most highly prized boron compounds and associated delivery systems are those that enter the cell and bind to intracellular components. Furthermore, the visualization of boron within individual cells has great importance as an aid to boron compound design.

In addition to the heterogeneous nature of the ${ }^{10} \mathrm{~B}$ distribution within and immediately surrounding individual tumor cells, additional heterogeneity introduced by the actual tumor morphology must be recognized. Large tumors are often characterized by a necrotic center that is poorly nourished surrounded by a vasculature that is capable of supplying nutrients for rapid peripheral tumor growth. This peripheral array of blood vessels is pressed into service as rapidiy 
as it can be created. As a consequence of this type of tumor growth and morphology, considerable macroscopic heterogeneity exists within the BNC target volume. The resulting gross inhomogeneity of ${ }^{10} \mathrm{~B}$ distribution must be quantitatively evaluated and taken into account during therapy experiments and in the in vivo evaluation of new boron compounds and their associated delivery systems.

\subsection{Methods of Analysis for Boron Pertinent to BNCT}

Analytical methods for boron required for BNCT research, boron compound evaluation, and eventual clinical applications span a broad spectrum of useful and some not yet perfected procedures. These include the macroscopic and noninvasive estimation of ${ }^{10} \mathrm{~B}$ concentrations in tumor and surrounding tissue with ${ }^{10} \mathrm{~B}$ magnetic resonance imaging (MRI), which is under development, and the microscopic visualization of ${ }^{10} \mathrm{~B}$ localization in tumor and tissue sections with $\alpha$-track autoradiography. Intracellular boron distribution and quantification is another subject that has begun to yield important results. These and other related subjects are discussed in the following sections.

\subsubsection{Noninvasive Macroscopic Estimation of ${ }^{10} \mathrm{~B}$ Concentrations In Vivo}

As pointed out above, safe and effective BNCT requires knowledge of the tumor's location, the concentrations of ${ }^{10} \mathrm{~B}$ in tumor, blood, and normal tissues, and the rate at which the ${ }^{10} \mathrm{~B}$-containing species is eliminated from these biological compartments. Noninvasive methods for both tumor visualization and the determination of ${ }^{10} \mathrm{~B}$ concentrations will be required. In principle, a chemically stable radiolabel having suitable characteristics for imaging and attached to a small fraction of the ${ }^{10} \mathrm{~B}$ compound in question ${ }^{[31]}$ could be employed, coupled with planar imaging as well as single photon emission computer tomography (SPECT) to visualize the compound in both primary and secondary metastatic tumors. ${ }^{[32]}$ However, each double-labeled ${ }^{10} \mathrm{~B}$ delivery system introduces complications and time constraints that are best eliminated.

Very recent research has explored the potential capability of NMR-active ${ }^{11} \mathrm{~B}$ and ${ }^{10} \mathrm{~B}$ nuclei in MRI and in the determination of the boron content of tumors loaded with the appropriate isotope. ${ }^{1} \mathrm{H}$ NMR imaging would be employed as an adjunctive modality. Continued development of this technique has achieved some success ${ }^{[33-36]}$ with the more responsive ${ }^{11} \mathrm{~B}$ nucleus, and the ${ }^{10} \mathrm{~B}$ nucleus may prove to be useful in the near future. It may eventually be possible not only to locate tumors with ${ }^{10} \mathrm{~B} /{ }^{1} \mathrm{H}$ MRI methods, but also to visualize in a quantitative fashion the local ${ }^{10} \mathrm{~B}$ concentrations in tumor and normal tissue. These analytical methods would be noninvasive, relatively rapid, and involve the ${ }^{10} \mathrm{~B}$ BNCT target nuclei as the signalgenerating species. The reader is referred to the original literature ${ }^{[33-36]}$ for the details of this developing technology.

\subsubsection{Methods for the Macro and Micro Determination} of Boron in Biological Samples

The need exists to determine accurately the concentration of boron present in biological research samples taken at biopsy and tissues obtained from animal biodistribution experiments. Such samples are often available in macroscopic quantities of 50 to $100 \mathrm{mg}$ or more. Following the oxidative dissolution of weighed tissue samples, boron analysis of the aqueous sample is accomplished with the newly developed, inductively coupled or direct current plasma-atomic emission spectroscopy (ICP-AES ${ }^{[37]}$ and DCP-AES, ${ }^{[38]}$ respectively) methods. These AES methods determine boron in tissue reliably at levels of $1 \mathrm{ppm}$. Extension of this method to microscopic tissue samples of $1 \mathrm{mg}$ obtained by needle biopsy is feasible with a modified sample preparation procedure.

An alternative to ICP-AES and DCP-AES for the analysis of biological samples utilizes the quantification of the prompt $\gamma$-photons emitted during thermal neutron capture by the ${ }^{10} \mathrm{~B}$ present in the sample, ${ }^{[39-41]}$ and comparison of the result with calibration measurements. This method requires a source of thermal neutrons and lacks the precision at low ${ }^{10} \mathrm{~B}$ concentrations characteristic of the plasma-AES methods.

\subsubsection{The Visualization and Microdosimetry of Boron in Biological Specimens}

The discussion of the stochastic nature of the inter- and intracellular BNC processes in Section 2.4 pointed up the need for experimental methods capable of providing the spatial distribution of boron in tumor and surrounding tissue sections taken from experimental animals or humans at biopsy. The inhomogeneous structure of tumors makes such studies vital to the understanding of the effectiveness of candidate boron-containing compounds in reaching individual tumor cells in the tumor structure. The $\alpha$-track autoradiography method ${ }^{[42,43]}$ is ideally suited for this purpose. The procedure involves the placement of very thin $(6-10 \mu \mathrm{m})$ tissue sections upon nitrocellulose sheets followed by exposure of the array to a thermal neutron source. The high LET $\alpha$ and ${ }^{7} \mathrm{Li}$ products of the resulting ${ }^{10} \mathrm{~B}(\mathrm{n}, \alpha)^{7} \mathrm{Li}$ reaction that are generated within the tissue sample sensitize the nitrocellulose for hydrolytic attack by strong aqueous base. Because of the limited track range of these particles and their high LET characteristics, individual neutron capture events are recorded and converted into visible tracks on the base-developed nitrocellulose film. Superposition of this track data upon that obtained from conventional optical representations of the same tissue section gives the relative density of ${ }^{10} \mathrm{~B}$ nuclei with respect to the histology of the sample. By using calibration samples, this method can also be employed to obtain the ${ }^{10} \mathrm{~B}$ concentration in tissue from track density data at ${ }^{10} \mathrm{~B}$ concentrations as low as $1 \mu \mathrm{g}$ per $\mathrm{g}$ sample. ${ }^{[44]}$

In addition to conventional histological samples taken from tumors, the $\alpha$-track radiographic method has often been applied to whole-body cross sections of experimental mice and rats. 


\subsubsection{Techniques for Imaging Boron on a Subcellular Scale}

The design of new boron-containing drugs and the elucidation of their associated tumor localization mechanisms could best be served if the movement and relative concentration of the drug within the targeted tumor cells could be followed in time-course experiments. The importance of such a capability to drug design derives from the fact that the most effective drugs will be those with the ability to enter tumor cells and bind with the cytoplasm, organelles, or even the nucleus. For example, if one were to synthesize a candidate DNA-binding boron system, it would be important to confirm this proposed intracellular targeting by direct observation of boron within the cell nucleus. At present, methods for such subcellular imaging have demonstrated their usefulness in specific examples and await further development.

The first method referred to here is the secondary-ion mass spectroscopic (SIMS) microscopy method, ${ }^{[45,46]}$ which may be applied to the quantitative determination of boron in single, freeze-fractured and dried, boron-containing cells taken from culture. This method has been applied to the imaging of cells targeted by efficacious boron drugs; it has a spatial resolution of $1 \mu \mathrm{m}$.

The second method is electron energy loss spectroscopic (EELS) imaging, ${ }^{[47]}$ which employs elemental mapping with an analytical electron microscope or an energy-filtering transmission electron microscope. Preliminary results for boron with EELS have been reported, ${ }^{[48]}$ but as in the case of the SIMS method, further development is required before this method of drug evaluation becomes generally useful.

\section{Early Clinical Trials of BNCT and the Related Search for Suitable Boron-Containing Target Species}

\subsection{Initial Clinical Investigations (1951-1962)}

The idea of actually applying Locher's binary BNCT concept $^{[6]}$ to medicine originated ${ }^{[49]}$ with William $\mathrm{H}$. Sweet, neurosurgeon at the Massachusetts General Hospital (MGH), Boston, and L. E. Farr, of the Brookhaven National Laboratory (BNL) Medical Department. Sweet and Farr proposed to improve the prognosis for brain tumor patients by employing BNCT to destroy the fibrils of residual tumor cells, which always remained following radical surgery to remove the accessible tumor. ${ }^{[50,51]}$ This procedure would remove the fibrils as foci for the reestablishment of the tumor. The life expectancy of patients with such malignant gliomas was usually less than six months, even with surgery. The fact that gliomas did not metastasize, the protection provided normal brain by the blood-brain barrier, and the resistance of these malignancies to all known forms of chemo- or radiotherapy made them excellent candidates for BNCT trials.

Earliest thoughts regarding the enhancement of distribution ratios for boron compounds in tumor and brain evolved from the concept that cerebral gliomas were accessible to the blood or "wet" side of the blood-brain barrier and would thus favor the uptake of hydrophilic species, while normal brain was relatively "dry" and less hydrophilic. Therefore, hydrophilic, nontoxic species were sought. ${ }^{\text {[52] }}$

Volunteer patients undergoing neurosurgical biopsy or resection were found to tolerate sodium borate $\left(\mathrm{Na}_{2} \mathrm{~B}_{4} \mathrm{O}_{7}\right)$ in concentrations of up to $200 \mathrm{mg}$ per $\mathrm{kg}$ body weight without severe side effects..$^{[3]}$ Biodistribution and time-course data indicated that shortly after intravenous injection of borate the concentration of boron in tumor was three times that found in normal brain and could be as high as $50 \mu \mathrm{g}$ per $\mathrm{g}$ tumor. Boron was present in blood at concentrations as high, or higher, than those found in tumor.

Initial clinical trials were conducted by Farr and Sweet in 1954 with a thermal neutron beam from the Brookhaven Graphite Research Reactor and ${ }^{10} \mathrm{~B}$-enriched sodium borate on ten patients with glioblastoma multiforme. ${ }^{[54]}$ Five patients received a single radiation dose, and five received multiple fractionated doses. No statistical prolongation of life was achieved. In 1959 the then new Brookhaven Medical Research Reactor was employed to treat sixteen more patients ${ }^{[55,56]}$ with ${ }^{10} \mathrm{~B}$-enriched borates as the target species. As before, no enhancement of survival times was observed, nor was $\alpha$-particle-induced radiation damage observed in patients' brains at autopsy.

In the early 1960 s the MGH/MIT Reactor, complete with a surgery located below a thermal beam port, became operational. Also during the 1950 s and early 1960 s, boron compounds were synthesized by several research groups and evaluated by Soloway et al. ${ }^{[57}$ of the MGH, who used murine models such as implanted ependymoblastomas for biodistribution experiments and time-course experiments. Many of the approximately 140 compounds evaluated by Soloway et al. were substituted phenylboronic acids (including 4-dihydroxyborylphenylalanine (BPA), which is of great interest today (see Section 7.9.1). During this same period the polyhedral borane anions $\mathrm{B}_{10} \mathrm{H}_{20}^{2-[58]}$ and $\mathrm{B}_{12} \mathrm{H}_{12}^{2-[59]}$ were discovered and their derivative chemistry extensively explored. In addition, the icosahedral carboranes ${ }^{[60]}$ closo1,2- and closo-1,7- $\mathrm{C}_{2} \mathrm{~B}_{10} \mathrm{H}_{12}$, were synthesized and derivatives prepared. The unusual kinetic stabilities of these polyhedral ions and carboranes immediately made them and their derivatives attractive candidates as BNCT target compounds. These developments coincided with the beginning of a new area of inorganic cluster chemistry. This subject is more fully described in Section 5.

Soloway evaluated the salt, $\mathrm{Na}_{2} \mathrm{~B}_{10} \mathrm{H}_{10}$, as a potential $B N C T$ target species in animals and humans. Large quantities of $\mathrm{Na}_{2} \mathrm{~B}_{10} \mathrm{H}_{10}$ could be administered by intracarotid injection to humans $\left(\mathrm{LD}_{50} 1.0 \mathrm{~g}\right.$ per $\mathrm{kg}$ in $\mathrm{C} 3 \mathrm{H}$ mice) and later recovered and identified by ${ }^{11} \mathrm{~B}$ NMR in urine specimens. In another experiment with. CD1 Swiss mice ${ }^{[61]}$ (male), the observed $\mathrm{LD}_{50}$ of $\mathrm{Na}_{2} \mathrm{~B}_{12} \mathrm{H}_{12}$ was $1025 \pm 15 \mathrm{mg}$ per $\mathrm{kg}$ body weight. Toxicity of the polyhedral ions was low, whereas the substituted phenylboronic acids often presented toxic reactions in animals.

Two boron compounds in ${ }^{10} \mathrm{~B}$-enriched form, 4-carboxyphenylboronic acid and $\mathrm{Na}_{2} \mathrm{~B}_{10} \mathrm{H}_{10}$ were chosen for a new set of human trials to be carried out at the MGH/MIT Reactor and MGH during 1961 and 1962 . Eighteen patients ${ }^{\text {[62] }}$ were subjected to a tumor "debulking" procedure, which removed extraneous tumor tissue and opened the skull for exposure to the poorly penetrating thermal neutron beam. 
Two of these patients were given $\mathrm{Na}_{2}{ }^{10} \mathrm{~B}_{10} \mathrm{H}_{10}{ }^{[*]}$ by intracarotid injection, while sixteen received ${ }^{10} \mathrm{~B}$-enriched 4-carboxyphenylboronic acid. Patients were irradiated after reopening the craniotomy site. Again, no therapeutically useful effect was observed, and patients died between 10 days and 11.5 months post-irradiation. Neuropathological examination of patients at autopsy indicated radiation necrosis with vascular lesions of different types in the brains of 9 of 14 patients. ${ }^{[62]}$ The cause of these effects was attributed to high concentrations of boron in blood at the time of neutron irradiation. Henceforth, it was thought to be desirable to employ boron reagents that were selectively accreted by tumor to ensure a mininal boron concentration in blood at the time of neutron irradiation. A second generation of candidate compounds were synthesized both by Soloway and by workers at the Du Pont Central Research Laboratory with this objective. For the most part these compounds were designed to function as tumoristatic alkylating agents or compounds capable of other covalent-bond-forming reactions with protein, and were based upon polyhedral borane anion $^{[58.59]}$ and carborane ${ }^{[60]}$ structures. The reader is referred to Sections 5.0 and 7.1 for a fuller description of these species. Of the compounds evaluated in biodistribution studies by Soloway for selective tumor accretion and effective clearance of the vasculature, the sodium salts of $\mathrm{B}_{12} \mathrm{H}_{11} \mathrm{SH}^{2-}$ and $1,10-\mathrm{B}_{10} \mathrm{Cl}_{8}(\mathrm{SH})_{2}^{2-}$ stood out, ${ }^{[61]}$ but only the former salt was sufficiently nontoxic $\left(\mathrm{LD}_{\mathrm{so}}\right.$ in male $\mathrm{CD} 1$ Swiss mice was $73 \pm 4 \mathrm{mg}$ per $\mathrm{kg}$ body weight) to warrant further study. It was given the acronym BSH.

\subsection{The Emergence of a BNCT in Japan: Hiroshi Hatanaka and $\mathrm{Na}_{2} \mathrm{~B}_{12} \mathrm{H}_{11} \mathrm{SH}$}

After spending several years at the MGH working with the BNCT team headed by Sweet, the neurosurgeon Hiroshi Hatanaka returned to Japan in 1968 and created a new chapter in the BNCT story. Hatanaka initiated a clinical trial with Japanese glioblastoma patients using ${ }^{10} \mathrm{~B}$-enriched $\mathrm{BSH}$ and primitive thermal neutron beams of low intenisty, which were available in the Tokyo area. The Shionogi Research Laboratories, Osaka, supplied $\mathrm{Na}_{2}{ }^{10} \mathrm{~B}_{12} \mathrm{H}_{12} \mathrm{SH}$ for these studies and synthesized the sodium salts of 1- and 2$\mathrm{B}_{10} \mathrm{H}_{9} \mathrm{SH}^{-}$as alternatives (see Section 7.1).

The procedure developed in Japan was similar to that employed by Sweet in the $1961 / 1962$ clinical trials. Patients with tumors near the periphery of the brain underwent a craniotomy followed by surgical debulking of the tumor mass. One to two weeks following this procedure the patients were given an intracarotid infusion of ${ }^{10} \mathrm{~B}$-enriched $\mathrm{BSH}$ at dosages between 30 and $80 \mathrm{mg}$ per $\mathrm{kg}$ body weight delivered over one to two hours. Approximately twelve hours later, the cranium was opened and the surgical site exposed for thermal neutron irradiation. Only reactors of low flux were available $\left(10^{9}\right.$ neutrons per $\mathrm{cm}^{2}$ per $\left.\mathrm{sec}\right)$, and irradiation times were of the order of three to five hours on a patient under remotely controlled anesthesia. Protection of the scalp was necessary to prevent radiation damage, since skin con-

[०] ${ }^{10} \mathrm{~B}$ in constitutional formulas is used here to indicate that the compound contains $95-96 \%{ }^{10}$ B. See Section 5.1 . tained a moderate concentration of ${ }^{10} \mathrm{~B}$. Tumor samples taken at the time of irradiation indicated the presence of 13 to $60 \mu \mathrm{g}{ }^{10} \mathrm{~B}$ per $\mathrm{g}$ tumor. The boron concentration in normal brain was not measured. ${ }^{[63]}$ Because thermal neutrons were necessarily employed in these studies, penetration of the beam in tissue was less than $6 \mathrm{~cm}$ due to neutron scattering and attenuation.

Hatanaka's work has been published and widely discussed. ${ }^{[63-66]}$ Noteworthy is the fact that no necrosis of the normal brain was observed, except in one atypical patient, who received an extraordinarily large neutron dose. This suggests that BSH was cleared from the blood before neutron irradiation commenced. However, recent work by Gavin et al. ${ }^{[24]}$ suggests that the small diameter of the capillary vessels in normal brain coupled with the intact blood-brain barrier at all points other than tumor interfaces is the criterion for the blood-vessel-sparing mechanism, which effectively reduces the apparent boron concentration in blood. BSH is a small hydrophilic anion that does not cross the bloodbrain barrier; its apparent inability to do so must also be related to its moderately strong complexation with high molecular weight proteins present in blood serum ${ }^{[67]}$ that also do not penetrate the blood-brain barrier. This process was discussed above in Section 2.4. In general, the patients of Hatanaka fared well considering the deadly nature of their disease. ${ }^{[14]}$ Of thirty-eight patients with Grade III and IV glioblastoma multiforme who were treated and twelve with tumors in the cerebral mantle less than $6 \mathrm{~cm}$ from the cortical surface, the reported mean survival was 44 months, and the median was about 26 months. Among them are several long term survivors: a man now 65 years old is alive and unaffected twenty years later; a 70-year-old woman and a 13-yearold girl also appear to be completely cured.

Hatanaka's results are remarkable, but they have stimulated some criticism within the medical community. The questions raised are centered about the consistency of data collection regarding patients and procedures, and the poor performance of the neutron beams that had to be employed. One must realize that Hatanaka demonstrated the efficacy of $\mathrm{BNC}$ in glioma therapy under conditions that were far from ideal. It is clear that thorough documentation could have been obtained if greater resources had been available.

The demonstration that BSH could be successfully applied as a target compound in the BNCT of glioblastoma multiforme has stimulated an international effort with this species as the centerpiece. Research groups located in the EC countries and others associated with the Idaho National Engineering Laboratory have initiated detailed pharmacokinetic studies of BSH with the aim of elucidating the mechanism responsible for its accretion in cerebral gliomas. Other studies pertain to the use of BSH in neutron irradiation experiments with large animals and its pharmacokinetics in humans. Clinical trials are being prepared in which the European consortium will employ the Petten reactor with BSH.

One complication in the use of BSH in therapy is the ease with which air-oxidation, perhaps catalyzed by traces of redox-active metal ions, takes place to form the disulfide dimer $\left[\mathrm{B}_{12} \mathrm{H}_{11} \mathrm{~S}\right]_{2}^{4-}$ and the corresponding oxodisulfide. ${ }^{[68.69]}$ Animal experiments have shown the former ion to be superior to $\mathrm{BSH}$ in tumor accretion, ${ }^{[70]}$ but much more 
toxic. A blue, reactive radical is observed in acidified aqueous solutions of the disulfide ion. ${ }^{[68.69]}$ These oxidation reactions may be necessary in vivo to establish the presumed covalent coupling of BSH to cysteine residues. The reader is referred to Section 7.1 for futher information. These same reactions require tedious purification of BSH used for drug purposes. However, at the present time BSH is the only acceptable boron target compound that could be used today in clinical trials in Europe or the USA. As we shall see, other suitable target species are under rapid development, but BSH will probably remain as the baseline compound for clinical studies conducted in the immediate future.

\section{Chemical Intermezzo}

With the exception of certain of Hatanaka's patients, BNCT has thus far not proven to be an outright success. Aside from the rationale that relates the probable success of BNCT to the level of development of radiation oncology, the characteristics of available neutron beams, or the sophistication of dosimetry, the key to success was, and remains, the availability of nontoxic boron target compounds that selectively accumulate in neoplasms as opposed to normal tissues. The synthetic chemist encounters a major problem in the logical development of these useful neutron target species: the paucity of quantitative and even qualitative information pertaining to the chemical and biological differences between malignant and normal cells. Such information is vital, since these differences identify possible pathways for the selective uptake and retention of boron by tumor cells. However, two important areas of existing clinical practice make use of such tumor cell differentiation: chemotherapy and the radioimmunoimaging of tumors. Chemotherapeutic drugs are evaluated for their ability to kill cancer cells selectively without great concern for the concentration differences between malignant and neighboring normal tissues, since the effective differentiation between cancerous and normal cells is based upon disparate cell reproduction and metabolism rates. The precision of radioimmunoimaging techniques now available is possible, because radiopharmaceuticals, through their attachment to monoclonal antibodies, can differentiate between normal and tumor cells with such exquisite selectivity that high tumor-to-normal-cell concentration ratios are attained while the actual concentration of the radiopharmaceutical in tumor is miniscule.

Idealiy practiced, BNCT requires even more demanding characteristics: the selectivity for tumor displayed by immunomediated radiopharmaceuticals coupled with concentrations of tumor-bound boron drugs greater than those used in chemotherapy and persisting in tumor for a period sufficient for effective neutron irradiation to be safely accomplished. Toxicity issues must also be controlled. Thus, the initial challenges to the chemist are: 1) identificaton of biodelivery (bioutilization) pathways that effectively differentiate between the targeted tumor and other tissues, and 2) design and synthesis of boron compounds that may be coupled to these biodelivery systems without serious compromise of biodelivery performance, but that maintain at least the lowest practical concentration of ${ }^{10} \mathrm{~B}$ in tumor required for therapy (approximately $10^{9}$ target atoms per cell).
In addition, boron must accumulate in tumor, while its concentration in blood is simultaneously minimized. If these challenges can be met, the use of the blood-brain barrier to augment tumor cell differentiation is no longer necessary, and BNCT can be increased in scope to include malignancies other than cerebral gliomas. Thus, tumors such as melanoma, lymphoma, adenoma, sarcoma, and others, as well as their metastases, become candidate diseases for BNCT with epithermal neutron sources.

\section{The Timely Discovery of Modern Borane Chemistry}

The development of BNCT research beyond the level of effort seen in the 1950s is due to the discovery of new boron chemistry in the late 1950 s and early 1960 s that exceeded the greatest hopes of the chemists involved with the earliest clinical trials of BNCT. The discovery of whole new families of polyhedral borane derivatives that were stabilized by three-dimensional electron delocalization, and also capable of being incorporated into organic molecules as substituents, allowed the concept of BNCT to survive the early clinical trials that were conducted with inappropriate boron compounds and to progress to its present promising status.

These first aromatic polyhedral borane species to be discovered were the predicted icosahedral closo $-\mathrm{B}_{12} \mathrm{H}_{12}^{2-}$ ion ${ }^{[59]}$ and the unpredicted closo- $\mathrm{B}_{10} \mathrm{H}_{10}^{2-}$ ion ${ }^{[58.71]}$ having the structure of a bicapped, square Archimedian antiprism. These ions were soon followed by the icosahedral closo$\mathrm{C}_{2} \mathrm{~B}_{10} \mathrm{H}_{12}$ carborane isomers, ${ }^{[72,73]}$ which are isoelectronic and isostructural with the closo- $\mathrm{B}_{12} \mathrm{H}_{12}^{2-}$ ions. Other polyhedral anions of the general formula closo- $\mathrm{B}_{n} \mathrm{H}_{n}^{2-}$ were discovered, and the entire family with $n=6$ through 12 became available. ${ }^{[74]}$ Similarly, other closo- $\mathrm{C}_{2} \mathrm{~B}_{n-2} \mathrm{H}_{\mathrm{a}}$ carboranes, likewise with $n=6$ through 12, were synthesized. ${ }^{[7]}$ These carborane molecules reacted with organic molecules, and isomers could be generated by movement of the polyhedral carbon atoms ${ }^{[72,75]}$ relative to each other over the polyhedral surface (polytopal rearrangements). Later research uncovered the corresponding closo- $\mathrm{CB}_{n-1} \mathrm{H}_{n}^{-}$carborane series, ${ }^{[76,77]}$ whose members also form organic derivatives. Metallacarboranes in which one or more $\mathrm{BH}$ vertices of carboranes is replaced by a metal vertex were soon discovered, ${ }^{[78,79]}$ as were the corresponding metallaboranes. ${ }^{[80]}$ The familial relationships of these and many other borane cluster derivatives have been integrated with chemical bond theory ${ }^{[81]}$ and served as a generalized basis for the evolution of inorganic cluster chemistry ${ }^{[82]}$ as a whole. The scope of modern boron chemistry extends far beyond the boundaries suggested here, and the interested reader is referred to specialized reviews of the many aspects of borane cluster chemistry. ${ }^{[83.84]}$ These seminal discoveries in inorganic and organometallic chemistry have made the search for effective BNCT compounds a feasible proposition, since these borane cluster compounds offered high boron content, excellent kinetic stabilities, and relatively simple derivativization for incorporation in organic molecules. Scheme 4 outlines several major features of borane cluster interconversions and some numbering conventions. 


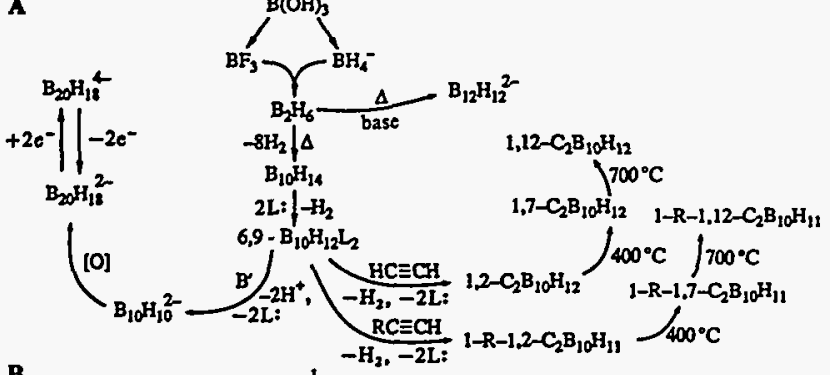

B
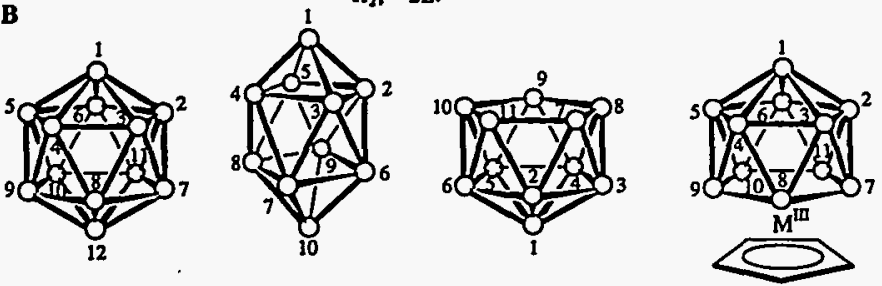

claso- $\mathrm{B}_{12} \mathrm{H}_{12}^{2}$ claso $\mathrm{CB}_{1}, \mathrm{H}_{12}^{-}$ claso- $\mathrm{B}_{10 \mathrm{H}} \mathrm{H}_{10}^{2}$ claso- $\mathrm{CB}_{2} \mathrm{H}_{10}^{-}$ closo- $\mathrm{C}_{2} \mathrm{~B}_{2} \mathrm{H}_{10}$ nedo $-\mathrm{B}_{11} \mathrm{H}_{12}^{4-}$ nido $\mathrm{CB}_{10} \mathrm{H}_{12}^{3-}$ nido- $\mathrm{C}_{2} \mathrm{~B}_{2} \mathrm{H}_{11}^{2-}$ claso- $\mathrm{CpM}^{n \mathrm{~B}_{21}} \mathrm{H}_{12}^{2-}$ claso- $\mathrm{CpM}^{20} \mathrm{CB}_{10} \mathrm{H}_{12}$ claso- $\mathrm{CPM}^{\mathrm{II}} \mathrm{C}_{2} \mathrm{~B}_{3} \mathrm{H}_{14}$

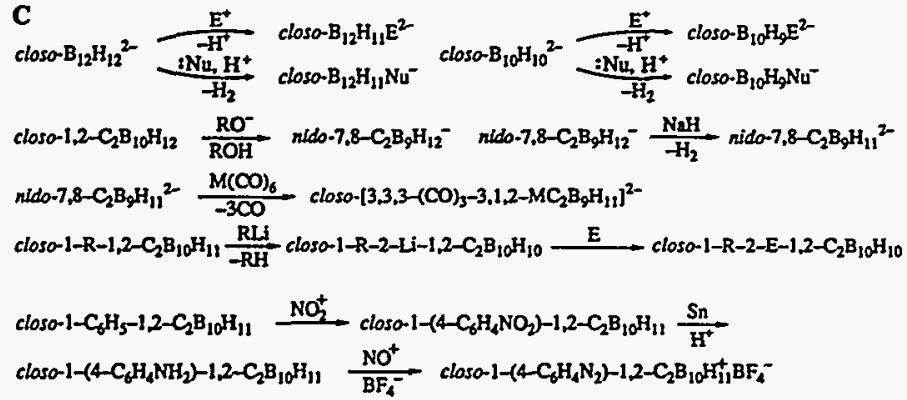

Scheme 4. An abbreviated presentation of some highlights of contemporary boron cluster chemistry of potential importance to BNCT. A: syntheses; B: structures; C: reactions. $\mathrm{B}^{\prime}=$ base, $\mathrm{E}=$ electrophile, $: \mathrm{Nu}=$ nucleophile, L: = ligand.

\subsection{The Synthesis of ${ }^{10} \mathrm{~B}$-Enriched Species Required for BNCT}

Scheme 4 is an abridged depiction of the familial relationship of the most accessible borane and carborane species that have been, or could be, employed in the synthesis of BNCT reagents. To achieve their full potential as ${ }^{10} \mathrm{~B}$ delivery vehicles these building-block molecules must be enriched in the ${ }^{10} \mathrm{~B}$ isotope. Enrichment to $95-96 \%{ }^{10} \mathrm{~B}$ is commonly employed, because the boric acid starting material is commercially available at this level of isotopic purity. In Scheme 4 , diborane, ${ }^{10} \mathrm{~B}_{2} \mathrm{H}_{6}$, is the key intermediate for the synthesis of the three major families of ${ }^{10} \mathrm{~B}$-enriched borane derivatives: ${ }^{10} \mathrm{~B}_{10} \mathrm{H}_{10}^{2-}, 1,2-\mathrm{C}_{2}{ }^{10} \mathrm{~B}_{10} \mathrm{H}_{12}$, and ${ }^{10} \mathrm{~B}_{12} \mathrm{H}_{12}^{2-}$ (see Scheme 4). Since the majority of the species that comprise the armamentarium of BNCT research. may be derived from one of the three precursors described above, the requirement for ${ }^{10} \mathrm{~B}$-enrichment does not present difficulties, but considerable additional costs may arise from the purchase of commercially available ${ }^{10} \mathrm{~B}$-enriched compounds. The need for ${ }^{10} \mathrm{~B}$-enrichment should be considered in the design of synthesis routes for new compounds.

\section{Classes of Boron Compounds and Bioutilization Pathways of Current Interest}

Although it may be possible to administer boron-containing target species to animals and humans by oral or perilesional pathways, the majority of boron biodistribution data are collected following intravenous injection of the experimental compound. The diagram presented in Scheme 5

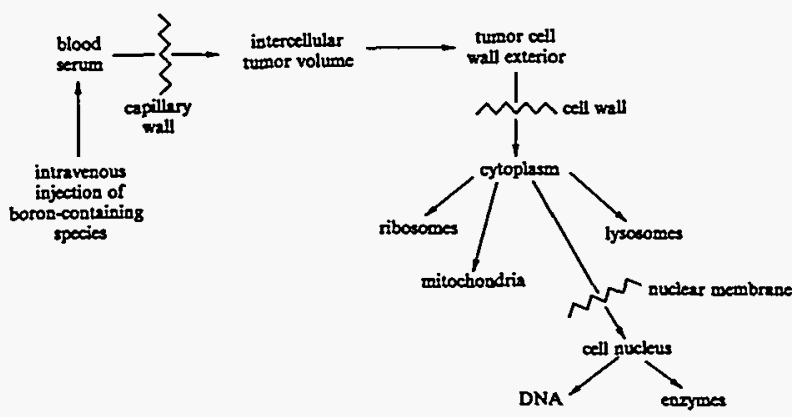

Scheme 5. A greatly simplified representation of the transport pathway required to bring a boron-containing species from the point of intravenous injection to the interior of a cell nucleus. 
traces the possible pathway of a hypothetical boron-containing species as it approaches, binds, and later enters a tumor cell. As previously pointed out, the lethal potential of a ${ }^{10} \mathrm{~B}$ nucleus increases markedly after entering the cell, and again after approaching the nucleus. Nuclear DNA is the target of greatest value, because in this case, only a single high LET neutron capture event within the DNA would be lethal to the cell.

The earliest attempts to correlate molecular structure and biodistribution of boron compounds ${ }^{[57]}$ were concerned with cerebral gliomas, normal brain tissue, blood, and the influence of the blood-brain barrier. Now that tumors other than those related to the brain are under investigation as BNCT subjects, a wider variety of tumor morphological and metabolic features are considered in the development of new boron carriers.

Carrier species under investigation today for the delivery of boron to some particular locus of the targeted tumor cell may have been designed to enter a specific bioutilization pathway as a mimic of a known cellular constituent or metabolite. Other molecules, exemplified by monoclonal antibodies and their fragments, are selectively bound to tumorcell receptor sites in the plasma membrane that are either overexpressed or uniquely expressed by tumor cells as opposed to normal cells. Such receptor-mediated molecules may be conjugated with boron-containing reagents and converted into very selective boron carriers. Small vesicles such as synthetic liposomes or modified low-density lipoproteins (LDLs), while not of molecular dimensions, may be employed as boron-carriers of a different type. Finally, some simple molecules such as porphyrins have proved to be useful for accumulation of boron in tumor (also termed boron localization), although the biological basis of this utility has not yet been clearly defined. Scheme 6 matches currently important boron-containing compounds with the biosynthesis of specific cellular components or the utilization of their characteristic receptors for BNCT. These items are found in the extracellular volume, the cell wall, the intracellular volume, and the cell nucleus.

\subsection{A Brief Overview of Biodelivery Schemes}

Molecular mimicry is employed in the design of compounds that may serve as boron-containing substitutes for molecules normally used in cell construction and metabolism. Species of this type have been employed, or proposed for use, in cellular phospholipid ${ }^{[85]}$ and protein synthesis. ${ }^{[86]}$ In addition, L-dopa surrogates ${ }^{[87]}$ are under investigation for incorporation in melanin synthesis by melanotic melanoma cells.

Recent advances in nucleic acid chemistry have stimulated efforts to incorporate boron-containing base molecules, nucleosides, and nucleotides in target cell DNA or its precursors during biosynthesis. ${ }^{[88]}$ The interaction of boron-containing "antisense" nucleotides and DNA ligands ${ }^{[89]}$ with existing nuclear DNA molecules provides related avenues to the radiologically vulnerable nucleus.

Specialized molecules that, after attachment of boroncontaining entities, bind with specific receptor sites of the tumor cell wall are also assumed, for the most part, to be endocytosed by the tumor cell. In some examples the receptor site is regenerated. This selective boron internalization process has broad scope and presents the capability of bringing a particular type of tumor cell under either successive or simultaneous attack by more than one type of receptorspecific boron carrier. An example would be two or more immunoglobulins targeted for different antigens which are

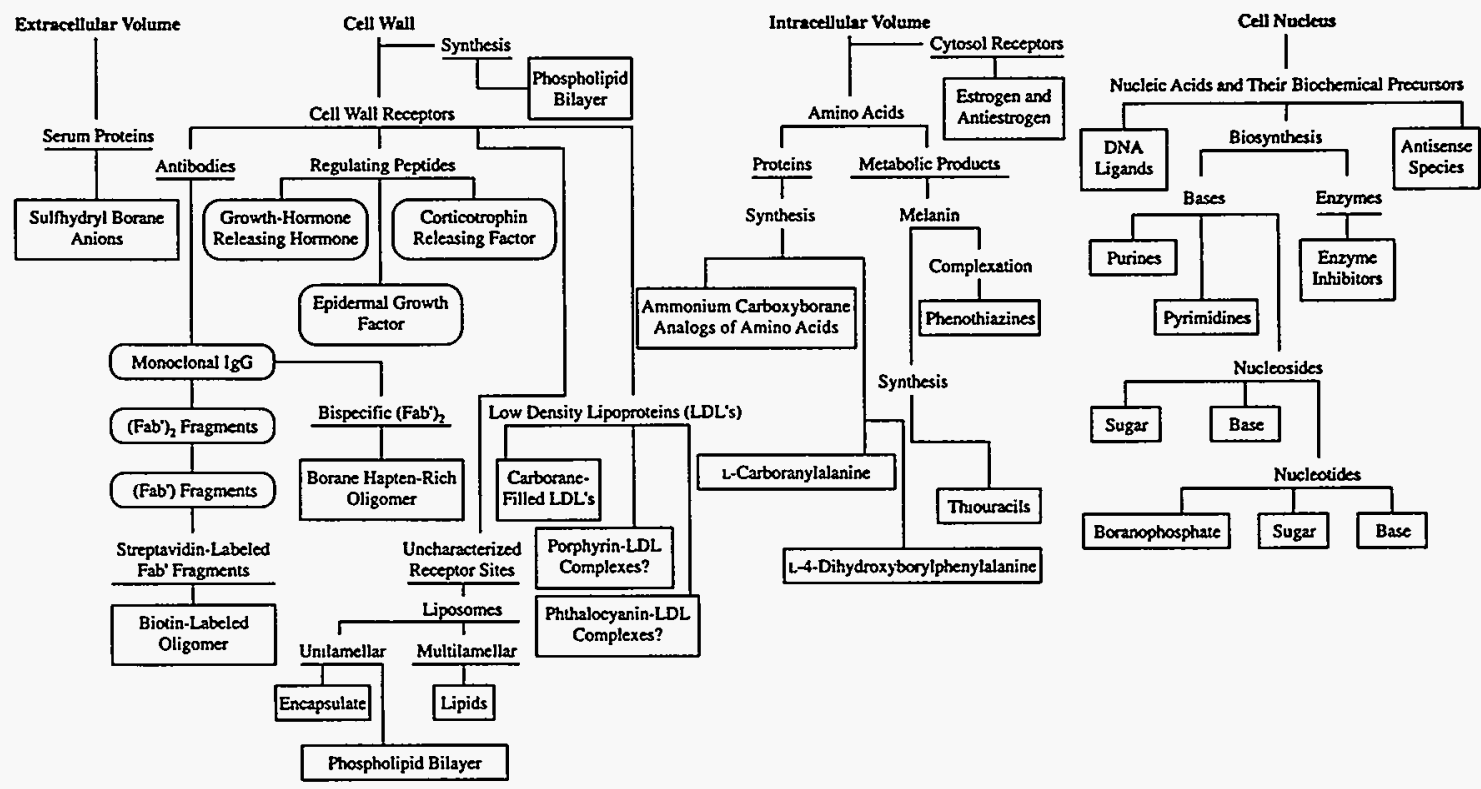

Scheme 6. A classification of boron-delivery vehicles based upon their method of incorporation into one of four biological compartments; extracellular volume, the cell wall, intracellular volume, and the cell nucleus. These assignments are subject to change as further progress is made. $\square$ : boron-containing species; 0 : conjugate of a boron-containing reagent. 
simultaneously expressed by the same tumor cell. The multiple attack, in principle, amplifies the effective concentration of ${ }^{10} \mathrm{~B}$ nuclei associated with specific cell types.

The requirement of $10^{9} \mathrm{~B}$ atoms per targeted cell for effective BNCT is difficult, but not impossible, to achieve by using individual molecules as the carrier species. However, organized aggregates of boron-rich molecules may enter delivery mechanisms specific to tumor cells and bring relatively huge quantities of boron to targeted cells with each delivery event. Such "supertanker" systems known at present are unilamellar liposomes ${ }^{[90]}$ containing polyhedral borane anion solutes within the liposome's aqueous core and reconstituted LDLs in which the natural cholesteryl esters have been replaced with hydrophobic esters derived from carborane. ${ }^{[91]}$ The reconstituted LDLs retain their ability to bind LDL receptor sites of tumor cells and become internalized through the still-functional apoprotein B-100 attached to the original LDL. Cell differentiation is observed, since compared to normal cells LDL receptor sites are extravagantly expressed on growing tumor cells. While the precise mechanisms responsible for selective liposome localization are presently unknown, receptor sites amenable to liposome binding and internalization must surely exist, since it is possible to observe fluorescent species ${ }^{\text {[92] }}$ within tumor cells following their in vivo delivery by liposomes. Furthermore, the results obtained with boron-containing liposomes are understandable only if liposomes are selectively internalized by tumor cells. The liposome cell differentiation process is thought to be based upon the immature ad hoc vasculature unique to rapidly growing tumors. The capillaries present in such tumors will be permeable to very small liposomes ( $<80 \mathrm{~nm}$ diameter), as observed, and allow their selective leakage into the tumor bed. ${ }^{[93]}$ This size selection process may be amplified by more rapid endocytosis of liposomes bound to tumor cells than of liposomes bound to normal cells.

The porphyrin macrocycle is capable of highly selective incorporation into tumor cells in very high concentrations. Although a specific receptor-driven process appears to be responsible for these results, as in the case of liposomes, no discrete receptor sites have been identified. It is possible, however, that the LDL receptor sites serve porphyrin endocytosis through initial hydrophobic binding of the porphyrin derivatives in LDL vesicles and incorporation of the resulting LDL-porphyrin host-guest complex in the usual fashion. ${ }^{[94]}$

\section{Boron-Containing Species and Their Delivery to Tumor Cells}

Specific classes of boron-containing molecules and delivery vehicles are described below organized according to their targeted locations within the tumor cell in accord with Scheme 6. Interestingly, the progression of boron compound development actually follows the organization of Scheme 6, proceeding sequentially from left to right. Thus, as time passes chemists are brought ever closer to the tumor-cell nucleus and increasingly sophisticated chemistry. This is possible due to continued development of borane cluster chemistry and its close alliance with organic and bioorganic chemistry.

\subsection{Species Localized in the Extracellular Volume}

As pointed out in Sections 3.1 and 3.2, the serious consideration of BNCT for tumors other than cerebral gliomas is of relatively recent origin. The presence of the blood-brain barrier, which protects normal brain tissue from the entry of very hydrophilic species and which is degraded at the tumor site, provided a mechanism for tissue differentiation. Nontoxic and very hydrophilic boron compounds were sought, which would be localized in useful amounts in tumor. ${ }^{[57]}$ With the level of sophistication of boron chemistry and related analytical methods of the early 1950 s, no consideration could be given to the design of boron compounds specifically targeted for intracellular localization in tumor cells or other such niceties. In fact, the class of boron compounds most readily available at the time were the series of arylboronic acids evaluated by Soloway. ${ }^{[57]}$ From a large number of candidates, ${ }^{10} \mathrm{~B}$-enriched 4-carboxyphenyl boronic acid was selected for use in clinical trials of 1959/1961 with terminal cancer patients, and DL-4-(dihydroxyboryl)phenylalanine (BPA), which is of current interest, ${ }^{[87]}$ was set aside. In general, the relatively high toxicity and low boron content of the arylboronic acids make them less attractive than compounds that were to appear following the discovery of the boron chemistry described in Sections 3.1 and 5.0.

The characteristics of the $\mathrm{B}_{10} \mathrm{H}_{10}^{2-}$ and $\mathrm{B}_{12} \mathrm{H}_{12}^{2-}$ ions ${ }^{[58,59]}$ suggested that their sodium salts would have the hydrophilic properties then sought for BNCT. Evaluation of these salts in animals proved that they display low toxicity, are incapable of crossing the blood-brain barrier, and have promising characteristics with respect to tumor localization and blood clearance rates. ${ }^{\text {(57) }} \mathrm{The} \mathrm{Na}_{2} \mathrm{~B}_{10} \mathrm{H}_{10}$ salt was employed in human trials (Section 3.1). Later work led to the synthesis of the polyhedral borane anion derivatives $\mathrm{B}_{12} \mathrm{H}_{11} \mathrm{SH}^{2-[95,69]}$ and $1,10-\mathrm{B}_{10} \mathrm{Cl}_{8}(\mathrm{SH})_{2} .^{[96]}$ The octachloro derivative proved to be too toxic for use, but the $\mathrm{B}_{12} \mathrm{H}_{12} \mathrm{SH}^{2-}$ ion has gained notoriety as the well-known BSH ion. The thiol function was introduced in order to provide covalent disulfide bonding with thiol groups presented by serum and tumor proteins. It has been established that approximately $60 \%$ of injected $\mathrm{Na}_{2} \mathrm{~B}_{12} \mathrm{H}_{11}$ SH is weakly and noncovalently bound to serum proteins. ${ }^{[67]}$ Neither free nor bound BSH crosses the intact blood brain barrier, although the tumor is accessible. Hatanaka's results in human glioma trials ${ }^{[33]}$ and other results with humans ${ }^{[97]}$ demonstrate that therapeutic quantities of BSH (>20 ppm) are present in tumor, while low concentrations are simultaneously present in normal brain. Blood concentrations of boron are comparable to or higher than simultaneous tumor concentrations.

The thiol derivatives of the borane anions are potentially unstable with respect to air-oxidation. As an example, BSH is easily oxidized to the corresponding disulfide $\mathrm{B}_{12} \mathrm{H}_{11} \mathrm{SSB}_{12} \mathrm{H}_{11}^{4-}$ and its oxide $\mathrm{B}_{12} \mathrm{H}_{11} \mathrm{SS}(\mathrm{O}) \mathrm{B}_{12} \mathrm{H}_{12}^{4-}, 168.691$ which has caused complications in product surety and analyses for its use as a drug. Acidified solutions of the disulfide rapidly turn blue, which indicates the presence of the radical 
anion ${ }^{168 .{ }^{69}} \mathrm{~B}_{12} \mathrm{H}_{11} \dot{\mathrm{S}} \mathrm{H}^{-}$and/or a related radical dianion $\mathrm{B}_{12} \mathrm{H}_{11} \dot{\mathrm{S}}^{2-}$.

The disulfide derived from BSH has been found to be taken up more efficiently than BSH itself by tumors in smail animals. ${ }^{[98]}$ Other in vitro data obtained by Fairchild et al. indicate that the disulfide, like BSH, is not taken up by the cell, but accumulates extracellularly. ${ }^{\text {[99] }}$ Very recently Coderre et al. demonstrated ${ }^{[100]}$ that the disulfide gave physically unrealistic RBE values of 1.1-1.4 for in vitro neutron irradiation experiments with $9 \mathrm{~L}$ gliosarcoma cells. These results suggest that the radiation damage that leads to tumor death is actually extracellular and most likely located in the endothelial cells of the vasculature sustaining tumor metabolism. The precise mechanism of BNCT with BSH is, of course, unknown and will remain so until detailed pharmacokinetic studies are completed. Perhaps BSH becomes bound to the rich tumor vasculature at a fairly rapid rate, but leaves this compartment at a reduced rate as BSH is cleared from blood. At some time an optimal tumor/blood BSH ratio will be reached; neutron irradiation can then be carried out within the time window during which these conditions exist.

Shionogi Research Laboratories, Osaka, have synthesized two isomeric $\mathrm{B}_{10} \mathrm{H}_{10}^{2-}$ derivatives that correspond to BSH: ${ }^{[101]} 1-$ and $2-\mathrm{B}_{10} \mathrm{H}_{9} \mathrm{SH}^{2-}$. Evaluation of these compounds is incomplete, but the $\mathrm{B}_{10} \mathrm{H}_{10}^{2-}$ framework probably offers no real advantage over $B_{12} H_{12}^{2-}$. Scheme 7 presents the synthesis of the $\mathrm{B}_{12} \mathrm{H}_{11} \mathrm{SH}^{2-}$ ion and its more common reactions.

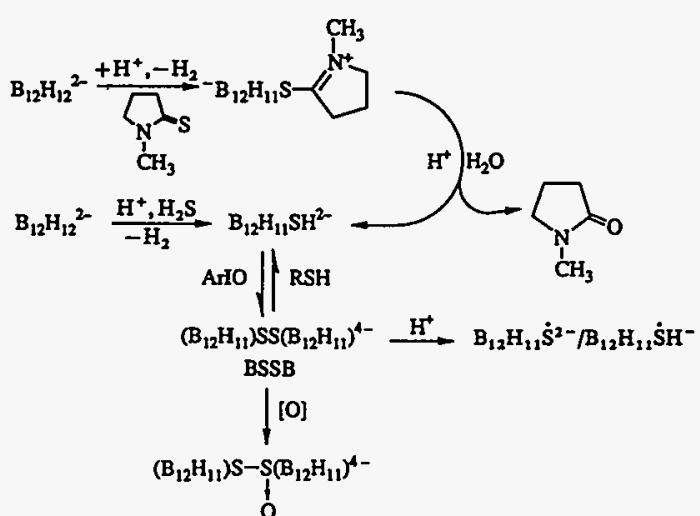

Scheme 7. A summary of $\mathrm{Na}_{2} \mathrm{~B}_{12} \mathrm{H}_{11} \mathrm{SH}$ (BSH) syntheses and reactions. $A r=$ aryl.

\subsection{Boron Localization Processes Utilizing Carborane Derivatives of Phospholipids as Constituents of Tumor Cell Walls}

Scheme 6 suggests that boron can be delivered to tumor cells by mechanisms that selectively introduce boron-containing phospholipids as components of the plasma membrane bilayer of malignant cells. The accelerated rate of cell reproduction in tumor compared to that of normal tissue causes selective accretion of boron in tumor cells.

Carborane derivatives of phosphocholine glyceryl ethers have been proposed as boron carriers, ${ }^{[85]}$ since it has been shown that naturally occuring lipid ethers such as 1 selectively accumulate in the plasma membrane of tumor cells giving attractive tumor/normal-tissue concentration ratios. In fact, $8-18 \%$ of the total phospholipid contained in the plasma membrane of cells grown in vitro can consist of the synthetic phopholipid ether 1. Consequently, Lemmen and Werner ${ }^{[85]}$ have synthesized DL-2 with a 1,2-carboranyl group appended as shown. Results pertaining to the efficacy of 2 have not been reported.

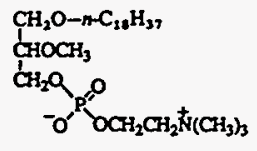

1

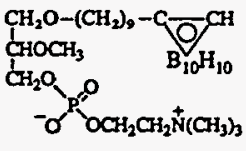

2
7.3. Boron Localization Through Selective Targeting of Antigens of the Tumor Cell Wall with Immunoglobulins

\subsubsection{Boron-Conjugated Antibodies and Their Fragments as Boron Carriers}

As suggested by Scheme 6, tumor cell walls carry a variety of receptor sites (antigens) for the recognition and binding of specific immunoglobulins. The existence of antigens which are specific to certain types of tumor cells or the overexpression of other antigens by tumor cells compared to normal cells allows the selective targeting of antigens in the tumor cell wall with the corresponding antibodies. Bale and Spar ${ }^{\left[{ }^{102]}\right.}$ first pointed out in 1967 that such tumor-targeted antibodies could probably be conjugated with suitable ${ }^{10} \mathrm{~B}$ species and employed to deliver these boron atoms to the corresponding antigen sites of the tumor cell wall. The very selective immunoglobulin would then become the carrier of therapeutic quantities of boron. Since a maximum of about $10^{6}$ antigenic sites of any one idiotype are present on each tumor cell and each malignant cell must carry about $10^{910} \mathrm{~B}$ atoms to achieve therapeutic levels, it is clear that each antigen-bound antibody must carry about $10^{3}{ }^{10} \mathrm{~B}$ atoms. This assumes $^{[103]}$ that the equilibrium constant for antibodyantigen complexation is at least $10^{9} \mathrm{M}^{-1}$. The fact that the binding constants associated with currently available antibodies can be as large as $10^{11}$ is encouraging. In addition, the observed internalization of antibody-antigen complexes on the cell surface offers hope that the boron delivered to the cell surface will actually move toward more effective regions of the cell, thus reducing the high density of boron atoms required if situated on the surface alone. ${ }^{[104]}$ The simultaneous expression of several specific types of potentially useful antigenic sites by tumor cells suggests the possible use of "cocktails" of boron-conjugated antibodies, which would concurrently target several different antigens. This option effectively increases the total number of useful antigenic binding sites, which, in turn, could allow a reduction of the number of ${ }^{10} \mathrm{~B}$ atoms required per antibody molecule.

These immunochemical guidelines could not be achieved until the early 1980s when Milstein and Köhler ${ }^{[105]}$ developed hybridomas as a source of unlimited quantities of chemically homogeneous monoclonal IgG antibodies 
(Mabs). Prior to this development, research workers hoping to employ immunoglobulins as boron delivery vehicles were restricted to the use of chemically heterogeneous polyclonal IgG antibodies tediously isolated from the sera of immunized animals. ${ }^{1061}$ Developments in genetic engineering have led to the essentially unlimited availability of a wide variety of antibody types such as IgG, $\left(\mathrm{Fab}^{\prime}\right)_{2}$, and $\mathrm{Fab}^{\prime}$ species, which may contain large segments of human immunoglobulin as well as customized functional groups for the directed conjugation of drugs, radioisotopes, and toxins. The humanized (chimeric) immunoglobulins offer a reduced risk of illiciting an unwanted immune response when administered to humans. ${ }^{[107]}$ Figure 2 illustrates the general relationship of IgG Mabs and their $\left(\mathrm{Fab}^{\prime}\right)_{2}$ and $\mathrm{Fab}^{\prime}$ fragments, which may be obtained by enzymatic and chemical cleavage of parent IgG Mabs, or most conveniently, from engineered clones.

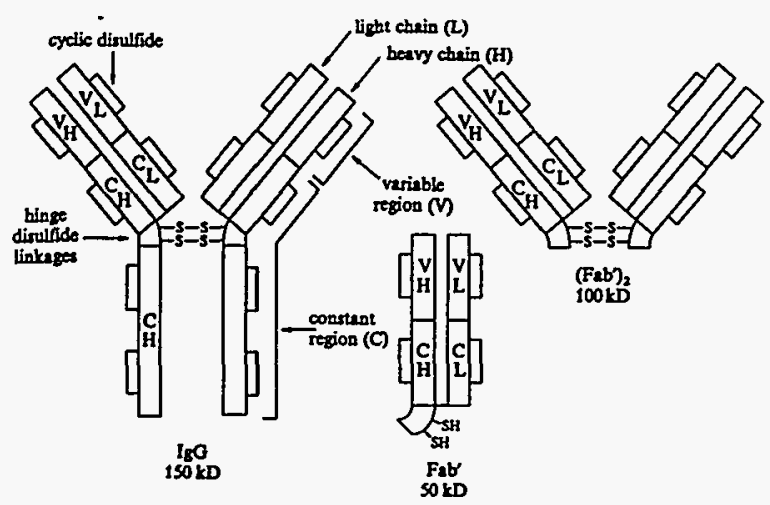

Fig. 2. Simplified representations of the immunoglobulins $\mathrm{IgG}_{4}\left(\mathrm{Fab}^{\prime}\right)_{2}$, and Fab' and their structural relationships. Antigen binding occurs at the terminus of the VH and VL chains in each of the immunoglobulin types represented here. $V_{H}$ and $V_{L}$ chains are joined by $S-S$ bonds represented by lines.

Scheme 8 diagrams the evolution of antibody conjugation and boron delivery methods as they have evolved with time. The initial investigation of boron-conjugation chemistry with model protein substrates such as bovine serum albumin and human $\gamma$-globulin was initiated nearly simultaneously in several laboratories in the 1970s. The initial chemical goal was the synthesis of useful boron-rich reagent molecules that contained nine to ten boron atoms each and displayed good reactivity for pendant $\varepsilon-\mathrm{NH}_{2}$ or $-\mathrm{SH}$ groups of lysine and cysteine residues contained in the antibody structure. ${ }^{[108]}$ Scheme 9 illustrates several representative examples of these early reagents together with others that were developed at a later date. Work of this sort progressed slowly with polyclonal IgG antibodies of various types and large protein molecules such as bovine serum albumin as model substrates. ${ }^{[109]}$
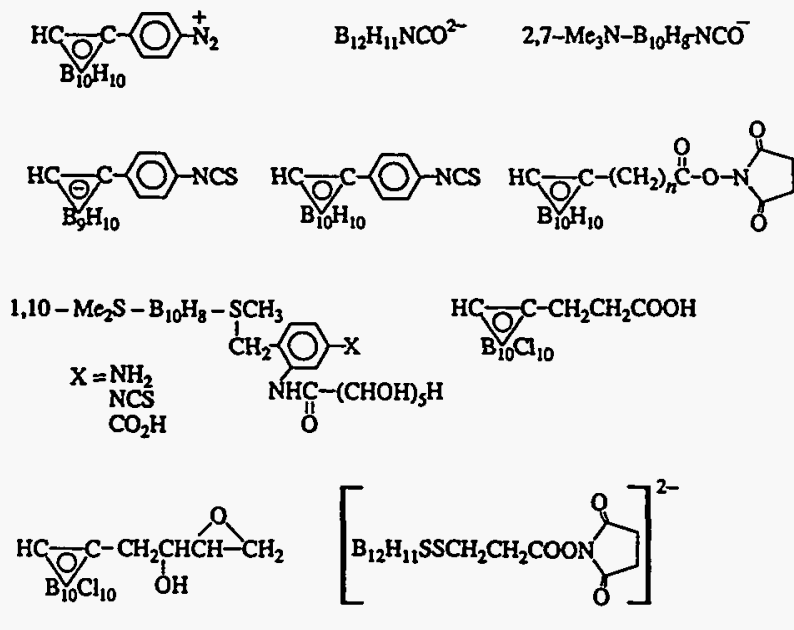

$\mathrm{B}_{12} \mathrm{H}_{11} \mathrm{~S}\left(\mathrm{CH}_{2}\right)_{n} \mathrm{COOH}^{2-}$

Scheme 9. Representative reagents investigated in past studies of immunoglobulin conjugation. Certain of these species are discussed elsewhere in this article. $n=1-4$

The earliest attempt to demonstrate the feasibility of the antibody approach using polyclonal antibodies and simple conjugation reagents such as the diazonium salt 4 derived from 1-(4-aminophenyl)-1,2-carborane ${ }^{[110]}$ (3) were seemingly successful. The diazonium salt 4 was conjugated with polyclonal antibodies directed against both human and murine histocompatibility antigens, and the conjugate employed for in vitro cell-killing of peripheral blood lymphocytes with a thermal neutron beam. Specific, antibody-directed cytotoxicity was observed, although the number of boron atoms attached to each antibody molecule was only of the order of 30 to 50 and these were of natural isotopic abundance $\left(20 \%{ }^{10} \mathrm{~B}\right)$. In another experiment, identical cell-killing ( $30 \%$ survival after two minutes of radia-

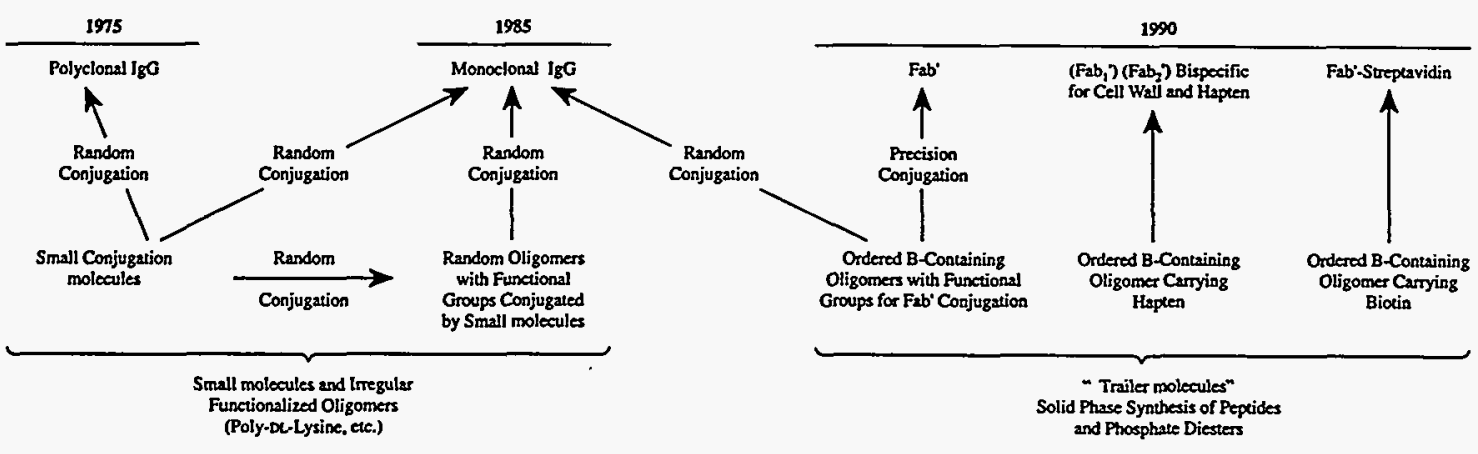

Scheme 8. The evolution of immunoglobulin conjugation methods to the discovery of highly specific bispecific antibodies potentially useful for the delivery of ${ }^{10}{ }_{B}$ to tumor cells without resort to covalent conjugation. 
tion) was observed with lymphocytes in contact with a solution of $\mathrm{K}_{2} \mathrm{~B}_{10} \mathrm{H}_{10}$ containing $2 \mu \mathrm{g}$ of $\mathrm{B}$ per $\mu \mathrm{L}$ or $3.8 \times 10^{-3} \mu \mathrm{g}$ of $\mathrm{B}$ per $\mu \mathrm{L}$ conjugated to $1.9 \mu \mathrm{g}$ antibody per $\mu \mathrm{L}(0.2 \% \mathrm{~B}$ of natural abundance). The efficacy of boron attached to antibody associated with the cell thus appears to be 500 times greater than when present in solution as $\mathrm{K}_{2} \mathrm{~B}_{10} \mathrm{H}_{10}$.

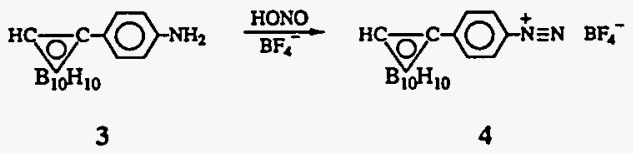

Later the diazonium salt 4 was conjugated with polyclonal caprine antibodies targeted at carcinoembryonic antigen (CEA). The conjugates, which contained up to eighty $B$ atoms per antibody molecule, were employed in both in vitro ${ }^{[1062]}$ and biodistribution studies with human colon tumor xenografts in golden hamsters. ${ }^{[106 \mathrm{~b}]}$ The results of these experiments demonstrated the immunological integrity of the boron conjugates, since good tumor localization was observed in the in vivo experiments. However, the injected dose of antibody conjugate was very low in all of these experiments and the boron content of the conjugate was relatively small. If attempts were made to increase the boron content of the antibody conjugates by increasing the extent of the conjugation reaction, the protein was denatured, precipitated, and lost immunoreactivity. ${ }^{[106.111]}$ In later work, reagents such as the anionic 5 were synthesized, ${ }^{[112]}$ as well as other carborane derivatives of simple sugars ${ }^{(113)}$ (for example 6), in order to enhance the water solubility of the carborane derivative with hydrophilic groups. These attempts were unsuccessful.

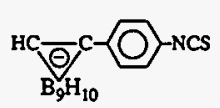

5

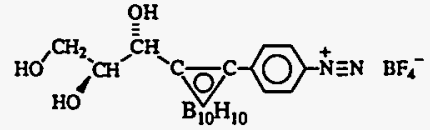

6
The complexity and heterogeneity of highly burdened antibody conjugates of either polyclonal or monoclonal origin led to the adoption of an alternative approach to boron-carrying Mabs having at least $10^{3} \mathrm{~B}$ atoms per molecule. This new tactic recognized that large numbers of boron atoms might be attached to Mabs with minimal alteration of the Mab structure and function by connecting boron-rich oligomer molecules to only a few random sites on the Mab surface. For example, approximately five oligomer molecules carrying 200 boron atoms each might be randomly attached to available $\mathrm{NH}_{2}$ or $\mathrm{SH}$ groups of the $\mathrm{Mab}$ and the resulting mixture of conjugates separated from unreacted Mab. As indicated in Scheme 8, this approach was first investigated in the early 1980 s by several investigators. ${ }^{[14,115]}$ With the exception of the dextran-bridged systems of Gabel, ${ }^{[16]}$ oligomeric boron-rich conjugation reagents were themselves randomly constructed species, prepared by reacting poly-DL-lysine ${ }^{[114]}$ or poly-L-ornithine ${ }^{[115]}$ with reagents

such as DL-7 or 8 . The chain lengths of the resulting conjugated oligomers were not known, and the number of unchanged $\mathrm{NH}_{2}$ groups was variable. Further coupling of oligomers of this sort with Mabs of various idiotypes produced conjugated Mabs with average boron atom populations of $1 \times 10^{3}-2 \times 10^{3}$. Unfortunately, the immunoreactivities of these conjugated Mabs were markedly lower than those of the parent Mabs. Also, time course experiments with mice proved the modified Mabs to be avidly bound to liver, whereas uptake by tumor was diminished. Recent studies with a starburst dendrimer bearing 48 amino groups as a substrate for reaction with DL-7 gave similar results after conjugation with Mab. ${ }^{[117]}$

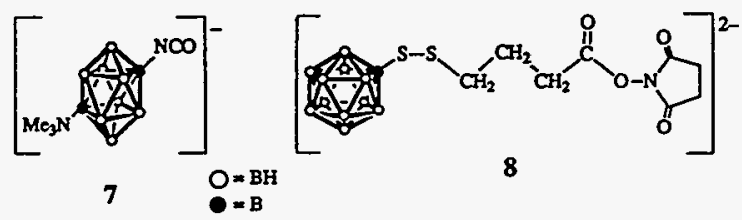

Contemporary with this approach through randomly constructed oligomers, we $e^{[1186.119]}$ began the investigation of precisely synthesized oligomeric peptides ("boron trailers") constructed from boron-rich $\alpha$-amino acids using Merrifield's solid-phase synthesis method. Scheme 10 illustrates the amino acids employed in these studies: ${ }^{[120.121]}$ the hy-

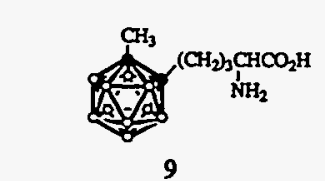

claso cage - very hydrophobic group

C. OBH

Scheme 10. DL-9 and one diastereomer of 10 , the amino acids employed in the synthesis of peptide oligomers used for antibody conjugation.

drophobic closo species 9 and its ambiphilic nido derivative 10. Scheme 11 depicts the synthesis of a fluorescence-labeled undecapeptide 11 from 9 and its conversion to the more hydrophilic structure 12 containing nido 10 residues. A similar fluorescence-labeled nido dipeptide 13 was prepared analogously. Random conjugation of 13 and 11 with anticarcinoembryonic antigen IgG Mab T84.66 through active ester methods produced conjugated $\mathrm{Mab}$ molecules having an average of 63 and 490 boron atoms, respectively. ${ }^{[121]}$ Biodistribution data were collected with nude mice bearing LS $174 \mathrm{~T}$ xenografts and ${ }^{125} \mathrm{I}$-labeled Mab conjugates. While the dipeptide conjugate gave biodistribution results that resembled those obtained with native Mab, the undecapeptide conjugate displayed greatly enhanced liver uptake and decreased tumor accretion. These observations ${ }^{[121]}$ suggested that as the boron loading of the conjugate is increased the loss of circulating conjugate to liver effectively competes with localization in tumor. A smaller immunoglobulin 

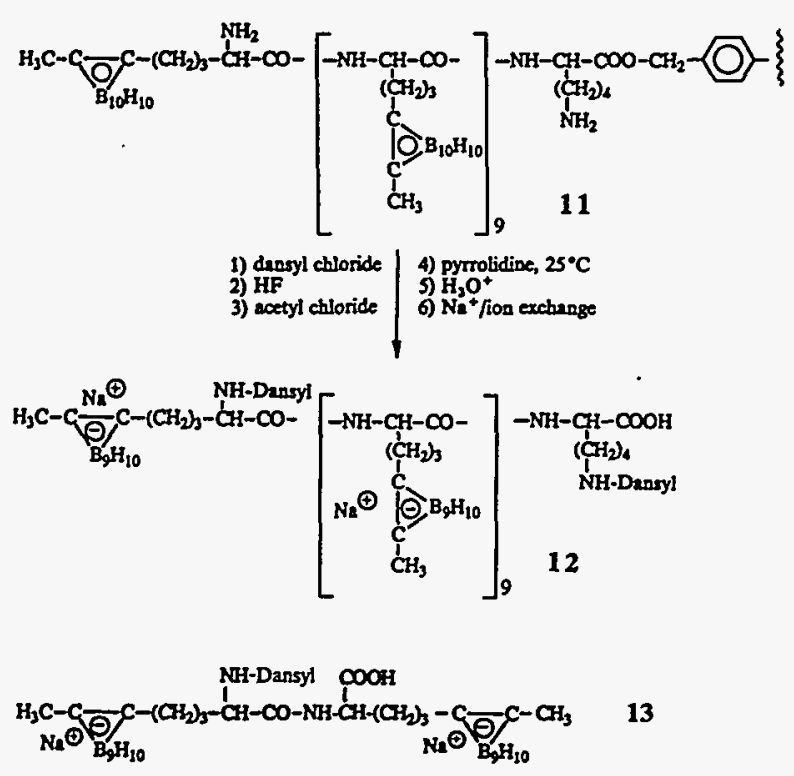

13

Scheme 11. The peptide 11 and its nido derivative 12, prepared by solid-phase synthesis. Peptide 12 was conjugated with antibody, and the conjugate evaluated in biodistribution studies (see Section 7.3.1). In 12 and 13 dansyl (a 5dimethylamino-1-naphthylsulfonyl) can be partly replaced by acetyl.

specifically labeled with a definite small number of hydrophilic oligomer reagent molecules would reduce the heterogeneity and complexity of the conjugate and perhaps lead to selective localization in tumor rather than in liver. ${ }^{[119]}$

The generalized Fab' molecule depicted in Figure 2 represents a class of immunoglobulins that may be obtained from reductive cleavage of the two disulfide linkages in the hinge region of the corresponding $\left(\mathrm{Fab}^{\prime}\right)_{2}$ species or possibly from engineered clones designed to express Fab' or customized variants of its basic structure. The Fab' derived from an IgG Mab having an antigen binding constant of $10^{11} \mathrm{M}^{-1} \mathrm{might}$ exhibit a binding constant of $10^{9}-10^{10} \mathrm{M}^{-1}$ and thus be useful as a carrier molecule. The two SH groups of each Fab'
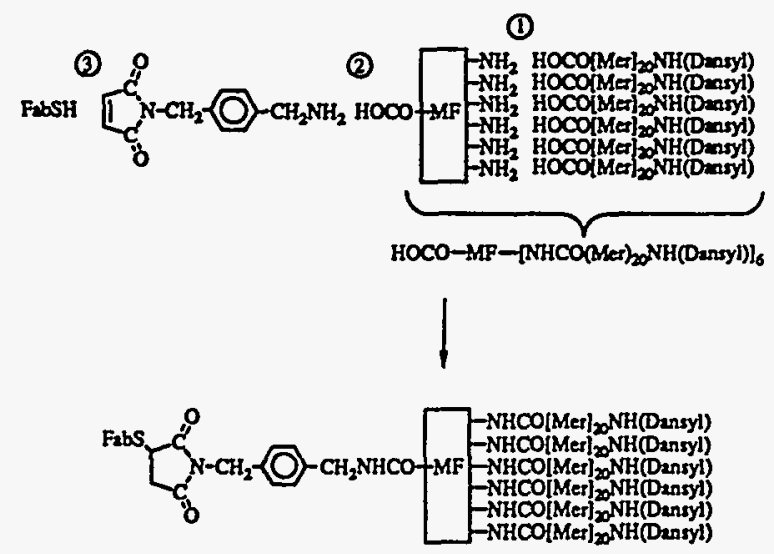

Scheme 12. Schematic representation of the assembly of a conjugate from Fab-SH and a linker molecule, a small lysine oligomer (manifold, MF) and boron-rich oligomers bearing fuorescent dansyl groups. The numerals indicate the order of assembly. Mer = repeating unit. The conjugate contains about $10^{3}$ boron atoms. molecule or the single SH group of a Fab-SH molecule may be employed for the conjugation ${ }^{[19]}$ of arrays of precisely synthesized, boron-rich trailer molecules collected upon small precisely structured poly-L-lysine manifold molecules as shown in Scheme 12. Although the first trailer molecules such as 12 were diastereomeric mixtures of small amphiphilic peptides derived from DL- $\alpha$-amino acids bearing anionic nido-carborane residues and fluorescent marker groups, ${ }^{[19-121]}$ it is possible to assemble oligomers based upon the diphosphate esters of boron-rich diols ${ }^{[122]}$ as shown in Scheme 13. The hydrophilic phosphate ester link-

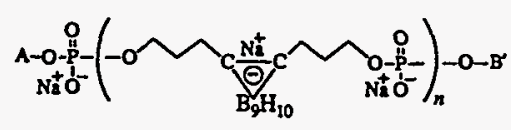

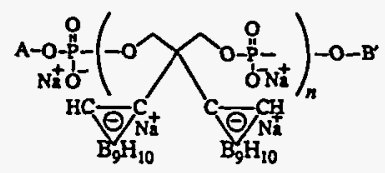

$\mathrm{A}=$ thymidine, $\mathrm{B}^{\prime}=\left(\mathrm{CH}_{2}\right)_{6} \mathrm{NH}_{2}, n=10$

$\mathrm{A}=$ biotio- $\mathrm{C}(\mathrm{O}) \mathrm{NH}\left(\mathrm{CH}_{2}\right)_{6}, \mathrm{~B}^{\prime}=\left(\mathrm{CH}_{2}\right)_{6} \mathrm{NH}_{2}, n=20$ $\mathrm{A}=$ thymidine, $\mathrm{B}^{\prime}=\left[\mathrm{CH}_{2} \mathrm{CH} \text { (nuorescein) } \mathrm{CH}_{2} \mathrm{OP}(\mathrm{O})_{2} \mathrm{O}_{2}\left(\mathrm{CH}_{2}\right)_{6} \mathrm{NH}_{2}\right]^{-}, n=40$ $\mathrm{A}=\left[\text { thymidineOP(O) }{ }_{2} \mathrm{OCH}_{2} \mathrm{CH} \text { (nluorescein) } \mathrm{CH}_{2}\right]^{-}, \mathrm{B}^{\prime}=\left(\mathrm{CH}_{2}\right)_{5} \mathrm{SH}, n=50$

Scheme 13. An illustration of but a few of a myriad of possible structural motifs available through automated oligonucleotide methods for the synthesis of oligomeric boron-containing phosphate diesters.

age combined with anionic nido-carborane residues ensures hydrophilicity of the oligomeric reagents. The attractiveness of phosphate oligomers is further enhanced by their rapid automated synthesis with experimental procedures developed for DNA syntheses coupled with the ease with which UV-absorbent nucleoside residues, fluorescent marker groups, biotin, segments of polynucleotides, and terminal linker groups may be built into the oligomeric structure. ${ }^{[23]}$ In one day it is possible to construct a phosphate trailer reagent, which could, in principle, contain many hundreds of boron atoms and be directly or indirectly linked to the SH functions of a Fab' molecule. ${ }^{[19]}$ Scheme 13 illustrates the structural scope of this new trailer chemistry and suggests a phosphate oligomer for linkage to Fab' species through a suitable linker molecule. Research in this new area of chemistry is being actively pursued.

\subsection{Binding of Boron and Hapten-Rich Oligomers to Cell Wall Antigen with Bispecific Antibodies and Other Host-Guest Interactions}

Bispecific antibodies ${ }^{[124]}$ such as the unsymmetrical $\left(\mathrm{Fab}^{\prime}\right)_{2}$ species, $\left(\mathrm{Fab}_{\text {nted }}^{\prime}\right)\left(\mathrm{Fab}_{\mathrm{CEN}}^{\prime}\right)$, offer the opportunity of binding a cell wall antigen, such as $\mathrm{CEA}$, to a nido-carborane anion hapten group present in high concentration within a boron-rich hydrophilic trailer species. Immunochemical interactions of this sort would allow the concentration of large quantities of boron at cell wall antigenic sites without the use of covalent bonds. In practice the bispecific antibody would 
be injected intravenously into the experimental tumor-bearing animal. Complexation of the Fab ${ }_{C E A}^{\prime}$ segment of the bispecific antibody with CEA antigen sites ensues, the blood is cleared of excess circulating antibody, and then the boronand hapten-rich trailer species is injected. Trailer-haptenFab', binding occurs, and the selective (antigen$\left.\mathrm{Fab}_{\mathrm{CEN}}^{\prime}\right)\left(\mathrm{Fab}_{\text {ntao }}^{\prime}\right.$-hapten-trailer) bond formation process is completed. Scheme 14 illustrates the completed antigen complex. The boron-rich trailer molecule could contain an anionic nido-carborane-hapten group in each repeating unit and at least $10^{3}$ boron atoms per trailer.

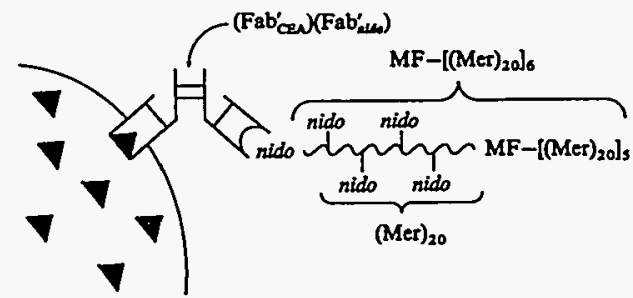

Scheme 14. The schematic representation of the use of a bispecific antibody

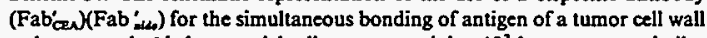
to boron- and nido-hapten-rich oligomer containing $10^{3}$ boron atoms, similar to the manifold shown in Scheme 12.

The synthesis of a bispecific antibody of the type described here ultimately requires a source of the $\mathrm{Fab}_{\text {nido }}^{\prime}$ fragment such as the parent $\mathrm{IgG}_{\text {nido }}$. Such a Mab is now available from a murine hybridoma. ${ }^{[251}$ Scheme 15 illustrates the synthesis route for the bispecific antibody. Alternatively, this antibody might also be available from quadroma sources formed by gene fusion of available anti-CEA Mab and $\operatorname{IgG}_{n i a}$ hybridomas. $^{[126]}$

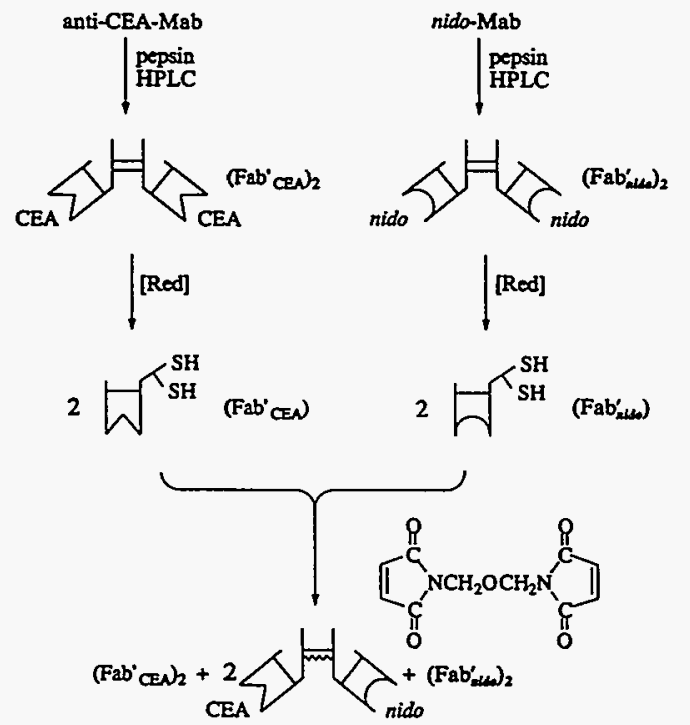

Scheme 15. The synthesis of a bispecific antibody from its IgG precursor molecules, anti-CEA-Mab and $I_{g} G_{m a n}-M a b$. Such a bispecific system is also illustrated in Scheme 14. [Red] = reduction.
A variant of the bispecific antibody concept is the application of the tightly associated streptavidin-(biotin) ${ }_{4}$ complex. ${ }^{[127]}$ In this instance Fab $^{\prime}{ }_{C E A}$ is conjugated with two streptavidin molecules (one per SH group), and the experimental tumor-bearing animal injected intravenously. Following accretion of the Fab' ${ }_{\mathrm{CEA}}$ at tumor and blood clearance, a boron-rich trailer species bearing a terminal biotin residue is administered in the same fashion. Ensuing in vivo complex formation would localize the boron-rich trailer molecule at antigenic sites occupied by $\mathrm{Fab}_{\mathrm{CEA}}^{\prime}$. Recent progress toward the immunological approach to boron localization at tumors suggests that such delivery methods may eventually be useful with some tumor types. The use of antibody "cocktails" comprising mixtures of immunoglobulin carriers, each directed to a different antigen present on the same tumor cell, may be employed to enhance tumor boron concentrations. Difficulties such as tumor heterogeneity and uneven vasculaturization remain, but immunotransport methods may be combined with other completely different BNCT protocols.

\subsection{Receptor-Mediated Bioregulatory Peptides as Boron Transport Vehicles}

Related to the immunoglobulin carriage of boron to tumor cell antigens is the possible use of selected bioregulatory peptides as transport vehicles. The density of their receptor sites is far higher in tumor cell membranes than in normal cells. ${ }^{[128,129]}$ Although the actual number of such receptors present on each tumor cell may be as low as $10^{4}-10^{5}$, receptors internalize their bound regulatory peptide and are regenerated. In effect, a pumping action exists for peptide internalization, which is itself regulated by the intracellular concentration of peptide. The useful rate of regeneration, coupled with the closer approach of internalized boron to the cell nucleus, lends validity to this boron delivery concept. Peptides such as epidermal growth factor (EGF) ${ }^{[128]}$ and corticotropin-releasing hormone $(\mathrm{CRH})^{[129]}$ are candidate peptides having 53 and 41 amino acid residues, respectively. Both EGF and CRH are under evaluation as receptor-mediated boron delivery vehicles of relatively low molecular weight. EGF receptors are overexpressed by many human tumors found in the lung, breast, bladder, head, and neck. CRH is similarly targeted for pituitary tumors involving corticotrophic cells (Cushings disease). A third peptide of interest is growth hormone releasing hormone (GHRH), which, like $\mathrm{CRH}$, is a pituitary-targeting peptide specific for somatotrophs (acromegaly) and composed of $\mathbf{4 0}$ amino acids. The successful application of CRH and GHRH delivery to BNCT of Cushing's disease and acromegaly would offer noninvasive procedures for these malignancies.

\subsubsection{EGF Conjugation}

Carlsson, Sjöberg et al..$^{[128]}$ and Barth, Carlsson et al. ${ }^{[130]}$ have begun the investigation of EGF conjugation with primary carboranyl amines ${ }^{(131)}$ such as 14 and its anionic nido analog 15 as well as $(R)$ - and $(S)$-carboranylalanine (16). ${ }^{[86]}$ Their conjugation procedure utilizes the coupling of dextran 


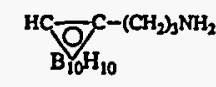

14

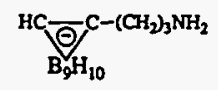

15

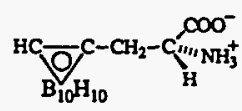

$(R)-16$ with the amino groups present in EGF and the amino groups of the amino carborane derivative, with 1-cyano-4-dimethy!aminopyridinium tetrafluoroborate, as in Scheme 16, and provides relatively small conjugates $(20-30 \mathrm{kD})$. They will be evaluated for boron delivery to glioma cells.
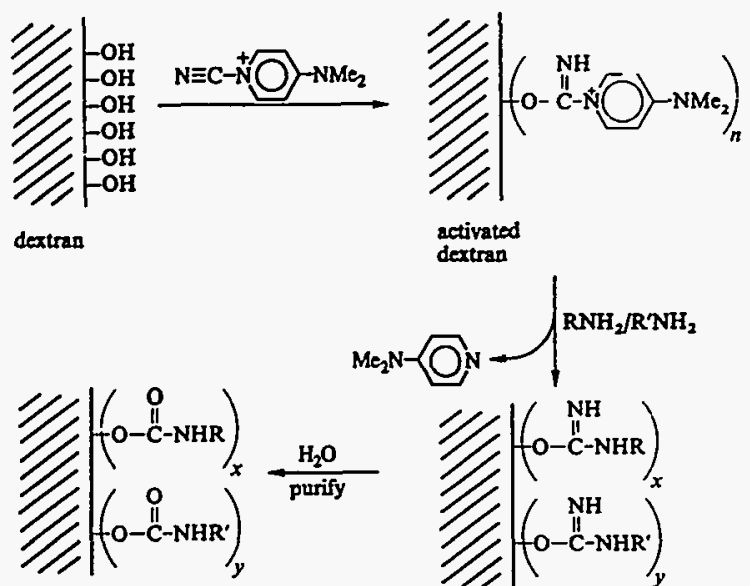

activated<smiles></smiles>

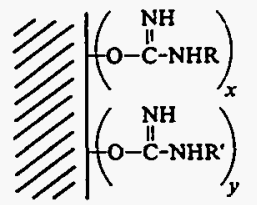

conjugate

Scheme 16. Mechanism of the conjugation of $\operatorname{EGF}\left(\mathrm{RNH}_{2}\right)$ with an aminofunctionalized carborane derivative, $\mathrm{RNH}_{2}$ by means of dextran.

\subsubsection{The Incorporation of Boron in CRH and GHRH Structures}

CRH and GHRH are of similar size, but they differ with respect to their biologically important termini; $\mathrm{CRH}$ may be modified by the addition of an extraneous amino acid to its amino terminus without serious loss of corticotrophic cell activation. ${ }^{[129]}$ GHRH may be similarly modified at its carboxyl terminus without significant loss of biological activity. Loriaux and Albertson et al. ${ }^{[29]}$ have evaluated carboranemodified GHRH and ovine CRH species prepared by Chen et al. ${ }^{[132]} \mathrm{CRH}$ was synthesized with standard Merrifield solid-phase procedures except that the serine amino terminus was acylated with the carboranylacetic acid 17. GHRH

$\underset{\mathrm{B} / 0}{\mathrm{O}} \mathrm{H}_{10}-\mathrm{CH}_{2} \mathrm{COOH}$

was modified at its carboxyl terminus by the addition of a lysine residue whose $\varepsilon$-amino group was acylated with 17 . Both the modified peptides evoked biological responses in vitro similar to that of the unmodified species. ${ }^{[129]}$ Their efficacy of boron delivery remains to be determined. The boron content of the peptides could most reasonably be in- creased by the attachment of small boron trailer molecules rather than 17 . The size, charge, and hydrophobicity of the trailer will require careful selection to retain receptor recognition and binding by the conjugate.

\subsection{Cell-Wall Receptor-Mediated Low-Density Lipoprotein (LDL) Vesicles as Boron Carriers}

The LDL vesicle ${ }^{[133-135]}$ illustrated in Figure 3 and briefly discussed in Section 6.1 comprises a phospholipid/ cholesterol shell with a diameter of approximately $15-$ $20 \mathrm{~nm}$, filled with cholesteryl esters of long-chain alkyl carboxylic acids. Apoprotein B-100 is attached to the external

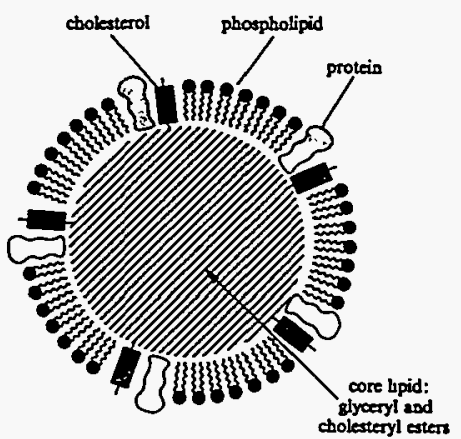

Fig. 3. A stylized cross-sectional view of a low-density lipoprotein vesicle filled with glyceryl and cholesteryl esters of fatty acids. The protein exposed on the vesicle surface responds to the $\mathrm{LDL}$ receptor sites present in the plasma mernbrane of cells.

surface of the vesicle and serves as a specific anchor for receptor sites located on the external cell walls of both malignant and normal cells. Receptor-LDL complexes are internalized by endocytosis, the LDL is degraded to cholesterol by lysosomes, and the receptor returns to the external cell wall. This pathway provides about $90 \%$ of the cholesterol necessary for cellular membrane synthesis. ${ }^{[136]}$ Malignant cells are expected to utilize cholesterol (and LDLs) at a much higher rate than normal cells because of higher replication rates, and therefore to have correspondingly higher receptor turnover rates. This provides a basis for cellular differentiation and the targeting of tumor cells with boron if the cholesteryl esters of the LDL core are replaced with a boronrich material. This concept was proposed by Kahl et al., ${ }^{\text {91.137] }}$ who exploited it through the substitution of esters derived from fatty alcohols and carborane carboxylic acid, such as 18 , for the native cholesteryl esters ${ }^{[138]}$ after removal of the latter material from harvested LDL vesicles by solvent extraction. The reconstituted boron-containing LDL vesicles contained approximately $12 \times 10^{3}$ boron atoms each. ${ }^{[91.137]}$ Later work has shown ${ }^{\text {[91b] }}$ that simple hydrophobic carborane derivatives such as 1-hexyl (19) or 2 propenyl (20) derivatives efficiently reconstitute LDL vesicles. In vitro evaluation of these novel boron carriers on CHO and V-79 Chinese hamster cells in conjunction with thermal neutron irradiation at the Brookhaven Medical Research Reactor established that boron was taken up intracellularly in concentrations as high as $240 \mu \mathrm{g}$ boron per $\mathrm{g}$ cells. ${ }^{[12]}$ This will be recognized as a spectacular result! 


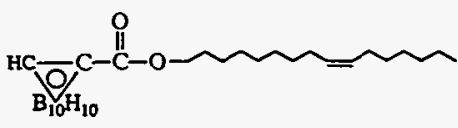

18

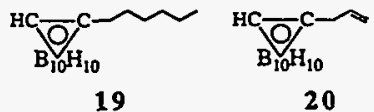

Kahl et al. have recently initiated biodistribution studies in small animals, but no results are available at this time.

7.6. LDL Complexes of Porphyrins and Phthalocyanines as Receptor-Mediated Boron Carriers

It has long been known that porphyrin, phthalocyanine, and related heterocyclic molecules have a propensity to localize in solid tumors and persist there in the long term. This property of porphyrins provides the basis of photodynamic therapy (PDT), ${ }^{[139]}$ another binary therapeutic method that utilizes porphyrin derivatives localized in tumor as in situ photosensitizers for the production of singlet oxygen on irradiation with red light. ${ }^{[140]}$ Candidate porphyrin derivatives that contain boron for BNCT purposes are also of interest for potential PDT applications. As pointed out in Section 6.1, the mechanism of this localization process or processes remains unknown, although the differentiation between uptake by normal and malignant tissues suggests that a receptor-mediated mechanism driven by very high turnover rates in tumor cells is involved. Porphyrins observed to be most effective in tumor localization are water soluble, amphiphilic species carrying hydrophobic substituents like BOPP (23). A plausible receptor-mediated mechanism might involve LDL vesicles, ${ }^{[94]}$ which utilize their hydrophobic constituents to solvate and encapsulate circulating porphyrin. Rapid uptake of the porphyrin-loaded LDLs would then internalize the heterocycle.

The possible use of boron-containing porphyrins as BNCT agents has been explored from the early 1980 s onward. Representative derivatives that have been synthesized are based upon tetraphenylporphyrin (e.g. BTPP (21)) ${ }^{[141]}$ and hematoporphyrin (e.g. VCDP (22) ${ }^{[142]}$ and BOPP (23)). ${ }^{[1431}$ The nido-7,8- $\mathrm{C}_{2} \mathrm{~B}_{9} \mathrm{H}_{11}^{-}$residues present in 21 and 22 are necessary to lend water solubility to the array. Since the anionic nido-carboranyl substituent is an amphiphilic ${ }^{[119]}$ rather than a hydrophilic substituent, this watersolubilizing tactic does not totally degrade the hydrophobic character of 21 and 22 . The porphyrin 23 would be expected to be more hydrophobic than 21 and 22 , since it contains four very hydrophobic closo-carboranyl groups, ${ }^{[1442]}$ and only two hydrophilic carboxylate substituents enhance hydrophilic properties. The greater hydrophobicity of 23 may be a direct cause of its superior tumor localization and retention $^{[144 b, c]}$ relative to 21 and 22 .

The biodistribution of $21,{ }^{[145]} 22,,^{[142,146]}$ and $23^{[143,147]}$ was evaluated with a variety of murine tumor models. Tumor types included gliomas and melanomas. The porphyrin derivatives were administered by either intravenous or intraperitoneal injections. Although the boron localization data obtained in these experiments was dependent upon experimental details and the identity of the porphyrin derivative, all three compounds were taken up and retained for days, whereas 23 was retained for weeks. Concentrations of boron in liver were generally about twice that simultaneously observed in the tumor, and when measured, boron levels in spleen and kidney were also elevated. Boron cleared from blood with time. Injected doses of $25-50 \mathrm{mg} \mathrm{B}$ per kg body weight were often employed, and boron concentrations of 15 to $40 \mu \mathrm{g}$ per $\mathrm{g}$ tumor were observed with carcinomas. Among porphyrin derivatives, $23^{[147]}$ provides the highest boron uptake by tumor combined with long retention times.

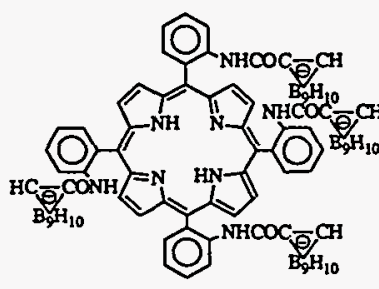

21 BTTP

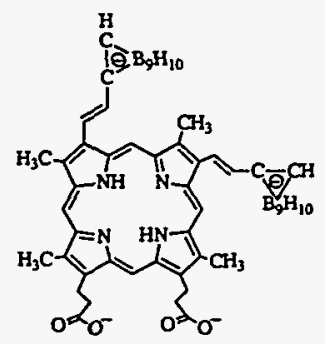

22 VCDP

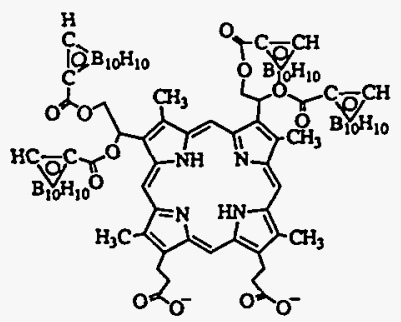

23 BOPP

Recent work with intracerebral glioma xenografts in CBA mice by Hill, Kahl et al. ${ }^{[18]}$ proved that 23 injected intravenously or intraperitoneally ( $30 \mathrm{mg} \mathrm{B}$ per $\mathrm{kg}$ body weight) could be localized in glioma at concentrations greater than $20 \mu \mathrm{g}$ B per $\mathrm{g}$ tumor, while the concentration in blood was less than half this value and in normal brain immeasurably low. Fluorescence studies with confocal laser scanning microscopy for 23 demonstrated that this compound was localized intracellularly and almost exclusively in organelles, both in vitro and in vivo. Density gradient ultracentrifugation studies proved these organelles to be mitochondria. It was calculated that this intracellular location of $\mathbf{2 3}$ could result in an up to tenfold reduction in the amount of boron required to achieve tumor necrosis by BNCT $(2-3 \mu \mathrm{g} \mathrm{B} \mathrm{per}$ $\mathrm{g}$ glioma). Porphyrin $\mathbf{2 3}$ is clearly an attractive candidate for a variety of tumors, and work pertinent to its adoption by the BNCT community is proceeding at a rapid pace.

Radiometal complexes of the phthalocyanines have been employed as diagnostic reagents, since they localize in tumors in a fashion reminiscent of porphyrins. ${ }^{[149]}$ The sul- 
fonated, water-soluble copper phthalocyanine has apparently been prepared by Soloway ${ }^{[149]}$ through the reaction of 1-(4-aminophenyl)-closo-1,2- $\mathrm{C}_{2} \mathrm{~B}_{10} \mathrm{H}_{10}$ with copper tetra(chlorosulfonyl)phthalocyanine followed by hydrolysis. No details on the purification, characterization, and efficacy of this species in tumor localization have yet been presented.

\subsection{Synthetic Liposomes as Tumor-Differentiating "Supertankers" for Boron Delivery}

In Sections 6.1 and 7.5, LDLs $^{[91]}$ and their cell wall receptors were discussed as potential systems for the delivery of boron in relatively large increments. Related to LDLs is the family of vesicles identified as synthetic unilamellar liposomes. ${ }^{[90]}$ These liposomes consist of a phospholipid bilayer that forms a spherical shell surrounding an aqueous core. The liposomes that have been applied to BNCT have, for the most part, carried hydrophilic sodium salts of polyhedral borane anions as solutes in the aqueous center of the vesicle, although, in principle, the phospholipid bilayer could also carry dissolved lipophilic species. Small multilamellar liposomes having several discrete layers of phospholipids might be utilized with boron-containing lipids as constituents. Figure 4 presents a cross-sectional view of a typical unilamellar liposome on the right and details of the bilayer structure. The

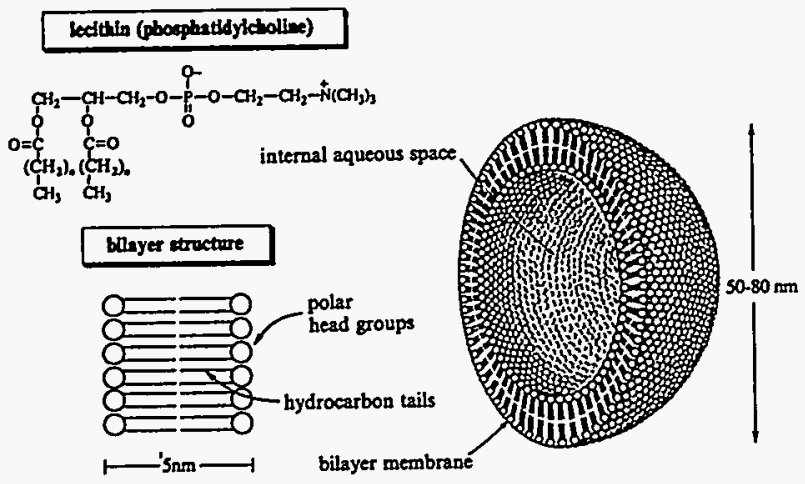

Fig. 4. Cross-sectional view of a liposome of the type found to be useful for boron delivery to tumors in mice. Synthetic phosphatidylcholine forms the bilayer membrane of these synthetic liposomes. Cholesterol is present in the bilayer, as well.

unilamellar liposomes employed for the localization of boron in tumor ${ }^{1901}$ range from $30-80 \mathrm{~nm}$ in diameter, and utilize cholesterol-rich bilayers of pure synthetic phospholipid to lend mechanical strength required for long-term circulation in the vascular system. Although the liposome shown in Figure 4 consists of a phospholipid having no net charge on its polar terminus, liposomes with net negative $^{[150]}$ or positive charges ${ }^{[151]}$ can be prepared. Surface charge affects biodistribution and pharmacokinetics. As shown in Section 7.7.1, liposomes of the type employed for boron delivery are capable of excellent selective localization in a variety of tumors following intravenous injection. They have previously been employed as tumor-selective delivery vehicles for radiopharmaceuticals used in animal models ${ }^{[152]}$ and in human diagnosis. ${ }^{[153]}$ Unlike LDLs, synthetic liposomes carry no receptor-seeking appendages, although, as pointed out in Section 6.1, they are selectively internalized by tumor cells. This cellular differentiation process is believed to be due, at least to a large extent, to the immature and consequently abnormally porous vasculature of rapidly growing tumors. ${ }^{[93]}$ The internalization process itself could arise by nonspecific endocytosis of the coated-pit $\rightarrow$ coatedvesicle type. ${ }^{[92.154]}$ Alternatively, LDL receptor sites may in some way be available to small liposomes and thereby provide a selective internalization pathway as in the case of LDLs themselves.

\subsubsection{Unilamellar Liposomes}

We have carried out extensive studies ${ }^{[90.155]}$ of unilamellar liposomes of the type illustrated in Figure 4 as boron delivery vehicles. Earlier results obtained by workers at Vestar, Inc. proved that liposomes of this type preferentially deliver their contents to tumor cells in animals and humans to provide levels of effector molecules in tumor five to ten times that of normal tissue, including blood. ${ }^{[151-153]}$ Sodium salts of polyhedral borane anions based upon $\mathrm{B}_{10} \mathrm{H}_{10}^{2-}$, its oxidation product, $\mathrm{n}-\mathrm{B}_{20} \mathrm{H}_{18}^{2-},{ }^{[*]}$ and their derivatives have provided water solutions $(250-300 \mathrm{~mm})$ of sodium salts with excellent hydrolytic stabilities, which were employed as the aqueous cores of $40-80 \mathrm{~nm}$ liposomes. ${ }^{[90.155]}$ These hyperosmotic vesicles proved to be generally stable; they can be stored for months and remain in circulation for 3-4 days. However, the stabilities observed in vivo were always a function of the identity of the solute present in the core. Biodistribution studies with many boron - containing liposomes of the type used here proved that liver and spleen (reticuloendothelial cells) accreted appreciable quantities of liposomes in competition with tumor. The initial liver-to-tumor boron concentration ratios were often about $2: 1$, but decreased with time. Blood always cleared with time, and tumor-toblood boron ratios of 5:1 or higher were not uncommon when the concentration of boron in tumor was in the therapeutic range (20-40 $\mu \mathrm{g}$ B per $\mu \mathrm{g}$ tumor). Protocols exist for the suppression of liposome uptake by reticuloenthelial cells. ${ }^{[151]}$ The effective toxicity of all compounds ${ }^{190.155]}$ delivered by liposomes should be decreased as a result of encapsulation. Although detailed toxicity data is not yet available for the majority of the more interesting compounds studied, no acute effects have been observed in mice whose tumors acquire therapeutic levels of boron. The majority of the biodistribution work was accomplished with BALB/c mice bearing implanted EMT 6 murine adenocarcinomas. ${ }^{[90,155]}$

$\mathrm{Na}_{2} \mathrm{~B}_{10} \mathrm{H}_{10}$ was the first polyhedral borane anion to be encapsulated as outlined above. ${ }^{[90.155]}$ Time-course evaluation with low total injected boron doses $(6 \mathrm{mg} \mathrm{B}$ per $\mathrm{kg}$ body weight) proved that although the $\mathrm{B}_{10} \mathrm{H}_{10}^{2-}$ ion was entering tumor, liver, and spleen, all of these tissues were cleared at similar rates, and boron was not retained in the tumor. BSH $\left(\mathrm{Na}_{2} \mathrm{~B}_{12} \mathrm{H}_{11} \mathrm{SH}, 7 \mathrm{mgB}\right.$ per kg body weight) gave essentially

["] $n$ here represents the "normal" isomer synthesized first and $i$ its photoisomer. 
the same result as that obtained with $\mathrm{Na}_{2} \mathrm{~B}_{10} \mathrm{H}_{10}$. These initial results suggested that in order to be effective the borane derivative must be capable of reaction with components of the cytoplasm such as the ubiquitous amino groups indigenous to intracellular proteins.

The interconversion of the isomeric $n-\mathrm{B}_{20} \mathrm{H}_{18}^{2-}(24)^{[157]}$ and $\mathrm{i}-\mathrm{B}_{20} \mathrm{H}_{18}^{2-}(25)^{[158]}$ ions, depicted in Scheme 17, and their chemistry, ${ }^{[156]}$ illustrated with 24 in Scheme $18,{ }^{[90.155]}$ suggested their possible intracellular incorporation as shown in Scheme 19. The $\mathrm{B}_{20} \mathrm{H}_{18}^{2-}$ ions deliver twice as many boron

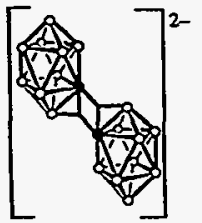

24
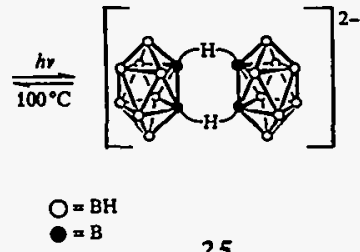

25

Scheme 17. Reversible interconversion of thermodynamically favored $n$ $\mathrm{B}_{20} \mathrm{H}_{18}^{2-}(24)$ and its less stable photoisomer, $\mathrm{i}-\mathrm{B}_{20} \mathrm{H}_{18}^{2-}$ (25).

atoms per three ions as $\mathrm{Na}_{2} \mathrm{~B}_{10} \mathrm{H}_{20}$ thus increasing boron availability for the same osmotic stress on the liposome. Scheme 4 indicates the preferred synthesis ${ }^{[71]}$ of $\mathrm{B}_{10} \mathrm{H}_{10}^{2-}$ from $B_{10} H_{14}$, which could be adapted to the synthesis of ${ }^{10} \mathrm{~B}$-enriched $\mathrm{B}_{10} \mathrm{H}_{10}^{2-}$ to serve as a precursor for a series of compounds including $\mathrm{n}$ - and $\mathrm{i}-\mathrm{B}_{20} \mathrm{H}_{18}^{2-}$.

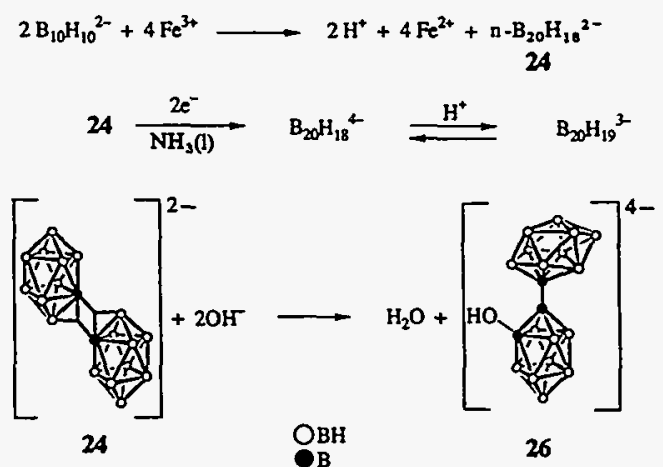

Scheme 18. The formation of $\mathrm{n}-\mathrm{B}_{20} \mathrm{H}_{18}^{2-}(24)$ and its subsequent two-electron reduction to form $\mathrm{c}^{2}-\mathrm{B}_{20} \mathrm{H}_{18}^{4-}(\mathrm{c}=$ equatorial), which undergoes acid-catalyzed rearrangement to the ae and $\mathrm{a}^{2}$ isomers $\left(\mathrm{a}=\right.$ apical). The $\mathrm{B}_{20} \mathrm{H}_{19}^{3}$ - intermediates are observed, and the most stable $\mathrm{a}^{2}-\mathrm{B}_{20} \mathrm{H}_{19}^{3-}$ isomer is easily isolated. The apical and equatorial positions of the component $\mathrm{B}_{10} \mathrm{H}_{9}^{2-}$ cages are joined by a normal two-election bond, which generates the three isomeric frameworks of $\mathrm{B}_{20} \mathrm{H}_{10}^{3-}$ and $\mathrm{B}_{20} \mathrm{H}_{18}^{4-}$. The kinetically controlled reaction of 24 with $\mathrm{OH}^{-}$yields the 2-hydroxy isomer 26 , which is isoelectronic with ae $-\mathrm{B}_{20} \mathrm{H}_{13}^{4-}$.

As expected, liposomes containing $\mathrm{Na}_{2}\left(\mathrm{n}-\mathrm{B}_{20} \mathrm{H}_{18}\right)$ (15 mg B per $\mathrm{kg}$ body weight) exhibited improved performance by increasing the quantity of boron available in a standard liposome dose. More significantly, the $n-\mathrm{B}_{20} \mathrm{H}_{18}^{2-}$ ion was retained by tumor, while other tissues were more rapidly cleared. The boron concentration in tumor was $24 \mu \mathrm{g}$ $\mathrm{B}$ per $\mathrm{g}$, and the tumor-to-blood ratio was 1.2:1 twenty-four hours after injection. These values changed to $3.3 \mu \mathrm{g}$ and $14: 1$ after $48 \mathrm{~h}$.

The more reactive photoisomer $\mathrm{Na}_{2}\left(\mathrm{i}-\mathrm{B}_{20} \mathrm{H}_{18}\right)(11 \mathrm{mg}$ $\mathrm{B}$ per $\mathrm{kg}$ body weight) was quite persistent. The boron concentration in tumor after $48 \mathrm{~h}$ ( $14 \mu \mathrm{g}$ per g) was still $71 \%$ of the value at $6 \mathrm{~h}$. During this period the tumor-to-blood concentration ratio increased to $12: 1$. Free $\mathrm{Na}_{2}\left(\mathrm{i}-\mathrm{B}_{20} \mathrm{H}_{18}\right)$ in buffer was not retained when injected (11 $\mathrm{mg} \mathrm{B}$ per kg body weight), but rapidly excreted; the tumor, blood, and liver concentrations of boron were only $1.9,1.9$, and $8.9 \mu \mathrm{g} \mathrm{B} \mathrm{per}$ $\mathrm{g}$, respectively, after $24 \mathrm{~h}$. An experiment in which two injections of $\mathrm{Na}_{2}\left(\mathrm{i}-\mathrm{B}_{20} \mathrm{H}_{18}\right.$ )-containing liposomes $(15 \mathrm{mg} \mathrm{B}$ per $\mathrm{kg}$ body weight injected at 0 and $24 \mathrm{~h}$ ) were administered yielded similar results after $24 \mathrm{~h}$. After the second injection and a total time of $48 \mathrm{~h}$, the boron concentration in tumor was $27 \mu \mathrm{g} \mathrm{B}$ per $\mathrm{g}$, and the tumor-to-blood concentraton ratio was $2.6: 1$. These are therapeutically useful values. The concentration of boron in the liver was about $60 \mu \mathrm{g} \mathrm{B}$ per $\mathrm{g}$.

Evaluation of liposomes that contained derivatives of the isomeric $\mathrm{B}_{20} \mathrm{H}_{28}^{4-}$ ions provided the basis of new localization concepts. The isomeric hydrolysis products $\mathrm{B}_{20} \mathrm{H}_{17} \mathrm{OH}^{4-\{156 \mathrm{a}\}}$ of 24 (26 in Scheme 18) or 25 are isoelectronic with the $\mathrm{B}_{20} \mathrm{H}_{18}^{4-}$ ions ${ }^{[159]}$ produced by two-electron reduction of 24 or 25 . The potassium salt of 26 was evaluated for use in BNCT in the usual fashion and shown to be rapidly cleared from tumor as in the case of $\mathrm{Na}_{2} \mathrm{~B}_{10} \mathrm{H}_{10}$. However, $\mathrm{Na}_{3} \mathrm{~B}_{20} \mathrm{H}_{19}{ }^{[159]}$ produced by protonation of $\mathrm{a}^{2} \cdot \mathrm{B}_{20} \mathrm{H}_{18}^{4-}$ ( $8 \mathrm{mg} \mathrm{B}$ per $\mathrm{kg}$ body weight) behaved differently. Nearly $30 \mu \mathrm{g} \mathrm{B}$ per $\mathrm{g}$ tumor was found after $6 \mathrm{~h}$. Concentrations of boron in tumor decreased at a moderate rate, while concentrations in liver increased for $30 \mathrm{~h}$. Boron levels in blood reached $7.5 \mu \mathrm{g} \mathrm{B}$ per $\mathrm{g}$ after $48 \mathrm{~h}$, while the tumor concentration fell to $12 \mu \mathrm{g}$ per $\mathrm{g}$. These results preface the discovery of the most efficacious polyhedral borane anion yet evaluated.

The ammonia-substituted ae- $\mathrm{B}_{20} \mathrm{H}_{18}^{4-}, \mathrm{B}_{20} \mathrm{H}_{17} \mathrm{NH}_{3}^{3-}(27$, see Scheme 20) is similar to the parent anion except that an uncharged $\mathrm{NH}_{3}$ molecule has been formally substituted for hydride ligand, thus reducing the ionic charge to -3 . Evaluation of $\mathrm{Na}_{3} \mathrm{~B}_{20} \mathrm{H}_{17} \mathrm{NH}_{3}$ in liposomes (11 mg B per kg body weight) produced spectacular results as shown in the time course plot of Figure $5 .{ }^{[160,161]}$ Even with the low injected dose employed, the boron concentration of $25 \mu \mathrm{g} \mathrm{B}$ per $\mathrm{g}$

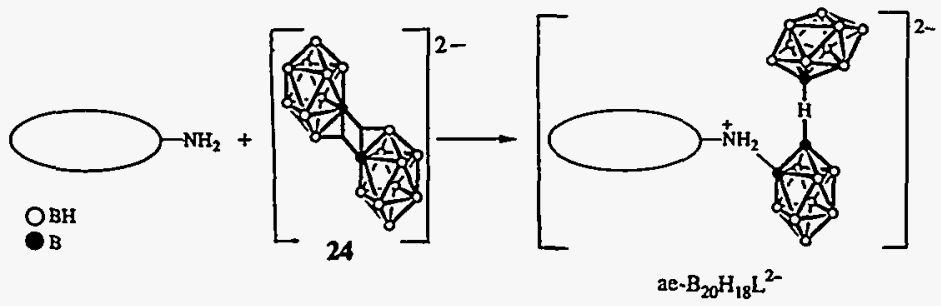

Angew. Chem. Int. Ed. Engl. 1993. 32, 950-984
Scheme 19. Proposed reaction of an intracellular protein molecule, symbolnzed as $\mathrm{O}-\mathrm{NH}_{2}$, with 24 to form an ae derivative. The $\mathrm{i}-\mathrm{B}_{20} \mathrm{H}_{15}^{2-}$ isomer (25) would provide a similar, but more facile reaction. 


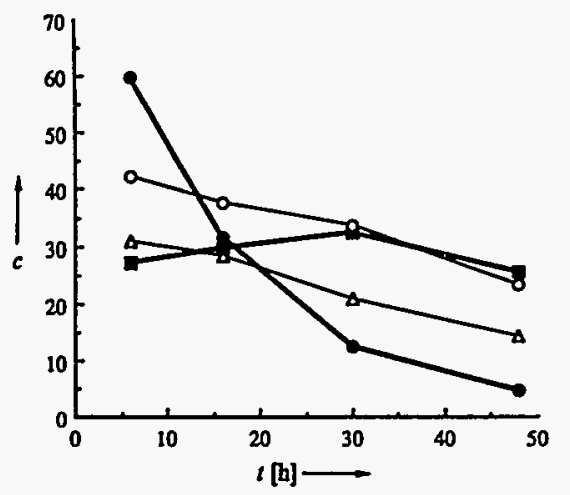

Fig. 5. Data from a time-course study on the distribution of liposomal $\mathrm{Na}_{3} \mathrm{~B}_{20} \mathrm{H}_{1}, \mathrm{NH}_{3}$ (27) in BALB/c mice bearing EMT6 mammary adenocarcinomas. $c=$ boron concentration in tissue in $\mu \mathrm{g}$ per $\mathrm{g}$. in blood, $n$ in tumor. 0 in liver, $\Delta$ in spleen. The injected dose was $11 \mathrm{mg}$ per $\mathrm{kg}$ body weight.

respectively. In fact, the boron concentration of tumor increases during the first $30 \mathrm{~h}$ and reaches a maximum of $35 \mu \mathrm{g}$ per g. One plausible explanation of these results is based upon the possible intracellular two-electron oxidation of 27 to $\mathrm{B}_{20} \mathrm{H}_{17} \mathrm{NH}_{3}^{-}$(28) within tumor cells as shown in Scheme 20 . The single negative charge of 28 would enhance

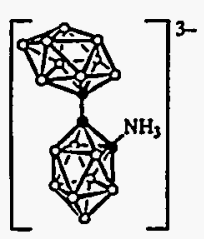

27

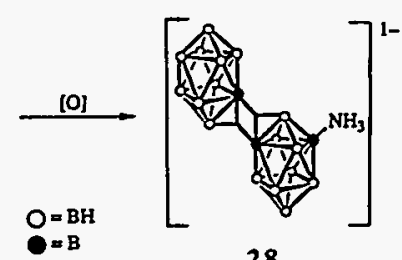

28
Scheme 20. Proposed intracellular oxidation of 27 to the very reactive, but hypothetical $\mathrm{B}_{20} \mathrm{H}_{17} \mathrm{NH}_{3}^{-}$(28), which should rapidly bind with intracellular protein. $[\mathrm{O}]=$ intracellular oxidation.

the electrophilicity of this ion compared to the $\mathrm{B}_{20} \mathrm{H}_{18}^{2-}$ ions, and could thus improve the intracellular bonding (see Scheme 19). In addition some other feature prevents the extensive uptake of 27 by liver and promotes the concomitant low final blood levels of boron. Extensive evaluation of 27 is underway, since its liposomes offer one of the most effective boron delivery systems yet described in small animals.

Another reactive polyhedral borane anion derivative has been recently described and evaluated for suitability for BNCT: ${ }^{[162]}$ This species is 2-isocyanato-closo- $\mathrm{B}_{10} \mathrm{H}_{9}^{2-}$ (29) ${ }^{[163]}$, whose isocyanate function is expected to form the corresponding urea derivative upon intracellular reaction with amino groups of protein as described in Scheme 21. Data from preliminary biodistribution studies with liposomes that contain the disodium salt of $29(9 \mathrm{mg} \mathrm{B}$ per $\mathrm{kg}$ body weight) are presented in Figure 6 along with a comparable set of results obtained with $\mathrm{Na}_{2} \mathrm{~B}_{10} \mathrm{H}_{10}(6 \mathrm{mg} \mathrm{B}$ per $\mathrm{kg}$ body weight). These results prove that 29 is better retained by tumor, liver, and spleen than its unsubstituted parent, $\mathrm{Na}_{2} \mathrm{~B}_{10} \mathrm{H}_{10}$. One may conclude that the isocyanate function of 29 serves, as expected, by linking to intracellular protein.

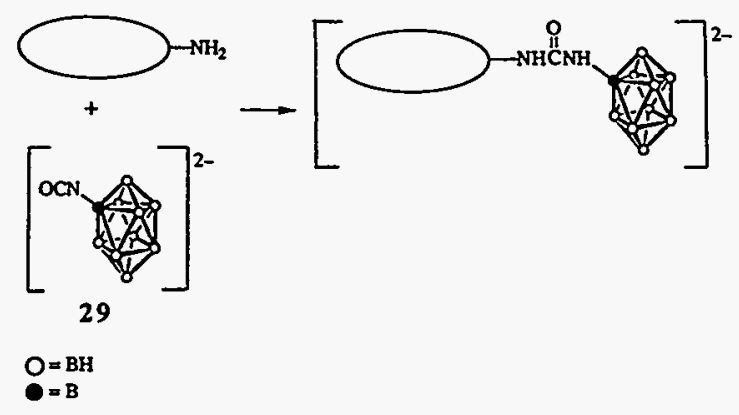

Scheme 21. Proposed reaction of 29 with an intracellular protein molecule o- $\mathrm{NH}_{2}$.

The synthesis and evaluation of liposomes containing other polyhedral anions that bear functional groups continues.

Although Moore et al. ${ }^{\left[{ }^{64]}\right.}$ reported the delivery of hydrophobic thiouracil derivatives of carborane (see Section 7.10.1) dissolved in lipid bilayer to murine melanoma xenografts on use of $100 \mathrm{~nm}$ phospholipid-supported liposomes, the efficacy of this procedure could not be deduced from the reported results except that high tumor selectivity was observed.

The simplicity, broad scope, and exciting preliminary results obtained with liposomes have established them as one of the most promising boron delivery methods yet devised.
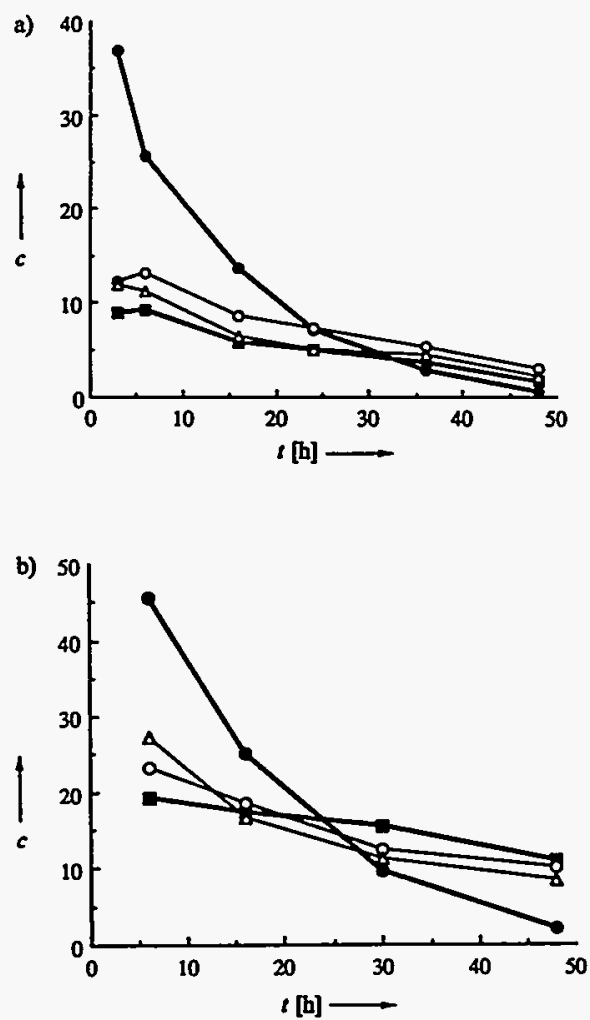

Fig. 6. Comparison of the distribution of liposomal $\mathrm{Na}_{2} \mathrm{~B}_{10} \mathrm{H}_{30}$ (a) and $\mathrm{Na}_{2}\left[2-\mathrm{OCN}-\mathrm{B}_{30} \mathrm{H}_{9}\right]$ (b) from time-course studies on EMT6 mammary adenocarcinomas grown in BALB/c mice. The injected dose was $6 \mathrm{mg}$ per $\mathrm{kg}$ body weight in a) and $9 \mathrm{mg}$ per $\mathrm{kg}$ body weight in b). For explanation of other symbols, see Figure 5. 
7.8. The Potential of Sex Hormone Receptors as Vehicles for the Delivery of Boron to the Cell Nucleus

Both breast and prostate cancer have predominant cell lines that depend upon the constant uptake of estrogen and dihydrotestosterone, respectively. These hormones become effective following complexation with their respective receptors present in the cytosol of the proper cell, followed by translocation of the resulting hormone-receptor complex to the chromatin within the cell nucleus. Both Hadd ${ }^{[165]}$ and Kahl ${ }^{[166]}$ have synthesized carborane derivatives of estradiol (30 and 31, among others). Hechter and Schwart ${ }^{[167]}$ used available data pertinent to receptor site densities to calculate the probable concentration of boron that could be obtained with receptors of this type. With efficacious hormone surrogates carrying one carborane cage per molecule (ten boron atoms) only about $0.015 \mu \mathrm{g} B$ per $\mathrm{g}$ tissue could be delivered to chromatin in this fashion. The goal of $1-2 \mu \mathrm{g} B$ per $g$ tissue was thought to be reasonable, since the boron targets were placed in the cell nucleus.

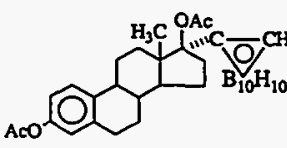

30

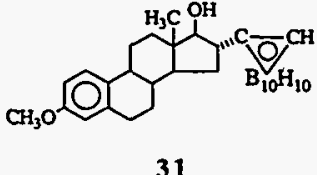

31<smiles>Oc1ccc2c(c1)CCC2c1ccccc1</smiles>

32

Work has apparently been abandoned with this aspect of selective boron delivery. However, Gabel et al. ${ }^{[168]}$ synthesized a boron-rich analog, 32, of the antiestrogen molecule U23 469-M that contained a decachlorocarborane substituent. This compound was taken up nonspecifically by ZR 75-1 breast cancer cells and partially inhibited the uptake of estradiol in these cells. The estimated $10^{4}-10^{5}$ receptor sites available per cell are far less than the number required to achieve therapeutic levels of boron. This line of endeavor has been abandoned as well. Even though boron was placed within the cell nucleus, it has been impossible to approach the concentrations required for BNCT.

\subsection{Boron-Containing Amino Acids as Prescursors in Protein Synthesis}

Recent emphasis has been placed upon the evaluation of a variety of boron-containing amino acid derivatives that may carry boron to tumors by becoming incorporated in protein synthesis or metabolism within the rapidly growing tumor cell. 2 -4-Dihydroxyborylphenylalanine ${ }^{[87]}$ has received particular attention. L-Carboranylalanine, ${ }^{[144 a]}$ an analog of phenylalanine, was only recently synthesized by improved methods. ${ }^{[862,86 \mathrm{~b}]}$ Spielvogel pioneered the synthe- sis of natural amino acid surrogates ${ }^{[169]}$ in which a carbon atom has been replaced by an isoelectronic and isosteric boron atom having four valence electrons and a negative formal charge. These studies constitute one of the most dynamic areas of BNCT research currently under investigation.

\subsubsection{Studies with L-4-Dihydroxyborylphenylalanine}

Snyder et al. ${ }^{[170]}$ first reported DL-4-dihydroxyborylphenylalanine (BPA (DL-33); see Scheme 23), which was subsequently examined in animals by Soloway in the 1950s and found to provide the most favorable tumor/brain selectivity (8.5:1) of all the boronic acids similarly screened at that time. ${ }^{[5]}$ Interest in BPA as a BNCT target compound was dormant until Mishima et al..$^{[87,171]}$ discovered its efficacy against B-16 melanoma in vitro and in hamsters bearing Greene's melanoma during neutron irradiation experiments ${ }^{[11]}$ with ${ }^{10} \mathrm{~B}$-enriched 33. Further work with Duroc pigs $^{[171]}$ and humans ${ }^{[172]}$ afflicted with melanoma proved that ${ }^{10} \mathrm{~B}$-enriched DL- or L-33, was effective in BNCT and that primary lesions that were highly pigmented were destroyed. Because of the extremely low solubility of $\mathbf{3 3}$ in water, the compound was administered by perilesional injection (i.p.) or orally. Esterification of 33 with sugars improved its solubility. ${ }^{[17]}$ This use of 33 against melanoma was developed by Mishima et al. ${ }^{[87]}$ based upon the fact that the melanin pigment normally present in active melanoic neoplasms is synthesized from natural amino acids containing aromatic rings such as L-dopa, L-tyrosine, and L-phenylalanine. Presumably, this process involves 5,6-indolequinone as an intermediate. It was assumed ${ }^{(87,171)}$ that 33 would fall into this class of melanin precursors ${ }^{[14]}$ and enter the characteristic melanin produced by intracellular melanosomes. Scheme 22 illustrates the biosynthesis of melanin. However, the insertion of 33 in this mechanism would require that the

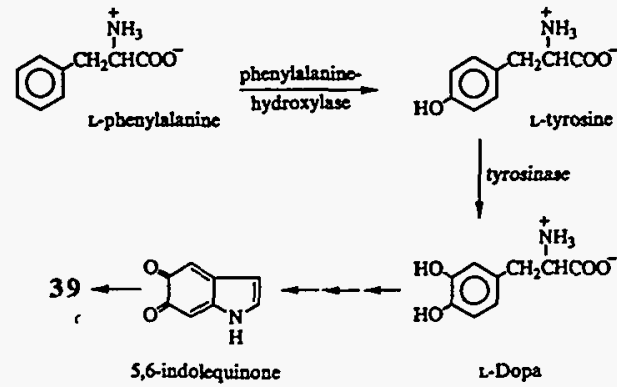

Scheme 22. The stepwise enzymatic oxidation of L-phenylalanine leading to the 5,6 -indolequinone polymer melanin 39 . The structure of 39 has not been precisely determined.

4-dihydroxyboryl group be removed ${ }^{[175]}$ prior to the formation of the quinone, presumably as boric acid. This loss of boric acid could occur through acid-catalyzed hydrolysis of the $\mathrm{B}-\mathrm{C}$ bond or by oxidative cleavage of the $\mathrm{C}-\mathrm{B}$ bond (Scheme 23). Migration of boric acid from the cell interior to the extracellular volume is a facile process that should preclude the accumulation of boron within tumor. The accre- 
tion of therapeutic boron concentrations in melanomas (and other tumor types) is therefore likely to be due to another bioutilization process.

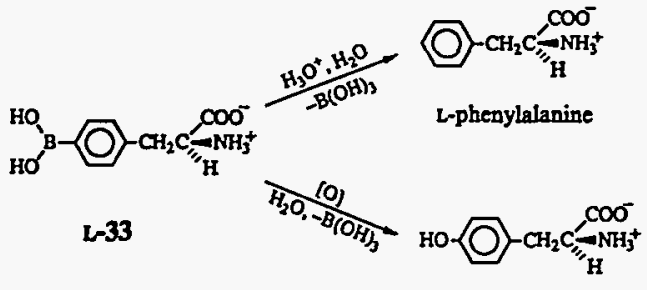

L-tyrosine

Scheme 23. Known reactions of L-33, which may occur in vivo and lead to the loss of boric acid.

Other experimental observations made with ion microscopy that shed light on this problem have been reported by Morrison et al. ${ }^{[46]}$ These workers developed a cell-culture system for the study of drug uptake into a malignant, highly pigmented M-3 murine melanoma clonal strain. The pigment level (extent of melanization) could be matched, area for area, to ion intensities from superimposed photomicrographs and ion images by using the aryl-bound deuterium present in $\left[\mathrm{D}_{4}\right]$-L-tyrosine and boron in $\mathrm{DL}-33$ as sources of the imaged ions. In any given area of the cell culture the increase in melanin concentration correlated linearly with an increase in deuterium concentration due to the uptake of tyrosine, a known melanin precursor (Scheme 22). The boron concentration did not change as melanin was produced; DL-33 consequently does not deposit its boron in the melanin of developing melanoma cells.

Coderre et al. demonstrated that 33 is accreted by the weakly pigmented B-16 melanoma carried by C57BL mice. ${ }^{[176]}$ This result suggests that 33 uptake, which according to other experiments favors the L-stereoisomer, ${ }^{[174]}$ proceeds through active transport of the amino acid for purposes other than the synthesis of melanin. This is important to therapy since many forms of human melanoma are variably pigmented, and very dangerous metastases are not pigmented early in their development.

In addition, Coderre et al. ${ }^{[174]}$ demonstrated that $L-33$ was much more effective than its enantiomer against HardingPassey melanoma, that oral administration of ${ }^{10} \mathrm{~B}$-enriched 33 achieved tumor concentrations of boron as high as $40 \mu \mathrm{g}$ per $\mathrm{g}$ in BALB/c mice,,$^{[176]}$ and that the growth inhibition of these tumors was directly related to the neutron fluence with the result that longer exposure time caused a longer delay tumor growth, as expected. More recent work by this same group $^{[177]}$ showed that boron was selectively delivered to tumors other than melanomas; the KHJJ murine mammary carcinoma in mice and GS-9L gliosarcoma grown in rat brain are representative of such tumors. Boron concentrations as high as $20 \mu \mathrm{g} \mathrm{B}$ per $\mathrm{g}$ tumor were attained in the gliosarcomas after oral administration of $\mathrm{L}-33$. Other studies of this type are too numerous to mention, but they all support the useful application of $\mathrm{L}-33$ to amelanoic tumors.

Recently Coderre et al. reported ${ }^{[100]}$ the experimentally determined RBE values of the BSH disulfide dimer BSSB
(Scheme 7) and L-33, obtained from cell-survival data following thermal neutron irradiation of cells that were enriched in the boron compounds. Gliosarcoma cells (GS-9L) were employed both in vitro and in intracerebral rat tumors. The in vitro $R B E$ values (Section 2.4) ranged from 3.5 to 4.1 for $L-33$ and from 1.1 to 1.4 for BSSB. It was pointed out that these BSSB values were physically unrealistic and characteristic of a mechanism for in vivo tumor cell death dependent upon the destruction of vascular endothelial cells through $\mathrm{BNC}$ and a resultant loss of vasculature function at the tumor. On the other hand, the higher RBE values observed with L-33 were consistent with neutron capture by intracellular L-33. The destruction of endothelial cells of the vasculature as the principal killing mechanism for BNCT with BSH and BSSB has been noted in Section 7.1.

As pointed out above, the low water solubility of $\mathrm{L}-33$ hydrochloride at physiological $\mathrm{pH}$ has complicated the use of L-33 in experiments with large animals. This problem has been largely overcome $e^{[173]}$ by converting L-33 to water soluble boronate esters with fructose, diethanolamine, and cyclodextrin derivatives. These species offer the promise of an intravenous injection protocol.

A phase I clinical trial for $L-33^{(178)}$ is planned by the New England Medical Center-MIT Program in Neutron Capture Therapy. This clinical trial will join another in progress at the School of Medicine at Kobe University, Japan.

Both DL-2- and DL-3-dihydroxyborylphenylalanine have been synthesized and await thorough evaluation. ${ }^{[19]}$ In addition, the 4-closo (34) and 4-nido (35) derivatives of DL-4carboranylphenylalanine have been prepared ${ }^{[180]}$ and are undergoing biological evaluation. An asymmetric synthesis
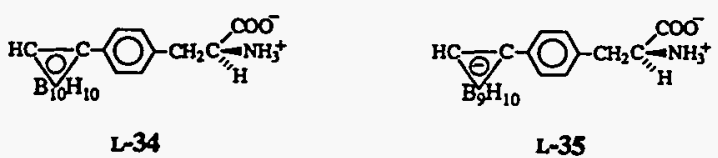

of L-33 has also been developed, ${ }^{[181]}$ which involves the hydrogenation, catalyzed by a chiral rhodium complex, of a substituted $\alpha$-amino acrylic acid derivative.

\subsubsection{L-Carboranylalanine}

The accelerated rate of synthesis of proteins and amino acid metabolites exhibited by a malignant cell, coupled with the active transport mechanisms available for amino acid accumulation within cells, provides a bioutilization process that may allow boron-containing $\alpha$-amino acids to enter tumor cells preferentially. The selective uptake of $\mathrm{L}-33$ by several types of malignant cells appears to follow this cell differentiation mechanism (see Section 7.9.1).

Since one of the most chemically attractive packages for the delivery of boron is the closo-1,2- $\mathrm{C}_{2} \mathrm{~B}_{10} \mathrm{H}_{12}$ cage, it is not surprising that the $\mathrm{L}$-phenylalanine surrogate, $\mathrm{L}$-carboranylalanine (16) is a highly desired species for biological evaluation. The initial synthesis of 16 was reported ${ }^{[144]}$ in 1976. 
The asymmetric syntheses of the $D$ and $L$ enantiomers have recently become the goal of several research groups, most notably those of Kahl et al. ${ }^{[86 a]}$ and Sjöberg et al. ${ }^{[86 \mathrm{~b}]}$ (Schemes 24 and 25, respectively). It was noted by the discov-
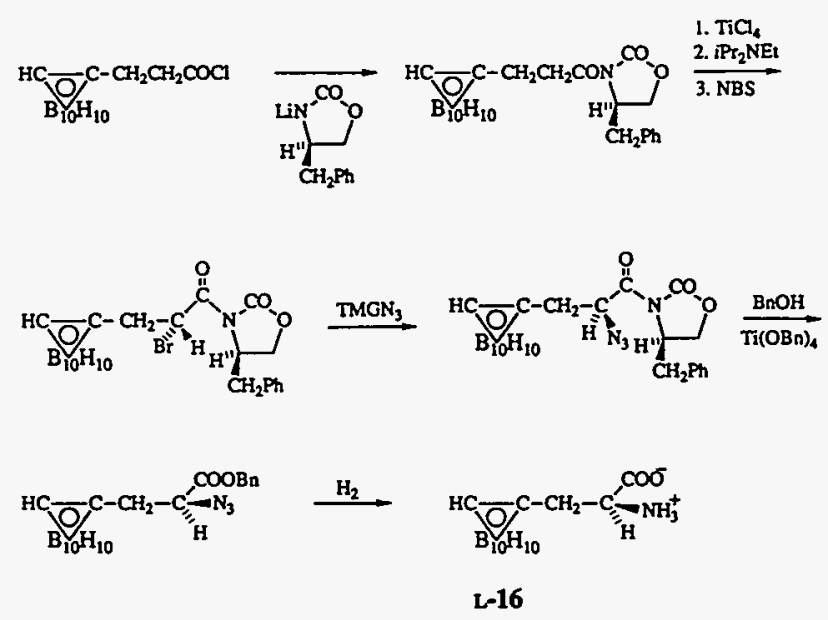

Scheme 24. The asymmetric synthesis of D- and L-16. devised by Kahl and Radel [86a]. NBS $=N$-bromosuccinimide. TMGN $_{3}=$ tetramethyl guanidinium azide. The subsequent hydrogenation proceeds catalytically.

erers of 16 that the carborane cage imparted extreme hydrophobicity upon this amino acid, ${ }^{[1442]}$ and Kahl et al. ${ }^{[862,128]}$ has pointed out that the steric requirements of the carborane group in $\mathbf{1 6}$ is approximately the same as the volume swept out by the rotation of the phenyl group in phenylalanine. Small hydrophilic peptides that incorporate L-carboranylalanine may prove useful to enhance its effective water solubility and bioavailability. Alternatively, its anionic nido-
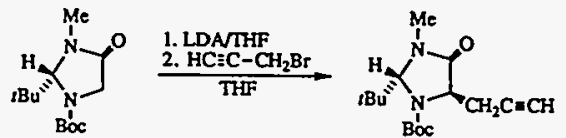

(R)

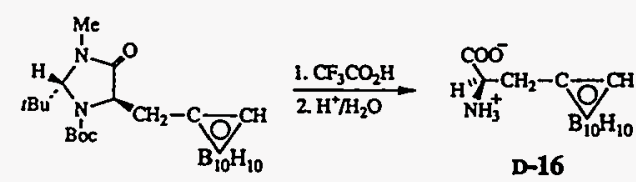

Scheme 25. An asymmetric synthesis of D- and L-16, deseribed by Sjöberg et al. $[86 \mathrm{~b}, 128] . \mathrm{LDA}=$ lithium diisopropylamide. The last step takes place on a cation exchange resin.

$\mathrm{C}_{2} \mathrm{~B}_{9} \mathrm{H}_{11}^{-}$derivative will, at the least, be amphiphilic. Nonetheless, the synthesis of 16 makes an important biomolecule readily available for the first time. The results of in vitro and in vivo evaluation of 16 and its derivatives are awaited with great anticipation.

Related to 16 are the amino acids $9^{[120,121]}$ and 10 (Section 7.3.1), which have not been investigated as enantiomer- ically pure compounds. The asymmetric synthesis of Sjöberg et al. ${ }^{[86 \mathrm{bl}}$ makes these and related species generally available.

\subsubsection{Ammonium Carboxyborane Analogs of Amino Acids}

Replacement of a neutral carbon atom by an anionic boron atom bearing four valence electrons results in an isoelectronic and isosteric replacement as in the formal relationship of closo- $\mathrm{B}_{12} \mathrm{H}_{12}^{2-}$ and closo-1,2- $\mathrm{C}_{2} \mathrm{~B}_{10} \mathrm{H}_{12}$ (see Section 5). This process also provides a pathway for the introduction of boron into simple organic compounds such as $\alpha$-amino acids and their derivatives. Spielvogel et al. ${ }^{[169,182]}$ have applied this principle to the BNCT of cerebral tumors by developing a synthesis of 36 , the ammonium carboxyborane analog of glycine. This particular molecule was of importance, because glycine has the lowest rate of transport across the blood-brain barrier of the amino acids, and the ${ }^{14} \mathrm{C}$-labeled glycine molecule was shown to give a tumor/ brain concentration ratio of $10: 1$ and a tumor/blood concentration ratio of 5:1 twenty-four hours after injection in BALB/c mice carrying brain inplants of Harding-Passey melanoma. ${ }^{[182]}$ Injection of sufficient 36 to provide $40 \mathrm{mg}$ per $\mathrm{kg}$ body weight in mice identical to those used in the ${ }^{14} \mathrm{C}$-labeled glycine experiment gave very disappointing results; rapid total clearance of 36 from the animals and $2 \mathrm{~h}$ postinjection tumor, brain, and blood concentrations of $16.2,7.6$, and $8.8 \mu \mathrm{g}$ B per $\mathrm{g}$ tissue, respectively. The bloodbrain barrier was apparently more permeable to 36 than to glycine. Additional data obtained by Porschen et al. ${ }^{[183]}$ on adenocarcinoma in C57 mice was consistent with these results. Even more recent data ${ }^{[184]}$ pertaining to small peptide derivatives such as $\mathbf{3 7}$ and $\mathbf{3 8}$ were equally discouraging from the viewpoint of BNCT. However, it is now well estab-

\section{$\mathrm{H}_{3} \stackrel{+}{\mathrm{N}} \overline{\mathrm{B}} \mathrm{H}_{2} \mathrm{COOH}$}

36 $\mathrm{B}_{10} \mathrm{H}_{12}\left(\mathrm{CH}_{3} \mathrm{CN}\right)_{2}$ $\mathrm{H}_{3} \mathrm{CN}$

$$
\left(\frac{10}{2}\right.
$$

(n)

lished ${ }^{[169]}$ that a variety of ammonium carboxyborane derivatives of the type discussed here have great promise as antineoplastic, hypolipidemic, and anti-inflammatory agents and are currently under development.

\subsection{Melanoma Differentiation Based Upon Boron-Containing Melanin Precursors and Complexation Agents}

The production of the complex pigment, melanin (39), by melanosomes present in a preponderance of melanoma types was previously proposed as a tumor cell differentiation process $^{[185]}$ that rationalized the efficacy of L-33 as a tumortargetting amino acid. ${ }^{1871}$ Although this suggestion was not supported by accumulated evidence, and although the true mechanism of boron accretion in tumor with L-33 is probably related to the accelerated uptake of amino acids such as tyrosine by tumor cells (Section 7.9.1), the actual incorporation of 2-thiouracil (40) into the melanin structure during its 
in vivo synthesis has been demonstrated. ${ }^{[186-188]}$ This provided an attractive possibility for the selective delivery of boron to the intracellular volume.

The ability of melanin and melanoproteins present in murine melanoma cells to tightly bind phenothiazine derivatives such as the potent tranquilizer chloropromazine ${ }^{[189]}$ $(41$, known under the trade name Thorazine) also suggested a pathway for the selective deposition of boron into melanoma cells. ${ }^{[190)}$ In this case preformed melanin must be available for reaction with the phenothiazine derivative.<smiles>O=C1C=CNC1=O</smiles>

40<smiles>ClC(Cl)(Cl)CCCN1Cc2ccc(cc2)Sc2ccccc21</smiles>

41

\subsubsection{2-Thiouracil Derivatives as Melanoma-Selective Boron Delivery Species}

Although it had been determined by Dencker et al. ${ }^{[186.187]}$ that 2-thiouracil (40) is selectively incorporated into melanin during its biosynthesis in melanoma cells, Fairchild et al. ${ }^{[188]}$ quantified this process for the first time in 1982 by using ${ }^{14} \mathrm{C}$-labeled 2-thiouracil $\left.\left({ }^{14} \mathrm{C}\right] \mathrm{TU}\right)$ administered intraperitoneally or intravenously in $\mathrm{BALB} / \mathrm{c}$ mice bearing HardingPassey melanoma. The tumor uptake of TU was correlated with melanin content. The tumor uptake of $\left[{ }^{14} \mathrm{C}\right] \mathrm{TU}$ was as high as 9 to $12 \%$ of the injected dose per $g$ tumor. Ratios of the concentration in tumor and normal tissue varied from 50:1 in metabolizing organs such as liver and lung, to 80:1 in pigmented whole eye, and 300:1 in muscle and brain. The percentage of uptake of $\left[{ }^{14} \mathrm{C}\right] \mathrm{TU}$ by tumor was virtually unchanged as the administered dose was varied by a factor of 5000 to give as much as $300 \mu \mathrm{g}\left[{ }^{14} \mathrm{C}\right] \mathrm{TU}$ incorporated per gram of tumor. This is equivalent to $26 \mu \mathrm{g} \mathrm{B}$ per $\mathrm{g}$ tumor if only one boron atom were incorporated in each molecule of 40. These initial results stimulated a major effort to develop new boron-containing delivery agents based upon derivatives of 2-thiouracil and other compounds having at least a loose structural relationship to thiourea. Compounds that have been explored carry boron as a dihydroxyboryl group, an uncharged 1-(closo-1,2- $\left.\mathrm{C}_{2} \mathrm{~B}_{10} \mathrm{H}_{11}\right)$ appendage or its anionic 7-(nido-7,8- $\mathrm{C}_{2} \mathrm{~B}_{9} \mathrm{H}_{11}^{-}$) counterpart, which enhances hydrophilicity. The research groups responsible for the

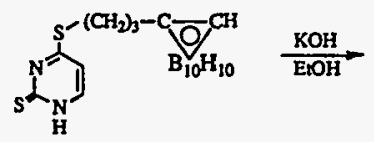

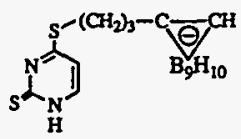

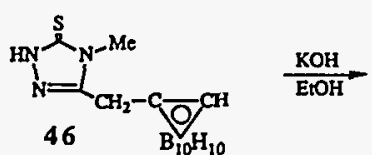<smiles></smiles>

Scheme 26. Thiouracil and thiotriazol derivatives synthesized and evaluated by Gabel et al. [191,192]. synthesis of this library of derivatives were headed by Gabel. ${ }^{[191.192]}$ and Wilson, ${ }^{[193,194]}$ whose representative contributions are illustrated in Schemes 26 and 27, respectively. In addition to carborane-substituted 2-thiouracil species, Wilson also synthesized the useful carboranylalkylamine building blocks 42 and 43 , which were in turn converted into the corresponding amides ${ }^{[194]} 44$ and 45 by reaction with 5-carboxy-2-thiouracil. Gabel et al. ${ }^{[192]}$ reported the synthesis of 46 and 47 carborane derivatives of 5-thio-1,2,4triazole, as well as of 48 and 49 .

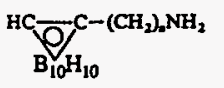

42, $n=1$

$43, n=3$

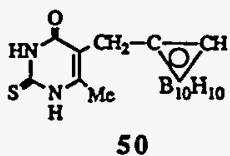

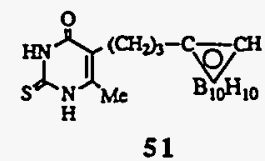

53

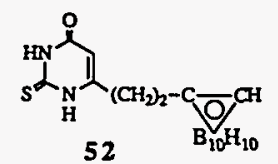<smiles>O=C(NCCC1CC1Br)c1c[nH]c(=S)[nH]c1=O</smiles>

44, $n=1$ $45, n=3$<smiles>[B]C1CC1CC1C(=O)C=CNC1=S</smiles>

Scheme 27. Carboranylalkylamine and thiouracil derivatives, synthesized and evaluated by Wilson et al. [193,194].

Evaluation of the selective uptake of these candidate species by murine melanoma models has proceeded slowly. Gabel et al. ${ }^{[191.1921}$ report that the dihydroxyboryl derivatives $48 \mathrm{a}$ and $48 \mathrm{~b}$ gave boron concentrations in B16 melanomas grown in $\mathrm{C} 57 / \mathrm{bl}$ mice ( $4 \mathrm{~h}$ after intraperitoneal administration of $9-16 \mathrm{mg} \mathrm{B}$ per $\mathrm{kg}$ body weight) of about $30 \mathrm{\mu g}$ B per $\mathrm{g}$ tumor with tumor-to-blood boron concentrations ratios of $4: 1$ to $7: 1$. The more hydrophobic 6-propyl<smiles>CC1NOC(=O)C(O)N1</smiles>

$48 \mathrm{a}$<smiles>CCCCOC1=C(O)NC(C)N1</smiles>

$48 b$<smiles></smiles>

$49 \mathrm{a}$

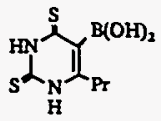

$49 b$ derivative $49 \mathrm{~b}$ was taken up to the extent of only $1.7 \mu \mathrm{g} \mathrm{B} \mathrm{per}$ $\mathrm{g}$ tumor, while $49 \mathrm{a}$ gave intermediate results. The 5 -thiotriazole species $\mathbf{4 6}$ and $\mathbf{4 7}$ gave poor selectivity and accumulated in the liver. The remaining carborane derivatives of Scheme 26 were less effective than the dihydroxyboryl compounds 48 and 49.

The carborane derivatives 50 through 53 of Wilson et al. ${ }^{\text {1193.194] }}$ were quite hydrophobic and too slowly taken up by nude mice bearing Harding-Passey melanoma xenografts to be useful. Incorporation of 51 in the phospholipid bilayer of a liposome (Section 7.7.1) was investigated by 
Moore et al. ${ }^{[164]}$ as a means of delivery for this hydrophobic species. High tumor specificity was observed, but no other data were reported.

While the efficacy of boron-containing 2-thiouracil and related compounds remains to be demonstrated and optimized by further molecular design and synthesis work, promising results were indeed obtained, and further work is warrranted. However, the presumed dependence of this delivery mode upon melanogenesis would seem to rule out the ability of these compounds to accumulate in amelanoic melanoma lines and immature premelanoic melanoma metastases having low tyrosinase activity.

\subsubsection{Phenothiazine Charge-Transfer Complexes with Existing Melanin as a Boron Delivery Mechanism}

Investigation of the tranquilizing drug 41 known as chloropromazine in the early 1960 s uncovered its remarkable ability to accumulate in the melanin-bearing eye tissues of experimental animals. ${ }^{[18]}$ This apparently selective complexation of 41 and other phenothiazine derivatives was demonstrated with melanin and melanoproteins obtained from many other sources. ${ }^{[189]}$ Melanin and its variations are complex polymeric pigments apparently derived from 5,6indolequinone and other precursors, ${ }^{[195,196]}$ some of which contain sulfur (cysteine). It is now accepted that the melanin-phenothiazine complexation reaction is the result of electron-transfer reactions in which melanin serves as the electron acceptor. ${ }^{[197]}$ The chemistry based upon these and other interesting observations is beyond the scope of this article, and the reader is referred to the original literature.

The accumulation of 41 in melanoproteins attracted the interest of Mishima et al. ${ }^{[190]}$ with respect to the possibility of selective transport of boron to melanin-rich melanomas with boron-substituted derivatives of 41 . The biodistribution of ${ }^{35} \mathrm{~S}$-labeled $\mathbf{4 1}$ was thoroughly examined by Fairchild et al. ${ }^{[198]}$ in a series of time-course experiments. Both Greene melanoma in Syrian golden hamsters (melanin content about $0.4 \%$ by weight of tumor) and Harding-Passey melanoma in BALB/c mice (melanin content about $0.7 \%$ by weight of tumor) were employed, since these models closely resembled human melanoma. The drug was administered by intraperitoneal injection. Twenty-four hours postinjection of up to $50 \mathrm{mg} 41$ per $\mathrm{kg}$ body weight, absolute concentrations of 41 in tumor that exceeded $100 \mu \mathrm{g}$ per $\mathrm{g}$ tumor could be obtained; the concomittant tumor-to-liver concentration ratio is about $10: 1$. The ratios of boron concentration in tumor to that in other tissues such as blood and muscle were greater than 100:1. Computation of hypothetical tissue concentrations of ${ }^{10} \mathrm{~B}$ were made with compound 54 as an example $\left(25.4 \%{ }^{10} \mathrm{~B}\right.$ by weight as sodium salt) and found to be sufficiently high ( $>20 \mu \mathrm{g}$ B per $\mathrm{g}$ tumor) for BNCT if this species provided the same performance as 41 .

The results of earlier investigators buttressed by the data of Fairchild et al. ${ }^{[198]}$ stimulated a search for suitable boroncontaining derivatives of phenothiazine. Consequently, Nakagawa and Aono ${ }^{[199]}$ reported the syntheses of the polyhedral borane anion derivatives 54 and 55 (Scheme 28). Both 52 and 53 were investigated in BNCT experiments ${ }^{[200]}$ with Greene's melanoma grown in Syrian golden hamsters. Al-

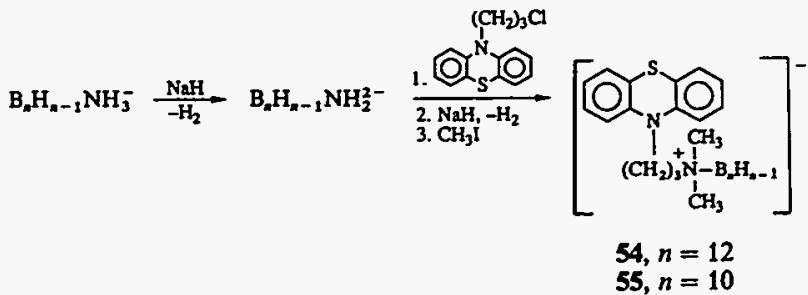

Scheme 28. Phenothiazine derivatives of the polyhedral $\mathrm{B}_{\mathrm{to}} \mathrm{H}_{10}^{2-}$ and $\mathrm{B}_{12} \mathrm{H}_{12}^{2-}$ ions, synthesized by Nakagawa and Aono [199] and evaluated by Mishima et al. [200].

though increased tumor destruction was observed in the presence of these compounds, their cell selectivity significantly differs from that of $\mathbf{4 1}$, and they were not pursued. The compounds 52 and 53 both carried their borane groups as coordination centers on the pendant dimethylaminopropyl group of 41. ${ }^{[199]}$ Soloway et al. ${ }^{[201\}}$ attached 1-(closo$\left.1,2-\mathrm{C}_{2} \mathrm{~B}_{10} \mathrm{H}_{11}^{-}\right)$carborane cages to the phenothiazine structure (Scheme 29). The anionic 7-(nido-7,8- $\left.\mathrm{C}_{2} \mathrm{~B}_{9} \mathrm{H}_{11}\right)$ carbor-
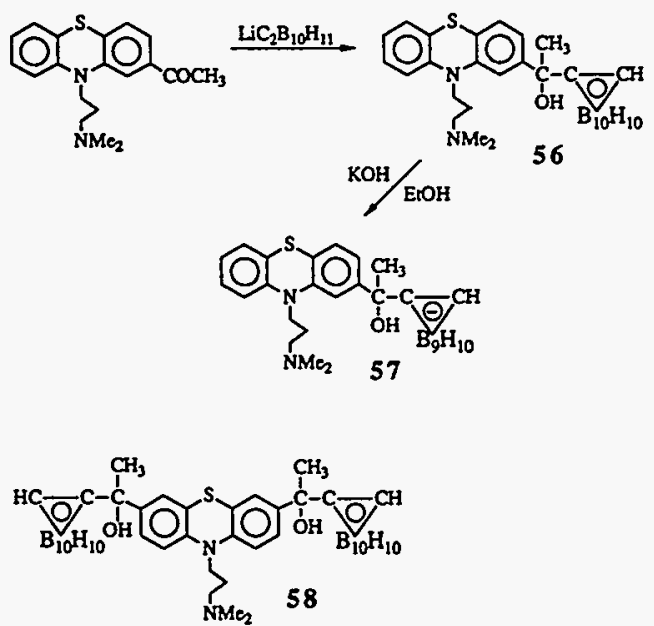

Scheme 29. Phenothiazine derivatives synthesized and evaluated by Soloway et a1. $[201,202]$.

ane derivative 57 was obtained by the usual route: removal of a $\mathrm{BH}^{2+}$ vertex from 56 with base. The compounds 56 through 58 gave preliminary in vivo evaluation results which suggested that they were too hydrophobic to be of interest, and efforts are underway to synthesize derivatives with improved water solubilities. ${ }^{\text {[202] }}$

The results reviewed above suggest that phenothiazine derivatives may have a place in BNCT if the search for a properly solvated compound is continued. Nonetheless, the fact that $\mathbf{4 1}$ and other phenothiazines can be localized only with preformed melanin puts limitations upon the actual therapeutic value of such a compound, even if it were discovered. It is interesting to note that research with phenothiazine derivatives has not been presented at either of the last two international meetings of the International Society for Neutron Capture Therapy convened in 1990 (Sydney) and 1992 (Columbus, Ohio). 
7.11. Approaches to the Selective Destruction of the Tumor Cell Nucleus

The spectacular development of the synthetic chemistry of nucleic acids and their derivatives ${ }^{[203]}$ has led to the discovery of new therapeutic agents and useful biological probes based upon modified synthetic nucleosides, mononucleotides, and oligonucleotides. Among these new DNA and RNA precursors are found species that serve as antiviral and anticancer agents either through enzyme inhibition or "antisense" activity, which blocks oncogene expression. ${ }^{[204]}$ Mono- and oligonucleotides have been synthesized which contain altered bases, sugars, and phosphorus-containing linker groups. It is, therefore, not surprising to discover research activities aimed at the modification of nucleotides by the incorporation of boron-containing motifs into all three of these essential structural components. While these boroncontaining nucleic acid components are important as such, they also offer new methodologies for the localization of boron in the tumor cell nucleus for purposes of BNCT.

Selectivity for nuclear accretion of boron in tumor cells is provided by the very rapid rate of tumor cell reproduction compared to that of normal cells. An important aspect of this observation is the fact that glioma cells undergo mitosis at a much greater rate than surrounding normal glial cells. Thus, it is possible that boron-containing species useful in BNCT and based upon DNA incorporation might be developed if such an ideal species were capable of Watson-Crick base-pairing, were not susceptible to nuclease activity, and could penetrate cell membranes. Alternatively, if a BNCT reagent were a cytotoxic enzyme inhibitor or "antisense" species, the effective lethality of BNCT would be further enhanced. As stated previously, the nucleus of a malignant cell is the target of highest value, since ${ }^{10} \mathrm{~B}$ is at least twice as effective in the cell nucleus as an equal quantity distributed throughout the cytoplasm.

Another approach to the intranuclear accretion of boron is the development of DNA ligand molecules that contain boron. ${ }^{[205.206]}$ The huge number of available DNA binding sites per tumor cell amplified by the very effective localization of the boron target atoms in the cell nucleus makes this an attractive approach.

The description of a representative variety of boron-containing nucleic acid components is presented in the following section. The mechanisms that provide cellular uptake of these species, where observed, is normally not known nor is their distribution within the cell's interior. Such problems are current research topics of great importance.

\subsubsection{Nucleosides Modified by Base Complexation with Cyanoborane, $\mathrm{BH}_{2} \mathrm{CN}$}

In keeping with their interest in synthesizing $\alpha$-amino acids in which single carbon atoms are formally replaced by an anionic boron atom having four valence electrons (see Section 7.9.3), Spielvogel et al. ${ }^{[207.208]}$ have modified the base residues present in representative nucleosides by complexation of specific nitrogen centers with the Lewis acid, $\mathrm{BH}_{2} \mathrm{CN}$. The attachment of $\mathrm{BH}_{2} \mathrm{CN}$ to the base residues was accomplished by the displacement of $\mathrm{Ph}_{3} \mathrm{P}$ from $\mathrm{Ph}_{3} \mathrm{PBH}_{2} \mathrm{CN}$ with a base nitrogen center present in $\mathrm{dG}, \mathrm{dI}, \mathrm{dA}$, and $\mathrm{dC}$ in which the hydroxyl groups are protected followed by deprotection of the nucleoside complex (Scheme 30). The $\mathrm{BH}_{2} \mathrm{CN}$ moiety was selectively placed on $\mathrm{N}^{7}$ of $\mathrm{dG}$ and $\mathrm{dI}, \mathrm{N}^{1}$ of $\mathrm{dA}$, and $\mathrm{N}^{3}$ of $\mathrm{dC}$. The site of coordination in both $\mathrm{dG}$ and $\mathrm{dI}$ is well

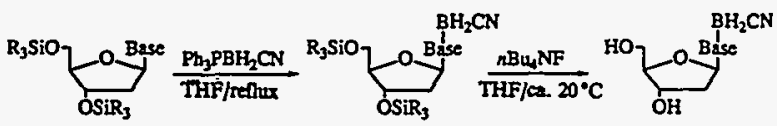

Bese $\cdot \mathrm{BH}_{2} \mathrm{CN}$ :

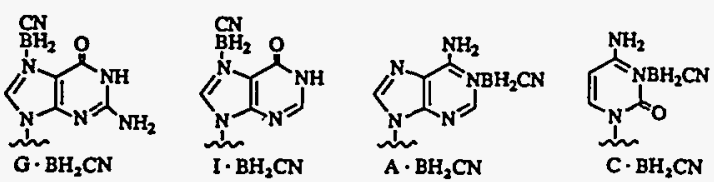

Scheme 30 . Nucleoside derivatives synthesized by Spielvogel et al., in which $\mathrm{BH}_{2} \mathrm{CN}$ is coordinated to base nitrogen atoms $[207,208]$.

separated from the sites involved in Watson-Crick basepairing. The $\mathrm{dG}-\mathrm{N}^{7}-\mathrm{BH}_{2} \mathrm{CN}$ adduct was shown by ${ }^{1} \mathrm{H} N M R$ experiments to form base pairs with $\mathrm{dC}$ as effectively as unmodified $\mathrm{dG}^{[208]}$ Attempts to prepare the $\mathrm{BH}_{2} \mathrm{CN}$ complex of $\mathrm{dT}$ were unsuccessful. In mice the $\mathrm{dC}-$ $\mathrm{N}^{3}-\mathrm{BH}_{2} \mathrm{CN}$ complex demonstrates biological activity as an inhibitor of inflammation, while dG-N $-\mathrm{N}^{7}-\mathrm{BH}_{2} \mathrm{CN}$ lowers serum cholesterol. ${ }^{[208]}$ Tumor localization in mice bearing Ehrlich ascites carcinoma was demonstrated with ${ }^{14} \mathrm{C}$ labeled $\mathrm{dC}-\mathrm{N}^{3}-\mathrm{BH}_{2} \mathrm{CN}$ and gave a tumor-to-blood concentration ratio of $8.5: 1$ four hours postinjection. ${ }^{1208)}$ The quantity of boron taken up by tumor was not determined.

\subsubsection{Nucleotide Analogs Containing Boranophosphate Linking Groups}

The formal replacement of the coordinated oxygen atom and its six valence electrons in a nucleotide phosphate linkage by a $\mathrm{BH}_{3}$ molecule results in the formation of a negatively charged boranophosphate moiety. Such modified nucleotide linkers are isoelectronic with uncharged methylphosphonate linking groups, which have found use in "antisense" constructs. ${ }^{[204]}$ The boranophosphates offer the advantage of a formal negative charge, largely on boron, and greater hydrophobicity than that presented by phosphate. The latter property is thought to be useful in assisting cellmembrane penetration. Modified nucleotides of the boranophosphate type were first prepared by Spielvogel et al. ${ }^{[208,209]}$ and proposed as boron delivery agents.

Scheme 31 describes the synthesis of typical boranophosphate oligonucleotides and suggests the feasibility of greatly extending the chains of these oligomers by the application of solid phase mediated synthesis methods.

The scope of the biological activity of compounds of the boranophosphate type, including cytotoxicity and enzymatic incorporation into DNA, is presently undergoing evaluation. ${ }^{[210]}$ Superior candidates for BNCT have not yet been identified. 


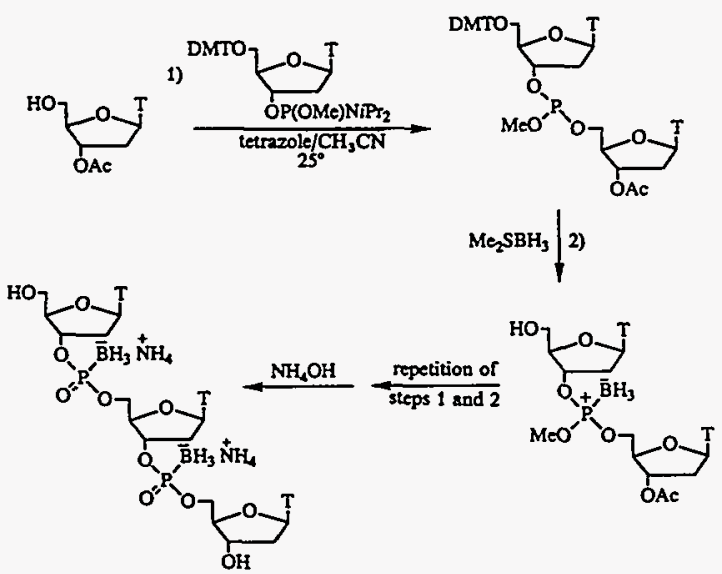

Scheme 31 . The synthetic route to nucleotides containing boranophosphate linking groups, which was devised by Spielvogel et al. [208,209].

\subsubsection{Boronic Acid Derivatives of Pyrimidine and Purine Nucleosides}

The first DNA precursor to be synthesized that contained a carbon-boron bond was 5-dihydroxyboryluracil (59, Liao et al. $\left.{ }^{[211]} 1964\right)$. This was followed by the synthesis of the 5-dihydroxyboryl-2'-deoxyuridine (DBDU, (60)) first described by Schinazi and Prusoff ${ }^{[212]}$ in 1978. The syntheses of 59 and 60 were accomplished by lithiation of suitable 5-bromo precursors in which the hydroxyl groups are protected, followed by reaction of the resulting lithium moiety with a trialkyl borate ester. ${ }^{[213]}$ The stereochemical similari-

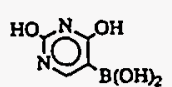

59

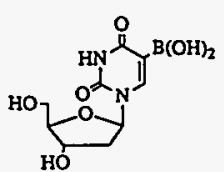

60 DBDU ty of 60 and natural thymidine (5-methyl-2'-deoxyuridine) suggested this synthesis. The possible modes of incorporation of 60 into the nucleus of tumor cells was envisioned by Schinazi et al. ${ }^{[214]}$ to possibly involve one or more of the following phenomena: 1) 60 is taken up selectively by malignant cells, and trapped within the cell as the $5^{\prime}$-monophosphate. This selectivity for tumor cells could be amplified if 60 were a better substrate for the thymidine kinase present in tumor cells than for the corresponding enzyme found in normal cells. 2) 60 could function as a inhibitor of enzymes involved in pyrimidine nucleoside biosynthesis. 3) 60 might become incorporated in DNA of tumor cells as a substitute for a pyrimidine nucleotide. 4) Any or all of the events listed above could provide ${ }^{10} \mathrm{~B}$-labeled 60 to the nucleus of the tumor cell for selective destruction through neutron capture. In principle, these mechanistic possibilities exist, to varying degrees, in every ${ }^{10} \mathrm{~B}$-labeled nucleoside or nucleotide molecule described here (Section 7.11).

Evaluation of the biological activity of 60 in vitro proved $^{[214]}$ that it was a good analog of thymidine and in- hibited the replication of HSV-1. This inhibition of viral replication was nullified by added thymidine. Preliminary in vitro neutron irradiation experiments with solutions of ${ }^{10} \mathrm{~B}$ enriched 60 containing varying amounts of thymidine and V79 chinese hamster cells (washed) were carried out at the Brookhaven Medical Research Reactor. Pre-equilibration of the subject cells with 60 decreased their survival rate upon exposure to thermal neutrons, and the addition of thymidine to the $\mathbf{6 0}$ solution mitigated this effect. These results suggested to the authors that 60 was bound during DNA synthesis, but that it could not effectively compete with thymidine.

Using newly developed synthesis methods Yamamoto has obtained a wide variety of new base and nucleoside derivatives that contain either a 3- or 4-(dihydroxyboryl)phenyl ${ }^{[215]}$ or an $\alpha$-hydroxy-4-(dihydroxyboryl)benzyl ${ }^{[216]}$ substituent. Representative syntheses are outlined in Scheme 32 . The dihydroxyboryl derivative prepared by these

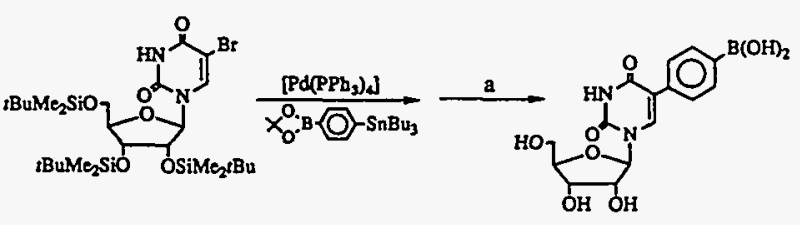

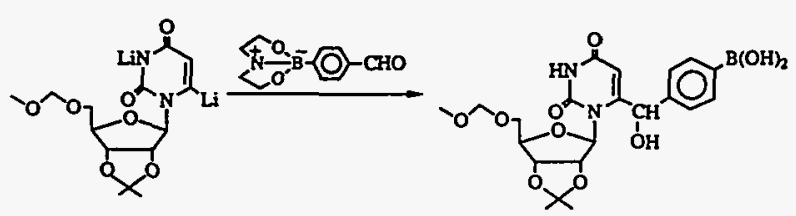

Scheme 32. Examples of two general synthetic routes leading to dihydroxyboryl derivatives of nucleosides, demonstrated by Yamamoto et a]. $[215,216]$. a: Deprotection.

new procedures are numerous; however, they are most likely susceptible to acid-catalyzed deboronation to form the corresponding phenyl- or $\alpha$-hydroxybenzyl-substituted nucleoside and boric acid. In addition, the added aryl groups will increase the hydrophobicity of the desired nucleosides with unpredictable results. Selected members of this group of nucleosides are undergoing evaluation with respect to their biological activities including efficacy in BNCT.

\subsubsection{Carborane-Substituted Nucleosides}

The relatively simple compounds described in the previous section which contain the dihydroxyboryl function are low in boron content and subject to hydrolytic deboronation reactions. As an alternative to these simpler boron compounds, the closo-1,2- $\mathrm{C}_{2} \mathrm{~B}_{10} \mathrm{H}_{12}$ carborane has been functionalized to produce carborane-substituted ribose and base components of nucleosides. These species may become trapped in the nucleus of the tumor cell as described in Section 7.11.3. Whereas the boron-rich closo-carborane substituent will markedly decrease the hydrophilicity of the substrate to which it is attached, conversion of the closo-carborane structure to the corresponding anionic nido-carborane structure 
may produce an amphiphilic derivative. Because the carborane substituent is comparatively large and has hydrophobic properties, it would not be expected that carborane-substituted nucleotides would actually become incorporated as surrogates in oligomeric DNA or RNA molecules of natural origin. However, intranuclear phosphorylation of a carboranyl-substituted nucleoside may serve to immobilize the resulting monophosphate within the nucleus.

Soloway et al. ${ }^{[217]}$ recently described the first carboranesubstituted nucleoside, 2'-O-(1,2-carboran-1-ylmethyl)uridine (61), which has been designated CBU-2' by its discoverers. Both the $3^{\prime}-O$ - and $5^{\prime}-0-(1,2-$-carboran-1-ylmethyl)uridine isomers have been described, ${ }^{[218]}$ as well as the anionic nido-carborane analog of 61. More recently, Tjarks et al. have described ${ }^{[219]}$ what may become a general synthesis of nucleosides, which they demonstrated with the synthesis of 61 (Scheme 33). The key precursor in these po-

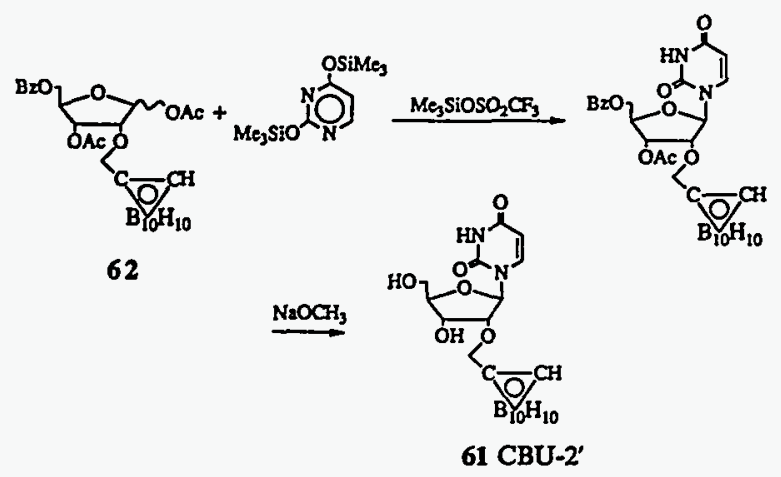

Scheme 33. An example of the generalized synthesis of carborane derivatives of nucleosides by Tjarks et al. in which 62 is a common precursor [219].

tential syntheses is $1,3-$ di- $O$-acetyl-5- $O$-benzoyl-2-O-(1',2'carboran-1'-ylmethyl)-D-ribofuranose (62), which has been shown to afford 61 by reaction with trimethylsilyl-protected uracil in the presence of trimethylsilyl trifluromethanesulfonate followed by alkaline hydrolysis. Only the $\beta$-configuration was observed at the 1-position of the ribose moiety. One hopes that this synthesis route will find wide application.

The carborane-substituted nucleoside 61 and its $3^{\prime}$ and $5^{\prime}$ isomers were efficiently taken up by F98 glioma cells in vitro. ${ }^{[218.220]}$ Morrison et al. ${ }^{[221]}$ have recently shown by SIMS (see Section 2.5.4) that the concentration of 61 in F98 glioma is higher in the cytoplasm than in the cell nucleus by a factor which varies from 1.4 to 2.6. The mechanism of cellular localization of $\mathbf{6 1}$ is under investigation. ${ }^{\text {(222) }}$

Yamamoto et al. ${ }^{[223]}$ has recently developed a route to uridine and 2'-deoxyuridine derivatives bearing either a 1,2carboran-1-yl or 2-hydroxymethyl-1,2-carboran-1-yl substituent in the 5-position of the uracil ring. These syntheses were initiated by using the Pd-catalyzed coupling of 5iodouridine or 5-iodo-2'-deoxyuridine in which the hydroxyl groups are protected with $\mathrm{HC} \equiv \mathrm{C}-\mathrm{SiMe}_{3}$ or $\mathrm{HC} \equiv \mathrm{C}-$ $\mathrm{CH}_{2} \mathrm{OAc}$. The resulting 5-alkynyl nucleoside derivatives were converted into the desired products, for example 64 , by the usual reaction with $\mathrm{B}_{10} \mathrm{H}_{14}$ and a Lewis base. Prelimi-

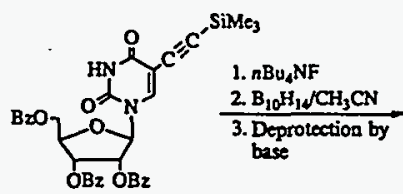

63

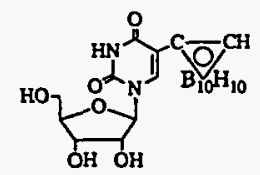

64 nary findings indicate that 5-carboranyluridine is cytotoxic against P-388 murine leukemia cells in vitro. Schinazi et al. ${ }^{[224]}$ have independently synthesized 5-carboranyl-2'-deoxyuridine (65) by a route similar to that reported by Yamamoto et al. Schinazi's group has studied the metabolites produced in CEM cells treated with 65. A new metabolite was isolated which displays the biochemical properties expected for a $5^{\prime}$-monophosphate of 65 , which is believed to arise by intracellular phosphorylation. ${ }^{[224]}$

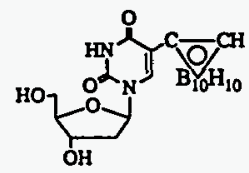

A synthesis of racemic 5-carboranyl-2',3'-dideoxy-3'-thiacytidine (6) has been developed by using the Pd-catalyzed alkynylation of 5-iodo-2', $3^{\prime}$-dideoxy-3'-thiacytidine. ${ }^{[224]}$ This and related compounds are undergoing biological evaluation.

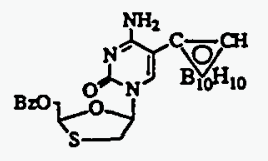

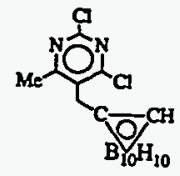

Reynolds et al..$^{[225]}$ have described the synthesis of 5-(1,2carboran-1-ylmethyl)-2,4-dichloro-6-methylpyrimidine (67), which may prove to be useful as the synthetic equivalent of substituted pyrimidines.

\subsubsection{Intranuclear Localization of Boron with DNA Ligands}

The possibility of localizing boron in the DNA of target cells by complexing it with boron-containing ligand molecules has recently been investigated by Whittaker et al. [205] and Corder et al. ${ }^{[206]}$ Dibenzimidazole reagents from Hoechst effectively bind to the minor groove of nuclear DNA, and a variety of reagents based upon this ligand sys-

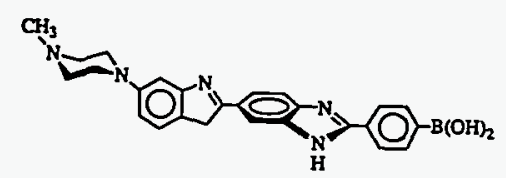

68 
tem have been developed. The simple phenylboronic acid derivative 68 has been the subject of preliminary in vitro investigations of cellular boron uptake. Fluorescence microscopy and spectrophotometric analyses of the ligand in isolated nuclei have demonstrated uptake of 68 by a variety of viable cells. Neutron capture experiments demonstrated enhanced cell killing in the presence of $2 \times 10^{-5} \mathrm{M} 68$. The observed effect was equivalent to that obtained with a solution of $4 \times 10^{-6} \mathrm{~g}{ }^{10} \mathrm{~B}(\mathrm{OH})_{3}$ per $\mathrm{mL}$ in contact with the cells during irradiation. This result was viewed as quite encouraging, and work is underway with other boron-containing ligands carrying several ${ }^{10} \mathrm{~B}$ atoms per ligand.

\subsubsection{Selective Delivery of Boron-Containing Species to the Nucleus}

Scheme 5 depicts the transport processes involved in bringing a boron-containing agent from blood serum into the nucleus of a tumor cell. Each of the methods outlined in Section 7.11.3 for the intranuclear accretion of boron depends upon the enhanced rate of mitosis of tumor cells, as opposed to normal cells, to provide the required bias for selective tumor cell destruction. In addition, the fact that the walls of malignant cells are penetrated may place structural limitations upon the reagent to be delivered that mitigates its desired function of intranuclear localization. These problems may be circumvented by coupling intranuclear reagents to delivery systems that are known to be biased toward malignant cells. Of the available tumor-selective delivery methods discussed in this review, unilamellar liposomes (Section 7.7.1) are distinguished by their apparent effectiveness in selectively penetrating plasma membranes of tumor cells and by their ability to carry a wide variety of hydrophilic species to the cell's interior. Hydrophilic bases, nucleosides, and nucleotides of all sizes that contain appreciable numbers of boron atoms per molecule are candidates for this mode of delivery. Such research has been initiated in my laboratory.

\section{The Needs and Prospects of BNCT}

In principle, Locher's original proposal has been demonstrated. BNCT offers great promise for the treatment of cancers not amenable to current management and characterized by high mortality rates such as cerebral glioma and metastatic melanoma. The war on cancer has evolved into trench-warfare, and therapeutic methods that depart from presently accepted therapeutic modalities must be brought to bear if progress is to be resumed. As a therapeutically unique, but superficially complex method, BNCT stands out as just such an opportunity if the research momentum of the present is further increased and applied to the problems that have been identified in this review.

The foremost prerequisite for the adaptation of BNCT as a readily accessible medical tool available for the treatment of patients from the general population requires that pure epithermal neutron beams must be made available from economical sources other than nuclear reactors and placed in urban areas. Such advanced neutron generators could be based upon the spallation of neutrons from a metallic target by accelerated protons (Section 2.3.2). Such devices could possibly be available in prototype form within two to five years.

Also associated with the clinical practice of BNCT is the potential use of real-time NMR imaging techniques (MRI), which employ ${ }^{10} \mathrm{~B}$ as the active nucleus, to visualize and quantify ${ }^{10} \mathrm{~B}$-loaded tumor and surrounding tissues (Section 2.5.1). Such a rapid and reliable noninvasive method of obtaining data on ${ }^{10} \mathrm{~B}$ targets would be invaluable for determining the required neutron dose, orienting the patient in the epithermal beam, and monitoring changes that occur at the tumor site between the application of fractional doses. Work is now in progress with the more NMR amenable ${ }^{11} \mathrm{~B} \mathrm{nu}$ cleus, which, if successful, will be applied to the development of a more valuable ${ }^{10} \mathrm{~B}$ MRI capability.

The outline of biodelivery modes presented in Scheme 6 is based upon data of variable reliability. Consequently, the precise determination of the positioning of ${ }^{10} \mathrm{~B}$ target nuclei both on, or in, malignant cells is vital to the intelligent design of new boron-containing compounds. While the subcellular distribution of boron may now be visualized (Section 2.5.4) if the subject cells are derived from an in vitro source, it would be especially useful to have similar data available from cells harvested from in vivo experiments. Data now available from in vitro preparations have been extremely enlightening and prompt further elaboration.

As seems likely, future development of boron-containing compounds will be increasingly directed toward systems designed to enter the tumor-cell nucleus on a cell-selective basis (Section 7.11). In addition to the biosyntheses of DNA or other biochemical processes that take place in the nucleus, the requirement of bringing the molecular tools such as boron-containing DNA precursors, DNA ligands, or antisense species to bear selectively upon malignant cells remains a major challenge. Receptor-mediated species, which may provide a selective means of penetration of the plasma membrane, might be coupled to the nucleus-binding reagent and enzymatically decoupled following cell entry. Cellular selectivity would be provided by the enhanced rate of mitosis and overexpression of receptor sites characteristic of tumor cells. On the other hand, it might prove possible to deliver the nucleus-binding reagent to the cell's cytoplasm through tumor-selective liposomes (Section 7.7.1) followed by migration of the reagent to the nucleus. Regardless of the novelty and elegance of new reagents that specifically interact with nuclear components, the problem of assuring selective binding to tumor celis demands consideration during the conception of the boron-containing agent itself.

The future development of BNCT clearly depends upon the design and synthesis of boron-containing species capable of extensive accretion within tumor cells and the related bioutilization systems that assure their selective delivery to malignant cells. The discoveries of modern microbiology and immunology that are related to practical bioutilization pathways (Section 6) have appeared at such a great rate that the corresponding chemical research effort may have fallen behind. A plea is made here for an extensive expansion of synthesis efforts aimed at the development of new synthesis methodology in the organoborane area and at its interface with bioorganic chemistry. A need exists for boron-contain- 
ing molecules and oligomers that may be coupled to biodelivery molecules in a modular fashion. Similarly, modular groups that may be used to overcome the innate hydrophobicity of the closo-carborane and the amphiphilic nido-anion substituents are needed.

The proper biological evaluation of a candidate boroncontaining system is vital to the correlation of chemical structure with biological activity and provides the guidance for further molecular design and synthesis efforts. It may very well be, as shown by recent work with boron-containing nucleosides, that aside from obvious applications in BNCT, some of the new compounds may have biological response profiles that make them desirable for other important applications. The work of Schinazi, Soloway, Spielvogel, and Yamamoto (Sections 7.9.3 and 7.11) provide examples of antiviral, anti-inflammatory, antineoplastic, and hypolipidemic compounds originally developed for BNCT research.

At this time, the concept of BNCT is closer to realization than ever before. New clinical trials with the thioborane anion BSH and dihydroxyborylphenylalanine (BPA, 33) are beyond the advanced planning stages in Europe and the United States. Trials with melanoma by Mishima and his colleagues continue in Japan, as do the therapeutic procedures of Hatanaka. It must be pointed out, however, that the clinical trials now in progress and those to be initiated in the near future, by necessity, employ boron delivery agents such as BSH (Section 7.1) and BPA (Section 7.9.1). These are not truly high-performance compounds, but they are reasonably well understood and offer reliability. The tumors to be attacked are cerebral gliomas and primary melanoma lesions; the nuclear reactors at Petten and MIT will serve as neutron sources.

Longer range plans for BNCT must proceed irregardless of the upcoming clinical trials, since by the time these trials are completed greatly improved boron delivery agents and new neutron sources could be available, provided increased financial support from both government and industry is forthcoming. It seems certain that the future of BNCT will brighten as more medical practitioners become aware of its virtues. The meeting of knowledge derived from physics, chemistry, biology, and medicine at one point in the multidimensional field of science is a unique circumstance that cries out for exploitation by mankind. Perhaps it was really planned!

I wish to thank my students, postdoctoral associates, and colleagues, both past and present, for their contributions to our group's efforts in BNCT chemistry, and boron cluster chemistry in general. Drs. Debra A. Feakes and Kenneth J. Shelly have made vital contributions to this article by collecting information and critically reviewing the product. I am grateful to Mrs. Diane Gromelski for the preparation of the manuscript, Mr. Albert Calleros for the artwork, and Karin Drechsel for proofreading. I also wish to thank my many friends in the $B N C T$ community for stimulation and the National Institutes of Health (RO1-CA31753 and RO1-CA53870) and the U.S. Department of Energy Idaho National Engineering Laboratory, Office of Energy Research, under DOE Field Office, Idaho, Contract DE-ACO7-761DO1570 for their support of our $B N C T$ research efforts.

Received: March 1, 1993 [A 910IE] German version: Angew. Chem. 1993, 105, 997
[1] J. Chadwick, Nature 1932, 129,312

[2] J. R. Dunning, G. B. Pegram, G. A. Fink, Phys. Rev. 1935, 47, 325.

[3] E. Fermi, O. Amaldi, F. D'Agostino, F. Rasetti, E. Segre, Proc. R. Soc. London $A$ 1934, 146, 483

[4] I. R. Dunning, G. B. Pegram, G. A. Fink, D. P. Mitchell, Phys. Rev. $1935,48,265$.

[5] H. J. Taylor, Proc, R. Soc London A 1935, 147, 873.

[6] G. L. Locher, Am. J. Roentgenol. Radium Ther. 1936, 36, 1.

[7] a) M. Javid, G. L. Brownell, W. H. Sweet, J. Clin. Invest. 1952, 31, 603 b) W. H. Sweet, N. Engl. J. Med. 1951, 245,875; c) W. H. Sweet, M. Javid. J. Neurosurg. 1952, 9, 200; d) L. E. Farr, W. H. Sweet, J. S. Robertson, C. G. Foster, H. B. Locksiey, D. L. Sutherland, M. L. Mendelsohn, E. F Stickley, Am. J. Roenigenol. Radium Ther. Nucl. Med. 1954, 71, 279; e) J. T. Godwin, L. E. Farr, W. H. Sweet, J. S. Robertson, Cancer 1955, \&, 601.

[8] 2) Proc. Third Int. Symp. Neutron Capture Ther. Cancer (Strahlenther. Onkol. 1989, 165); b) R. M. Brugger, J. A. Shih in [8z], p. 153; c) R. F. Martin, G. D'Cunha, M. Pardec, B. I Allen, Int. J. Radiat. Biol. 1988, 54, 205.

[9] a) Boron-Neutron Capture Therapy for Tumors (Ed.: H. Hatanaka) Nishamura, Nigata, Japan, 1986); b) H. Hatanaka in (9a], p. 5.

[10] R. G. Fairchild, V. P. Bond. Int. J. Radiat. Oncol. Biol. Phys. 1985, 11, 831

[11] F. Pfister, G. Hehn, F. El-Husseini in [8a], p. 107.

[12] a) Abstr. Fifth Int. Symp. Neutron Capture Ther. Cancer 1992; b) R Huiskamp, P. R. Gavin, A. S. Siefert, K. H. I. Phillips in [12a], p. 23.

[13] a) Proc. Second Int. Symp. Neutron Copture Ther. Concer (Neutron Capture Therepy (Ed.: H. Hatanaka), Nishamura, Nigata, Japan, 1986); b) R. M. Brugger, T. J. Less in [13a], p. 110.

[14] R. F. Barth, A. H. Soloway, R. G. Fairchild, Cancer Res. 1990, 50, 1061.

[15] a) Proc. Workshop Neutron Capture Ther. (Brookhaven Nat. Lab. Rep. 1986, BNL 51995; b) R. M. Brugger, T. J. Less, G. G. Passmore in [15a] p. 32 .

[16] a) Proc. Fourth Int. Symp. Neutron Capture Ther. Cancer (Progress in Neutron Capture Therapy for Cancer (Ed.: B. J. Allen, D. E. Moore, B. V. Harrington), Plenum, New York, 1992); b) V. Benary, R. G. Fairchild, Kalef-Ezra, D. Greenberg, Y. Kamen, S. Fiarman, L. Wielopolski in Kalef-Ezra,

[17] a) D. W. Nigg, F. J. Wheeler, P. D. Randolf in [16a], p. 145; b) F. J. Wheeler, M. L Griebenow, D. E. Wessol, D. W. Nigg in [8a], p. 186

(18] J. C. Yanch, O. K. Harling in [16a], p. 133.

[19] J. C. Yanch, X-L. Zhou, R. E. Shefer, R. E. Klinkowstein, H. Zhuh in [12a], p. 45.

[20] C.-K. C. Wang. T. E. Blue, R. A. Gabbauer in [8a], p. 75.

[21] a) J. C. Yanch, X.-L. Zhou, R. E. Shefer, R. E. Klinkowstein, G. L. Brownell in [16a], p. 107; b) T. E. Blue, T.X. B. Que, R. N. Christensen, P. Guo, J. W. Blue in [16a], p. 113; c) R. E. Shefer, R. E. Klinkowstein, J. C. Yanch, G. L. Brownell in (16a], p. 119; d) E. Grusell in [16a] p. 123 e) J. F. Crawford, H. Reiste, H. Conde, K. Elmgren, T. Ronnqvist, E Grusell, B. Nillsson, O. Pettersson, P. Stromberg, B. Larsson in [16a] p. 129.

[22] R. G. Zamenhof, B. W. Murray, G. L. Brownell, G. R. Wellum, E. I. Tolpin, Med. Phys. 1975, 2, 47.

[23] R. G. Fairchild in [9a], p. 24

[24] P. R. Gavin, R. Huiskamp, F. I. Wheeler, M. L. Griebenow in [12a], p. 34.

[25] D. W. Nigg. F. J. Wheeler, P. D. Randolph in [16a], p. 145.

[26] F. J. Wheeler, C. A. Atkinson, P. R. Gavin in [16a], p. 137.

[27] a) R. F. Barth, A. H. Soloway, R. G. Fairchild, Cancer Res. 1990, 50 1061; b) Proc. First Int. Symp. Neutron Capture Ther. (Brookhaven Nat. Lab. 1983, BNL 51730); c) R. G. Fairchild, V. P. Bond in (27 b], p. 1.

[28] The author is indebted to D. W. Nigg and F. J. Wheeler of the Idaho National Engineering Laboratory for these data.

[29] A. Wambersie, L. E. Feinendegen, Clinical Aspects of Neutron Capture Therapy (Ed.: R. G. Fairchild, V. P. Bond, A. D. Woodhead), Plenum, New York. 1989, p. 277

[30] D. Gabel, S. Foster, R. G. Fairchild, Radiat. Res. 1987, 111, 14

[31] M. Ljungberg, S.-E. Strand, J. Nucl. Med. 1990, 31, 493.

[32] R. J. Paxton, B. G. Beatty, M. F. Hawthome, A. Varadarajan, L. E Williams, F. L. Curtis, C. B. Knobler, J. D. Beatty, J. E. Shively, Proc Natl. Acad. Sci. USA 1991, 88, 3387.

[33] G. W. Kabalka, M. Davis, P. Bendel, Magn. Reson. Med. 1988, 8, 231.

[34] T. L. Richards, K. M. Bradshaw, D. M. Freeman, C. H. Stoak, P. R Gavin in [8a], p. 179.

[35] K. M. Bradshaw, T. L. Richards, S. L. Kraft in [16a], p. 325.

[36] K. M. Bradshaw, M. P. Schweizer, G. H. Glover, J. K. Thomas in [12 a] p. 52.

[37] a) S. R. Tamat, D. E. Moore, B. J. Allen, Anal. Chem. 1987, 59, 2161; b) W. F. Bauer, J. A. Wishard, C. Rae, N. Lassahn in [16a], p. 297

[38] a) R. F. Barth, A. H. Soloway, D. M. Adams, F. Alam, A. K. M. Anisuz zaman in [16a], p. 305; b) R. F. Barth, D. M. Adams, A. H. Soloway, E. B. Mechetner, F. Alam, A. K. M. Anisuzzaman, Anal. Chem. 1991, 63, 890.

139] R. Rogus, O. K. Harling, I. Olmez, S. Wirdzek in [16a], p. 301

[40] T. Matsumoto, O. Aizawa in [16a], p. 313. 
[41] Y. Nakagowa, T. Kobayashi, Y. Ueno, H. Hatanoka, K. Mukai, K. Matsumoto in [16a], p. 283.

[42] M. Abe, K. Amano, K. Kitamura, J. Tateshi, H. Hatanaka, J. Nucl. Med. $1986,27,677$.

[43] D. Gabel, H. Holstein, B. Larsson, L. Gille, G. Erikson, D. Sacker, P. Som, R. G. Fairchild, Cancer Res. 1987, 47, 5451.

[44] J. E. Woollard, Y. Jiang, J. F. Curran. T. E. Blue, R. F. Barth in [16a], p. 317

[45] B. D. Bennett, X. Zha. I. Gay, G. H. Morrison, Biol. Cell (1981) 1992 $74,105$.

[46] X. Zha, B. D. Bennett, W. A. Ausserer, G. H. Morrison in [16a], p. 331.

[47] R. D. Leapman, R. L. Ormberg, Ultramicroscopy 1988, 24, 251.

[48] D. E. Moore, J. R. Stretch, A. L. Dawes, D. J. H. Cockayne, B. J. Allen, G. Constantine in [16a], p. 335 .

[49] For the history of BNCT, see: D. N. Slatkin, Brain 1991, 114, 1609.

[50] $\operatorname{Sec}[7 b]$.

[51] $\operatorname{Sec}[7 \mathrm{c}]$.

[52] A. H. Soloway, personal communication, 1958.

[53] W. H. Sweet, M. Javid, Trans. Am. Neurol. Assoc. 1951, 60.

[S4] Sec also [7d].

[SS] L. E. Farr, W. Haymaker. T. Konikowski, S. W. Lippincott. Int. J. Neurol. 1962, 3, 564 .

[56] I. D. Archarnbeau, Radiology 1970, 94, 179.

[57] A. H. Soloway, R. L. Wright, J. R. Messer, J. Pharm. Exp. Ther. 1961, $134,117$.

[58] a) M. F. Hawthorne, A. R. Pitochelli, J. Am. Chem. Soc. 1959, 81, 5519; b) W. N. Lipscomb, A. R. Pitochelli, M. F. Hawthome, ibid. 1959, 81, 5833.

[59] a) A. R. Pitochelli, M. F. Hawthome, J. Am. Chem. Soc. 1960, 82, 3228; b) H. C. Miller, N. E. Miller, E. L. Muetterties, ibid. 1963, 85, 3885

[60] a) T. L. Heying, J. W. Ager, S. L. Clark, D. J. Mangold, H. L. Goldstein. M. Hillman, R. J. Polak, J. W. Szymanski, Inorg. Chem. 1963, 2, 1089; b) D. Grafstein, J. Dvorak, ibid. 1963, 2,1128.

[61] A. H. Soloway, H. Hatanaka, M. A. Davis, J. Med. Chem. 1967, 10, 714.

[62] A. K. Asbury, R. G. Ojean, S. L. Nielsen, W. H. Sweet, J. Neuropathol. Exp. Neurol. 1972, 31, 278.

[63] H. Hatanaka, S. Kamano, K. Amano, S. Hojo, K. Sano, S. Egawa, H. Yasukochi in [9a], p. 349.

[64] H. Hatanaka, Y. Urano in [9a], p. 381

(65) H. Hatanaka, K. Amano, S. Kamano, K. Sano in [13a], p. 447.

[66] H. Hatanaka, J. Neurol. 1975, 209, 81.

[67] W. F. Bauer, K. M. Bradshaw, T. L. Richards in [16a], p. 339.

[68] G. R. Wellum, E. I. Tolpin, A. H. Soloway, A. Kaczmarezyk, Inorg. Chem. 1977, 16, 2120

[69] E. I. Tolpin, G. R. Wellum, S. A. Berley, Inorg. Chem. 1978, 17, 2867.

[70] D. Slatkin, P. Micca, A. Forman, D. Gabel, L. Wielopolski, R. Fairchild, Biochem. Pharmacol. 1986, 35, 1771.

[71] M. F. Hawthome, R. L. Pilling, The Preparation of the $B_{10} H_{10}^{2-}-I o n$ (Inorg. Synth. 1967, 9, 16).

[72] See $[60 \mathrm{a}, \mathrm{b}]$

[73] R. N. Grimes, Carboranes, Academic Press, New York, 1970.

[74] E. L. Muetterties, W. H. Knoth, Polyhedral Boranes, Dekker, New York, 1968.

[75] G. B. Dunks, M. F. Hawthorne, Ace. Chem. Res. 1973, 6, 124.

[76] W. H. Knoth, J. Am. Chem. Soc, 1967, 89, 1274.

[77] J. H. Morris, S.-A. Khan, F. Mair, G. Peters in [16a], p. 215

[78] M. F. Hawthorne, Acc. Chem. Res. 1968, 1, 281

[79] M. F. Hawthorne, G. B. Dunks, Science 1972. 78, 462.

[80] R. N. Leyden, B. P. Sullivan, R. T. Baker, M. F. Hawthorne, J. Am. Chem. Soc. 1978, 100,3758

[81] W. N. Lipscomb, Boron Hydrides, W. A. Benjamin, New York, 1963.

[82] K. Wade, Eleciron Deficient Compounds, Appleton-Century-Crofts, New York, 1971.

[83] Boron Hydride Chemistry (Ed.: E. L. Muetterties), Academic Press, New York, 1975.

[84] Metal Interactions with Boron Clusters (Ed.: R. N. Grimes), Plenum, New York, 1982.

[85] P. Lemmen, B. Werner in [12a], p. 9.

[86] a) P. A. Radel. S. B. Kahl in [12a], p. 5; b) S. Sjōberg, M. F. Hawthorne, P. Lindstrom, J. Malmquist, J. Carlsson, A. Andersson, O. Pettersson in [12a], p. 2.

[87] M. Ichihashi, T. Nakanishi, Y. Mishima, J. Invest. Dermatol. 1982, 78, 215.

[88] R. F. Schinazzi, W. H. Prusoff, Tetrahedron Letl. 1978, 4981

[89] B. F. Spielvogel, A. Sood, B. R. Shaw, I. H. Hall, Pure Appl. Chem. 1991, 63,415 .

[90] K. Shelly, D. A. Feakes, M. F. Hawthorne, P. G. Schmidt, T. A. Krisch, W. F. Bauer, Proc. Natl. Acad. Sci. USA 1992, 89, 9039.

[91] a) B. H. Laster, S. B. Kahl, E. A. Popenoe, D. A. Pate, R. G. Fairchild, Cancer Res. 1991, 51, 4588; b) S. B. Kahl, D. W. Pate in [12a], p. 10.

[92] R. M. Straubinger, D. Papahadjopoulis, K. Hong, Biochemistry 1990, 29, 4929.

[93] R. K. Jain, L. E. Gerlowski, Crit. Rev. Oncol. Hematol. 1986, 5, 115.

[94] B. A. Allison, E. Waterfield, A. M. Richter, J. T. Ledy, Photochem. Photo blol. 1991, 54, 709

[95] W. H. Knoth, J. C. Sauer, D. C. England, W. R. Hertler, E. L. Muetterties. J. Am. Chem. Soc. 1964, 86. 3973.
[96] W. H. Knoth, J. Am. Chem. Soc. 1966, 88, 935.

[97] D. Haritz, K. Piscol, D. Gabel in [16a], p. 557.

[98] D. N. Slatkin, D. D. Joel, R. G. Fairchild, P. L. Micca, K. M. Nawrocky. B. H. Laster, J. A. Coderre, C. C. Finkel, C. E. Poletti, W. H. Sweet, Basic Life Sei. 1989, S0, 179.

[99] R. G. Fairchild, S. B. Kahl, B. H. Laster, J. Kalef-Ezra, E. A. Popenoe, Cancer Res. 1990, 50, 4860.

[100] J. A. Coderre, M. S. Makar, P. L. Micea, M. M. Nawrocky, D. D. Joel, D. N. Slatkin in [12a], p. 26

[101] M. Komura, H. Nakai, M. Shiro, J. Chem. Soc. Dalton Trans. 1987, 1953.

[102] W. F. Bale, I. L. Spar, Adv. Biol. Med. Phys. 1967, S, 286.

[103] E. I. Tolpin, G. R. Wellum, F. C. Dohan, P. L. Kornblith, R. G. Zamenhof, Oncology 1975, 32, 223.

[104] R. F. Barth, N. Mafune, F. Alam, D. M. Adams, A. H. Soloway, G. E. Makroglow, O. A. Oredipe, T. E. Blue, Z. Steplewski in [8a], p. 142.

[105] G. Köhler, C. Milstein, Nature 1975, 256, 495; see also G. Köhler, Angew. Chem. 1985, 97, 829; Angew. Chem. Int. Ed. Engl. 1985, 24, 827 (Nobel Lecture).

[106] a) E. Mizusawa, H. L. Dahlman, S. J. Bennett, D. M. Goldenberg, M. F. Hawthorne, Proc. Natl. Acad. Sci. USA 1982, 79, 3011; b) D. M. Goldenberg. R. M. Sharkey. F. J. Primus, E. Mizusawa, M. F. Hawthorne, ibid. $1984,81,560$.

[107] For an example A. R. Horwitz, C. P. Chang, M. Better, K. E. Hellstrom, R. R. Robinson, Proc. Natl. Acad. Sci. USA 1988, 85, 8678.

[108] For a perspective of immunochemistry till 1968, see D. Pressman, A. L. Grossberg, The Structural Basis of Antibody Specificity, W. A. Benjamin, New York, 1968.

[109] a) A. G. Mallinger, E. L. Jozwiak, J. C. Carter, Cancer Res. 1976, 32, 1947; b) E. I. Tolpin, H. S. Wong, W. N. Lipscomb, J. Med. Chem. 1974, 17, 792; c) R. L. Szeath, A. H. Soloway, A. S. Dey, ibid. 1974, 17, 796; d) P. M. Jacobs, R. L. Sneath, A. H. Soloway, A.S. Dey, J. Pharm. Sci. 1976, 65, 604; e) R. L. Sneath, J. E. Wright, A. H. Soloway, S. M. O'Keefe, A. S. Dey, W. D. Smolnycki, J. Med. Chem. 1976, 19, 1290.

[110] M. F. Hawthorne, T. E. Berry, P. A. Wegner, J. Am. Chem. Soc. 1965, 87, 4746.

[111] M.F. Hawthome, R. J. Wiersema, J. Med. Chem. 1972, 15, 449

(112] a) E. A. Mizusawa, M. R. Thompson, M. F. Hawthorue, Inorg. Chem. 1985, 24, 1911; b) A. Varadarajan, R. M. Sharkey, D. M. Goldenberg. M. F. Hawthome, Bioconjugate Chem. 1991, 2, 102.

[113] J. L. Maurer, F. Berchier, A. J. Serino, C. B. Knobler, M. F. Hawthorne, J. Org. Chem. 1990, 55, 838 and previous publications in this series.

[114] F. Alam, A. H. Soloway, R. F. Barth, N. Mafune, D. M. Adams, W. H. Knoth, J. Med. Chem. 1989, 32, 2326.

[115] S. R. Tamat, A. Patwardhan, D. E. Moore, A. Kabral, K. Bradstock, P. Hersey, B. J. Allea in [8 a], p. 145.

[116] R. Abraham, R. Müller, D. Gabel in [8a]. p. 148

[117] R. F. Barth, A. H. Soloway, D. M. Adams, F. Alam in [16a], p. 265.

[118] a) Boron Compounds Suitable for Neutron Capture Therapy for the Treatment of Cancer (Workshop) (Ed.: A. H. Soloway), Annapolis, MD, USA, 1988; b) M. F. Hawthome, A. Varadarajan, J. L. Maurer in [118a]

[119] M. F. Hawthome, Pure Appl. Chem. 1991, 63, 327.

[120] A. Varadarajan, M. F. Hawthorne, Bioconjugale Chem. 1991, 2, 242.

[121] R. J. Paxton, B.-G. Beatty, A. Varadarajan, M. F. Hawthorne, Bioconjugate Chem. 1992, 3, 243.

[122] M. F. Hawthorne, R. R. Kane, C. S. Lee, C. L. Coe, M. A. St. Rose in [12a], p. 14.

[123] M. F. Hawthorne, R. R. Kane, unpublished results.

[124] C. H. Chang, C. N. Ahlem, B. Wolfert, S. M. Hochschwender, R. Jue, J. M. Frincke, D. J. Carlo, J. Nucl. Med 1986, 27, 1041 ; L. D. Anderson, D. L. Meyer, T. R. Battersby, I. M. Frincke, D. Mackensen, S. Lowe, P. Connolly, ibid. 1988, 29,835.

[125] F. J. Primus, M. F. Hawthome et al., unpublished results, 1991

[126] Research in progress: F. J. Primus et al.

[127] D. J. Hnatowich, F. Virzi, M. Ruschowski, J. Nucl. Med, 1987, 28, 1294.

[128] A. Andersson, I Andersson J-O. Burgman J Capola J Carlsson $H$ Conde, I. Crawford, S. Graffmann, E. Grusell, A. Holmburg, E. Johansson, B. S. Larsson, B. Larsson, T. Liljefors, P. Lindstrom, J. Malmquist, L. Pellettieri, O. Pettersson, J. Ponten, A. Roberti, K. Russel, H. Reist, L. Salford, S. Sjōberg, B. Stemerlöw, P. Strömberg, B. Westermark in [16a], p. 41.

[129] B. D. Albertson, G. Willeke, M. Millan, S. Binney, S. Martsolf, D. L Loriaux in [12a], p. 22.

[130] J. Capala, R. F. Barth, D. M. Adams, J. Carlsson in [12a], p. 30.

[131] a) S. Sjöberg. J. Malmquist, P. Lindstrom, J. Carlsson, A. Andersson, 0 . Pettersson in [12a], p. 6; b) J. Malmquist, S. Sjöberg, Inorg. Chem. 1992 $31,2534$.

[132] H.C. Chen et al., unpublished results.

[133] M. S. Brown, R. T. Kovanen, J. L. Goldstein, Science 1981, 212, 628 J. L. Goldstein, R. G. W. Anderson, M. S. Brown, Nature 1979, 279, 679.

[134] J. L. Goldstein, J. H. S. Holgeson. M. S. Brown, J. Biol. Chem. 1979, 254 ,

[135] See also M.S. Brown, J. L. Goldstein, Angew. Chem. 1986, 98, 579; Angew. Chem. Int. Ed. Engl. 1986, 25, 583 (Nobel Lecture). 
[136] Y. U. Ho, R. G. Smith, M. S. Brown, J. L. Goldstein, Blood 1978, S2. 1099.

[137] S. B. Kahl, J. C. Callaway in [8a], p. 137

[138] S. B. Kahl, Tetrahedron Lett. 1990, 31, 1517

[139] a) T. Dougherty, Photochem. Photobiol. 1987, 45, 879; b) T. F. Delaney, E. Glatstein, Compr. Ther. 1989, 14, 43.

[140] T. Dougherty, K. R. Weishaupt, C. J. Gomer, Cancer Res. 1976, 36, 2326

[141] a) S. B. Kah] in [27b], p. 294; b) S. B. Kahl, D. D. Joel, G. C. Finkel P. L. Micea, M. M. Nawrocky, J. A. Coderre, D. N. Slatkin, Basic Life Sel. $1989,50,325$

[142] a) M. Miura, D. Gabel, R. G. Fairchild, B. H. Laster, L. S. Warkentien in [8a], p. 131; b) M. Miura, D. Gabel, G. Oenbrink, R. G. Fairchild, Tetrahedron Lett, 1990, 31, 2247.

[143] S. B. Kabl, M.-S. Koo in [16a], p. 223.

[144] a) R. Schwyzer, K. Q.Do, A. N. Eberle, J. L. Fauchere, Helv. Chim. Acto 1981, 64, 2078; b) G. Oenbrink, P. Jürgenlimke, D. Gabel, Photochem. Pholoblol. 1988, 48, 51 ; c) I. Moan, S. Somer, Cancer Letl. Shannon Irel. $1983,21,167$.

[145] S. B. Kahl, D. D. Joel, M. M. Nawrocky, P. L. Micca, K. P. Tran, G. C. Finkel, D. N. Slatkin, Proc, Natl. Acad. Sci. USA 1990, 87, 7265.

[146] M. Miura, P. Micca, D. Gabel in [16a], p. 455.

[147] R. G. Fairchild, S. B. Kahl, B. H. Laster, J. Kajef-Ezra, E. A. Popenoe, Cancer Res. 1990, 50, 4860 .

[148] J. S. Hill, S. B. Kahl, A. H. Kaye, M. F. Gonzales, N. J. Vardaxis, C. I. Johnson, S. S. Stylli, Y. Nakamura in [16a], p. 501.

[149] F. Alam, A. H. Soloway, B. V. Bapat, R. F. Barth, D. M. Adams, Basic Life Sel. 1989, 50, 107.

[150] R. M. Straubinger, N. G. Lopez, R. J. Debs, K. Hong, D. Papahadjopoulos, Cancer Res. 1988, 48, 5237 .

[151] R. T. Profitt, L. E. Williams, C. A. Presant, G. W. Tin, I. A. Uliana, R. C. Gamble, J. D. Baldeschwieler, Science 1983, 220, 502.

[152] R. T. Proffitt, L. E. Williams, C. A. Presant, G.W. Tin, J. A. Utiana, R. C. Gamble, J. D. Baldeschwicler, J. Nucl. Med. 1983, 24, 4S.

[153] a) C. A. Presant, R. T. Proffit, A. F. Turner, L. E. Williams, D. Windsor, J. L. Werner, P. Kennedy, C. Wiseman, K. Gala, R. J. McKenna, Cencer 1988, 62, 905; b) C. A. Presant, D. Blayney, R. T. Proffith, A. F. Turner, L. E. Williams, H. I. Nadel, P. Kennedy, C. Wiseman, K. Gala, R. J Crossley, S. J. Preiss, G. E. Ksionski. S. L. Presant, Lancet 1990, 335. 1307.

[154] R. M. Straubinger, K. Hong, D. S. Friend, D. Papahadjopoulos, Cell Cambridge Mass. 1983, 32, 1069.

[155] K. Shelly, M. F. Hawthorne, P. G. Schmidt in [16a], p. 259.

[156] a) R. L. Pilling, M. F. Hawthome, E. A. Pier, J. Am. Chem. Soc. 1964, 86, 3568; b) M. F. Hawthorne, R. L. Pilling, P. M. Garrett, ibid. 1965, 87, 4740 .

[157] B. L. Chamberland, E. L. Muetterties, Inorg. Chem. 1964, 3, 1450.

[158] M. F. Hawthorne, R. L. Pilling, J. Am. Chem. Soc. 1966, 88, 3873.

[159] M. F. Hawthorne, R. L. Pilting. P. F. Stokely, J. Am. Chem. Soc. 1965, 87, 1893.

[160] D. A. Feakes, K. J. Shelly, M. F. Hawthome, P. G. Schmidt, T. A Krisch, W. F. Bauer, Boron USA Workshop III, Pullmann, WA, USA. 1992.

[161] D. A. Feakes, K. J. Shelly, M. F. Hawthorne, P. G. Schmidt, C. A. Elstad, G. G. Meadow, W. F. Bauer in [i2a], p. 10.

[162] K. J. Shelly, M. F. Hawthorne, C. B. Knobler in [12a], p. 14.

[163] K. J. Shelly, C. B. Knobler, M. F. Hawthorne, Inorg. Chem. 1992, 31 , 2889.

[164] D. E. Moore, A. K. Chandler, S. Corderoy-Buck, J. G. Wilson, B. J. Allen in [16a], p. 451 .

[165] H. E. Hadd in [27b], p. 281

[166] S. B. Kahl in [27 b], p. 294.

[167] O. Hechter, I. L. Schwartz in [27b], p. 197.

[168] F. Wellmann, R. Abraham, R. Müller, D. Gabel, Z. Narurforsch. C 1991 , $46,252$.

[169] B. F. Spiclvogel, Mol. Struct. Energ. 1988, 5, 329

[170] H. R. Snyder, A. J. Reedy, W. J. Lennar, J. Am. Chem. Soe. 1958, 80, 835.

[171] Y. Mishima, M. Ichihashi, T. Nakanishi, M. Tsuji, M. Ueda, T. Nakagawa in [27 b], p. 355.

[172] a) Y. Mishima, C. Honda, M. Ichihashi, H. Obara, J. Hiratsuka, H. Fukuda, H. Karashima, T. Kobayashi, K. Kanda, K. Yoshino, Lancet 1989,388 ; b) Y. Ujeno, K. Akuta, H. Hatanaka, Y. Mishima, Y. Oda, Y. Nakagawa in [16a], p. 593; c) Y. Mishima, M. Ichihashi, C. Honda, $M$. Shiono, T. Nagogawa, H. Obara, J. Shirkawa, J. Hiratsuka, K. Kanda, T. Kobayashi, T. Nozaki, O. Aizawa, T. Sao, H. Karoshima, K. Yoshino, H Fukuda in [16a], p. 577.

(173] a) Y. Kinoshita, K. Yoshino, Y. Mori, H. Kakihana, Y. Mishima in [16a] p. 243; b) C. Honda, M. Shiono, N. Wadabayashi, M. Ichihashi, $Y$ Mishima, T. Kobayashi, K. Kanada, Y. Hori, K. Yoshino in [16a], p. 421.

[174] J. A. Coderre, J. D. Glass, R. G. Fairchild, U. Roy, S. Cohen, I. Fand, Cancer Res. 1987, 47, 6377.

(175) D. C. Roberts, K. Suda, J. Samanen, D. S. Kemp, Tetrahedron Lett. 1980 , 21,3435 .

[176] J. A. Coderre, J. A. Kalef-Ezra, R. G. Fairchild, P. L. Micea, L. E. Reinstein, J. D. Glass, Cancer Res. 1988, 48, 6313.
[177] J. A. Coderre, J. D. Glass, R. G. Fairchild, P. L. Micea, I. Fand, D. D. Joel, Cancer Res, $1990,50,138$.

[178] R. G. Zamenhof, D. E. Wazer, H. Madoc-Jones, O. K. Harling in [12a] P. 58.

[179] K. Yoshino, T. Maruyama, H. Takahashi, Y. Mori, H. Kakihana, Y Mishima, M. Ichihashi, C. Honda, M. Shiono in [12a], p. 4.

[180] J. K. Prashar, D. E. Moore, J. G. Wilson, B. I Allen in [12 a], p. 2.

[181] E. G. Samsel, B. M. Simpson in [16a], p. 251.

[182] B. F. Spielvogel, A. T. McPhail, I. H. Hall, R. G. Fairchild, P. L. Micea in $[27 \mathrm{~b}]$, p. 245 .

[183] W. Porschen, J. Marx, H. Müblensiepen, L. E. Feinendegen, F. Dallacker, H. Mückter, W. Müllners, T. Böhmel in [27 b], p. 331.

[184] B. F. Spielvogel, A. Good, J. Tomasz, S. Kathikeyan, W. Powell in [12a], p. 6.

[185] Y. Mishima, M. Ichihashi, S. Hatta, C. Honda, K. Yamamura, T. Nakagawa, Pigm. Cell Res. 1987, 2, 226.

[186] L. Dencker, B. Larsson, K. Olander, S. Ullberg, Br. J. Cancer 1982, 45, 95 .

[187] L. Dencker, B. Larsson, K. Olander, S. Ullberg, M. Yokota, Pharmarol. Toxicol. 1981, 49, 141.

[188] R. G. Fairchild, S. Packer, D. Greenberg, S. Prantika, A. B. Brill, I Fand, W. P. McNally, Cancer Res. 1982, 42, 5126.

[189] A. G. Bolt, I. S. Forrest, Recent Adv. Biol. Psychiatry 1968, 10, 20

[190] Y. Mishima, Pigm. Cell 1973, 1, 215.

[191] W. Tjarks, D. Gabel, J. Med. Chem. 1991, 34, 315

[192] H. Ketz, W. Tjarks, D. Gabel, Tetrahedron Lett. 1990, 31,4003

[193] J. G. Wilson, Pigm. Cell Res. 1987, 2, 297.

[194] J. G. Wilson in [16a], p. 227.

[195] H. S. Mason, D. J. E. Ingram, B. Allen, Arch. Biochem. Biophys. 1960, 86, 225.

[196] M. S. Blois, A. B. Zahlan, J. E. Maling, Biophys. J. 1964, 4, 471

[197] S. Packer, R. G. Fairchild, K. P. Watts, D. Greenberg. S. I. Hannon, Radiopharmaceuticals: Structure - Activity Relationships (Ed.: R.P. Spencer), Grune and Stratton. New York, 1981, Chapter 12, p. 251.

[198] R. G. Fairchild, D. Greenberg. K. P. Watts, S. Packer, H. L. Alkins, P. Som, S. J. Hannon, A. B. Brill, I. Fand, W. P. McNally, Cancer Res. 1982. 42.556.

[199] T. Nakagawa, K. Aono, Chem. Pharm. Bull. 1976, 24, 778.

[200] T. Nakanishi, M. Ichihashi, Y. Mishima, T. Matsuzawa, H. Fukuda, Int. J. Radiat. Biol. 1980, 37, 573.

[201] F. Alam, A. H. Soloway, R. F. Barth, D. M. Adams in [13 a], p. 8.

[202] F. Alam, B. V. Bapot, A. H. Soloway, R. F. Barth, N. Mafune, D. M. Adams in [8a]. p. 121.

[203] 3. Goodchild Bioconjugate Chem. 1990, 1, 165

[204] Oligodeoxynucleotides: Antisense Inhibisors of Gene Expression (Ed.: J. S. Cohen), CRC Press, Boca Raton, FL, USA, 1989.

[205] A. D. Whittaker, D. P. Kelly, M. Pardee, R. F. Martin in [16a], p. 231.

[206] A. Corder, R. F. Marin, A. Whittaker, D. P. Kelly, H. Meriaty, B. J Allen in [12a], p. 12.

[207] A. Sood, B. F. Spielvogel, B. R. Shaw, J. Am. Chem. Soc. 1989, 111, 9234

(208] a) B. F. Spielvogel, A. Sood, B. R. Shaw, I. H. Hall, Pure Appl. Chem $1991,63,415$; b) B. F. Spielvogel, A. Sood, B. R. Shaw, I. H. Hall, R. G. Fairchild, B. H. Laster, C. Gordon in [16a], p. 211.

[209] a) A. Sood, B. R. Shaw, B. F. Spielvogel, J. Am. Chem. Soc. 1990, 112, 9000 ; b) see also I Tomasz, B. R. Shaw, K. Porter, B. F. Spielvogel, A Sood, Angew. Chem. 1992, 104, 1404; Angew. Chem. Int. Ed. Engl. 1992, 31,1373 .

[210] B. F. Spielvogel, A. Sood, W. Powell, I Tomasz, K. Porter in [12a], p. 17.

[211] T. K. Liao, E. C. Podrebarac, C. C. Cheng, J. Am. Chem. Soc. 1964, 86, 1869.

[212] R. F. Schinazi, W. H. Prusorf, Tetrahedron Lett. 1978, 4981.

[213] R. F. Schinazi, W. H. Prusoff, J. Org. Chem. 1985, 50, 841.

[214] R. F. Schinazi, S. Kusuma, B. H. Laster, E. Popenoc, R. G. Fairehild in [118a].

[215] Y. Yamamoto, T. Seko, H. Nemoto, J. Org. Chem. 1989, 54, 4734.

[216] a) Y. Yamamoto, T. Seko, F. Rong, H. Nemoto, Tetrahedron Lett. 1989, 30, 7191; b) Y. Yamamoto, Pure Appl. Chem. 1991, 63, 423.

[217] A. K. M. Anisuzzaman, F. Alam, A. H. Soloway, Polyhedron 1990, 9. 891.

[218] W. Tjarks, A. K. M. Anisuzzaman, L. Liang, A. H. Soloway, R. F. Barth, D. I. Perkins, D. M. Adams, J. Med. Chem. 1992, 35, 1628.

[219] W. Tjarks, A. K. M. Anisuzzaman, A. H. Soloway, Nucleosides Nucleotides 1992, 11, 1765.

[220] a) L. Liu, R. F. Barth, A. H. Soloway, A. K. M. Anisuzaman, F. Alam, W. Tjarks, X.H. Zha, G. H. Morrison, Proc. Am. Assoc, Cancer Res. 1991, 34, 2418; b) W. Tjarks, A. K. M. Anisuzeaman, A. H. Soloway, L. Liu, R. F. Barth in [12a], p. 11.

[221] See [45].

[222] A. H. Soloway, personal communication, 1992

[223] Y. Yamamoto, T. Seko, H. Nakamura, H. Nemoto, H. Hojo, N. Mukai, Y. Hashimoto, J. Chem. Soc. Chem. Commen. 1992, 157.

[224] R. F. Schinazi, N. Goudgaon, J. Soria, D. C. Liotta in [12a], p. 11

[225] R.C. Reynolds, T. W. Trask. W. D. Sedwick, J. Org. Chem. 1991, S6, 2391 
, 


\section{Large Animal Normal Tissue Tolerance Using an Epithermal Neutron Beam and Borocaptate Sodium}

P. R. Gavin, R. Huiskamp, F. J. Wheeler, S. L. Kraft, C. E. DeHaan

This article is a reprint from Strahlentherapie und Onkologie, 169, 48-56, (1993). 
$\because$

, 


\title{
Large animal normal tissue tolerance using an epithermal neutron beam and borocaptate sodium
}

\author{
P. R. Gavin' ${ }^{1}$, R. Huiskamp², F. J. Wheeler ${ }^{3}$, S. L. Kraft ${ }^{4}$, C. E. DeHaan' \\ Department of Veterinary Clinical Medicine and Sugery ${ }^{1}$, Washington State University, Pullman, WA, USA, Radiobiology \\ and Radioecology, Netherlands Energy Research Foundation (ECN) ${ }^{2}$, Petten, the Netherlands, Idaho National Engineering \\ Laboratory, EG \& G Idaho Inc. ${ }^{3}$, Idaho Falls, ID, USA, and Department of Veterinary Clinical Sciences, Kansas State \\ University ${ }^{4}$, Manhattan, KS, USA
}

Irradiation of the canine head following intravenous $\mathrm{Na}_{2} \mathrm{~B}_{12} \mathrm{H}_{11} \mathrm{SH}(\mathrm{BSH})$ administration has provided useful information concerning the tolerance of skin and brain to the resultant complex form of irradiaton. The effect of the boron capture reaction in skin and brain has provided estimates of the influence of the microscopic dosimetry involved. Dogs irradiated with the epithermal beam alone provided valuable insight into the relative biological effectiveness (RBE) of the fast neutron component $(>10 \mathrm{keV})$ of the epithermal beam. When compared with literature values for X-rays for the occurrence of skin necrosis in dogs, an RBE of 4.5 was derived. Previous pharmacokinetic data concerning the distribution of $\mathrm{Na}_{2} \mathrm{~B}_{12} \mathrm{H}_{11} \mathrm{SH}$ (BSH) to blood and brain has been used to obtain input parameters for computer models of the microvasculature of the brain. Monte Carlo com. puter models were used to simulate the microscopic distribution of BSH in the normal brain. The term compound factor describes the product of the microscopic boron fission fragment dose hitting the nucleus and the relative biologic effectiveness divided by the macroscopic equilibrium dose of the boron reaction in the tissue of interest. The computed compound factor for $\mathrm{Na}_{2} \mathrm{~B}_{12} \mathrm{H}_{11} \mathrm{SH}$ (BSH) in normal brain was 0.37 . This factor agreed very well with the value of 0.32 obtained for the brain necrosis with the dog irradiations. The compound factor for the dog's skin was experimentally derived from the dog experiments and was equal to 0.5 .

Strahlentoleranz normalen Gewebes großer Tiere bei Anwendung eines epithermischen Neutronenstrahls und $\mathrm{Na}_{2} \mathrm{~B}_{12} \mathrm{H}_{11} \mathrm{SH}$

Die Bestrahlung des Hundekopfes nach intravenöser Gabe von $\mathrm{Na}_{2} \mathrm{~B}_{12} \mathrm{H}_{11} \mathrm{SH}$ (BSH) ergab wertvolle Hinweise zur Beurteilung der Empfindlichkeit von Haut und Gehirn gegenulber der resultierenden komplexen Strahlung. Der Einfluß der damit zusammenhängenden mikroskopischen Dosisverteilung konnte aufgrund der Auswirkungen der Boreinfangreaktion in Haut und Gehirn bestimmt werden. Mit Hilfe der nur mit einem epithermischen Strahl behandelten Tiere konnten Einblicke in die relative biologische Wirksamkeit (RBW) des Anteils schneller Neutronen ( $>10 \mathrm{keV}$ ) im epithermischen Strahl gewonnen werden. Im Vergleich mit Angaben der Literatur uber das Auftreten von Hautnekrosen bei Hunden nach Röntgenbestrahlung wurde eine RBW von 4,5 abgeleitet. Auf der Grundlage frilherer pharmakokinetischer Daten uber die Verteilung von $\mathrm{Na}_{2} \mathrm{~B}_{12} \mathrm{H}_{11} \mathrm{SH}$ (BSH) in Blut und Gehirn konnten Eingangsparameter für Computermodelle der Mikrogefäßversorgung des Gehirns gewonnen werden. Die mikroskopische Verteilung von BSH im normalen Gehirn wurde mit Hilfe von Monte-Carlo-Computermodellen simuliert. Der Ausdruck ,compound factor" bezeichnet das Produkt der Dosis der auf den Kern auftreffenden mikroskopischen Kernfragmente und der relativen biologischen Wirksamkeit, geteilt durch die makroskopische Gleichgewichtsdosis der Borreaktion im betreffenden Gewebe. Der errechnete compound factor für $\mathrm{Na}_{2} \mathrm{~B}_{12} \mathrm{H}_{11} \mathrm{SH}$ im normalen Gehirn lag bei 0,37 . Dieser Faktor stimmte sehr gut mit dem bei den Bestrahlungen von Hunden erhaltenen Hirnnekrosewert von 0,32 uberein. Der compound factor der Hundehaut wurde experimentell aus den Versuchen mit Hunden abgeleitet und betrug 0,5. 
Introduction

Epithermal neutron beams, defined as having energies above thermal neutron energy $(<0.5 \mathrm{eV})$ and below 10 $\mathrm{keV}$, are being developed for potential boron neutron capture therapy (BNCT) to allow treatment of deep seated tumors. like glioblastoma multiforme, through the intact skin. The neutron capture cross-sections for elements in normal tissue are several orders of magnitude lower than that for boron. However, due to the relative high concentrations of hydrogen and nitrogen in normal tissue, their neutron capture reactions through the ${ }^{1} \mathrm{H}(\mathrm{n}, \gamma)^{2} \mathrm{H}$ and the ${ }^{14} \mathrm{~N}(\mathrm{n}, \mathrm{p}){ }^{14} \mathrm{C}$ reaction respectively contribute significantly to the total radiation absorbed dose. Additional sources which contribute to the absorbed radiation dose are incident $\gamma$-radiation and fast neutrons, i.e. contamination components in the epithermal beam.

The resultant radiation consists of a complex mixture of low and high energy photons and atomic particles. The primary radiation, the fission fragments resulting from neutron capture by boron, have effective ranges in tissue of $10 \mu \mathrm{m}$ or less. Therefore, the distribution of the boron atoms has a marked effect on the radiation dose to the critical tissues. A large animal model has to be used to study the tolerance of critical normal tissues to this form of radiotherapy using an epithelial neutron beam since in rodents and other small animals, the total body dose is intolerable and prevents studies on late effects. This total body irradiation is mainly caused by the gamma components coming from the ${ }^{1} \mathrm{H}(n, \gamma)^{2} \mathrm{H}$ reaction in the irradiation field.

The pharmacokinetics of the boron compound borocaptate sodium $\left(\mathrm{Na}_{2} \mathrm{~B}_{12} \mathrm{H}_{11} \mathrm{SH}\right)$ hereafter called $\mathrm{BSH}$ have been studied extensively in the dog [14]. The knowledge of the macrodistribution of this compound in almost all normal tissues allows estimates of the radiation dose contribution of the boron neutron capture reaction to critical normal tissues [16]. Complete sampling of all normal tissues in the dog augments the limited sampling that is being obtained from human pharmacokinetic studies [4].

Large animal normal tissue tolerance studies play an important role in the design of patient irradiations using new radiation modalities $[2,5,10]$. Knowledge of tolerance of the superficial overlying tissues and most importantly of the brain have to be determined prior to initiation of any new radiation treatments for brain

Strahlenther. Onkol. 169 (1993), 48-56 (Nr. 1) tumors $[5,22]$. Readily determined gross tissue boron concentrations and subsequent tumor to blood and tumor to normal tissue ratios may be extremely misleading about the radiation dose to these various tissues. At this time, experimental studies and the empirical results are needed to verify the complex radiation dose distribution that occurs during neutron capture radiation therapy.

The total physical dose required to reach a biological isoeffect appears to increase directly as the proportion of boron capture dose increases [7]. This effect together with knowledge of the macrodistribution, led to estimates of the influence of the microdistribution of the BSH compound. It has been suggested that the term compound factor should be used to describe the product of the microscopic physical radiation dose and relative biologic effectiveness (RBE) of the ${ }^{10} \mathrm{~B}(\mathrm{n}$, $\alpha)^{7} \mathrm{Li}$, divided by the macroscopic physical dose to predict the normal tissue responses [6].

The following study documents the initial studies of epithermal irradiation and boron neutron capture using a canine model and BSH as the boron carrier and compares the biologic observations with a computer model which is used to predict the compound factor and hence the equivalent radiation in normal tissues. This computer model could also be used for other boron compounds with a different macroscopic distribution. This information is needed to design appropriate normal tissue tolerance studies for different organ systems and/or different boron compounds.

\section{Materials and methods}

\section{Pharmacokinetics}

The pharmacokinetics of BSH was studied in normal laboratory dogs and in dogs with spontaneous occurring brain tumors which were referred by private veterinary practitioners. The animal experiments were approved by the appropriate animal care and use committees at Washington State University and Brookhaven National Laboratory. The amount of compound administered intravenously was varied from 20 to 100 $\mathrm{mg}$ boron $/ \mathrm{kg}$ body weight. Sampling of the blood and body tissues was during two to twelve hours post administration. Detailed descriptions of the protocol have been previously reported [14]. The boron levels were determined by inductively coupled plasma-atomic emission spectroscopy (ICP-AES) as described elsewhere [3]. 


\section{Irradiation procedures}

Twenty-five labrador-type dogs have been irradiated with the epithermal neutron beam at the Brookhaven Medical Research Reactor (BMRR) Brookhaven, N.Y., USA, using a $5 \times 10 \mathrm{~cm}^{2}$ portal. Ten of the dogs irradiated at BMRR were with the epithermal beam alone (EPI [epithermal neutron only irradiation] dogs), five dogs were irradiated to a dose of $11 \mathrm{~Gy}$, and five to 16.5 Gy. The other 15 dogs at BMRR were irradiated with epithermal neutron beams with blood boron concentrations of 31 to $62 \mu \mathrm{g}$ boron/g blood (BORON [epithermal neutron irradiation with boron capture] dogs). The dogs had BSH (enriched to $95 \%{ }^{10} \mathrm{~B}$ ) administered intravenously at a rate of $1 \mathrm{mg} \mathrm{B} \mathrm{kg} \cdot$ body weight $-1 \cdot \min -1$. The dose varied from 0 to $64 \mathrm{~Gy}$. The dosage groups are defined by the peak physical dose that occurred at 2 to $3 \mathrm{~cm}$ beneath the surface. The doses were adjusted to skin dose for the determination of skin RBE. Details of the skin and peak physical dosages have been provided in earlier papers $[7,8]$.

All dogs had serial physical, and neurological examinations, complete blood counts, cerebrospinal fluid analysis, and brain imaging via computed tomography (CT) and/or magnetic resonance imaging (MRI). The brain imaging was performed prior and following the administration of appropriate contrast agents. The protocol details have been reported previously [7]. This report will limit the findings to the observed acute and late skin reactions and the brain reactions seen with MRI.

The skin and brain changes that occurred were compared to historical values reported in the literature for conventional photon irradiation in order to estimate the RBE of the fast neutron ( $>10 \mathrm{keV}$ ) component of the epithermal beam component $[5,17]$. The complexity of boron reaction involving microdosimetry have necessitated the term "compound factor" that represents the product of the geometric effects of microdosimetry and the RBE of the fission fragments. The method of the radiation dose component calculation has been reported previously [16].

The RBE for last neutrons was derived using the EPI dog tolerance studies by subtracting the physical doses multiplied by their RBE of the various beam components. The RBE's used in the calculations were equal to $1.0,2.7$ and 1.0 for all gamma sources, the ${ }^{14} \mathrm{~N}(\mathrm{n}, \mathrm{p}){ }^{14} \mathrm{C}$ reaction and other capture reactions respectively [1].

The "compound factor" for the ${ }^{10} \mathrm{~B}(\mathrm{n}, \alpha)^{7} \mathrm{Li}$ reaction was derived using a similar procedure as described for the calculation of the fast neutron RBE. The fast neutron RBE derived from the EPI dog tolerance study was used as additional input leaving only the equivalent boron capture dose as variable. The value of 4.5 for the fast neutron RBE was chosen for these calculations.

\section{Computer modelling}

A Monte Carlo computer model of the capillaries was used to determine the physical and equivalent dose to the endothelial cell nucleus as a function of the boron concentration and distribution pattern. The input parameters for the model were diagrammed (Figure 1). Charged particle transport in tissue for the fission fragments was used [21]. Known macroscopic and inferred microscopic information from the canine studies was used in the models to predict the effect of the epithermal beam and the ${ }^{10} \mathrm{~B}(\mathrm{n}, \alpha)^{7} \mathrm{Li}$ reaction on the endothelium. The RBE values used for ${ }^{14} \mathrm{~N}(n, p){ }^{14} \mathrm{C}$ and ${ }^{10} \mathrm{~B}(\mathrm{n}, \alpha)^{7} \mathrm{Li}$, and fast neutrons were $2.7,2.3$, and 4.5 respectively. The first two values were obtained from the literature [1] and the fast neutron RBE was derived from skin reactions in the dogs described above.

\section{Results}

The biodistribution of BSH was studied in both normal laboratory and dogs referred with spontaneous brain tumors [14]. No difference in normal tissue concentration was seen between these groups of dogs. The boron concentration in the skin of the head was slightly lower than the blood boron concentration (Figure 2). However, the brain parenchyma had significantly less boron than the blood. All concentrations decreased with time and the slopes of the decrease in the three tissue components were not statistically different.

The observed skin changes were biphasic with the early epidermal changes most prominent at three to four

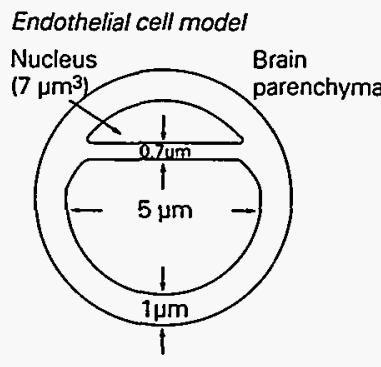

Strahlenther. Onkol. 169 (1993), 48-56 (Nr. 1)
Figure 1. Parameters used for the Monte Carlo model of a brain capillary. 


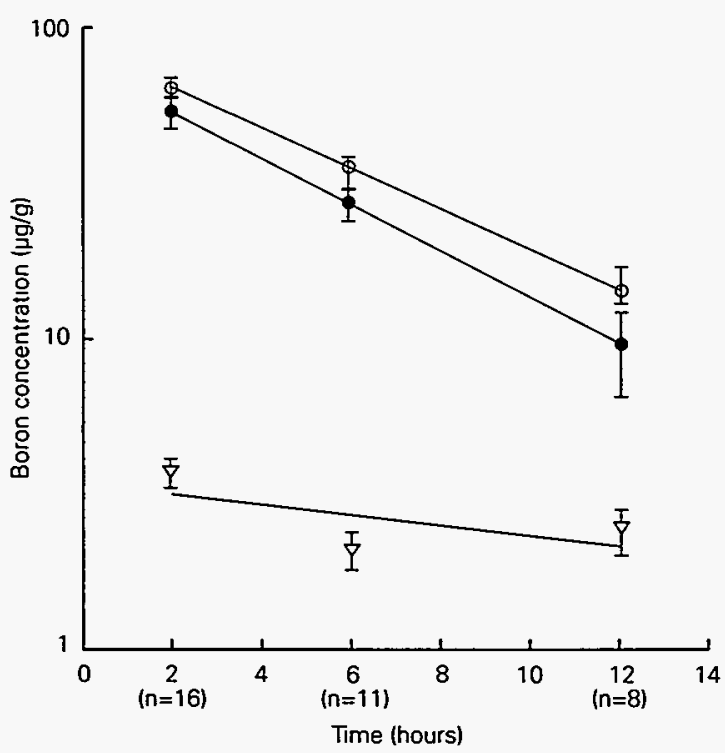

Figure 2. Mean boron concentration $(\mu \mathrm{g} / \mathrm{g})$ in blood $(O)$, skin of the head $(\odot)$ and brain $(\nabla)$ versus time after end of intravenous BSH infusion $(55 \mathrm{mg} \mathrm{10B} / \mathrm{kg}$ ).

weeks and the dermal necrosis occurred at twelve weeks post-irradiation. Dry desquamation with small patchy areas of moist desquamation and epithelial ulceration occurred in the EPI dogs exposed to 11 Gy peak ( $3 \mathrm{~cm}$ depth) physical dose. Moist desquamation with ulceration in greater than $50 \%$ of the incident radiaton field, were observed in the 16.5 Gy EPI dogs and in the BORON dogs that received more than $38 \mathrm{~Gy}$ peak physical dose. The late skin reaction, i.e. dermal necrosis, occurred in the 16.5 Gy EPI dogs and in the BORON dogs that received 64 Gy peak physical dose. The 64 Gy BORON dogs were euthanitized following dermal necrosis and several unsuccessful surgical attempts to alleviate the problem. The 16.4 Gy EPI dogs were euthanitized following dermal necrosis.

The 11 Gy EPI dogs did not develop any visible CNS lesions within one year post-irradiation. The $38 \mathrm{~Gy}$ BORON dogs had fatal white matter necrosis of the cerebrum that appeared at 22 weeks post-irradiation. Two of three 27 Gy BORON dogs developed small contrast enhancing lesions of the cerebral white matter at six months post-irradiation (Figure 3 ). However, the

Figure 3. Transverse (coronal) magnetic resonance image in a dog of the 27 Gy BORON group at six months post-irradiation. Arrows indicate an area of contrast enhancement using Gd-DTPA (Magnevist $^{\circledast}$ ) at dosage of $0.1 \mathrm{mmol} / \mathrm{kg}$.
27 Gy BORON dogs were clinically normal at that time and remained so for the twelve months of the study. The contrast enhancing lesions were not present on the MRI examination twelve months post-irradiation.

The fast neutron RBE for the acute and late skin effects were derived using published data for the other beam components and compared with skin tolerance doses determined for $200 \mathrm{kVp}$ X-rays. Separate values equal to 3.0 to 4.9 and $\geq 4.9$ were calculated for the acute and late skin effects respectively (Table 1).

The dose modification of the boron capture dose, or compound factor, was determined by using published and the above calculated RBE's and comparison of the remaining boron capture physical dose component with published X-ray tolerance doses for the skin and brain of the dog. Separate compound factors equal to 0.5 and 0.32 were derived for the skin and CNS respectively (Table 2). Using the derived RBE and compound factors, a summary of the experimental groups physical and equivalent dose is given in Table 3.

Using the BMRR epithermal beam operating at $3 \mathrm{MW}$, and a $5 \times 10 \mathrm{~cm}$ field, the capillary model with a boron distribution of $50 \mathrm{ppm}$ in the lumen and $1 \mathrm{ppm}$ in the endothelial cell cytoplasm and surrounding brain parenchyma resulted in a capillary endothelial-nuclear physical dose of $6.44 \mathrm{~Gy}$ with a peak physical dose of $18.30 \mathrm{~Gy}$. The calculated equivalent dose at the thermal neutron peak and to the endothelial nuclei was 39.28 and 12.0 equivalent $\mathrm{Gy}$, respectively (Table 4 ).

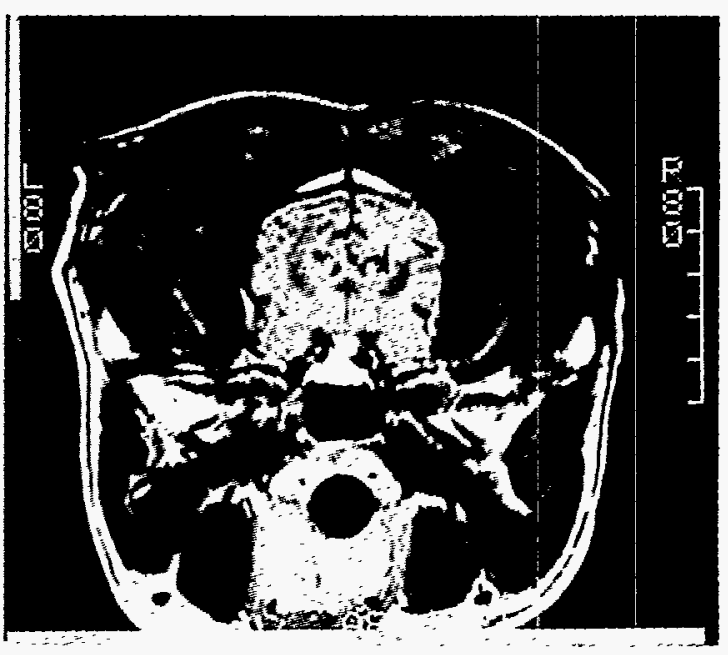


The fraction of the physical boron dose that hits the endothelial nucleus multiplied by the RBE of 2.3 resulted in a compound factor of 0.37 for BSH in the normal brain vasculature.

\section{Discussion}

Some inferences of the microscopic distribution can be drawn from the macroscopic distribution of BSH in the blood and brain (Figure 2). The normal blood-brainbarrier excluded the boron from the normal brain parenchyma following an intravenous administation of BSH. The tight junctions between the endothelial cells form the primary portion of the barrier [19]. This fact together with the hydrophilic nature of the compound indicate that the compound is also excluded from the endothelial cytoplasm. In contrast, the skin contained boron levels similar to that observed in the blood and this would necessitate a large extravascular component for the skin boron values. The fenestrated capillaries found outside the brain prevent use of the same logic to analyze the gross brain boron concentration.

The normal tissue tolerance observed in the EPI dogs revealed important information about the fast neutron components in the epithermal beam. The comparison of the observed skin reactions listed in Table 1 with those observed for X-rays are difficult and have limita- tions. The reactions seen in the present study occurred in either none or all of the dogs per dose group. The referenced study used a similar incident field size, had small numbers of dogs and did not have dermal necrosis in $100 \%$ of any of the dosage groups [17]. Based on these differences of the compared biological endpoints, the calculated RBE for the fast neutron component in the epithermal beams may be conservative. In addition, the authors are aware that the RBE equal to 1.0 chosen for gamma photons may be too high especially in light of the applied dose rate of about $0.02 \mathrm{~Gy} / \mathrm{min}$. However, the gamma photon fraction consists of incident and generated gamma photons of several energies and again a conservative approach was taken pending additional studies to further refine these values. The various components of the radiation field were considered as independent factors. The authors are aware of the findings indicating the dependence of the components and the effect of the dependence on calculated values like RBE [20]. However, the complexity of the radiation field, coupled with a relative lack of precise information concerning some of the components, necessitated the simple additive approach at this time.

In this paper, the term fast neutron physical dose is used for all reactions from neutrons with energies greater than $10 \mathrm{keV}$. There is a lack of information concerning the RBE's of neutrons in the energy ranges found

\begin{tabular}{|c|c|c|c|c|c|c|}
\hline Response type & Biological endpoint & $\begin{array}{l}\text { Ref. X-ray dose } \\
(\mathrm{GY})^{*}\end{array}$ & Radiation component & $\begin{array}{l}\text { Phys. dose } \\
\text { (Gy) }\end{array}$ & $\begin{array}{l}\text { Eq. dose } \\
(\mathrm{RBE} \text {. Gy })^{* * *}\end{array}$ & $\begin{array}{l}\text { Fast neutron } \\
\mathrm{RBE}^{* * * *}\end{array}$ \\
\hline \multirow[t]{8}{*}{ Early skin reactions } & \multirow[t]{4}{*}{ Moist desquamation } & \multirow[t]{4}{*}{18.3} & Other ${ }^{* *}$ & 0.52 & 0.52 & \\
\hline & & & $N(n, p)$ & 1.35 & 3.65 & \\
\hline & & & Gamma & 4.49 & 4.49 & \\
\hline & & & Fast $n$ & 3.18 & & 3.0 \\
\hline & \multirow{4}{*}{$\begin{array}{l}\text { Moist desquamation } \\
\text { + ulceration }\end{array}$} & \multirow[t]{4}{*}{24.4} & Other** & 0.52 & 0.52 & \\
\hline & & & $N(n, p)$ & 1.35 & 3.65 & \\
\hline & & & Gamma & 4.49 & 4.49 & \\
\hline & & & Fast n & 3.18 & & 4.9 \\
\hline \multirow[t]{8}{*}{ Late skin reactions } & \multirow[t]{4}{*}{$50 \%$ skin necrosis } & \multirow[t]{4}{*}{36.6} & Other** & 0.78 & 0.78 & \\
\hline & & & $N(n, p)$ & 2.03 & 5.48 & \\
\hline & & & Gamma & 6.76 & 6.76 & \\
\hline & & & Fast $\mathbf{n}$ & 4.78 & & 4.9 \\
\hline & \multirow[t]{4}{*}{$>50 \%$ skin necrosis } & \multirow[t]{4}{*}{$>36.6$} & Other** & 0.78 & 0.78 & \\
\hline & & & $N(n, p)$ & 2.03 & 5.48 & \\
\hline & & & Gamma & 6.76 & 6.76 & \\
\hline & & & Fast $\mathbf{n}$ & 4.78 & & $>4.9$ \\
\hline
\end{tabular}

* Park et al. [17], ** Other: dose coming from neutron capture reactions by other elements, primarily $\mathrm{Na}$ and $\mathrm{Cl} .{ }^{* * *} \mathrm{RBE}$ gamma and other $=1.0 ; \operatorname{RBE} N(n, p)=2.7$; Archambeau [1]. ${ }^{* * *} \operatorname{RBE}_{\text {fast neutrons }}=\{$ Ref. X-ray dose- $[($ Eq. dose gamma $)+($ Eq. dose $N(n, p))+($ Eq. dose other)]]/Phys, dose fast neutron.

Table 1. Calculation of fast neutron RBE. 


\begin{tabular}{|c|c|c|c|c|c|}
\hline $\begin{array}{l}\text { Biological endpoint } \\
\text { (dose group)* }^{*}\end{array}$ & $\begin{array}{l}\text { Ref. X-ray dose } \\
(G y)^{* *}\end{array}$ & Radiation component & $\begin{array}{l}\text { Phys. dose } \\
\text { (Gy) }\end{array}$ & $\begin{array}{l}\text { Eq. dose } \\
\left(\mathrm{RBE}^{*} \mathrm{~Gy}\right)^{* * * *}\end{array}$ & $\begin{array}{l}\text { BSH compound } \\
\text { factor }{ }^{* * * * *}\end{array}$ \\
\hline \multirow{5}{*}{$\begin{array}{l}\text { Skin moist des- } \\
\text { quamation w \& } \\
\text { w/o ulceration } \\
\text { ( } 38 \text { Gy dog group) }\end{array}$} & \multirow[t]{5}{*}{24.4} & Other $* * *$ & 0.36 & 0.36 & \\
\hline & & $(N(n, p)$ & 0.92 & 2.48 & \\
\hline & & Fast $n$ & 2.17 & 9.77 & \\
\hline & & Gamma & 3.06 & 3.06 & \\
\hline & & $(B(n, \alpha)$ & 17.05 & & 0.51 \\
\hline \multirow[t]{2}{*}{ Dermal necrosis } & \multirow[t]{5}{*}{36.6} & Other*** & 0.48 & 0.48 & \\
\hline & & $N(n, p)$ & 1.24 & 3.35 & \\
\hline \multirow[t]{3}{*}{ (64 Gy dog group) } & & Fast $n$ & 2.91 & 13.10 & \\
\hline & & Gamma & 4.11 & 4.11 & \\
\hline & & $B(n, \alpha)$ & 29.90 & & 0.52 \\
\hline \multirow[t]{2}{*}{ CNS necrosis } & \multirow[t]{5}{*}{14.9} & Other ${ }^{* * *}$ & 0.42 & 0.42 & \\
\hline & & $N(n, p)$ & 0.68 & 1.84 & \\
\hline \multirow[t]{3}{*}{ (27 Gy dog group) } & & Fast $n$ & 0.57 & 2.57 & \\
\hline & & Gamma & 3.19 & 3.19 & \\
\hline & & $(B(n, \alpha)$ & 21.58 & & 0.32 \\
\hline
\end{tabular}

* From Table 3. ** Skin effects: Park et al. [17]. CNS effects: Fike et al. [5].*** Other: dose coming from neutron capture reactions by other elements, primarily $\mathrm{Na}$ and $\mathrm{Cl}$. $* * * * \operatorname{RBE}$ gamma and other $=1.0 ; \operatorname{RBE~N}(\mathrm{n}, \mathrm{p})=2.7 ;$ Archambeau [1]. ${ }^{* * * * *}$ Compound factor ${ }_{2 . . . .}=\{$ Ref. $\mathrm{X}$-ray dose- $[($ Eq. dose gamma $)+($ Eq. dose $N(n, p))+($ Eq. dose other $)+($ Eq. dose fast $n)]\} /$ Phys. dose $B(n, \alpha)$.

Table 2. Compound factor.

in these epithermal beams. However, a value of 4.5 or greater is in general agreement with other reports [5, $12,15]$. In this report the use of large single doses obtained an RBE for fast neutrons similar to that observed in fractionated studies with $15 \mathrm{MeV}$ to $22 \mathrm{MeV}$ neutrons in large animals and humans $[5,12,15]$. The increase in the apparent $\mathrm{RBE}$ with late tissue reactions of skin (dermal necrosis) compared to the early responses in skin was in agreement with the radiation biologic premise that early responding tissues have a lower RBE for fast neutrons than late responding tissues [9]. The RBE calculated for the more clinically important late skin reaction was used for the subsequent calculation of equivalent doses.

Attempts to date to obtain brain tolerance values for the epithermal beam with acceptable skin reactions has failed, and this has necessitated the use of the same vascular endothelial RBE for the fast neutron dose component in the skin and brain since these late effects are both determined by vasculature. The peak equivalent dose of the 11 Gy EPI dogs exceeded the reported tolerance for the brain. However, a large fraction of the equivalent dose was due to fast neutrons and their effect diminishes rapidly with increasing depth. Therefore, it is proposed that only the surface of the brain was irradiated to the peak dose and that the majority of the brain was exposed to tolerable equivalent doses. Severe atrophy of the irradiated temporal muscle in the 11 Gy EPI dogs was observed at twelve months postirradiation. This fact may indicate an increased contribution of the fast neutron component to the human brain, that lies much closer to the surface than in the labrador dog.

\begin{tabular}{|c|c|c|c|c|c|c|}
\hline $\begin{array}{l}\text { Peak phys. } \\
\text { dose group }\end{array}$ & $\begin{array}{l}\text { Peak phys. } \\
\text { dose range } \\
(\mathrm{Gy})\end{array}$ & $\begin{array}{l}\text { Peak eq. } \\
\text { dose range } \\
(\text { Eq. Gy)* }\end{array}$ & $\begin{array}{l}\text { Skin phys. } \\
\text { dose range } \\
\text { (Gy) }\end{array}$ & $\begin{array}{l}\text { Skin eq. } \\
\text { dose range } \\
\text { (Eq. Gy)* }\end{array}$ & $\begin{array}{l}{ }^{10} \mathrm{~B} \text { Blood } \\
\text { range } \\
(\mu \mathrm{g} / \mathrm{g}) 0\end{array}$ & $\begin{array}{l}\text { No. of } \\
\text { dogs }\end{array}$ \\
\hline 0 & 0 & 0 & 0 & 0 & 0 & 2 \\
\hline 11 & 11 & 18 & 9.5 & 23 & 0 & 5 \\
\hline 16.5 & 16.5 & 27.2 & 14.3 & 25.9 & 0 & 5 \\
\hline 15 & $12.5-14.6$ & $8.1-8.6$ & $7.8-8.1$ & $8.8-9.3$ & $31-47$ & 5 \\
\hline 27 & $25.5-27.2$ & $14.8-15.4$ & $15.7-16.4$ & $15.3-16.5$ & $45-60$ & 3 \\
\hline 38 & $38.2-38.6$ & $21.4-24.5$ & $23.3-24.0$ & $22.5-25.8$ & $38-57$ & 3 \\
\hline 64 & $64.4-64.5$ & $34.5-34.5$ & 38.6 & $35.9-36.0$ & $62-63$ & 2 \\
\hline
\end{tabular}

*Equivalent dose calculated using RBE for fast neutrons equal to 4.5 (Table 1) and compound factors from Table 2.

Table 3. Experimental groups irradiated at the BMRR epithermal beam.

Strahlenther. Onkol. 169 (1993), 48-56 (Nr. 1) 


\begin{tabular}{|c|c|c|c|c|c|c|c|}
\hline Dose description & Type of calculation & $\begin{array}{l}\text { Dose } \\
{ }^{10} \mathrm{~B} \\
(\mathrm{n}, \text { alpha) }\end{array}$ & ${ }^{14} \mathrm{~N}(\mathrm{n}, \mathrm{p})$ & $\begin{array}{l}\text { Fast } \\
\text { neutron }\end{array}$ & Gamma & Other & Total \\
\hline Peak, equilibrium, physical & Macroscopic, physical (Gy)* & 14.1 & 0.49 & 0.50 & 2.83 & 0.34 & 18.30 \\
\hline Peak, equilibrium, equivalent & Macroscopic, equivalent (eq. Gy) *.** $^{*}$ & 3.25 & 1.33 & 2.27 & 2.83 & 0.34 & 39.28 \\
\hline Capillary, endothelial, physical & Microscopic, physical $(\mathrm{Gy})^{* * *}$ & 2.28 & 0.49 & 0.50 & 2.83 & 0.34 & 6.44 \\
\hline Capillary, endothelial, equivalent & 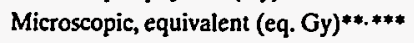 & 5.24 & 1.33 & 2.27 & 2.83 & 0.34 & 12.00 \\
\hline
\end{tabular}

*Peak physical dose in center of blood vessels greater or equal $20 \mu \mathrm{m}$, boron concentration in normal brain of: $50 \mathrm{ppm}$ boron in the endothelial lumen, $1 \mathrm{ppm}$ in the endothelial cytoplasm and brain parenchyma. **RBE's used for equivalent dose calculation: 2.3 for $10 \mathrm{~B}(\mathrm{n}$, alpha); 2.7 for $14 N(n, p)$; and 4.5 for fast neutrons. ***Capillary at peak dose depth, boron concentration in normal brain of: 50 ppm boron in the endothelial lumen, $1 \mathrm{ppm}$ in the endothelial cytoplasm and brain parenchyma. eq. $\mathrm{Gy}=$ physical dose (Gy) multiplied by RBE or compound factor.

Table 4. Overall peak equilibrium vasculature dose and capillary nuclear dose in brain with BSH.

The fast neutron contamination of the available epithermal beams and the incident and induced gamma components indicate a need for high boron concentrations to effectively use the boron capture reactions for radiotherapy. The fast neutron and gamma dose are nontargeted irradiations and contribute significantly to the normal tissue dose. The fast neutron dose could make the superficial tissues the limiting tissue at low blood boron concentrations.

In the BORON dogs peak equilibrium physical radiation dose levels far in excess of those found with conventional X-ray irradiation, resulted in normal tissue reactions similar to those seen in the previous studies $[5,22]$. The sublethal contrast enhancing lesion in the cerebral cortex (Figure 3) observed in the 27 Gy BORON dogs, was chosen as the endpoint to compare to a previous study (Table 2) [5]. While the $39 \mathrm{~Gy}$ BORON dogs had the "early late" change described in that report, it occurred in three of three dogs and it was reasoned that this dose exceeded the $50 \%$ tolerance dose reported by Fike et al. [5]. In subsequent experiments, the sublethal change (Figure 3) occurs at a dose that can be lethal in some dogs and was therefore chosen as the reference dose for our calculation of the compound factor. This fact together with the high RBE's for the proton and fast neutron components readily indicate that the compound factor for the boron fission reaction products is less than one. A compound factor equal to 0.32 for the boron reaction in the cerebral vasculature is a general agreement with mathematical models that predict this degree of sparing due to the compound distribution determined and inferred by measurements following $\mathrm{BSH}$ infusion $[13,18]$. The main geometrical effects or this sparing effect are: lack of charged particle equilibrium in vessels $<20 \mu \mathrm{m}$ in diameter, and the upstream, downstream energy depositions not contributing to the capillary endothelial nuclear dose. These factors result in a large dose sparing effect for the ${ }^{10} \mathrm{~B}(n, \alpha)^{7} \mathrm{Li}$ reaction in the capillaries.

The compound factor equal to 0.5 for skin cannot be confirmed or analyzed further prior to the acquisition of data concerning quantitative microscopic localization data of boron in the skin. At this time, we are unable to determine the microscopic distribution of boron in skin but this distribution would have a major effect of the dose distribution of the boron capture fission fragments. Cellular and subcellular information is needed to allow further analysis of the compound factors and to permit computer modelling of the microscopic boron dose distribution that occurs with $\mathrm{BSH}$ in normal skin. However, assuming that skin necrosis is mediated through radiation damage to the vasculoconnective tissue [10], the observed compound factor for BSH in the skin of 0.5 suggest a similar sparing phenomenon as observed in the cerebral capillaries.

The computer model of the capillary (Figure 1) depicts the parameters used to help understand the radiation dose to the endothelial nucleus of the cerebral capillaries. The boron distribution found in the blood and brain, following BSH administration, support the parameters used. The vascular computer model for the endothelial response resulted in a compound factor for the brain endothelium that was markedly similar to that calculated for the brain necrosis reaction in the boron capture dog head irradiation study. Given the BSH distribution in the normal brain, brain necrosis is logically due to endothelial damage, and has been supported by histologic analysis of the affected brains. This nonuniform boron distribution between blood and brain resulted in a modelled compound factor of 0.37 for BSH in the brain. The endothelial dose response oberved in the canine brain gave a compound factor of 
0.32. The compound factor is the product of RBE and microscopic physical dose divided by the macroscopic equilibrium physical dose. This compound factor would indicate that approximately $83 \%$ of the peak physical boron capture dose (roughly five of six fission events) do not contribute to the endothelial nuclear dose. This sparing is equal to that seen in the canine normal tissue brain studies but is greater than that previously reported $[13,18]$. The results listed in Table 4 indicate the importance of the difference between macroscopic physical and equivalent radiation doses and microscopic physical and equivalent radiation doses. An equivalent and endothelial nuclear dose of 12 eq.Gy (physical dose [Gy] multiplied by RBE or compound factor) was chosen to represent a dose below the threshold for detectable damage [5]. To achieve this dose, the calculated macroscopic physical dose at the thermal neutron peak would be equal to $18.3 \mathrm{~Gy}$ while the macroscopic equivalent dose atthis site would exceed $39 \mathrm{eq} \cdot \mathrm{Gy}$. The parameters chosen 50 ppm boron in the blood, $1 \mathrm{ppm}$ in the endothelial cell cytoplasm and surrounding brain prenchyma and the BMRR epithermal beam with a $5.10 \mathrm{~cm}$ portal must be emphasized.

The vascular sparing of the capillary endothelial nuclei has been predicted $[13,18]$. The results from the dog irradiations with BSH support this sparing effect. However, different distributions of other compounds will give different sparing effects. Thus, compound factors for each tissue and compound need to be determined [6]. The Monte Carlo simulation mode of cerebral capillaries can help design future experiments of normal tissue tolerance of various organ systems for various beams and boron compounds following acquisition of boron tissue distribution data.

Table 3 summarizes the physical and equivalent dose components for all groups using the above mentioned RBE's and compound factors for BSH. This information was listed to put the radiaton doses used into proper perspective. The equivalent doses must be used to make meaningful comparisons between different epithermal beams and with previous studies using conventional radiation modalities. The values derived have been used to analyze two normal dogs recently irradiated with an epithermal beam with other beam characteristics than the EMRR epithermal beam, at the $45 \mathrm{MW}$ High Flux Reactor at Petten, the Netherlands with BSH as boron compound. The dogs were irradiated during the same time using a $8 \mathrm{~cm}$ diameter beam. One dog had a mean blood boron concentration of $62 \mathrm{ppm}$ boron and the other $38 \mathrm{ppm}$ yielding macroscopic peak physical doses of 24.9 and $17.5 \mathrm{~Gy}$, respectively. The macroscopic peak equivalent doses were derived using the RBE's and compound factor derived from the BMRR experiments and were equal to 17.7 and $15.2 \mathrm{eq} \cdot \mathrm{Gy}$. These equivalent doses predicted correctly that both dogs would have CNS necrosis. The skin physical doses for these two dogs were 12.3 and $9.6 \mathrm{~Gy}$. The derived skin equivalent doses, 17.2 and 15.8 eq. $\mathrm{Gy}$ also agreed well with the observed skin effects, i.e. dry desquamation and hair loss.

These results stress the complexity of assessing the normal tissue tolerance with boron capture treatments and epithermal neutron beams. Additional large animal model studies using epithermal beams and the various boron carriers are needed to clarify the relative reactions and will give useful definitions of equivalent dose. The authors believe this information is needed prior to human patient treatment with this complex bimodal therapy.

Copyright: The submitted publication has been authorized by a contractor of the U.S. Government under DOE contract No. DE-AC07-761D01570. Accordingly, the U.S. Government retains a nonexclusive, royalty-free License to publish or reproduce the published form of this contribution, or allows others to do so, for U.S. Government purposes.

\section{References}

1. Archambeau, J. O.: Swine skin: a model to evaluate dose recovery from different radiations. In: Fairchild, R. G., V. P. Bond, A. D. Woodhead (eds.): Clinical aspects of neutron capture therapy. Basic Life Sci. 50 (1989), 9-20.

2. Archambeau, J. O., A. Ines: Response of swine skin microvasculature to acute single exposures of $X$ rays: quantification of endothelial changes. Radiat. Res. 98 (1988), 32-51.

3. Bauer, W. F. D. A. Johnson, S. M. Steele, K. Messeck, D. L. Miller, W. A. Propp: Gross boron determination in biological samples by inductively coupled plasma atomic emission spectroscopy. Strahlenther. Onkol. 165 (1988), 176-179.

4. Fankhauser, H., G. Stragliotto: Biodistribution of known sulfhydryl (BSH) on patients with intracranial tumors. In: Allen, B. J., D. E. Moore, B. U. Harrington (ed): Progress in neutron capture therapy for cancer, Plenum Press, New York, London (1992), 551-556.

5. Fike, J. R., G. T. Gobbel: Central nervous system radiation injury in large animals models. In: Gutin, P. H., S. A. Leibel, G. E. Sheline (eds.): Radiation injury to the nervous system. Raven Press, New York 1991, p. 113-135.

6. Gahbauer, R.: RBE in normal tissue. In: Proc. European collaboration on Boron Neutron Capture Therapy, Petten. Plenum Press, New York (in press). 
7. Gavin, P. R., S. L. Kraft, C. E. DeHaan, M. L. Griebenow, M. P. Moore: $A$ large animal model for boron neutron capture therapy. In: Allen, B. J., D. E. Moore, B. U. Harrington (ed): Progress in neutron capture therapy for cancer, Plenum Press. New York, London (1992), 479-484.

8. Gavin, P. R., F. J. Wheeler, R. Huiskamp, A. Siefert, S. L. Kraft, C. E. DeHaan: Large animai model studies of normal tissue tolerance using an epithermal beam and borocaptate sodium. In: Proc. European Collaboration on Boron Neutron Capture Therapy, Petten. Plenum Press, New York (in press).

9. Hall, E. T., D. K. Novak, A. M. Kellerer, H. M: Rossi, S. Morino, L. G. Goodman: RBE as a function of neutron energy experimental observations. Radiat. Res. 64 (1975), 245-255.

10. Hopewell, J. W.: The importance of vascular damage in development of late effects in normal tissues. In: Meyer, R. E., H. R. Withers: Radiation biology in cancer research. Raven Press. New York 1980, p. 449-459.

11. Hopewell, J. W.: The skin: it's structure and response to ionizing radiation. Int. J. Radiat. Biol. 57 (1990), 751-773.

12. Hornsey, S.: Experimental central nervous system injury from fast neutrons. In: Gutin, P. H., S. A. Leibel, G. E. Sheline (eds.): Radiation injury to the nervous system. Raven Press, New York 1991, p. 137-148.

13. Kitao, T.: Vascular wall dose from boron neutron capture reaction. In: Hatanaka, H. (ed.): Boron neutron capture therapy for tumors. Nishimura Co. Ltd., Niigata, Japan 1986, p. 191-197.

14. Kraft, S. L., P. R. Gavin, C. E. DeHaan, C. W. Leathers, W. Bauer, R. V. Dorn III: The biodistribution of boron in canine spontaneous intracranial tumors following borocaptate sodium infusion. Allen, B. J., D. E. Moore, B. U. Harrington (ed): Progress in neutron capture therapy for cancer, Plenum Press, New York, London (1992), 537-540.
15. Laramore, G. E.: Injury to the central nervous system after high LET radiation. In: Gutin, P. H., S. A. Leibel, G. E. Sheline (eds): Radiation injury to the nervous system. Raven Press, New York 1991, p. 341-360.

16. Nigg, P. W., P. D. Randolph. F. J. Wheeler: Demonstration of three dimensional deterministic radiation transport theory dose distribution analysis for boron neutron capture therapy. Med. Phys. 18 (1991), 43-53.

17. Park, R. D., T. R. O'Brien, B. B. Baker, J. C. Morgan: Single dose irradiation of canine skin. Vet. Radiol. 15 (1974). 108-111.

18. Rydin. R. A., O. L. Deutsch. B. W. Muray: The effect of geometry on capillary wall dose for boron neutron capture therapy. Phys. Med. Biol. 21 (1976), 134-138.

19. Stewart. P. A.. C. L. Farrell. R. F. Del Macstro. C. G. Ellis: The effect of cellular microenvironment on vessels in the brain. Part 1: Vessel structure in tumor, peritumor, and brain from humans with malignant glioma. Int. J. Radiat. Biol. 60 (1991), 125-130.

20. Zaider, M.: Concepts for describing the interaction between two agents. Radiat. Res. 123 (1990), 257-262.

21. Ziegler. J. F.. J. P. Biersack. U. Littmark: The stopping and range of ions in solids. Pergamon Press. New York 1970.

22. Zook, B. C., E. W. Bradley, G. W. Csarett, C. C. Rodgers: Pathologic findings in canine brain irradiated with fractionated fast neutrons or photons. Radiat. Res. 84 (1980), 526-528.

For the authors: P. R. Gavin. M. D., Dept. of Veterinary Clinical Medicine and Surgery, McCoy Hall, Washington State University, Pullman. WA 99164-6610, U.S.A. 
, 


\section{Creating and Using a Type of Free-Form Geometry in Monte Carlo Particle Transport}

D. E. Wessol and F. J. Wheeler

This article is a reprint from Nuclear Science and Engineering, 113, 314-323, (1993). 
, 


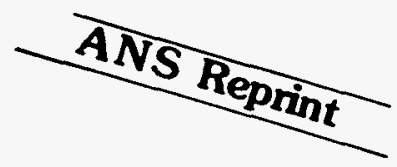

\title{
Creating and Using a Type of Free-Form Geometry in Monte Carlo Particle Transport
}

\author{
Daniel E. Wessol* and Floyd J. Wheeler \\ Idaho National Engineering Laboratory, EG\&G Idaho, Inc. \\ P.O. Box 1625, Idaho Falls, Idaho 83415-1575
}

Received January 2, 1992

Accepted August 5, 1992

\begin{abstract}
While the reactor physicists were fine-tuning the Monte Carlo paradigm for particle transport in regular geometries, the computer scientists were developing rendering algorithms to display extremely realistic renditions of irregular objects ranging from the ubiquitous teakettle to dynamic Jell-O. For many years, the modeling commonality of these apparently diverse disciplines was either largely ignored or unnoticed by many members in both technical communities. Apparently, with the exception of a few visionaries such as the Mathematical Application Group, Inc. (MAGI), each community was not sufficiently aware of what the other was doing. This common basis included the treatment of neutral particle transport through complicated geometries in three-dimensional space. In one instance, it is called the Boltzmann transport equation, while in the other, it is commonly referred to as the rendering equation.

Even though the modeling methods share a common basis, the initial strategies each discipline developed for variance reduction were remarkably different. Initially, the reactor physicist used Russian roulette, importance sampling, particle splitting, and rejection techniques. In the early stages of development, the computer scientist relied primarily on rejection techniques, including a very elegant hierarchical construction and sampling method. This sampling method allowed the computer scientist to viably track particles through irregular geometries in three-dimensional space, while the initial methods developed by the reactor physicists would only allow for efficient searches through analytical surfaces or objects.

As time goes by, it appears there has been some merging of the variance reduction strategies between the two disciplines. This is an early (possibly first) incorporation of geometric hierarchical construction and sampling into the reactor physicists' Monte Carlo transport model that permits efficient tracking through nonuniform rational B-spline surfaces in three-dimensional space. After some discussion, the results from this model are compared with experiments and the model employing implicit (analytical) geometric representation.
\end{abstract}

\section{INTRODUCTION}

With motivation from the Idaho National Engineering Laboratory's (INEL) boron neutron capture therapy (BNCT) program to model irregularly shaped biological systems, INEL and the University of Utah jointly developed a Monte Carlo-based radiation transport tool that is capable of tracking neutrons, photons, and charged particles through nonuniform rational

*Present address: Montana State University, CS Department, 410 Roberts Hall, Bozeman, Montana 59715.
B-spline surfaces ${ }^{1}$ (NURBS), a class of free-form geometry. The authors make the assumption that the readers of this paper have sufficient knowledge of the Monte Carlo method and will, as the title suggests, elaborate on the geometric considerations and construction of the free-form NURBS geometry, as well as the strategies employed to track through this geometry.

When modeling anatomical detail for the INEL BNCT program, a capability was required to model irregularly shaped boundaries more precisely than was available with just combinatorial or constructive solid geometry. At this point, the smooth surfaces that the 
mechanical designers were able to achieve with splinebased, computer-aided design tools caught our attention. After an extensive search for the right method, the authors found that NURBS would meet the requirements. Having reached this conclusion at INEL, we were pleasantly surprised to discover that world-class NURBS experts resided in our backyard, a mere 200 miles away at the University of Utah. The decision was made to modify their Alpha_1 geometric spline-based modeling system ${ }^{2}$ to create B-spline geometries suitable for INEL's Monte Carlo transport code. These modifications resulted in a spline-based geometric editor, named BNCT_Edit. In addition to interactively carving out three-dimensional geometry from an arbitrary image space (in our case, medical images), BNCT_Edit will also graphically display the results of the Monte Carlo analysis.

Now that free-form geometries are available, we are faced with the issue of how to intersect the spline surfaces so that we can return boundary-intersection distances to the Monte Carlo collision routines. Since a ray tracer was developed at the University of Utah ${ }^{3}$ that already could compute the surface intersection distances for the free-form geometries, it was a relatively straightforward task to pass this information to the Monte Carlo process analogously to the way the distance to boundary is passed back by the combinatorial geometry routines.

The Monte Carlo method for neutron transport has been under development and refinement since the 1930s, when Enrico Fermi was conducting numerical experiments showing how the newly discovered neutron might interact with condensed matter. ${ }^{4}$ Although ray tracing has been in use for some time to study geometric optics, its development and refinement for computer graphics began to mature only in the 1960 s with the development of Syntha Vision by the Mathematical Application Group, Inc. (MAGI). In the late 1970s, Whitted ${ }^{5}$ demonstrated the viability of using ray tracing for shading irregular surfaces.

This introduction is, by necessity, a vast oversimplification of the many years of development and refinement in both disciplines to get us to this point in time. That is why we would like to take some time explaining NURBS and their properties in Sec. II, and we discuss in Sec. III the way intersection distances are found efficiently by using the ray-tracing algorithm. Finally, we present some results from this newly developed model along with some current and future considerations in Sec. IV.

\section{NONUNIFORM RATIONAL B-SPLINE SURFACES TUTORIAL}

A powerful, free-form curve and surface representation system, NURBS is an involved mathematical formalism that can produce striking and beautiful object renditions. The NURBS representation incorporates the properties of B-splines, interpolating splines, and Bezier curves and surfaces. NURBS may be best described as a naturally smooth curve (or surface) represented as a piecewise polynomial expression. NURBS are easy to form into complex, sculptured shapes and require much less storage than the polygonal representation they have replaced. Because of the rational property of NURBS, they can exactly represent conic sections.

\section{II.A. B-Spline Basis}

The curve generated by use of the B-spline basis is given by $\alpha(t)$, the position vector along a curve as a function of the parameter $t$ :

$$
\alpha(t)=\sum_{i=0}^{n} P_{i} B_{i, k}(t) \quad\left(t_{\min } \leq t<t_{\max }\right),
$$

where $P_{i}$ are the user-specified control points chosen to fit an object of interest and $B_{i, k}(t)$ are the $i$ 'th weighting or basis functions of order $k$ (degree $k-1$ ). In this case, the curve is represented by $n$ segments and $n+1$ points. Simply stated, the position vector is a weighted sum of the control points at parameter $t$.

The B-spline basis uses an elaborate weighting scheme developed to compute the contribution each control point makes to the position vector at a particular value of the parameter. The weighting functions are derived from the following ${ }^{6,7}$ recurrence relationships:

$B_{i, 1}(t)= \begin{cases}1 & \text { for } x_{i} \leq t<x_{i+1} \\ 0 & \text { otherwise }\end{cases}$

and

$B_{i, k}(t)=\frac{B_{i, k-1}(t)\left(t-x_{i}\right)}{\left(x_{i+k-1}-x_{i}\right)}+\frac{B_{i+1, k-1}(t)\left(x_{i+k}-t\right)}{\left(x_{i+k}-x_{i+1}\right)}$,

where $x_{i}$ is an element of the so-called knot vector with the property $\left(x_{i} \leq x_{i+1}\right)$. The knot vector is a series of nondecreasing scalar values that steer the curve about the control points. Equation (2) provides for local control of the curve by providing for zero weighting of points far from the parameter. These knot elements determine both the parametric range and the smoothness in the transition between each of the polynomial pieces. Each knot element corresponds parametrically to a place on the curve where two of the polynomial pieces are joined together. The number of elements in the knot vector is determined from the indices in the recurrence relationships. The maximum parametric value for an open knot vector is defined to be

$$
t_{\max }=n-k+2 \text {. }
$$

It is interesting to note at this point that the nonuniform part of the NURBS acronym refers to the knot

NUCLEAR SCIENCE AND ENGINEERING VOL. 113 APR. 1993 
vector. The nonuniform knot vector allows treatment of a collection of polynomial pieces as a single entity, automatically maintaining curvature continuity. Duplicate values within the knot vector are allowed, and this multiplicity controls how smooth the transition is between polynomial pieces, drawing the curve closer to the corresponding control point. The beginning and end of an open knot vector must have a multiplicity equal to the order, and the number of intermediate values in the vector depends on the number of control points. As the order decreases, the curve will draw closer to the defining polygon until a second-order (or linear) expression is obtained, resulting in a linear fit between control points.

Most of what has been stated thus far has been in the form of terse definition rather than reason. A complete treatise on B-splines would consume far more space than this journal would allow, so we defer reasoning to Ref. 8. Instead, we now illustrate how a knot vector is constructed, then illustrate the relationships among the control points, the knot vector, and curve.

\section{II.B. Knot Vector Example}

Consider the construction of an open knot vector for a third-order (quadratic) curve $(k=3)$ with five control points $(n=4)$. As stated before, the beginning and end of the open knot vector must have a multiplicity equal to the order, or three in this case. Since the parameter ranges from 0 to $t_{\max }$ with $t_{\max }=4-3+2=3$, our knot vector will be $\left[\begin{array}{llllllll}0 & 0 & 0 & 1 & 2 & 3 & 3 & 3\end{array}\right]$.

\section{II.C. Control Points, Knot Vector, and Curve Relationship}

Figures 1, 2, and 3 show a fourth-order (cubic) spline described by five control points and open-end conditions. Note that the knots have a multiplicity of four at either end of the knot vector and a tangency of the curve to the control polygon at both end points. The middle control point is moved in Fig. 2, which, in turn, changes the curve in an intuitive manner. Figure 3 shows how a change in the knot vector alters the curve in a less intuitive way. Also note the nonuniform spacing of the knot vector. Figure 4 demonstrates how changes in the order are reflected in the curve shape. The control points are left the same, but the order is reduced to three. Note the change in the knot vector and how the curve draws closer to the control polygon, recalling the property that, as the order decreases, the curve lies closer to the control polygon.

\section{II.D. Surface Representation}

The next increment beyond the curve is the surface, which is described by the tensor-product spline as a function of two parameters $(\mu, v)$ for two curves with orders $k$ and $l$, respectively:

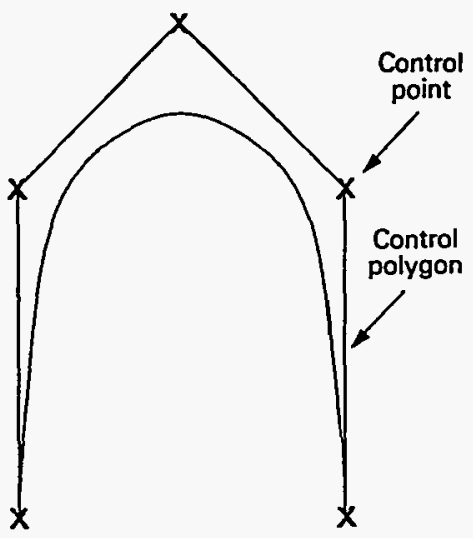

Knot vector $-\left[\begin{array}{lllllllll}0 & 0 & 0 & 0 & 1 & 2 & 2 & 2 & 2\end{array}\right]$ Order $(k)=4$

Fig. 1. Simple B-spline curve. ${ }^{2}$

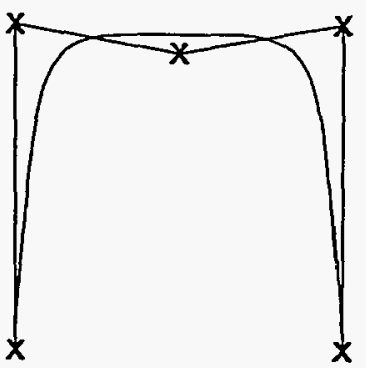

Knot vector - $\left[\begin{array}{lllllllll}0 & 0 & 0 & 0 & 1 & 2 & 2 & 2 & 2\end{array}\right]$

Order $(k)=4$

Fig. 2. Middle control point relocated. ${ }^{2}$

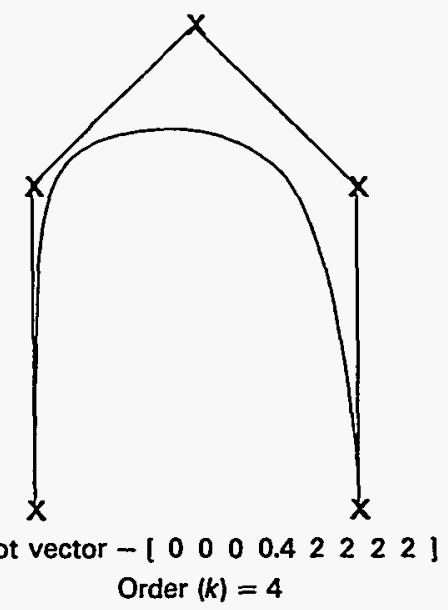

Fig. 3. Simple curve with new knot vector. ${ }^{2}$ 


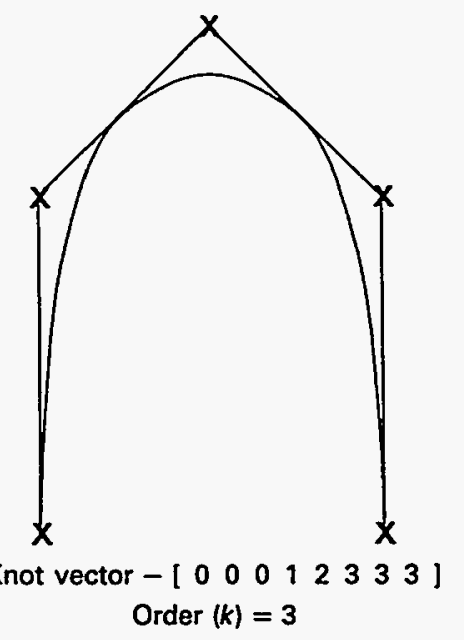

Fig. 4. Simple curve with different order. ${ }^{2}$

$$
\beta(\mu, v)=\sum_{i=0}^{n} \sum_{j=0}^{m} P_{i+1, j+1} B_{i, k}(\mu) C_{j, l}(v),
$$

where

$$
\begin{aligned}
B_{i, 1}(\mu)= & \begin{cases}1 & \text { for } x_{i} \leq \mu<x_{i+1} \\
0 & \text { otherwise }\end{cases} \\
B_{i, k}(\mu)= & \frac{B_{i, k-1}(\mu)\left(\mu-x_{i}\right)}{\left(x_{i+k-1}-x_{i}\right)} \\
& +\frac{B_{i+1, k-1}(\mu)\left(x_{i+k}-\mu\right)}{\left(x_{i+k}-x_{i+1}\right)}
\end{aligned}
$$

and

$$
\begin{aligned}
C_{j, 1}(v)= & \begin{cases}1 & \text { for } y_{j} \leq v<y_{j+1} \\
0 & \text { otherwise }\end{cases} \\
C_{j, l}(v)= & \frac{C_{j, l-1}(v)\left(v-y_{j}\right)}{\left(y_{j+l-1}-y_{j}\right)} \\
& +\frac{C_{j+1, l-1}(v)\left(y_{j+l}-v\right)}{\left(y_{j+l}-y_{j+1}\right)}
\end{aligned}
$$

Figure 5 shows an example of a surface generated with this spline. The tensor-product spline, which maps a rectangle into Euclidean three-dimensional space, can be regarded as a mathematical extension of the curve to the surface. A convenient way to think of this spline surface is to consider the rows or columns of the control mesh as a set of individual B-spline control curves analogous to the control points of the previous discussion. The second knot vector and order then describe how these curves will be blended to form a surface just as points were weighted to form a curve.

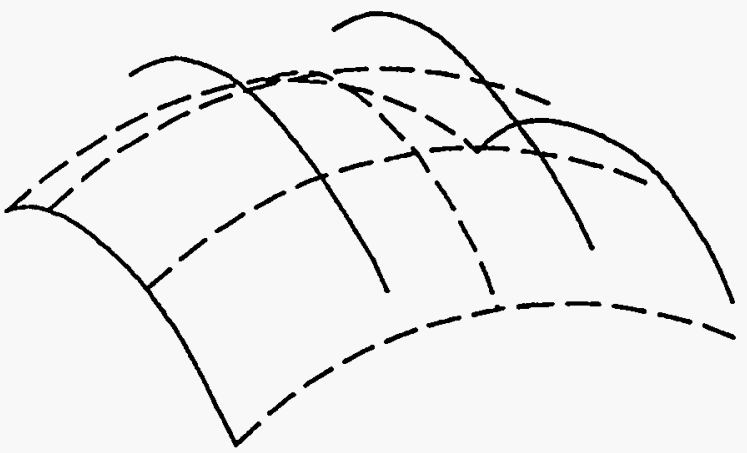

Fig. 5. Control curves for a surface.

Our tutorial ends here; bear in mind the superficial treatment given to the subject. Important discussions, e.g., refinement (where additional control points are added $a$ posteriori) and adaptive subdivision (a technique used for converting a surface mesh into polygonal form for rendering), were deliberately excluded.

\section{RAY TRACING NONUNIFORM RATIONAL B-SPLINE SURFACES}

In this section, as with Sec. II, only a functional description of the topic is provided; the references provide the theoretical descriptions. In the context of this treatise, the expressions "ray tracing" and "surface intersection" are used interchangeably since it is the raytracing algorithm that produces the distance to the next surface intersection, given an arbitrary point in threedimensional space along a ray with prespecified direction cosines.

The underlying issue with ray tracing can almost always be attributed to efficiency concerns. This is especially true when the geometries are constructed of free-form surfaces. The next issue is that of robustness. Are the results correct and reproducible? The next two sections address these issues.

According to Whitted, ${ }^{5}$ the cost of computing the point of surface intersection closest to the current ray position accounts for $95 \%$ of the total cost of the raytracing application in computer graphics. This percentage may not be as high for the neutron transport application because of considerations for the various reaction mode decisions, cross-section computation (or lookup), and energy exchange phenomena. It is still likely to consume well over $50 \%$ of the computational resource.

\section{III.A. Hierarchical Bounding}

One of the oldest and most fundamental acceleration techniques employed by the computer scientist is 
the bounding volume. The object of interest is surrounded by a bounding volume that permits a simpler intersection test than for the object itself. If it is determined that the ray does not intersect the bounding volume, there is no need to further check the object for an intersection. While this strategy substitutes a simpler intersection test for a more costly one, it does not reduce the number of tests.

Hierarchical bounding 9 reduces the number of tests by enclosing several nested bounding volumes into a larger (or parent) bounding volume. If the ray fails to intersect the parent volume, there is no need to consider any of the other enclosed volumes for subsequent intersection tests. If the ray does intersect a bounding volume, the process is continued until the ray has failed to intersect any of the inner bounding volumes or until the smallest bounding volume (or leaf node) is reached. Each leaf node is a single geometric object and will ultimately contain the ray-object intersection. Each interior node consists of a bounding volume and a list of pointers to other nodes in the tree or hierarchy. A bounding volume may contain more than one sibling bounding volume or geometric object. This is similar to some of the rejection strategies used in the neutron transport problem without the notion of nested or hierarchical objects. A simplified example of hierarchical bounding is illustrated in Fig. 6 for two rays. Assuming all objects of interest are bounded by volume $A$, then, if it is determined that ray 1 fails to intersect volume $A$, there is no further need to follow the ray. If it has been determined that ray 2 intersects volume B,

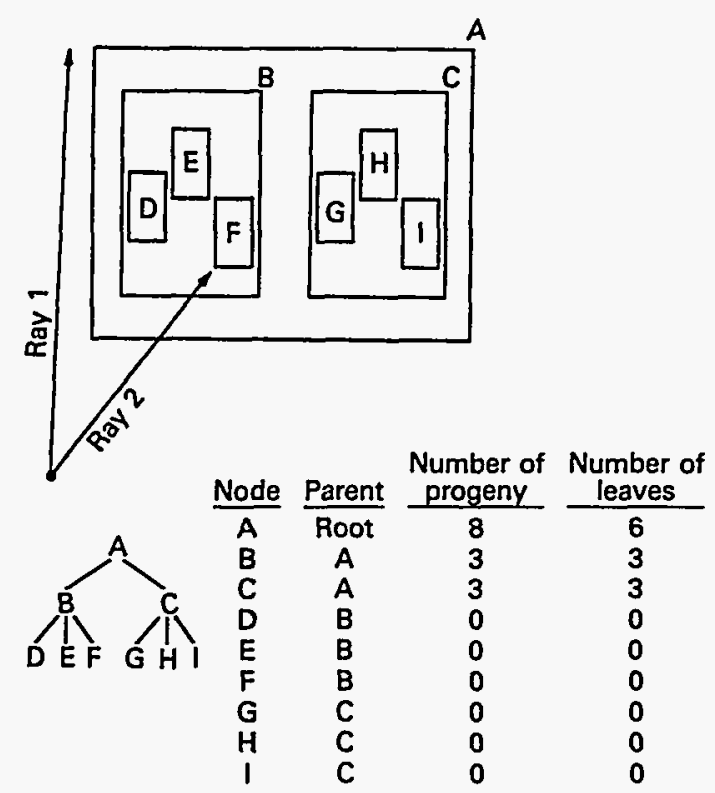

Fig. 6. Hierarchical bounding. only volumes $\mathrm{D}, \mathrm{E}$, and $\mathrm{F}$ (leaf nodes) require intersection tests, as well as an exit test from volume $B$.

The cost of a successful intersection is the sum of the cost of the number of tests against the bounding volume and the number of tests against the geometric objects within. There is always a trade-off between minimizing the number and the cost of each of these tests. By minimizing the size of the bounding volume, the number of intersections with it are reduced. This will, however, tend to increase its geometric complexity, resulting in a more costly intersection, but will also result in fewer tests against the usually more complex object within.

Construction of the hierarchical nesting is a crucial step for efficient execution. In the earlier methods, this was done interactively via a structure editor. In addition, these early methods worked only with implicit surfaces. Current methods for the automatic construction of bounding volumes for free-form surfaces is primarily based on the methods developed by Sweeney, ${ }^{10}$ with significant developments by Kay and Kajiya," Kajiya, ${ }^{12}$ Arvo and Kirk, ${ }^{13}$ and others.

\section{III.B. Implementation}

Numerical methods for intersecting B-spline surfaces generally include the following steps:

1. Preprocess a set of hierarchical bounding boxes around each spline surface and store as the geometry file.

2. Determine which bounding box contains the raysurface intersection.

3. Use this bounding box location as the starting point for the direct numerical solution, e.g., Newton's method, of the remainder of the intersection distance.

The University of Utah's method for intersecting the B-spline surfaces ${ }^{3}$ that we use here consists of the following steps:

1. Using the Oslo algorithm, ${ }^{1}$ subdivide the surface into polygons until each polygon passes the criterion for flatness.

2. As the surface is subdivided, build a tree of hierarchical bounding volumes with the polygons at the leaf nodes.

3. Intersect the ray with the bounding volumes, and test the ray against the polygons at the leaves.

The first two steps are performed as the preprocessing step, and a geometry file is created for the transport computation. This has to be done only once for each calculation.

\section{III.C. Preprocessing}

Each vertex of a polygonal element must be coplanar to construct a normal for that polygon. If the surface control mesh does not meet the flatness criterion, 
it is subdivided in half until each subdivided portion is flat. The subdivision is performed by adding a knot with multiplicity $k$ (if subdividing along the $\mu$ direction) or $l$ (if subdividing along the $v$ direction) in the center of the knot vector. If the surface is flat enough in both directions, a polygon is formed that becomes the leaf node of the hierarchical bounding volumes. Each parent node will form two children nodes until the flatness criterion is achieved. In practice, the flat polygon is further divided into two triangles so the barycentric coordinates may be used for the final ray-polygon intersection test. We will not elaborate how the final intersection test in barycentric coordinates is conducted, but the interested reader should consult Ref. 3 .

Our basic objective is to restrict the ray tracer's interest to the more promising object candidates for intersection. As the surface is subdivided, a tree is constructed with the original surface as the root node, and two child nodes are generated for each surface division. When the trees are finally built, they are surrounded by sets of hierarchical bounding boxes constructed by examining the extents of the geometry. The boxes and polygons are tagged with material identifications and stored as a geometry file for the Monte Carlo computation, which uses the boundary information obtained from the ray-tracing algorithms.

\section{III.D. Surface Intersection}

The bounding volumes provide an efficient means of finding the ray-surface intersections. As the ray traverses the bounding volume hierarchy, a sorting operation is performed with respect to each ray, based on the estimated distances to the points of intersection with the bounding volumes. Arranging these volumes by distance from the ray's origin ensures that the volumes are processed in the same order encountered by the ray. If, during this traversal, a leaf node containing triangles is encountered, a ray-object intersection test is performed; otherwise, the next bounding volume is processed until either a leaf node is encountered or the ray exits the model. The ray-triangle intersection tests are more easily performed in barycentric coordinates. The point representing the intersection lying on the same plane as the triangle to be tested for the intersection is mapped into barycentric space. If the point lies within the vertices of the triangle, an intersection with that triangle occurred. This is by no means the only method, nor necessarily the best method, for intersecting splined surfaces. As our methods evolve, we will search for that best method. Future considerations will include an evaluation of Arvo and Kirk's ${ }^{13}$ five-dimensional space subdivision as well as others.

\section{RESULTS AND DISCUSSION}

The NURBS ray-tracing capability was incorporated into the rtt_MC (radiation transport in tissue by
Monte Carlo) computer code. This module is an extensive modification of the RAFFLE general-purpose Monte Carlo computer code, ${ }^{14}$ designed specifically to address the modeling, transport, and display requirements of BNCT patient treatment planning. Unlike conventional photon therapy, where scatter corrections to first-flight distributions provide useful results, BNCT requires a rigorous three-dimensional solution of the Boltzmann transport equation for each case. The threedimensional model is typically constructed based on medical image slices through the appropriate anatomical features. The rtt_MC models are based on regular geometry primitives or combinations of regular geometry primitives and NURBS bodies. All NURBS bodies are enclosed by combinations of regular geometry primitives forming a non-reentrant bounding feature.

The rtt_MC results have been compared with experimental and three-dimensional transport results ${ }^{15}$ in regular geometry. For initial validation and evaluation of NURBS geometry, a simple benchmark was defined. This benchmark is a cube of Lucite irradiated by an epithermal neutron beam. The beam source was a $10-x$ $10-\mathrm{cm}^{2}$ plane with isotropic angular distribution and a characteristic energy spectrum calculated for the Brookhaven Medical Research Reactor's (BMRR's) epithermal neutron beam, ${ }^{16}$ located at Brookhaven National Laboratory. Figure 7 shows a sketch of the configuration. A 5.08-cm-thick, lithiated, polyethylene beam delimiter with a $10-\times 10-\mathrm{cm}^{2}$ opening separates the cube and the planar source. The delimiter hole and cube centers are aligned with the beam axis, and one surface of the cube is coplanar with the outer face of the beam delimiter. Actual irradiations at the BMRR have greater complexity in geometric and source representation but are not dissimilar from the simple benchmark used here.

Two cases are presented in this paper: The first case uses only regular geometry primitives, and the second case has the cube modeled with NURBS surfaces. In the

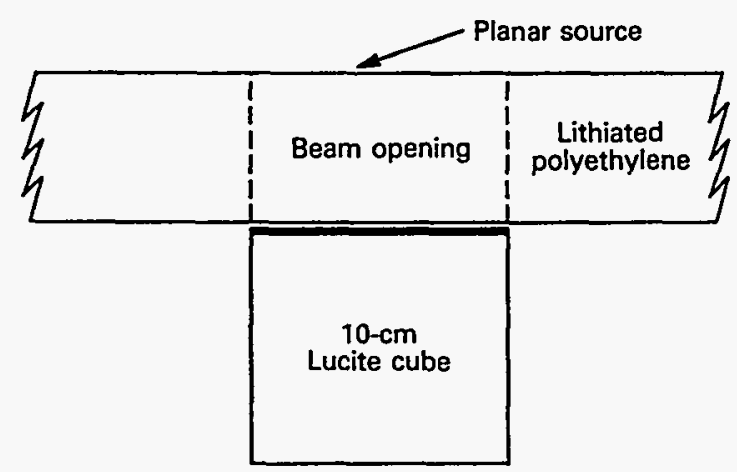

Fig. 7. Benchmark problem for $10-\mathrm{cm}$ Lucite cube irradiated in the BMRR epithermal neutron beam.

NUCLEAR SCIENCE AND ENGINEERING $\quad$ VOL. $113 \quad$ APR. 1993 
TABLE I

Comparison of rtt_MC Results for the Cube Benchmark

\begin{tabular}{|c|c|c|}
\hline \multirow[b]{2}{*}{ Parameter } & \multicolumn{2}{|c|}{ Geometry } \\
\hline & Regular & NURBS \\
\hline $\begin{array}{l}\text { Total neutron absorption in cube } \\
\text { (absorptions per source neutron) }\end{array}$ & $4.67( \pm 0.02) \times 10^{-2}$ & $4.68( \pm 0.02) \times 10^{-2}$ \\
\hline $\begin{array}{l}\text { Peak thermal neutron flux } \\
(<0.414 \mathrm{eV})\left(\mathrm{n} / \mathrm{cm}^{2} \cdot \mathrm{s} \cdot \mathrm{MW}\right)\end{array}$ & $5.43( \pm 0.03) \times 10^{8}$ & $5.46( \pm 0.03) \times 10^{8}$ \\
\hline $\begin{array}{l}\text { Peak gamma dose rate } \\
(\mathrm{cGy} / \mathrm{min} \cdot \mathrm{MW})\end{array}$ & $1.800( \pm 0.0087)$ & $1.791( \pm 0.0087)$ \\
\hline Neutron histories & 2500000 & 2500000 \\
\hline Photon histories & 2500000 & 2500000 \\
\hline Run time (CPU min) & 51.7 & 237.1 \\
\hline
\end{tabular}

NURBS representation, the cube is reconstructed from three pseudo-image slices. Each slice has eight control points, two slightly separated points at each corner. The resulting reconstruction provides an excellent representation of the cube with very little distortion, even at the corners. Computer runs were performed on a single CPU HP 9000/750. Table I shows a comparison of these two cases. These results show good agreement and represent partial validation of the NURBS ray-tracing algorithms as employed in rtt_MC. The CPU time required for the NURBS representation is much greater (a factor of 4 to 5) than for the regular geometry representation. This factor may be reduced as the code is optimized and more effective use of the hierarchical strategies are utilized.

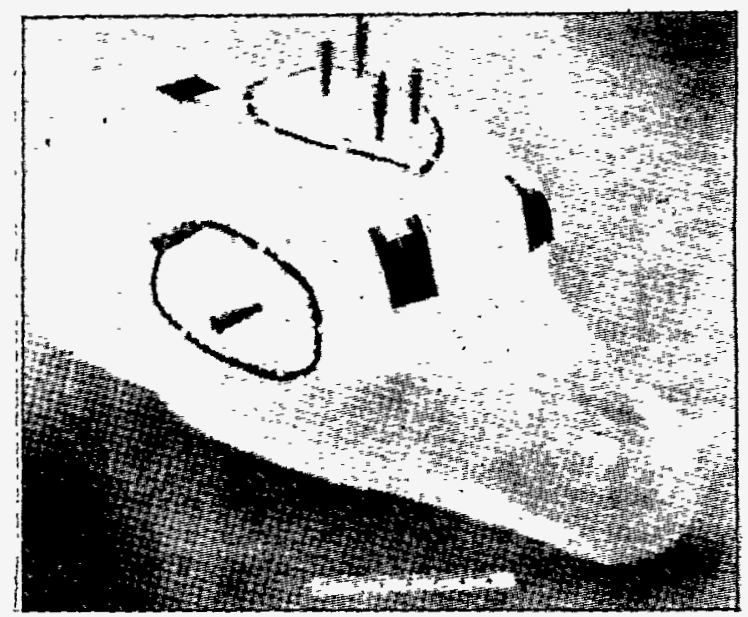

Fig. 8. Photograph of Lucite dog head phantom with dosimetry wires.

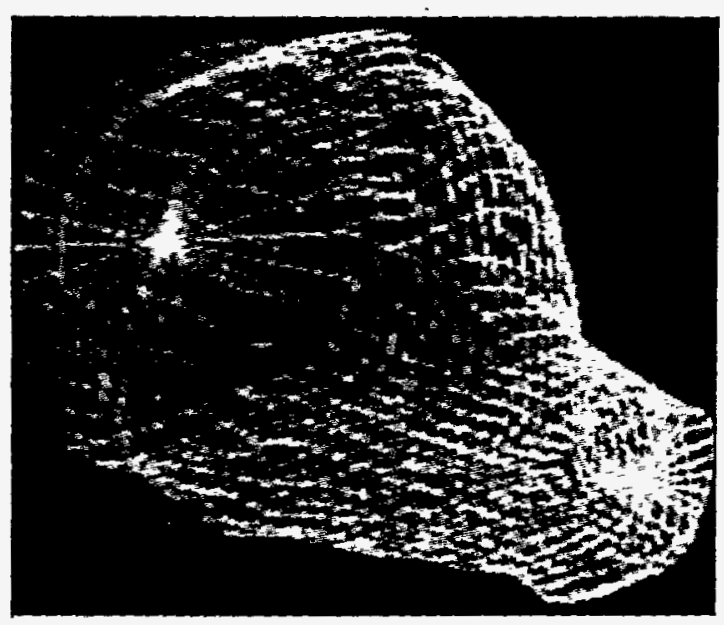

Fig. 9. B-spline representation of the dog head phantom.

The large-animal program for BNCT uses healthy dogs and dogs with spontaneously occurring brain tumors. These animals are administered intravenous injections of the boron compound and are irradiated in the BMRR beam. Validation of the calculational models is partially provided by irradiations of a realistic Lucite dog head phantom (Fig. 8). Dosimetry during these irradiations provided neutron flux and gamma dose as a function of depth at various locations in the head. The NURBS representation of the head phantom was based on magnetic resonance images taken every centimetre in planes perpendicular to the spinal cord. Figure 9 shows a NURBS representation of the three-dimensional model reconstructed from these image slices. The beam is represented as a disk source where the neutron and gamma intensities and

NUCLEAR SCIENCE AND ENGINEERING VOL. 113 APR. 1993 
angular distributions are given by two-dimensional deterministic calculations based on first principles. ${ }^{16}$ Therefore, both the measured and calculated values reported here are absolute with no adjustment. The

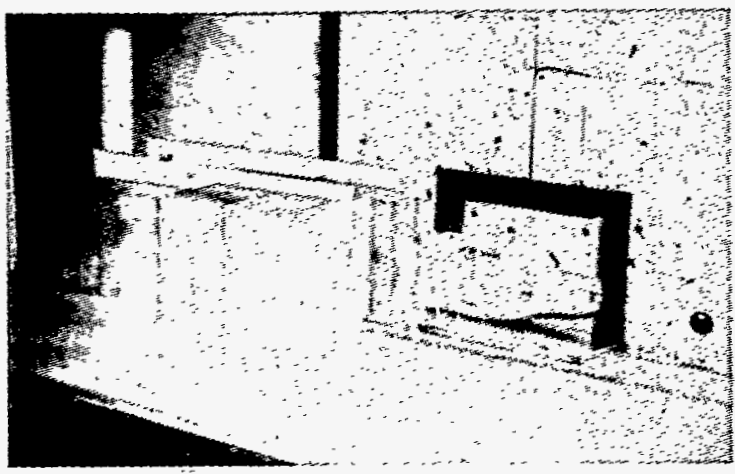

Fig. 10. Experimental configuration for dog head phantom irradiation at BMRR. head phantom was placed in the beam such that the beam axis was perpendicular to the top of the head. A $5.08-\mathrm{cm}$ beam delimiter with a $9.7-\times 9.8-\mathrm{cm}^{2}$ opening restricted the size of the beam. The experimental configuration is shown in Fig. 10. Holes were drilled into the phantom from the top of the head, allowing placement of flux wires and thermoluminescent dosimeters for neutron flux and gamma dose measurements. Calculated values were obtained with the $\mathrm{rtt}_{-} \mathrm{MC}$ code using the NURBS geometry representation.

Figure 11 shows the calculated isoflux distribution for a transverse plane just behind the eyes at beam center. Figure 12 shows the calculated gamma isodose plot for the same plane. These plots are obtained from the rtt_MC calculation by use of a three-dimensional edit grid for detailed tallies throughout the NURBS geometry. The Monte Carlo calculations represented 1000000 neutron histories and 1000000 photon histories and required $126 \mathrm{~min}$ of CPU time on the HP workstation.

References 15 and 17 describe these measurements in more detail along with calculated results using threedimensional deterministic methods and regular geometry

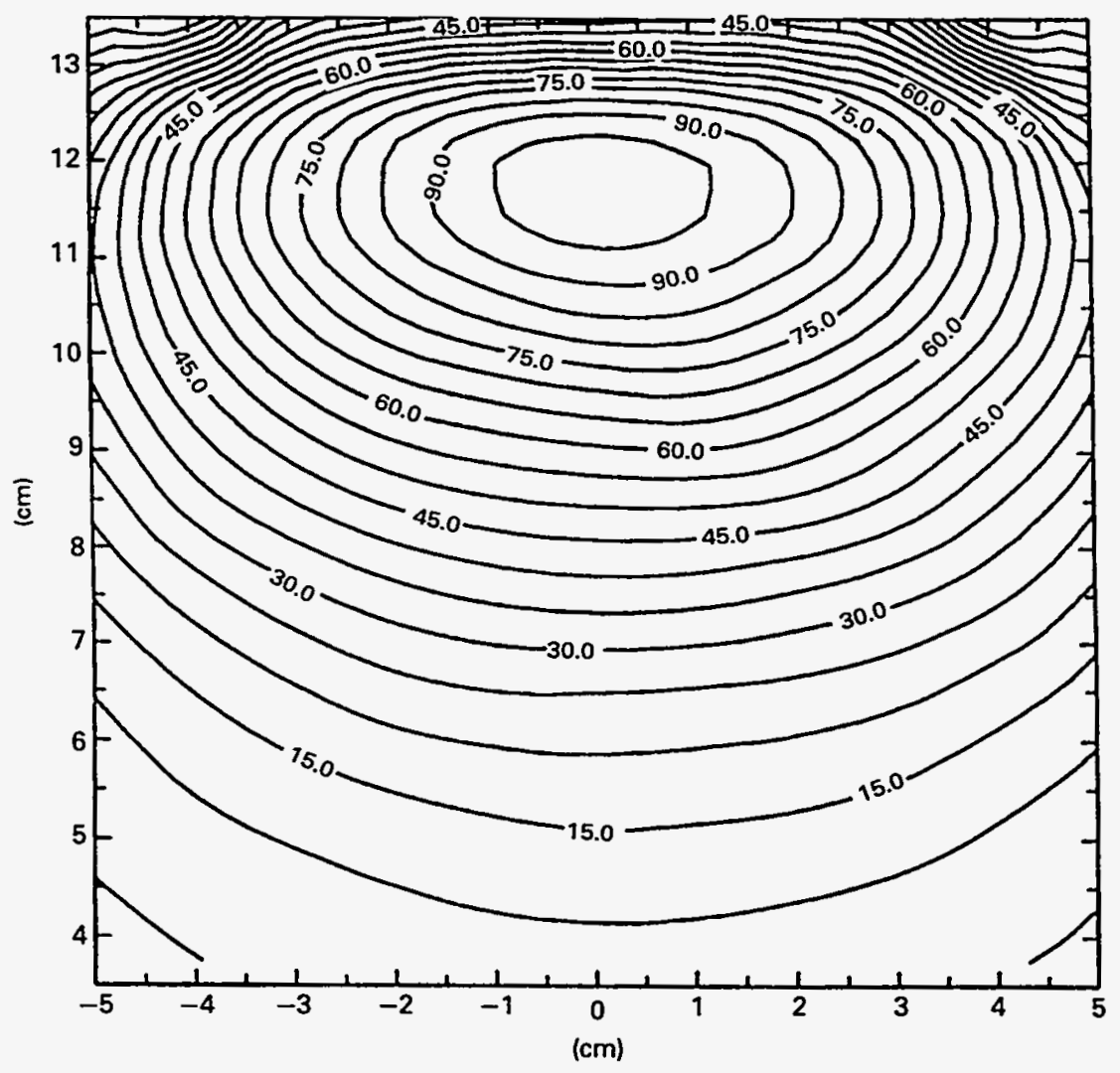

Fig. 11. Dog head phantom calculation; relative thermal flux (contours at $x$ plane $=11.10$ ).

NUCLEAR SCIENCE AND ENGINEERING VOL. $113 \quad$ APR. 1993 


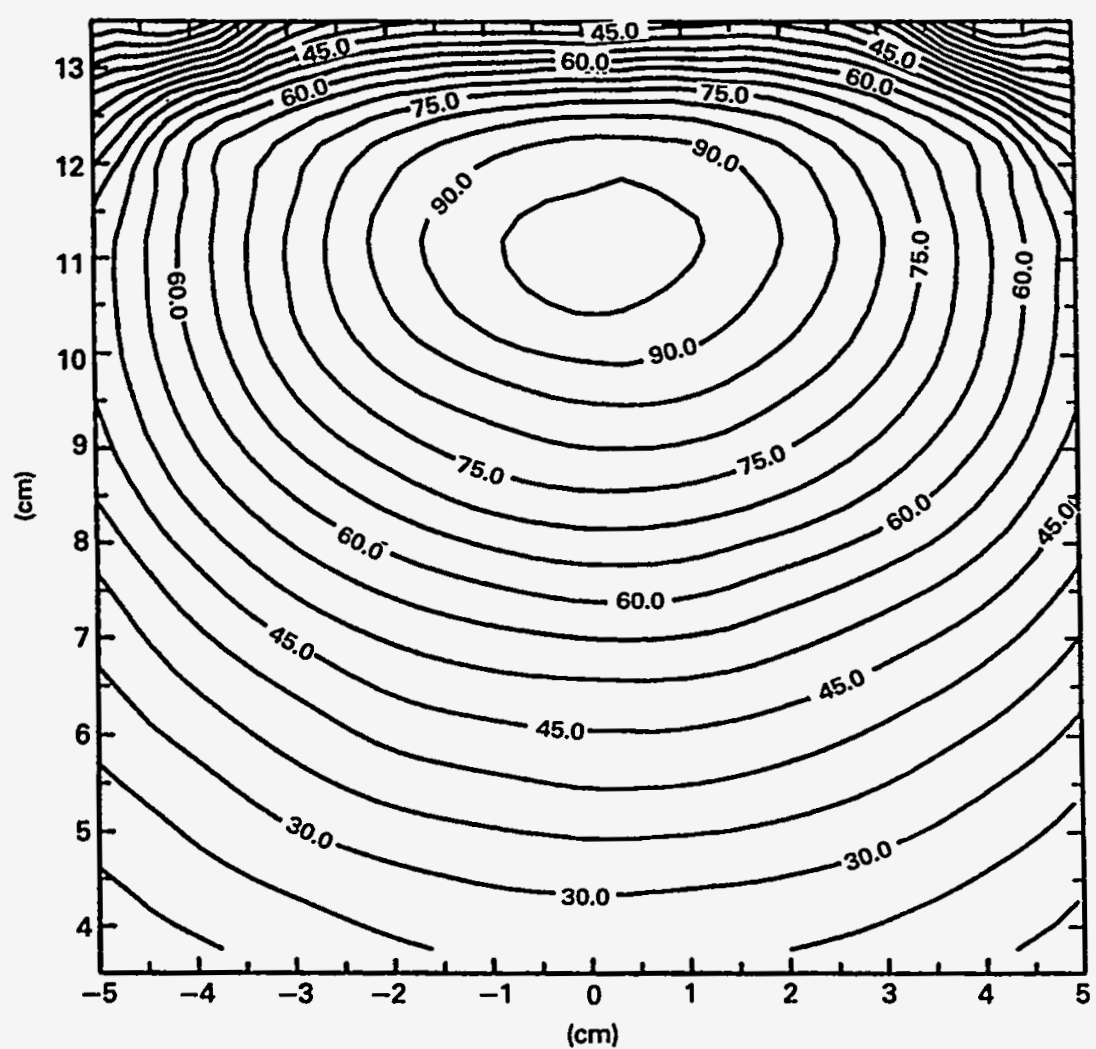

Fig. 12. Dog head phantom calculation; relative gamma dose (contours at $x$ plane $=11.10$ ).

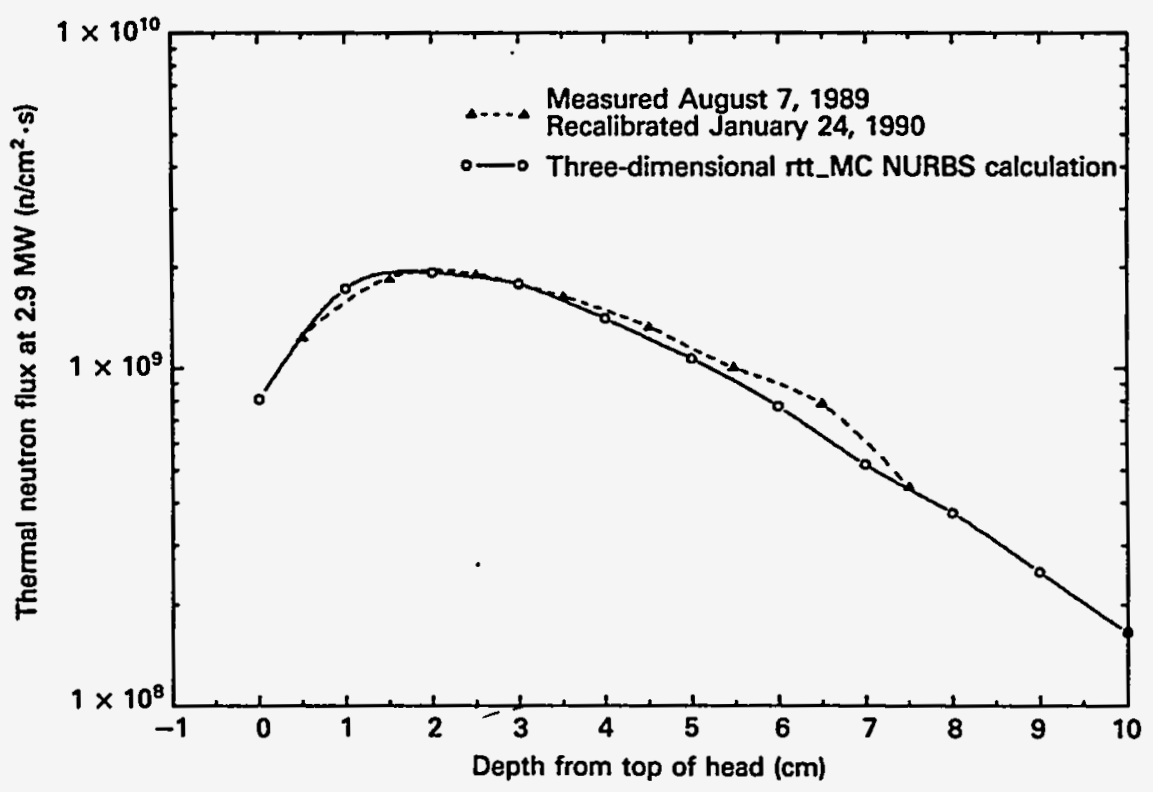

Fig. 13. Measured and calculated depth profiles of BMRR (2.9-MW) thermal flux; dog head phantom at $x=11.5 \mathrm{~cm}$, $y=0.5 \mathrm{~cm}$ (flux wire 3).

NUCLEAR SCIENCE AND ENGINEERING VOL. $113 \quad$ APR. 1993 
Monte Carlo methods. The measured thermal neutron flux for a flux wire near the beam center (flux wire 3) is shown in Fig. 13 along with the calculated results using the NURBS model described in this paper.

The peak thermal neutron flux measured for flux wire 3 was $1.91 \times 10^{9} \mathrm{n} / \mathrm{cm}^{2} \cdot \mathrm{s}$ at a reactor power of 2.9 MW. The calculated peak thermal neutron flux at this position was $1.97 \times 10^{9}\left( \pm 0.02 \times 10^{9}\right) \mathrm{n} / \mathrm{cm}^{2} \cdot \mathrm{s}$. The calculated values are, in general, within $10 \%$ of measurement for all flux wires. Again, this is with no adjustment of the calculational model or measured values. The NURBS model results compare more favorably with measurement than regular geometry Monte Carlo results ${ }^{17}$; however, they are both within experimental error.

As we gain more experience tracking particles through the free-form geometries, it is planned to optimize current methods and obtain and implement improved methods for intersecting free-form surfaces. We have also distributed (parallelized) the Monte Carlo code so it will run on several computers (workstations) simultaneously, allowing viable tracking through very complicated geometries. Using this capability, throughput rate is limited only by the number of computers available and the suitability of the random number generator. Our future plans also include more tightly integrating the free-form and combinatorial geometries.

\section{ACKNOWLEDGMENT}

This study was performed under the auspices of the U.S. Department of Energy (DOE), Office of Energy Research, DOE Field Office, Idaho, under contract DE-AC07$76 I D 01570$.

\section{REFERENCES}

1. E. COHEN, T. LYCHE, and R. RIESENFELD, "Discrete B-Splines and Subdivision Techniques in ComputerAided Geometric Design and Computer Graphics," Comput. Graphics Image Process., 14, 2, 87 (1980).

2. "Alpha_1 Users' Manual," Utah Alpha_1 Project, University of Utah, Department of Computer Science (1986).

3. J. W. PETERSON, "PRT: A High Quality Image Synthesis System for B-Spline Surfaces," Master's Thesis, University of Utah (1988).
4. M. H. KALOS and P. A. WHITLOCK, Monte Carlo Methods, Volume I: Basics, John Wiley \& Sons, New York (1986).

5. T. WHITTED, "An Improved Model for Shaded Display," Commun. ACM, 23, 6, 343 (1980).

6. M. G. COX, "The Numerical Evaluation of B-Splines," DNAC 4, National Physical Laboratory (Aug. 1971).

7. C. DE BOOR, "On Calculation with B-Splines," J. Approx. Theory, 6, 50 (1972).

8. R. H. BARTELS, J. C. BEATTY, and B. A. BARSKY, "An Introduction to the Use of Splines in Computer Graphics," Technical Report TR CS-83-09, University of Waterloo (May 1985).

9. S. RUBIN and T. WHITTED, "A Three-Dimensional Representation for Fast Rendering of Complex Scenes," Comput. Graphics, 14, 3, 100 (1980).

10. M. A. SWEENEY and R. H. BARTELS, "Ray Tracing Free-Form B-Spline Surfaces," IEEE Computer Graphics and Applications, 6, 2, 41 (1986).

11. T. L. KAY and J. T. KAJIYA, "Ray Tracing Complex Scenes," Comput. Graphics, 20, 4, 269 (1986).

12. J. T. KAJIYA, "The Rendering Equation," presented at Hewlett-Packard Computer Graphics Symposium, Fort Collins, Colorado, 1988.

13. J. A. ARVO and D. KIRK, "Fast Ray Tracing by Ray Classification," Comput. Graphics, 21, 4, 55 (1987).

14. F. J. WHEELER et al., "The RAFFLE V General Purpose Monte Carlo Code for Neutron and Gamma Transport," EGG-PHYS-6003, Rev. 1, EG\&G Idaho, Inc. (1983).

15. F. J. WHEELER and D. W. NIGG, "Three-Dimensional Radiation Dose Distribution Analysis for Boron Neutron Capture Therapy," Nucl. Sci. Eng., 110, 16 (1992).

16. F. J. WHEELER, D. K. PARSONS, B. L. RUSHTON, and D. W. NIGG, "Epithermal-Neutron Beam Design for Neutron Capture Therapy at the PBF and BMRR Reactor Facilities," Nucl. Technol., 92, 106 (1990).

17. D. W. NIGG, P. D. RANDOLPH, and F. J. WHEELER, "Demonstration of Three-Dimensional Deterministic Radiation Transport Theory Dose Distribution Analysis for Boron Neutron Capture Therapy," J. Med. Phys., 18, 1, 44 (1991). 


\section{Nuclear Characterization of the HFR Petten BNCT Facility}

P. Watkins, Y. Harker, C. Amaro, W. Voorbraak, F. Stecher-Rasmussen, H. Verhagen, C. Perks, H. Delafield, G. Constantine, and R. Moss

This article is a reprint from Advances in Neutron Capture Therapy, pp. 59-65, (1993). 
NUCLEAR CHARACTERISATION OF THE

\title{
HFR PETTEN BNCT FACILITY
}

\author{
P. Watkins', Y. Harker ${ }^{2}$, C. Amaro', W. Voorbraak ${ }^{3}$, \\ F. Stecher-Rasmussen ${ }^{3}$, H. Verhagen ${ }^{3}$, C. Perks ${ }^{4}$, \\ H. Delafield ${ }^{4}$, G. Constantine' and R.L. Moss ${ }^{1}$ \\ 'Institute for Advanced Materials \\ JRC Petten, The Netherlands \\ ${ }^{2}$ Idaho National Engineering Laboratory \\ Idaho Falls, USA \\ ${ }^{3}$ The Netherlands Energy Research Foundation \\ ECN Petten, The Netherlands \\ ${ }^{4}$ AEA Technology, Harwell, UK
}

\section{INTRODUCTION}

The major work at Petten over the last few years has been the design and construction of an epithermal neutron beam facility in the HB11 beam tube. Following installation of the filter assembly in 1990, and the first operation of the beam at full reactor power in June 1991 , it was finally possible to start the planned series of irradiation experiments. These include phantom irradiations, cell culture experiments and a healthy tissue tolerance study using beagle dogs. Prior to this work a considerable amount of neutron and photon dosimetry was performed to measure various parameters of the beam. Apart from providing definite values for such data, these measurements were essential to validate the calculational models which were used in the design and later for the treatment planning.

The campaign of measurements at HB11 has continued in parallel with the other experiments noted above. In this paper the various measurements that have been performed are reviewed both for the free beam and also within phantoms. These were undertaken as part of an intensive collaborative effort between INEL, AEA Harwell, ECN and JRC Petten and included: stacked resonance-energy activation foils for measuring the neutron spectrum in the thermal energy range; threshold foils and proton recoil proportional counters for the fast neutron energies; and thermoluminescence detectors (TLD) for the gamma field. 
In addition to measurements the foil configurations and phantoms have been modelled using the Monte Carlo code MCNP. Wherever possible the results from these calculations are compared with the measured values and the reasons for any discrepancies are discussed.

\section{STANDARD DOSIMETRY MEASUREMENTS}

After some initial, tentative experiments it became clear that it was important to create a set of well defined dosimetry measurements which would be repeated at regular intervals. Such an approach minimises the possible sources of variation and has the additional benefit of providing data which shows the time dependent behaviour of the beam. This latter point was considered to be of very great importance, first because one of the requirements for future clinical trials would be evidence to demonstrate the stability of the beam in time and secondly, because the HFR is a "working" reactor with many experiments within the core which vary between reactor cycles. The variation of the beam parameters with reactor cycle is therefore of considerable importance.

The possible dosimetric measurements that could be included in such a database is immense but a decision was taken to define a minimum set of simple measurements which could be extended as required. This basic set of measurements has already been supplemented by a number of separate dosimetry measurements which were needed to determine specific features of the beam.

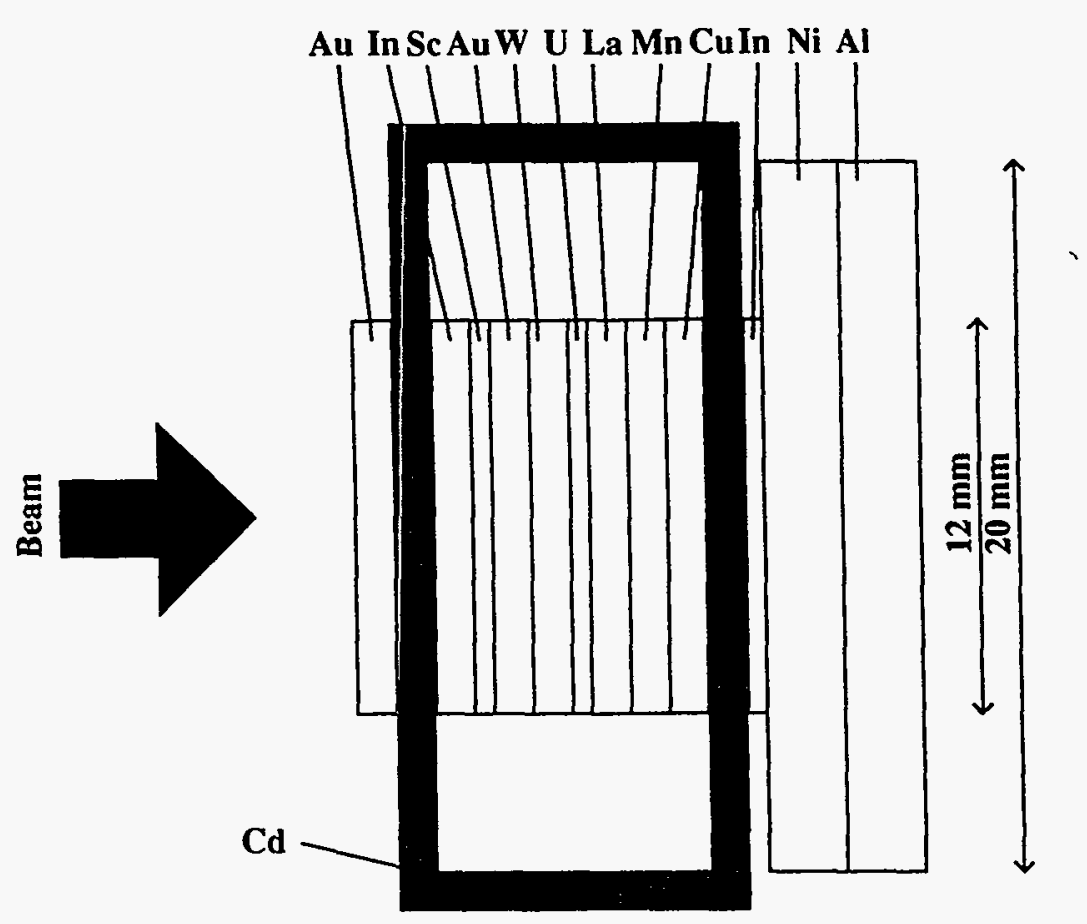

Figure 1. Free beam foil set. 
The standard neutron measurement is that of a stack of resonance and threshold foils, Fig 1, which is positioned at the beam centre immediately on exit from the final aperture. The foils used are responsive to the thermal, low epithermal and fast neutron energies. The important epithermal region from $1 \mathrm{keV}$ to about $0.5 \mathrm{MeV}$ is relatively untouched by this measurement. This is recognised as a major deficiency and is being remedied by the development of a technique which uses resonance foils within a moderating medium. However the existing foil set has the advantage of being straightforward to irradiate and is capable of detecting any gross changes in the beam parameters.

The photon component of the beam is measured using 5 TLDs placed at the same position as the foil set and arranged in the form of a cross. The TLDs are of the Harshaw type 700 using enriched ${ }^{7} \mathrm{Li}$ and are encapsulated in perspex boxes for the irradiations.

\section{CALCULATED SPECTRUM AND FOIL RESPONSES}

To compare the neutron measurements with calculations the foil sets were modelled using the Monte Carlo code MCNP. The MCNP model is shown in Fig 2. The comparisons with measurement are shown in Table 1 under the column $\mathrm{C} / \mathrm{M}$ where it can be seen that in most cases the agreement with measurement is quite good except for the fast neutron energy range which is discussed below. The stability of the beam as a function of time is also shown in the table and is seen to be very good.

Table 1. Free beam foil set results over time, normalised to 1.0 for $2 / 4 / 92$ and ratio of calculated to measured values(C/M).

\begin{tabular}{|c|c|c|c|c|c|c|c|}
\hline \multirow[b]{2}{*}{ Foil } & \multirow[b]{2}{*}{ Composition } & \multirow{2}{*}{$\begin{array}{c}\text { Energy } \\
\text { Response }\end{array}$} & \multicolumn{4}{|c|}{ Date of Irradiation } & \multirow[b]{2}{*}{$\mathrm{C} / \mathrm{M}$} \\
\hline & & & $3 / 91$ & $2 / 4 / 92$ & $21 / 5 / 92$ & $10 / 7 / 92$ & \\
\hline${ }^{197} \mathrm{Au}$ & $1 \% \mathrm{Au}$ in $\mathrm{Al}$ & Thermal & 1.04 & 1.00 & 0.95 & 0.95 & 0.96 \\
\hline${ }^{115}$ In & $0.2 \%$ In in $\mathrm{Al}$ & Epi & 0.96 & 1.00 & 0.94 & 0.94 & 0.69 \\
\hline${ }^{45} \mathrm{Sc}$ & $99.99 \%$ Sc & Epi & 0.99 & 1.00 & 0.95 & 0.97 & 0.78 \\
\hline${ }^{199} \mathrm{Au}$ & $1 \% \mathrm{Au}$ in $\mathrm{Al}$ & Epi & 1.01 & 1.00 & 0.95 & 0.96 & 0.94 \\
\hline${ }^{186} \mathrm{~W}$ & $1 \% \mathrm{~W}$ in $\mathrm{Al}$ & Epi & 0.96 & 1.00 & 0.94 & 0.95 & 1.36 \\
\hline${ }^{238} \mathrm{U}$ & $23 \% \mathrm{U}$ in $\mathrm{Al}$ & Epi & 0.98 & 1.00 & 0.94 & 0.95 & 1.08 \\
\hline${ }^{139} \mathrm{La}$ & $5 \% \mathrm{La}$ in $\mathrm{Al}$ & Epi & 0.98 & 1.00 & 0.95 & 0.96 & 1.07 \\
\hline${ }^{\mathrm{ss}} \mathrm{Mn}$ & $1 \% \mathrm{Mn}$ in $\mathrm{Al}$ & Epi & 1.00 & 1.00 & 0.92 & 0.96 & 1.43 \\
\hline${ }^{63} \mathrm{Cu}$ & $10 \% \mathrm{Cu}$ in $\mathrm{Al}$ & Epi & 1.01 & 1.00 & 0.94 & 0.95 & 1.16 \\
\hline${ }^{1115} \mathrm{In}$ & $100 \%$ In & Fast & 1.22 & 1.00 & 0.87 & 0.92 & 2.43 \\
\hline${ }^{58} \mathrm{Ni}$ & $100 \% \mathrm{Ni}$ & Fast & 2.63 & 1.00 & 0.93 & 0.94 & 3.24 \\
\hline${ }^{27} \mathrm{Al}$ & $100 \% \mathrm{Al}$ & Fast & --- & 1.00 & 0.88 & 1.03 & 5.26 \\
\hline
\end{tabular}

Initial calculations using an MCNP model of the complete HFR core and HB11 facility had been used to design the filter components and to generate neutron and photon sources just behind the final aperture. These sources have subsequently been used in the treatment planning models and for the foil and phantom simulations reported here. 
When the beam was first opened in 1991 a series of measurements were undertaken which suggested that the very fast component of the beam $(>0.5 \mathrm{MeV})$ was greater than predicted by the MCNP calculations. In the light of this result the calculated spectrum was adjusted to give a "standard" spectrum that has been used in the work reported here.

In retrospect these early measurements have been shown to be misleading. One of the major components of the filter, the argon cryostat, was very unstable during this initial operational period. Considerable changes have since been made and the argon system now behaves in a far more consistent manner. However as a consequence of these improvements all the measurements now show a very much reduced fast neutron component, indicating a spectrum much closer to that of the original calculations. For this reason the data in Table 1 is normalised to the measured values of April 1992 and the earlier values can be considered as atypical for the existing configuration.

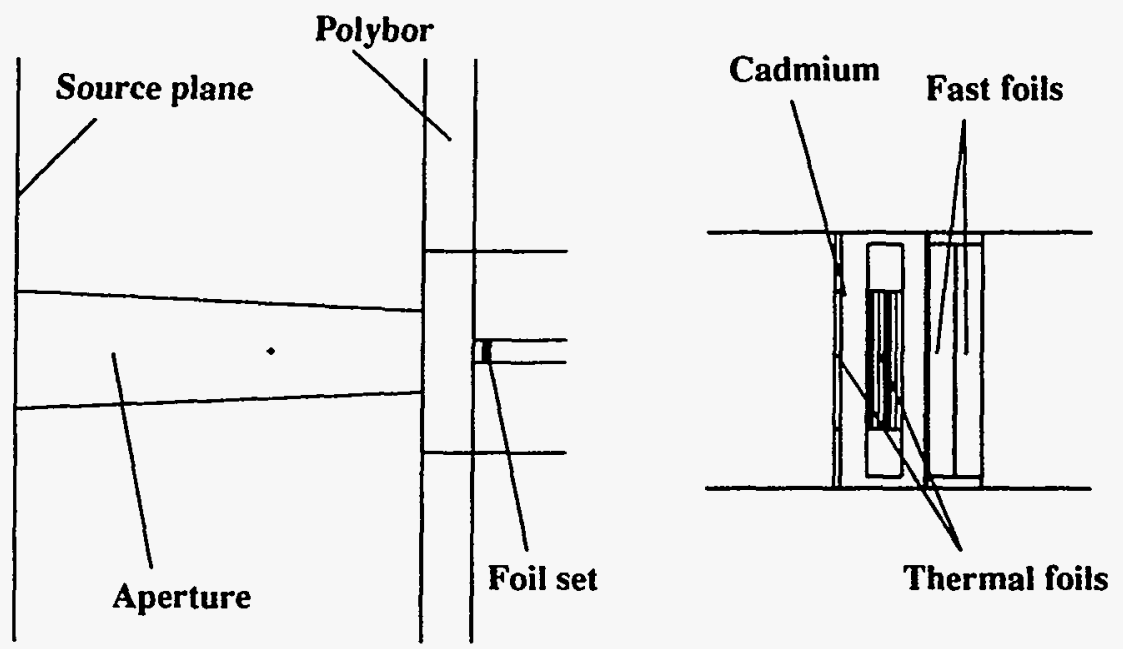

Figure 2. MCNP model of the free beam foil set.

The "standard" spectrum used in the calculations was not changed as a result of these revised measurements and consequently there remains a significant discrepancy with the calculated values for those foils responding to fast neutrons. However the effect of this reduction in flux above $0.5 \mathrm{MeV}$ on the fast neutron dose is minimal since flux levels in this energy range are very low.

The results from the TLD irradiations show the same sort of stability over time although there is a dependency upon the condition of the argon cryostat. For fresh, unirradiated argon the gamma dose in the free beam is measured at about $1 \mathrm{~Gy} \mathrm{hr}^{-1}$. However after operation the argon becomes activated and the gamma contamination of the free beam rises by about $25 \%$. 


\section{PHANTOM MEASUREMENTS AND CALCULATIONS}

In addition to the free beam measurements several different phantoms have also been used to investigate the beam parameters. These included two lucite phantoms constructed by collaborators from INEL, a full sized beagle head phantom and a cylindrical phantom. Both phantoms have provisions for the insertion of neutron activation wires or TLDs.

Various hollow perspex phantoms have also been used. These can be filled with water or tissue equivalent liquid (TEL) and are designed to accept simple activation foil packs at a number of positions throughout their volume. Many of the cell irradiation studies at Petten have used these same phantoms.

Two further phantoms are available which have also been used in conventional photon and neutron therapy. The first of these is a cuboidal tank of water into which $\mathrm{BF}_{3}$, ionisation or fission counters may be inserted. A tracking device allows these detectors to be moved remotely thus providing a means of rapidly determining flux contours within the tank. A second phantom is constructed from a solid cube of polythene with holes bored at a number of positions to accept ionisation or fission counters. In both cases these phantoms are being used to validate the treatment planning calculations ${ }^{2}$. The polythene block phantom is also being developed as a means of rapidly checking the epithermal component of the beam prior to every irradiation.

In all cases both measurements and calculations have been performed with these phantoms. The beagle phantom is now described in more detail. This phantom is of a size comparable to that of a beagle dog and contains a number of finely bored holes that can accept $\mathrm{Au} / \mathrm{Cu}$ wires for neutron activation analysis or TLDs for the determination of the gamma component. The phantom was provided by INEL and formed part of an intensive measurement campaign performed at Petten in $1991^{2}$. Since that time the beagle phantom has been irradiated on several occasions and forms a central part of the validation programme for the treatment planning model. It has the important benefit of providing a means of measuring the thermal neutron flux at depth in a body that is of similar size and composition to the beagles used in the healthy tissue tolerance study.

As with the free beam measurements the beagle phantom was also modelled with MCNP, Fig 3. From the measured copper and gold activation rates estimates were made of the thermal neutron flux as a function of depth. Fig 4 compares the measured and calculated estimates of thermal neutron flux where the agreement is seen to be very good, especially as no renormalisation of measured nor calculated values has been performed.

\section{PROTON RECOIL MEASUREMENTS}

Free beam measurements of the neutron spectrum have also been performed by collaborators from AEA Technology using gas filled proton recoil counters ${ }^{4}$. These measurements have been be undertaken at low reactor power to avoid saturating the counters. Potentially these measurements could provide spectral information from energies of about $10 \mathrm{keV}$ upwards. the results from these measurements agree with the calculated spectrum and also with the foil measurements. However there remain some discrepancies with regard to the absolute intensity which have yet to be resolved.

Unfortunately it has not been possible to perform these measurements very frequently and there remains a paucity of proton recoil data for the HB11 beam, especially for the time period after the improvements to the argon system. 

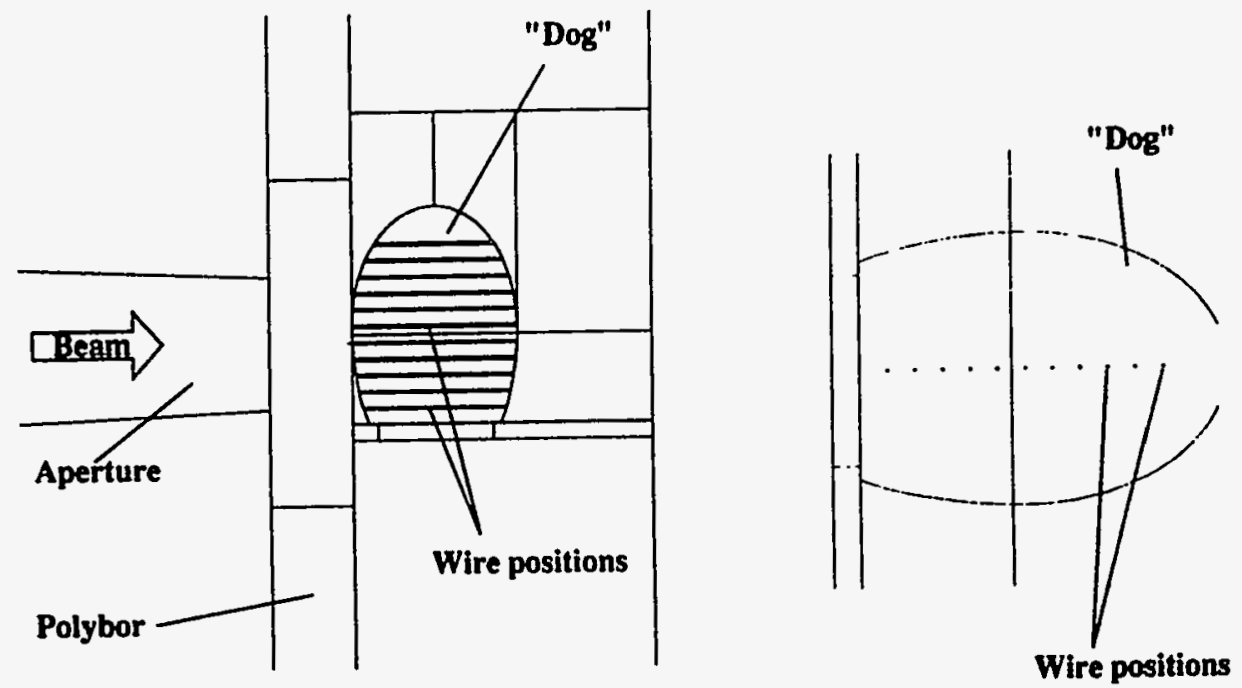

Figure 3. MCNP model of the lucite beagle phantom.

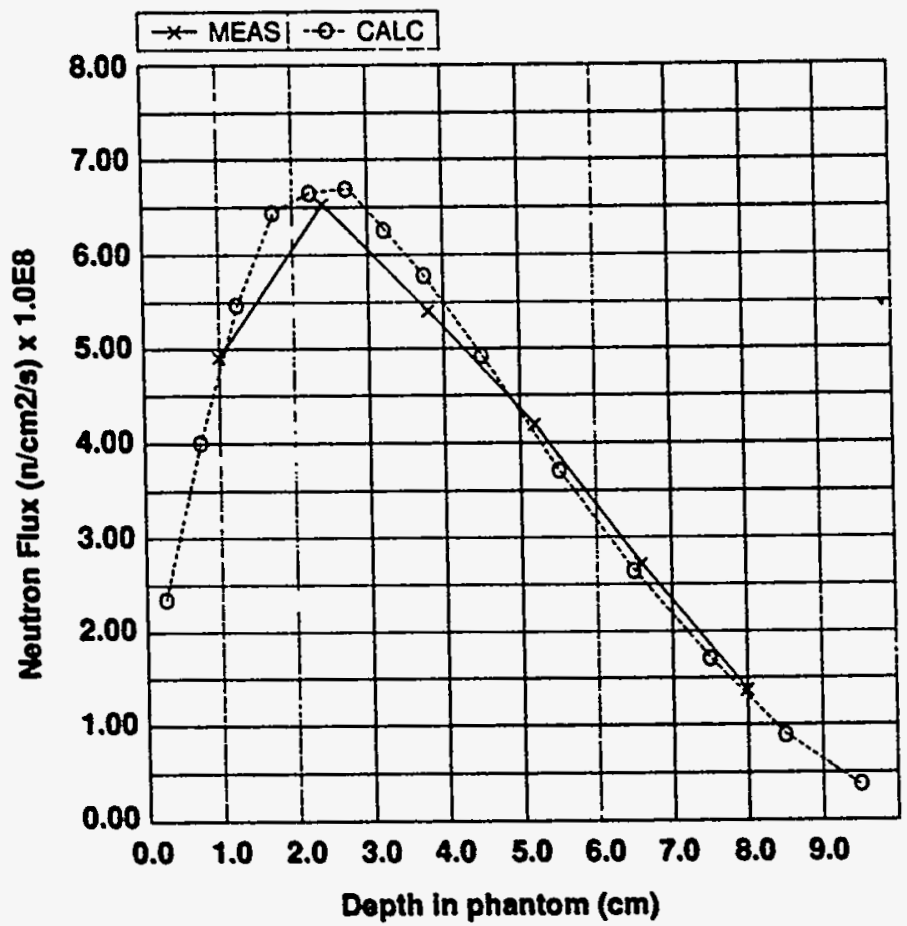

Figure 4. Comparison of thermal neutron flux from measurement and as calculated from MCNP. 


\section{CONCLUSIONS}

The present series of free beam dosimetry measurements at the HFR Petten show that the BNCT beam remains very stable. No strong cycle-to-cycle variations have been observed. However the existing neutron foil measurements are incapable of responding to neutrons in the important epithermal energy range and further measurements are being developed to investigate this region.

Calculations with MCNP have been shown to reproduce the measured values reasonably well although there are some unacceptable discrepancies for some of the free beam foil materials. Measured and calculated values of the thermal neutron flux within the various phantoms agree well with each other.

It is intended to obtain further measured data at most neutron energies in the near future, particularly using the proton recoil method. These will be used in a spectrum adjustment procedure, currently under development, to correct the calculated spectrum.

\section{REFERENCES}

1. Briesmeister, J.F(Ed), "MCNP - A General Purpose Monte Carlo Code for Néutron and Photon Transport", LA-7396-M, Revision 2, September 1986.

2. Konijnenberg, M., et al, "Measurements and Calculations for Boron Neutron Capture Therapy Treatment Planning", This meeting.

3. Harker,Y., et al , "Neutron Spectrum Measurements at the Petten HFR and at Brookhaven BMRR", This meeting.

4. Perks, C., amd Delafield, H.J., "Neutron Spectrometry Measurements of the Petten HFR, HB11 Neutron Beam", International Workshop and Plenary Meeting, 'Towards Clinical Trials of Glioma with BNCT', Petten, The Netherlands, September 1991. 



\section{Physics Parameters for an Epithermal Neutron Beam at the Georgia Institute of Technology}

D. Nigg, G. Storr, and F. Wheeler

This article is a reprint from Advances in Neutron Capture Therapy, pp. 71-74, (1993). 
PHYBICB PARAMETERB FOR AN EPITHERMAL-NEUTRON BEAM AT THE

GEORGIA INBTITUTE OF TECENOLOGY

\author{
David W. Nigg, Gregory J. Storr', and Floyd J. Wheeler \\ National Center for BNCT Measurement and Development \\ Idaho National Engineering Laboratory \\ EG\&G Idaho, Inc. \\ P.O. Box 1625 \\ Idaho Falls, ID 83415
}

\begin{abstract}
Boron Neutron Capture Therapy (BNCT) research in the United States has focused on the use of epithermal $(0.5 \mathrm{eV}$ to $10 \mathrm{keV})$ neutron beams for treatment of certain highly-malignant primary brain tumors and possibly for treatment of metastatic malignant melanoma. Various types of epithermal neutron sources for BNCT have been proposed over the years. Reactor-based sources, accelerator-based sources, and radioactive neutron sources have all been extensively examined. The first practical, large-scale, epithermal neutron beam for BNCT was installed at the Brookhaven Medical Research Reactor (BMRR)'. This beam was designed and constructed in a cooperative effort between Brookhaven National Laboratory (BNL) and the Idaho National Engineering Laboratory (INEL). It has been used extensively for BNCT research activities conducted by INEL, BNL, and others.
\end{abstract}

Although the BMRR beam has served the BNCT research community well, and will continue to be a valuable research tool for the foreseeable future, there are also reasons to seriously consider the construction of an advanced epithermal neutron beam facility at the Georgia Institute of Technology Research Reactor (GTRR). The neutronic characteristics of the GTRR are such that a properly-designed epithermal neutron filter in the existing large horizontal medical irradiation port at this facility would offer certain significant improvements in performance relative to the BMRR beam.

The GTRR is a heavy-water moderated and cooled plate-fuel-type reactor with a graphite neutron reflector. The proposed GTRR epithermal neutron beam facility developed in this work would be installed in the existing large biomedical irradiation port as shown in Figure 1. In this conceptual design an in-vessel filter/moderator structure, composed of 908 aluminum and $108 \mathrm{D}_{2} \mathrm{O}$ by volume, occupies the space between the core and the reactor vessel on the side of the reactor adjacent to the biomedical beam port. Outside the vessel, the existing large reflector and shield penetration is lined with a laminated bismuth-lead-cadmium filter housing. Within this housing is a region of dry aluminum plates occupying a volume fraction of 908 . This region is approximately $0.7 \mathrm{~m}$ in thickness. It is followed by a $0.076 \mathrm{~m}$ region of lithiated-aluminum plates and a $0.01 \mathrm{~m}$ titanium plate for spectral shaping in the 0-1 eV and the 10-100 keV ranges, respectively. Finally, there is a $0.076 \mathrm{~m}$ bismuth-lead gamma shield. The movable concrete shield block is also lined with laminated bismuth, lead and cadmium to help provide collimation of the neutron beam and to suppress contamination from concrete capture gamma radiation. A bismuth and lithiated-polyethylene thermal neutron and gamma shield is positioned on the exit port wall of the currently-existing treatment room. Provision can be made for a variety of beam delimiter inserts to control the size and shape of the beam exit port.

1 On attachment from the Australian Nuclear sciences and Technology organisation (ANSTO), PMB 1, Menai, NSW, Australia.

Advances in Neutron Capture Therapy, Edited by

A.H Soloway et al., Plenum Press, New York, 1993 
Calculations to estimate the neutronic performance of the proposed epithermal beam at the GTRR were performed by a combination of two- and three-dimensional discrete-ordinates techniques using local adaptations of the well-known DORT ${ }^{2}$ and TORT ${ }^{3}$ computer codes, with cross sections taken from the BUGLE80 coupled neutron-gamma library. These calculations were supplemented by confirmatory Monte Carlo analyses performed using the MCNPs code. This approach is basically the game as was successfully employed for physics design of the BMRR epithermal neutron beam, although extra emphasis is being placed on accurately calculating the fast neutron component of the beam since all methods tend to underestimate this component if one is not careful. As an added check, the exit port spectrum was also calculated using the DORT code and an 80-neutron, 20-gamma group cross section library that was independently-generated for this application using the Aus ${ }^{6}$ system. The resultg were in agreement with the results obtained using the BUGLE-80 library.

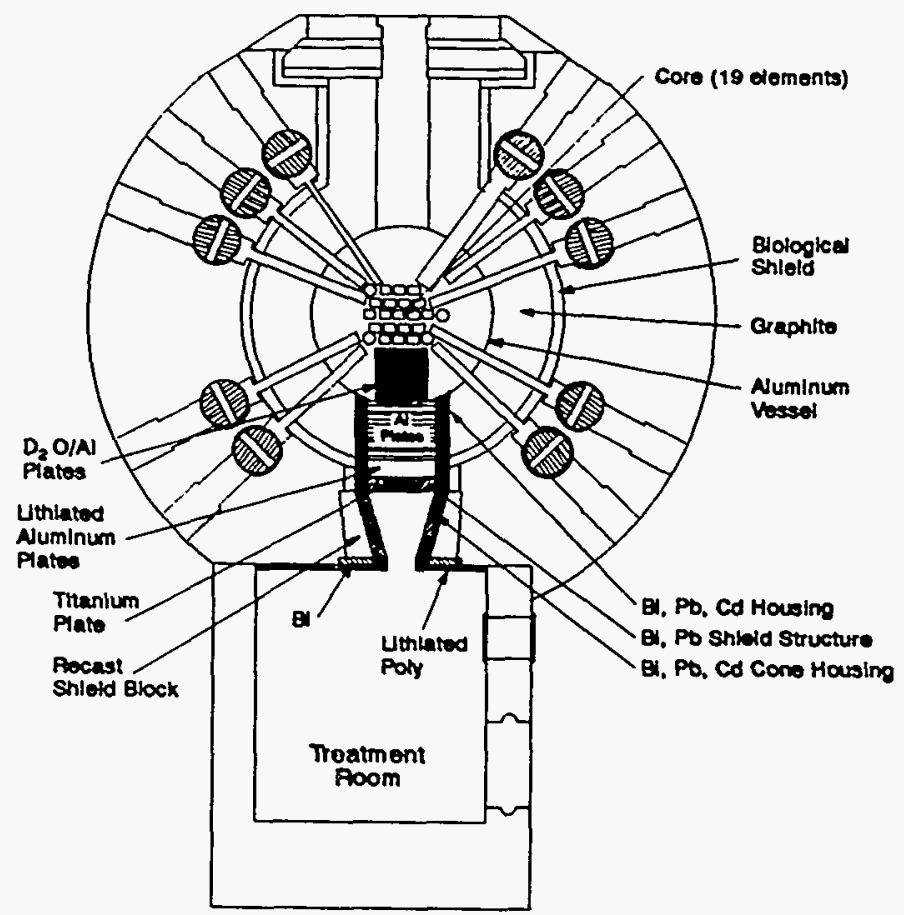

Figure 1. Conceptual Epithermal Neutron Facility for Boron Neutron Capture Therapy at the Georgia Institute of Technology Research Reactor.

The calculated exit port neutron flux spectra for the BMRR and the GTRR neutron beams are compared in Figure 2. The integrated epithermal intensity of the GTRR beam (about $4 \times 10^{9} \mathrm{n} / \mathrm{cm}^{2}-8$ at the rated reactor power of $5 \mathrm{MW}$ ) is about a factor of 2 greater than that of the BMRR beam at its rated power of $3 \mathrm{MW}$. The calculated fast neutron component for the GTRR beam (about $1.5 \times 10^{-11}$ CGy per unit epithermal flux) is, radiobiologically, very significantly smaller than that of the current BMRR beam. The proposed GTRR beam is also well-collimated, with a current to flux ratio of about 0.85 compared to 0.6 for the BMRR beam. A well-collimated beam produces less undesirable healthy-tissue dose near the surface relative to the desirable boron capture dose at depth. 
The results of clinically realistic phantom dosimetry calculations based on the methods outlined in References 7 and 8 show that the desirable boron dose at depth that can be generated with the GTRR beam is generally about a factor of three times larger per $M W$ of reactor power than is the case for the BMRR beam. For a given total therapeutic dose, the treatment time for GTRR is therefore about a factor of 5 shorter at the rated reactor power of $5 \mathrm{MW}$ than is the case for BMRR at its rated power of 3 MW. In addition, the fast neutron dose component of the GTRR beam relative to that of the BMRR beam is much reduced, as expected. The GTRR beam also offers a more favorable boron dose-depth profile than the BMRR beam. When the various calculated space-dependent physical dose components that are generated within the irradiation volume are weighted by appropriate Relative Biological Effectiveness (RBE) factors, it can be shown that the so called "advantage depth" for the GTRR beam is about $1 \mathrm{~cm}$ greater than that of the BMRR beam for clinically realistic tumor boron concentrations. This is radiobiologically very significant.

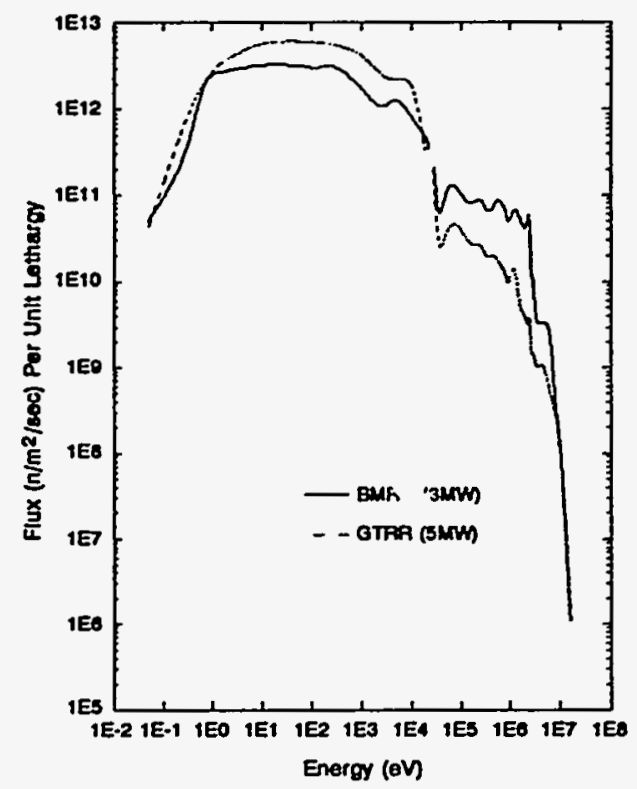

Figure 2. Comparison of calculated BMRR and GTRR neutron beam spectra.

When considered on the combined basis of physics, radiobiology, economics, and geographic location, the proposed GTRR beam offers, overall, the most desirable known source of epithermal neutrons for BNCT that is available, or can be made available, in the U.S., using present reactor or accelerator technology. The best currently operating epithermal neutron source in the U.S. is the BMRR beam, which will continue to see heavy uge for BNCT research for the foreseeable future. The BMRR beam, however, has a radiobiologically significant level of fast neutron contamination, which has led to surface tissue damage in some canine experiments?. With the GTRR beam, this contamination can be suppressed to a level such that dose near the surface is not treatment-limiting. This would allow some types of advanced animal experimentation to be done without surgically reflecting the scalp. Also, extrapolation of animal BNCT irradiation data for the purpose of deciding whether to proceed to human clinical trials would be facilitated by the availability of the proposed GTRR beam because this beam has spectral qualities that are more typical of what would be required for routine human treatment should BNCT become a widespread modality. 
This study was performed under the auspices of the U.S. Department of Energy, Office of Energy Research, DOE Field Office, Idaho, Contract Number DE-AC07-76IDO1570.

\section{REFERENCES}

1. F.J. Wheeler, et.al., Epithermal neutron beam design for neutron capture therapy at the power burgt facility and the Brookhaven Medical Research Reactor, Nuclear Technology 92, 106, October, 1990.

2. W.A. Rhoades, and R.L. Childs, An updated version of the DOT-4 oneand two-dimensional neutron/photon transport code, Oak Ridge National Laboratory, ORNL-5851, April 1982.

3. W.A. Rhoades, and R.I. Childs, The TORT three-dimensional discreteordinates neutron/photon transport code, Oak Ridge National Laboratory, ORNL-6268, 1987.

4. R.W. Roussin, BUGLE-80 coupled 47-neutron, 20 gamma-ray P3 crosg section library, Radiation Shielding Information Center, DLC-75, 1980 .

5. MCNP Monte Carlo neutron and photon transport code systems, Radiation Shielding Information Center, CC-200A.

6. G.S. Robinson, A guide to the AUS modular neutronics code Bystem, AAEC/E645, (Australian Atomic Energy Commission), April 1987.

7. D.W. Nigg, et. al., Demonstration of three-dimensional deterministic radiation transport theory dose distribution analysis for Boron Neutron Capture Therapy, Medical Physics, 18:1, 43-53, January 1991.

8. F.J. Wheeler and D.W. Nigg, Three-dimensional radiation dose distribution analysis for neutron capture therapy, Nuclear science and Engineering, 110, 16-31, January 1992.

9. P.R. Gavin, et. al. Large animal model - the biologic effects of mixed irradiation fields utilizing BSH and epithermal neutron irradiation, Proceedings of International Workshop on Macro- and Microdosimetry and Treatment Planning for Neutron Capture Therapy, MIT, October 1991, (To be published). 


\section{PLENARY TALK: Analytical Dosimetry and Treatment Planning for BNCT}

D. Nigg

This article is a reprint from Advances in Neutron Capture Therapy, pp. 143-146, (1993).

83 
v 
AMALYTICAL DOBIMETRY AND TREATMENT PLANAING FOR BNCT

David w. Nigg

National Center for BNCT Measurement and Development Idaho National Engineering Laboratory BGEG, Idaho, Inc.

P.O. Box 1625

Idaho Falls, Idaho 83415

Calculation of radiation dose distributions for Boron Neutron Capture Therapy (BNCT) is an essential supporting function for in vivo BNCT radiobiological research. Such calculations will also ultimately be routinely required for human treatment planning. Radiation transport and dose distribution analysis for BNCT is much more complex than is the case for standard photon therapy. There are several different physical radiation dose components associated with BNCT, each of which has it's own characteristic spatial distribution and Relative Biological Effectiveness (RBE). Host of these radiation dose components result from neutron interactions that take place after the incident neutrons from the treatment beam have undergone several scattering interactions. Accordingly, many of the simplifying assumptions that work well for radiation transport calculations associated with treatment planning for standard photon therapy are not appropriate for BNCT. Full three-dimensional calculations with an explicit treatment of particle scattering and tissue heterogeneities are required.

In standard photon therapy, the absorbed radiation dose is produced by energetic electrons that result from various well-known interactions between photons and tissue components. In BNCT, on the other hand, most of the absorbed dose is deposited by High Iinear Energy Transfer (High IET) secondary charged particles produced by various neutron interactions in tissue and in the incorporated boron. The most important of these is, of course, the therapeutically-desirable boron $(n, a l p h a)$ interaction. All of the other neutron-induced dose components arise from neutron interactions that take place with the normally-present tissue constituents. These components are non-selective and are therefore not desirable. The most significant of these is the proton recoil dose produced by elastic scatter of fast ( $>10 \mathrm{keV}$ ) neutrons on hydrogen. This component is believed to have a relatively high RBE for acute normal tisgue damage. The proton recoil dose component can be suppressed by the use of a properly-designed epithermal neutron beam but, as a practical matter, it cannot be totally eliminated. Other significant unavoidable neutron-induced background dose components include the dose that is deposited as a result of the nitrogen $(n, p)$ interaction and several other smaller components. There is also a photon dose component in BNCT that is produced largely as a result of the radiative capture of neutrons by hydrogen as well as from the small incident photon component of the incident treatment beam itself.

Figure 1 shows the essential elements that must be present in any calculational system used for BNCT analytic dosimetry and treatment planning. At the heart of the system is a module for calculating the solution of the three-dimensional Boltzman transport equation for neutral particles, given certain descriptive information as input. This descriptive information consists of a geometric model of the irradiation volume (ideally constructed directly from medical image data), a mathematical description of the treatment beam, and a complete set of coupled neutron-photon cross 


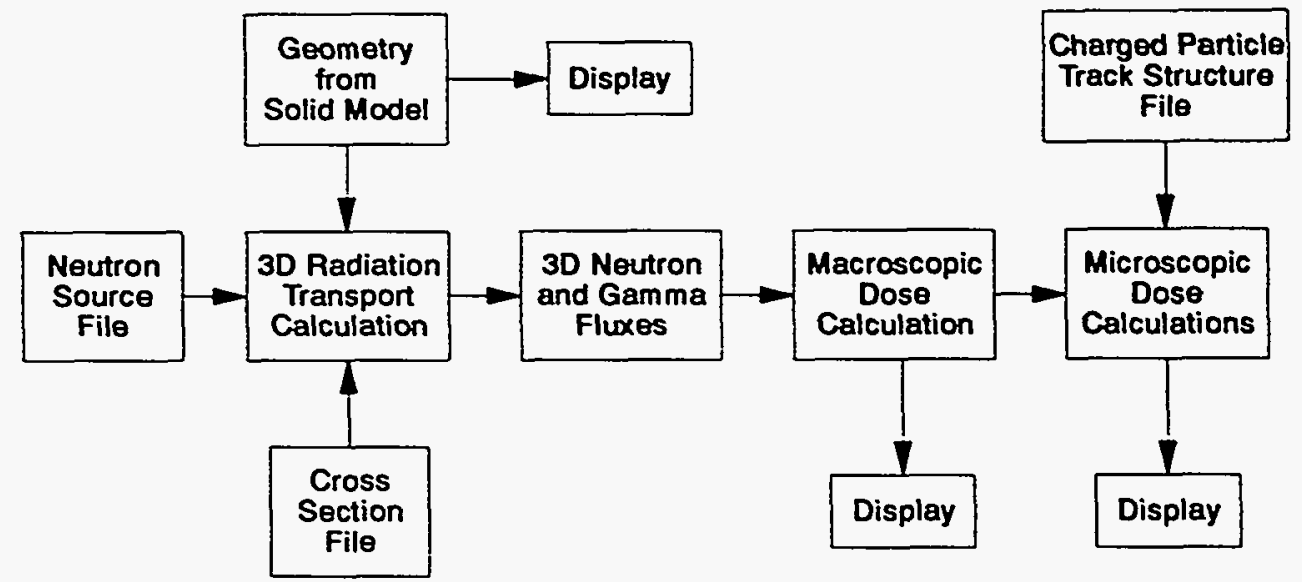

Figure 1. Flow diagram for BNCT neutral particle transport and microdosimetry analysis.

section data for all elements that are present. Geometric descriptions may be constructed by any of three different methods. Each of these methods will be described in detail below. The beam description consists of the spectrum, angular distribution, and the spatial intensity distribution across the plane of incidence, of both the neutron and the photon flux components of the treatment beam. This information is usually constructed from a combination of calculational and experimental data pertinent to the beam of interest. Neutron and photon cross sections are typically taken from standard data collections such as the Evaluated Nuclear Data File' and preprocessed into an appropriate multigroup or continuous-energy format. Given the calculated neutron and photon fluxes throughout the treatment volume, it is a relatively simple matter to construct the resulting macroscopic absorbed dose distribution by folding the calculated flux data with flux-to-dose conversion factor data for each radiation dose component, integrating over the neutron or photon energy range as appropriate, and summing all components. This method is acceptably accurate for all neutron-induced dose components in BNCT since the charged particles that result from these interactions have very short ranges in tissue (on the order of a few micrometers). Thus it is appropriate to assume that, on a macroscopic scale, the spatial distribution for each of these components follows the weighted spatial distribution of the original neutron flux. For the photon-induced dose components, this approximation is, for well-known reasons, less-accurate, especially near voids and material interfaces, but it is currently commonly used in BNCT since the photon dose is typically small compared to the total dose. Standard void and interface correction methods could be used for this dose component in situations where greater accuracy is desired.

As mentioned above, the ranges of the charged particles that produce most of the absorbed dose in BNCT are comparable to typical tissue cell dimensions. As a result it is also necessary to take microdosimetric considerations into account when correlating radiation dose with observed radiobiological response and when attempting to estimate the RBE of the various BNCT dose components under different conditions. For example, the RBE of the boron ( $n$, alpha) dose component can vary widely depending on the intracellular distribution of the particular boron-carrying pharmaceutical that is employed. It is not enough just to know the macroscopic absorbed dose. One must look at what is occurring on a microscopic scale in order to realistically estimate cell survival fractions under various conditions of irradiation. This situation is further complicated by the fact that there are relatively few boron interactions needed per cell to produce a therapeutic dose in BNCT. Thus the statistical nature of neutron interactions with matter must also be explicitly considered. Some cells will experience radiobiologically-significant deviations from the average 
absorbed dose simply because of this phenomenon. Accordingly, the capability to perform charged particle transport calculations on a microscopic scale and to combine the reaulte with variou postulated radiobiological response functions in a statistically correct manner must also be included in calculational systems used for BNCT dosimetry analysis. Two fundamentally different methods are available for computing the basic neutron and photon flux distributions needed for BNCT. The Monte Carlo stochastic simulation method is the current method of choice, since the complex geometries that are characteristic of biological syeteme can be very accurately represented using this technique and one can obtain integral information about the radiation dose distribution (e.g. integrated dose over a particular tissue compartment) in quite reasonable (few-minute) computing times. Deterministic solution methods are also feasible. These methods, by their nature, automatically provide very detailed apatial dose distributions as well as integral data, but this is (currently) accomplished at the expense of somewhat longer computing times and some loss of geometric precision. The two different methods therefore complement each other and each has its own appropriate uses.

Three basic methods have been developed for constructing the geometric description of the irradiation volume for use in BNCT dosimetric modeling. The first, and computationally the simplest (and therefore the fastestrunning), method is to approximate the irradiation volume using combinations of various geometric primitives (spheres, cubes, cylinders, etc.) described by simple surface equations. This method is incorporated into the wellknown MCNP code" and has been widely used for all types of radiation transport calculations over the years. In BNCT applications, however, the geometric primitive method has largely been superseded by two independent methods for constructing the necessary geometric descriptions directly from medical image data.

The first of the direct geometric reconstruction methods employs a "voxel reconatruction" technique whereby each plane of medical image data is partitioned into gquare (typically 1-centimeter) areas, each of which is assigned material properties based on CT density or some other appropriate criterion ${ }^{34}$. One then mathematically stacks the square regions generated in each medical image plane to construct a three-dimensional array of parallelpipeds that can be input to a standard Monte Carlo code such as MCNP. The various tisgue compartments are represented as combinations of adjacent parallelpipeds. This method permits reconstructions to be generated relatively quickly and it is very amenable to automation. In addition the same method can be used in exactly the same form to construct geometry for a deterministic radiation transport calculation if this is desired. The primary disadvantage of the method stems from the fact that complex curved surfaces are represented by a series of orthogonal voxel facetg. This can adversely affect the accuracy of radiation transport calculations based on this technique, unless one employs a relatively large number of small voxels and accepts the longer computer execution times that result.

The second method for direct construction of geometry from medical images is based on the calculation of non-uniform rational B-spline fits to the various tisgue compartment (or any desired subcompartment) aurfaces. With this method, one first electronically outlines the regions of interest (e.g. skin, skull, brain, various tumor regions, etc.) on each medical image plane. These outlines are then mathematically combined to produce detailed B-Spline equations describing the three-dimensional surfaces that enclose each volume of interest. These surface equations completely describe the geometry and can subsequently be used in a Monte Carlo radiation transport calculation. The spline surfaces can also be combined with geometric primitive surfaces to further specify the calculational geometry if desired. A spline surface reconstruction capablilty has been incorporated into the special-purpose bnct edit/rtt MC code sequence for medical dosimetry ${ }^{3,6}$. (The rtt MC code will aIso accepE parallelpiped arrays constructed using the voxel Eechnique if desired). The primary advantage of the surface equation reconstruction technique is that it is very efficient and accurate. The complex curved surfaces of the varlous anatomical structures of interest in BNCT dosimetry are very faithfully represented and particle tracking can be accomplished with the minimum number of boundary crossings that is consistent with the number of tissue compartments modeled. The primary disadvantage is that, unlike the 
situation with voxel-based reconstructions, one does not automatically obtain spatially-detailed dose distributions. This problem has been overcome without significant $108 s$ of calculational efficiency, however, with the development of the so-called "Subelement Monte-Carlo" technique that is available in the rtt MC code. ${ }^{5}$ A secondary disadvantage of the surface reconstruction metho $\bar{d}$ is that it may not be easily adaptable to deterministic methods for calculating radiation transport. This remains to be seen.

In conclusion, it may be stated that the technology for performing radiation dose distribution analysis and treatment planning calculations for BNCT is well into the advanced stage. The necessary three-dimensional radiation transport calculations can be accurately performed using any of several different combinations of geometric models and mathematical techniques for obtaining the solution to the governing equations. Currently-ongoing efforts have focused on providing detailed, understandable output displays (dose contours superimposed on medical image data, dosevolume histograms, 3-D visualizations, etc.) , medical image manipulations, tumor location capabilities and other such "user-friendly" features, similar to those that are available with advanced photon treatment planning systems.

\section{ACKNOWLEDGWENTS}

The author wishes to express his gratitude to Dr. R. Barth for the opportunity to present this Plenary lecture at the Fifth International Symposium on Neutron Capture Therapy and to Dr. R. Zamenhof, Dr. $I$. Reinstein, and Dr. H. Liu for providing some of the visual aides that were used in the oral presentation.

This study was performed under the auspices of the U.S. Department of Energy, Office of Energy research, DOE Field office, Idaho, under Contract Number DE-AC07-76ID01570.

\section{REFEREHCES}

1. R. Kinsey, Data formats and procedures for the evaluated nuclear data file ENDF, Brookhaven National Laboratory, BNL-NCS-50496, 1979.

2. MCNP monte carlo neutron and photon transport code systems, Radiation Shielding Information Center, Oak Ridge National Laboratory, U.S.A., CCC-200A.

3. R. Zamenhof, et. al., Treatment planning for neutron capture therapy of glioblastoma multiforme using epithermal neutron beam from the MITRII research reactor and monte carlo simulation, in: "Progress In Neutron Capture Therapy for Cancer," B.J. Allen, et. al. (ed), Plenum Press, New York, NY (1992).

4. L.E. Reinstein, et. al., SBNCT-PLAN: A 3-dimensional treatment planning system for boron neutron capture therapy, Proceedings of the Fifth International symposium on Neutron Capture Therapy, Osu, September 1992, (To be published).

5. F.J. Wheeler and D.W. Nigg, Three-dimensional radiation dose distribution analysis for boron neutron capture therapy, Nuclear Science and Engineering, 110, 16-31, January 1992.

6. D.E. Wessol and F.J. Wheeler, Methods for creating and using free-form geometries in monte carlo particle transport, Nuclear science and Engineering, (To be published). 
Post Treatment Dose Distribution Evaluation for a Recent NCT Patient

\author{
G. Storr and F. Wheeler
}

This article is a reprint from Advances in Neutron Capture Therapy, pp. 153-157, (1993). 


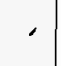




\section{POST TREATMENT DOSE DISTRIBUTION EVALUATION FOR A RECENT NCT}

\section{PATIENT}

Gregory J. Storr, ${ }^{1}$ and Floyd J. Wheeler

Idaho National Engineering Laboratory, EG\&G Idaho, PO Box 1625 , Idaho Falls, ID 83415-1575, USA

${ }^{1}$ On attachment from Nuclear Technology Program, Ansto, PMB 1, Menai 2234, NSW, Australia

\section{INTRODUCTION}

As a prelude to establishing, (1) criteria for selection of Boron Neutron Capture Therapy (BNCT) epithermal beam clinical trial patients and (2) treatment planning strategies, posttreatment evaluations of dose distributions in several BNCT patients are being done using the Idaho National Engineering Laboratory (INEL) BNCT Program's patient treatment planning software (bnct_edit and rtt_MC) $)^{1,2}$.

The objectives of this work are :

- calculate the dose delivered to the BNCT treated patients

- calculate the possible dose distribution for the patients using the proposed Georgia Institute of Technology's reactor epithermal beam ${ }^{3}$

- correlate calculated dose with effect

\section{PATIENT DATA}

We have data for seven recent Australian and US BNCT patients treated in Japan and at this stage we report in detail on one patient study. As well as individual patient medical images, accurate calculations of dose also relies on knowledge of boron concentrations in the tumor, tissue surrounding the tumor, normal tissue, and blood, heavy water concentrations in the irradiation volume, and dose modification factors (e.g. radiation component RBE's). The patient (\# 1) we report on was diagnosed with Glioblastoma Multiforme in the left occipital lobe in February 1991, was treated with BNCT in May 1991 at the JAERI Research Reactor No. 2 (JRR-2), and at September 1992 was surviving.

Table 1 lists the information on patient 1's treatment at JRR-2. The boron tumor concentration compares well with three Australian patients blood boron levels, (measured between $12 \mu \mathrm{g} / \mathrm{g}$ and $35 \mu \mathrm{g} / \mathrm{g}$ during irradiation) as does the $\mathrm{D}_{2} \mathrm{O}$ concentration of $9 \mathrm{wt} \%$ in four Australian patients ${ }^{4}$. 
Table 1. Patient $1 \mathrm{BNCT}$ treatment data

\begin{tabular}{cr}
\hline Irradiation time $\quad(\mathrm{min})$ & 270.0 \\
Reactor Power $\quad(\mathrm{MW})$ & 10.0 \\
Max. th. flux - dura $\left(\mathrm{n} \mathrm{cm}^{-2} \mathrm{~s}^{-1}\right)$ & $7.39 \times 10^{8}$ \\
${ }_{10} \mathrm{~B}$ concentration - tumor $(\mu \mathrm{g} / \mathrm{g})$ & 18.0 \\
$10^{0} \mathrm{~B}$ concentration - blood $(\mu \mathrm{g} / \mathrm{g})$ & 0.3 \\
$\mathrm{D}_{2} \mathrm{O}$ concentration (wt \%) & 9.0 \\
Tumor dose - brain surface (cGy) & 4600.0 \\
Tumor dose - 5cm deep (cGy) & 746.0 \\
\hline
\end{tabular}

\section{MODELING STRATEGY}

Figure 1 shows the modeling strategy used to obtain the dose distributions. The method adopted in this work was to prepare burnup dependent smeared fuel element cross sections and all other material cross sections with the AUS neutronics system ${ }^{5}$. As all the Japanese reactors used for BNCT have beams of mostly thermal energy neutrons, the cross section energy group structure selected comprised 26 neutron groups ( 10 groups $<0.414 \mathrm{eV}$ ) and 4 photon groups. These cross sections were then used in discrete ordinates models, using DORT $^{6}$, of the reactor core (XY) and filter (RZ). The calculated neutron and gamma angular fluxes at the patient irradiation position were then used as a source input to the patient treatment Monte Carlo model.

\section{PATIENT MODELING}

Modeling the irradiation volume of a patient is achieved by using the two modules of the INEL patient treatment planning software; (1) bnct_edit ${ }^{1}$, a geometry reconstruction

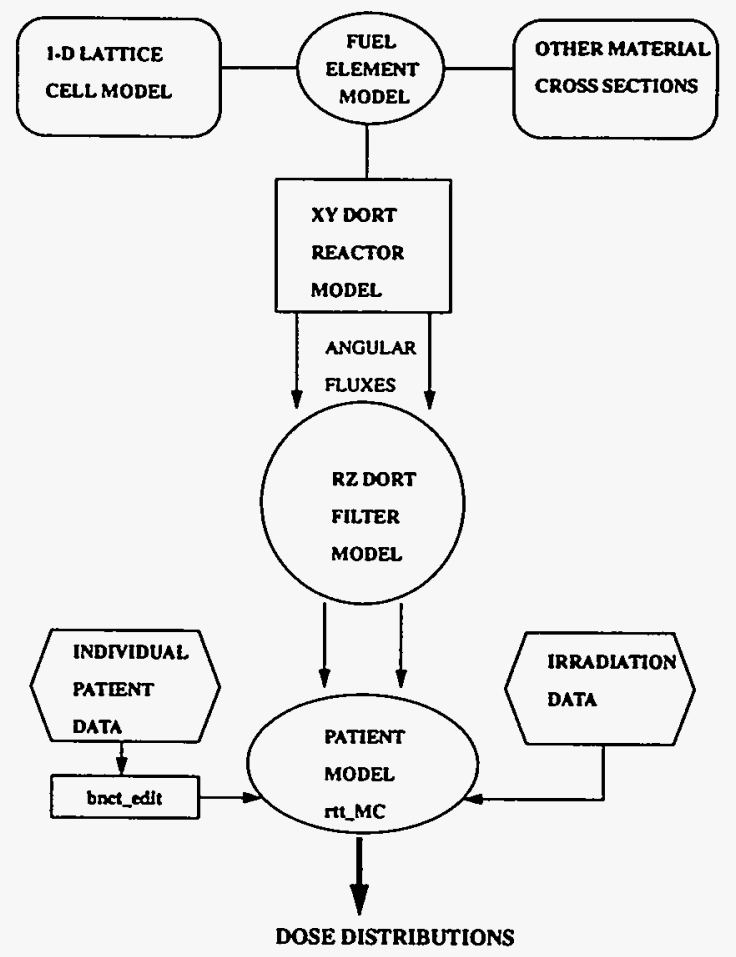

Figure 1. Modeling strategy for dose distribution analysis 
package for modeling individual patient geometries as a series of surface equations, and (2) $r t t \_M C^{2}$, a neutron and photon Monte Carlo code developed for BNCT dose distribution calculations. The modules are linked through the surface geometry description of the treatment volume, in this case the patient's head. The angular flux data from the filter model edit plane (the beam hole plane) and geometry descriptions for the beam and the patient are combined to form the overall problem. Material assignments are made from standard libraries and for patient 1 a special brain material that includes $9 w t \% D_{2} \mathrm{O}$ replacement was used.

Four bodies were constructed in the three-dimensional geometry description of patient 1 , corresponding to the head, brain, area of edema and swelling (designated as the target) and the tumor. The body representing the head was modified to include the surgical craniotomy for the patient. The surgical debulking is not represented in this model however, the approximation that the tumor still exists at the time of therapy is valid provided the material composition and boron concentration in this region are represented accurately.

\section{RESULTS}

\section{JRR-2 irradiation}

The calculated thermal flux was normalized to the measured value at the position adjacent to the treatment position - i.e. $1.0 \times 10^{9} \mathrm{n} \mathrm{cm}^{-2} \mathrm{~s}^{-1}$. When the calculated total thermal flux was normalized to the reactor power, the thermal flux $u s$ approximately twice the measurement. Comparison of in-core thermal fluxes showed intensities consistent with measurement in this reactor. There are uncertainties in the exact dimension and composition of the graphite and lead shielding layers adjacent to the reactor aluminium tank and

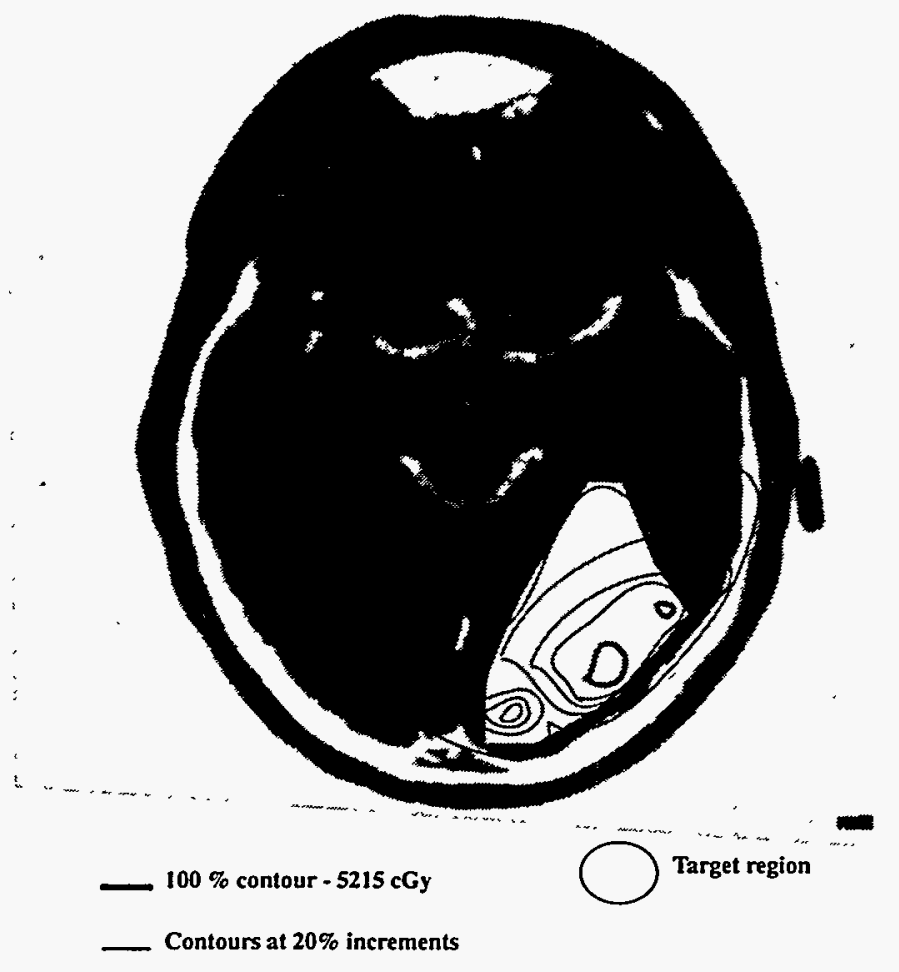

Figure 2. Total RBE-dose contours for JRR-2 irradiation of patient 1. 
between the patient treatment position. We believe this to be the cause of the difference between the measurement and calculation. Scoping calculations varying the thickness of the graphite and lead layers outside the reactor tank, showed that while neutron intensity is affected neutron spectral changes are slight for various lead and graphite combinations. The large heavy water moderator layer principally determines the neutron spectrum however, the activation gamma's from the lead and graphite layers change significantly dependent on the layer thicknesses. The bismuth shield helps to reduce this effect significantly.

Figure 2 shows an axial MR image of patient 1 close to the center-line of the target and tumor volumes, with calculated RBE-dose contours overlaying the image. The maximum $\mathrm{RBE}$-dose of $8500 \mathrm{cGy}$ lies within the tumor volume. The target volume which would have contained edema, normal brain and presumably tumor, appears on the MR images as a single structure. Variability in boron concentrations within the tumor and target volumes is well recognized, ${ }^{7}$ and this presented us with a range of choices for assigning boron concentrations in the target volume. In Figure 2 the RBE-doses correspond to a target boron level half that measured in the tumor. Note that this calculation gives a secondary peak in the target and outside the tumor volume of approximately $5300 \mathrm{RBE}$ cGy. Because of the presumed mixture of normal tissue, edema and tumor in the target this value is considered in this calculation to be the peak dose to healthy tissue. At the maximum depth of the target volume the RBE-dose is calculated to be approximately $1000 \mathrm{cGy}$.

Table 2 gives a comparison of physical dose from the calculation reported here and data from Japan. The total physical dose comparison is excellent, however there is a significant difference when comparing the gamma and boron dose components. The possibility exists that the Japan gamma dose does not include the induced gamma component.

\section{GTRR calculation}

Patient l's geometry was modified from the thermal beam case to fill in the "craniotomy", the input source was changed to the proposed Georgia Tech Research Reactor (GTRR) epithermal neutron beam ${ }^{3}$ and the $\mathrm{D}_{2} \mathrm{O}$ was removed from the tissue materials. Table 3 shows a comparison of percentage of dose for the major dose components at increasingly deep locations in patient l's reconstructed geometry. In Table $3100 \%$ dose at a specified location equals the total RBE-dose calculated with the JRR-2 beam. The epithermal beam RBE-dose peak is deeper than for the thermal beam however, for this patient whose tumor was located close to the brain surface, a favorable dose distribution is achieved from the thermal beam. The deepest portions of the target volume, which would have been likely to contain tumor cells have a larger dose for the epithermal beam than the thermal beam. Normal brain at depth receives less radiation with the thermal beam. A larger advantage for the epithermal beam in patients with deep tumors is expected.

A correlation of dose with effect will only be possible when more patients are studied. However, 14 months post-BNCT patient 1 underwent a full neurological exam, MR and positron emission tomography (PET) studies and the evidence from the MR study states, ".. no evidence of recurrent tumor." ${ }^{18}$ As this patient was diagnosed with Glioblastoma Multiforme, and the early prognosis was 6 months of life, it seems obvious that therapeutic benefit was gained from this patient's decision to undergo Neutron Capture Therapy.

Table 2. Tumor Physical Dose Comparison - Patient 1

\begin{tabular}{lccccc}
\hline & B-10 & Gamma & N-14 & Fast & Total \\
\hline ttt_MC & 2949 & 901 & 278 & 364 & 4492 \\
Japan & 3700 & 162 & 238 & 463 & 4563 \\
\hline
\end{tabular}


Table 3. Percentage of JRR-2 RBE dose components with location, for modeled thermal and epithermal beams

\begin{tabular}{|c|c|c|c|c|c|c|c|c|}
\hline & \multicolumn{4}{|c|}{ JRR-2 } & \multicolumn{3}{|c|}{ GTRR } & \multirow[b]{2}{*}{ Fast } \\
\hline & B-10 & Gamma & N-14 & Fast & B-10 & Gamma & N-14 & \\
\hline Tumor & 80 & 6 & 6 & 8 & 63 & 22 & 5 & 1 \\
\hline Target (1) & 65 & 13 & 10 & 12 & 55 & 35 & 8 & 2 \\
\hline Target (2) & 59 & 19 & 9 & 13 & 73 & 47 & 11 & 1 \\
\hline Brain & 4 & 46 & 17 & 33 & 6 & 125 & 25 & 1 \\
\hline
\end{tabular}

\section{CONCLUSIONS}

This study has modeled the fuel, reactor and filter of the JRR-2 reactor in Japan, and applied the calculated neutron and gamma fluxes to a model of a BNCT patient treated at that reactor. The physical dose distribution compares well with data from Japan. The calculated doses for this patient show a favorable distribution using a thermal energy neutron beam. A comparison with the proposed GTRR epithermal neutron beam, showed that dose at depth was larger for the higher energy beam for the same peak healthy tissue dose, even where the patient model did not include a craniotomy or tissue loaded with $\mathrm{D}_{2} \mathrm{O}$.

\section{ACKNOWLEDGEMENTS}

Ron Dorn MD, from the Mountain States Tumor Institute in Boise, Idaho, provided the patient dose information. Dave Madden MD, from the Eastern Idaho Regional Medical Center assisted with patient tumor and target location. Robert Weaver of EG\&G Idaho provided software and computer assistance crucial to this work. This study was performed under the auspices of the U.S. Department of Energy, Office of Energy Research, DOE Field Office, Idaho, under Contract Number DE-AC07-76IDO1570.

\section{REFERENCES}

1. D. Wessol, J. Cobb, F. Wheeler, E. Cohen, B. Cobb, and D. Barber, Interactive Generation of 3-D B-Spline Objects from Planar Image Data for Multidimensional Analysis, presented at Society of Exploration Geophysicists 60ih Ann. Int. Mtg. and Exposition, San Francisco, CA,(1990).

2. F.J. Wheeler and D.W. Nigg, Three-Dimensional Radiation Dose Distribution Analysis for Boron Neutron Capture Therapy, Nucl.Sci.Eng.110:16(1992).

3. D.W. Nigg, G.J. Stor, and F.J. Wheeler, Physics Parameters for an Epithermal- Neutron Facility at the Georgia Institute of Technology Research Reactor, These proceedings.

4. N. Blagojevic, G. Storr, B. Allen, H. Hatanaka, and H. Nakagawa, Role of Heavy Water in Boron Neutron Capture Therapy, presented at Intemational Workshop on Macro and Microdosimetry and treatment planning for Neutron Capture Therapy, Cambridge, MA,(1991).

5. G.S. Robinson, "A Guide to the AUS Modular Neutronics Code System," AAEC/E645 (1987)

6. W.A. Rhoades and R.L. Childs, DORT - Two-Dimensional Discrete Ordinates Transport Code, Oak Ridge National Laboratory, CCC-484,(1989).

7. D. Haritz, Results of Continued Clinical Investigations of BSH in Patients with Malignant Glioma, These proceedings.

8. W. Sweet, Massachusetts General Hospital, [private communication].

\section{COPYRIGHT}

The submitted publication has been authored by a contractor of the U.S. Government under DOE Contract Number DE-AC07-76IDO1570. Accordingly, the U.S. Government retains' a royalty-free license to publish or reproduce the published form of this contribution, or allow others to do so, for U.S. Government purposes. 



\title{
Enantioselective Synthesis of an Unnatural Amino Acid, L-Carboranylalanine
}

\author{
P. Radel and S. Kahl
}

This article is a reprint from Advances in Neutron Capture Therapy, pp. 277-280, (1993). 


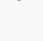




\title{
ENANTIOSELECTIVE SYNTHESIS OF AN UNNATURAL
}

\section{AMINO ACID, L-CARBORANYLALANINE}

\author{
Peggy A. Radel and Stephen B. Kahl \\ Department of Pharmaceutical Chemistry \\ University of California, San Francisco, CA 94143-0446
}

\section{BACKGROUND AND TARGET SELECTION}

Boron neutron capture therapy (BNCT) holds great promise as a radiochemotherapeutic treatment for various cancers, including malignant melanoma and glioblastoma multiforme. Its appeal lies in the potential for great selectivity in cell killing due to its binary nature. A noncytotoxic compound containing $10_{\mathrm{B}}$ is first introduced to the tumor. Subsequent exposure to an epithermal neutron beam will cause a uniquely efficient neutron capture by $10_{\mathrm{B}}$. The ${ }^{11} \mathrm{~B}$ intermediate undergoes rapid decomposition to an $\alpha-$ particle and recoil lithium atom. The sizeable kinetic energy of these particles ( $2.8 \mathrm{MeV}$ ) is deposited within 10-15 $\mu \mathrm{m}$, roughly one cell diameter. The two independently manipulable requirements for this therapy allow great precision in treatment of neoplasms.

There is a need to develop compounds that would be more selective for tumor uptake and which would maximize the amount of $10_{\mathrm{B}}$ introduced. Compounds incorporating carborane cages are attractive as these functionalities import ten boron atoms per cage, are very stable, and possess interesting physical characteristics. The initial target compound, L-carboranylalanine (1), was chosen as an isostere for phenylalanine.
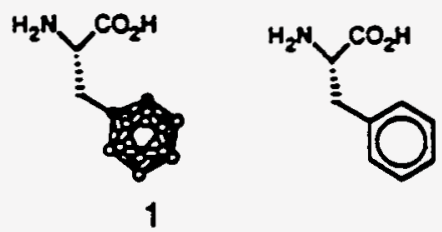

Figure 1. Structures of carboranylalanine and phenylalanine.

An initial area of application is in melanoma, as a false substrate for the abnormally high aromatic amino acid transport process involved in melanin biosynthesis. Carboranylalanine could provide much greater intracellular importation of $10_{\mathrm{B}}$ than $\mathrm{p}$ boronophenylalanine, which is currently in Phase I trials (1). We believe that carboranylalanine is a good biomimetic of phenylalanine based on its stability, hydrophobicity, and space filling characteristics. We conclude that carboranylalanine will 
resemble phenylalanine (rotating freely about the 1,4 axis of its aromatic ring) and will be actively imported.

The use of peptide analogs in receptor mediated importation processes will be explored. We will be investigating several systems known to be upregulated in neoplastic cells relative to normal cells. Even with the increased bulk of the carborane cage, we believe highly active peptide analogs can be produced upon substitution with carboranylalanine. The increased hydrophobicity and increased area of contact with cell surface receptors may increase the binding of carboranylalanyl-substituted peptide analogs, leading to increased uptake and retention of these drugs. Design of peptide analogs bearing carboranylalanine will allow us to address specific cancers, such as prostate and breast, which presently have no well-localized drugs for BNCT. Use of peptide analogs does require that both L- and D- carboranylalanine be readily available.

\section{SYNTHESIS}

L-carboranylalanine has been synthesized by Schwyzer et al, in low yield (2). We wanted to find a higher yielding, enantioselective route that would also allow general access to other homologs. These improvements are required as the expensive, less preponderant natural isotope, ${ }^{10} \mathrm{~B}$, is the only species that will undergo neutron capture. In evaluating synthetic routes, brevity and late introduction of the carborane cage were attractive. Our attempts to utilize a strategy involving a stereoselective serine lactone ring opening with a nucleophilic metallocarborane in the last step were not successful (3). The little explored reactivity of that species was not sufficient to effect the transformation under the required conditions. A longer but potentially high yielding alternative route was then examined. Diastereoselective introduction of an azido group to imide systems had been reported by Evans and coworkers; the azidoimide was then converted to the free amino acid (4). Our investigation was performed with the natural isotope distribution (80\% $11_{\mathrm{B}}$ and $20 \% 10_{\mathrm{B}}$ ), to be repeated with $10_{\mathrm{B}}$ enriched materials, if warranted.

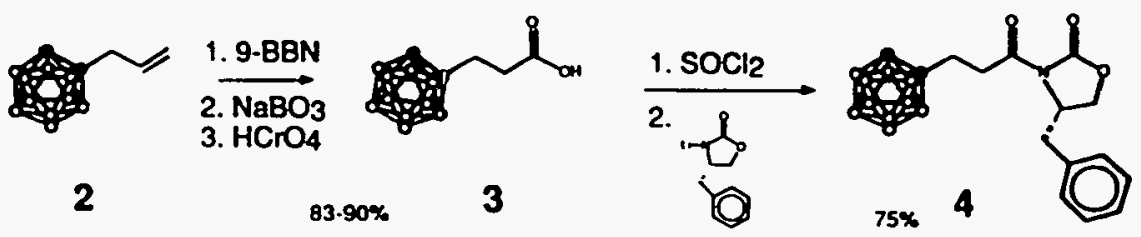

Scheme 1

The required imide was synthesized starting from the commercially available allyl carborane(2) (Scheme 1). Hydroboration of this molecule required very mild alkaline peroxidic workup to prevent carborane cage destruction (5). The results illustrate the interesting balance between steric and electronic directing effects in this reaction. Use of diborane in THF led to isolation of 35\% of the secondary alcohol and $65 \%$ of the desired primary alcohol. The inductive directing effect could be overcome by steric effects when a bulky hydroborating agent, 9-BBN, was used. Using this reagent, none of the secondary alcohol was isolated by chromatography nor detected in the $13 \mathrm{C}$ NMR of the crude reaction product. The crude alcohol was oxidized with Jones reagent to yield the acid 3 in 

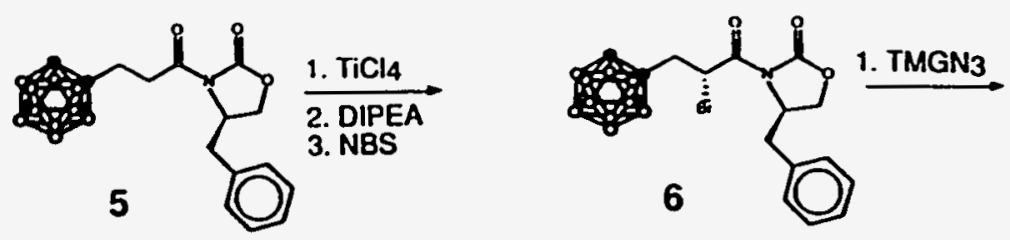

98:2

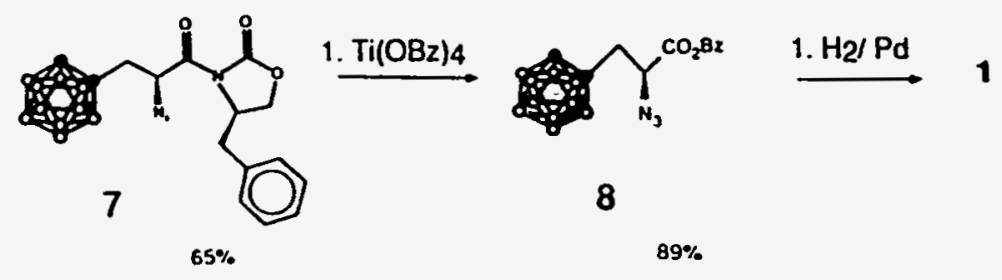

Scheme 2

$90 \%$ yieid from 2 . The acid was converted to the acid chloride which was reacted with the lithium salt of R- or S-4-benzyl-2-oxazolidone to yield imide 4 or 5 in $75 \%$ yield.

Direct azidization was attempted, but it was shown that the imide 4 did not survive exposure to potassium amide bases, even for very short periods at low temperature. Indirect, two step introduction of the $\alpha$-azido group was therefore examined. Stereoselective bromination of imide 5 (Scheme 2) was attempted with di-nbutylborontriflate, diisopropyl-amine and $\mathrm{N}$-bromosuccinimide. Irresolvably incomplete enolization was observed with commercially available triflate. We decided to apply another enolization not previously reported in asymmetric brominations, which utilized titanium tetrachloride to chelate the imide system (6). This allowed highly diastereoselective bromination, yielding a 98:2 ratio of the desired isomer 6 . This reaction still requires optimization; at present $10-20 \%$ may not enolize. Azide displacement, utilizing tetramethylguanidinium azide, proceeded with inversion at $-23^{\circ} \mathrm{C}$ and was completely stereoselective. Highly purified ( $>99: 1$ by HPLC analysis) azidoimide 7 could be isolated in $65 \%$ over-all yield from 5 .

Removal of the oxazolidone "template" was accomplished under mild conditions by transesterification of 7 with titanium tetrabenzyloxide in $89 \%$ yield (7). The more usual peroxidic alkaline hydrolysis would destroy the carborane cage. Hydrogenolysis of azidobenzyl ester 8 proceeds without difficulty to the desired amino acid in $89 \%$ yield. Similar yields and diastereoselectivites were obtained in using imide 5 as a starting material to obtain D-carboranylalanine.

Analysis to quantify the ratio of enantiomers present in the final product has not been straight-forward. The published optical rotation was not reproducible. Conversion of the amino acid to the $\alpha$-N-Mosher's amides, with methyl or benzyl ester of the carboxylic acid, did not yield base line separation of the $19 \mathrm{~F}$ NMR signals of the diastereomeric amides. GLC separation of the azidobenzylester 8 on a chiral cyclodextrin column was not successful and HPLC separation on a Nucleosil Chiral-2 or a Chiralcel OB column did not yield resolved enantiomers. However, use of a Chiralcel OJ column did permit baseline resolution of enantiomers. From the reaction sequence utilizing carefully chromatographed intermediates, a 98:2 ratio of isomers for the R-azido-benzylester 8 was found. Less careful chromatography of the corresponding intermediates led to a 5:95 ratio in the Sazidoester. 
Once the stereochemical analysis has been satisfactorily completed, cell culture studies will be conducted with L-carboranylalanine to ascertain the extent of uptake in B16 melanoma cells. Assay will be by the ICP-AAS method for boron content. Cell survival studies upon thermal neutron irradiation can then be performed.

Peptides will be synthesized on supported systems, purified, then studied in similar fashion for tumor uptake in appropriate cell lines. If significant uptake has occurred, appropriate binding assays may be used to ascertain the mode of entry.

\section{CONCLUSION}

We have synthesized L-carboranylalanine enantioselectively and in good yields. The route lends itself to eventual gram scale synthesis. D-carboranylalanine has also been synthesized, in similar yields and diastereoselectivities. The introduction of the $\alpha$-amino group is accomplished via highly diastereoselective bromination (98:2) of an imide system with subsequent azide displacement to yield an azidoimide, which can be converted without significant erosion of stereointegrity to the desired amino acid.

L-carboranylalanine may find an immediate application as a selective tumor localizing drug for melanoma in BNCT. Both $\mathrm{L}$ - and D-carboranylalanine will be utilized by incorporation into peptide analogs targeted for receptor mediated processes, to develop new tissue specific localizing drugs for various cancers in BNCT treatment. L- and Dcarboranylalanine will also prove to be useful structure/activity probes in general peptide design/ function elucidation work.

\section{ACKNOWLEDGEMENTS}

The authors wish to acknowledge support from the Department of Energy, in the form of grant DE-FG03-89ER6073. Partial funding was also made available from E.G. and G., Idaho, C91-103476. P. A. Radel wishes to thank Larry Wainschel and David Pate for their assistance.

\section{REFERENCES}

1. Mishima, Y., Ichihashi, M., Honda, C., Shono, M., Nakagawa, T., Obara, H., Shirakawa, J., Hiratsuka, J., Kanda, K., Kobayashi, T., Nozaki, T., Aizawa, O., Sato, T., Korashima, H., Yoshino, K.,\& Fukuda, H. (1992) in Proc. of the Fourth International Symposium on Neutron Capture Therapy for Cancer, (4th: 1990: Sydney, Australia)

ed. B. J. Allen, D. E. Moore, and B. V. Harrington, Plenum Press.

2. Leukart, O., Caviezel, M., Eberle, A., Escher, E., Tun-Kyi, A. \& Schwyzer, R. (1976) Helv. Chim. Acta . 59, 2184-2187.

3. Arnold, L. D., Drover, J. C. G., Vederas, J. C. (1987) J. Am Chem. Soc. 109,4649-4659.

4. Evans, D. A., Britton, T. C., Ellman, J. A., \& Dorow, R. L., (1990) J. Am. Chem Soc. 112, 4011-4030.

5. Kabalka, G. W., Shoup, T. M., \& Goudgaon, N. M., (1989) J. Org. Chem. 54, 59305933.

6. Evans, D. A., Urpi, F., Somers, T. C., Clark, J. S., \& Bilodeau, M. T. (1990)

J.Am. Chem. Soc. 112, 8215-8216.

7. Evans, D. A., Ellman, J. A., \& Dorow, R. L. (1987) Tetrahedron Let. 28, 1123-1126. 
Carbonyl Nonahydrodecaborate(1-): A Versatile Synthon for Functionalized Borane Anions

K. Shelly, M. F. Hawthorne, and C. Knobler

This article is a reprint from Advances in Neutron Capture Therapy, pp. 325-328, (1993). 

CARBONYL NONAHYDRODECABORATE(1-): A VERSATILE SYNTHON FOR FUNCTIONALIZED BORANE ANIONS

\author{
Kenneth Shelly, M. Frederick Hawthorne, and Carolyn B. Knobler \\ Department of Chemistry and Biochemistry \\ University of California at Los Angeles \\ Los Angeles, CA 90024 USA
}

\title{
INTRODUCTION
}

Despite the surging interest in neutron capture therapy, the development and testing of new boron containing compounds has not received as much attention as other areas of BNCT. The most intensively studied boron agents are the mercaptoundecahydro-closododecaborate dianion $\left(\left[\mathrm{B}_{12} \mathrm{H}_{11} \mathrm{SH}\right]^{2-}\right.$, "BSH") and 4-(dihydroxyboryl)phenylalanine (BPA), yet both of these species have been known for decades. ${ }^{1,2}$ The development of new boron agents for BNCT requires convenient high-yield methods for the introduction of boron moieties into suitable chemical structures.

Common strategies employed in the synthesis of new materials for BNCT include the attachment of boron-containing residues to existing compounds known to accumulate in tumor tissues or polymeric carrier delivery agents. The most popular large boron-rich moiety used to accomplish this is the carboranyl group derived from ortho-carborane, $\mathrm{C}_{2} \mathrm{~B}_{10} \mathrm{H}_{12}{ }^{3}$ This molecule offers the advantages of relative stability, high boron content, and the versatility of its derivative chemistry. The use of the carboranyl moiety may be limited, however, due to its extreme hydrophobicity, although in many cases it may be transformed to the more hydrophillic $-\mathrm{C}_{2} \mathrm{~B}_{9} \mathrm{H}_{10}{ }^{-}$group.

The development of water-soluble boron carriers would be facilitated by a readily available boron-rich hydrophilic group capable of derivatization and conjugation. Polyhedral boranes such as the $\left[\mathrm{B}_{10} \mathrm{H}_{10}\right]^{2-}$ ion ${ }^{4}$ and the $\left[\mathrm{B}_{12} \mathrm{H}_{12}\right]^{2-}$ ion ${ }^{5}$ are good candidates because of their hydrolytic stability and high boron content, and they are easily synthesized from decaborane in good yield. Their rich derivative chemistry, however, is usually of limited utility because of low yields or the production of multiple products or isomers that may be difficult to separate. $1,6-8$

Carbon monoxide is one of the most versatile derivatives known in polyhedral borane chemistry, capable of a wide variety of functional group transformations. ${ }^{8-10}$ Unfortunately the synthesis of the carbonyl boranes themselves is usually difficult.8,11 The direct substitution with carbon monoxide on $\left[\mathrm{B}_{10} \mathrm{H}_{10}\right]^{2-}$ and $\left[\mathrm{B}_{12} \mathrm{H}_{12}\right]^{2-}$ is shown in Eqn. 1 and 2. These reactions require severe conditions (high temperature and pressure) and produce 

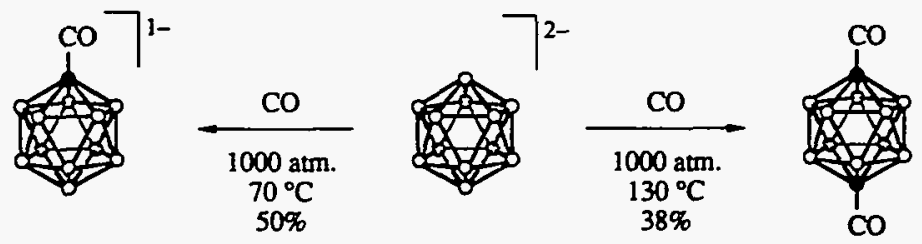

(Eqn. 1)
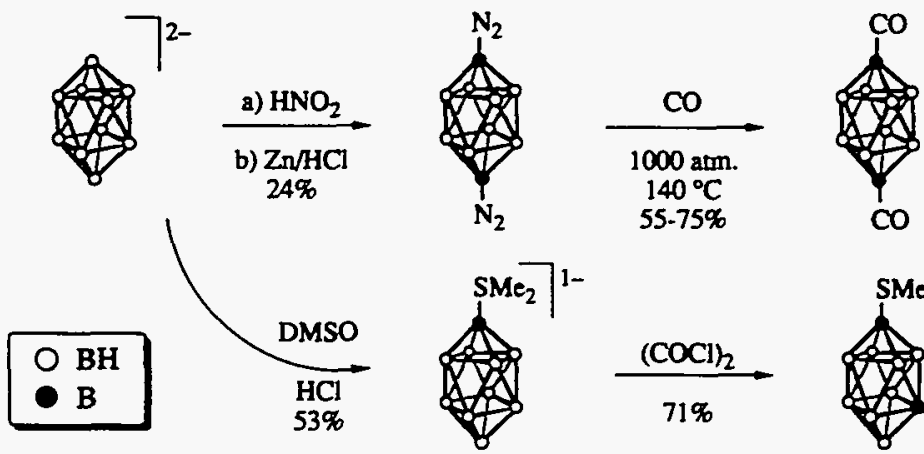

(Eqn. 2)

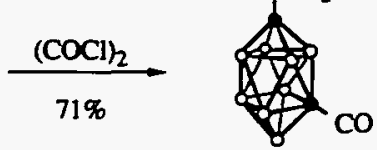

(Eqqn. 3)

relatively low yields of product. A more convenient method using oxalyl chloride ${ }^{12}$ (Eqn. 3 ) to produce monocarbonyl derivatives of ligand substituted $\left[\mathrm{B}_{10} \mathrm{H}_{10}\right]^{2-}$ ions proceeds in more reasonable yield; however the substituted $\left[\mathrm{B}_{10} \mathrm{H}_{9} \mathrm{~L}\right]^{-}$precursors are available in only low to moderate yields. 6.7

Our interest in the production of a variety of water-soluble boron species for encapsulation in liposomes ${ }^{13,14}$ prompted us to reexamine the carbonylation of polyhedral borane anions. This has led to the development of a convenient method to produce monofunctionalized derivatives of $\left[\mathrm{B}_{10} \mathrm{H}_{10}\right]^{2-}$ in high yield.

\section{DISCUSSION}

The reaction of oxalyl chloride with $\left[\mathrm{B}_{10} \mathrm{H}_{10}\right]^{2-}$ proceeds rapidly and essentially quanttatively at room temperature with the evolution of carbon monoxide to produce [2$\left.\mathrm{B}_{10} \mathrm{H}_{9} \mathrm{CO}\right]^{-}$. The cleanest reaction is observed employing $\left[\mathrm{Ph}_{3} \mathrm{PMe}\right]\left[\mathrm{B}_{10} \mathrm{H}_{10}\right]$ in $\mathrm{CH}_{2} \mathrm{Cl}_{2}$, but the reaction has been used with several salts of $\left[\mathrm{B}_{10} \mathrm{H}_{10}\right]^{2-}$ (cesium, tetramethylammonium, triethylammonium) and with other solvents such as acetonitrile and tetrahydrofuran. The reaction product exhibits a strong infrared peak at $2129 \mathrm{~cm}^{-1}$. This absorption lies in the frequency range typical of carbon monoxide coordinated to boron, which is higher than that of metal carbonyls due to the lack of d orbital back-bonding. The ${ }^{11} \mathrm{~B}\left[{ }^{1} \mathrm{H}\right]$ spectrum of the reaction mixture in $\mathrm{CH}_{2} \mathrm{Cl}_{2}$ exhibits seven signals, consistent with equatorial substitution, and shows only traces of byproducts. The substituted boron is indicated by a high field resosance at $-43.8 \mathrm{ppm}$ (relative to $\mathrm{BF}_{3} \cdot \mathrm{Et}_{2} \mathrm{O}$ ) which is a singlet in the proton coupled spectrum. [ $\left.\mathrm{Ph}_{3} \mathrm{PMe}\right]\left[2-\mathrm{B}_{10} \mathrm{H}_{9} \mathrm{CO}\right.$ ] may be isolated as a light tan solid in $85 \%$ yield, or the reaction mixture may be used directly in further reactions. The structure of [2$\left.\mathrm{B}_{10} \mathrm{H}_{9} \mathrm{CO}\right]^{-}$has been determined by $\mathrm{X}$-ray crystallography ${ }^{15}$ and shows a linear $\mathrm{B}-\mathrm{C}-\mathrm{O}$ array with a C-O distance of $1.13 \AA$.

The utility of the $\left[2-\mathrm{B}_{10} \mathrm{H}_{9} \mathrm{CO}\right]^{-}$anion results from its ease of conversion to a variety of other species as depicted in Figure 1. Although $\left[2-\mathrm{B}_{10} \mathrm{H}_{9} \mathrm{CO}\right]^{-}$is relatively insensitive to moisture in the solid state or in solution, it is easily hydrated in aqueous solvent mixtures to form [2- $\left.\mathrm{B}_{10} \mathrm{H}_{9} \mathrm{CO}_{2} \mathrm{H}\right]^{2-}$ in accordance with Equation 4, which lies predominantly to the 


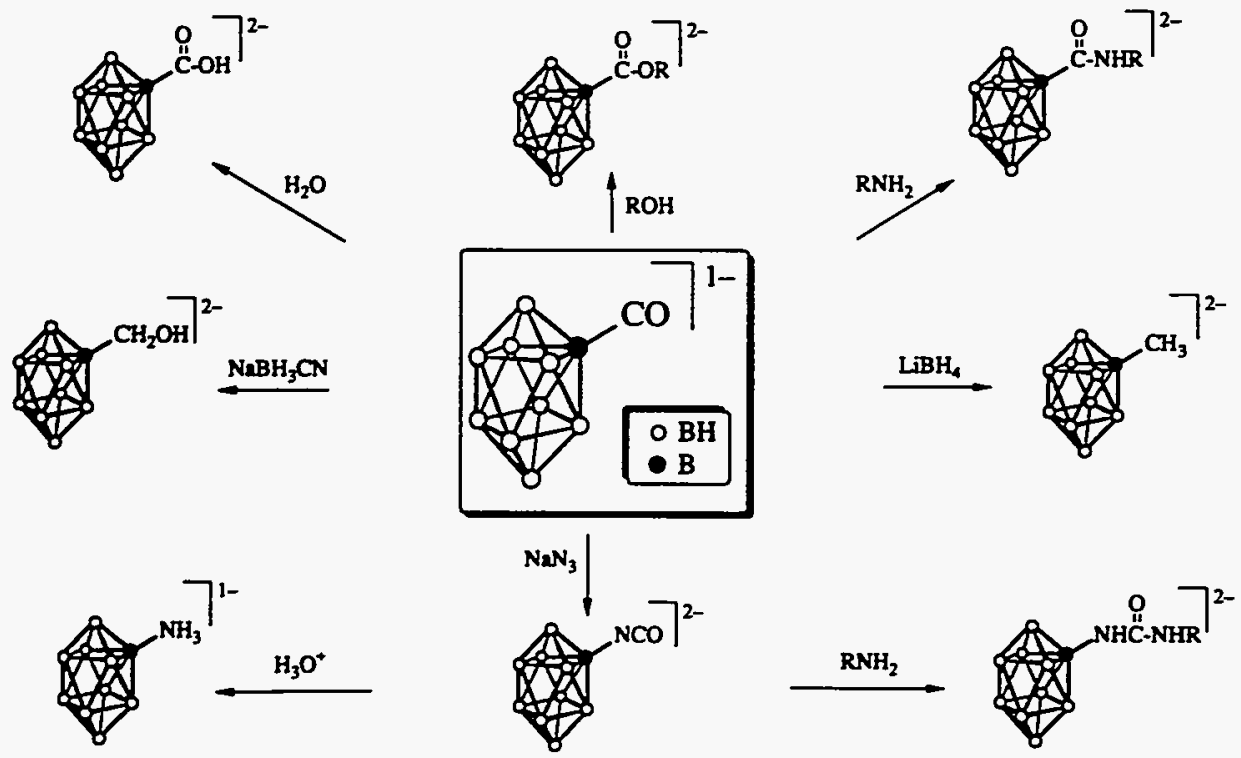

Figure 1. Transformations of the $\mathrm{B}_{10} \mathrm{HgCO}_{9}^{-}$ion.

$$
\left[2-\mathrm{B}_{10} \mathrm{H}_{9} \mathrm{CO}\right]^{-}+\mathrm{H}_{2} \mathrm{O} \rightleftharpoons\left[2-\mathrm{B}_{10} \mathrm{H}_{9} \mathrm{CO}_{2} \mathrm{H}^{2-} \mathrm{H}^{+}\right.
$$

right in aqueous solutions. The transformation of the carbonyl group from $-\mathrm{C}=\mathrm{O}$ to $>\mathrm{C}=\mathrm{O}$ is indicated by the appearance of an infrared stretch at $1690 \mathrm{~cm}^{-1}$ as well as a-C-O stretch at $1256 \mathrm{~cm}^{-1}$. The identity of $\left[\mathrm{Ph}_{3} \mathrm{PMe}\right]\left[2-\mathrm{B}_{10} \mathrm{H}_{9} \mathrm{CO}_{2} \mathrm{H}\right]$ has been confirmed by crystallographic analysis.

The equilibrium in Equation 4 suggests the formation of other acyl derivatives of the decahydrodecaborate anion. The reaction of $\left[2-\mathrm{B}_{10} \mathrm{H}_{9} \mathrm{CO}^{-}\right.$with an amine $\left(\mathrm{RNH}_{2}\right)$ results in the formation of an amide $\left(\left[2-\mathrm{B}_{10} \mathrm{H}_{9} \mathrm{CONHR}\right]^{2-}\right)$ as long as an excess of the amine is present to absorb the acid formed by the reaction. Esters are produced by the combination of [2- $\left.\mathrm{B}_{10} \mathrm{H}_{9} \mathrm{CO}\right]^{-}$with alcohols; in this case, the reaction is slow or incomplete unless an auxiliary base is added to absorb the acid formed.

The reduction of $\left[2-\mathrm{B}_{10} \mathrm{H}_{9} \mathrm{CO}\right]^{-}$with borohydride ion $\left(\mathrm{BH}_{4}{ }^{-}\right)$in refluxing THF produces $\left[2-\mathrm{B}_{10} \mathrm{H}_{9} \mathrm{CH}_{3}\right]^{2-}$ (confirmed by $\mathrm{X}$-ray crystallography). Although this is a novel species, as an easily synthesized single alkyl isomer of substituted $\left[\mathrm{B}_{10} \mathrm{H}_{10}\right]^{2-}$, it has little utility as a route to other derivatives. Milder reaction conditions employing cyanoborohydride $\left(\mathrm{BH}_{3} \mathrm{CN}^{-}\right)$as reducing agent result in a mixture of products including [2$\left.\mathrm{B}_{10} \mathrm{H}_{9} \mathrm{CH}_{2} \mathrm{OH}\right]^{2-}$, an ion with the potential of derivatization or conjugation with many other moieties. The optimized production and isolation of this species is in progress.

A very useful transformation of $\left[2-\mathrm{B}_{10} \mathrm{H}_{9} \mathrm{CO}\right]-$ occurs from its reaction with azide ion in acetonitrile. The carbonyl undergoes a Curtius-type rearrangement to form the isocyanate [2- $\mathrm{B}_{10} \mathrm{H}_{9} \mathrm{NCO}^{2-}$, indicated by an infrared absorption at $2304 \mathrm{~cm}^{-1}$. This ion, whose structure has been determined by $\mathrm{X}$-ray crystallography, is stable in neutral aqueous solution but is rapidly hydrolyzed in acidic media. The hydrolysis product, [2- $\left.\mathrm{B}_{10} \mathrm{H}_{9} \mathrm{NH}_{3}\right]^{-}$, has been previously synthesized by other more direct methods but in reduced yield compared to the present route. The isocyanate ion is also reactive with alcohols and amines, enabling the synthesis of a variety of amine derivatives of $\left[\mathrm{B}_{10} \mathrm{H}_{10}\right]^{2-}$. 
The [2- $\left.\mathrm{B}_{10} \mathrm{H}_{9} \mathrm{CO}\right]-$ is conveniently prepared in high yield from the decahydrodecaborate anion. This carbonyl species may be conjugated to existing species or derivatized to a variety of other soluble $\left[\mathrm{B}_{10} \mathrm{H}_{10}\right]^{2-}$ derivatives. The $\left[2-\mathrm{B}_{10} \mathrm{H}_{9} \mathrm{CO}\right]-$ ion therefore offers a high-yield route to the production of many boron-rich species for boron neutron capture therapy.

\section{ACKNOWLEDGMENTS}

This research was conducted as part of the BNCT program of the Idaho National Engineering Laboratory (INEL) and was performed under the auspices of the U.S. Department of Energy, Office of Energy Research, under DOE Field Office, Idaho, Contract No. DE-AC07-76ID01570.

\section{REFERENCES}

1. Knoth, W.H. (1964) J. Am. Chem. Soc. 86, 3973-3983.

2. Snyder, H.R., Reedy, A.J. \& Lennarz, W.J. (1958) J. Am. Chem. Soc. 80, 835-838.

3. Hawthome, M.F. (1991) Pure \& Appl. Chem. 63, 327-334.

4. Hawthorne, M.F. \& Pitochelli, A.R. (1959) J. Am. Chem. Soc. 81, 5519.

5. Pitochelli, A.R. \& Hawthome, M.F. (1960) J. Am. Chem. Soc. 82, 3228-29.

6. Hertler, W.R. \& Raasch, M.S. (1964) J. Am. Chem. Soc. 86, 3661-3668.

7. Hertler, W.R., Knoth, W.H. \& Muetterties, E.L. (1965) Inorg. Chem. 4,280-287.

8. Knoth, W.H., Sauer, J.C., Balthis, J.H., Miller, H.C. \& Muetterties, E.L. (1967) J. Am. Chem. Soc. 89, 4842-4850.

9. Haslinger, F., Soloway, A.H. \& Butler, D.N. (1966) J. Med. Chem. 9, 581-584.

10. Alam, F., Soloway, A.H., Barth, R.F., Mafune, N., Adams, D.M. \& Knoth, W.H. (1989) J. Med. Chem. 32, 2326-2330.

11. Knoth, W.H., Sauer, J.C., England, D.C., Hertler, W.R. \& Muetterties, E.L. (1966) J. Am. Chem. Soc. 88, 935-939.

12. Hertler, W.R., Knoth, W.H. \& Muetterties, E.L. (1965) Inorg. Chem. 4, 288-293).

13. Shelly, K., Hawthome, M.F. \& Schmidt, P.G. (1992) in Progress in Neutron Capture Therapy for Cancer, eds. Allen, B.J., Moore, D.E. \& Harrington, B.V. (Plenum Press, New York), p. 259-264.

14. Shelly, K., Feakes, D.A., Hawthorne, M.F., Schmidt, P.G., Krisch, T.A. \& Bauer, W.F. (1992) Proc. Natl. Acad. Sci. USA 89, 9039-9043.

15. Shelly, K., Hawthome, M.F. \& Knobler, C.B. (1992) Inorg. Chem. 31,2889-2892. 
PLENARY PRESENTATION: Boron Delivery to Tumors Mediated by Macromolecules and Vesicles

M. F. Hawthorne

This article is a reprint from Advances in Neutron Capture Therapy, pp. 345-350, (1993). 

BORON DELIVERY TO TUMORS MEDIATED BY MACROMOLECULES AND VESICLES

\author{
M. Frederick Hawthorne \\ University of California at Los Angeles, Los Angeles, CA
}

\title{
INTRODUCTION
}

During the past decade many types of macromolecular species potentially capable of selectively transporting large quantities of ${ }^{10} \mathrm{~B}$ to tumor have been examined for the purposes of BNCT. These large boron carriers have either been obtained by the conjugation of highmolecular weight biomolecules, such as antibodies, with small boron-containing reagents or the purposeful synthesis of large boron-rich oligomers which are then attached as reagents to antibodies or other peptides targeted for tumor cell receptor sites. In addition to the use of large molecules as boron carriers, recent research has demonstrated the utility of synthetic unilamellar liposomes as tumor cell-selective vesicles capable of the in vivo delivery of therapeutic quantities of boron to tumor with low injected doses of boron. A group of receptor-mediated vesicular delivery systems are under preliminary examination which are comprised of low density lipoproteins filled with hydrophobic carborane derivatives. The characteristic common to all of these macromolecular and vesicular tumor targeting boron delivery systems is their use of receptor sites that are either unique to tumor cells or grossly overexpressed by tumor cells relative to the cells which comprise normal tissues.

\section{BORON DELIVERY USING IMMUNOPROTEINS}

Bale and $\mathrm{Spar}^{1}$ in 1967 proposed the use of tumor associated antibodies as means to deliver ${ }^{10} \mathrm{~B}$ to malignant cells for BNCT. At the time, the only antibodies available for this purpose were polyclonal, structurally heterogeneous and not available in large quantities. Nonetheless, $\mathrm{we}^{2}$ and others ${ }^{3}$ successfully conjugated available antibodies in a random manner using small boron-rich reagents such as the 4-carboranyldiazonium cation ${ }^{2}$ and functionalized polyhedral borane anions ${ }^{3}$. These earliest attempts to produce viable boroncontaining polyclonal (and monoclonal ${ }^{4}$ at a later date) conjugates were generally unsuccessful since only a few conjugation reagents could be attached to the antibody before immunoreactivity was lost. In addition, the boron contents of the conjugates were much too low for further consideration. Water-solublizing groups ${ }^{5}$ including sugars 6 were incorporated into boron conjugation reagent structures with little avail. Interest in the use of 
immunoproteins as boron delivery vehicles waned until the discovery of hybridoma procedures by Millstein and Köhler which made monoclonal antibodies (Mabs) plentiful ${ }^{7}$.

Simple arithmetical considerations led to the conclusion that each of the $10^{6}$ antigenic sites assumed to be located on each tumor cell would require approximately $10^{3} 10 \mathrm{~B}$-atoms in order to achieve the $10^{9} 10 \mathrm{~B}$-atoms per cell estimated for effective BNCT. This result could only be achieved if each Mab molecule were bonded to at least $10^{3} 10 \mathrm{~B}$-atoms. The obvious method for achieving such a high boron loading of protein was to attach boron-rich oligomers to only a few random points in the Mab structure, usually indigenous lysine $\varepsilon$-amino groups. The first efforts in this direction are exemplified by the work of Barth and Soloway ${ }^{8}$ in which the $\varepsilon$-amino groups of poly-L-lysine were reacted with boron-rich conjugation reagents derived from the $\mathrm{B}_{10} \mathrm{H}_{10}{ }^{2-}$ polyhedral ion. The conjugated oligomer was then coupled to Mab to achieve a product with $2 \times 10^{3} \mathrm{~B}$-atoms per Mab molecule, but with greatly reduced immunoreactivity. In this instance the nonuniformity of the poly-L-lysine oligomer, the randomness of the conjugation of these species with boron reagent and the similar randomness of the conjugation of the oligomeric reagent with Mab lysine $\varepsilon$-amino groups yields a highly heterogeneous product which would be difficult to reproduce if desired and imposible to structurally modify in a controlled manner. Very recent work ${ }^{9}$ with a "starburst" dendrimer carrying 48 primary amino groups provided an oligomer of uniform molecular weight, but subsequent conjugation of the polyamine to boron reagent and coupling of the product to Mab introduced structural uncertainties. However, the product contained over $2 \mathrm{x}$ $10^{3} \mathrm{~B}$-atoms per mab molecule while the immunoreactivity was about $80 \%$ of that presented by the native antibody. Animal experiments 10 with these dendrimer-containing conjugates gave excessive liver uptake relative to tumor; behavior characteristic of every highly substituted Mab examined thus far.

Rather than attack pristine Mab molecules with a randomized array of oligomeric conjugation reagents we have synthesized designed oligomeric structures for this purpose. ${ }^{11,12}$ These precisely synthesized oligomers were peptides comprised of boron-rich $\alpha$-amino acids and amino-terminated with a fluorescent group. The carboxyl terminus was employed for active ester mediated conjugation with Mab lysine residues in a random fashion. The peptides themselves were trivially designated as "boron trailer reagents" or simply, "trailers" and synthesized from the amino acids illustrated in Figure 1 using Merrifield solid phase syntheses. Figure 2 depicts the structure of an undecapeptide which was conjugated with T84.66 anti-CEA Mab and studied with nude mice bearing ten-day-old LS 174T tumor xenografts. A fluorescent conjugate bearing $600 \mathrm{~B}-$ atoms per Mab was obtained ${ }^{12.13}$ and shown to have retained $95 \%$ of the original Mab immunoreactivity. In mice, ${ }^{13} 43$ and $4.3 \%$ of the injected dose of conjugate per gram of tissue localized in the liver and tumor tissues, respectively, 48 hours post injection ( $27 \mu \mathrm{g} /$ mouse). Blood contained only $1.2 \% \mathrm{ID} / \mathrm{g}$. These partial biodistribution results were in agrement with data obtained by others $8-10$ and the dramatic uptake of Mab conjugate by liver is identified as the single largest problem to be
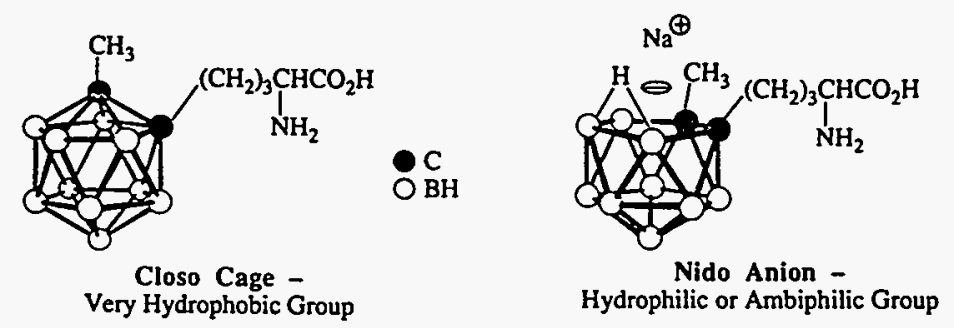

Figure 1. Two boron-rich $\alpha$-amino acids employed in peptide synthesis as their $t$-BOC derivatives. 


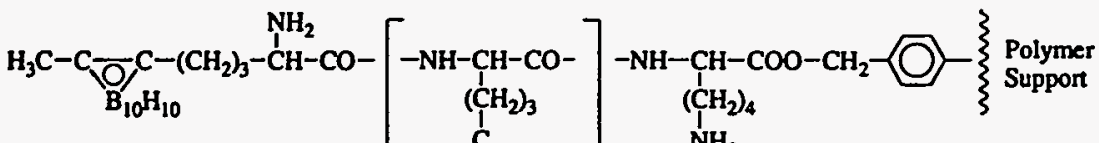

$$
\begin{aligned}
& {\left[\begin{array}{l}
1 \\
{ }_{C}^{C} \mathrm{~B}_{10} \mathrm{H}_{10} \\
\mathrm{C}_{3}
\end{array}\right]_{9}} \\
& \text { (closo-CB) }{ }_{10} \text { Lys }
\end{aligned}
$$

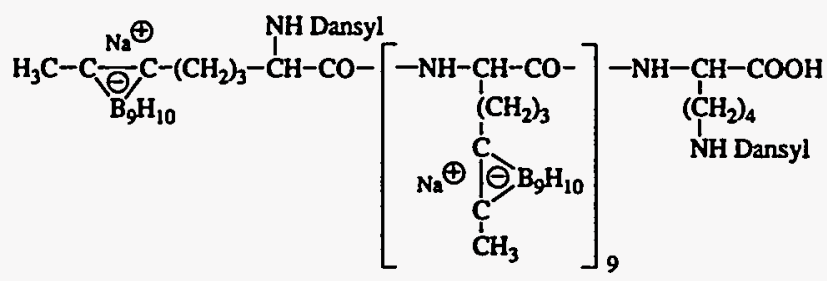

Acetyl may occasionally replace dansyl in these species.

Figure 2. An undecapeptide trailer reagent synthesiz $\mathrm{d}$ using the Merrifield method.

surmounted if immunoproteins are to ever succeed as boron carriers for BNCT. While Mabs coupled with trailers provide a less disordered immunoconjugate than those previously available, it is clear that the random attachment of trailers to Mab molecules is not amenable to complete structural control. Also, the use of a large IgG Mab as the basic molecular structure to be modified may contribute to excessive liver accretion. Accordingly, we have begun a new approach to this endeavor which makes use of an engineered FabSH anti-CEA fragment conjugated via its thiol group to a manifold peptide which, in turn, collects a fixed number of trailer oligomers. The latter species could be based upon either peptide or diphosphate ester structures. Figure 3 illustrates the construction of the complete FabSH conjugate. The total array could have a molecular weight of approximately $50 \mathrm{kD}$. At this time, work is progressing on the FabSH (and dithiol Fab') molecules and new hydrophilic trailers and manifold structures. Oligomeric diphosphate esters of carboranyl diols appear to be attractive candidates for trailer applications.

It has been pointed out by us 12 that bispecific antibodies might serve to initially attach large boron-rich arrays to tumor cells by serving as a bridge between tumor cell wall antigen and hapten groups present in free trailer species. Figure 4 presents a pictorial representation

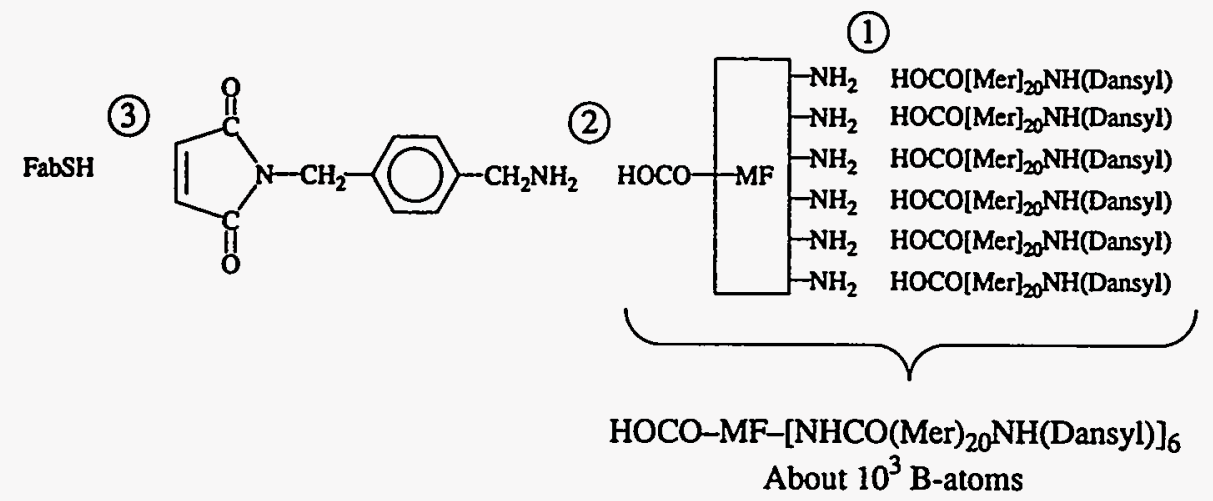

Figure 3. Sequence of reactions leading to immunodirected boron-rich oligomers. The HOCO-(Mer) $20 \mathrm{NH}_{2}$ precursor is a peptide or a polyamide with 20 boron-rich repeating units. The HOCO-(MF)(NH $)_{6}$ species is a peptide manifold containing five lysine residues. 


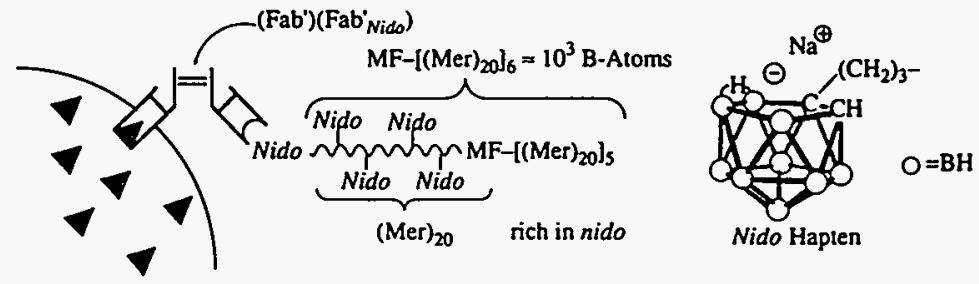

Figure 4. The bispecific antibody binding hapten-containing boron-rich molecule to cell wall CEA. The synthetic bispecific antibody is initially injected and binds to tumor cell wall CEA. The manifold trailer MF[(Mer) $\left.{ }_{20}\right]_{6}$ is injected later and the Nido hapten targets its specific antibody binding site now held to the cell wall by CEA antigen/antibody interaction.

of this concept which would hopefully attach $10^{3} \mathrm{~B}$-atoms to each bispecific antibody which is complexed with a cell wall antigen without the use of covalent chemical bonds. The bispecific antibody will be synthesized by linking a Fab'CEA to a Fab'nido. The required Fab' fragments are obtained from their respective monoclonal IgG by enzymatic cleavage. While the Fab'CEA is available from well known Mabs, the Fab'nido (which recognizes the [nido-7,8- $\left.\mathrm{C}_{2} \mathrm{~B}_{9} \mathrm{H}_{11^{-}}\right]^{-}$hapten) is derived from an $\mathrm{IgG}_{\text {nido }}$ available from a newly cloned, but productive hybridoma. The hapten employed in the trailer molecule also serves as the source of boron and could be of either the peptide or diol diphosphate type.

While one considers bridging, non-covalent bonding interactions which may secure a boron reservoir to a tumor cell wall antigen prior to endocytosis of the combined structure, one should not overlook the possible application of the classical four-fold streptavidin (biotin) 4 host-guest complex having an equilibrium constant for biotin binding of approximately $10^{15}$. The desired bispecific system could be constructed from the FabSH antibody fragment described above. Bonding of the thiol function of FabSH to streptavidin with a linker would provide an immunoprotein capable of both cell wall antigen complexation and binding with up to four biotin-terminated trailer molecules which are also fluorescence labeled for in vitro analytical purposes. Work has begun with component prototypes of this system.

\section{GROWTH FACTOR RECEPTORS AS TARGETS FOR BORON DELIVERY}

Although the cell wall antigens associated with a variety of tumor cell lines have been investigated as Mab conjugate receptor sites, it is possible to employ the receptors of growth and regulatory peptides, such as epidermal growth factor (EGF), as targets for Mabs ${ }^{14}$ conjugated with boron trailer molecules.

Since receptors such as those associated with EGF are extravagantly expressed by many types of tumor cells compared to nonmalignant cells, it is also possible that the parent peptide such as the 53-amino acid EGF can be conjugated with boron-rich trailer reagents and employed for boron delivery through the normal EGF pathway. ${ }^{15}$ Since the EGF and related growth factor molecules are relatively small, the size of the attached trailer molecules would be critical. A small trailer would be less perturbing to the biological function of the EGF, but the boron-delivery capability of such a conjugate would be compromised. A large trailer would provide more boron per conjugated EGF molecule, but receptor binding and cell entry of the conjugate col:ld be impaired. Cultured glioma cells have been employed to demonstrate the cytotoxicity of EGF-131 I-dextran conjugates. 16

\section{NATURAL AND SYNTHETIC VESICLES AS BORON DELIVERY VEHICLES}

Vesicles, such as the naturally occurring low density lipoproteins ${ }^{17}$ (LDL's) and 
synthetic species such as uni-and multilamellar liposomes ${ }^{18}$ have the potential to deliver relatively huge quantities of boron-containing species to tumor cells. The LDL delivery mechanism is well understood and is mediated by apoprotein B-100, imbedded in the phospholipid shell of the LDL, which binds to the tumor cell LDL receptor site. Such receptor sites are overexpressed by rapidly growing tumor cells which require LDL-delivered cholesterol for their continued growth. Replacement of the cholesteryl fatty acid esters, which fill the interior of the phospholipid shell of the LDL, with hydrophobic fatty alcohol esters of carborane carboxylic acids provides an LDL which is rich in boron and apparently unchanged in its ability to bind LDL receptor sites. Following complexation of the boroncontaining LDL with the tumor cell wall receptor, the receptor-LDL array is internalized, stripped of the LDL and the receptor returned to the cell wall surface. This pumping action will proceed as long as LDL's are available and the cells internal regulatory mechanism perceives a need for additional cholesterol. Since the boron-rich LDL's contain little available cholesterol, active LDL uptake may proceed in an essentially unfettered manner until terminated by another means. This LDL delivery method was devised by Kahl ${ }^{17}$ and recently shown to provide as much as $240 \mathrm{ppm}$ of intracellular boron in tumor cells grown in culture. Although the required LDL's must be harvested from a compatible donor and converted to the desired product, this inconvenience is mitigated by the excellent tumor cell boron levels achieved.

Figure 5 depicts the cutaway structure of a synthetic lip.ssome and the structure of the phospholipid which, when mixed with an equal weight of cholesterol, provides the lipid bilayer. Such unilamellar liposomes have been under investigation in our laboratories for the past five years. The selective delivery of therapeutic quantities of boron to tumor (EMT 6 murine adenocarcinoma and P1798 murine lymphosarcoma) in mice with high tumor/blood ratios and low injected doses $(5-15 \mathrm{mg} / \mathrm{kg}$ body wt.) has been demonstrated 18 . The selective accretion of boron in tumors is thought to be the result of liposome leakage from immature ad hoc tumor vasculature into the tumor bed. The migration of the free liposomes to tumor cells followed by endocytosis has been demonstrated using fluorescence microscopy with fluorescent liposomes. The boron-containing species introduced as a solute in the aqueous core of the liposome is the sodium salt of a polyhedral borane anion. Considerable resources have been devoted to the discovery of efficacious borane derivatives and this effort continues along with a search for suitable boron-containing compounds which may be added to the lipid bilayer without compromise of the structural integrity of the liposome. Borane derivatives capable of reaction with indigenous intracellular protein amino groups have been designed

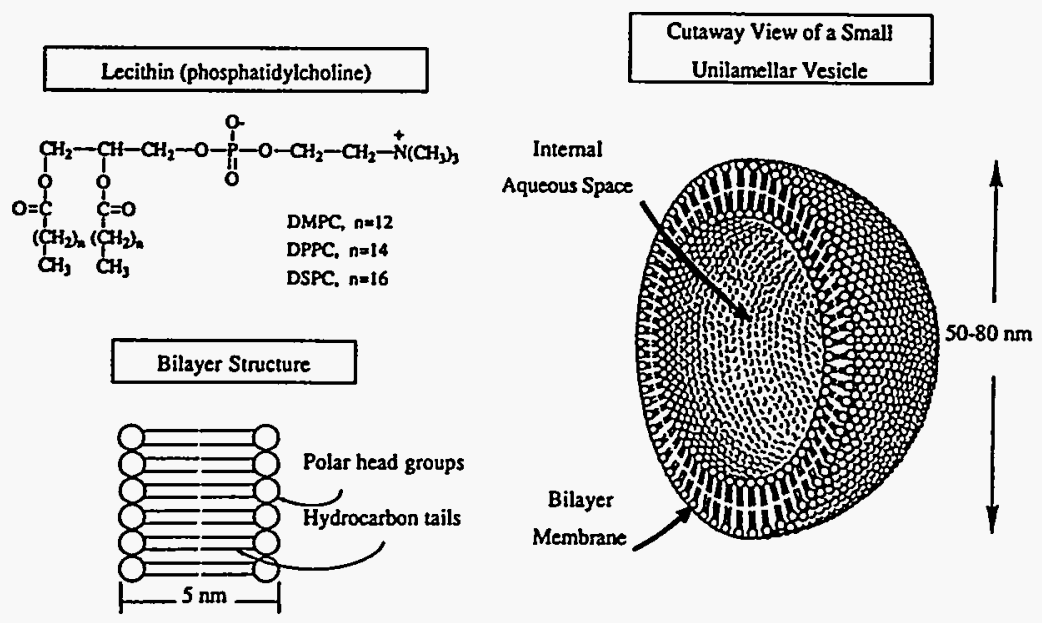

Figure 5. Cutaway cross-sectional view of a typical liposome, its bilayer construction and the phospholipid used in the bilayer. 
and synthesized. Such reactivity insures retention of the boron species within the cell while blood, liver and spleen are cleared. Figure 6 presents a time point biodistribution experiment. Only tumor, blood, liver and spleen data are presented because all other tissues do not take up appreciable quantities of boron. The ease with which liposomes can be prepared coupled with the freedom they provide for the development of reactive boron derivatives make them among the most attractive known candidates for the realization of BNCT.

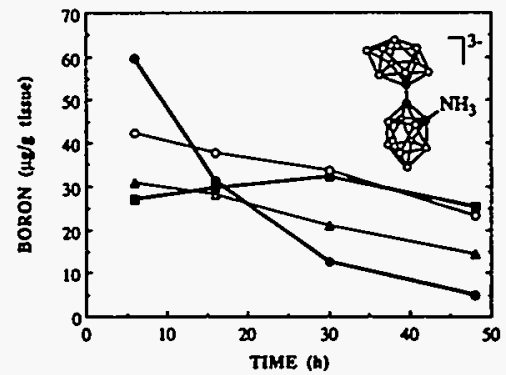

Figure 6. Biodistribution of liposomal $\mathrm{Na}_{3} \mathrm{~B}_{20} \mathrm{H}_{17} \mathrm{NH}_{3}$ in Balb/c Mice bearing EMT 6 tumors. Injected dose $11 \mathrm{mg} / \mathrm{kg}$ body weight. $O$, Blood; $\square$, tumor; $O$, liver and $\Delta$, spleen. After 48 hrs. tumor contains 25.4ppm B and tumor/blood $=5.3$.

\section{ACKNOWLEDGEMENTS}

The author wishes to thank his coworkers and colleagues in the BNCT community for their direct and indirect contributions to this paper. He also thanks the National Cancer Institute for continuing support through grant R01-CA31753 and the Idaho Engineering Laboratory of the U.S. Department of Energy under DOE field Office, Idaho, Contract DE-AC07-76IDO1570.

\section{REFERENCES}

1. Bale, W.F. \& Spar, I.L. (1967). Adv. Biol. Med. Phys. 5, 286-356.

2. Hawthome, M.F., Wiersema, R.J. \& Takasugi, M. (1972). J. Medicinal Chem. 15, 449-452.

3. Barth, R.F., Johnson, C.W., Wei, W-Z., Carey, W.E., Soloway, A.H. \& McGuire, J. (1982). Cancer Detect. Prevent. 5, 315-323.

4. Mizusawa, E., Dahlman, H.L., Bennet, S.J., Goldenberg, D.M. \& Hawthome, M.F. (1982).Proc. Nall. Acad. Sci. USA, 79, 3011-3014.

5. Sneath, R.L., Wright, J.E., Soloway, A.H., O'Keefe, S.M., Dey, A.S. \& Smolnycki, W.D. (1976). J. Medicinal Chem. 19, 1290-1294.

6. Maurer, J.L., Berchier, F.B., Serino, A.J., Knobler, C.B. \& Hawthome, M.F. (1990). J. Org. Chem., 55, 838-842.

7. Kohler, G. \& Milstein, C. (1975). Nature, 256, 495-497.

8. Alam, F., Soloway, A.H., Barth, R.F., Mafune, N., Adams, D.M. \& Knoth, W.H. (1989). J. Medicinal Chem., 32, 2326-2330.

9. Barth, R.F., Soloway, A.H., Adams, D.M. and Alam, F. Delivery of boron-10 for neutron capture therapy by means of monoclonal antibody-starburst dendrimer immunoconjugates in: "Progress in Neutron Capture Therapy for Cancer", Allen, B.J., Moore, D.E. and Harrington, B.V. eds., Plenum Publishing Corp., New York (1992).

10. Soloway, A.H., Private communication.

11. Varadarjan, A., \& Hawthome, M.F. (1991). Bioconjugate Chemistry, 2, 242-247.

12. Hawthome, M.F. (1991). Pure Appl. Chem. 63(3), 327.

13. Paxton, R.J., Beatty, B.G., Hawthorne, M.F., Varadarajan, A., Williams, L.E., Curtis, F.L., Knobler, C.B., Beatty, J.D., \& Shively, J.E. (1991). Proc. Nall. Acad. Sci. USA, 88, 3387-3391.

14. Mendelsohn, J. (1990). Seminars in Cancer Biology, 1, 339-344.

15. Malmquish, J. \& Sjöberg, S. (1992). Inorg. Chem., 31, 2534-2537.

16. Andersson, A., Capola, J., Carlsson, J. (1992). Neuro-Oncology, in press.

17. Laster, B.H., Kahl, S.B., Popenoe, E.A., Pate, D.A., \& Fairchild, R.G. (1991). Cancer Res. 51, 4588-4593.

18. Shelly, K., Feakes, D.A., Hawthome, M.F., Schmidt, P.G., Krisch, T.A., \& Bauer, W.F. (1992). Proc. Natl. Acad. Sci. USA, 89, 9030-9043. 


\section{Liposomal Delivery of Boron to Murine Tumors for Boron Neutron Capture Therapy}

D. Feakes, K. Shelly, M. F. Hawthorne, P. Schmidt, C. Elstad, G. Meadows, and W. Bauer

This article is a reprint from Advances in Neutron Capture Therapy, pp. 395-398, (1993). 
, 


\title{
LIPOSOMAL DELIVERY OF BORON TO MURINE TUMORS FOR BORON NEUTRON CAPTURE THERAPY
}

\author{
D.A. Feakes, ${ }^{1}$ K.J. Shelly, ${ }^{1}$ M.F. Hawthorne, ${ }^{1}$ P.G. Schmidt, ${ }^{2}$ C.A. \\ Elstad, ${ }^{2}$ G.G. Meadows ${ }^{3}$ and W.F. Bauer ${ }^{4}$ \\ ${ }^{1}$ University of California at Los Angeles, Los Angeles, CA \\ 2 Vestar, Inc., San Dimas, CA \\ ${ }^{3}$ Washington State University, Pullman, WA \\ ${ }^{4}$ Idaho National Engineering Laboratory, Idaho Falls, ID
}

Liposomes, of a specific size and composition, have demonstrated unique ability to selectively deliver significant quantities of boron to murine tumors. Aqueous solutions of water soluble compounds with a high boron content have been encapsulated into the liposomes and studied in vivo. Although the liposomes deliver their contents to the interior of the tumor cells, the characteristics of the boron compound determine whether boron will be retained by the tumor cells or simply cleared from the system. Investigation of a series of compounds which vary in characteristics such as size, charge, and reactivity have allowed us to develop a working hypothesis which predicts a priori, with some degree of success, the ability of these boron compounds to be retained by tumor cells.

\section{MATERIALS AND METHODS}

The polyhedral borane anion derivatives used were prepared by published methods or slight modifications thereof. ${ }^{1-5}$ Distearoyl phosphatidylcholine was obtained from Avanti Polar Lipids, and cholesterol was supplied by Calbiochem or Sigma.

Liposome emulsions were prepared by probe sonication of a dried film composed of equimolar amounts of the phospholipid and cholesterol with the hydrating solution (typically $5 \mathrm{~mL}, 250-300 \mathrm{mM}$ in the boron-containing salt) at $65^{\circ} \mathrm{C}$ for $15-30 \mathrm{~min}$. The vesicles were separated from the remaining free borane salt by eluting through a column of Sephadex G-25 (medium) with isotonic phosphate-buffered saline or lactose. Liposomal preparations were diluted with the appropriate buffer to a lipid concentration of $23-24 \mathrm{mg} / \mathrm{mL}$ and sterilized by filtration through a $0.22 \mu \mathrm{m}$ Millipore membrane. The integrity of the encapsulated borane salt was confirmed by ${ }^{11} \mathrm{~B}$ NMR at $162 \mathrm{MHz}$.

All murine biodistribution studies utilized female Balb/c mice (16-20 g), with EMT6 tumors implanted in the right flank 7-10 days prior to the experiment. Tumor mass at the time of sacrifice was $125-350 \mathrm{mg}$. Injections of liposome emulsions $(200 \mu \mathrm{L})$ were made in the tail vein. Boron analyses were perfomed by inductively coupled plasma atomic emission spectroscopy (ICP-AES) ${ }^{6}$ at the Idaho National Engineering Laboratory. Each data point represents the average of five mice. For clarity, error bars are not shown in the graphical data; standard deviations were typically $5-15 \%$ of the average values. 


\section{BIODISTRIBUTION STUDIES IN MICE}

Murine biodistribution results for liposomes containing $\mathrm{Na}_{2} \mathrm{~B}_{10} \mathrm{H}_{10}$ and $\mathrm{Na}_{2} \mathrm{~B}_{12} \mathrm{H}_{11} \mathrm{SH}$ have been reported. ${ }^{7}$ They are characterized by the delivery of the boron species to the tumor (approximately $10 \mu \mathrm{g}$ boron/g tissue at 6 hours) followed by rapid clearance. Although neither species provides therapeutically useful boron accumulation in tumor, these initial experiments verified that liposomes could be used to deliver their contents to tumor cells although the compounds themselves were not suitable for in vivo retention of the boron and BNCT.

The murine biodistributions of the isomers, $\mathrm{Na}_{2}\left(n-\mathrm{B}_{20} \mathrm{H}_{18}\right)$ and $\mathrm{Na}_{2}\left(i-\mathrm{B}_{20} \mathrm{H}_{18}\right)$, are quite similar to each other. ${ }^{7}$ Six hour tumor boron values are approximately $20 \mu \mathrm{g}$ boron/g tissue or twice that of the previously studied ten-boron species. The boron tumor concentration is essentially maintained over the 48 hour time period, especially in the case of the $\mathrm{Na}_{2}\left(i-\mathrm{B}_{20} \mathrm{H}_{18}\right)$ species.

$\mathrm{Na}_{2}\left(i-\mathrm{B}_{20} \mathrm{H}_{18}\right)$ in buffer solution was injected in concentrations equivalent to the encapsulated species (Table 1). Although the unencapsulated species had some affinity for liver, accumulation was not as high as that observed with the encapsulated species. The remaining tissues examined contained only trace amounts of boron 6 hours post-injection.

The murine biodistribution of $\mathrm{K}_{4} \mathrm{~B}_{20} \mathrm{H}_{17} \mathrm{OH}$ is shown in Figure 1A. The blood loses $80 \%$ of its boron content between 6 and 30 hours, resulting in a 48 hour tumor to blood ratio of 3.4. Although the liver and spleen rapidly clear, the tumor loses boron as well. There is a $57 \%$ decrease in the tumor boron concentration over the 48 hour time period resulting in a final boron concentration of $7.3 \mu \mathrm{g}$ boron $/ \mathrm{g}$ tissue.

The double injection experiment (Figure $1 \mathrm{~B}$ ) with $\mathrm{Na}_{2}\left(i-\mathrm{B}_{20} \mathrm{H}_{18}\right)$ was performed by injecting liposomes at 0 and 24 hours $(280 \mu \mathrm{g}$ boron each injection, mice receiving both injections received $560 \mu \mathrm{g}$ boron). The first 24 hour time period is analogous to the single injection experiment. ${ }^{7}$ At 24 hours, the second injection was administered, increasing the boron concentration in all of the tissues. Boron cleared from the various tissues at the usual rates, resulting in a 48 hour tumor boron concentration of $26.6 \mu \mathrm{g}$ boron/g tissue and a tumor to blood ratio of 2.6 .

Liposomes loaded with $\mathrm{Na}_{2} \mathrm{~B}_{10} \mathrm{H}_{9} \mathrm{NCO}$ displayed the biodistribution shown in Figure 1C. The 6 hour tumor boron concentration $(19.2 \mu \mathrm{g}$ boron/g tissue) only slowly decreases with time (20\% loss between 6 and 30 hours) as compared to the rapid clearance of $\mathrm{Na}_{2} \mathrm{~B}_{10} \mathrm{H}_{10}$ (46\% loss between 6 and 24 hours). Blood boron levels drop rapidly, $79 \%$ between 6 and 30 hours, resulting in a 48 hour tumor to blood ratio of 5.0.

The murine biodistribution of $\mathrm{Na}_{3} \mathrm{~B}_{20} \mathrm{H}_{17} \mathrm{NH}_{3}$ is shown in Figure 1D. The tumor boron concentration increases over a period of approximately 30 hours and then slowly decreases, resulting in a final tumor boron concentration which is still $94 \%$ of the initial tumor boron concentration. All other tissues clear steadily over the 48 hour time period. The low blood boron concentration at 48 hours yields a tumor to blood boron ratio of 5.3.

Table 1. Murine tissue boron concentrations after injection of free $\mathrm{Na}_{2}\left(i-\mathrm{B}_{20} \mathrm{H}_{18}\right)(200 \mu \mathrm{g}$ of boron, $\approx 11 \mathrm{mg} / \mathrm{kg}$ of body weight) in buffered solution.

Boron concentration, $\mu \mathrm{g} / \mathrm{g}$ of tissue $\pm \mathrm{SD}$

\begin{tabular}{lrr} 
& \multicolumn{1}{c}{$6 \mathrm{~h}$} & $24 \mathrm{~h}$ \\
\hline Bissue & & \\
\hline Blood & $0.9 \pm 0.1$ & $1.9 \pm 0.7$ \\
Spleen & $18.1 \pm 0.8$ & $8.9 \pm 1.5$ \\
Tumor & $0.9 \pm 0.1$ & $1.5 \pm 0.5$ \\
& $1.2 \pm 0.3$ & $1.9 \pm 0.6$
\end{tabular}



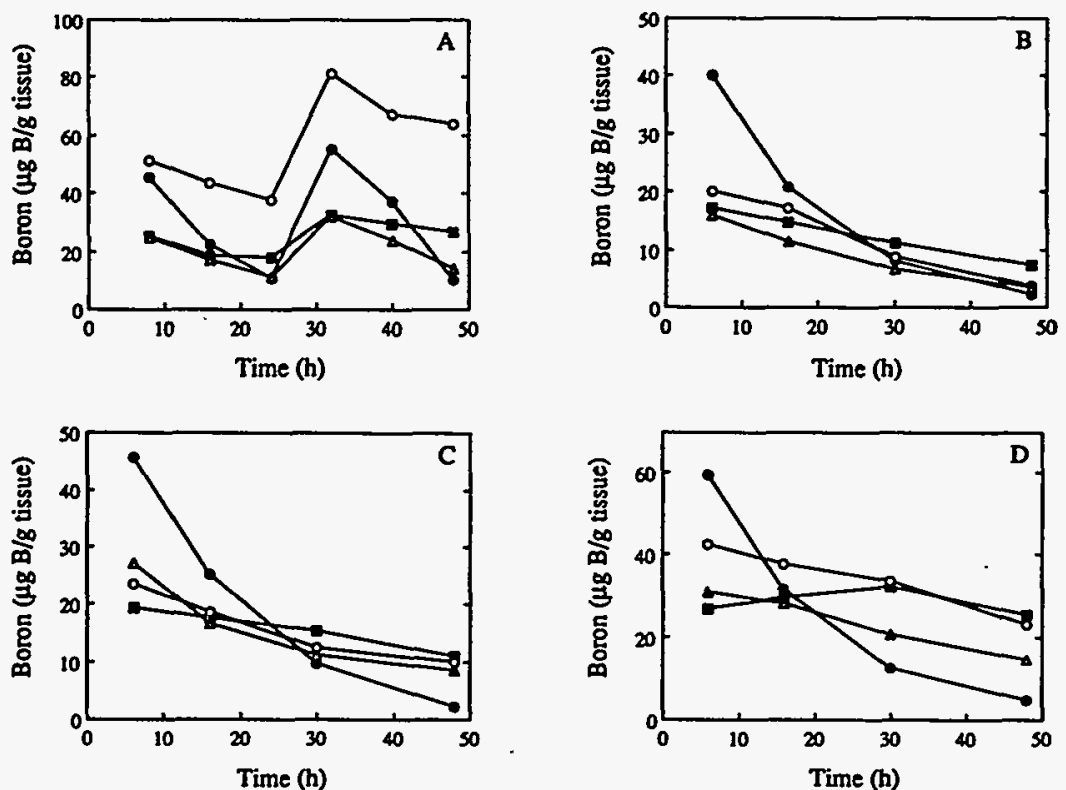

Figure 1. Murine tissue boron concentrations from delivery of borane salts by liposomes. $\bullet, B l o o d ; a$, tumor; O, liver; $\Delta$, spleen; Borane salts, with the injected doses were $\mathrm{Na}_{2}\left(i-\mathrm{B}_{20} \mathrm{H}_{18}\right), 280 \mu \mathrm{g}$ of boron each injection ( $\approx 15 \mathrm{mg} / \mathrm{kg}$ of body weight) $(A), \mathrm{K}_{4} \mathrm{~B}_{20} \mathrm{H}_{17} \mathrm{OH}, 2 \Gamma, \mu \mathrm{g}$ of boron ( $\approx 11 \mathrm{mg} / \mathrm{kg}$ of body weight) $(B)$, $\mathrm{Na}_{2} \mathrm{~B}_{10} \mathrm{H} \mathrm{NCO}, 166 \mu \mathrm{g}$ boron $\left(\approx 9 \mathrm{mg} / \mathrm{kg}\right.$ of body weight) $(C), \mathrm{Na}_{3} \mathrm{~B}_{20} \mathrm{H}_{17} \mathrm{NH}_{3}, 198 \mu \mathrm{g}$ of boron $\Leftrightarrow 11$ $\mathrm{mg} / \mathrm{kg}$ of body weight) (D).

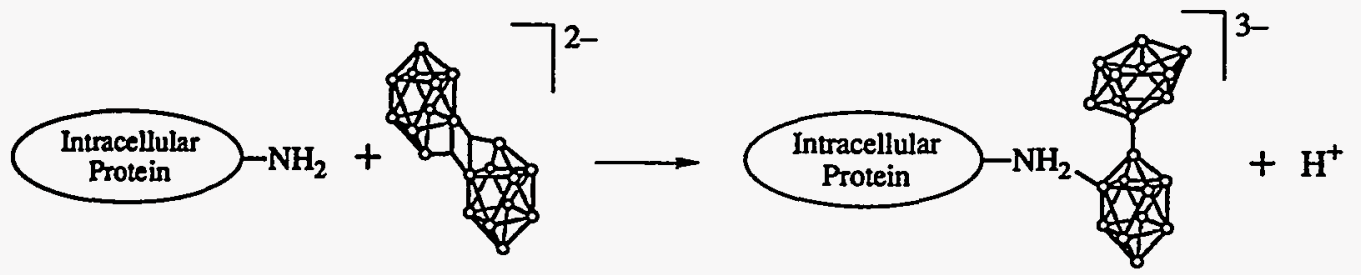

Figure 2. Proposed reaction of $\mathrm{Na}_{2}\left(n-\mathrm{B}_{20} \mathrm{H}_{18}\right)$ with the amino group of an intracellular protein moiety.

\section{DISCUSSION}

Initial investigations of the liposomal delivery of boron to murine tumors utilized $\mathrm{Na}_{2} \mathrm{~B}_{10} \mathrm{H}_{10}$ and $\mathrm{Na}_{2} \mathrm{~B}_{12} \mathrm{H}_{11} \mathrm{SH}$ because of their high boron content, ease of synthesis, water solubility, hydrolytic stability, and lack of toxicity. These compounds were successfully delivered to the tumor, but quickly cleared due to their inability to be retained by the tumor cells. ${ }^{7}$ Emphasis changed to the product resulting from the oxidative coupling of $\mathrm{Na}_{2} \mathrm{~B}_{10} \mathrm{H}_{10}, \mathrm{Na}_{2}\left(n-\mathrm{B}_{20} \mathrm{H}_{18}\right)$, and its photoisomer, $\mathrm{Na} 2\left(i-\mathrm{B}_{20} \mathrm{H}_{18}\right)$, since these compounds are known to undergo nucleophilic attack. 3,4 Additionally, the osmotic stress upon the liposomes encapsulating the $\mathrm{Na}_{2} \mathrm{~B}_{20} \mathrm{H}_{18}$ species is equivalent to those encapsulating $\mathrm{Na}_{2} \mathrm{~B}_{10} \mathrm{H}_{10}$ even though twice the concentration of boron is delivered. These compounds possess the potential to react with intracellular protein moieties as illustrated in Figure 2.

Biodistribution studies of both the normal and the photoisomer demonstrated retention of the delivered boron by the tumor cells over the 48 hour period, suggesting an intracellular reactivity suitable for tumor retention. ${ }^{7}$ Further investigations, including the determination of the biodistribution of unencapsulated $\mathrm{Na}_{2}\left(i-\mathrm{B}_{20} \mathrm{H}_{18}\right)$ and its encapsulated hydrolysis product, $\mathrm{Na}_{4} \mathrm{~B}_{20} \mathrm{H}_{17} \mathrm{OH}$, have established that liposomal $\mathrm{Na}_{2}\left(i-\mathrm{B}_{20} \mathrm{H}_{18}\right)$ is the active species in vivo. Neither the unencapsulated $\mathrm{Na}_{2}\left(i-\mathrm{B}_{20} \mathrm{H}_{18}\right)$ nor encapsulated $\mathrm{Na}_{4} \mathrm{~B}_{20} \mathrm{H}_{17} \mathrm{OH}$ are retained by the tumor. 
Double injection experiments are of interest because they enable a second dose of the boron compound to be administered once the initial blood boron has cleared. In the case of $\mathrm{Na}_{2}\left(i-\mathrm{B}_{20} \mathrm{H}_{18}\right)$, the second dose was administered at 24 hours. All normal tissues then cleared at their usual rates. However, because the tumor retains boron, the tumor boron concentration is further increased by the second dose, resulting in a final tumor boron concentration of $26.6 \mu \mathrm{g}$ boron $/ \mathrm{g}$ tissue.

The biodistribution of two other compounds believed to possess the potential for intracellular protein reaction, $\mathrm{Na}_{2} \mathrm{~B}_{10} \mathrm{H}_{9} \mathrm{NCO}$ and $\mathrm{Na}_{3} \mathrm{~B}_{20} \mathrm{H}_{17} \mathrm{NH}_{3}$, were examined. Compared to its unsubstituted precursor, $\mathrm{Na}_{2} \mathrm{~B}_{10} \mathrm{H}_{10}, \mathrm{Na}_{2} \mathrm{~B}_{10} \mathrm{H}_{9} \mathrm{NCO}$ retained the boron delivered to the tumor ( $80 \%$ versus $54 \%$ over the first 24 hours). Since the only difference between the two compounds is the amine reactive isocyanate moiety, the proposed intracellular protein reaction appears to be substantiated.

The reduced amine derivative of $\mathrm{Na}_{2}\left(n-\mathrm{B}_{20} \mathrm{H}_{18}\right), \mathrm{Na}_{3} \mathrm{~B}_{20} \mathrm{H}_{17} \mathrm{NH}_{3}$, is the only compound studied to date which acccumulates in tumor over approximately 30 hours. This easily oxidized compound probably reverts to the more reactive $\mathrm{B}_{20} \mathrm{H}_{17} \mathrm{NH}_{3}{ }^{1-}$ species in vivo. The tumor boron concentration after 48 hours is essentially equal to the boron concentration after 6 hours.

Both the normal isomer, $\mathrm{Na}_{2}\left(n-\mathrm{B}_{20} \mathrm{H}_{18}\right)$, and the reduced amine, $\mathrm{Na}_{3} \mathrm{~B}_{20} \mathrm{H}_{17} \mathrm{NH}_{3}$, have recently been investigated in Balb/c mice bearing P1798 murine lymphosarcoma. The biodistributions are similar to the EMT6 results.

\section{CONCLUSION}

Based on the data accumulated to date, a working hypothesis has been proposed which allows the prediction, a priori and with some degree of success, of which boron compounds will be retained by the tumor when delivered by liposomes. This hypothesis is that compounds possessing the potential to react with intracellular protein moieties will be retained within the tumor cells. This hypothesis is substantiated by the biodistributions of $\mathrm{Na}_{2}\left(n-\mathrm{B}_{20} \mathrm{H}_{18}\right), \mathrm{Na}_{2}\left(i-\mathrm{B}_{20} \mathrm{H}_{18}\right), \mathrm{Na}_{2} \mathrm{~B}_{10} \mathrm{H}_{9} \mathrm{NCO}$, and $\mathrm{Na}_{3} \mathrm{~B}_{20} \mathrm{H}_{17} \mathrm{NH}_{3}$. The retention in tumor of the reduced amine species, $\mathrm{Na}_{3} \mathrm{~B}_{20} \mathrm{H}_{17} \mathrm{NH}_{3}$, is attributed to its ability to be oxidized to a more reactive $\mathrm{Na}_{3} \mathrm{~B}_{20} \mathrm{H}_{17} \mathrm{NH}_{3}$ species. Compounds which do not possess either a reactive functional group or the ability to be oxidized intracellularly to a more reactive species, such as $\mathrm{K}_{4} \mathrm{~B}_{20} \mathrm{H}_{17} \mathrm{OH}$, are not retained by the tumor. Double injection experiments, such as the one reported for $\mathrm{Na}_{2}\left(i-\mathrm{B}_{20} \mathrm{H}_{18}\right)$, can be used to further increase the boron concentration retained by the tumor once the initial boron has cleared from normal tissues. The diversity and utility of liposomal delivery of boron compounds to tumors is one of the most promising systems known to date.

\section{ACKNOWLEDGEMENTS}

This research was conducted as part of the BNCT program of the Idaho National Engineering Laboratory and was performed under the auspices of the U.S. Department of Energy (DOE), Office of Energy Research, under DOE Field Office, Idaho, Contract DE-AC07-76ID01570.

\section{REFERENCES}

1. Hawthome, M.F. \& Pilling, R.L. (1967) in Inorganic Syntheses Vol. IX, ed. Tyree, Jr., S.Y. (McGraw-Hill Publishing Co. Inc., New York), pp. 16-19.

2. Hawthorne, M.F. \& Pilling, R.L. (1966) J. Am. Chem. Soc. 88, 3873.

3. Hawthome, M.F., Pilling,R.L. \& Garrett, P.M. (1965) J. Am. Chem. Soc. 87, 4740-4746.

4. Hawthorne, M.F., Pilling, R.L., Stokely, P.F. \& Garrett, P.M. (1963) J. Am. Chem. Soc. 85, 3704.

5. Shelly, K., Knobler, C.B. \& Hawthome, M.F. (1992) Inorg. Chem. 31, 2889-2892.

6. Tama, S.R., Moore, D.E. \& Allen, BJ. (1987) Anal. Chem. 59, 2161-2164.

7. Shelly, K., Hawthome, M.F. \& Schmidt, P.G. (1992) in Progress in Neutron Capture Therapy for Cancer, ed. Allen, B.J., Moore, D.E. and Harrington, B.V. (Plenum Publishing Corp., New York), pp. 259-263. 


\section{Low Density Lipoprotein Reconstitutions with Alkyl and Aryl}

Carboranes

S. Kahl, D. Pate, and L. Wainschel

This article is a reprint from Advances in Neutron Capture Therapy, pp. 399-402, (1993). 

LOW DENSITY LIPOPROTEIN RECONSTITUTIONS WITH ALKYL AND ARYL CARBORANES

\author{
Stephen B. Kahl, David W. Pate, and Larry A. Wainschel \\ Department of Pharmaceutical Chemistry \\ University of California, San Francisco \\ San Francisco, CA 941 .3-0446
}

\title{
INTRODUCTION
}

Low density lipoproteins (LDL's) are among the most attractive of possible boron delivery methodologies by virtue of their high carrying capacity for hydrophobic boron compounds and their cellular internalization through receptor-mediated processes. Previous work in our laboratory has demonstrated that long chain unsaturated fatty alcohol esters of carborane carboxylic acid can be very efficiently exchanged for the cholesterol ester core of human LDL. In collaboration with coworkers at Brookhaven National Laboratory using in vitro techniques, we have found that: (a) boron concentrations exceeding 10 times that needed for therapy were easily obtained; (b) boron remained firmly bound despite repeated washings and suspension in boron-free medium; (c) boron distribution was intracellular, with a biological efficacy indicative of a cytoplasmic location; (d) in vitro uptake and efficacy studies were consistent with a receptor-mediated binding mechanism; (e) boron uptake, retention, and efficacy were better by far than those obtained with any other boron compound previously studied in our in vitro screening system. (1) The present study was undertaken to determine whether simple alkyl and aryl carboranes could be similarly exchanged for LDL cholesterol and, if so, whether any identifiable structure activity relationships among the compounds could be identified. We also wished to determine whether reconstituted LDL's suffered serious aggregation and to demonstrate that ICP-AES and prompt gamma spectrometric boron assay procedures produced coincident numbers.

\section{MATERIALS AND METHODS}

Allyl, methyl, phenyl and butenyl orthocarborane and metacarborane were purchased from Dexsil Chemical Corp. and required further purification (either vacuum distillation or recrystallization) before use. Pure hexyl carborane was a generous gift from Callery Chemical Co. Elaidyl carborane carboxylate (ECC) was synthesized by the method Kahl(2) from elaidyl alcohol (Nu-Chek Prep) and carborane carboxylic acid chloride. Low density lipoprotein was isolated from an overweight but otherwise healthy volunteer by density gradient ultracentrifugation and reconstituted with boron compounds by the method of Kahl and Callaway. (3) Protein content of reconstituted solutions was determined by the micro-Lowry method. Boron analyses were carried out at Brookhaven National Laboratory (prompt gamma) and at Idaho National Engineering Laboratory (ICP-AES). 


\section{RESULTS}

Table 1 shows the results of one LDL reconstitution. Each entry represents the average \pm standard deviation for three reconstitutions of each compound. The boron analyses were carried out at BNL using the prompt gamma technique. All samples were made using unenriched material. The boron content is reported here as natural boron so the errors associated with the phenyl, methyl and meta carborane points are substantial. Table 2 displays the boron/protein ratios obtained for a series of samples either singly or doubly filtered through a 0.45 micron filter. Each number represents the average of four boron/protein ratios with each of these ratios in turn obtained using prompt gamma boron values.

Table 3 presents a comparison of boron analytical data obtained from prompt gamma and ICP-AES analyses of identical reconstituted LDL samples. The ICP-AES data were obtained using a longer and more forceful bomb digestion technique than is normally used. Prompt gamma data are again based on ${ }^{10} \mathrm{~B}$ determinations of natural abundance samples.

\section{DISCUSSION}

Low density lipoproteins are attractive targets as tumor-selective boron carriers for a number of reasons. Their oily core provides a favorable domain for lipophilic drugs and prodrugs, and it has been demonstrated that the natural core components can be replaced, in part or in toto, with lipophilic drugs. $(1,4)$ They are constituted of natural components able to survive in tissue and plasma for significant time periods. Their small particle size $(17-25 \mathrm{~nm})$ allows them to diffuse from vascular to extravascular compartments. Interaction of the surface protein, Apo B-100, with receptors on the target surface results in endocytosis of the LDL particle and delivery of its core contents to the

Table 1. LDL reconstitutions with alkyl and aryl carboranes

\begin{tabular}{lccc}
\hline Compound & Ave.ug B/gm & Ave. Hg protein/gm & Ave. Ug B/ug protein \\
\hline 1-hexyl carborane & $472 \pm 8$ & $660 \pm 15$ & $0.71 \pm .05$ \\
1-allyl carborane & $379 \pm 125$ & $673 \pm 100$ & $0.55 \pm .11$ \\
1-butenyl carborane & $67 \pm 20$ & $508 \pm 100$ & $0.13 \pm .04$ \\
1-phenyl carborane & $59 \pm 35$ & $492 \pm 60$ & $0.11 \pm .06$ \\
1-methyl carborane & $39 \pm 10$ & $224 \pm 13$ & $0.11 \pm .06$ \\
meta carborane & $22 \pm 5$ & $511 \pm 60$ & $0.04 \pm .02$ \\
\hline
\end{tabular}

Table 2. Boron/protein ratios for singly vs. doubly filtered samples

\begin{tabular}{lcc}
\hline & \multicolumn{2}{c}{$\mu \mathrm{g} / \mathrm{B} / \mathrm{g}$ Prolein } \\
Compound & single filtered & double filtered \\
1-hexyl carborane & $0.71 \pm 0.05$ & $0.68 \pm 0.04$ \\
1-allyl carborane & $0.34 \pm 0.10$ & $0.30 \pm 0.04$ \\
ECC & $0.25 \pm 0.02$ & $0.22 \pm 0.02$ \\
1-methyl carborane & $0.21 \pm 0.05$ & $0.39 \pm 0.15$ \\
1-phenyl carborane & $0.15 \pm 0.05$ & $0.14 \pm 0.04$ \\
1-butenyl carborane & $0.06 \pm 0.02$ & $0.09 \pm 0.03$ \\
\hline
\end{tabular}


Table 3. Comparison of prompt gamma and ICP-AES measurements of boron content in identical samples.

\begin{tabular}{ccr} 
sample\# & prompt gamma & ICP \\
1 & & \\
2 & 512.5 & 590.0 \\
3 & 484.5 & 526.4 \\
4 & 418.5 & 480.6 \\
5 & 30.5 & 39.2 \\
6 & 49.0 & 47.3 \\
7 & 38.0 & 42.1 \\
8 & 68.0 & 92.7 \\
9 & 82.0 & 125.1 \\
10 & 49.5 & 46.9 \\
11 & 113.0 & 68.3 \\
12 & 30.0 & 39.6 \\
13 & 34.0 & 40.0 \\
14 & 22.5 & 33.7 \\
15 & 27.0 & 32.4 \\
16 & 17.0 & 43.8 \\
17 & 520.5 & 547.6 \\
18 & 370.5 & 400.1 \\
\hline
\end{tabular}

peri-nuclear region of the cell. This receptor-mediated process is significantly enhanced in certain pathophysiologic states including many cancers and the atherosclerotic plaques characteristic of arteriosclerosis. We have previously demonstrated that hydrophobic boron compounds, particularly ortho-carborane derivatives of unsaturated long chain fatty acids, can be efficiently introduced into the LDL core and that the resultant boronated LDL particles behave in cell culture in a manner consistent with receptor-mediated uptake.(1) In fact, the very high degree of core substitution obtained with these derivatives appears to negate the normal negative feedback regulation by cholesterol and results in a non-saturable uptake process.

In a preliminary report several years ago, we noted that several alkyl and aryl carboranes could also serve as reconstitution agents. (3) The present study was undertaken to confirm these findings and to seek evidence of any relationship between the nature of the side chain substitutent and the reconstituting ability of the compound. Table 1 presents results from a representative set of these reconstitutions. These quantitative results support our previous findings that $n$-hexyl and allyl carborane reconstitute into LDL far more efficiently than do other substituted and unsubstituted carboranes. With the exception of meta carborane, the trend in measured protein values is mimicked by overall reconstitution efficiency as expressed in $\mu \mathrm{g}$ boron/ $\mu \mathrm{g}$ protein, i.e., high measured protein in a reconstituted sample generally reflects high boron content. Moreover, the variance of both boron and protein values within a given set of reconstitutions as expressed as a percentage of the mean value is invariably low for "good" reconstituters and high for "poor" ones. These data suggest that "poor" reconstituters disrupt the Apo B-100 protein which makes up $40 \%$ of the spherical particulate surface. The molecular causes of this disruption are certainly not clear, but may be related to the physicochemical nature of the carboranes. Those carboranes which are crystalline at room temperature (ortho, meta, methyl, butenyl and phenyl) do not reconstitute well, whereas those which are oils (hexyl and allyl) do. All of the carborane esters of fatty alcohols which were previously found to be "good" reconstituters were oils. Whatever the mechanism; it is clear that only hexyl and allyl carborane are viable candidates among this series for LDL-mediated tumor boron delivery.

It was also of interest to determine whether the single filtration following reconstitution to remove the starch support was sufficient to remove LDL aggregates. There had been some suggestion in the literature that reconstituted LDL aggregated and 
that these aggregates were rapidly removed by the reticuloendothelial system when injected into animals. Table 2 presents the results of our study of this phenomenon. The singly filtered samples all have boron/protein ratios within experimental error of similar determinations from Table 1 with the exception of allyl carborane which usually is associated with a large standard deviation. The elaidyl carborane carboxylate has been our standard for all previous in vitro work, and the ratio reported here is consistent with values for a number of previous samples. It is clear that the second filtration step has a relatively minor effect on the over-all boron/protein ratio of the boronated LDL. Indeed, in each of the pairs of ratios both values are within experimental error of each other. However, the raw data (not shown) indicate an interesting effect. For the better compounds, i.e. hexyl, allyl and ECC, both the absolute boron content and the absolute protein content are reduced by approximately $10 \%$ during the second filtration. In contrast, in the poorer compounds the drop in boron content varies widely while the protein content falls predictably by $10-15 \%$. This is further confirmation that such compounds are unsuitable for LDL incorporation and disrupt the LDL in an as yet unknown fashion.

The third goal of this study was to assess the comparability of the two most commonly used boron assays, prompt gamma and ICP-AES spectrometry. Since the tissue boron content of a series of planned animal biodistribution experiments using boronated LDL is to rely on the ICP-AES method, it is important to demonstrate that both methods give comparable numbers. An early attempt at this comparison gave wildly different values for the same sample with significant carry over from one analysis to the next. This was attributed to the very "greasy" nature of the LDL matrix and high thermal stability of the carborane cage. The results presented in Table 3 were generated after increasing the severity and length of the sample bomb digestion procedure. For samples containing up to $\sim 600 \mathrm{ppm}$ boron, the ICP technique gives boron concentration values that are generally higher than with prompt gamma analysis. Interestingly, the degree of agreement is greatest at the highest values, e.g. samples 1-3 and 16-18. Perhaps not coincidentally, these samples contained hexyl and allyl carborane, respectively. The samples with greatest divergence, i.e. samples 13-15, contained meta carborane. This may indicate that the new sample digestion procedure is still not adequate to overcome the greater chemical stability of meta carborane compared to ortho carborane.

Although the present study does suggest that n-hexyl or allyl carboranes could be used for small animal studies of boronated LDL, we believe it more prudent to continue using ECC. Animal distribution studies with this compound encapsulated in LDL have begun and will be reported upon at a later date.

\section{ACKNOWLEDGMENTS}

The authors wish to thank Dr. Brenda H. Laster of BNL and Dr. William F. Bauer of INEL for carrying out the boron analyses. The submitted paper has been authorized by a contractor of the US government under DOE Contract No. DE-ACO7-76IDO1570. Accordingly, the US government retains a non-exclusive, royalty-free license to publish or reproduce the published form of this contribution, or allow others to do so, for U.S. government purposes.

\section{REFERENCES}

1. Laster, B.H., Kahl, S.B., Popenoe, E.A., Pate, D.W., and Fairchild, R.G., Cancer Res. 51:4588-4593 (1991).

2. Kahl, S.B., Tetrahedron Lett. 31:1517-1520 (1990).

3. Kahl, S.B. and Callaway, J.C., Strahlenther. Onkol. 165:137-139 (1989).

4. Firestone, R.A., Pisano, J.M., Falek, J.R., McPhaul, M.M. and Krieger, M., J. Med. Chem. 27:1037-1043 (1984). 


\section{A Rapid Method for the Direct Analysis of Boron in Whole Blood by Atomic Emission Spectroscopy}

W. Bauer, P. Micca, and B. White

This article is a reprint from Advances in Neutron Capture Therapy, pp. 403-407, (1993). 
, 


\title{
A RAPID METHOD FOR THE DIRECT ANALYSIS OF BORON IN WHOLE BLOOD BY ATOMIC EMISSION SPECTROSCOPY
}

\author{
W.F. Bauer ${ }^{1 *}$, P.L. Micca ${ }^{2}$ and B.M White ${ }^{1}$ \\ ${ }^{1}$ Idaho National Engineering Laboratory, EG\&G Idaho, Inc. \\ Idaho Falls, ID 83402 \\ ${ }^{2}$ Medical Department, Brookhaven National Laboratory \\ Upton, NY 11973
}

\section{INTRODUCTION}

The INEL BNCT program has been involved in normal tissue dose tolerance studies using dogs as the large animal model ${ }^{1}$. In order to accurately predict when to begin an irradiation and then determine the length of the radiation required to achieve the desired radiological dose at a specified mean boron concentration, a rapid technique for the determination of boron is required. It has previously been shown that after administration and distribution of $\mathrm{Na}_{2} \mathrm{~B}_{12} \mathrm{H}_{11} \mathrm{SH}(\mathrm{BSH})$, the elimination phase has $\mathrm{aT}_{1 / 2}$ of $\sim 300$ minutes ${ }^{2}$ and one should be able to roughly predict blood $\mathrm{B}$ concentrations at specified times during the elimination. However, the physiology can be expected to vary somewhat from animal to animal and the blood B concentrations are expected to vary as well. These variations mean that target B concentrations can easily be missed.

There are numerous methods available for B analysis in biological samples, however, most require some form of time consuming digestion procedure (20 minutes minimum) to eliminate the matrix and, in many cases, to convert the $B$ to a single form. Rapid analysis of animal tissues by atomic emission spectroscopy has been performed by suspending homogenized tissue in a deionized water slurry ${ }^{3}$. This analysis procedure, including tissue homogenization, could be performed in $\sim 10$ minutes. Since a heparinized blood sample would not require homogenization, it should readily be suspendable in solution.

This paper describes the analysis of heparinized whole blood using slurry sample introduction into both an inductively-coupled plasma and a direct current plasma atomic emission spectrometer (ICP-AES and DCP-AES, respectively). A non-ionic surfactant (Triton $\mathrm{X}-100^{\mathrm{m}}$ ) is used to form a stable slurry of suspended blood cells. The non-ionic surfactant was selected to minimize cell destruction and ICP or DCP problems caused by excess alkali metal content. 
Table 1. Results of an initial study comparing slurry nebulization and acid digestion of whole blood spiked with either BSH or tetraborate. Analysis was by ICP-AES.

\begin{tabular}{lcccc}
\hline \hline & $\begin{array}{c}\text { Slurry } \\
\mu \mathrm{g} \mathrm{B} / \mathrm{g}\end{array}$ & $\begin{array}{c}\text { Digestion } \\
\mu \mathrm{g} \mathrm{B} / \mathrm{g}\end{array}$ & Difference $^{*}$ & RPD $^{\mathrm{b}}$ \\
\hline Blood & $<0.42$ & $<0.40$ & & \\
$15 \mu \mathrm{L} \mathrm{BSH}$ & 9.50 & 9.57 & -0.08 & -0.8 \\
$30 \mu \mathrm{L} \mathrm{BSH}$ & 18.43 & 17.96 & 0.47 & 2.6 \\
$45 \mu \mathrm{L} \mathrm{BSH}$ & 30.16 & 29.76 & 0.40 & 1.3 \\
$60 \mu \mathrm{L} \mathrm{BSH}$ & 39.16 & 38.02 & 1.14 & 2.9 \\
$75 \mu \mathrm{L} \mathrm{BSH}$ & 49.20 & 48.38 & 0.82 & 1.7 \\
$60 \mu \mathrm{L} \mathrm{B} \mathrm{O}_{7}^{2}$ & 53.02 & 50.68 & 2.34 & 4.5 \\
$40 \mu \mathrm{L} \mathrm{B} \mathrm{O}_{7}^{2}$ & 36.27 & 34.24 & 2.02 & 5.7 \\
$30 \mu \mathrm{L} \mathrm{B} \mathrm{O}_{7}^{2}$ & 27.70 & 26.31 & 1.40 & 5.2 \\
$20 \mu \mathrm{L} \mathrm{B} \mathrm{O}_{7}^{2}$ & 19.39 & 18.25 & 1.14 & 6.1 \\
$10 \mu \mathrm{L} \mathrm{B} \mathrm{O}_{7}^{2}$ & 9.69 & 9.60 & 0.09 & 1.0 \\
Mean & & & 0.97 & 3.0 \\
Standard Deviation & & 0.79 & 2.3 \\
\hline
\end{tabular}

- difference $=$ digestion - slurry

Relative $\%$ difference $=$ difference $\times 100 /$ mean

\section{EXPERIMENTAL}

Samples were prepared as slurries simply by weighing a $300 \mathrm{mg}$ aliquot into a $10 \mathrm{~mL}$ volumetric flask, adding $0.2 \mathrm{~mL}$ of a $2.5 \%$ solution of Triton X-100 ${ }^{\mathrm{mu}}$, and diluting to the mark with deionized, boron free water. Standards were prepared in a similar $0.05 \%$ solution of Triton X-100 NIST). Salts of BSH were from Callery Chemical Company and Centronics Limited and p-boronophenylalanine (BPA) was obtained from Callery Chemical Company. The $\mathrm{Na}_{4} \mathrm{~B}_{24} \mathrm{H}_{22} \mathrm{~S}_{2}$ (BSSB) was prepared from BSH by the method described in reference 4 . Standard solutions containing ${ }^{10} \mathrm{~B}$ were prepared from dried $95 \%{ }^{10} \mathrm{~B}$ enriched $\mathrm{B}(\mathrm{OH})_{3}$ obtained from NIST. Boron analysis was performed by ICP-AES at both the 208.959 and $249.678 \mathrm{~nm}$ emission lines while the DCP-AES analysis used the 208.959 and $249.778 \mathrm{~nm}$ emission lines of boron.

\section{RESULTS AND DISCUSSION}

Initial experimentation was performed using blood obtained from a healthy labrador retriever. Aliquots $(1 \mathrm{~mL})$ of the heparinized whole blood were spiked with differing quantities of a BSH stock solution $(691 \mu \mathrm{g} \mathrm{B} / \mathrm{mL})$ and a commercial standard containing $\mathrm{B}(1000 \mu \mathrm{g} / \mathrm{mL})$ as ammonium tetraborate. The resulting solutions were analyzed by standard ICP-AES procedures after digestion of $200 \mathrm{mg}$ with nitric acids. Slurries for ICPAES consisted of $200 \mathrm{mg}$ aliquots of the spiked blood that had been suspended in $0.05 \%$ Triton X-100 ${ }^{\mathrm{ra}}$. The results of this experiment are shown in Table 1. Plotting the ICPAES analysis results for slurry nebulization versus the standard digestion procedure results in the regression line $C_{\text {slurry }}=\left(1.03_{8} \pm 0.01_{4}\right) C_{\text {digest }}-(0.09 \pm 0.43)$. The results from the two sample preparation techniques agree very well, however, a slight $(-3 \%)$ positive bias may be present in the slurry results. This slight bias was not a great concern because it most likely was the result of having mismatched standard and sample matrices. This initial study 
Table 2. Effect of blood volume on the recovery of B by DCP-AES with slurry sample introduction.

\begin{tabular}{lcccccc}
\hline mg Blood & 0 & 109 & 219 & 300 & 400 & 500 \\
\% Recovery & 100 & 97 & 101 & 103 & 101 & 101 \\
\hline
\end{tabular}

Table 3. Comparison of typical results for B analysis by prompt $\gamma$, DCP-AES and ICP-AES.

\begin{tabular}{|c|c|c|c|c|}
\hline \multirow[b]{2}{*}{ Sample } & \multicolumn{4}{|c|}{ Concentrations in $\mu \mathrm{g}{ }^{10} \mathrm{~B} / \mathrm{g}$} \\
\hline & Prompt $\gamma^{2}$ & Prompt $\boldsymbol{\gamma}^{\mathbf{b}}$ & DCP-AES & ICP-AES ${ }^{e}$ \\
\hline BPA Solution & 26.9 & & 27.1 & \\
\hline $9.5 \mu \mathrm{g}{ }^{10} \mathrm{~B} / \mathrm{mL}$ Standard & 9.6 & 9.5 & 9.7 & \\
\hline Blank Blood Spike (Borate) & 12.5 & & 12.2 & \\
\hline BSSB Solution & 122 & 125 & 114 & \\
\hline A $300 \mathrm{~min}$ Blood & 48.5 & 47.3 & 43.2 & 50.5 \\
\hline A $360 \mathrm{~min}$ Blood & 43.6 & 41.8 & 37.7 & 42.2 \\
\hline A $420 \mathrm{~min}$ Blood & 37.5 & 38.9 & 33.3 & 38.0 \\
\hline A $489 \mathrm{~min}$ Blood & 32.3 & 32.2 & 28.8 & 32.5 \\
\hline A $558 \mathrm{~min}$ Blood & 29.0 & 29.5 & 26.3 & 26.6 \\
\hline A $627 \mathrm{~min}$ Blood & 24.3 & & 22.6 & 26.4 \\
\hline B $360 \mathrm{~min}$ Blood & 44.2 & & 40.0 & 45.7 \\
\hline B $420 \mathrm{~min}$ Blood & 40.3 & & 34.8 & 40.5 \\
\hline B $496 \mathrm{~min}$ Blood & 36.0 & & 31.7 & 37.1 \\
\hline B $562 \min$ Blood & 33.3 & & 29.1 & 33.6 \\
\hline B $622 \min$ Blood & 30.2 & & 26.5 & 30.8 \\
\hline C $240 \mathrm{~min}$ Blood & 60.7 & & 54.0 & 62.7 \\
\hline C $300 \min$ Blood & 54.1 & & 47.2 & 56.0 \\
\hline C $360 \mathrm{~min}$ Blood & 45.9 & & 42.0 & 48.4 \\
\hline C $420 \mathrm{~min}$ Blood & 39.3 & & 34.7 & 39.4 \\
\hline C $506 \mathrm{~min}$ Blood & 33.2 & & 28.1 & 32.3 \\
\hline C $571 \mathrm{~min}$ Blood & 29.9 & & 26.3 & 30.7 \\
\hline C $639 \mathrm{~min}$ Blood & 26.8 & & 24.4 & 27.6 \\
\hline
\end{tabular}

- Using multipoint calibration at a later date

- Using only a 2 point calibration on the day of the infusion

- Same slurries as for DCP but analysis was several days later

utilized calibration standards that were prepared in only a deionized water matrix. To avoid this potential problem, all subsequent standards were prepared in $0.05 \%$ Triton X$100^{\mathrm{m}}$.

Since the aspiration and nebulization of the blood slurries for boron analysis by ICPAES worked well for both BSH and a simple borate, the technique was taken to BNL to be used with the DCP-AES in direct support of the large animal irradiations. Because the detection limits for the $208.959 \mathrm{~nm}$ B emission on the DCP-AES were only $\sim 0.023 \mu \mathrm{g} / \mathrm{mL}$, it was decided to use $300 \mathrm{mg}$ of heparinized whole blood instead of the $200 \mathrm{mg}$ used in the ICP-AES experiments. To be sure that there were no significant physical or spectral interferences associated with this change, increasing quantities of B free whole dog blood 
Table 4. Mean \% errors for whole blood B analysis by prompt $\gamma$, ICP-AES and DCPAES.

\begin{tabular}{lcccc}
\hline & \multicolumn{4}{c}{ Mean \% Errors } \\
\cline { 3 - 5 } Sample Type & Prompt $\gamma^{b}$ & ICP-AES & DCP-1 & DCP-2 $^{c}$ \\
\hline Overall Error & $0.1 \pm 2.8$ & $1.6 \pm 3.7$ & $-9.5 \pm 4.4$ & $-3.9 \pm 6.4$ \\
Cage Compounds & $0.2 \pm 3.0$ & $1.6 \pm 3.7$ & $-11.0 \pm 2.3$ & $-5.8 \pm 4.1$ \\
Borate Compounds & -0.8 & & $0.0 \pm 1.9$ & \\
\hline
\end{tabular}

- Assumes prompt $\gamma$ with multipoint calibration is true value

${ }^{b}$ Prompt $\gamma$ with 2 point calibration

c DCP-AES analysis prior to $(-1)$ and after $(-2)$ viewing area adjustments

were spiked with B from a commercial standard. The results of this experiment are shown in Table 2 and indicate that no significant interferences exist with as much as $5 \%$ blood in the slurry when using DCP-AES.

Some typical analysis results for the same blood samples by prompt $\gamma^{6}$ and slurry nebulization with DCP-AES and ICP-AES are shown in Table 3. The ICP-AES analyses were performed at the INEL on the same slurry preparations that were used for DCP-AES at BNL. Prompt $\gamma$ was in some cases done twice, once the day the sample was taken using only a blank and a single standard for calibration and a second time when a multipoint calibration curve could be obtained. Examination of Table 3 indicates that the DCP-AES results are always lower for blood and the BSSB compound and near normal for BPA and boric acid. Assuming that the multipoint prompt $\gamma$ results are near the true values, the $\%$ errors were calculated and averaged as presented in Table 4. The precisions for all methods and B compounds are quite comparable, however the first DCP-AES analysis shows a mean $-11 \%$ error for boron cage compounds. This discrepancy was not noted in other DCP-AES analyses when the samples were digested?. Viewing a cooler region of the DCP appeared to give an enhanced recovery for B as BSSB so some blood slurry samples were rerun viewing this region. The results of this run are presented in the last column of Table 4. Even though the recovery of the cage compounds was somewhat better, the mean error was still $\sim-6 \%$ for B in cage compounds. The reason for the lower DCP-AES

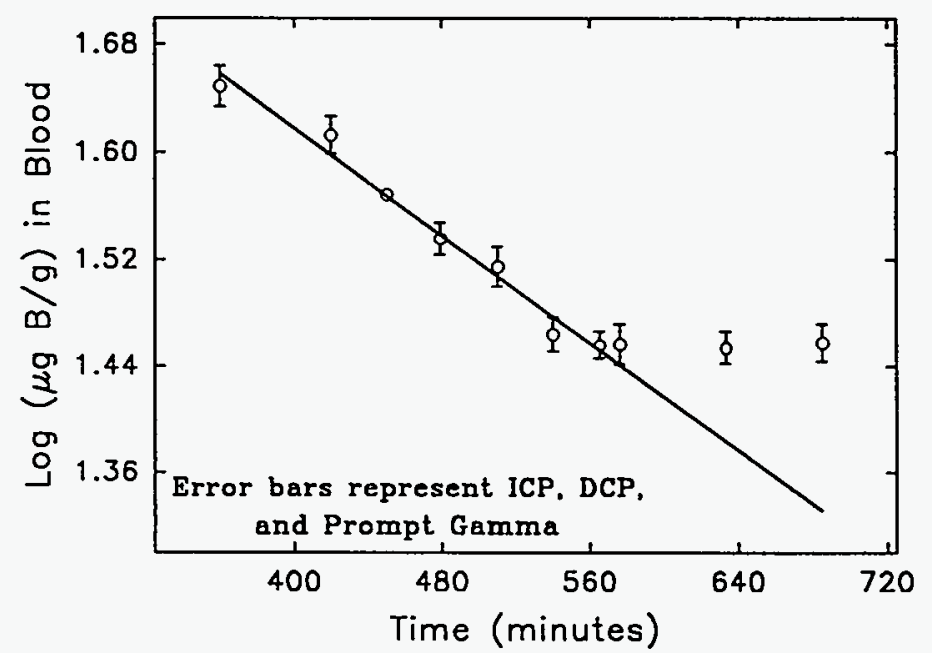

Figure 1. Elimination of $B$ in an atypical dog following administration of BSH. Each point represents the mean of the DCP-AES analysis and ICP-AES and prompt $\gamma$ performed at a later date. 
results is not known but believed to be related to the fact that the sample never reaches the core of the DCP and is not contained within the plasma. All subsequent B analysis by DCP-AES were corrected for this negative bias after the analysis of a reference standard or sample.

Figure 1 indicates the utility of the rapid analytical technique in treatment planning. In this dog, the B elimination was quite normal until $\sim 560$ minutes. At this point the dog was taken to the reactor and anaesthetized. This, in some way, disrupted the normal elimination and during the course of the irradiation there was no net change in the blood B level. By using the rapid DCP-AES analysis of the blood slurries, adjustments could be made in the irradiation schedule knowing that the elimination had ceased.

\section{CONCLUSIONS}

Blood samples can be prepared and analyzed for B as slurries within 5-15 minutes of receipt. The analysis does not appear to be affected by blood slurry concentrations of up to $5 \%$ and the slurries are stable for several days. When $B$ is present as a cage type compound, DCP-AES results are typically 5-15\% low. This negative error can easily be corrected for if a suitable reference standard or a reference analysis of a sample is available. Boron analysis in the slurries by ICP-AES is unaffected by the compound type, however, caution should be used in order to assure that pr jitive errors do not result from volatile B compounds.

\section{ACKNOWLEDGEMENTS}

This work was performed under the auspices of the U.S. Department of Energy, Office of Energy Research, under DOE Field Office, Idaho, Contract No. DE-AC07$761 D 01570$.

\section{REFERENCES}

1. P.R. Gavin, C.E. DeHaan, S.L. Kraft, M.P. Moore, L.R. Wendling, and R.V. Dom, Acute and late reactions following boron neutron capture epithermal neutron therapy in dogs with spontaneous brain tumors, (1992) in Progress in Neutron Capture Therapy for Cancer, eds B.J. Allen et. al. ( Plenum Press, NY) pp. 507-514.

2. P.R. Gavin, S.L. Kraft, L.R. Wendling, and D.L. Miller, Canine spontaneous brain tumors - a large animal model for BNCT (1989) Strahlenther. Onkol. 165, 225.

3. M.D. Wichman, R.C. Fry, and M.K. Hoffman, Slurry method for rapid DCP atomic emission determination of residual bone fragments in mechanically separated turkey (1986) Appl. Spectr. 40, 351.

4. G.R. Wellum, E.I. Tolpin, A.H. Soloway, and A. Kaczmarczyk, Synthesis of $\mu$-disulfidobis(undecahydro-closo-dodecaborate)(4-) and of a derived free radical (1977) Inorg. Chem. 16, 2120.

5. W.F. Bauer, D.A. Johnson, S.M. Steele, K. Messick, D.L. Miller, and W.A. Propp, Gross boron determination in biological samples by inductively coupled plasma-atomic emission spectroscopy (1989) Strahlenther. Onkol. 165, 176.

6. R.G. Fairchild, D. Gabel, B.H. Laster, D. Greenberg, W. Kiszenick, and P.L. Micca, Microanalytical techniques for boron analysis using the ${ }^{80} \mathrm{~B}(\mathrm{n}, \alpha)^{7} \mathrm{Li}$ reaction $(1986) \mathrm{Med}$. Phys. 13, 50.

7. R.F. Barth, D.M. Adams, A.H. Soloway, E.B. Mechetner, F. Alam, and A.K.M. Anisuzzaman, Determination of boron in tissues and cells using direct-current plasma atomic emission spectroscopy (1991) Anal. Chem. 63, 890. 
, 


\section{Quantification of Boron in Biological Tissue by Secondary Ion Mass Spectrometry}

V. Chia, R. Bleiler, D. Sams, C. Jones, R. Odom, W. Bauer, A. Gianotto, C. Swartz, and $\mathrm{J}$. Weidner

This article is a reprint from Advances in Neutron Capture Therapy, pp. 409-412, (1993). 


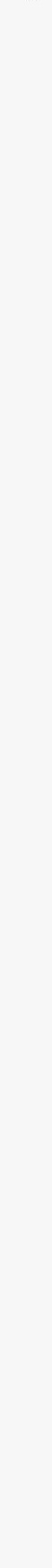




\title{
QUANTIFICATION OF BORON IN BIOLOGICAL TISSUE BY SECONDARY ION MASS SPECTROMETRY
}

\author{
V.K.F. Chia, ${ }^{1}$ R.J. Blieler, ${ }^{1}$ D.B. Sams, ${ }^{1}$ C.M. Jones, ${ }^{1}$ R.W. Odom, ${ }^{1}$ \\ W.F. Bauer, ${ }^{2}$ A.K. Gianotto, ${ }^{2}$ C.D. Swartz, ${ }^{3}$ and J.P. Weidner ${ }^{3}$ \\ ${ }^{1}$ Charles Evans \& Associates, Redwood City, CA 94063 \\ ${ }^{2}$ Idaho National Engineering Laboratory, Idaho Falls, ID 83415-3519 \\ ${ }^{3}$ Washington State University, Pullman, WA 99163
}

\section{INTRODUCTION}

The combination of boron-containing drugs and neutron irradiation to combat certain brain cancers has proven to be a viable method for cancer treatment. The effectiveness of this treatment depends on the selectivity of the carrier compound delivering boron to the tumor cells. To date, the most effective brain tumor treatment drug is borocaptate sodium $\left(\mathrm{Na}_{2} \mathrm{~B}_{12} \mathrm{H}_{11} \mathrm{SH}\right.$ or $\left.\mathrm{BSH}\right)$. As new drugs are constantly being synthesized to improve selective delivery of boron to cancer cells any analytical technique capable of quantifying boron at the subcellular level will play an important role in determining the drug's potency for boron neutron capture therapy (BNCT).

Electron probe X-ray microanalysis (EPXMA) is an established technique for obtaining quantitative information on the distribution of elements at the ultrastructural level. ${ }^{1}$ However, EPXMA is unable to detect boron because of X-ray absorption in the boron region by the beryllium window of the detector. The complimentary technique of electron energy loss spectroscopy (EELS) is less often used primarily because analyses must be performed on extremely thin ( $<60 \mathrm{~nm}$ ) uniform sections of tissue. Secondary ion mass spectrometry (SIMS), although universally recognized as a sensitive quantitative technique by the semiconductor industry, is often not exploited by the biological and medical community even though ion microscopy has been utilized sucessfully for a variety of biological applications. ${ }^{2}$

This paper describes the application of SIMS using a Cameca mass spectrometer to localize and quantify boron in rat tissue after BSH intravenous delivery. The methodology for preparing tissue samples suitable for SIMS analysis is described and quantitative elemental images from the liver tissue are presented. It should be noted that this is our first SIMS experiment with infused rats and these boron localization results should be treated as preliminary.

Advances in Neutron Capture Therapy, Edited by

A.H Soloway et al., Plenum Press, New York, 1993

409 


\section{EXPERIMENTS AND RESULTS}

A laboratory rat was intravenously infused with ${ }^{11} \mathrm{BSH}$ (100 $\mathrm{mg} \mathrm{B} / \mathrm{kg}$ body weight) and the boron allowed to distribute to the tissues for about one hour. This rat and a control rat (not administered with boron) were then euthanized and the liver and three regions of the brain (cerebral hemispheres and brainstem) removed and immediately cryopreserved using a liquid nitrogen metal mirror fixation unit. ${ }^{1}$ At the time of writing this paper, only the liver tissues from the infused and control rats were analyzed by SIMS and no inductively coupled plasma atomic emission spectrocopy (ICP-AES) data were available for comparison.

Cryofixation, as opposed to chemical fixation, is the only method by which both ultracellular and intracellular element contents can be simultaneously preserved. The frozen hydrated tissues were then cryoultramicrotomed at about $-140^{\circ} \mathrm{C}$ using no embedding medium, including the "low temperature" types or freeze substitution technique, which may inadvertently cause some form of chemical change to occur in the tissues. Freeze-drying was performed on the cryosections and the freeze-dried sections placed onto high-purity silicon wafers for SIMS analysis. Although not performed during this test experiment, it is imperative that conventional transmission electron microscopy (CTEM) is performed in parallel to the SIMS analysis to evaluate the quality of the crysections with respect to induced structural artifacts from the crypreparation procedures.

We have used brain homogenate as the matrix standard in this study because our primary goal is to accurately quantify boron in brain tumors. Figure 1 shows boron calibration curves of ${ }^{11} \mathrm{~B}$-boric acid and ${ }^{11} \mathrm{BSH}$ in brain homogenate and ${ }^{11} \mathrm{BSH}$ in gelatin. The absolute boron concentrations were quantified by ICP-AES. A similar experiment using the isotopically enriched form of these compounds was also performed. Irrespective of the boron isotope used, the slope of the calibration plots for BSH in brain homogenate was consistently lower than with boric acid. However, similar slopes are observed for the plots of ${ }^{11} \mathrm{~B}$-boric acid in brain homogenate and ${ }^{11} \mathrm{BSH}$ in gelatin suggesting that the sampled homogenate analysed was more aqeous containing unbound boron. ${ }^{3}$ Similar experiments in aqueous media are being performed to confirm this hypothesis.

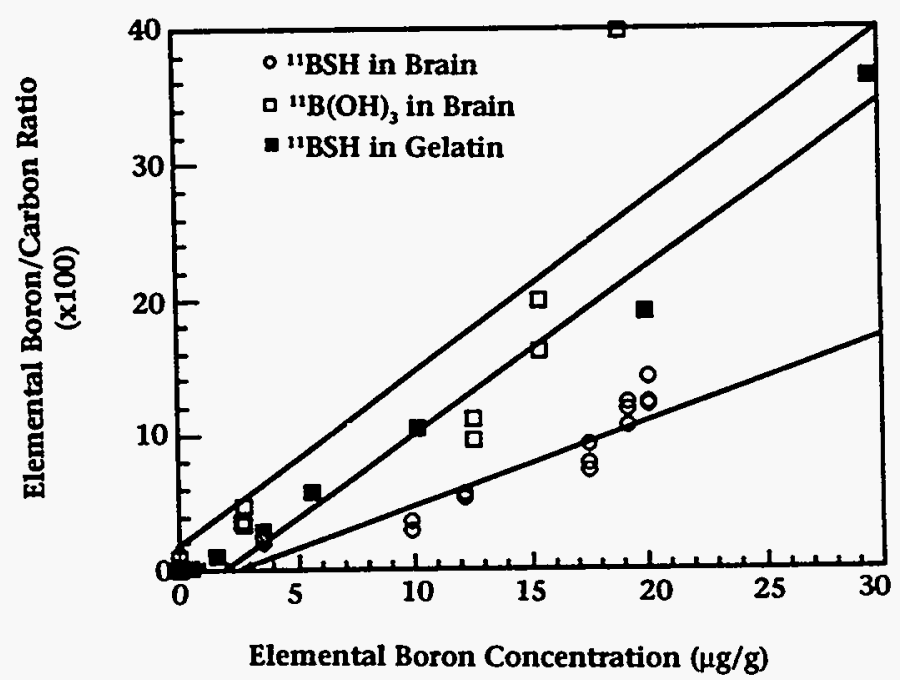

Figure 1. Boron calibration curves of $\mathrm{BSH}$ and boric acid in brain bomogenate and gelatin. 
For the purpose of this study, an average value of the brain homogenate and gelatin calibration results was used for SIMS quantification in the liver tissue. Dynamic SIMS profiles acquired from the livers of the diffused and control rat are shown in Figure 2. Boron concentrations of $53 \mu \mathrm{g} \mathrm{B} / \mathrm{g}$ in the diffused rat and $13 \mu \mathrm{g} \mathrm{B} / \mathrm{g}$ in the control rat were observed. An optical micrograph of the freeze-dried section and a boron secondary ion image of the infused rat's liver are shown in Figure 3. Boron is homogeneously distributed and is not observed in the tissue voids indicating that no diffusible boron was lost during sample preparation. The potassium secondary ion image in Figure 4 shows clusters of high intensity spots probably corresponding to cells. The observed potassium/sodium ratio of about eight suggests that the tissue was viable at the time of crypreservation.

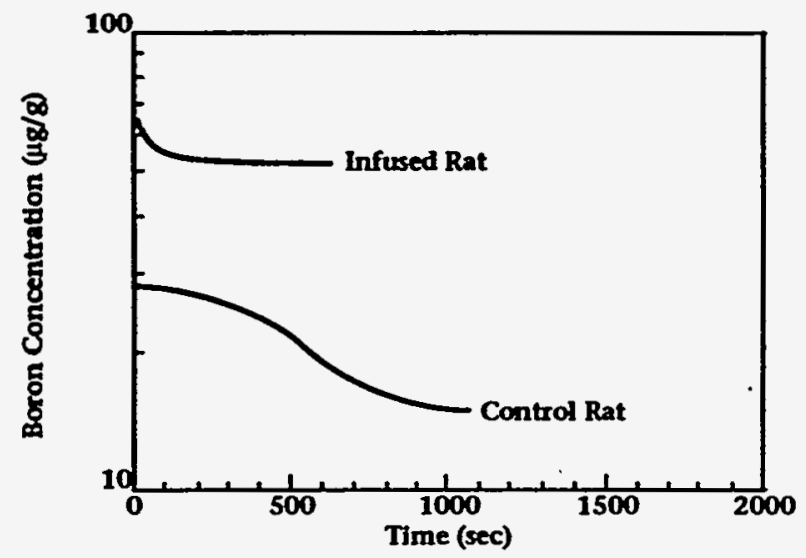

Figure 2. SIMS depth profiles of boron in the liver of an infused BSH rat and control rat.

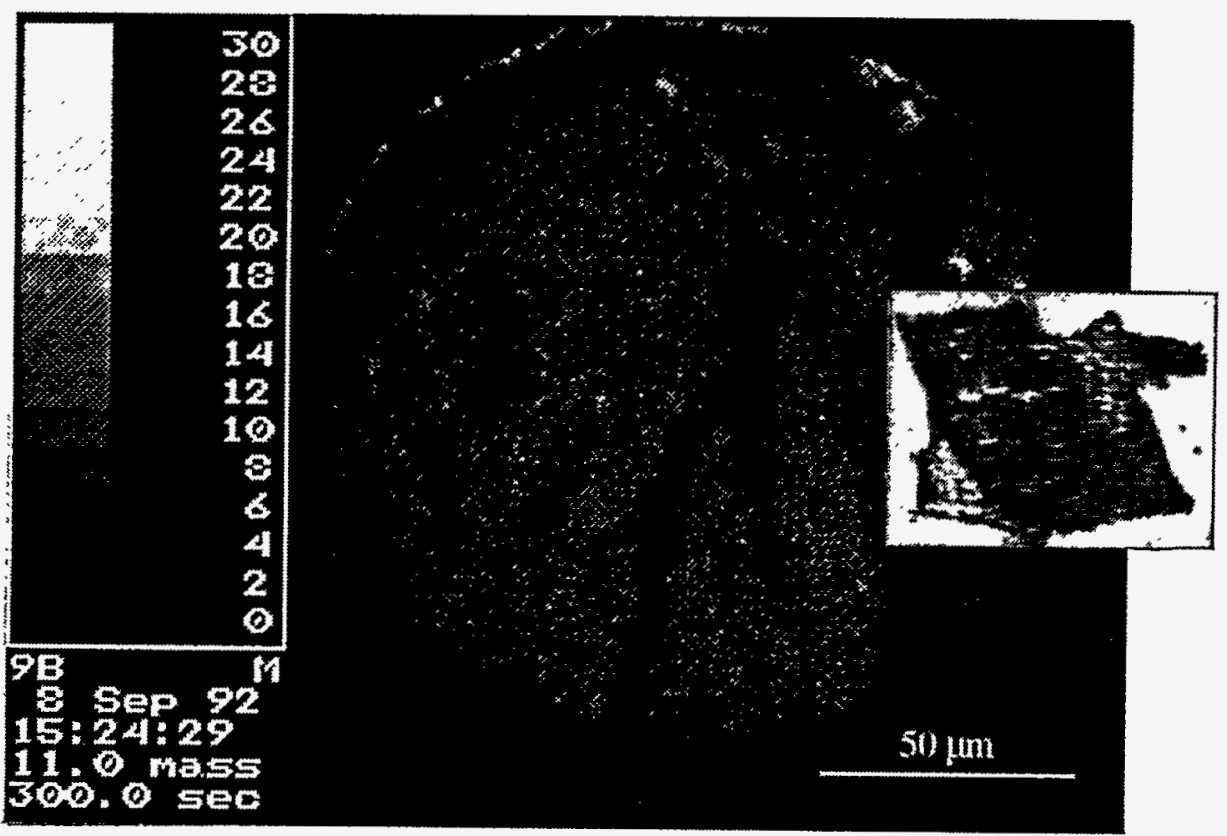

Figure 3: Boron secondary ion image of liver tissue from the infused rat. Insert shows an optical micrograph of the freeze-dried liver section prepared at Duke University Medical Center. 


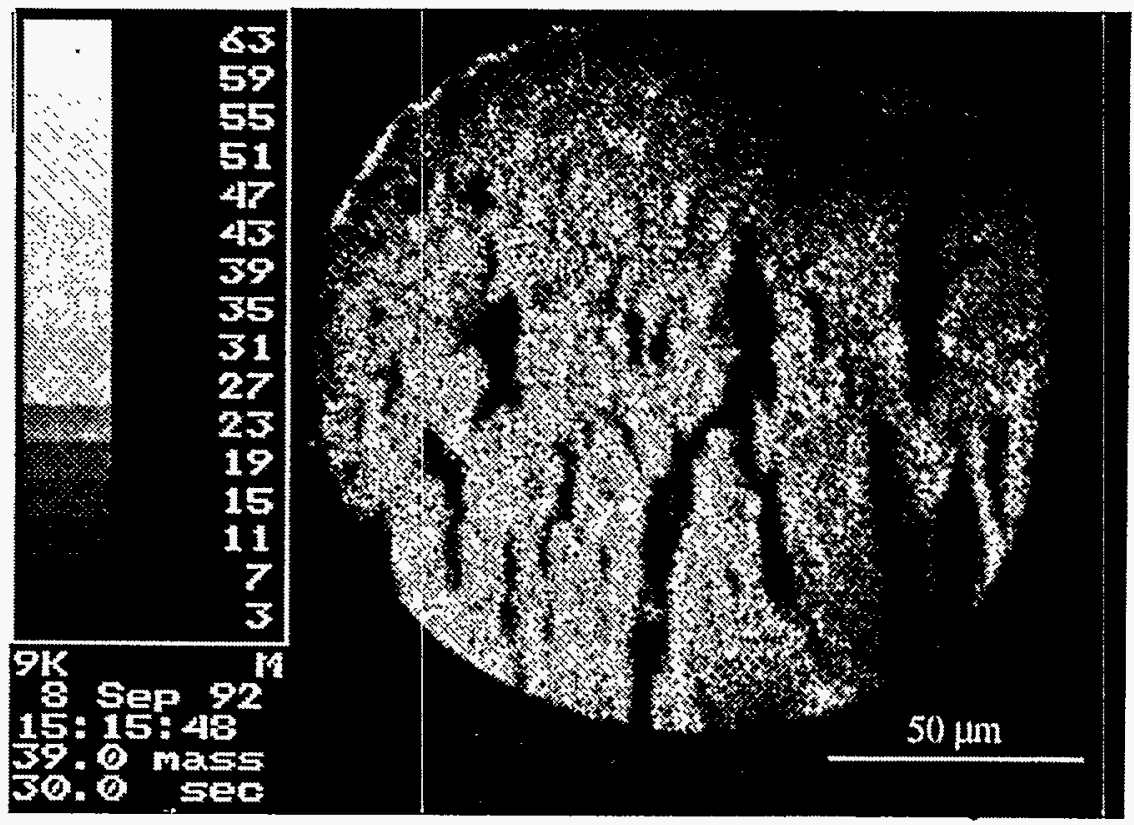

Figure 4. Potassium secondary ion image of liver tissue from the infused rat.

\section{CONCLUSIONS}

These preliminary results illustrate that with appropriate cryopreservation of tissue quantitative imaging is possible by SIMS. Quantification using brain homogenate gave reasonable boron concentration values in the liver; a comparison with ICP-AES results will, confirm the accuracy. Boron was homogenously distributed in the freeze-dried liver section of the infused rat. The viability of the tissue at the time of cryopreservation was confirmed by the high potassium to sodium ratio. The high intensity potassium spots observed probably correspond to individual cells. Further improvements in the cryopreservation methodology is required to obtain the higher resolution images needed for this localization study.

\section{REFERENCES}

1. A. LeFurgey, M. Bond and P. Ingram, Frontiers in Electron Probe Microanalysis: Applications to Cell Physiology, Ultramicroscopy, 24: 185 (1988)

2. R.W. Linton, I.H. Musselman, S.R. Bryan, Laser and Ion Microprobe Mass Spectrometry - Applications to Human Tissues, in: "Microprobe Analysis in Medicine," P. Ingram, J.D. Shelburne and V.L. Roggli, eds., Hemisphere Publishing Co., NY (1989)

3. W.F. Bauer, K.M. Bradshaw and T.L. Richards, Interaction Between Boron Containing Compounds and Serum Albumin Observed by Nuclear Magnetic Resonance, in: "Progress in Neutron Capture Therapy for Cancer, " B.J. Allen et al, eds., Plenum Press, NY (1992) 
Determination of Strongly Protein-Bound Borocaptate Species by High Performance Liquid Chromatography with Online Inductively Coupled Plasma Atomic Emission Spectroscopy Detection of Boron

N. Hotz and W. Bauer

This article is a reprint from Advances in Neutron Capture Therapy, pp. 439-443, (1993). 
, 


\title{
DETERMINATION OF STRONGLY PROTELN-BOUND BOROCAPTATE SPECIES BY HIGH PERFORMANCE LIQUID CHROMATOGRAPHY WITH ON-LINE INDUCTIVELY COUPLED PLASMA ATOMIC EMISSION
} SPECTROSCOPY DETECTION OF BORON

\author{
N.J. Hotz and W.F. Bauer \\ Idaho National Engineering Laboratory, EG\&G Idaho, Inc. \\ Idaho Falls, ID 83415
}

\section{INTRODUCTION}

Borocaptate compounds have been studied for many years as a means of delivering ${ }^{10} \mathrm{~B}$ to brain tumors prior to neutron exposure for BNCT. The actual nature of the observed binding of mercapto-undecahydrododecaborate $\left(\mathrm{B}_{12} \mathrm{H}_{11} \mathrm{SH}^{2-}, \mathrm{BSH}\right)$ anion and it's dimer $\left(\mathrm{B}_{24} \mathrm{H}_{22} \mathrm{~S}_{2}{ }^{4}\right.$, BSSB) to plasma proteins has been of interest as this will affect both the toxicity and the biodistribution of these anions throughout the body. The toxicity of the borocaptate species may be related to the ability to bind to proteins and compete for sites which normally bind metabolic by-products.

Early work concerning the protein binding of BSH indicated that covalent binding via a disulfide linkage may occur between $\mathrm{BSH}$ and serum protein $\mathrm{s}^{1,2}$. If this is true, a minimum amount of $\mathrm{BSH}$ would be required to fill the strong covalent binding sites and only BSH in excess of this level would be available for tumor uptake. However, nuclear magnetic resonance (NMR) spectroscopy does not show evidence of covalent binding of $\mathrm{BSH}$ to albumins via a covalent disulfide bond ${ }^{3}$, therefore indicating that binding is primarily ionic. In an experiment performed in our laboratory, phosphate buffered saline rinses performed on bovine serum albumin/BSH retained above ultrafiltration membranes were successful in removing some of the borocaptate that was considered "bound" to the protein. The dilution of the BSH and BSA, which is believed to shift the equilibrium and result in the release of the ionically bound $\mathrm{BSH}$, could be performed by gel filtration chromatography (GPC). Since GPC is not an equilibrium method, the drug-protein complex will dissociate and the drug separated from the protein ${ }^{4}$. In the case of BSH binding to protein, only strongly associated or covalently bound compounds would be expected to co-elute with the high molecular weight proteins.

The primary disadvantage with GPC to determine the protein binding of BSH and related compounds is the typical UV-detectors used for liquid chromatography cannot distinguish between protein and protein with bound boron. To determine the $\mathrm{B}$ containing proteins, fractions can be collected and $B$ determined by a separate technique such as 
inductively-coupled plasma atomic emission spectroscopy (ICP-AES). Two modes of on line HPLC detection are clearly needed, one to detect the protein and one to detect the boron. To perform this dual detection, an ICP-AES has been coupled to a high performance liquid chromatograph with a UV detector to allow serial detection of the protein (UV at $280 \mathrm{~nm}$ ) and boron (ICP-AES at $249.77 \mathrm{~nm}$ ).

\section{MATERIALS AND METHODS}

Solutions of bovine serum albumin (BSA, Sigma A-6793) and human serum albumin (HSA, Sigma A-1667) were made at $4 \% \mathrm{w} / \mathrm{w}$ in $\mathrm{pH} 7.3,0.10 \mathrm{M}$ phosphate buffer. Varying amounts of $\mathrm{Li}_{2} \mathrm{~B}_{12} \mathrm{H}_{12}(\mathrm{BH}), \mathrm{Na}_{2} \mathrm{~B}_{12} \mathrm{H}_{11} \mathrm{SH}(\mathrm{BSH}), \mathrm{Cs}_{4} \mathrm{~B}_{24} \mathrm{H}_{22} \mathrm{~S}_{2}$ (BSSB) and $\mathrm{B}(\mathrm{OH})_{3}$ were added to the albumin solutions and allowed to incubate for 24 hours before GPC. The binding of the boron compounds was also performed at different incubation temperatures and incubation times. Twenty microliter volumes were injected into a two column series of Macrosphere GPC 300 columns (Altech \& Associates) with a flow rate of $0.5 \mathrm{~mL} / \mathrm{min}$ of $\mathrm{pH} 7.3,0.10 \mathrm{M}$ phosphate buffer. Deionized water containing $0.1 \%$ Triton X-100 (Sigma X-100R-S) at $2.0 \mathrm{~mL} / \mathrm{min}$ was added to the UV detector effluent in order to stabilize the ICP and to maintain the optimum delivery flow to the nebulizer on the ARL 3520 ICP-AES. The conventional ICP-AES sample introduction system with a concentric glass nebulizer and spray chamber were utilized. Protein peaks were monitored at $280 \mathrm{~nm}$ and the B emission line at $249.77 \mathrm{~nm}$ was monitored to detect any eluting boron compounds. The diagram in Figure 1 shows a general schematic of the instrumental setup. Ultrafiltration and equilibrium dialysis experiments were done with 30000 molecular weight cutoff membranes.

\section{RESULTS AND DISCUSSION}

A decrease of the baseline noise in the ICP-AES signal during elution of the protein peak was observed during early experiments. In order to maintain the lower noise level, a $0.1 \%$ aqueous solution of Triton $\mathrm{X}-100$ was added to the carrier flow immediately after

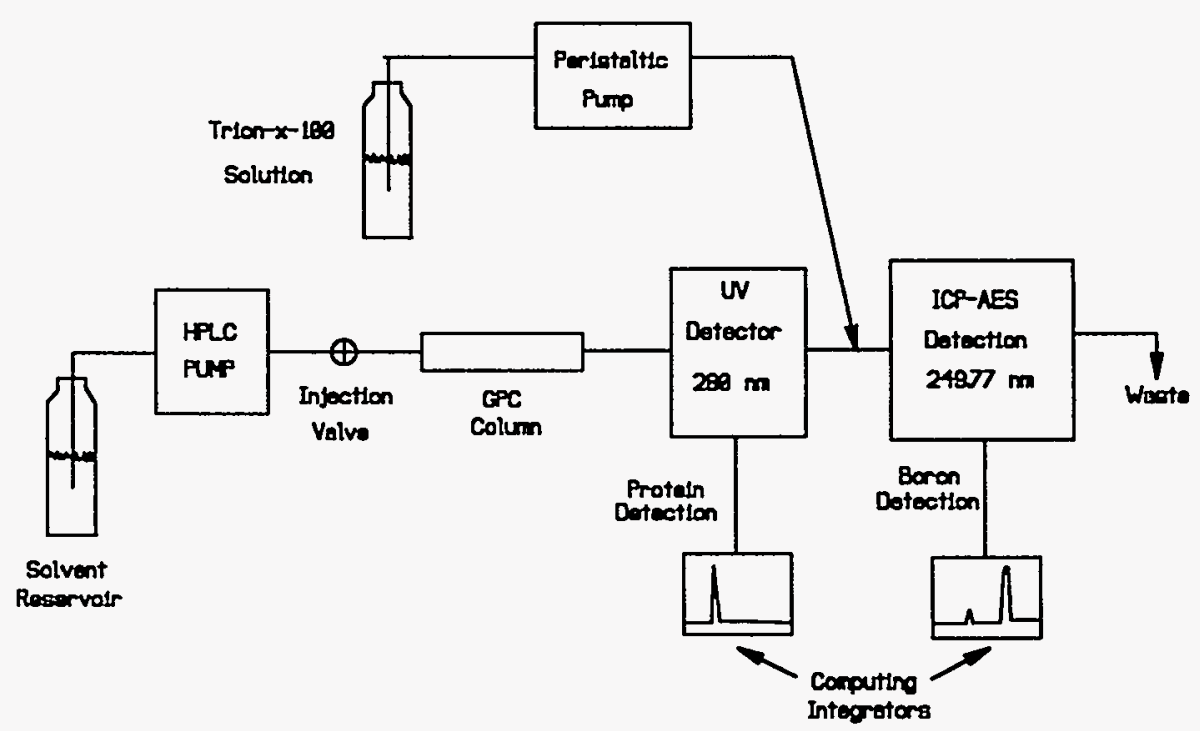

Figure 1. HPLC apparatus for the separation and detection of boron containing proteins. 

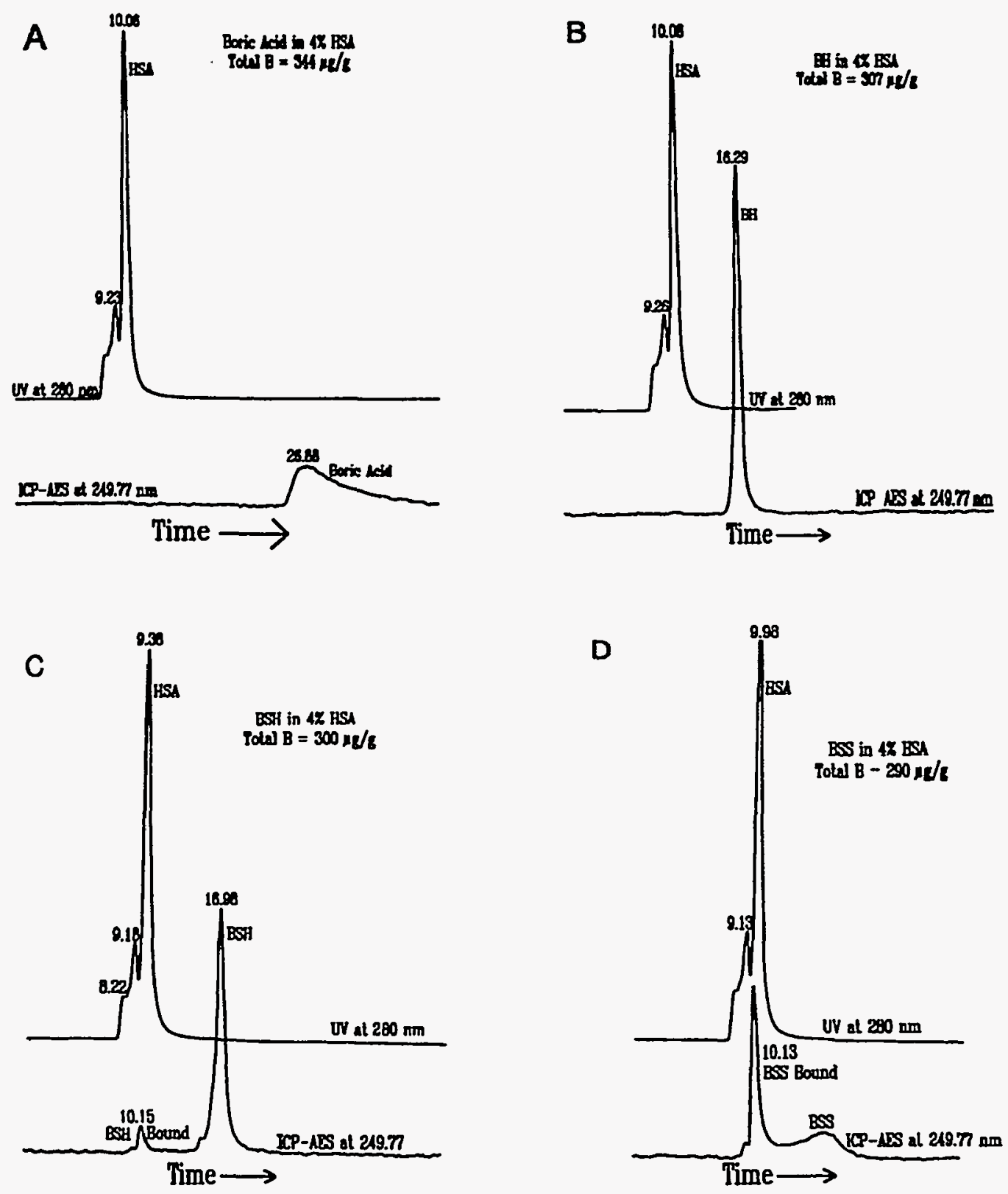

Figure 2. Chromatograms showing the nature of the interaction of $\left.\left.A) B(O H)_{3}, B\right) B_{12} H_{12}{ }^{-2}, C\right) B S H$, and D) BSSB with HSA. Detection is via UV (top) and ICP-AES (bottom).

the UV-detector. This "makeup" flow was necessary to prevent significant band broadening caused by the dead volume of the spray chamber and the addition of the surfactant served to noticeably reduce the noise levels.

Figures 2A through 2D demonstrate the binding differences found between the various B compounds with HSA after a 24 hour incubation period at $37^{\circ} \mathrm{C}$. Boric acid (Figure 2A) and BH (Figure 2B) are completely separated from any association with the albumin molecule. Binding of $\mathrm{BH}$ to HSA has been observed during ultrafiltration experiments, however, no binding is observed during GPC. The binding of these compounds appears to be solely ionic as previously reported ${ }^{1,2}$. The BSH anion, however, shows only a small quantity of B that is tightly bound to the albumin molecule (Figure 2C), 
most likely due to the BSSB and BSSOB already present in the BSH stock solution. This conclusion is supported by the fact that the peak areas indicated $\sim 7 \%$ of the B was bound to the protein at all total B concentration levels and by Figure 2D which shows almost complete binding of the BSSB boron to the albumin. The tailing and poor peak shape of the "free BSSB" may indicate that the binding is via a covalent disulfide exchange type reaction. This strong binding of BSSB to albumin has been previously noted ${ }^{5}$ and the reactivity may be partially explained by the ability of BSSB to form free radicals under certain conditions ${ }^{6}$ and the importance of such free radicals in the formation of disulfide bonds ${ }^{7}$. The binding of BSH to albumin is essentially complete within the time of mixing ( $\sim 5$ minutes) therefore, BSH would not be expected to react significantly with serum proteins during the course of a normal infusion and elimination period. The observed levels of BSH binding to HSA are at significantly lower levels than those determined by ultrafiltration or equilibrium dialysis (65-85\% bound). The ICP-AES (boron) chromatographs in Figure 3 demonstrate that only a limited number of sites are available for covalent binding and that subsequent concentration doublings of BSSB do not lead to an appreciable increase in "covalently" bound boron.

\section{CONCLUSIONS}

A method for determining protein bound boron has been described and demonstrated. The BSH anion is easily separated from its association with albumin molecules indicating that the binding is primarily of an ionic nature. The BSH dimer, BSSB, is quite strongly associated with the albumin molecule due to the ability to attack sulfhydryl and disulfide groups on the parent protein and create a series of disulfide exchange reactions. The results presented here suggest that no significant binding of BSH to albumin would be expected during the course of an infusion and BNCT treatment. Future work will examine BSH binding in real animal systems after infusion of the compound, i.e. in situ incubation of BSH with blood proteins.

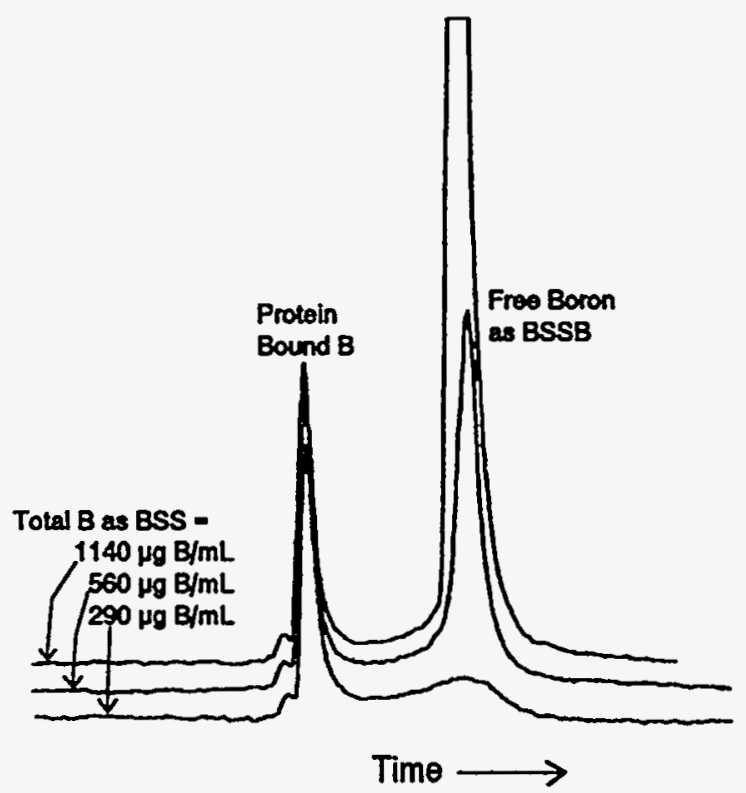

Figure 3. GPC chromatograms with ICP-AES detection of BSSB incubated with $4 \%$ HSA. The albumin molecule becomes saturated with covalently bound B at $\sim 1.3$ BSSB per albumin. 


\section{ACKNOWLEDGEMENTS}

This work was performed under the auspices of the U.S. Department of Energy, Office of Energy Research, under DOE Field Office, Idaho, Contract No. DE-AC07$761 D 01570$.

\section{REFERENCES}

1. A. H. Soloway, H. Hatanaka and M. A. Davis (1967) J. Med. Chem. 10, 714-717.

2. T. Nakagawa, T. Nagai. Parts I, II, and III (1976) Chem. Pharm. Bull. 24, 2934-2954.

3. W. F. Bauer, K. M. Bradshaw, and T. L. Richards (1992) in Progress in Neutron Capture Therapy for Cancer, ed. ed. B.J. Allen et al. ( Plenum Press, New York), pp. 339 -343.

4. T. C. Pinkerton and K. A. Koeplinger (1990) Anal. Chem. 62, 2114-2122.

5. D. Slatkin, P. Micca, A. Forman, D. Gabel, L. Wielopolski, and R. Fairchild (1986) Biochemical Pharmacology, 35, 1771-1776.

6. G. R. Wellum, E. I. Tolpin, A. H. Soloway, and A. Kaczmarczyk (1977) Inorganic Chemistry 16, 2120-2122.

7. J. March (1977) Advanced Orgainic Chemistry, 2nd ed. (McGraw-Hill Book Company, New York) p. 1115. 

NMR Studies of the Interaction of Borocaptate Sodium with Serum Albumin

P. Tang, M. Schweizer, W. Bauer, and K. Bradshaw

This article is a reprint from Advances in Neutron Capture Therapy, pp. 453-457, (1993). 


\section{,}




\title{
NMR STUDIES OF THE INTERACTION OF BOROCAPTATE SODIUM WITH SERUM ALBUMIN
}

\author{
Peng-Peng Z. Tang ${ }^{1}$, Martin P. Schweizer ${ }^{1}$, William F. Bauer ${ }^{3}$ \\ and Kenneth M. Bradshaw ${ }^{1,2}$ \\ 1. Dept. of Medicinal Chemistry and Dept. of Radiology, University of Utah, \\ Salt Lake City, UT 84112 \\ 2. Neutron Technology, Boise, ID 83702 \\ 3. Idaho National Engineering Laboratory, Idaho Falls, ID $83415-3519$
}

\section{INTRODUCTION}

The interaction between BSH and serum albumin is of interest because it is related to the pharmacokinetics of BSH. Early research ${ }^{(1)}$ suggested that covalent disulfide bridge might be involved in the interaction, but the idea was contradicted by subsequent studies ${ }^{[2.3,4]}$ using ${ }^{11} \mathrm{~B}$ Nuclear Magnetic Resonance (NMR) spectroscopy. NMR has been proved to be a very powerful technique to study the protein binding of a small molecule, such as $\mathrm{BSH}^{[\mathrm{s}]}$, and boron NMR is typically suitable to study the binding effect of the boronated agent. Both ${ }^{11} \mathrm{~B}$ and ${ }^{10} \mathrm{~B}$ have NMR activity, but ${ }^{11} \mathrm{~B}$ has better NMR sensitivity $\left(16.5 \%\right.$ vs. $2 \%$ relative to $\left.{ }^{1} \mathrm{H}\right)$ and a larger natural abundance ( $81 \%$ vs. $19 \%)^{[6]}$. Even though ${ }^{10} \mathrm{~B}$ is the neutron active nucleus for BNCT, the more sensitive nucleus " $\mathrm{B}$ is more appropriate for NMR studies. Since the isotopic difference does not make any differences in the structure and binding effect of a compound, the result obtained from the ${ }^{11} \mathrm{~B}$ NMR of BSH can hold for the ${ }^{10} \mathrm{~B}$ enriched agent.

The chemical shift of boron NMR is very sensitive to the chemical environment. According to chemical differences, the 12 boron nuclei of BSH can be classified into 4 groups, as indicated in Fig. 1. Previous research indicated that the formation of a covalent disulfide bridge moves the "1B chemical shift of sulfhydryl boron (B1) $3 \mathrm{ppm}$ downfield[2] since a covalent binding to BSH changes the chemical environment around the boron nuclei.

The relaxation rates of a quadrupole nucleus are also verry sensitive to protein binding. The binding can produce large changes in the quadrupole relaxation rate as a result of changes in the electric field gradients and the correlation times. In the interaction of albumin with BSH, the ${ }^{11} \mathrm{~B}$ linewidth, $\mathrm{W}^{\mathrm{b}, e}$, of protein bound boron is obtained by subtracting the ${ }^{11} \mathrm{~B}$ linewidth, $W^{u}$, of unbound BSH (obtained from free BSH solution) from the observed "B linewidth, $W^{\circ}$, of $B S H$ in albumin solution $\left(W^{b}, e=W^{\circ}-W^{u}\right)$. The ${ }^{11} B$ spin-spin relaxation rate of protein bound BSH and the rate of exchange between bound and unbound BSH contribute to $W^{b, e}$. When the bound and unbound BSH are under fast exchange, the linewidth $W^{b, e}$ decreases as the temperature increases ${ }^{[3]}$.

The relaxation rate of a nucleus is also related to its correlation time $\left(\tau_{c}\right)$. The correlation time, according to Stoke's Law, is proportional to the solvent viscosity[s]. When more than $1 \%(w / v)$ aqueous solution of protein is used, the effect of viscosity $(\eta)$ on relaxation rate cannot be neglected. Under the so called 'extreme narrowing condition', the quadrupole relaxation rate $(R)$ is linearly related to the correlation time $\left(\tau_{c}\right)^{[s]}$. Assuming that this condition applies to our system, the "B longitudinal relaxation rate $\left(R_{1}\right)$ is proportional to the 
viscosity. Since the flow times of water $\left(F^{*} t\right)$ and that of the aqueous solutions of serum albumin $\left(\mathrm{Ft}_{\mathrm{t}}\right)$ measured with an Otswald viscometer are proportional to their viscosities, the ratio $\mathrm{f}^{\mathrm{v}}$ is proportional to the ratio of the viscosities of the solutions (eq. 1), and therefor can be applied to the measured ${ }^{11} B$ longitudinal relaxation rate $\left(R_{1}\right)$ as a correction coefficient to account for the viscosity effect (eq. 2).

$$
\begin{aligned}
& \mathrm{F}_{\mathrm{t}}=\mathrm{C} \frac{\eta}{\xi}==\Rightarrow \frac{\eta^{*}}{\eta}=\frac{\xi * \mathrm{~F}_{\mathrm{t}}^{*}}{\xi}=\mathrm{f}^{\mathrm{v}} \\
& \mathrm{R}_{\mathrm{t}}^{*}=\mathrm{f}^{\mathrm{v}} \mathrm{R}_{1}
\end{aligned}
$$

where $F_{t}, R_{1}$ and $f^{v}$ are defined as above, $C$ is an apparatus constant for the viscometer, $\xi$ is the density of the solution, and * corresponds to values for water.

After correction for the viscosity effect, the observed "B longitudinal relaxation rate of BSH upon titration with protein reflects the interaction between BSH and the protein, having contributions from both bound and unbound BSH as follows:

$$
R_{1}{ }^{\circ}=R_{1}{ }^{b} f+R_{1}{ }^{u}(1-f)
$$

where $R^{b}$ and $R^{u}$ are the relaxation rates for bound and unbound $B S H$, respectively, $R^{\circ}$ is the observed relaxation rate with albumin present after correction for viscosity, and $\mathrm{f}$ is the fraction of bound BSH at a chosen concentration of protein. If the relaxation rate of bound BSH can be obtained, the fraction $\mathrm{f}$ can be derived from this equation and the concentrations of bound and unbound BSH ([B] $]^{\mathrm{b}}$ and $[\mathrm{B}]^{\mathrm{u}}$ ) can also be obtained .

With known concentrations of bound and unbound BSH, one can evaluate the binding constant $\mathrm{K}$ (mole $\mathrm{e}^{-1}$ ) and the number of binding sites, $\mathrm{n}$, on serum albumin according to the binding equation $[7$, assuming there is only one type of binding site:

$$
\frac{[B]^{b}}{[B]^{u}[S A]_{0}}=K n-K \frac{[B]^{b}}{[S A]_{0}}
$$

(where $[\mathrm{SA}]_{0}$ is the concentration of serum albumin).

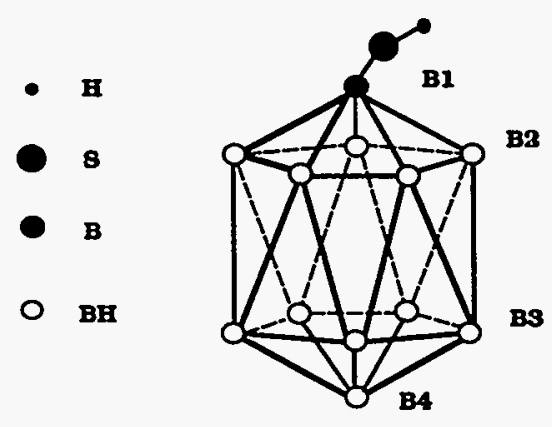

Figure 1. Structure of BSH Anion

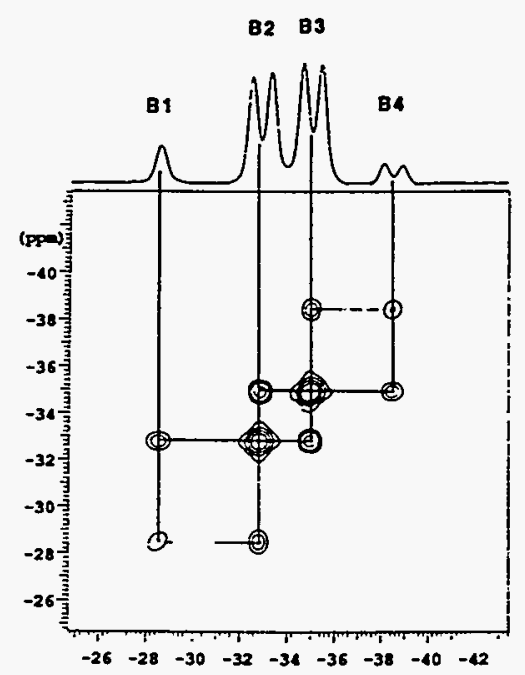

Figure 2. "1B COSY spectrum. The connections among the boron nuclei are indicated.

\section{EXPERIMENTAL PROCEDURES}

All NMR studies were carried out on a Varian UNITY spectrometer (11.7 Tesla, 160 $\mathrm{MHz}$ for ${ }^{11} \mathrm{~B}$ ). The chemical shifts of four types of "B in BSH (see Fig. 1). were measured 
with variation of temperature and concentration of serum albumin. Three types of serum albumin - Human (HSA), Dog (DSA) and Bovine (BSA)- were used at concentrations of $1 \%$ to $5 \%(w / v)$. The proteins were purchased from Sigma and used without further purification. Saturated boric acid solution was used as an external reference for the chemical shift. The boron connectivities were determined by a ${ }^{11} \mathrm{~B}$ chemical shift correlation spectrum (Fig. 2). The " ${ }^{13}$ and proton coupling constant was measured. The concentration of BSH was $200 \mathrm{ugB} / \mathrm{mL} \mathrm{D}_{2} \mathrm{O}$, at boron natural abundance, in phosphate buffer at $\mathrm{pD}=7.4$. A $3000 \mathrm{~Hz}$ sweep width and $8 \mathrm{k}$ data points were used. Quartz NMR sample tubes and baseline correction were used to exclude the background interference of the borosilicate glass. The ${ }^{11} \mathbf{B}$ linewidths and longitudinal relaxation rates of BSH were studied at $295 \mathrm{~K}$ and $310 \mathrm{~K}$ with protein titration from $1 \%$ to $5 \%(w / v)$. Two to 3 samples were used for each protein concentration. The ${ }^{11} B$ linewidth and $R_{1}$ were measured 2 to 3 times for each sample, and calculated 2-3 times with different phase and baseline correction. The calculated values were then analyzed statistically. The "${ }^{1 B} \mathrm{~B}$ linewidths, $W^{b}, e$, were obtained by subtraction ( $W^{b}, e=W^{0}-$ $\mathrm{W}^{\prime \prime}$ ) as discussed above. The viscosity effect on the longitudinal relaxation rates was corrected as follows. The flow times of water and of $1 \%-4 \%$ aqueous solution of BSA were measured using an Otswald viscometer immersed in a water bath at $295 \mathrm{~K}$ and $310 \mathrm{~K}$. Two $\mathrm{mL}$ of each sample was transferred into the viscometer. The flow time of each solution were averaged from 5 to 6 measurements. The densities of water and protein solutions were taken from literature ${ }^{[8]}$. Since the molecular weights of the three proteins are similar, the flow times measured for BSA were also used for HS/ ind DSA. The viscosity correction coefficient, $\mathrm{f}^{\mathrm{v}}$, and the longitudinal relaxation rates, $R *_{1}$, were then calculated using equations (1) and (2).

\section{RESULTS AND DISCUSSION}

The "B chemical shifts of BSH are listed in table 1. The chemical shifts remain the same for various temperatures (295 to $310 \mathrm{~K}$ ) and varying concentrations of BSA, HSA and DSA (1\% to 5\%). The "B chemical shift of B1 moves $3 \mathrm{ppm}$ downfield when a disulfide bridge is formed due to BSH reacting with cysteine ${ }^{[2]}$. However, the observation here indicates that there is a non-covalent binding, with no disulfide bond formed between BSH and these proteins. The observed ${ }^{1} \mathrm{~J}_{1_{1}-I_{\mathrm{H}}}$ is $126 \mathrm{~Hz}$ for all the proton bonded borons and does not change with protein titration and temperature variation which is consistent with non-covalent binding.

\section{Table 1. The Chemical Shift* of BSH}

$\begin{array}{llll}\text { B1 } & \text { B2 } & \text { B3 } & \text { B4 } \\ -28.41 \pm 0.08 & -32.80 \pm 0.04 & -34.92 \pm 0.03 & -38.50 \pm 0.00 \\ \text { * Saturated } \mathrm{H}_{3} \mathrm{BO}_{3}=0 \text { as external reference. } & \end{array}$

Table 2 shows the subtracted linewidths, $W^{b, e}$, for $B 2$ and $B 3$ of BSH in the presence of $1 \%$ to $3 \%$ concentrations of HSA. The fact that $\mathrm{W}^{\mathrm{b}, \mathrm{e}}$ decreases as temperature increases from $295 \mathrm{~K}$ to $310 \mathrm{~K}$ indicates a fast exchange between the unbound and bound $\mathrm{BSH}$ as discussed above. A similar behavior was observed during the titration of all three types of serum albumin, which indicates a fast exchange in the BSH binding with all these proteins.

Table 2. The Line width Wb,e $(\mathrm{Hz})$ of BSH $v s$, temperature change

$\begin{array}{lllllll} & \text { B2 } & \text { B2 } & \text { B2 } & \text { B3 } & \text { B3 } & \text { B3 } \\ & \text { 1\%HSA } & \text { 2\%HSA } & \text { 3\%HSA } & 1 \% \text { HSA } & 2 \% \text { HSA } & 3 \% H S A \\ \text { 295K } & 19.83 \pm 1.77 & 46.03 \pm 2.82 & 61.33 \pm 3.42 & 15.57 \pm 0.55 & 37.97 \pm 2.37 & 56.92 \pm 2.77 \\ 310 \mathrm{~K} & 4.33 \pm 3.22 & 16.33 \pm 1.59 & 26.33 \pm 7.35 & 1.60 \pm 3.61 & 14.50 \pm 2.51 & 20.47 \pm 2.75\end{array}$

The longitudinal relaxation times of boron were measured at 295 and $310 \mathrm{~K}$ with the titration of serum albumin. After correction for the viscosity, the longitudinal relaxation rate, $\mathrm{R}^{*}$, of the four types of boron are plotted vs. the concentration of protein (Fig. 3). Similar patterns are observed from the plots of all three types of protein. The $\mathrm{R}^{*}{ }_{1}$ of $\mathrm{Bl}$ is less affected by the 

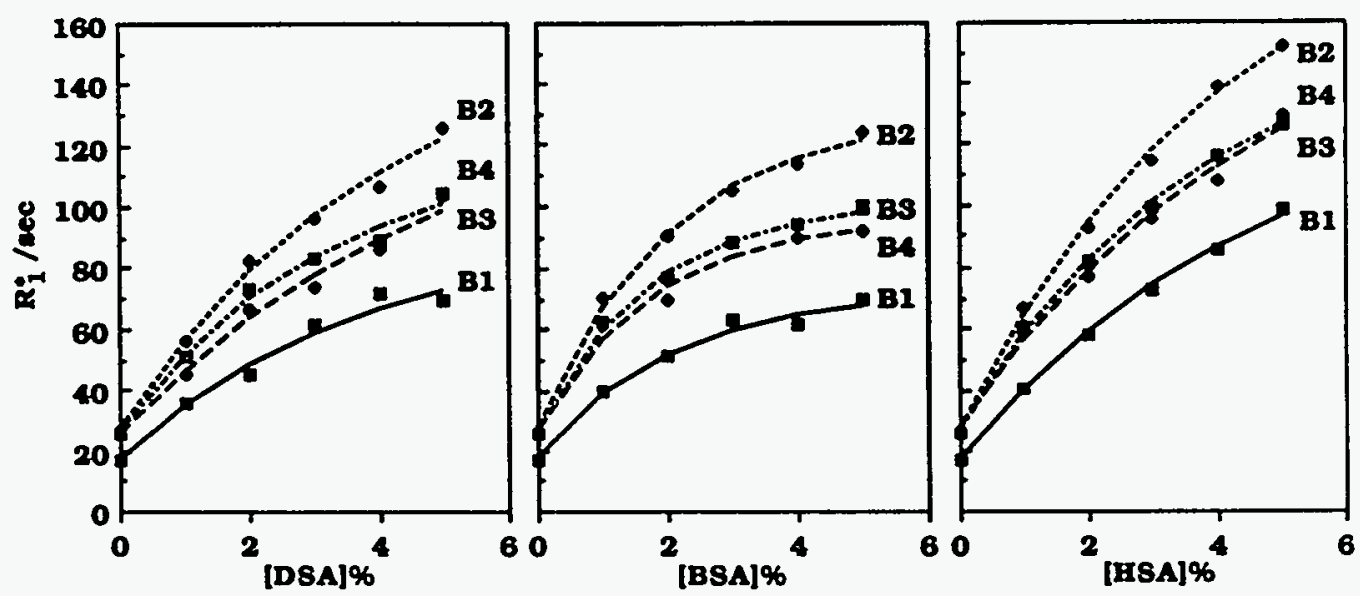

295K
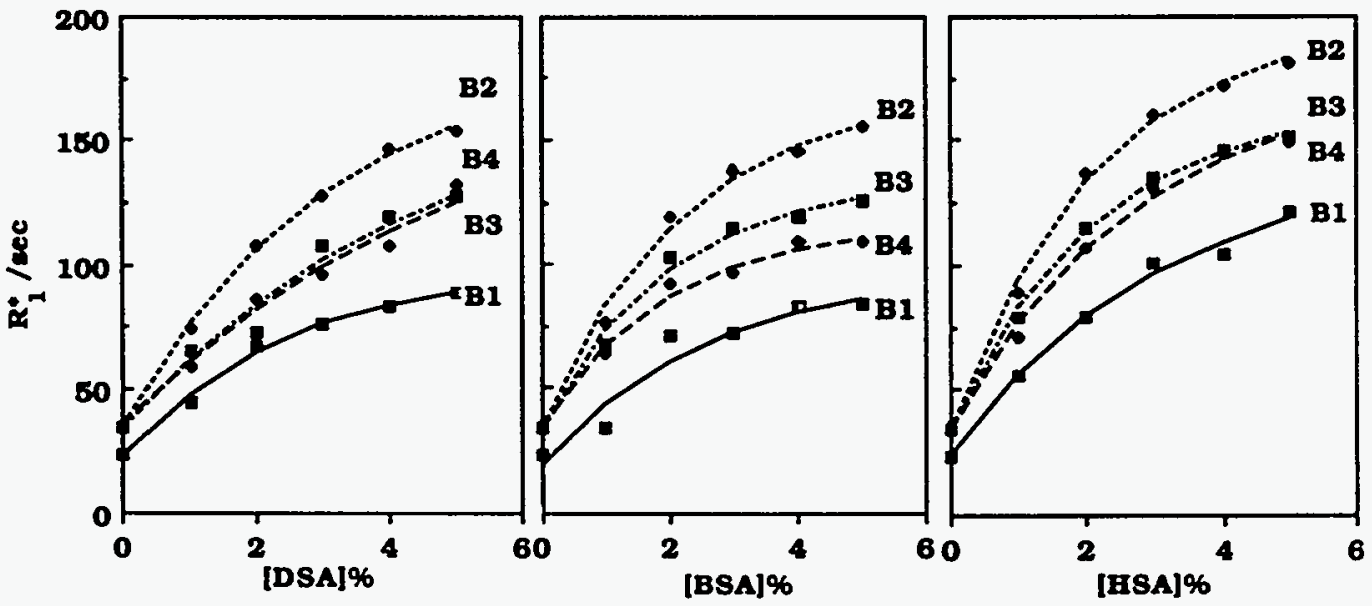

Fig 3. The longitudinal relaxation rate $v s$ the concentration of the serum albumin. The points are the measured rate after viscosity correction and the lines are that calculated using the fitting function.

titration, as indicated by the lowest position in these plots. This also indicates that there is no formation of a disulfide bridge, since B1 would be most affected by such a event.

These plots also show that the longitudinal relaxation rate linearly increases with the concentration of serum albumin up to about $3 \%$, then reaches a maximum value. This behavior may indicate that the interaction of BSH and serum albumin is saturated at about $3 \%$ protein, with the limited amount of BSH available. To prove that saturation occurs, a saturation function was used to fit the observed data:

$$
\mathrm{Y}=\mathrm{Y}_{\mathrm{o}}-\mathrm{ae}-\mathrm{bX}
$$

where $\mathrm{Y}$ is the observed relaxation rate at albumin concentration $\mathrm{X}, \mathrm{Y}_{\mathrm{o}}$ is the relaxation rate as $X$ approaches infinity, and $a$ and $b$ are the fitting coefficients. According to the binding equilibrium, as the concentration of serum albumin approaches infinity, the BSH can be considered as all bound to albumin, therefore $Y_{0}$ corresponds to the longitudinal relaxation rate of bound $\mathrm{BSH}$. With the measured relaxation rate of unbound BSH and the calculated rate of bound BSH, the binding fraction is calculated using equation 3 , and the binding constant $\mathrm{K}$ and the number of binding sites $n$ are evaluated using equation 4 . Table 3 lists the binding parameters. 
Table 3. The Binding Parameters

A. The binding fraction ( $f$ ) of BSH at various concentration of serum albumin

\begin{tabular}{lllllll} 
& \multicolumn{3}{l}{$295 \mathrm{~K}$} & & \multicolumn{3}{c}{$310 \mathrm{~K}$} \\
& DSA & HSA & BSA & DSA & HSA & BSA \\
$1 \%$ & $.26 \pm .01$ & $.31 \pm .04$ & $.34 \pm .03$ & $.23 \pm .02$ & $.21 \pm .02$ & $.42 \pm .03$ \\
$2 \%$ & $.46 \pm .03$ & $.55 \pm .07$ & $.63 \pm .03$ & $.41 \pm .03$ & $.35 \pm .03$ & $.63 \pm .02$ \\
$3 \%$ & $.60 \pm .03$ & $.70 \pm .06$ & $.74 \pm .05$ & $.54 \pm .05$ & $.48 \pm .03$ & $.79 \pm .03$ \\
$4 \%$ & $.71 \pm .03$ & $.79 \pm .03$ & $.83 \pm .03$ & $.64 \pm .05$ & $.59 \pm .03$ & $.87 \pm .03$ \\
$5 \%$ & $.79 \pm .02$ & $.85 \pm .05$ & $.89 \pm .03$ & $.74 \pm .02$ & $.68 \pm .03$ & $.93 \pm .01$
\end{tabular}

B. The binding constant $(\mathrm{K})$ and the number of binding sites (n) on albumin

$\begin{array}{lllllll} & & 295 K & & & 310 \mathrm{~K} & \\ & \text { DSA } & \text { HSA } & \text { BSA } & \text { DSA } & \text { HSA } & \text { BSA } \\ \mathrm{K}\left(\text { mole }^{-1}\right) & 2.89 & 3.11 & 3.69 & 1.80 & 1.27 & 4.87 \\ \mathrm{n} & 3.40 & 4.15 & 4.70 & 3.48 & 3.42 & 5.14\end{array}$

The obtained number of binding sites is consistent with that obtained using other methods ${ }^{[9]}$.

\section{CONCLUSIONS}

1) There is non-covalent binding and no formation of a disulfide bridge between BSH and the three types of albumin.

2) The bound and unbound BSH are under fast exchange.

3) The number of binding sites on albumin is about 3 to 5 .

4) The binding constant is about $3\left(\mathrm{~mole}^{-1}\right)$ at $295 \mathrm{~K}$ and $1.5\left(\mathrm{~mole}^{-1}\right)$ at $310 \mathrm{~K}$, which means that the binding effect decreases as temperature increases. The exception was observed for BSA, which gives a binding constant $4.87\left(\mathrm{~mole}^{-1}\right)$ at $310 \mathrm{~K}$. The inconsistency may result from the purity and water content of the sample.

\section{ACKNOWLEDGMENT}

This project was supported in part by the US. Dept. of Energy, contract \# DE-AC07$76 \mathrm{ID} 01570$.

\section{REFERENCES}

1. T. Nakagawa and T. Nagai, Interaction between serum albumin and mercaptoundecahydrododecaborate ion (an agent for boron-neutron capture therapy of brain tumor), Chem. Pharm. Bull. 24:2934 (1976).

2. E. G. Samsel and D. L. Miller, High resolution ${ }^{10} B$ and ${ }^{11} B$ nuclear magnetic resonance (NMR) spectroscopy of $\mathrm{Na}_{2} \mathrm{~B}_{12} \mathrm{H}_{11} \mathrm{SH}$ impurities and metabolites, Strah. Onkol. 165:140 (1989).

3. W. F. Bauer, K. M. Bradshaw and T. L. Richards, Interaction between boron containing compounds and serum albumin observed by nuclear magnetic resonance, in 'Progress in Neutron Capture Therapy for Cancer' Ed: B. J. Allan, D. E. Moore and B. V. Harrington. Plenum Press, p 339, 1990.

4. K. M. Bradshaw, W. F. Bauer and T. L. Richards, Observation of the $\mathrm{Na}_{2} \mathrm{~B}_{12} \mathrm{H}_{11} \mathrm{SH}$ binding to serum albumin species, presented at the boron USA II workshop, Research Triangle Park, NC (1990).

5. R. A. Dwek, in 'Nuclear Magnetic Resonance in Bio-Chemistry', Clarendon Press Oxford, (1975).

6. F. A. Bovey, in 'Nuclear Magnetic Resonance Spectroscopy' 2nd Ed. p3, Acad. Press, (1988).

7. A. Z. Khan and L. Aarons, Design and analysis of protein binding experiments, J. Theo. Biol. 140:145 (1989).

8. CRC Handbook of Chemistry and Physics, Ed. R. C. Weast, CRC Press, 60: F11 and D258, (1980).

9. W. F. Bauer, INEL Internal Report, (1991). 



\title{
Purity and Quality Determinations of Borocaptate Sodium
}

\author{
A. Gianotto and W. Bauer
}

This article is a reprint from Advances in Neutron Capture Therapy, pp. 459-463, (1993). 



\author{
A. K. Gianotto and W. F. Bauer \\ Idaho National Engineering Laboratory, EG\&G Idaho, Inc. \\ Idaho Falls, ID 83415
}

\title{
INTRODUCTION
}

The chemical purity or "quality" of any drug can influence it's action in the body. Knowledge of the chemical purity of the boron delivery agent borocaptate sodium $\left(\mathrm{Na}_{2} \mathrm{~B}_{12} \mathrm{H}_{11} \mathrm{SH}\right.$ or $\left.\mathrm{BSH}\right)$ can have a direct effect upon it's toxicity ${ }^{1}$, biodistribution ${ }^{2}$ and the proper interpretation of experimental data ${ }^{3}$. The FDA requires that drugs seeking Orphan Drug status or an investigational new drug (IND) be chemically characterized ${ }^{4}$. The purity of BSH is most often determined by liquid chromatographic procedures ${ }^{1,4}$. The purity and "quality" of any chemical compound is only as good as the techniques used to characterize it and the number of potential impurities actively looked for in the sample. For these reasons, the somewhat more rigorous approach to purity determinations of BSH described here has been adopted by our laboratory.

\section{EXPERIMENTAL}

High performance liquid chromatography (HPLC) was performed with 20-50 $\mu \mathrm{L}$ injections onto a 300 or $250 \times 8 \times 4$ or $3 \mathrm{~mm}$ column packed with 5 or $10 \mu \mathrm{m}$ particles of Nucleosil ${ }^{\oplus} C_{18}$ (octadecyl) from Machery-Nagel. The mobile phase was a 50:50 $\mathrm{CH}_{3} \mathrm{OH}: \mathrm{H}_{2} \mathrm{O}$ solution with $5 \mathrm{mM}$ TBAS ion pairing reagent (Alltech Associates, Inc.) adjusted to $\mathrm{pH} 8.1$ and flowing at $1.0 \mathrm{~mL} / \mathrm{min}$ with the $4 \mathrm{~mm}$ inner diameter columns. Detection was performed from 200-300 nm with a Waters 991 Photodiode Array Detector.

Elemental determinations of boron, sulfur, and sodium were performed by inductively coupled plasma-atomic emission spectroscopy (ICP-AES). Aliquots (150 $\mu \mathrm{L})$ of samples and standards previously prepared for HPLC were either simply diluted or were digested with $3 \mathrm{~mL}$ of $\mathrm{HNO}_{3}$ prior to the final dilutionto $10 \mathrm{~mL}$. Analyses of carbon, hydrogen and nitrogen were performed on a Model EA 1108, Carlo Erba CHNS Analyzer. A nitrogen atmosphere was utilized as much as possible for preparation of the samples used in the combustion analyses to avoid additional contamination with water.

The ${ }^{10} \mathrm{~B}$ and ${ }^{11} \mathrm{~B}$ nuclear magnetic resonance (NMR) spectra were obtained on a Bruker Model AC300 NMR. The sample was simply diluted with water and spiked with $\mathrm{D}_{2} \mathrm{O}$ to provide a lock signal. 
Samples were prepared and handled for Fourier transform infrared (FTIR) spectroscopic analysis entirely under nitrogen as potassium bromide $(\mathrm{KBr})$ pellets. The actual sample mass in each pellet was not accurately determined, consequently, the spectra were normalized to offset sample mass differences. All spectra were collected on a Digilab Model 65 FTIR.

\section{DISCUSSION}

Normally, HPLC is used to determine the "purity" of BSH salts. The primary analytes are usually the dimer (BSS) and oxidized dimer (BSSO). Typical chromatograms showing the separation of the oxidized species from the BSH parent peak are shown in Figure 1 and some actual BSS and BSSO concentrations are listed in Table 1. A variety of problems with the chromatography and it's use in determining contamination levels are also demonstrated in Figure 1. Sample AA has an obvious interference on the leading edge of the BSS peak which can be expected to bias the results high. Sample AA also has significant levels of some contaminant that elutes very late ( $>25$ minutes) in the chromatogram. These late eluting components have not yet been identified but show up consistently in this sample and in many others to varying degrees. There are also additional contaminants that elute in and around the BSS and BSSO peak and some possibly even before the BSH parent peak. These components have not been positively identified but are believed to be byproducts of the reaction to produce BSH (see for example ref. 5 and 6).

Because of the possibility of serious contamination by other chemicals going undetected by HPLC, ICP-AES was used to determine elemental concentrations of S, B and $\mathrm{Na}$ in the BSH samples. Table 2 gives some typical results from these determinations. The utility of this analysis is demonstrated by sample FF which shows a noticeable problem with an excess of $\mathrm{Na}(\mathrm{Na} / \mathrm{S}=2.47$ and $\mathrm{B} / \mathrm{Na}=5.23)$. The HPLC chromatograms of FF were unremarkable, however this sample obviously contains a nonboron containing sodium salt.

The $\mathrm{B} / \mathrm{S}$ ratios in Table 2 should be at or near 12, however these ratio's are typically between 12 and 13 , possibly partially due to a slight bias (1-2\%) in the B

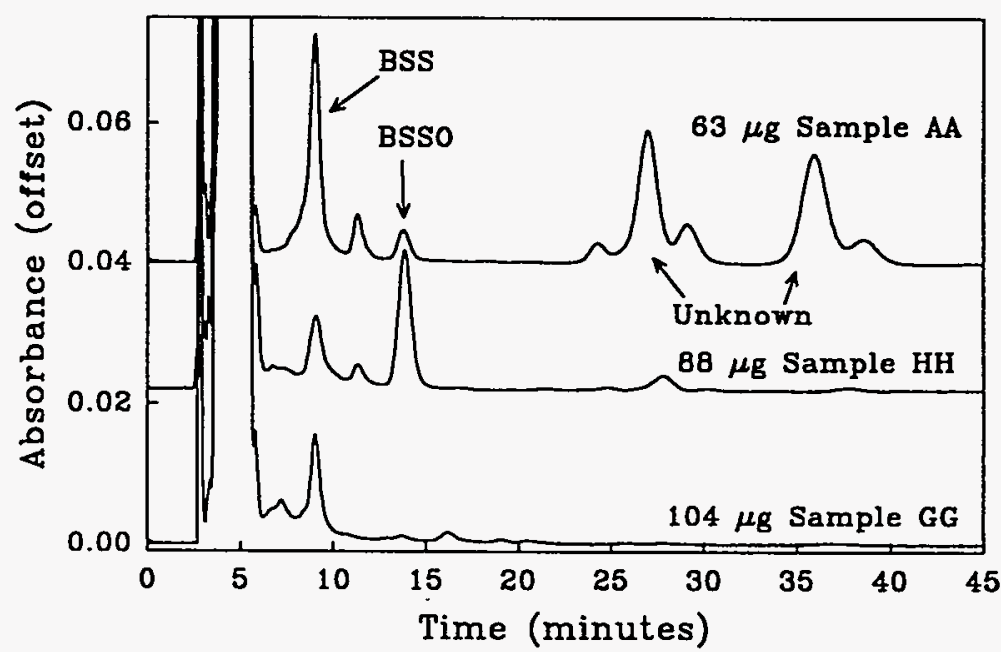

Figure 1. Chromatograms of BSH samples. $20 \mu \mathrm{L}$ injection into $5 \mathrm{mM}$ TBAS in $50: 50 \mathrm{CH}_{3} \mathrm{OH}: \mathrm{H}_{2} \mathrm{O}$ flowing at $1 \mathrm{~mL} / \mathrm{min}$. Chromatogram plotted at $215 \mathrm{~nm}$. 
Table 1. Typical results of HPLC analyses for BSS and BSSO contamination of BSH salts.

\begin{tabular}{|c|c|c|c|c|}
\hline Sample & Compound" & $\begin{array}{l}\text { BSS }^{4} \\
\text { (wt\%) } \\
\end{array}$ & $\begin{array}{l}\text { BSSO }^{4} \\
\text { (wt\%) }\end{array}$ & $\begin{array}{l}\mathrm{B}_{12} \mathrm{H}_{12}{ }^{2} \\
(w t \%)^{6} \\
\text { (wt }\end{array}$ \\
\hline AA & $\mathrm{Na}_{2} \mathrm{~B}_{12} \mathrm{H}_{11} \mathrm{SH}$ & $0.9 \pm 0.1$ & $0.097 \pm 0.004$ & ND \\
\hline BB & $\mathrm{Na}_{2}{ }^{10} \mathrm{~B}_{12} \mathrm{H}_{11} \mathrm{SH}$ & $0.07 \pm 0.02$ & $0.277 \pm 0.009$ & ND \\
\hline $\mathrm{CC}$ & $\mathrm{Na}_{2}{ }^{10} \mathrm{~B}_{12} \mathrm{H}_{11} \mathrm{SH}$ & $1.1 \pm 0.1$ & $0.34 \pm 0.01$ & $\sim 37$ \\
\hline $\mathrm{DD}$ & $\mathrm{Cs}_{2} \mathrm{~B}_{12} \mathrm{H}_{11} \mathrm{SH}$ & $0.35 \pm 0.05$ & $0.29 \pm 0.02$ & $\sim 3$ \\
\hline EE & $\mathrm{Cs}_{2} \mathrm{~B}_{12} \mathrm{H}_{11} \mathrm{SH}$ & $0.40 \pm 0.06$ & $0.14 \pm 0.02$ & $<1$ \\
\hline FF & $\mathrm{Na}_{2}{ }^{10} \mathrm{~B}_{12} \mathrm{H}_{11} \mathrm{SH}$ & $0.09 \pm 0.02$ & $\mathrm{ND}^{\mathrm{e}}$ & ND \\
\hline GG & $\mathrm{Na}_{2}{ }^{10} \mathrm{~B}_{12} \mathrm{H}_{11} \mathrm{SH}$ & $0.23 \pm 0.05$ & $\mathrm{ND}^{\mathrm{c}}$ & ND \\
\hline $\mathbf{H H}$ & $\mathrm{Na}_{2}{ }^{10} \mathrm{~B}_{12} \mathrm{H}_{11} \mathrm{SH}$ & $0.20 \pm 0.07$ & $0.35 \pm 0.02$ & \\
\hline
\end{tabular}

ND $=$ Not detected

- All concentrations have been corrected for isotopic abundance.

- Single chromatograms with a single $\mathrm{B}_{12} \mathrm{H}_{12}{ }^{2}$ standard.

- Not detected at less than $\sim 0.05$ weight percent.

determinations. The $\mathrm{B} / \mathrm{S}$ and $\mathrm{Na} / \mathrm{S}$ ratios of sample $\mathrm{CC}$ are excessively high even though the $\mathrm{B} / \mathrm{Na}$ ratio is near normal. This is an obvious indication of a boron containing sodium salt, most likely to be $\mathrm{Na}_{2} \mathrm{~B}_{12} \mathrm{H}_{12}$. A closer look at the HPLC chromatogram results indicated that the $\mathrm{B}_{12} \mathrm{H}_{12}{ }^{2-}$ anion may have been present but eluted just slightly before, but was not completely resolved from the BSH peak. Since the HPLC conditions used to determine BSS and BSSO were unable to separate $\mathrm{B}_{12} \mathrm{H}_{12}{ }^{2-}$ from $\mathrm{BSH}$, the conditions were adjusted and the chromatograms of $\mathrm{CC}$ in Figure 2 were obtained. The presence of $\mathrm{B}_{12} \mathrm{H}_{12}{ }^{2-}$ was confirmed. The utility of the photodiode array detection is also demonstrated in Figure 2. Both chromatograms were from a single run and the increased detectibility of $\mathrm{B}_{12} \mathrm{H}_{12}{ }^{2-}$ at $205 \mathrm{~nm}$ could be exploited. The NMR spectra in Figure 3 also indicates the significant $\mathrm{B}_{12} \mathrm{H}_{12}{ }^{2-}$ contamination of sample CC. Most NMR spectra of $\mathrm{BSH}$ show a slightly larger peak than expected peak at $\delta-15 \mathrm{ppm}$. Could this peak height enhancement be due in part to non BSH contaminants?

Table 2. Mole ratios determined from elemental analyses by ICP-AES.

\begin{tabular}{ccccc}
\hline \hline & & \multicolumn{3}{c}{ Mole Ratios } \\
\cline { 3 - 5 } Sample & Compound & $\mathrm{B} / \mathrm{S}$ & $\mathrm{B} / \mathrm{Na}$ & $\mathrm{Na} / \mathrm{S}$ \\
\hline \hline $\mathrm{AA}$ & $\mathrm{Na}_{2} \mathrm{~B}_{12} \mathrm{H}_{11} \mathrm{SH}$ & 13.3 & 6.4 & 2.1 \\
$\mathrm{BB}$ & $\mathrm{Na}_{2}{ }^{10} \mathrm{~B}_{12} \mathrm{H}_{11} \mathrm{SH}$ & 12.7 & 6.5 & 2.0 \\
$\mathrm{CC}$ & $\mathrm{Na}_{2}{ }^{10} \mathrm{~B}_{12} \mathrm{H}_{11} \mathrm{SH}$ & 19.1 & 5.2 & 3.7 \\
$\mathrm{DD}$ & $\mathrm{Cs}_{2} \mathrm{~B}_{12} \mathrm{H}_{11} \mathrm{SH}$ & $13.2 \pm 0.2$ & & \\
EE & $\mathrm{Cs}_{2} \mathrm{~B}_{12} \mathrm{H}_{11} \mathrm{SH}$ & $12.6 \pm 0.3$ & & \\
FF & $\mathrm{Na}_{2}{ }^{10} \mathrm{~B}_{12} \mathrm{H}_{11} \mathrm{SH}$ & $12.83 \pm 0.06$ & $5.23 \pm 0.06$ & $2.47 \pm 0.06$ \\
$\mathrm{GG}$ & $\mathrm{Na}_{2}{ }^{10} \mathrm{~B}_{12} \mathrm{H}_{11} \mathrm{SH}$ & $12.8 \pm 0.6$ & $6.4 \pm 0.1$ & $2.0 \pm 0.1$ \\
$\mathrm{HH}$ & $\mathrm{Na}_{2}{ }^{10} \mathrm{~B}_{12} \mathrm{H}_{11} \mathrm{SH}$ & 13 & 6.5 & 2.0 \\
\hline \hline
\end{tabular}




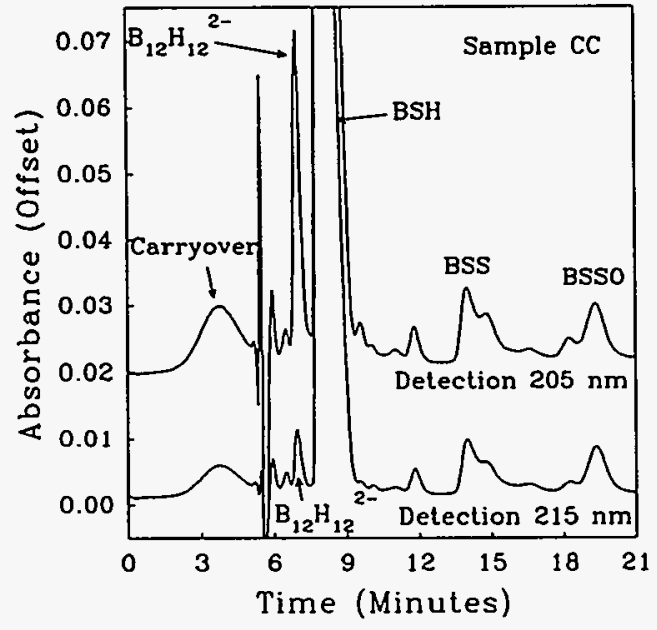

Figure 2. HPLC showing major contamination of $\mathrm{CC}$ with $\mathrm{B}_{12} \mathrm{H}_{12}{ }^{2}$. Conditions same as in Figure 1 but flowing at $0.5 \mathrm{~mL} / \mathrm{min}$.

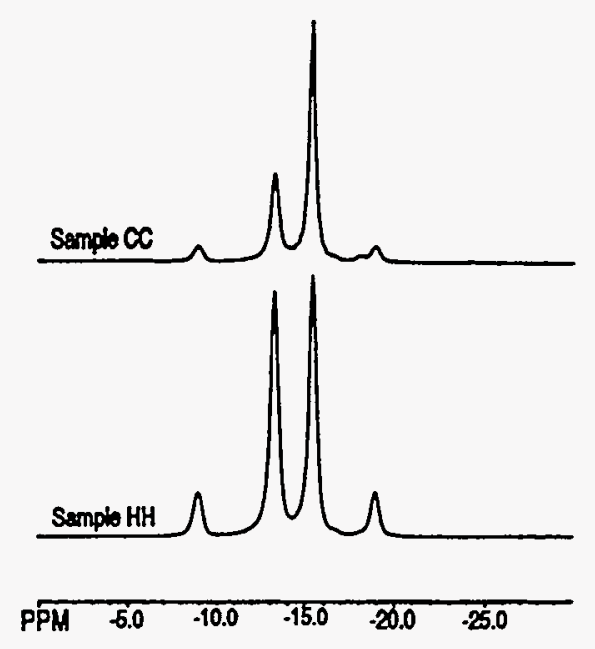

Figure 3. ' $\mathrm{H}$ decoupled ${ }^{10} \mathrm{~B}$ NMR spectra for ${ }^{10} \mathrm{BSH}(\mathrm{HH})$ and ${ }^{10} \mathrm{BSH}$ with significant ${ }^{10} \mathrm{~B}_{12} \mathrm{H}_{12}{ }^{2}$ (CC).

Many of the potential contaminants are organic in nature such as reagents used in the synthesis and some intermediate products. Elemental CHN analysis should point out significant contamination of $\mathrm{BSH}$ with these materials. The $\mathrm{CHN}$ analysis results indicated that sample FF had a relatively high level of $C$ contamination $(-1.7 \mathrm{wt} \% \mathrm{C})$ and sample $\mathrm{EE}$ had excessive contamination by an organic material ( $\sim 5.89 \mathrm{wt} \% \mathrm{C})$. Subsequent Fourier Transform Infrared (FTIR) spectroscopy (Figure 4) confirmed the presence of an organic solvent in sample EE that was not identifible by HPLC and indicated that an unidentified hydrocarbon was present in FF. Also notable in the IR spectra are the obvious presence of significant water in samples FF and AA while water is absent in sample EE.

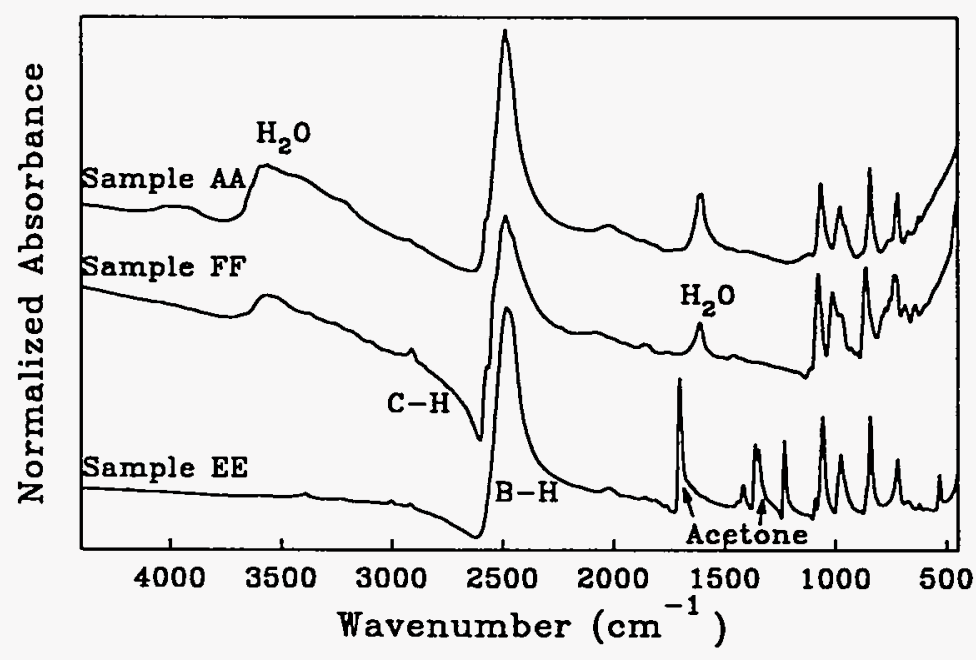

Figure 4. Infrared spectra of $\mathrm{BSH}$ samples obtained from $\mathrm{KBr}$ pellets. 


\section{CONCLUSIONS}

The value of a multitechnique approach to the chemical characterization of BSH salts has been demonstrated. Liquid chromatography may be used to quantitatively determine BSS and BSSO as well as to identify the presence of other unknown compounds. Elemental analysis may be used to confirm stoichiometry and can ultimately identify some impurities such as other salts containing B, S, or Na. Spectroscopic such as NMR and FTIR techniques can not only identify the presence of major contaminants but will also provide confirmation of the chemical nature of the contaminant. With the use of all of the analytical techniques described here, some unidentified impurities have been discovered and these impurities are known to exist in much of the $\mathrm{Na}_{2} \mathrm{BSH}$ used for BNCT research.

\section{ACKNOWLEDGEMENTS}

This work was performed under the auspices of the U.S. Department of Energy, Office of Energy Research, under DOE Field Office, Idaho, Contract No. DE-AC07$761 \mathrm{D} 01570$.

\section{REFERENCES}

1. T. Yoshizaki, 1. Ikechi, and K. Nagasawa (1986) in Neutron Capture Therapy, ed. H. Hatanaka (Nishimura Co., Ltd., Niigata, Japan), pp. 394-400.

2. D. Slatkin, P. Micca, A. Forman, D. Gabel, L. Wielopolski, and R. Fairchild (1986) Biochemical Pharmacology 35, 1771-1776.

3. A.H. Soloway, F. Alam, and R.F. Barth in Workshop on Neutron Capture Therapy, eds. R.G. Fairchild and B.P. Bond, (Brookhaven National Laboratory, Upton), BNL-51994, pp162-172.

4. J.M. Shremmer, D.J. Noonan, J.L. Russell, Jr., D.C. Johnson and T.Z. Polta (1986) in Neutron Capture Therapy, ed. H. Hatnaka (Nishimura Co., Ltd., Niigata, Japan) 388-393.

5. E. I. Tolpin, G.R. Wellum and S.A. Berley (1978) Inorg. Chem. 17, 2867-2873.

6. B. Gniner, Z. Plzák, and I.Viň̌ (1991) J. Chrom. 588, 201-210. 



\section{PLENARY PRESENTATION: Large Animal Studies on the Use of BNCT for the Treatment of Brain Tumors}

P. Gavin, C. DeHaan, M. Moore, J. Weidner, D. Swartz, S. Kraft, C. Atkinson,

C. Amaro, W. Bauer, and A. Siefert

This article is a reprint from Advances in Neutron Capture Therapy, pp. 469-475, (1993). 


\author{
P.R. Gavin, C.E. DeHaan, M.P. Moore, J.P. Weidner, C.D. Swartz, ${ }^{1}$ S.L. \\ Kraft, ${ }^{2}$ C.A. Atkinson, C.R. Amaro, W.F. Bauer, ${ }^{3}$ and A. Siefert ${ }^{4}$ \\ 'Washington State University, Pullman, WA, ${ }^{2}$ Kansas State University, \\ Manhattan, KS, ${ }^{3}$ Idaho National Engineering Laboratory, Idaho Falls, ID, ${ }^{4} \mathrm{Joint}$ \\ Research Centre, Petten, The Netherlands
}

\title{
INTRODUCTION
}

Animal models are useful to study the relative effects of BNCT on normal tissues and tumors. True efficacy studies of the modality on glioblastoma or other human malignancies requires human clinical trials. The use of large animals, primarily dogs, to study the effects of BNCT has been and continues to be of major interest. The use of large animals permits the study of normal tissue tolerance of the tissues of the head at an acceptable total body dose. These studies have been extended from normal dogs to dogs with induced and spontaneous tumors. While induced tumors in rodents are often used to study tumor response, the total body dose accompanying BNCT severely limits their use for normal tissue tolerance studies, especially with the epithermal-neutron beams.

\section{LITERATURE REVIEW}

Previous large animal model studies were done primarily with dogs, BSH, and thermalneutron beams. ${ }^{1-4}$ The reported tolerance of the normal canine brain was in the 35-90 Gy range, far exceeding the known tolerance of the canine brain of $15 \mathrm{~Gy}$ from a single fraction of megavoltage $\mathrm{x}$-rays. ${ }^{5}$ The authors reported the geometric sparing effects to the brain capillary endothelial cells may be responsible for the tolerance observed at these high physical radiation doses. However, the "contaminant" gamma and neutron irradiation should have exceeded tolerance. Several compounding factors must be considered. Some of the studies were done through the intact skin and overlying musculature, while some had the superficial tissues reflected to allow the thermal-neutron beam better penetration into the brain. Even with the reflected studies, the volume of the brain irradiated to these high doses would be small due to the extremely rapid fall-off of the thermal-neutron beam. Boron measurement accuracy and neutron dosimetry have also improved considerably since these irradiations were performed. In addition, the pharmacokinetics of the BSH compound have since been well defined following a single administration of intravenous BSH. ${ }^{6}$ It should also be recognized that the peak physical dose assumes this dose is delivered to a volume large enough to obtain charged particle equilibrium. Therefore, BNCT doses as quoted could only be achieved within the lumen of vessels measuring $20 \mu$ in diameter or greater. The capillary (5-7 $\mu$ diameter) dose would be substantially less.

Studies using thermal- and fast-neutron beams and BNCT following BSH administration have been performed using swine. ${ }^{8}$ Swine skin is an excellent model for human skin. However, the authors reported difficulty in obtaining a relative biological effectiveness (RBE) for the boron capture reactions due to insufficient knowledge of the BSH distribution in the skin of swine at that time.

Advances in Neutron Capture Therapy, Edited by A.H Soloway et al., Plenum Press, New York, 1993 
An interesting study was performed in neonatal beagles bearing an implanted, viral-induced, intracerebral tumor.9 There was a suggestion from the results that the BNCT was successful in eradicating some of these tumors. Unfortunately, this is a highly immunogenetic tumor and spontaneous regression was noted in the control group. Numbers of animals treated versus control were insufficient to establish statistical significance. In the treated group, there were 5 animals without residual tumor at necropsy and subsequent histopathologic examination. Two of the animals lived beyond 250 days and were sacrificed with no apparent complications. Two animals died 20 and 33 days post-treatment with no residual tumor but a suggestion of normal tissue damage. One animal that was not normal, and was sacrificed at 23 days post-treatment, also showed no residual tumor. This experiment would indicate that the treatment was successful in eradicating the tumors from these animals. The radiation dose to the normal tissues may have been excessive in some animals. The use of induced immunogenetic tumors in dogs is most appropriate for short-term studies. Due to the problem with spontaneous remission in some animals, the model is not well suited to look at tumor control and delayed normal tissue reactions that generally occur 5-6 months following treatment.

\section{CURRENT STUDIES}

Currently there are 2 active large animal BNCT programs--one, the Idaho National Engineering Laboratory (INEL) sponsored Washington State University (WSU) group using the Brookhaven Medical Research Reactor (BMRR) epithermal beam, and the other, a European community group using the Petten epithermal beam. The INEL group has irradiated 60 normal laboratory dogs to peak physical doses of 0-64.5 Gy with epithermal neutrons alone or after the intravenous infusion of BSH. The European group has irradiated 15 dogs to date with bloodboron concentrations of 0-62 $\mu \mathrm{g} / \mathrm{gm}$ and total peak physical doses of 6.5-23 Gy including a 4fraction study.

Prior to the normal tissue tolerance studies, a large study involving the pharmacokinetics of BSH in normal dogs and dogs with spontaneous brain tumors has been done. ${ }^{10}$ BSH acts in a predictable, 2-compartmental model during the first 10 hours post-administration. Boron concentrations in the normal tissues can be accurately predicted from blood-boron measurements. ${ }^{11}$ Most tissues of the head have similar concentrations on a $\mu \mathrm{g}$ basis with the exception of the normal brain. BSH appears to be excluded by the normal blood-brain barrier, and the boron within the normal brain can be largely accounted for by $4 \%$ blood volume.

Tumor concentrations in dogs with spontaneous tumors have been measured primarily at 3 post-administration time intervals. ${ }^{12}$ The time intervals studied were 2,6 , and 12 hours following the end of infusion. All animals received $50 \mathrm{mg}$ boron per $\mathrm{kg}$ body weight $\mathrm{BSH}$ intravenously at a rate of $1 \mathrm{mg}$ boron per $\mathrm{kg}$ body weight per minute. The mean tumor concentration was generally less than the blood concentration. Improvements in tissue boron analysis allowed tumor samples as small as $10 \mathrm{mg}$ to be accurately determined. A large degree of heterogeneity of the tumor blood-boron concentrations was detected.

The pharmacokinetic data allowed normal tissue concentrations to be readily determined from blood-boron concentrations, sampled following intravenous BSH administration, prior, during, and after irradiation. The INEL group utilizes a $5 \times 10 \mathrm{~cm}$ portal centered over the right hemisphere of large retriever-type dogs. The mean target blood-boron concentrations during irradiation were $0,25,50$, or $100 \mu \mathrm{g} / \mathrm{g}$. The European group utilizes an $8 \mathrm{~cm}$ diameter incident beam centered over the brain of beagles. The dogs irradiated Petten have a mean target of bloodboron concentration of 0,25 , or $50 \mu \mathrm{g} / \mathrm{g}$. Both studies utilize a single dorsal portal. As mentioned above, the Petten group is also looking at the effect of fractionation utilizing 4 fractions in some animals; all other animals were treated with a single fraction.

To date, the WSU dogs have been monitored for up to one year post-irradiation. The total body radiation effect can be detected by changes in the complete blood count. ${ }^{13}$ The changes in the circulating white blood cells could be due to the blood-pool irradiation that would occur during these lengthy irradiations. However, the depression of the platelets would indicate significant total-body bone marrow irradiation. Platelet counts as low as $40,000 / \mathrm{mm}^{3}$ have been observed. All changes in the blood count monitored to date have been responsible for no significant clinical problems, and have returned to normal values within 40 days post-irradiation. The Petten group has noted similar changes in the complete blood counts. ${ }^{14}$

Skin tolerance has been studied by both groups. The WSU dogs have reactions that have varied from mild epilation and hair color change to dermal necrosis. ${ }^{15}$ The group at Petten, to date, has only observed epilation, depigmentation, and dry desquamation. ${ }^{14}$ The higher range of doses used in the WSU dogs revealed some animals with a biphasic skin reaction. The initial 
epithelial reaction peaked at 3 weeks post-irradiation. In the highest dose groups, this reaction consisted of total epilation, depigmentation, and a moist desquamation. These areas healed and became re-epithelialized over the next several weeks. At 3 months post-irradiation, dermal necrosis ensued. The nature of the epithermal beams prevented surgical resection of the necrotic tissue and even prevented successful grafting techniques containing their own vascular supply. The BMRR reactor is an isotropic beam and the margins of the irradiation field become indistinct after scattering in the patient. While the moist desquamation in the early phase and the dermal necrosis in the later phase occur within the incident beam, significant irradiation spreads from the incident beam and easily-recognized histologic damage to the dermis was detected throughout the dorsal region of the head. This loss of sharp beam edges is readily seen in hair color changes. ${ }^{\text {is }}$ In contrast, the Petten beam is a collimated beam and the incident $8 \mathrm{~cm}$ field had the majority of the change and there was an additional $1 \mathrm{~cm}$ rim with milder change. The remaining edge is rather sharply defined. ${ }^{14}$ While this $1 \mathrm{~cm}$ rim increases the easily recognized change by $50 \%$ in volume, the remainder of the head and skin appear unaffected. At this time, it should be emphasized that dermal necrosis, while especially devastating in BNCT, should not be a problem when patients are irradiated with blood-boron concentrations between 25 and $100 \mathrm{ppm}$. At those blood-boron concentrations, brain necrosis occurs at a dose significantly below that required for dermal necrosis. The dermal necrosis observed was so severe that the animals could not be maintained long enough to ensure changes would occur within the CNS. However, groups with physical doses well below that seen in the dermal necrosis animals had the CNS effects mentioned below.

The irradiation effects on the central nervous system, in both WSU and Petten dogs, ranged from an elevated cerebral spinal fluid protein at 3 weeks post-irradiation to ischemic necrosis as early as 19 weeks post-irradiation. The ischemic necrosis seen at 5-6 months post-irradiation was rapidly progressive, could not be alleviated with supportive medical care, and resulted in the animal being humanely terminated. ${ }^{14,16}$

There is an inverse relationship between the blood-boron concentration and the peak physical dose required for an isoeffect. ${ }^{17}$ Evidence to date clearly indicates that there is significant "dose sparing" to the capillary endothelium from the boron fission fragments following neutron capture. The results from epithermal neutron irradiation alone, and with boron capture, have been used to arrive at RBE estimates for the fast-neutron component, and the compound effect (the product of the microdosimetry and RBE) for BSH. The low gamma dose rate may result in a dose reduction factor for the gamma contamination. ${ }^{18}$ These factors will require further study for better understanding. An excellent example of the dose sparing effects is illustrated in Table 1. The 5 dogs irradiated at $50 \mathrm{ppm}$ blood-boron concentration at $27 \mathrm{~Gy}$ showed no magnetic resonance changes at 6 weeks post-irradiation. Four of 4 dogs irradiated at the same peak physical dose at $25 \mathrm{ppm}$ blood-boron concentration had readily demonstrated magnetic resonance changes at 6 months.

Table 1. Six month post-irradiation MRI

\begin{tabular}{|c|c|c|c|c|}
\hline $\begin{array}{l}\text { Treatment } \\
\text { Parameters }\end{array}$ & $\begin{array}{c}\text { Dog } \\
\text { Number }\end{array}$ & \multicolumn{3}{|c|}{ MR Brain Changes } \\
\hline $\begin{array}{r}50 \text { ug/g Boron } \\
@ 27 \mathrm{~Gy}\end{array}$ & $\begin{array}{l}2314 \\
2452 \\
2746 \\
2763 \\
3290\end{array}$ & $\begin{array}{l}0 \\
0 \\
0 \\
0 \\
0\end{array}$ & $\begin{array}{l}0 \\
0 \\
0 \\
0 \\
0\end{array}$ & $\begin{array}{l}0 \\
0 \\
0 \\
0 \\
0\end{array}$ \\
\hline $\begin{array}{r}25 \text { ug/g Boron } \\
@ 27 \text { Gy }\end{array}$ & $\begin{array}{l}2311 \\
3300 \\
2320 \\
2465\end{array}$ & $\begin{array}{l}2 \\
1 \\
2 \\
1\end{array}$ & $\begin{array}{l}2 \\
2 \\
2 \\
2\end{array}$ & $\begin{array}{l}2 \\
1 \\
1 \\
2\end{array}$ \\
\hline $\begin{array}{l}T_{1}=T R 400 \\
T_{2}=T R 2000 \\
G d \text { - Gadolium- } \\
\text { Relative MR ch } \\
\text { Treatment paras } \\
G y=\text { total phy }\end{array}$ & $\begin{array}{l}\text { ent } \\
\text { ange, } 1 \\
\text { tean bloc } \\
\text { e at the }\end{array}$ & 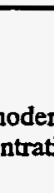 & $=$ & \\
\hline
\end{tabular}


The currently used RBEs and compound factors for BSH appear to be useful in predicting the outcome from experimentation as indicated in Table 1. In addition, these factors have been used to arrive at the dosage groups utilizing the Petten reactor in their dog irradiation studies. The peak dose takes into account the different beam parameters, and size of the dog, which will influence the depth of the peak radiation dose. Due to the complexity and number of factors involved, it should be emphasized that the totality of the factors involved appear to be predictive. Any individual item is not necessarily correct, and a change in any individual factor would necessitate changes in the other components. Figure 1 indicates the various equivalent doses used to predict the response from 4 dogs. Two of these dogs ( $\$ 997 \&$ 946) were irradiated at BMRR and 2 dogs ( $\$ 976$ \& 504) at Petten. These factors would have predicted that 3 dogs (\$976, 504 \& 946) would have exceeded the threshold dose of $14.8-15$ Gy that would result in a $50 \%$ lethality following gamma or megavoltage $x$-ray irradiation. It would have predicted that one animal was slightly beneath that level. The experimental evidence confirms that the same 3 of 4 animals were affected with a lethal ischemic brain necrosis, and the animal predicted to be spared showed only sublethal, clinically quiescent change that was only detected on routine magnetic resonance examination. Figure 2 illustrates the MRI of ischemic necrosis seen that is fatal in dogs at 5-6 months post-irradiation and Figure 3 is representative of the MRI of sublethal clinically quiescent change seen in some animals at the 6 month post-irradiation routine examination.

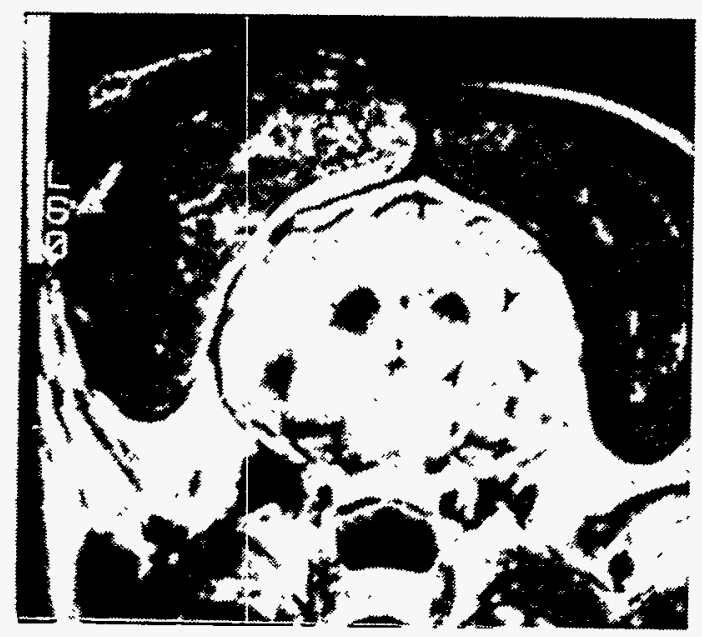

Figure 1. Equivalent Peak Radiation Dose. Dogs 976 and 504 were irradiated at Petten and dogs 997 and 946 at BMRR. The RBEs or compound factors used were: total gamma and other $=1 ; \mathrm{N}(\mathrm{n}, \mathrm{p})=2.7$; fast neutron $=4.5 ;$ and ${ }^{10} \mathrm{~B}(\mathrm{n}, \alpha)=0.33$ The reference line at 14.8 eq $\mathrm{Gy}$ represents the $\mathrm{LD}_{\mathrm{s0}}$ for brain necrosis from a single
exposure of $\mathrm{x}$-rays.

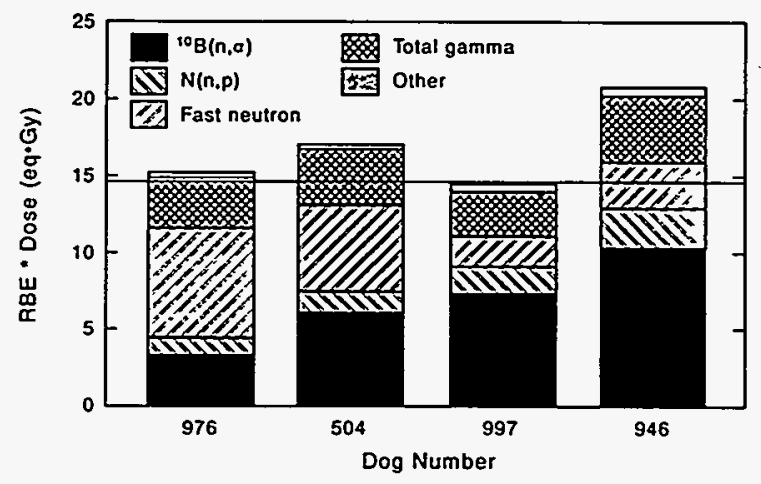

Figure 2. Lethal brain necrosis. Transverse section $T_{1}$ weighted MRI following contrast administration of a dog that developed fatal radiation necrosis 6 months post irradiation. Arrow indicates the contrast enhanced area that was histopathologically confirmed to be a hemorrhagic necrotic area. 


\section{SPONTANEOUS TUMOR DOG RESULTS}

Twenty-one (21) dogs with spontaneous brain tumors have also been irradiated with the BMRR epithermal beam following intravenous infusion of BSH (Table 2). These studies were an extension of the normal tissue tolerance study to detect any change in normal tissue tolerance that might occur in animals with spontaneous brain tumors. The study also measured objective changes within the tumor at acceptable normal tissue injury levels. All dogs were treated with a single, dorsal, $10 \times 10$ portal regardless of tumor location. The total peak physical dose varied from 16-26 Gy and the mean blood-boron concentration during irradiation varied from 14-85 $\mu \mathrm{g} / \mathrm{g}$. The post-irradiation followup period varied from hours to greater than 3 years. No known acute toxicities have been observed. The 2 animals that survived only hours following administration of the compound and epithermal irradiation might indicate an acute toxicity. However, no histopathologic evidence of acute toxicity was detected and it is felt that the deaths

Table 2. Canine Tumor Treatment Summary

\begin{tabular}{|c|c|c|c|c|c|c|c|c|c|}
\hline \multirow{2}{*}{$\frac{\text { DOG }}{50 \mathrm{mg}}$} & $\begin{array}{l}\text { SEXI } \\
\text { AGE }\end{array}$ & \multirow{2}{*}{ DIAGNOSIS } & $\begin{array}{c}\text { SURVIVAL } \\
\text { DAYS }\end{array}$ & \multirow[t]{2}{*}{$\begin{array}{l}\text { CAUSE } \\
\text { OF DEATH } \\
\end{array}$} & \multicolumn{2}{|c|}{$\frac{{ }^{10} \mathrm{~B} \text { AVG. (ppm) }}{\text { TARGET MEAS. }}$} & \multicolumn{2}{|c|}{$\begin{array}{l}\text { DOSE (CGY) } \\
\text { TARGET CALC. }\end{array}$} & $\begin{array}{l}\text { TOLERANCE } \\
\text { EXCEEDED }\end{array}$ \\
\hline & ${ }^{10} \mathrm{~B} / \mathrm{k}$ & & tered (V) & & & & & & \\
\hline-79 & F/7 & Oligodendrogliome & 348 & Died-Urinary obstruction & 25 & 18.5 & 1900 & 1923 & \\
\hline-80 & $M / 4$ & Astrocytoma & 12 & Diod-Anesthesia & 25 & 21.6 & 1900 & 1722 & \\
\hline .83 & $M / 1\}$ & Meningioma & 170 & Died-Focal encephalomalacia & 25 & 21.4 & 1900 & 1829 & + \\
\hline-87 & $\mathrm{M} / \mathrm{R}$ & Moningioma & 140 & Euthonasia-PND & 25 & 20.6 & 1900 & 1653 & \\
\hline-93 & F/6 & Oligodondroglioma & 300 & Diod-PND & 25 & 24.9 & 1900 & 1912 & + \\
\hline .94 & F/5 & Meningioma & $>1022$ & & 25 & 14.3 & 1900 & 1887 & + \\
\hline-95 & $M / 6$ & Moningioma & 0.5 & Died-AND following irradiation & n 25 & 24.5 & 1900 & 1743 & \\
\hline-100 & $\boldsymbol{F} \boldsymbol{\eta}$ & Meningioma & 43 & Euthenasia-PND & 25 & 28.9 & 2300 & 2185 & \\
\hline-108 & $F / 4$ & Choroid plexus pepilloma & 115 & Unknown & 50 & 51.9 & 1900 & & \\
\hline-118 & $F / 9$ & Astrocytoma & 158 & Died-PND & 50 & 85.3 & 2300 & & \\
\hline-129 & $F / 3$ & Choroid ploxus pepilloma & 386 & Euthanasia-PND & 50 & 56.9 & 2300 & 2141 & \\
\hline-132 & $M / 13$ & Moningioma & 54 & Died-Surgery & 50 & 34.5 & 2300 & & \\
\hline-134 & F/8 & Meningioma & 33 & Died-PND & 50 & 20.6 & 1900 & 1729 & \\
\hline-139 & $M / 11$ & Meningioma & 13 & Died-Log infection & 50 & $\mathbf{5 7 . 5}$ & 2300 & & \\
\hline .140 & $F / 3$ & $\begin{array}{l}\text { Choroid plexus papilloma } \\
\text { or Meningioma }\end{array}$ & 532 & Euthanasia-PND & 50 & 40.3 & 2300 & & \\
\hline .142 & $F / 6$ & Meningiome & 181 & Euthanasia-PND & 50 & 54.2 & 2300 & 2708 & + \\
\hline-147 & M/9 & Malignant meningioma & 102 & Euthanasia-PND & 50 & 37.1 & 2300 & & \\
\hline-151 & M/6 & Intre-axial onhancing mass & $>531$ & & 50 & 67.6 & 2300 & & \\
\hline-152 & $M / 10$ & Meningioma & 99 & Euthanasia-Unrelated tumors & 50 & 60.8 & 2300 & & \\
\hline 100 & & & ared $\mathbf{N}$ & & & & & & \\
\hline-155 & $F / 11$ & Meningiome & 3 & Died-Unidentified & 100 & 101.6 & 2600 & & \\
\hline-161 & M/8 & Choroid plexus papilloma & 22 & Euthenasia-PND & 100 & 102.2 & 2600 & & \\
\hline
\end{tabular}

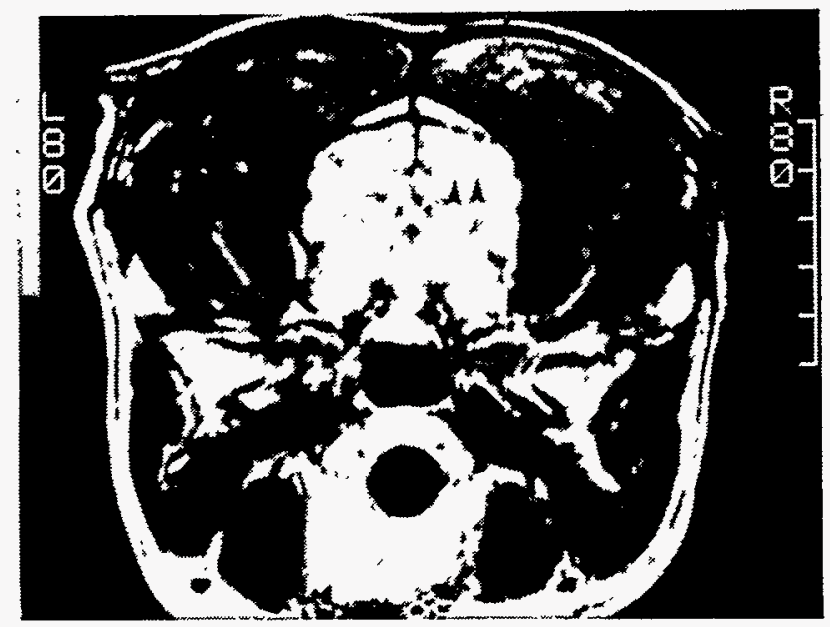

Figure 3. Sublethal brain necrosis. Transverse section of a $T_{1}$ weighted MRI following contrast administration. The areas (arrow) were detected on the 6 moeth post radiation exposure examination. The areas were not detected on the 12 month post exposure examination. Histopathologic examination at that time revealed a gial scar. 
were attributed to the combined brain tumor and/or anesthesia required for treatment. Several animals did have changes in the peripheral blood similar to that described above, as well as elevation of the cerebral spinal fluid protein 3 weeks post-irradiation. In addition, most of the animals had epilation from partial to complete with pigmentation reversal in the hair that regrew. The most severe skin change seen was a moist desquamation in the center of the incident field. No dermal necrosis was encountered. Ischemic necrosis of the normal brain occurred in 2 animals (\#83 and 142). Animal \#83 had considerable peritumor edema at the time of treatment that could not be controlled or could not be eliminated with corticosteroids. It is felt that the edematous brain contained significant amounts of boron at the time of irradiation and led to ischemic necrosis in that region. ${ }^{12}$ Dog \#142 had ischemic necrosis similar to that seen in the normal dogs and would be indicative of an apparent excessive dose to the normal capillary endothelium and the calculated dose received was significantly higher than planned. Dogs \#93 and 94 had magnetic resonance visible change at the 6 month post-treatment evaluation similar to that described in the sublethal changes in the normal dogs. These changes subsequently were not visible as was seen in the normal dogs at 1 year post-irradiation.

It can be seen that the BNCT as delivered in a single dose through a dorsal portal was not effective in controlling all of the tumors. The response of the tumors was at least equal to that available from conventional therapy in the dog recently reported. ${ }^{19}$ The response to intraaxial glial tumors appeared better than that reported for the aggressive conventional therapy and included surgery, irradiation, and chemotherapy. The current studies should be viewed in a phase-I/phaseII trial perspective. Subsequent irradiation of dogs with spontaneous brain tumors will tailor the portal size and location and may involve multiple ports to achieve a better physical dose distribution than the initial studies reported above. While proper controls are difficult in spontaneous tumor work of large animals, certainly objective tumor response and long term survival would be noteworthy. It should be emphasized that of the dogs in the initial study of spontaneous tumors, only one animal was lost to followup in eventual histopathologic examination following treatment, thus providing a wealth of material to study the effect of BNCT on the tumor and normal tissues.

The dog has proven to be an excellent model to study the normal tissue tolerance to BNCT with BSH. Purpose-bred dogs and dogs with spontaneous tumors will be used to study other boron compounds with potential for BNCT. The results from the large animal study form a basis for eventual human clinical trials. The same clinical examination and treatment planning tools will be directly adapted to the human trials.

ACKNOWLEDGEMENT Work supported in part by the U.S. Department of Energy, Office of Energy Research, under DOE Field Office, Idaho, Contract No. DE-AC07-76IDO1570.

\section{REFERENCES}

1. R.G. Zamenhof, A.H. Madoc-Jones, and O.K. Harling, A clinical trial of neutron capture therapy for brain tumors at New England Medical Center and the Massachusetts Institute of Technology, Proceedings of Workshop on Neutron Capture Therapy, Cambridge, MA, January 22-23 (1986).

2. J.E. Kirsch, G.L. Brownell, R.G. Zamenhof, W.H. Sweet, J.R. Messer, E.P. Richardson, A. Tateuchi, and $\mathbf{H}$. Hatanaka, Irradiation of the normal canine brain after craniectomy with the current boron neutron capture therapy regimen at MTT-reactor, in: Boron-Neutron Capture Therapy for Tumors, H. Hatanaka, ed., Nishimura Co., Ltd., Niigata (1986).

3. Y. Ushio, T. Hayakawa, K. Morimoto, H. Mogami, T. Takeuchi, K.Amano, T. Miobe, and H. Hatanaka, Pathologic study of the effect of boron-neutron capture radiation on normal brains of dogs, in: Boron-Neutron Capture Therapy for Tumors, H. Hatanaka, ed., Nishimura Co., Ltd., Niigata (1986).

4. A. Takeuchi, and H. Hatanaka, Tolerance of canine brain to the revised boron neutron capture therapy, in: Boron-Neutron Capture Therapy for Tumors, H. Hatanaka, ed., Nishimura Co., Ltd. (1986).

5. J.R. Fike, C.E. Cann, R.L. Davis, J.K. Borcick, T.L. Phillips, and L.B. Russel, Computed tomographic analysis of the canine brain: Effects of hemobrain x-irradiation, Rad Res 99:294310 (1984).

6. S.L. Kraft, P.R. Gavin, C.E. DeHaan, W.F. Bauer, and T.E. Ary, The biodistribution of boron in normal canine tissues following borocaptate sodium administration and the effect of plasma exchange, in: Progress in Neutron Capture Therapy for Cancer, Allen, Moore, and Harrington, eds., Plenum Press, New York (1992). 
7. P.R. Gavin, C.D. DeHaan, S.L. Kraft, M.P. Moore, F.J. Wheeler, and D.L. Miller, Dosimetric considerations: Radiation tolerance of the normal canine brain following boron neutron capture therapy, Proceedings of the 38th Annual Scientific Meeting, Radiation Research Society, New Orleans, Poster Abstract (1990).

8. J.O. Archambeau, K.G. Yuirchild, and H.J. Brenneis, The response of the skin of swine to increasing absorbed doses of radiation from a thermal neutron beam, a degraded fission neutron beam, and the ${ }^{10} \mathrm{~B}(\mathrm{n}, \alpha)^{7} \mathrm{Li}$ reactor, Rad Res 45:145-164 (1971)

9. C.L. Brownell, J.E. Kirsch, J.C. Murphy, M. Ashtari, W.C. Schoene, C. Rumbaugh, and G.R. Wellum, Pre-clinical neutron capture therapy trials at MIT using $\mathrm{Na}_{2} \mathrm{~B}_{12} \mathrm{H}_{11} \mathrm{SH}$, Proceedings of First International Symposium on BNCT, Boston, MA, October 12-14, Brookhaven National Laboratory, Long Island, NY (1983).

10. S.L. Kraft, P.R. Gavin, C.E. DeHaan, C.W. Leathers, W.F. Bauer, D.L. Miller, and R.V. Dorn III, Borocaptate sodium: A potential delivery compound for boron neutron capture therapy evaluated in dogs with spontaneous intracranial tumors, Proceedings National Academy of Science, in press, (1992).

11. S.L. Kraft, P.R. Gavin, W.F. Bauer, D.L. Miller, and M.L. Griebenow, Biodistribution of boron in dogs with spontaneous intracranial tumors following borocaptate sodium administration Part II: Boron concentrations in normal canine tissues. Submitted to Rad Res.

12. S.L. Kraft, P.R. Gavin, C.W. Leathers, C.E. DeHaan, W.F. Bauer, D.L. Miller, and R.V. Dorn III, Biodistribution of boron in dogs with spontaneous intracranial tumors following borocaptate sodium administration Part I: Tumor boron concentrations. Submitted to Rad Res.

13. P.R. Gavin, C.E. DeHaan, S.L. Kraft, Y.D. Harker, P.D. Randolf, and F.J. Wheeler, Regional and total body dose following BNCT epithermal head irradiation: Biologic and dosimetric evaluation, in: Progress in Neutron Capture Therapy for Cancer, Allen, Moore, and Harrington, eds., Plenum Press, New York (1992).

14. K.H.I. Philipp, R. Huiskamp, J. Casado, A.S. Siefert, P.R. Gavin, and H.L. Moss, Developments in healthy tissue tolerance studies as a prerequisite for clinical application of BNCT with borocaptate sodium at the high flux reactor in Petten. In these proceedings.

15. C.E. DeHaan, P.R. Gavin, S.L. Kraft, F.J. Wheeler, and C.A. Atkinson, Qualitative dose response of the normal canine head to peithermal irradiation with or without boron capture, in: Progress in Neutron Capture Therapy for Cancer, Allen, Moore, and Harrington, eds., Plenum Press, New York (1992).

16. A. Siefert, J. Casado, K. Philipp, R. Huiskamp, P. Gavin, E. Duhmke, and R.L. Moss, Brain effects observed in the canine healthy tissue tolerance studies for BNCT with borocaptate sodium at the epithermal neutron beam of the HFR Petten. In these proceedings.

17. P.R. Gavin, S.L. Kraft, C.E. DeHaan, M.L. Griebenow; and M.P. Moore, A large animal model for boron neutron capture therapy, in: Progress in Neutron Capture Therapy for Cancer, Allen, Moore, and Harrington, eds., Plenum Press, New York (1992).

18. R. Huiskamp, P.R. Gavin, F.J. Wheeler, A.S. Siefert, and K.H.I. Philipp, Dose effect comparisons between HFR and BMRR irradiated dogs with respect to bealthy tissue tolerance. In these proceedings.

19. G.L. Heidner, J.N. Kornegay, R.L. Page, R.K. Dodge, and D.E. Thrall, Analysis of survival in a retrospective study, J Vet Intern Med 5:219-226 (1991). 
" 


\section{Cardiovascular Toxicities Associated with Intravenous Administration of $\mathrm{p}$-Boronophenylalanine Formulations}

T. LaHann, C. Sills, G. Hematillake, T. Dymock, and G. Daniell

This article is a reprint from Advances in Neutron Capture Therapy, pp. 513-517, (1993). 



\title{
CARDIOVASCULAR TOXICITIES ASSOCIATED WITH INTRAVENOUS
}

\section{ADMINISTRATION OF P-BORONOPHENYLALANINE FORMULATIONS}

\author{
T. R. LaHann, C. Sills, G. Hematillake, T. Dymock and G. Daniell \\ Center for Toxicology Research \\ Department of Pharmaceutical Sciences \\ College of Pharmacy \\ Idaho State University \\ Pocatello, Idaho, 83209
}

\section{INTRODUCTION}

${ }^{10}$ Boronophenylalanine (BPA) has been shown to be an effective boron delivery drug for boron neutron capture therapy (BNCT) of experimental and spontaneously occurring cancers ${ }^{1}$. For successful BNCT, tumor boron concentrations of at least $20 \mathrm{ppm}$ are thought to be necessary, but higher tumor boron levels are desirable. Calculations ${ }^{2}$ indicate that for a given neutron exposure, each doubling of the tumor boron concentration should increase tumor cell kill by a factor of about 10,000 . Thus, significant therapeutic benefit would be achieved by delivering the largest possible dose of BPA to tumor. Factors that limit the amount of BPA deliverable to tumor are 1) the aqueous solubility and 2) the intrinsic toxicity of the BPA. BPA is poorly soluble in aqueous solution ( $<4 \mathrm{~g} / \mathrm{L}$ at $\mathrm{pH} 7.4$ ), but formation of a BPA-fructose complex dramatically improves water solubility ${ }^{3}$ and allows delivery of high levels of boron to tissues ${ }^{3}$. Little is known about the toxicity of BPA, in part because its poor water solubility has made it difficult to deliver large amounts of drug to animal tissues. Soloway et al. ${ }^{4}$, Mishima et al..$^{5}$, Taniyama et al. ${ }^{6}$ and Meek ${ }^{7}$ have all studied the acute toxicity of BPA. Reported $L D_{s 0}$ values for BPA ranged from $640 \mathrm{mg} / \mathrm{kg}^{6}$ (i.p., pH 1 solution, rats) to $1,520 \mathrm{mg} / \mathrm{kg}^{d}$ (i.v., pH 10 solution, mice). Chronic oral and s.c. dosing of BPA suspensions was well tolerated ${ }^{7}$, although the apparent safety of BPA suspensions may more reflect BPA's relative insolubility at physiological pH than its intrinsic toxicity profile. Nevertheless, the prevailing view seems to be that BPA is a relatively safe drug. While assessing the biodistribution of novel BPA-cyclodextrin formulations, we noted that i.v. infusion of this formulation could impair cardiovascular and pulmonary function in dogs ${ }^{8}$. To better understand the significance of such an observation, we undertook a study of the effects of high dose BPA infusions on the function of the cardiovascular and pulmonary systems. In this paper, we report the approximate maximally tolerated dose for i.v. infusion of a BPA-fructose complex and the effects of high dose BPA infusions on cardiovascular function in the rat.

\section{METHODS}

Male Sprague-Dawley and Long-Evans rats were anesthetized (pentobarbital $50 \mathrm{mg} / \mathrm{kg}$ i.p or urethane $1.2 \mathrm{~g} / \mathrm{kg}$ i.p.). Arterial blood pressure was monitored via an indwelling cannula (carotid or femoral artery). Cardiac left ventricular pressure was measured from a cannula threaded through 
the right carotid artery. Drug was infused via either the right jugular or femoral vein, and the left jugular vein was cannulated for measurement of central venous pressure. Heart rate was calculated from the EKG R-wave, 3-lead EKG being recorded from subdermal electrodes. The trachea was cannulated for artificial respiration (70 breaths/minute, tidal volume: $2.5-3.0 \mathrm{cc} / \mathrm{stroke}$ ). Rectal temperature was clamped at $37.5 \pm 1$ degree $C$. Cardiac and stroke output were monitored by a $2 \mathrm{~mm}$ transit-time ultrasound flow probe (Transonic 6E) placed around the ascending aorta. D,L-pboronophenylalanine (Callery Chemical Company, Pittsburgh PA) was prepared as a $6.0 \pm 0.1$ $\mathrm{mg} / \mathrm{ml}$ (mean $\pm \mathrm{SEM}, \mathrm{N}=4$ ) solution in $\mathrm{pH} 7.4,0.1 \mathrm{M}$ phosphate buffered saline by transiently increasing the $\mathrm{pH}$ of the suspension to about 10 and then readjusting $\mathrm{pH}$ to 7.4. BPA was prepared as a $173.2 \pm 3.8 \mathrm{mg} / \mathrm{ml}$ (mean $\pm \mathrm{SEM}, \mathrm{N}=18$, range: $148.6-196.9$ ) solution in $\mathrm{pH} 7.4$ fructose/distilled water by adding fructose to a suspension of $200 \mathrm{mg} / \mathrm{ml} \mathrm{BPA}$ in a $1: 1 \mathrm{molar}$ ratio, transiently increasing the $\mathrm{pH}$ of the suspension to about 10 and then readjusting the $\mathrm{pH}$ to 7.4. A solution osmotically equivalent to $186 \mathrm{mg} / \mathrm{ml}$ BPA was prepared by dissolving mannitol and fructose (1:1 molar ratio) in $\mathrm{pH} 7.4$ distilled water. Formulations were passed through a 0.22 micron filter and were prepared immediately prior to use. Reported concentrations of BPA are based on measurement of boron (by inductively coupled plasma atomic emission spectrometry) in the final, filtered product. Target rates for i.v. infusion of BPA-fructose were 8.5, 12.8 or 17 $\mathrm{ml} / \mathrm{kg} / \mathrm{hr}$, and delivered volumes were within $7 \%$ of targeted volumes. The infused volumes, corrected for the actual concentration of the infused solutions corresponded to actual BPA doses of $1,694 \mathrm{mg} / \mathrm{kg}, 2,306 \pm 144 \mathrm{mg} / \mathrm{kg}$ or $2,972 \pm 124 \mathrm{mg} / \mathrm{kg}$. The target rate for infusion of the BPA-buffer formulation was $17 \mathrm{ml} / \mathrm{kg} / \mathrm{hr}$ for a target BPA dose of $102 \mathrm{mg} / \mathrm{kg}$ and an actual dose of $99.0 \pm 1.7 \mathrm{mg} / \mathrm{kg}(\mathrm{N}=5)$. When a continuous drug infusion protocol was not employed, rats dosed with BPA-fructose received a 35 minute post-BPA infusion of quarter-normal saline. Physiological data were recorded on an AstroMed MT-95000 thermal recorder and simultaneously digitized and stored on magnetic tape for later analysis on an 80486-type computer. Data were analyzed using algorithms developed with the software analysis program "DATAPAC II".

\section{RESULTS AND DISCUSSION}

\section{I.V. Infusion of BPA - Lethal Dose}

Anesthetized rats infused with an average of $2,972 \mathrm{mg} / \mathrm{kg}$ of BPA $(\mathrm{N}=5)$ over a one hour period, survived without grossly observable impairment of function, provided that surgical stress was minimized. BPA was delivered as a BPA-fructose complex, the observation period was 7 days and minimal stress was defined as no instrumentation of the animals except an i.v. cannula for infusion of drug. Animals dosed with $2,306 \mathrm{mg} / \mathrm{kg}$ BPA $(\mathrm{N}=7)$ survived until sacrifice on day 2 . Anesthetized rats infused with $4,334 \pm 56 \mathrm{mg} / \mathrm{kg}$ of BPA (target dose: $1,586 \mathrm{mg} / \mathrm{kg} / \mathrm{hr}$ for 3 hours, BPA-fructose complex) died $(\mathrm{N}=4)$. Three rats died by the end of the infusion and one rat died approximately $24 \mathrm{hrs}$ after the infusion. Rats infused with a mannitol/fructose/water solution that mimicked the volume and osmotic load of a $4,827 \mathrm{mg} / \mathrm{kg}$ dose of BPA (3 hour infusion, target rate: $8.5 \mathrm{ml} / \mathrm{kg} / \mathrm{hr}, \mathrm{N}=3$ ) survived the 7 day observation period without grossly observable impairment of function. Rats infused with saline $(8.5 \mathrm{ml} / \mathrm{kg} / \mathrm{hr}$ for 3 hours, $\mathrm{N}=2)$ or with fructose-distilled water (172 $\mathrm{mg}$ fructose $/ \mathrm{ml} ; 8.5 \mathrm{ml} / \mathrm{kg} / \mathrm{hr}$ for 3 hours, $\mathrm{N}=2$ ) also survived the 7 day observation period. These experiments indicate that death associated with the BPA infusions was not a result of volume loading or of altered systemic osmolarity. Rats instrumented for recording of cardiovascular and pulmonary function and infused with the BPA-fructose complex died after receiving doses of BPA that were non-lethal in minimally stressed rats. Three of five rats instrumented for recording of cardiovascular parameters and infused with $2,181 \pm 30 \mathrm{mg} / \mathrm{kg}$ of BPA died within 6 hours of infusion. Physiological stress apparently increases the toxicity of BPA, rendering smaller doses of BPA more likely to kill the rats. This suggests that patients afflicted with cancer may be more sensitive to the toxic effects of BPA than healthy subjects would be.

\section{Cardiovascular Effects of BPA}

The rapid death after infusion of high doses of BPA implicated cardiovascular or pulmonary 
dysfunction or a combination of both as the primary system failure responsible for death. ${ }^{\wedge}$ rtificial support of respiration failed to protect against the lethal effect of BPA, implicating cardiuvascular impairment as the causative factor. BPA formulations elevated arterial blood pressure (BP), partially as a result of the volume load infused, but BPA increased BP more than did equal volumes of control solution. After infusion of large amounts of BPA, the pressor response was followed by a gradual, sustained decline of BP (figure 1). Only minor changes in central venous pressure were observed. Like BP, cardiac output (CO) increased during BPA infusion, but then gradually and continuously declined until the animals died (figure 1). Peak stroke output (SO; maximum flow per cardiac contraction) initially increased, but then declined markedly. Total peripheral resistance (TPR, a measure of the extent of vascular constriction) increased over time (figure 1), possibly to compensate for the fall in $\mathrm{CO}$. $\mathrm{CO}$, SO and TPR were little affected by the mannitol infusion.
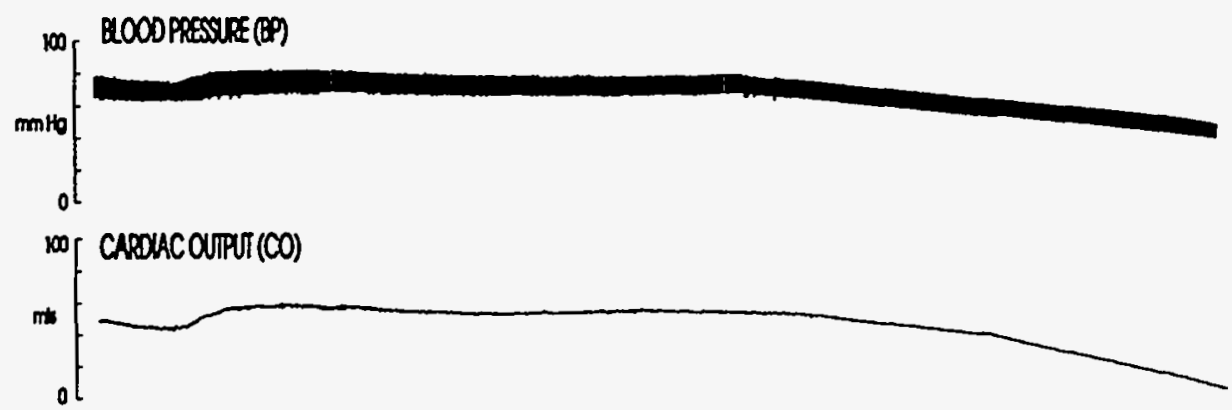

TOTLL PERPERLLRESSANCE (PP)

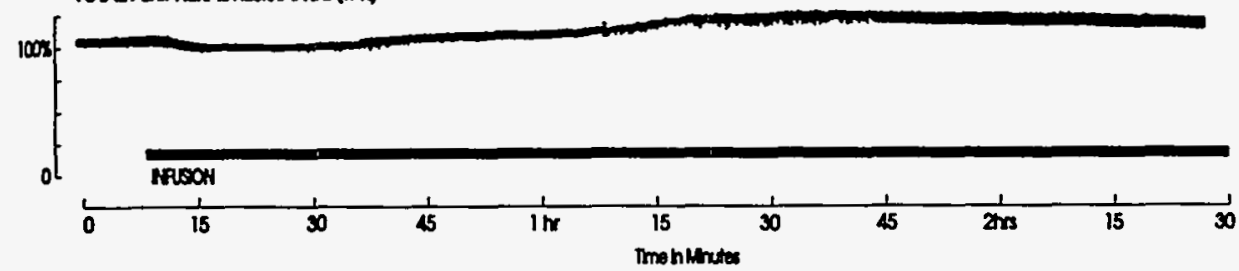

Figure 1. Blood pressure, cardiac output and total peripheral resistance before and during a 3 hour infusion (bar) of BPA $(4,394 \mathrm{mg} / \mathrm{kg})$ to a Long-Evans rat.
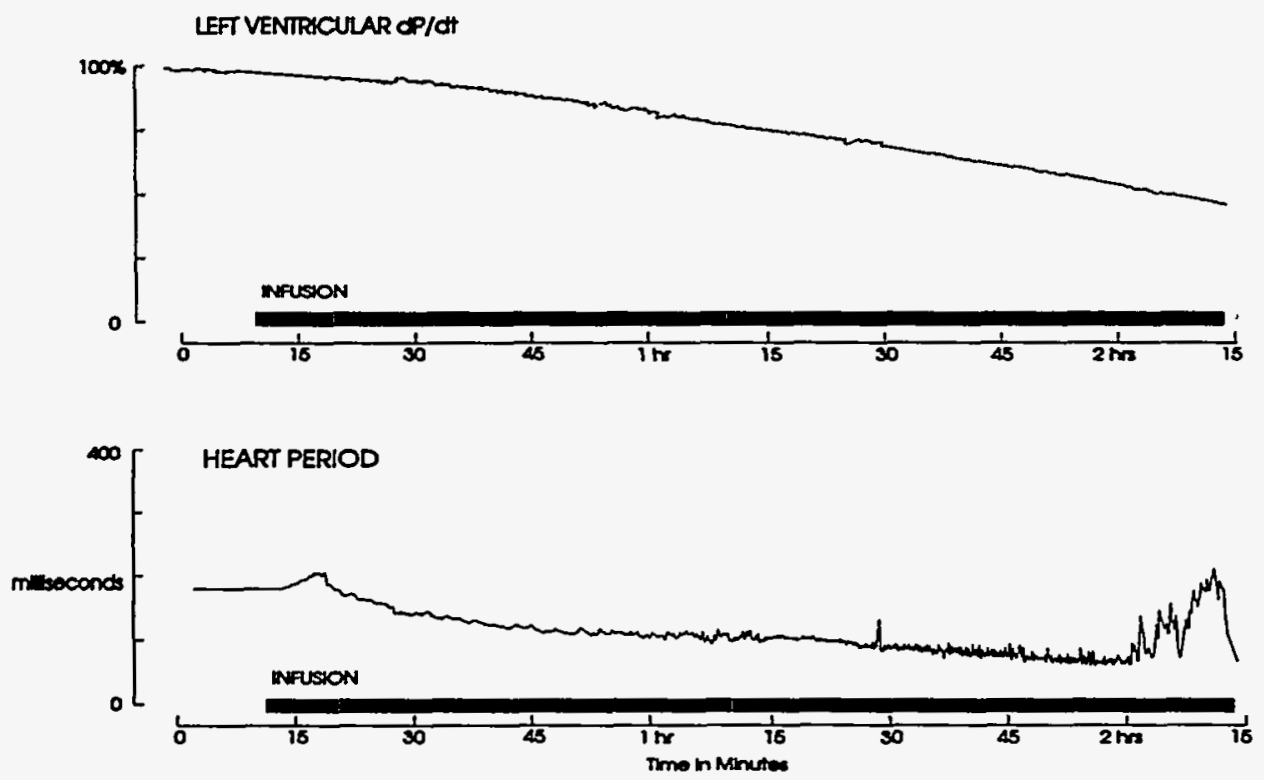

Figure 2. Left ventricular $\mathrm{dP} / \mathrm{dt}$ and heart rate before and during a 3 hour infusion (bar) of BPA $(4,394$ $\mathrm{mg} / \mathrm{kg}$ ) to a Long-Evans rat. 
BPA also altered left ventricular $\mathrm{dP} / \mathrm{dt}$ and heart period (the time between heart beats) (figure 2). In some animals, BPA exerted a positive inotropic effect, but as the infusion proceeded, cardiac contractility always diminished. Heart period decreased gradually (i.e., heart rate increased) until just prior to death, which always appeared to be preceded by ventricular fibrillation.

Usually, cardiovascular parameters were little affected in rats receiving doses of BPA less than $100 \mathrm{mg} / \mathrm{kg}$. However, there may be a small subpopulation of animals in which BPA doses as low as $100 \mathrm{mg} / \mathrm{kg}$ impair cardiovascular function. BPA-induced cardiovascular lability (figure 3 ) has been observed in only two rats, but an abnormal blood pressure response and a Biots-type breathing pattern were also observed in one of three dogs dosed with a BPA-Molecusol ${ }^{\Phi}$ formulation $^{8}$. Vehicle-induced cardiovascular lability has not been observed in either rats or dogs, suggesting that the lability associated with BPA infusions was unlikely to be a measurement artifact. Although only a few animals have displayed cardiovascular lability after BPA administration, because lability has been observed in both dogs and rats, it may be that a small subpopulation of animals are particularly sensitive to BPA. This sensitivity might have a genetic basis, or may reflect a reduction in the animal's functional reserve. If a reduced functional reserve is the predisposing factor, then compared to healthy subjects, cancer patients may be at greater risk of BPA-induced toxic reactions.

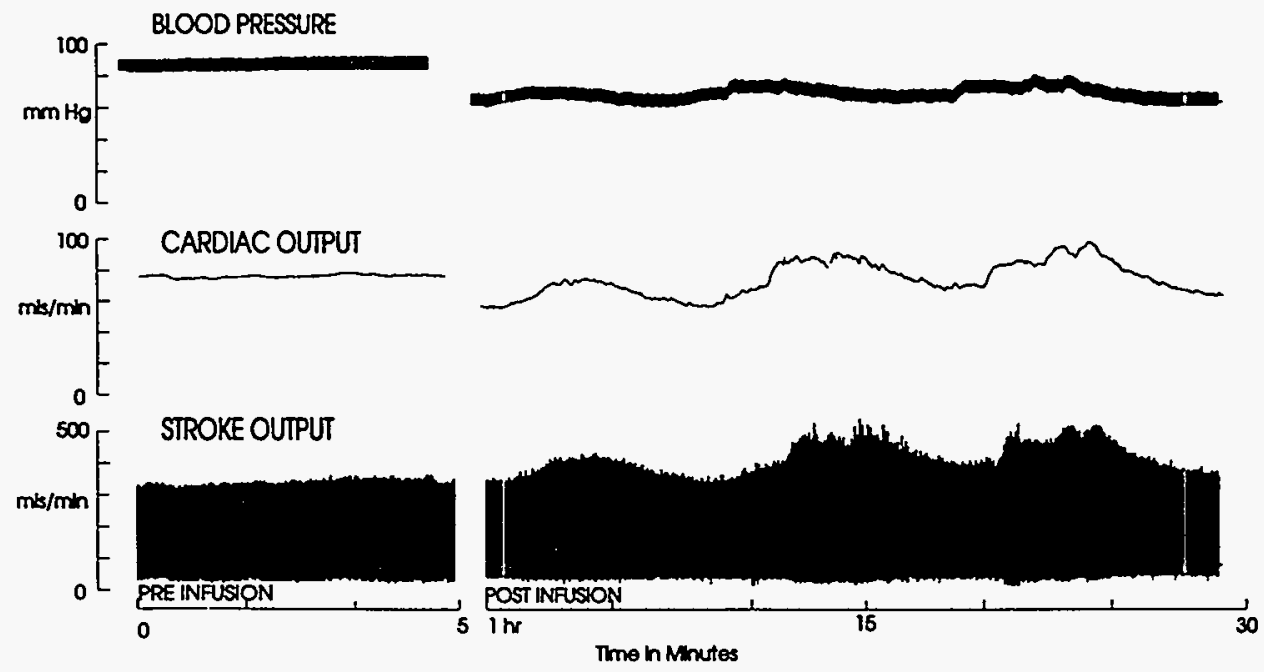

Figure 3. Blood pressure, cardiac output and stroke output before and after infusion of BPA (100 mg/kg) to a Sprague-Dawley rat.

\section{CONCLUSIONS}

Intravenous administration of BPA doses up to about $3,000 \mathrm{mg} / \mathrm{kg}$ appear to be well tolerated when delivered over a one hour infusion period to minimally stressed rats. Infusion of more than $4,000 \mathrm{mg} / \mathrm{kg}$ over a three hour time period resulted in death, probably as a result of BPA-induced impairment of cardiovascular function. Stress seemed to exacerbate BPA's toxicity so that doses of BPA that were not lethal in minimally stressed animals, did kill when administered in the presence of significant physiological stress. Finally, it is possible that a subpopulation of animals may exist that is abnormally sensitive to BPA and that administration of BPA doses as low as 100 $\mathrm{mg} / \mathrm{kg}$ to these animals may elicit cardiovascular lability.

\section{ACKNOWLEDGEMENTS}

This study was performed under the auspices of the U.S. Department of Energy, Office of Energy Research, DOE Idaho Field Office, Contract No. DE-AC07-76ID01570 


\section{REFERENCES}

1. Barth, R., Soloway, A. \& Fairchild, R. (1990) Boron Neutron Capture Therapy for Cancer. Scientific American 263, 100-107.

2. Wheeler, F., Griebenow, M., Wessol, D., Nigg, D. \& Anderl, R. (1989) A Stoichastic Model for High LET Response for BNCT, in Basic Life Sciences: Clinical Aspects of Neutron Capture Therapy, eds. Fairchild, R., Bond, V. \& Woodhead, A. (Plenum Press, New York) pp. 165-178. 3. LaHann, T., Lu, D., Sills, C., Daniell, G., Kraft, S., Gavin, P. \& Bauer, W. (in press) Bioavailability of Intravenous Formulations of p-BPA in Dog and Rat, in Proceedings of the Fifth International Symposium on Neutron Capture Therapy, eds. Barth, R. \& Soloway A. (Plenum Press, New York).

4. Soloway, A., Wright, R. \& Messer, J. (1961) Evaluation of Boron Compounds for Use in Neutron Capture Therapy of Brain Tumors. J. Pharmacol. Exp. Therap. 134, 117-122.

5. Mishima, Y., Kakihana, H., Okamoto, M., Yoshino, K. \& Konno, K. (filed 1979) Auxiliary Agents for Neutron Capture in Skin, Japanese Patent Application 30209-1979.

6. Taniyama, K., Fujiwara, H., Kuno, T., Saito, N., Shuntoh, H., Sakaue, M. \& Tanaka, C. (1989) Acute and Subacute Toxicity of ${ }^{10} \mathrm{~B}-$ Paraboronophenylalanine, Pig. Cell Res. 2, 291-296.

7. Meek, A. (1988) Investigational New Drug Application for p-Boronophenylalanine, United States Food and Drug Administration Investigational New Drug Application.

8. LaHann, T., Bauer, W., Gavin, P. and Lu, D. (in press) Pharmacokinetics and toxicity of a pboronophenylalanine-cyclodextrin formulation delivered by i.v. infusion to dogs, in Polymeric Drugs and Drug Delivery Systems, ed. Ottenbrite, R. (American Chemical Society, Washington).

\section{COPYRIGHT}

The submitted publication has been authored by a contractor of the U.S. Government under DOE Contract No. DE-AC07-76ID01570. Accordingly, the U.S. Government retains a nonexclusive, royalty-free license to publish or reproduce the published form of this contribution or allow others to do so, for U.S. Government purposes. 



\title{
Dose Effect Comparisons between HFR and BMRR Irradiated Dogs with Respect to Healthy Tissue Tolerance
}

\author{
R. Huiskamp, P. Gavin, F. Wheeler, A. Siefert, and K. Philipp
}

This article is a reprint from Advances in Neutron Capture Therapy, pp. 565-569, (1993). 



\title{
DOSE EFFECT COMPARISONS BETWEEN HFR AND \\ BMRR IRRADIATED DOGS WITH RESPECT TO \\ HEALTHY TISSUE TOLERANCE
}

\author{
René Huiskamp ${ }^{1}$, Patrick R. Gavin ${ }^{2}$, Floyd J. Wheeler ${ }^{3}$, \\ Axel Siefert ${ }^{4}$, and Katharina H.I. Philipp ${ }^{1}$ \\ 'Netherlands Energy Research Foundation ECN, Petten, The Netherlands \\ ${ }^{2}$ Washington State University, Pullman WA, USA \\ ${ }^{3}$ EG\&G Idaho Inc., Idaho Falls ID, USA \\ ${ }^{4}$ Commission of the European Communities, JRC, Petten, The Netherlands
}

\section{INTRODUCTION}

Epithermal neutrons beams are being developed for the application of boron neutron capture therapy (BNCT) of deep seated tumors, like glioblastoma and astrocytomas, through the intact skin. Epithermal neutrons will be moderated by the tissue mass between skin and tumour to produce the thermal neutrons necessary for the ${ }^{10} \mathrm{~B}(\mathrm{n}, \alpha)^{7} \mathrm{Li}$ reaction in the target tissue. Although the neutron capture cross-sections of elements in normal tissue are several orders of magnitude lower that for boron, the high abundance of hydrogen and nitrogen will cause a significant contribution to the total absorbed radiation dose through the ${ }^{1} \mathrm{H}(\mathrm{n}, \gamma)^{2} \mathrm{H}$ and the ${ }^{14} \mathrm{~N}(\mathrm{n}, \mathrm{p}){ }^{14} \mathrm{C}$ reaction, respectively. Due to inevitable incomplete filtration, an epithermal beam will also contain a fast neutron component, i.e. neutrons with energies $\geq$ $10 \mathrm{keV}$, and a $\gamma$-photon component originating from the reactor and produced in structural and filter materials. Therefore, the resultant radiation consists of a complex of low and high LET radiation of which the constituents vary rapidly with depth in tissue. Together with the dependence of the biological effects on the extra- and intracellular localization of boron, the assessment of the tolerance of normal tissues in large animal models necessary prior BNCT with borocaptate sodium (BSH) of brain tumors is very complicated.

Based on the ongoing canine healthy tissue tolerance study at the Brookhaven Medical Research Reactor (BMRR) using the epithermal beam without BSH, the relative biological effectiveness (RBE) of the fast neutron beam component has been determined for skin reactions. In addition, a "compound factor", i.e geometry $\times \mathrm{RBE}$, for the ${ }^{10} \mathrm{~B}(n, \alpha)^{7} \mathrm{Li}$ reaction was derived for dogs irradiated at the BMRR with the epithermal beam and BSH (Gavin et al., 1993a, 1993b, in press). Currently, a healthy tissue tolerance study with BSH is being carried out at the HB11 epithermal beam of the High Flux Reactor at Petten. The present papers describes prelimenary dose effect comparisons between High Flux Reactor

Advances in Neutron Capture Therapy, Edited by A.H Soloway et al., Plenum Press, New York, 1993 
(HFR) and BMRR irradiated dogs with respect to healthy tissue tolerance in order to refine the BSH compound factors and the fast neutron RBE for skin and brain.

\section{MATERIALS AND METHODS}

A total of thirty dogs have been irradiated at the Brookhaven Medical Research Reactor (BMRR) reactor. Fifteen of these dogs were irradiated with the epithermal neutron beam alone (EPI dogs). Fifteen dogs were irradiated with the epithermal beam while the blood boron concentration was $30-62 \mathrm{ppm}{ }^{10} \mathrm{~B}$ following an intravenous administration of BSH (BORON dogs). A description of the dosage groups as well as the observed biological effects have been previously reported (Gavin et al.,1992). To date, fifteen dogs have been irradiated at the High Flux Reactor in Petten, The Netherlands. Three dogs have been irradiated with the epithermal beam alone and twelve dogs have been irradiated at blood boron level varying from $25-62 \mathrm{ppm}{ }^{10} \mathrm{~B}$ (see Siefert et al., these proceedings).

The physical and dosimetric characteristics of both beams and field sizes used are reported elsewhere (Nigg et al., 1992; Harker et al., these proceedings; Watkins et al, these proceedings).

The relative biological effectiveness (RBE) for fast neutrons was derived using the EPI dog tolerance studies by subtracting the physical doses multiplied by their RBE of the various beam components and compared with historical values for conventional photon irradiation. The RBE's used in the calculations were equal to 2.7 and 1.0 for the ${ }^{14} \mathrm{~N}(\mathrm{n}, \mathrm{p})^{14} \mathrm{C}$

Table 1. Calculation of fast neutron RBE for skin reactions using epithermal beam irradiated only (EPI) dose groups.

\begin{tabular}{|c|c|c|c|c|c|c|}
\hline Response & $\begin{array}{c}\text { Biological } \\
\text { endpoint }\end{array}$ & $\begin{array}{l}\text { Ref } x \text { ray } \\
\text { oose }(G y) !\end{array}$ & $\begin{array}{l}\text { Radiation } \\
\text { component }\end{array}$ & $\begin{array}{l}\text { Priys dose } \\
\text { (oy) }\end{array}$ & 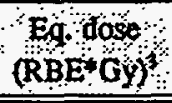 & $\begin{array}{l}\text { Fastineutron } \\
\text { RBBE }\end{array}$ \\
\hline \multirow{2}{*}{$\begin{array}{l}\text { Early skin } \\
\text { reactions }\end{array}$} & $\begin{array}{c}\text { Moist } \\
\text { desquamation } \\
\text { (11.0 Gy EPI dogs) }\end{array}$ & 18.3 & $\begin{array}{l}\text { Other }^{2} \\
\mathrm{~N}(\mathbf{n}, \mathbf{p}) \\
\text { Gamma } \\
\text { Fast } \mathrm{n}\end{array}$ & $\begin{array}{l}0.52 \\
1.35 \\
4.49 \\
3.18 \\
\end{array}$ & $\begin{array}{l}0.52 \\
3.65 \\
2.99\end{array}$ & 3.5 \\
\hline & $\begin{array}{c}\text { Moist } \\
\text { desquamation + } \\
\text { ulceration } \\
\text { (13.7 Gy EPI dogs) }\end{array}$ & 24.4 & $\begin{array}{l}\text { Other }^{2} \\
\mathrm{~N}(\mathbf{n}, \mathbf{p}) \\
\text { Gamma } \\
\text { Fast n }\end{array}$ & $\begin{array}{l}0.65 \\
1.69 \\
5.61 \\
3.97 \\
\end{array}$ & $\begin{array}{l}0.65 \\
4.55 \\
3.76\end{array}$ & 3.9 \\
\hline \multirow{2}{*}{$\begin{array}{l}\text { Late skin } \\
\text { reactions }\end{array}$} & $\begin{array}{c}50 \% \text { skin necrosis } \\
\text { (16.5 Gy EPI dogs) }\end{array}$ & 36.6 & $\begin{array}{l}\text { Other } \\
\text { N(n,p) } \\
\text { Gamma } \\
\text { Fast n }\end{array}$ & $\begin{array}{l}0.78 \\
2.03 \\
6.76 \\
4.78\end{array}$ & $\begin{array}{l}0.78 \\
5.48 \\
4.53\end{array}$ & 5.4 \\
\hline & $\begin{array}{c}>50 \% \text { skin } \\
\text { necrosis } \\
\text { (16.5 Gy EPI dogs) }\end{array}$ & $>36.6$ & $\begin{array}{l}\text { Other } \\
N(n, p) \\
\text { Gamma } \\
\text { Fast } n\end{array}$ & $\begin{array}{l}0.78 \\
2.03 \\
6.76 \\
4.78\end{array}$ & $\begin{array}{l}0.78 \\
5.48 \\
4.53\end{array}$ & $>5.4$ \\
\hline
\end{tabular}

'Park et al.(1974).

${ }^{2}$ Other: dose coming from neutron capture reactions by other elements, primarily $\mathrm{Na}$ and $\mathrm{Cl}$.

${ }^{3} \mathrm{RBE}$ other $=1.0 ; \operatorname{RBE} \mathrm{N}(\mathrm{n}, \mathrm{p})=2.7 ; \mathrm{DRF}$ gamma $=0.67$.

${ }^{4} R_{B E} E_{\text {fas narmons }}=\{$ Ref. X-ray dose- $[(E q$. dose gamma $)+(E q$. dose $N(n, p))+(E q$. dose other $)]\}$

Phys. dose fast neutron 
reaction and other capture reactions respectively. The "compound factor", i.e. geometry* $\mathrm{RBE}$, for the ${ }^{10} \mathrm{~B}(\mathrm{n}, \alpha)^{7} \mathrm{Li}$ reaction was derived from the BORON dog tolerance study using a similar procedure as described for the calculation of the fast neutron RBE: The fast neutron RBE derived from the EPI dog tolerance study was used as additional input leaving the equivalent boron capture dose as variable (Gavin et al., 1993a,1993b, in press).

For dogs irradiated with the HFR epithermal neutron beam, equivalent doses were calculated using BMRR derived dose-modifying factors and the observed biological effects obtained with both beams were compared.

\section{RESULTS AND DISCUSSION}

The fast neutron RBE for early and late skin reactions were derived from the epithermal neutron only irradiated dogs and using published data on the skin tolerance doses determined for $200 \mathrm{kVp} \mathrm{X}$-rays (Park et al.,1974). Since the gamma dose rate in the BMRR epithermal neutron beam is about $0.02 \mathrm{~Gy} / \mathrm{min}$, a gamma dose rate factor (DRF) for skin of 1.5 was introduced in the present calculations (Fu, 1991). RBE values equal to 3.4-3.9 and $\geq 5.4$ were calculated for early and late skin effects respectively (Table 1).

To date no detectable neurological signs have been observed in the $11 \mathrm{~Gy}$ and 13.7 Gy EPI dogs. The 16.4 Gy EPI dogs had to be euthanitized due to dermal necrosis. Since both skin and brain tolerance are determined by the vasculature response after irradiation, the RBE for fast neutron component in brain is assumed to be identical to that calculated for the skin. In addition, a gamma DRF of 1.5 for brain was used (Fu,1991). The resultant peak equivalent dose to brain for the $11 \mathrm{~Gy}$ and $13.7 \mathrm{~Gy}$ EPI dogs was equal to 16.4 and 20.4 RBE*Gy respectively which is far above the reported tolerance of the brain for Xirradiation (ED50=14.9 Gy, Fike and Gobbel, 1991). Since the peak equivalent dose in these animals is located at the surface of the brain and mainly determined by the fast neutron dose which rapidly declines with depth and volume effects may play a role, the majority of the brain will be exposed to tolerable equivalent doses. An equivalent dose estimation $1 \mathrm{~cm}$ beyond the peak equivalent dose, i.e. $1 \mathrm{~cm}$ within the brain of a Labrador dog, was calculated to get a more representative equivalent dose estimation for brain tissue. The equivalent dose calculated at a position $1 \mathrm{~cm}$ in the brain was equal to 13.5 and 16.7 $\mathrm{RBE}^{*} \mathrm{~Gy}$ for the 11 and $13.7 \mathrm{~Gy}$ EPI dogs respectively.

The compound factor for the ${ }^{10} \mathrm{~B}(\mathrm{n}, \alpha)^{7} \mathrm{Li}$ dose was also determined $1 \mathrm{~cm}$ in the brain, using the published and presently calculated dose- modifying factors for the other beam components and compared with published X-ray on skin and brain tolerance. The obtained compound factors for BSH for skin and brain damage were equal to 0.51 and 0.47 respectively (Table 2 ).

Table 2. Calculated BSH compound factor for CNS necrosis defined at $1 \mathrm{~cm}$ depth in the brain and skin reactions.

\begin{tabular}{|c|c|}
\hline Biological endpoint (dose group) & BSH Compound factor \\
\hline $\begin{array}{c}\text { Skin moist desquamation w \& w/o ulceration } \\
\text { (38 Gy BORON dog group) }\end{array}$ & 0.51 \\
\hline Dermal necrosis (64 Gy BORON dog group) & 0.52 \\
\hline \hline CNS necrosis (27 Gy BORON dog group) & 0.47 \\
\hline
\end{tabular}

'Dose-modifying factors used for compound factor calculation: $D R F$ Gamma $=0.67$; $R B E$ other $=1.0$; RBE fast $n=5.0 ; \operatorname{RBE} N(n, p)=2.7$ 
Table 3. Summary of the evaluation of HFR dog irradiations to date (October 1992).

\begin{tabular}{|c|c|c|c|c|c|}
\hline $\begin{array}{r}\text { iradiation } \\
\text { date }: \\
\because \quad \therefore \\
\end{array}$ & $\begin{array}{l}\text { exposure } \\
\therefore \text { time } \\
\therefore \quad\end{array}$ & $\begin{array}{c}\left.\text { blood }{ }^{10} \mathrm{~B}\right) \\
(\mathrm{ppm})\end{array}$ & $\begin{array}{c}\text { Peak phys. dose } \\
\text { (Gy) }\end{array}$ & $\begin{array}{c}\text { Equivalent dose at } \\
2.25 \mathrm{~cm} \text { depth } \\
\left(\mathrm{RBE}^{*} \mathrm{~Gy}\right)\end{array}$ & Observed effect \\
\hline $91 / 7$ & 1.77 hrs & 38.5 & 17.5 & 14.7 & brain necrosis \\
\hline $91 \pi$ & $1.77 \mathrm{hrs}$ & 62.3 & 24.9 & 18.2 & brain necrosis \\
\hline $92 / 4$ & $1.60 \mathrm{hrs}$ & 50.7 & 19.2 & 14.9 & non-lethal damage \\
\hline $92 / 4$ & $1.60 \mathrm{hrs}$ & 47.6 & 18.3 & 14.4 & non-lethal damage \\
\hline $92 / 4$ & $1.60 \mathrm{hrs}$ & 52.0 & 19.6 & 15.1 & none \\
\hline $92 / 4$ & $1.43 \mathrm{hrs}$ & 58.7 & 19.2 & 14.2 & non-lethal damage \\
\hline $92 / 4$ & $1.43 \mathrm{hrs}$ & 53.2 & 17.9 & 13.7 & none \\
\hline $92 / 4$ & $1.93 \mathrm{hrs}$ & 0 & 6.2 & 9.9 & none \\
\hline $92 / 4$ & $1.93 \mathrm{hrs}$ & 0 & 6.2 & 9.9 & none \\
\hline $92 / 4$ & 1.93 hrs & 0 & 6.2 & 9.9 & none \\
\hline
\end{tabular}

To date 15 Beagle dogs have been irradiated using the HFR epithermal neutron beam. Two dogs irradiated last year, developed lethal brain necrosis. Of the 13 dogs irradiated in April and May this year, 2 out of 3 dogs that were irradiated at $19 \mathrm{~Gy}$ physical dose with $50 \mathrm{ppm}{ }^{10} \mathrm{~B}$ showed lesions in their brain comparable with those observed in the 27 Gy BORON dogs irradiated at the BMRR. Their calculated equivalent doses, calculated also $1 \mathrm{~cm}$ in the brain, ranged from 14.4-14.9 RBE*Gy and are in good agreement with the calculated equivalent dose for the BMRR irradiated dogs. One out of 2 dogs that were irradiated at $17 \mathrm{~Gy}$ physical dose with $50 \mathrm{ppm}{ }^{10} \mathrm{~B}$. The calculated equivalent doses are listed in Table 3. Three dogs irradiated with the epithermal beam alone at $6.2 \mathrm{~Gy}$ physical dose did not show any lesions. The other dogs irradiated in May are currently under evaluation. Although the dose description used in this study is arbitrary, the resulting dosemodifying factors allow prediction of biological effects.

The observed skin effects of the HFR irradiated dogs are reported elsewhere in these proceedings (Philipp et al, these proceedings). The calculated equivalent doses for the skin and the observed effects are in agreement with historical $\mathrm{X}$-ray data.

In conclusion, the use of a dose rate factor for the gamma component and the derived fast neutron RBE and compound factors for skin and brain, and a dose description beyond the thermal peak allow a good equivalent dose description in relation to the observed effects. The used dose-modification factors allow inter-comparisons of healthy tissue tolerance studies at different beams. Future results from both healthy tissue tolerance studies will refine the dose-modifying factors used for the equivalent dose description in BNCT.

\section{REFERENCES}

Fike, J.R. and Gobbel, G.T., 1991, Central nervous system radiation injury in large animals models, in: Radiation Injury to the Nervous System,Gutin,P.H., S.A. Leibel, G.E. Sheline, ed., pp.69-87, Raven Press, New York.

Fu, K.K., 1991, Influence of dose rate on normal tissue tolerance, in: Radiation Injury to the Nervous System, Gutin,P.H., S.A. Leibel, G.E. Sheline, ed., pp.69-87, Raven Press, New York.

Gavin, P.R., Wheeler, F.J., Huiskamp, R., Siefert, A., Kraft, S.L., and DeHaan, C.E., 1992, Large animal 
model studies of normal tissue tolerance using an epithermal beam and borocaptate sodium. In: Proc. European Collaboration on Boron Neutron Capture Therapy, Petten, Plenum Press, New York, in press (1993a).

Gavin, P.R., Huiskamp, R., Wheeler, FJ., Kraft, S.L. and DeHaan, C.E., Large animal normal tissue tolerance using an epithermal neutron beam and borocaptate sodium. Strahlentherapie und Onkologie 169 , in press (1993b).

Gavin, P.R., Kraft, S.L., DeHaan, C.E., Griebenow, M.L., and Moore, M.P., 1992, A large animal model for boron neutron capture therapy. In: Proc. of the 4th Intem. Symposium on Neutron Capture Therapy, Sydney, B.J. Allen, D.E. Moore, and B.V. Harrington, B.V., ed., pp.479-484, Plenum Press, New York.

Harker, Y.D., Anderl, R.A., Amaro, C.R., Watkins, P.R.D., and Voorbraak, W.P., Neutron spectrum measurements at the High Flux Reactor (Petten, The Netherlands) and the Brookhaven Medical Research Reactor, Ibid.

Harker, Y.D., Amaro, C.R., Randolph, P.R., and Wheeler, F.J., Neutron measurements in canine and cylindrical phantoms at the Brookahven Medical Research Reactor and the High Flux Reactor (Petten), Ibid.

Nigg, D.W., Wheeler, F.J, and Randolph, D.J. Characterization of the BMRR and PPBF epithermal neutron beams in phantom using three-dimensional deterministic radiation transport theory, In: Proc. of the 4th Intern. Symposium on Neutron Capture Therapy, Sydney, BJ. Allen, D.E. Moore, and B.V. Harrington, B.V., ed., pp.479-484, Plenum Press, New York.

Park, R.D., T.R. O'Brien, Baker,B.B., and MorganJ.C., 1974, Single dose irradiation of canine skin. Vet. Radiol. 15: 108.

Philipp, K.H.I., Gavin, P.R., Casado, J., Siefert, A., Moss, R.L. and Huiskamp, R., Developments in the healthy tissue tolerance studies as a precondition for clinical application of boron neutron capture therapy, Ibid.

Siefert, A., Casado, J., Philipp, K., Huiskamp, R., Gavin, P.R., Dühmke, E., and Moss, R.L., Brain effects observed in the healthy tissue tolerance studies in BNCT with borocaptate sodium at the epithermal neutron beam of the HFR, Petten, Ibid.

Watkins, P., Moss, R.L., Siefert, A.S., Huiskamp, R., Gavin, P.R. and Konijnenberg, M., Evaluation of dose components for the healthy tissue tolerance studies on dogs at the HFR Petten, Ibid. 



\section{Pharmacokinetics of Borocaptate Sodium in Canine Head Determined by ${ }^{11} \mathrm{~B}$ Magnetic Resonance Imaging and Spectroscopy}

K. Bradshaw, M. Schweizer, G. Glover, and J. Hadley

This article is a reprint from Advances in Neutron Capture Therapy, pp. 579-583, (1993). 



\title{
PHARMACOKINETICS OF BOROCAPTATE SODIUM
}

IN CANINE HEAD DETERMINED BY 11B

MAGNETIC RESONANCE IMAGING AND SPECTROSCOPY

\author{
K. M. Bradshaw, ${ }^{1}$ M. P. Schweizer, ${ }^{2}$ G. H. Glover, ${ }^{3}$ and J. R. Hadley ${ }^{2}$ \\ ${ }^{1}$ Neutron Technology, Boise, ID, 83702 \\ 2Medical Imaging Research Laboratory, University of Utah \\ Salt Lake City, UT \\ ${ }^{3}$ Dept. of Radiology, Stanıord University, Stanford, CA
}

\section{INTRODUCTION}

As new boron compounds are introduced for BNCT, requirements to conduct pharmacokinetic studies more quickly and noninvasively will intensify. The purpose of this paper is to present a method for determining the in vivo distribution and kinetics of $\mathrm{Na}_{2} \mathrm{~B}_{12} \mathrm{H}_{11} \mathrm{SH}$ (Borocaptate Sodium or $\mathrm{BSH}$ ) and to monitor it's deployment in real time. To obtain pharmacokinetic data in "real time" the method must be non invasive and fast enough to sample the changing environment. Magnetic Resonance (MR) had been suggested from the beginning as an ideal non invasive method, but, because of the low signal-to-noise (SNR) for boron imaging (about $10^{6}$ times less than proton imaging) and fast relaxation of the nuclei (less than $1 \mathrm{msec}$ ), problems seemed insurmountable. With the advances in MRI technology, a solution has been found that makes the premise of this research feasible. Instead of using two-dimensional chemical shift imaging 1,2 (2D-CSI) which takes too long to acquire image data, we are proposing the use of a threedimensional (3D) projection reconstruction method. The details of this method have been reported elsewhere ${ }^{3}$ and will not be repeated here. The method meets the necessary requirements of being non invasive and fast, with data acquisition on the order of minutes instead of hours.

To demonstrate the viability of using boron $3 \mathrm{D}$ projection reconstruction imaging to conduct pharmacokinetic research, in vivo studies were conducted involving dogs with and without tumors. The BSH uptake and elimination of different tissue types were compared qualitatively by comparing the relative boron MRI signals.

\section{METHODS}

\section{MR Imaging Techniques}

Using an idea from computed tomography, projection reconstruction has been adapted to an MRI system allowing faster acquisition of the data within the constraints of the fast decay time of the signal. The boron data are acquired within $140 \mu \mathrm{sec}$ of the start of the pulse sequence. 
${ }^{11} \mathrm{~B}$ is used in all imaging and spectroscopy techniques reported here since ${ }^{10} \mathrm{~B}$ is eight times less sensitive for equal number of nuclei ${ }^{4}$. Still ${ }^{11} \mathrm{~B}$ imaging is about $10^{6}$ times lower in SNR than achieved with proton imaging requiring special attention to SNR. Using a $0.5 \mathrm{~dB}$ low noise preamplifier and quadrature techniques, sufficient improvements in the SNR were made that allowed imaging of ${ }^{11} \mathrm{~B}$ concentrations below $25 \mathrm{ppm}$ using projection reconstruction. The most difficult challenge we had was the acquisition of the fast decaying MRI signal. Because the decay of the MRI signal from ${ }^{11} \mathrm{~B}$ in $\mathrm{BSH}$ bound to protein (T2 relaxation time) is on the order of $600 \mu \mathrm{sec}$, the signal dies out before it can be acquired using normal imaging sequences such as "spin echo". Techniques that required less pre data acquisition time to establish the imaging environment such as whole head spectroscopy and chemical shift imaging were tried but did not meet our imaging requirements.

With our projection reconstruction technique we can achieve an anisotropic volume of $7.5 \mathrm{~mm} \times 7.5 \mathrm{~mm} \times 15.0 \mathrm{~mm}$. A typical image series (scan) takes approximately eight minutes, although faster scanning is possible. During an eight-minute scan, the $3 \mathrm{D}$ data are collected with a GE Signa ${ }^{\mathrm{TM}} 1.5$ Tesla MRI system and then reconstructed on a Sun ${ }^{\mathrm{TM}}$ workstation. The resulting boron images can be superimposed over corresponding proton images of higher resolution or displayed separately. Using region of interest (ROI) measurements, the intensity data of the boron image is correlated with a known reference within the imaging volume, and the relative boron concentration is then determined. With this technique, pharmacokinetic studies are performed by taking a series of scans over time and comparing the same locations from scan to scan. Whole head spectroscopy is used to determine large volume kinetics.

\section{In Vivo Study Design}

Dogs with and without gliosarcoma tumors have been tested for BSH uptake and distribution using the following protocol:

1. The dog is initially immobilized with Telazol and then maintained on $1.5-2 \%$ isoflurane anesthesia for the remainder of the experiment.

2. Proton sagittal and axial $\mathrm{T} 1$ and $\mathrm{T} 2$ weighted images are obtained for anatomical referencing and tumor enhancement.

3. BSH is infused by $I V$ over 1 hour. Solution is $100 \mathrm{mg}{ }^{11} \mathrm{~B} / \mathrm{kg}$ in $11 \mathrm{ml}$ saline $/ \mathrm{kg}(\mathrm{kg}-$ dog weight).

4. Blood is sampled every 10 minutes during infusion and every 20 minutes during elimination phase of BSH.

5. Whole head spectroscopy is performed before each blood sampling using 100 averages.

6. $11 \mathrm{~B}$ images are collected every 20 minutes after infusion.

7. Tissue taken at necropsy and blood sampling taken during the experiment are analyzed by Inductive Coupled Plasma-Atomic Emission Spectroscopy.

\section{RESULTS}

Differences between the blood and tissue kinetics are seen by comparing the boron in blood with the whole head spectroscopy (Figure 1). Both ${ }^{11} \mathrm{~B}$ concentration in blood and ${ }^{11} \mathrm{~B}$ MR signal intensity are normalized to the maximum level observed. There is strong correlation during uptake, indicative of a concentration driven gradient, and a longer retention of boron in tissue for the first hour of the elimination phase. This last observation infers that the MR signal comes primarily from the tissue and that there is some mechanism slowing the release of BSH from tissue. This general kinetic behavior is corroborated by the invasive studies performed by Kraft et. $\mathrm{al}^{5}$. 


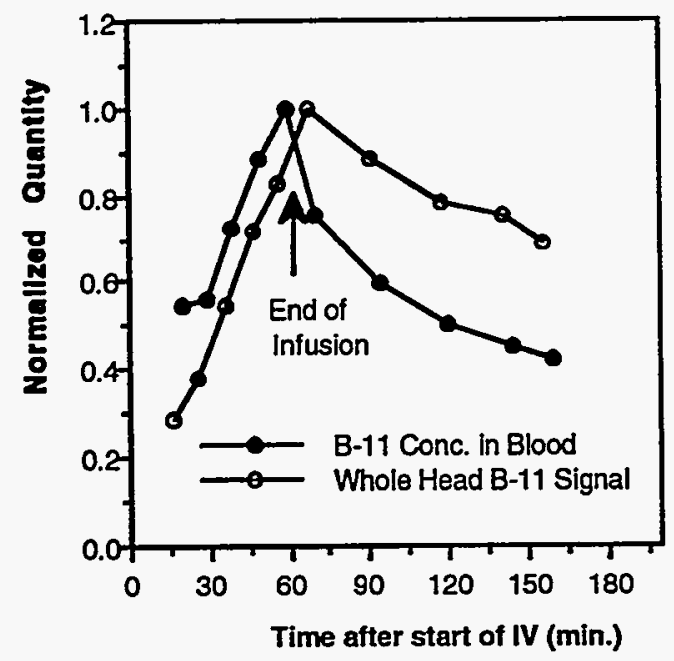

Figure 1. Comparison of boron spectroscopy signal from whole head with boron in whole blood.

ROI measurements of a $48 \mathrm{~cm}$ field of view (FOV) boron image with an isotropic volume (voxel) of $1.5 \mathrm{~cm} \times 1.5 \mathrm{~cm} \times 1.5 \mathrm{~cm}$ are shown in Figure 2. Here several different tissues are compared, with the more vascularized tissue showing faster changes over time, as expected. The intensity of each measurement was normalized against the average intensity from a vial filled with $50 \mathrm{ppm}$ boric acid which was placed on the dog's head over the eyes. Since these data re from a dog whose blood brain barrier was intact there was negligible concentration of BSH in the brain. For comparison, the end point of the graph for muscle (lines 3 and 4) corresponds to about $25 \mathrm{ppm}$ as measured by ICP-AES.

Examination of the images containing tumors offers additional understanding of the distribution of boron in the dog's head when the blood brain barrier has been destroyed by a

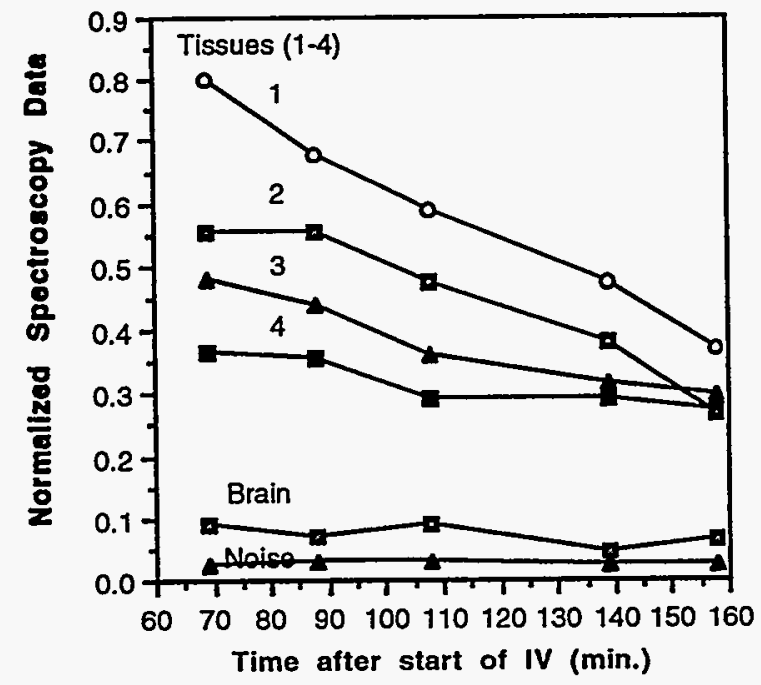

Figure 2. Comparison of boron signal intensity of different tissues of equal volumes after end of infusion of BSH. 1 and 2 are from tongue and nasal mucosa respectively, and 3 and 4 are from masticator muscle. Boron concentration in brain is negligible. 
tumor. Figure 3 shows a dog with a gliosarcoma located in the left hemisphere. A T2 weighted proton image on the right is provided for referencing anatomy. Comparing the intensity data from this image with data taken about one hour later shows that the boron in the surrounding tissue decreased by $30 \%$ whereas the boron decreased only by $14 \%$ in the tumor. This indicates a preferential retention of BSH in tumor. The normal brain still showed negligible amounts of boron.

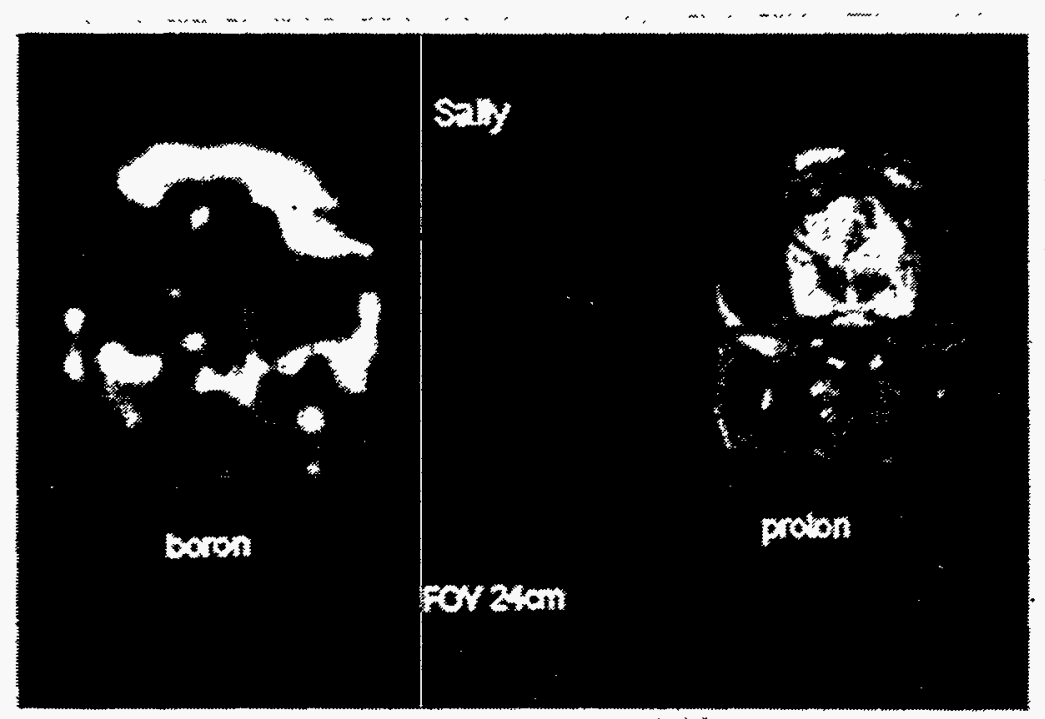

Figure 3. A $24 \mathrm{~cm}$ FOV boron image showing a gliosarcoma in the left hemisphere of the brain. Resolution is $7.5 \mathrm{~mm} \times 7.5 \mathrm{~mm} \times 15 \mathrm{~mm}$.

\section{CONCLUSIONS}

Using the projection reconstruction MR imaging technique, qualitative comparisons of boron in tissue (concentrations less than $25 \mathrm{ppm}$ ) can be obtained which also compares favorably with known invasive studies. With a scan time of $8 \mathrm{~min} .28 \mathrm{sec}$., the image data provide a fair "snap-shot" of the kinetic changes during the elimination phase. Since the biological half-life of BSH is about four hours, significant changes in boron concentration in tissue will not have occurred during each scan time. Future studies will focus on developing analytical methods to determine the absolute concentration of boron in vivo using the intensity of the MR signal. This will require using a standard boric acid reference that will be in the FOV of the boron image as well as investigation of potential intensity changes with different tissues.

The smallest anisotropic volume that has been achieved is $7.5 \mathrm{~mm} \times 7.5 \mathrm{~mm} \times 15.0$ $\mathrm{mm}$. Further improvements in the quadrature splitter and the elimination of extra coax cable, as well as faster repetition rates will improve the SNR making a smaller isotropic volume possible.

\section{ACKNOWLEDGMENTS}

This research was supported in part by the U.S. Department of Energy under contract DE-AC07-76ID01570 (Idaho National Engineering Laboratory). We thank Dr. John Hilton, and the Johns Hopkins University for the gliosarcoma cells. We appreciate the assistance of the following individuals from the University of Utah, Dr. Richard Tippets, 
Department of Neurosurgery, Suzanne Johnson and Tamer Ghanem (animal assistance), Dr. Cheryl Harris, Department of Pathology and Dr. Peng-Peng Tang, Department of Medicinal Chemistry.

\section{REFERENCES}

1. Kabalka, GW, Davis M, Bendel P, "Boron-11 MRI and MRS of Intact Animals Infused with a Boron Neutron Capture Agent," Magn Reson Med 1988;8:231-237.

2. Bradshaw, KM, Richards TL, "In vivo Chemical Shift Imaging of Canine Brain Tumors After Injection of Na2B12H11SH (abstr)," In: Book of abstracts; Society of Magnetic Resonance in Medicine 1990, Berkeley, Calif: Society of Magnetic Resonance in Medicine, 1990; 1166.

3. Glover, GH, Pauli, JM, Bradshaw, KM, "Boron-11 Imaging with a Three-dimensional Reconstruction Method," JMRI, Jan./Feb., pp. 47-52, 1992.

4. Bovey, FA; in "Nuclear Magnetic Resonance Spectroscopy," 2nd Ed. p3, Acad. Press, 1988.

5. Kraft SL, Gavin PR, DeHaan CE, Leathers CW, Bauer WF, Dorn RV. The biodistribution of boron in canine spontaneous intracranial tumors following borocaptate sodium infusion. In: Progress in NCT for Cancer. Allen BJ et al, Eds. Plenum Press, New York, 1992. pp. 479-484. 



\section{Bioavailability of Intravenous Formulations of p-Boronophenylalanine in Dog and Rat}

T. LaHann, D. Lu, C. Sills, G. Daniell, S. Kraft, P. Gavin, and W. Bauer

This article is a reprint from Advances in Neutron Capture Therapy, pp. 585-589, (1993). 

BIOAVAILABILITY OF INTRAVENOUS FORMULATIONS OF

\title{
P-BORONOPHENYLALANINE IN DOG AND RAT
}

\author{
T.R. LaHann, D.R. Lu, G. Daniell, C. Sills, ${ }^{1}$ S.L. Kraft, ${ }^{2}$ P.R. Gavin, ${ }^{3}$ and \\ W.F. Bauer ${ }^{4}$ \\ 'Center for Toxicology Research \\ Department of Pharmaceutical Sciences \\ Idaho State University \\ Pocatello, Idaho, 83209 \\ ${ }^{2}$ College of Veterinary Medicine \\ Kansas State University \\ Manhattan, Kansas, 66506 \\ ${ }^{3}$ College of Veterinary Medicine \\ Washington State University \\ Pullman, Washington, 99164 \\ 'Idaho National Engineering Laboratory \\ Idaho Falls, Idaho, 83415
}

\section{INTRODUCTION}

Boron neutron capture therapy (BNCT) is being developed as a treatment for malignant melanoma. P-boronophenylalanine (BPA) was initially proposed as a boron (B) delivery drug for BNCT of malignant melanoma because it was postulated that this B-containing amino acid, by mimicking a melanin precursor, would selectively accumulate in melanoma cells. BPA does seem to selectively accumulate in melanocytes, apparently as a result of uptake by an amino acid transport system ${ }^{1}$. For successful BNCT, tumor B concentrations of at least $20 \mathrm{ppm}$ are thought to be necessary, but higher tumor $B$ levels are desirable. Calculations ${ }^{2}$ indicate that for a given neutron exposure, each doubling of the tumor $B$ concentration should increase tumor cell kill by a factor of about 10,000 . Thus, even modest increases in the amount of $B$ in tumor cells can dramatically improve the effectiveness of BNCT as a cancer treatment.

If amino acid transport systems accumulate BPA, then high extracellular concentrations of BPA should increase the amount of BPA entering melanocytes. I.V. infusion of BPA is the simplest route for delivering BPA to the tumor cells, but is not widely used because at physiological pH, BPA exhibits poor water solubility $(<4 \mathrm{ppm})$. Possible options for increasing BPA solubility include host-guest formation ${ }^{3}$ and use of organic complexes ${ }^{4}$. The purpose of the work reported here was to 1) determine the extent to which the aqueous solubility of BPA is increased by hostguest complex formation with the cyclodextrin 2-hydroxypropyl- $\beta$-cyclodextrin and by organic complex formation with fructose and 2) test the hypothesis that i.v. formulations which enhance BPA solubility will increase the delivery of B to tissue sites. 


\section{METHODS}

D,L-p-boronophenylalanine was purchased from Callery Chemical Company (Pittsburgh PA) and 2-hydroxypropyl- $\beta$-cyclodextrin $\left(\mathrm{Molecusol}^{\oplus}\right.$ ) was purchased from Pharmatec, Inc. (Alachua, FL). For solubility determinations, an excess of BPA was always used. All formulations were sonicated, adjusted to a final $\mathrm{pH}$ of 7.4 and filtered through a 0.22 micron filter. The BPA concentration of filtered solutions was calculated from B levels which were measured by inductively coupled plasma atomic emission spectrometry. Formulations used in rat studies were prepared immediately before use; formulations for dog experiments were prepared within 3 days of use. High performance liquid chromatography studies demonstrated that aqueous BPA solutions were stable at physiological $\mathrm{pH}$ for at least 3 days. Male Sprague-Dawley rats $(300-400 \mathrm{~g})$ and female Beagle dogs (8.3-11.0 $\mathrm{Kg}$ ) were anesthetized, a vein cannulated for infusion of drug, and either the cephalic vein (dog) or the femoral artery (rat) cannulated for blood sampling. Drug was infused to dogs at a target rate of $17 \mathrm{ml} / \mathrm{kg} / \mathrm{hr}$ for a one hour period, while rats were infused at target rates of $8.5,12.8$ or 17 $\mathrm{ml} / \mathrm{kg} / \mathrm{hr}$ for 1-3 hours. Urine was collected by urinary catheter (dogs) or bladder cannulation (rats). Plasma and serum samples were collected from dogs prior to, during (every 20 minutes) and after the infusion (8 to 10 samples over $72 \mathrm{hrs).} \mathrm{Plasma} \mathrm{and} \mathrm{serum} \mathrm{samples} \mathrm{were} \mathrm{collected}$ from rats at hourly intervals for 3-7 hrs. Each dog received three treatments: 1) BPA in buffer, 2) BPA in Molecusol $^{\Phi}$ and 3) Molecusol $^{\oplus}$ alone, with at least a three week wash-out period between infusions. Each rat received only a single drug formulation. Pharmacokinetic analysis of the data was conducted using the computer program PharmK (v.1.2.). Unless otherwise specified, BPA and $B$ levels are reported as mean \pm SEM of three experiments.

\section{RESULTS AND DISCUSSION}

\section{Solubility Studies}

B levels in distilled water and in $0.1 \mathrm{M}, \mathrm{pH} 7.4$ phosphate buffer were below the limits of detection $(<0.008 \mathrm{mg} / \mathrm{ml} \mathrm{BPA})$. The solubility of BPA in the buffer was $2.2 \pm 0.4 \mathrm{mg} / \mathrm{ml}$ $(\mathrm{N}=7)$, but varied as a function of the lot of BPA used. Different BPA lots displayed aqueous solubilities ranging from 1.15 to $3.7 \mathrm{mg} / \mathrm{ml}$. By briefly increasing the $\mathrm{pH}$ of the BPA-buffer suspension to about 10 before adjusting it to 7.4, more BPA could be solubilized. A pH 7.4 BPA solution of $6.0 \pm 0.1 \mathrm{mg} / \mathrm{ml}(\mathrm{N}=4)$ prepared by manipulation of $\mathrm{pH}$ did not precipitate for several hours. BPA solubility in Molecusol ${ }^{\oplus} /$ neutral water solutions increased with increasing concentrations of Molecusol $^{\oplus}(10-45 \% \mathrm{w} / \mathrm{v})$ and since $45 \%$ was approximately iso-osmotic, this concentration was selected for in vivo experimentation. The BPA concentration in $45 \%$ Molecusol ${ }^{\Phi} /$ water was $6.4 \pm 0.1 \mathrm{mg} / \mathrm{ml}$. Briefly elevating the $\mathrm{pH}$ of the BPA-Molecusol ${ }^{\oplus}$ suspension to about 10 and then readjusting $\mathrm{pH}$ to 7.4 solubilized more $\mathrm{BPA}(11.5 \pm 1.0 \mathrm{mg} / \mathrm{ml})$. However, the greatest increase in solubility was achieved by complexing BPA with fructose. A 1:1 molar ratio of BPA and fructose in pH 7.4 distilled water solubilized less than $10 \mathrm{mg} / \mathrm{ml} \mathrm{BPA}$. Briefly increasing the $\mathrm{pH}$ of a $200 \mathrm{mg} / \mathrm{ml}$ BPA suspension to about 10 and then readjusting $\mathrm{pH}$ to 7.4 solubilized $173.2 \pm 3.8 \mathrm{mg} / \mathrm{ml}$ BPA $(\mathrm{N}=18$, range: 148.6 - 196.9). Neither BPA-fructose nor BPA-Molecusol ${ }^{\oplus}$ formulations showed signs of precipitation over a 7 day observation period and when added to rat plasma, showed no macroscopic evidence of precipitation.

\section{Dog Studies}

The plasma B concentrations, plotted as a function of time during and after i.v. infusion of BPAbuffer $(1.15 \mathrm{mg} / \mathrm{ml})$ and BPA-Molecusol ${ }^{\otimes}(5.7 \mathrm{mg} / \mathrm{ml})$ are shown in Figure 1 .

In previous dog experiments, either serum or plasma B levels were measured. Significant differences between serum and plasma B concentrations were not observed in the current experiments, indicating that either could be used. The peak serum concentration achieved with the BPA-Molecusol ${ }^{\Phi}$ formulation $(16.5 \pm 1.9 \mathrm{ppm} \mathrm{B})$ was higher than that achieved with the BPA- 


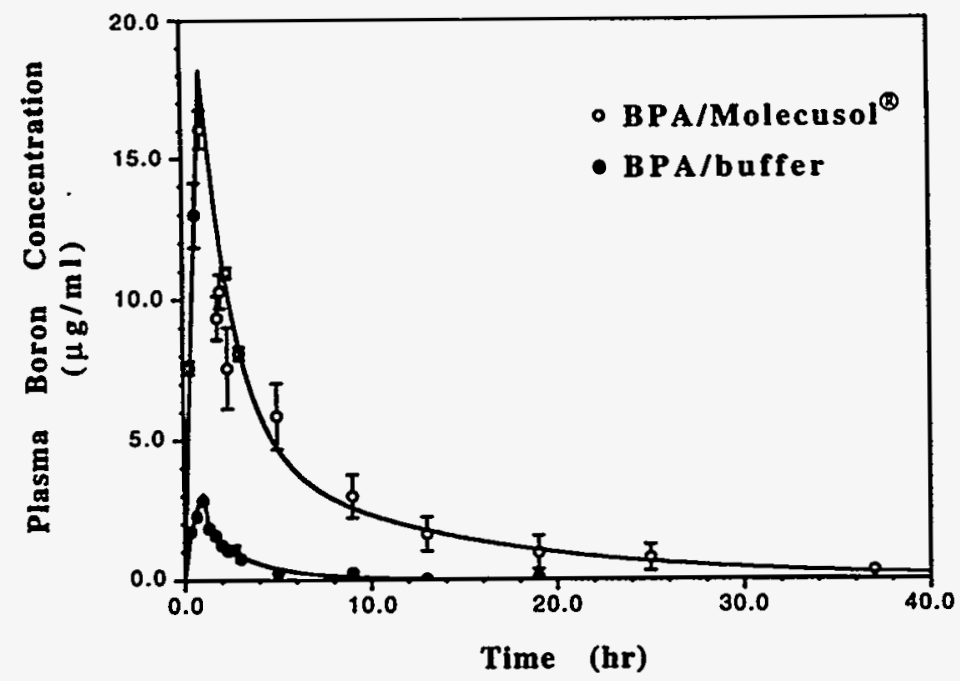

Figure 1. Plasma B profiles after infusion of BPA-water-Molecusol ${ }^{\bullet}(\bullet)$ and BPA-buffer $(0)$ formulations into dogs.

buffer formulation ( $3.8 \pm 0.3 \mathrm{ppm} \mathrm{B)}$ and higher than the peak values achieved with subcutaneous administration of a $200 \mathrm{mg} / \mathrm{kg}$ suspension (peak serum level: $3.4 \pm 0.5 \mathrm{ppm} \mathrm{B}, \mathrm{N}=5$ ) or oral administration of a $50 \mathrm{mg} / \mathrm{kg}$ suspension (peak serum level: $2.0 \pm 0.6 \mathrm{ppm} \mathrm{B}$ ). A two compartment model for i.v. infusion was used to fit the data. This model predicts that the plasma concentration versus time curve will be biexponential: $C_{p}=A\left(e^{a T}-1\right) e^{-t t}+B\left(e^{b T}-1\right) e^{-b}$. During the infusion period, $T=t$, and during the post-infusion period $T$ becomes a constant (i. e. the duration of the infusion). For the BPA-Molecusol ${ }^{\Phi}$ formulation, the parameters were calculated to be: $\mathrm{A}$ $=30.83 ; \mathrm{B}=59.27 ; \mathrm{a}=0.58$ and $\mathrm{b}=0.08$, while for the BPA-buffer formulation, the parameters were calculated to be: $A=1.28 ; B=5.67 ; a=4.43$ and $b=0.38$. The areas under the plasma concentration-time curve (AUCs), the half lives (b phase), apparent volumes of distribution and clearances were 90.10 and $6.95 \mu \mathrm{gr} / \mathrm{mL}, 8.43$ and $1.83 \mathrm{hr}, 0.20$ and $0.12 \mathrm{~L} / \mathrm{kg}$, and 0.05 and $0.14 \mathrm{~L} / \mathrm{hr} / \mathrm{kg}$ for the BPA-Molecusol ${ }^{\circ}$ formulation and for the BPA-buffer formulation, respectively. Measurement of urine and blood B levels suggested that over the first 4 hours post-infusion, most of the B remained in the body, but resided in tissues other than blood. Assuming that total tissue load 4 hours after termination of infusion (tissue $B$ (tso) can be calculated as: (total $B$ infused)-[(B in blood $\left.{ }_{4 \pi n}\right)+$ (urinary excretion of $\left.\left.B_{4 b a n}\right)\right]$, and that the drug distributes equally throughout the dog tissues (i.e., the dog mimics a single, well-stirred compartment), the average tissue $B$ ars achieved with the BPA-Molecusol ${ }^{\circledR}$ formulation can be estimated as $4.5 \pm 0.9$ $\mathrm{ppm}$. Blood B levels at this time were $3.4 \pm 1.1 \mathrm{ppm}$. For the BPA-buffer infusion, average tissue $B$ sare was $0.9 \pm 0.1 \mathrm{ppm}$, with blood $B_{4 \mathrm{hr}}$ of $0.4 \pm 0.2 \mathrm{ppm}$.

\section{Rat Studies}

Plasma, serum and tissue samples were collected from rats infused with buffer only, with BPA in buffer (mean BPA concentration: $1.2 \mathrm{mg} / \mathrm{ml}$ to mimic dog experiments), with BPA in pH adjusted buffer (mean BPA concentration: $6.0 \mathrm{mg} / \mathrm{ml}$ ), and with BPA in fructose/water (mean BPA concentration: $173.2 \mathrm{mg} / \mathrm{ml}$ ). BPA-buffer was infused at a target rate of $17 \mathrm{ml} / \mathrm{kg} / \mathrm{hr}$ for one hour and actual rates were within 5\% of the target value. The BPA-fructose complex was infused at target delivery rates of 12.8 and $17 \mathrm{ml} / \mathrm{kg} / \mathrm{hr}$, and delivered BPA doses were $2,217 \pm 39 \mathrm{mg} / \mathrm{kg}$ $(\mathrm{N}=15)$ and $2,917 \pm 61 \mathrm{mg} / \mathrm{kg}(\mathrm{N}=8)$. Actual infusion rates were within $7 \%$ of target values. Rats infused at target doses of 12.8 and $17 \mathrm{ml} / \mathrm{kg} / \mathrm{hr}$ of BPA survived if subject to minimal surgical stress, but rats infused with $4,334 \pm 56 \mathrm{mg} / \mathrm{kg}$ (target dose: $1,586 \mathrm{mg} / \mathrm{kg} / \mathrm{hr}$ for 3 hours) all died. Toxicities associated with this formulation are discussed in an accompanying paper ${ }^{5}$. Rats infused 


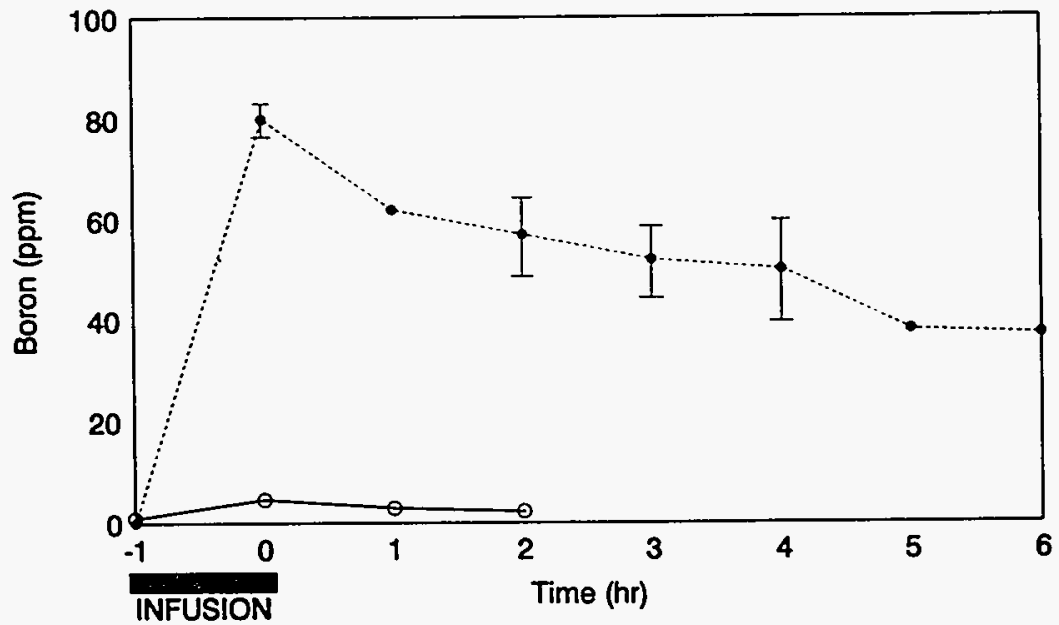

Figure 2. Time course of serum B after infusion of $2,063 \pm 55 \mathrm{mg} / \mathrm{kg}(\mathrm{N}=3)$ of BPA-fructose-water ( $\bullet$ and $99 \pm 1.7 \mathrm{mg} / \mathrm{kg}(\mathrm{N}=5)$ of BPA-buffer $(0)$ formulations into rats. Equal volumes of formulation were infused over a one-hour period (bar).

with BPA doses of $2,148 \pm 45 \mathrm{mg} / \mathrm{kg}(\mathrm{N}=6)$ were sacrificed 6 hours after termination of the BPA infusion, and tissue samples collected for boron analysis. Figure 2 illustrates the difference in the time course of serum B when equal volumes of the BPA-fructose complex and the BPA-buffer solution $(6 \mathrm{mg} / \mathrm{ml})$ were infused.

Serum B values were not significantly different from plasma $B$ levels. If the assumption is made that BPA distributes equally throughout the tissues of the rat (i.e., the rat is viewed as a single, well-stirred compartment) the average tissue distribution of BPA at the termination of the BPAfructose infusion can be estimated to be $1.58 \mathrm{mg} / \mathrm{g}$, for an average tissue $B$ concentration of 75.7 $\mathrm{ppm}$. Using the same assumption, the average tissue concentration of BPA after infusion of BPAbuffer, can be estimated to be $0.075 \mathrm{mg} / \mathrm{g}$, for an average tissue B concentration of $3.6 \mathrm{ppm}$. Twenty ppm B is considered the lower limit for effective BNCT. Analysis of tissue samples demonstrated that even 6 hours after i.v. infusion of target doses of $2,148 \pm 45 \mathrm{mg} / \mathrm{kg}$ BPA $(\mathrm{N}=6)$, tissue B levels greatly exceeded the requirement for effective BNCT (e.g., heart: $42 \pm 7$ ppm, muscle: $56 \pm 10 \mathrm{ppm}$, lung: $64 \pm 19 \mathrm{ppm}$, liver: $54 \pm 14 \mathrm{ppm}$ ). Tissue B levels achieved 2 hours after infusion of the $6 \mathrm{mg} / \mathrm{ml}$ BPA-buffer solution were much lower (e.g., heart: $2.3 \pm$ $0.2 \mathrm{ppm}$, muscle: $3.3 \pm 1.6 \mathrm{ppm}$, lung: $2.7 \pm 0.4 \mathrm{ppm}$, liver: $2.9 \pm 0.3 \mathrm{ppm}$ ) and tissue levels of B achieved 2 hours after infusion of $1.2 \mathrm{mg} / \mathrm{ml} \mathrm{BPA}$ in buffer were lower yet (heart: $1.0 \mathrm{ppm}$, muscle: $0.8 \mathrm{ppm}$, lung: $1.1 \mathrm{ppm}$, liver: $1.0 \mathrm{ppm}, \mathrm{N}=2$ ). Rats receiving control infusions (no $\mathrm{BPA}$ ) had tissue $B$ levels near or below the detection limit for B $(0.4 \mathrm{ppm})$.

\section{CONCLUSIONS}

Our studies suggest that novel formulations of BPA should increase the delivery of B (BPA) to malignant melanoma cells, while eliciting little or no systemic toxicity. Of the options investigated, BPA complex formation with fructose appears most promising. Using the fructose formulation, BPA doses of about $3,000 \mathrm{mg} / \mathrm{kg}$ could be administered to rats without inducing obvious toxicities. Tissue B levels measured 6 hours after termination of the infusion were still 2 to 4 fold greater than the minimum levels $(20 \mathrm{ppm})$ deemed necessary for effective BNCT. Given these results, we postulate that i.v. infusion of high doses of BPA delivered as a BPA-fructose complex will increase the B loading of melanoma cells and facilitate BNCT of malignant melanoma. Studies to test this hypothesis are underway. 


\section{ACKNOWLEDGEMENTS}

The authors wish to thank Dr. E. Jarvi (ISU) for performing the HPLC analysis, and Ms. C. Johnson and Mr. V. Sweet (WSU) for assisting with the animal experiments. This study was performed under the auspices of the U.S. Department of Energy, Office of Energy Research, DOE Idaho Field Office, Contract No. DE-AC07-76ID01570.

\section{REFERENCES}

1. Coderre J., Glass, J., Fairchild, R., Roy, U., Cohen, S. \& Faud, I. (1987) Selective Targeting of BPA to Melanoma for Neutron Capture Therapy, Cancer Res. 47, 6377-6383.

2. Wheeler, F., Griebenow, M., Wessol, D., Nigg, D. \& Anderl, R. (1989) A stoichastic model for high LET response for BNCT, in Basic Life Sciences: Clinical Aspects of Neutron Capture Therapy, eds. Fairchild, R., Bond, V. \& Woodhead, A. (Plenum Press, New York), pp 165-178. 3. Brewster, M., Simplins, J., Hora, M., Stern, W. \& Bodor, N. (1989) The Potential Use of Cyclodextrins in Parenteral Formulations, J. Parent. Sci. Technol. 43, 231-240.

4. Yoshino, K., Suzuki, A., Mori, Y., Kakihana, H., Honda, C., Mishima, Y., Kobayashi, T. \& Kanda, K. (1989) Improvement of Solubility of p-Boronophenylalanine by Complex Formation with Monosaccharides, Strahlenter. Onkol. 165, 127-129.

5. LaHann, T., Sills, C., Hematillake, G., Dymock, T. \& Daniell, G. (in press) Cardiovascular toxicities associated with i.v. administration of p-BPA formulations, in Proceedings of the Fifth International Symposium on Neutron Capture Therapy, eds. Barth, R. \& Soloway A. (Plenum Press, New York).

\section{COPYRIGHT}

The submitted publication has been authored by a contractor of the U.S. Government under DOE Contract No. DE-AC07-76ID01570. Accordingly, the U.S. Government retains a nonexclusive, royalty-free license to publish or reproduce the published form of this contribution or allow others to do so, for U.S. Government purposes. 
, 


\section{Computer Modeling the Boron Compound Factor in Normal Brain Tissue}

P. Gavin, R. Huiskamp, F. Wheeler, and M. Griebenow

This article is a reprint from Advances in Neutron Capture Therapy, pp. 591-595, (1993). 



\title{
IN NORMAL BRAIN TISSUE
}

\author{
P.R. Gavin, ${ }^{1}$ R. Huiskamp, ${ }^{2}$ F.J. Wheeler, ${ }^{3}$ and M.L. Griebenow ${ }^{3}$ \\ ${ }^{1}$ Washington State University, Department of Veterinary Clinical Medicine \\ \& Surgery, Pullman, WA 99164-6610 USA \\ ${ }^{2}$ Netherlands Energy Research Foundation, Petten, The Netherlands \\ ${ }^{3}$ Idaho National Engineering Laboratory, EG\&G Idaho, Inc., INEL BNCT \\ Program, P.O. Box 1625, Idaho Falls, ID 83415-1575 USA
}

\section{INTRODUCTION}

The macroscopic distribution of borocaptate sodium $\left(\mathrm{Na}_{2} \mathrm{~B}_{12} \mathrm{H}_{11} \mathrm{SH}\right.$ or $\left.\mathrm{BSH}\right)$ in normal tissues has been determined and can be accurately predicted from the blood concentration. ${ }^{1,2}$ The compound para-borono-phenylalanine (p-BPA) has also been studied in dogs and normal tissue distribution has been determined. ${ }^{3}$ The total physical dose required to reach a biological isoeffect appears to increase directly as the proportion of boron capture dose increases. ${ }^{4}$ This effect, together with knowledge of the macrodistribution, led to estimates of the influence of the microdistribution of the BSH compound. This paper reports a computer model that was used to predict the compound factor for BSH and p-BPA and, hence, the equivalent radiation in normal tissues. The compound factor would need to be calculated for other compounds with different distributions. This information is needed to design appropriate normal tissue tolerance studies for different organ systems and/or different boron compounds.

\section{MATERIALS AND METHODS}

A Monte Carlo computer model of the capillaries was used to determine the physical and equivalent dose to the endothelial cell nucleus as a function of the boron level and distribution pattern (Figure 1). Charged-particle transport methods used have been described. ${ }^{5}$

Known macroscopic and inferred microscopic information from the canine studies were used in the models to predict the effect of the epithermal beam and the ${ }^{10} \mathrm{~B}(\mathrm{n}, \alpha)$ reaction on the endothelium. The equivalent dose to the capillary nucleus was held constant at 12 equivalent Gy. The RBE values used for nitrogen capture (n,proton), ${ }^{10} \mathrm{~B}(\mathrm{n}, \alpha)^{7} \mathrm{Li}$, and fast neutrons were $2.7,2.3$, and 4.5 , respectively. The first two values are from the literature and the fast-neutron RBE was derived from skin reactions in dogs. 6,7 

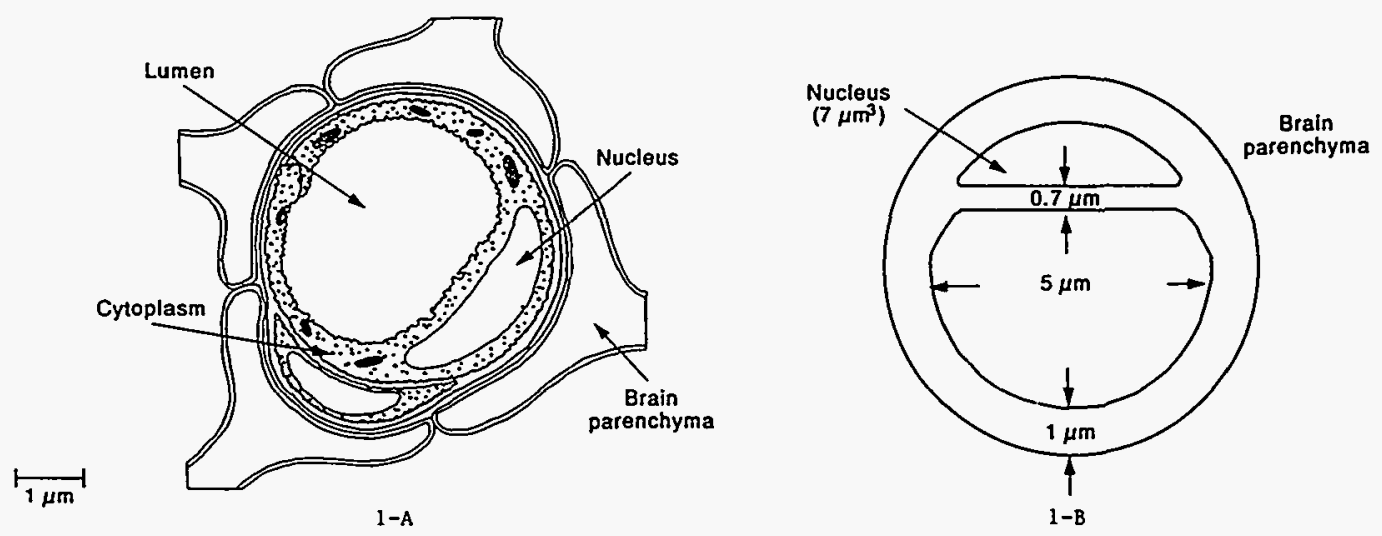

Figure 1. Brain capillary model. $A=$ artist's drawing of histologic structure; $\mathrm{B}=$ computer model.

\section{RESULTS}

Using the BMRR epithermal beam parameters, the capillary model, with a distribution similar to BSH of $25 \mathrm{ppm}$ in the lumen and $1 \mathrm{ppm}$ in the endothelial cell cytoplasm and surrounding parenchyma, resulted in a capillary endothelial-nuclear physical dose of 10.5 Gy with a peak physical dose of $20 \mathrm{~Gy}$. The calculated equivalent dose to the endothelial nuclei was 16.54 equivalent Gy (Table 1). The percentages of boron capture physical dose to the capillary endothelial nucleus from interregional transport of ${ }^{10} \mathrm{~B}$ capture fragments is illustrated in Figure 2-A.

The model resulted in a capillary endothelial-nuclear physical dose of $11.6 \mathrm{~Gy}$ when the boron was $8 \mathrm{ppm}$ and uniformly distributed in the blood, endothelial cell, and surrounding parenchyma (i.e., a distribution pattern observed with p-BPA). The calculated equivalent dose to the endothelial nuclei was 19.51 equivalent Gy (Table 1). The percentage physical dose contributions to the endothelial cell nucleus from the transport of ${ }^{10} \mathrm{~B}$ capture fragments is depicted in Figure 2-B.

The fraction of the total boron dose that struck the endothelial nucleus, multiplied by the RBE of 2.3, resulted in a compound factor of 0.40 for the first case with asymmetric microdistribution. The second case, with uniform microdistribution, resulted in a compound factor of 1.96 .

\section{DISCUSSION}

Some inferences of the microscopic distribution can be drawn from the macroscopic distribution of BSH and p-BPA in the blood and brain. The normal blood-brain-barrier excluded the boron from the normal brain parenchyma following an intravenous administration of BSH. The primary portion of the barrier is the tight junctions between the endothelial cells. ${ }^{8}$ This fact, together with the hydrophilic nature of the compound, would dictate that the compound is also excluded from the endothelial cytoplasm. The boron associated with p-BPA readily crossed the blood-brain-barrier and was present in the normal brain parenchyma in the same concentration as the blood, indicating a large extravascular component. Since it is the boron element that is analyzed rather than the parent phenylalanine compound, the mode of boron passage into the brain is unknown. Phenylalanine is a precursor to the neuroamines and there are active transport mechanisms for these compounds in the brain endothelial cells. In any case, since the boron was 
Table 1. Brain capillary endothelial dose

\begin{tabular}{|c|c|c|c|c|c|c|c|}
\hline \multirow{2}{*}{$\begin{array}{l}\text { Type of } \\
\text { compound } \\
\text { distribution }\end{array}$} & \multirow{2}{*}{$\begin{array}{l}\text { Type of } \\
\text { calculation }\end{array}$} & \multicolumn{6}{|c|}{ Dose } \\
\hline & & $10 \mathrm{~B}(\mathrm{n}$, alpha) & $14 N(n, p)$ & $\begin{array}{c}\text { Fast } \\
\text { neutron }\end{array}$ & Gamma & Other & Total \\
\hline Non-uniform" & $\begin{array}{c}\text { Physical } \\
\text { (Gy) }\end{array}$ & 2.20 & 0.88 & 0.90 & 5.04 & 0.60 & 9.62 \\
\hline (BSH in brain) & $\begin{array}{c}\text { Equivalent } \\
\text { dose } \\
\left(E_{q}^{*} \mathbf{G y}^{\mathrm{e}}{ }^{\mathrm{e}}\right.\end{array}$ & 5.07 & 2.38 & 4.05 & 5.04 & 0.60 & 17.14 \\
\hline Uniform & $\begin{array}{l}\text { Physical } \\
\text { (Gy) }\end{array}$ & 3.43 & 0.88 & 0.90 & 5.04 & 0.60 & 10.85 \\
\hline (p-BPA in Brain) & $\begin{array}{c}\text { Equivalent } \\
\text { dose } \\
\left(\mathbf{E q}^{*} \mathbf{G y}\right)^{\mathbf{c}}\end{array}$ & 7.89 & 2.38 & 4.05 & 5.04 & 0.60 & 19.96 \\
\hline
\end{tabular}

$25 \mathrm{ppm}$ boron in the endothelial lumen, $1 \mathrm{ppm}$ in the endothelial cytoplasm and brain parenchyma.

${ }^{6} 8 \mathrm{ppm}$ boron (l-BPA) in the endothelial lumen \& cytoplasm, \& $8 \mathrm{ppm}$ in the brain parenchyma.

'RBE's used for equivalent dose calculation: 2.3 for $10 \mathrm{~B}(\mathrm{n}, \mathrm{alpha}) ; 2.7$ for $14 \mathrm{~N}(\mathrm{n}, \mathrm{p})$; \& 4.5 for fast neutrons.
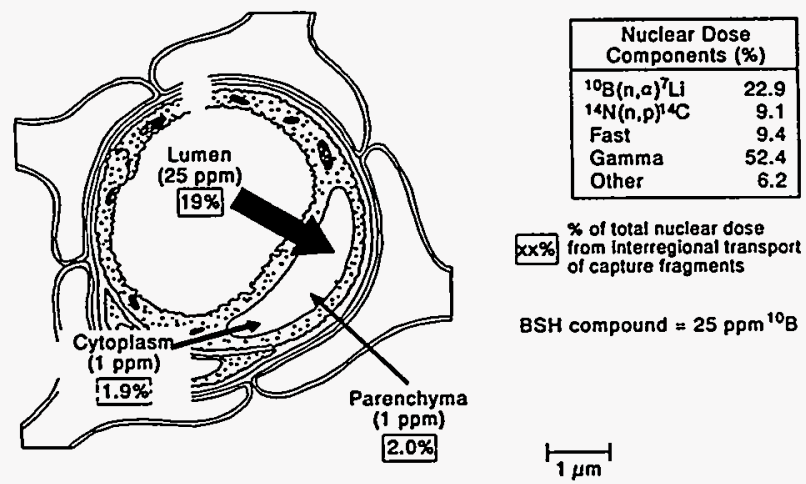

BSH compound $=25 \mathrm{ppm}^{10} \mathrm{~B}$

$\longmapsto$

$3-\AA$
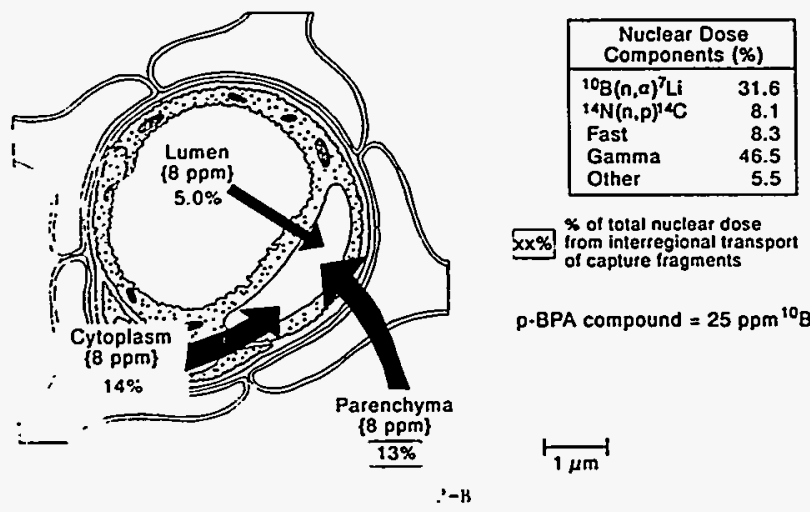

$x$ of total nuclear dose

x.\%. Trom interregional transpo

of capture tragments

p-BPA compound $=25 \mathrm{ppm}^{10} \mathrm{~B}$

$\longmapsto$

Figure 2. $A=$ Percentage of endothelial nucleus doses from interregional transport of ${ }^{10} \mathrm{~B}$ capture fragments. Nonuniform boron distribution that occurs in the brain following BSH infusion. With a tumor-to-blood ration of $1: 1$, the tumor concentration would be $25 \mathrm{ppm}$; $\mathrm{B}=$ Percentage of endothelial nuclear dose from interregional transport of ${ }^{10} \mathrm{~B}$ capture fragments. Uniform boron distributions that occur in the skin following BSH infusion, or in skin and brain following p-BPA administration. Calculations also used $25 \mathrm{ppm}$ in tumor (tumor-to-blood ratio of 3:1. 
present in approximately equal concentrations in the blood and brain, there must be similar concentrations in the endothelial cytoplasm.

The capillary endothelial model resulted in the endothelial nuclear radiation doses, as depicted in Figures 2-A and 2-B. The lumenal concentrations used for Figure 2-A would be similar to those that occur with a tumor concentration of $25 \mathrm{ppm}$ and a tumor-to-blood ratio of 1 , as observed with the BSH compound. The lumenal concentration used in Figure 2-B would be similar to those that occur with a tumor concentration of $25 \mathrm{ppm}$ and a tumor-to-blood ratio of 3 , as observed with the p-BPA compound.

The vascular computer model for the endothelial response resulted in compound factors for the two scenarios that were markedly similar to those calculated from the doghead irradiation studies using brain necrosis endpoints. ${ }^{6}$ The nonuniform compound distribution (BSH in brain) resulted in a computed compound factor of $0.33-0.40$ that would model endothelial dose response observed in the canine brain necrosis. ${ }^{6}$ The compound factor is the product of RBE and microscopic physical dose divided by total or macroscopic physical dose. This compound factor would indicate that approximately $83 \%$ of the peak physical boron capture dose (roughly 5 of 6 fission events) do not contribute to the BSH central nervous system endothelial nuclear dose. This sparing is greater than that previously reported, but less than that observed in the canine normal tissue brain studies. ${ }^{9,10}$

The uniform boron distribution (p-BPA in skin and brain) resulted in a compound factor of 1.96 that would model the endothelial dose seen in the canine skin necrosis (BSH in skin). ${ }^{6}$ Skin necrosis, that can occur approximately 3 months following irradiation, has been attributed to vascular damage to the dermis. ${ }^{11}$ The canine skin response indicated an increased compound factor of BSH in skin compared to brain, but a value of only 0.5 was obtained. ${ }^{6}$ The skin boron concentration is the total boron per gram of a complex tissue. The tissue analyzed contained hair, epidermis, dermis, and subcutaneous fat. The discrepancy between the biologic and computer compound factors for BSH in skin indicates that the boron was not uniformly distributed in relation to the dermal vessels. Microscopic quantification of the boron in skin is needed before this discrepancy can be fully explained.

Brain necrosis with BSH is logically due to the capillary BNCT endothelial dose and this has been supported by histologic analysis of the affected brains. Skin necrosis that can occur approximately three months following irradiation has also been attributed to vascular damage to the dermis. ${ }^{11}$ The uniform boron distribution model simulates the $p$ BPA distribution in the brain. Additional critical targets, including the myelin-producing oligodendrogliocytes, may decrease the tolerance of the brain with this compound. ${ }^{12}$ The calculated endothelial nuclear physical and equivalent doses shown in Table 1 were substantially higher in the uniform distribution model, even with one-third of the lumenal concentration of boron.

The vascular sparing of the capillary endothelial nuclei has been predicted. ${ }^{8,9}$ The results from the dog irradiations with BSH support this sparing effect; however, the different distribution of the compounds indicate a need to determine this effect, or compound factor, for each tissue and compound. ${ }^{13}$

ACKNOWLEDGEMENT: Work supported in part by the U.S. Department of Energy, Office of Energy Research, under DOE Field Office, Idaho, Contract No. DE-AC07$76 \mathrm{ID} 01570$.

\section{REFERENCES}

1. S.L. Kraft, P.R. Gavin, C.E. DeHaan, C.W. Leathers, W.F. Bauer, D.L. Miller, and R.V. Dorn III, Borocaptate sodium: A potential boron delivery compound for boron 
neutron capture therapy evaluated in dogs with spontaneous intracranial tumors, Proceedings, National Academy of Sciences, USA. 89:11973-11977 (1992).

2. A. Siefer, J. Casado, R.L. Moss, P.R. Gavin, K. Philipp, R. Huiskamp, and E. Duhmke, Healthy tissue tolerance studies for BNCT at the High Flux Reactor Petten: First results, Proceedings of the European Collaboration on Boron Neutron Capture Therapy Workshop. Petten. The Netherlands, September 18-20, 1991, Plenum Press, New York (1991).

3. P.R. Gavin, S.L. Kraft, C.E. DeHaan, R.D. Sande, M. Papageorges, and W.F. Bauer, Spontaneous canine oral melanoma: A large animal model for BNCT, Proceedings of the Fourth International Symposium on Neutron Capture Therapy, Sydney, New South Wales, Australia. December 4-7, 1991, Plenum Press, New York (1990).

4. P.R. Gavin, S.L. Kraft, C.E. DeHaan, M.L. Griebenow, and M.P. Moore, A large animal model for boron neutron capture therapy, Proceedings of the Fourth International Symposium on Neutron Capture Therapy, Sydney. New South Wales, Australia. December 4-7, 1991, Plenum Press, New York (1990).

5. D.W. Nigg, F.J. Wheeler, and P.D. Randolph, Characterization of the BMRR and PBF epithermal-neutron beams in phantom using three-dimensional derministic radiation transport theory, in: Progress in Neutron Capture Therapy for Cancer, B.J. Allen, D.E. Moore, B.V. Harrington, eds., Plenum Press, New York, pp 145-149 (1992).

6. P.R. Gavin, F.J. Wheeler, R. Huiskamp, A. Siefert, S.L. Kraft, and C.E. DeHaan, Large animal model studies of normal tissue tolerance using $\Omega$ epithermal beam and borocaptate sodium, Proceedings of the European Collaboration on Boron Neutron Capture Therapy Workshop. Petten. The Netherlands. September 18-20, 1991, Plenum Press, New York (1991).

7. J.O. Archambeau, Swine skin: A model to evaluate dose recovery from different radiations, in: Clinical Aspects of Neutron Capture Therapy, R.G. Fairchild, V.P. Bond, A.D. Woodhead, eds., Plenum Press, New York, Basic Life Sciences 50:9-20 (1989).

8. P.A. Stewart, C.L. Farrell, R.F. DelMaestro, and C.G. Ellis, The effect of cellular microenvironment on vessels in the brain, Part $\mathrm{I}$ : Vessel structure in tumor, peritumor, and brain from humans with malignant blioma, Int J Radiat Biol. 60:125130 (1991).

9. R.A. Rydin, O.L. Deutsch, and B.W. Muray, The effect of geometry on capillary wall dose for boron neutron capture therapy, Phys Med Biol, 21:134-138 (1976).

10. K. Kitao, Vascular wall dose from boron neutron capture reaction, in: Boron Neutron Capture Therapy for Tumors, H. Hatanaka, ed., Nishimura Co., Ltd., Nigata, Japan, pp 191-197 (1986).

11. J.W. Hopewell, The importance of vascular damage in development of late effects in normal tissues, Radiation Biology in Cancer Research, R.E. Meyer and H.R. Withers, eds., Raven Press, New York, pp 449-459 (1980).

12. A.J. van der Kogel, Effects of high-LET radiations on the CNS, Procedings of the European Collaboration on Boron Neutron Capture Therapy Workshop, Petten. The Netherlands. September 18-20, 1991, Plenum Press, New York (1991).

13. R. Gahbauer, R., RBE in normal tissue, Proceedings of the European Collaboration on Boron Neutron Capture Therapy Workshop, Petten. The Netherlands, September 1820. 1991, Plenum Press, New York (1991). 



\section{Computer Modeling the Boron Compound Factor in Brain Tumors}

P. Gavin, R. Huiskamp, F. Wheeler, and M. Griebenow

This article is a reprint from Advances in Neutron Capture Therapy, pp. 597-601, (1993). 


$$
\because
$$




\title{
COMPUTER MODELING THE BORON COMPOUND FACTOR
}

\section{IN BRAIN TUMORS}

\author{
P.R. Gavin, ${ }^{1}$ R. Huiskamp, ${ }^{2}$ F.J. Wheeler, ${ }^{3}$ and M.L. Griebenow ${ }^{3}$ \\ 'Washington State University, Department of Veterinary Clinical Medicine \\ Surgery, Pullman, WA 99164-6610 USA \\ ${ }^{2}$ Netherlands Energy Research Foundation, Petten, The Netherlands \\ ${ }^{3}$ Idaho National Engineering Laboratory, EG\&G Idaho, Inc., INEL BNCT \\ Program, P.O. Box 1625, Idaho Falls, ID 83415-1575 USA
}

\section{INTRODUCTION}

Dogs with spontaneous brain tumors have been used to study the potential of boron neutron capture therapy (BNCT) using an epithermal neutron beam with borocaptate sodium $\left(\mathrm{Na}_{2} \mathrm{~B}_{12} \mathrm{H}_{11} \mathrm{SH}\right.$ or $\left.\mathrm{BSH}\right){ }^{1,2}$ The compound para-borono-phenylalanine ( $\left.\mathrm{p}-\mathrm{BPA}\right)$ has also been studied in dogs with oral melanomas and normal tissue distribution has been determined. ${ }^{3}$ It has been suggested that the term "compound factor" be used to describe the product of the microscopic distribution and relative biologic effectiveness (RBE) of the ${ }^{10} \mathrm{~B}(\mathrm{n}, \alpha)$ reaction to predict the normal and tumor tissue responses. ${ }^{4}$ This paper reports the computer model that was used to predict the compound factor for BSH and p-BPA and, hence, the equivalent radiation in tumors and the relation to normal tissue response. The compound factor would need to be calculated for other compounds with different distributions.

\section{MATERIALS AND METHODS}

The charged-particle transport methods used for dose calculations are described in these proceedings. ${ }^{5}$ A Monte Carlo computer model was developed to represent a tumor. This model was one of a matrix of $10 \mu \mathrm{m}$-diameter spheres representing the tumor cells. The cell nucleus was a $5 \mu \mathrm{m}$-diameter sphere and the interstitial space had a thickness of $0.5 \mu \mathrm{m}$ (Figure 1). Therefore, the model is represented as concentric spheres with radii of the nucleus, cytoplasm, extracellular space, and cytoplasm of adjacent cells of 2.5, 5.0, 5.5 , and $11.0 \mu \mathrm{m}$, respectively. The tumor model was used for two different sets of parameters that placed $25 \mathrm{ppm}$ boron into the tumor. The first set (BSH) used a 


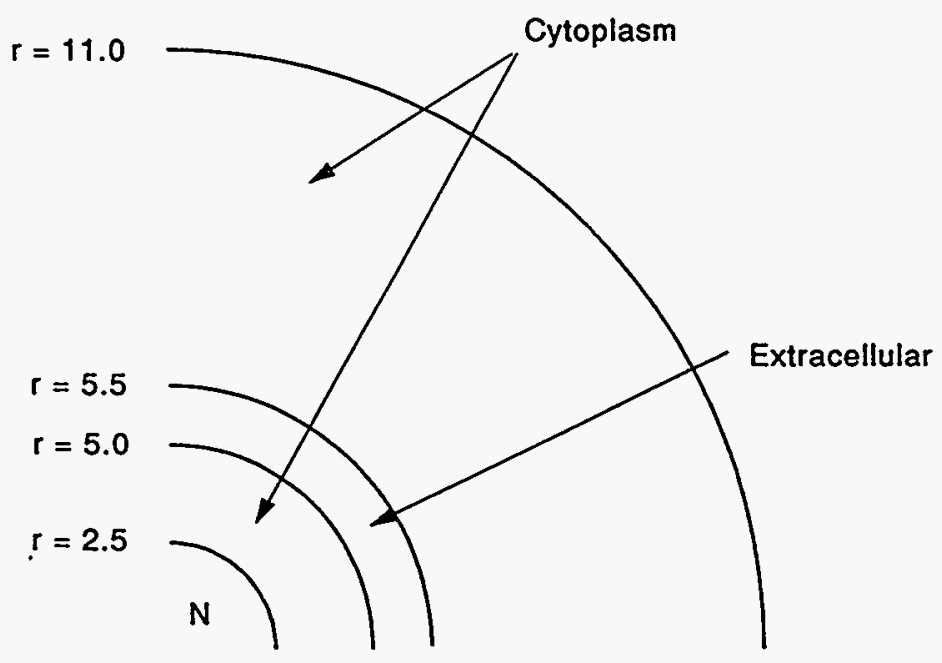

Figure 1. Computer model of tumor. Relative volumes of nucleus, cytoplasm, and extracellular space are $10.7 \%, 75.1 \%$, and $14.2 \%$ respectively.

tumor-to-blood ratio of 1 and limited the tumor distribution to $90 \%$ to the intercellular space and $10 \%$ to the tumor cell cytoplasm. The second set (p-BPA) used a tumor-toblood ratio of 3 and the tumor concentration was $80 \%$ in the extracellular space, $17.5 \%$ in the tumor cell cytoplasm, and $2.5 \%$ in the nucleus.

Known macroscopic and inferred microscopic information from the canine studies were used in the models to predict the effect of the epithermal beam and the ${ }^{10} \mathrm{~B}(n, \alpha)$ reaction on the tumor. The tumor depth for calculational purposes was $2 \mathrm{~cm}$ beyond the thermal neutron peak. The equivalent dose to the capillary nucleus was held constant at 12 equivalent Gy to calculate the relative effect on the tumor as the boron concentration was varied. The RBE values used for nitrogen capture (n,proton), ${ }^{10} \mathrm{~B}(\mathrm{n}, \alpha){ }^{7} \mathrm{Li}$, and fast neutrons were $2.7,2.3$, and 4.5 , respectively. The first two values are from the literature and the fast-neutron RBE was derived from skin reactions in dogs.,7

\section{RESULTS}

The tumor model with the first set of parameters matched the nonuniform boron normal tissue distribution, as found with BSH in the blood and brain. ${ }^{1,2}$ The tumor dose was calculated on the midline $2 \mathrm{~cm}$ distal from the peak physical dose. The tumor-cell nuclear equivalent dose was calculated using a constant brain capillary endothelial equivalent dose of 12 equivalent $\mathrm{Gy}$ with varying tumor-boron concentrations ranging from 10-100 ppm (Table 1). The equivalent dose to the tumor nuclei increased dramatically, compared to the capillary endothelial nuclei, as the boron concentration in the blood was increased (Figure 2). The calculated compound factor for the boron in the tumor was 0.91 .

The tumor model with the second set of parameters used a uniform boron distribution similar to that observed with BSH in the skin and blood and with p-BPA in the skin, brain, and blood. ${ }^{1-3,8,9}$ The tumor-cell nuclear equivalent dose was calculated as above with a constant equivalent brain endothelial cell nuclear dose of 12 equivalent $\mathrm{Gy}$ and for tumor-boron concentrations ranging from 10-100 ppm (Table 1). A less pronounced effect of tumor nuclei-equivalent dose to endothelial nuclei-equivalent dose was observed as the boron concentration was increased. The calculated compound factor for p-BPA in the tumor was 1.16 . 
Table 1. Brain tumor dose as a function of boron distribution normalized to endothelial Iso-dose

\begin{tabular}{|c|c|c|c|c|c|c|c|c|c|c|}
\hline $\begin{array}{l}\text { Type of } \\
\text { compound } \\
\text { distribution }\end{array}$ & $\begin{array}{l}\text { Tumor:blood } \\
\text { ratio }\end{array}$ & $\begin{array}{c}\% \\
\text { compound } \\
\text { extracellular }\end{array}$ & $\begin{array}{c}\% \\
\text { compound } \\
\text { in } \\
\text { cytoplasm }\end{array}$ & $\begin{array}{c}\% \\
\text { compound } \\
\text { in nucleus }\end{array}$ & $\begin{array}{l}\text { ppm } \\
\text { 10B } \\
\text { tumor }\end{array}$ & $\begin{array}{l}\text { Macro } \\
\text { peak } \\
\text { equiv. } \\
\text { dose } \\
\left(E q^{*} \mathbf{G} y\right)\end{array}$ & $\begin{array}{c}\text { Macro } \\
\text { tumor } \\
\text { equiv. } \\
\text { dose } \\
\left(\mathrm{Eq}^{*} \mathrm{~Gy}\right)\end{array}$ & $\begin{array}{c}\text { Micro } \\
\text { endothelial } \\
\text { nuclear } \\
\text { equiv. } \\
\text { dose } \\
\text { (Eq*Gy) }\end{array}$ & $\begin{array}{c}\text { Micro } \\
\text { tumor } \\
\text { nuclear } \\
\text { equiv. } \\
\text { dose } \\
(\mathbf{E q *} \mathbf{G y})\end{array}$ & $\begin{array}{l}\text { Tumor nuclear } \\
\text { equiv. } \\
\text { dose/endothelial } \\
\text { nuclear equiv. } \\
\text { dose }\end{array}$ \\
\hline \multirow{4}{*}{$\begin{array}{c}\begin{array}{c}\text { Non- } \\
\text { uniform }\end{array} \\
\text { BSH }\end{array}$} & \multirow{4}{*}{1.1} & \multirow{4}{*}{90} & \multirow{4}{*}{10} & \multirow{4}{*}{0} & 10 & 20.7 & 15.1 & 12.0 & 10.1 & 0.84 \\
\hline & & & & & 25 & 31.0 & 22.3 & 12.0 & 12.5 & 1.04 \\
\hline & & & & & 50 & 42.1 & 31.3 & 12.0 & 15.0 & 1.25 \\
\hline & & & & & 100 & 54.2 & 40.5 & 12.0 & 18.6 & 1.55 \\
\hline \multirow{4}{*}{ Uniform } & \multirow{4}{*}{$3: 1$} & \multirow{4}{*}{80} & \multirow{4}{*}{17.5} & \multirow{4}{*}{2.5} & 10 & 19.5 & 14.2 & 12.0 & 10.3 & 0.89 \\
\hline & & & & & 25 & 25.7 & 19.0 & 12.0 & 11.9 & 0.99 \\
\hline & & & & & 50 & 30.8 & 22.9 & 12.0 & 13.1 & 1.09 \\
\hline & & & & & 100 & 35.3 & 26.3 & 12.0 & 14.3 & 1.19 \\
\hline
\end{tabular}




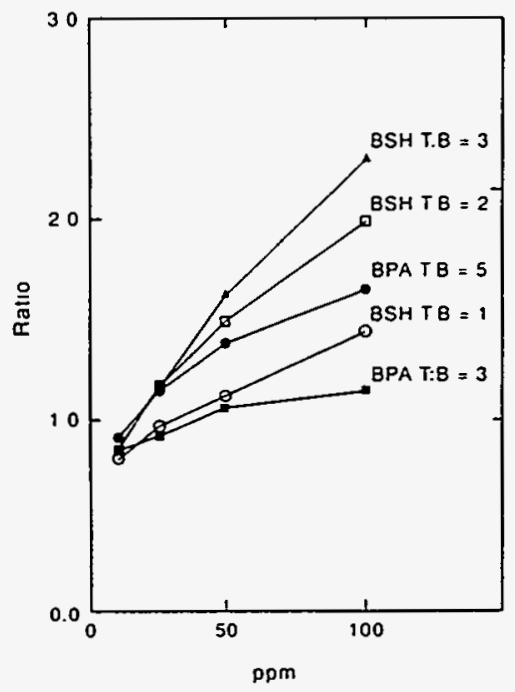

Figure 2. Plot of therapeutic advantages of brain tumor nuclear equivalent dose to brain vascular endothelial nuclear dose with various compounds and tumor-toblood ratios vs boron concentrations in the tumor.

\section{DISCUSSION}

The microscopic tumor-boron distribution of the compound was purposely favorably set for the model that would simulate the uniform normal brain-boron distribution and 3:1 tumor-to-brain ratios that occur with p-BPA. ${ }^{3.8 .9}$ The calculated compound factor for this scenario was 1.16 , substantially better than the nonuniform microscopic boron distribution compound factor of 0.91 calculated for BSH distribution. There is no conclusive evidence that BSH in tumors is intracellular. The hydrophilic nature and pharmacokinetic data would suggest that most of the compound may be extracellular in the interstitial space. ${ }^{1}$ Cell culture data has indicated some boron within the cells incubated in medium containing BSH. ${ }^{10}$ Therefore, the model parameters of $90 \%$ extracellular and $10 \%$ intracellular for the BSH compound were chosen. Pharmacokinetic and cell culture data have indicated that the boron associated with p-BPA is also probably not permanently incorporated into the cell cytoplasm;" however, the intracellular localization of the boron was artificially doubled for the p-BPA compound to compare the compound's effects on tumors and brain microvasculature.

The tumor-to-blood ratios reported by others ${ }^{8,9.12}$ are in excess of the $1: 1$ used for BSH and the 3:1 used for p-BPA. Therefore, additional data was obtained from the tumor model with the same nonuniform microscopic boron tumor distribution as before, but with tumor-to-blood ratios of 2:1 and 3:1 for BSH and 5:1 for p-BPA with the uniform distribution (Figure 2). Concentrations of boron in spontaneous canine brain tumors can exceed $50 \mathrm{ppm}$ with conventional dosages and administration schedules. ${ }^{1}$ Concentrations of boron following p-BPA administration have generally not been associated with tumor concentrations greater than $30-40 \mathrm{ppm}^{8,9}$ These results suggest that BSH may be the better compound for treating brain tumors.

The results shown in Figure 2 demonstrate that the increased equivalent dose to the endothelial cell nucleus with p-BPA overwhelms the increased tumor equivalent dose from improved tumor-to-blood ratios and improved microscopic tumor cell boron localization. The increase of boron concentrations in tumor (and blood) boron concentrations has a large effect on the equivalent tumor dose compared to the equivalent brain endothelial vascular dose. This increase in therapeutic gain was more pronounced with the nonuniform boron distribution observed with BSH in the brain. 
ACKNOWLEDGMENT. Work supported in part by the U.S. Department of Energy, Office of Energy Research, under DOE Field Office, Idaho, Contract No. DE-AC0776 IDO1570.

\section{REFERENCES}

1. S.L. Kraft, P.R. Gavin, C.E. DeHaan, C.W. Leathers, W.F. Bauer, D.L. Miller, and R.V. Dorn III, Borocaptate sodium: A potential boron delivery compound for boron neutron capture therapy evaluated in dogs with spontaneous intracranial tumors, Proceedings, National Academy of Sciences, USA. 89:11973-11977 (1992).

2. P.R. Gavin, S.L. Kraft, C.E. DeHaan, M.L. Griebenow, and M.P. Moore, A large animal model for boron neutron capture therapy, Proceedings of the Fourth International Symposium on Neutron Capture Therapy, Sydney, New South Wales, Australia, December 4-7, 1991, Plenum Press, New York (1990).

3. P.R. Gavin, S.L. Kraft, C.E. DeHaan, R.D. Sande, M. Papageorges, and W.F. Bauer, Spontaneous canine oral melanoma: A large animal model for BNCT, Proceedings of the Fourth International Symposium on Neutron Capture Therapy, Sydney, New South Wales, Australia, December 4-7,1991, Plenum Press, New York (1990).

4. R. Gahbauer, R., RBE in normal tissue, Proceedings of the European Collaboration on Boron Neutron Capture Therapy Workshop, Petten, The Netherlands, September 18-20, 1991, Plenum Press, New York (1991).

5. D.E. Wessol, F.J. Wheeler, R. Babcock, and J.D. Starkey, Medical image reconstruction and geometry generation software, Proceedings of the Sth International Symposium on Neutron Capture Therapy, Columbus, Ohio, September 14-17, 1992, Plenum Press, New York (1992).

6. P.R. Gavin, F.J. Wheeler, R. Huiskamp, A. Siefert, S.L. Kraft, and C.E. DeHaan, Large animal model studies of normal tissue tolerance using an epithermal beam and borocaptate sodium, Proceedings of the European Collaboration on Boron Neutron Capture Therapy Workshop, Petten. The Netherlands, September 18-20, 1991, Plenum Press, New York (1991).

7. J.O. Archambeau, Swine skin: A model to evaluate dose recovery from different radiations, in: Clinical Aspects of Neutron Capture Therapy, R.G. Fairchild, V.P. Bond, A.D. Woodhead, eds., Plenum Press, New York, Basic Life Sciences 50:9-20 (1989).

8. J.A. Coderre, J.D. Glass, R.G. Fairchild, U. Roy, S. Cohen, and I. Fand, Selective Targeting of boronophenylalanine to melanoma in BALB/c mice for neutron capture therapy, Cancer Res 47:6377-6388 (1987).

9. J.A. Coderre, J.D. Glass, R.G. Fairchild, P. Micca, I. Fand, and D.D. Joel, Selective delivery of boron by the melanin precursor analogue p-boronophenylalanine to tumors other than melanoma, Cancer Res, 50:138-141 (1990).

10. R. Verrijk, I.J.H. Smolders, R.H. Huiskamp, W.C. deBruijn, and A.C. Begg, Pharmacokinetics of boron in animals and cell cultures using ICP-AES and boron localization with electron spectroscopic imaging, Proceedings of the 5th International Symposium on Neutron Capture Therapy, Columbus, Ohio, September 14-17, 1992, Plenum Press, New York (1992).

11. M. Papageorges, C. Elstad, G.G. Meadows, and W.F. Bauer, Effect of tyrosine and phenylalanine deprivation on the uptake of para-boronophenylalanine in melanoma cells, Proceedings of the Fourth International Symposium on Neutron Capture Therapy. Sydney New South Wales, Australia, December 4-7, 1991, Plenum Press, New York (1990).

12. H. Fankhauser and G. Stragliotto, Biodistribution of known sulfhydryl (BSH) on patients with intracranial tumors, Proceedings of the Fourth International Symposium on Neutron Capture Therapy, Sydney, New South Wales, Australia, December 4-7, 1991, Plenum Press, New York (1990). 
, 


\title{
Determination of Fast-Neutron Dose Distributions in the Canine Central Nervous System
}

\author{
D. Nigg, J. Moran, and F. Wheeler
}

This article is a reprint from Advances in Neutron Capture Therapy, pp. 603-606, (1993). 
, 


\title{
DETERMINATION OF FABT-NEUTRON DOSE DIBTRIBUTIONS IN THE CANINE
}

\section{CENTRAT NERVOUB BYSTEM}

\author{
David W. Nigg, Jean M. Moran, and Floyd J. Wheeler \\ National Center for BNCT Measurement and Development \\ Idaho National Engineering Laboratory \\ EG\&G, Idaho, Inc. \\ P.O. Box 1625 \\ Idaho Falls, Idaho 83415
}

\begin{abstract}
The Idaho National Engineering Laboratory (INEL) Center for Boron Neutron Capture Therapy (BNCT) Measurement and Development conducts, sponsors, and coordinates research and development efforts in a variety of BNCT-related areas. Current activities include an ongoing series of largeanimal (canine) model irradiation experiments conducted in collaboration with the Washington State University School of Veterinary Medicinel. These experiments employ the epithermal-neutron beam at the Brookhaven Medical Research Reactor (BMRR) ${ }^{2}$ and have the purpose of determining normal tissue tolerance and spontaneous brain tumor response to the unique $\operatorname{mix}$ of radiation components that characterize BNCT.

The normal tissue tolerance experiments employ borocaptate sodium (BSH) as the boron carrying pharmaceutical. The experimental animals for the tolerance experiments are typically Labrador .Retrievers without brain tumors. Tolerance levels in skin and central nervous system (CNS) tissue with various concentrations of boron have been established and initial estimates of the relative biological effectiveness (RBE) of the various radiation dose components have been made'. Irradiations designed to bring the CNS of the dogs to expected tolerance levels without boron have, however, been hampered by skin damage caused by the moderately high fast neutron component of the BMRR beam. Surface tissue is dose-limiting for the zero-boron irradiations. In addition, the dogs employed for this experiment series have significantly more cover tissue over the brain (primarily becauge of the temporalis muscle) than is the case for humans. This cover tissue attenuates the fast neutron component in the treatment beam to levels in the brain itself that make it difficult to accurately estimate the RBE of this important beam component from the observed biological response of the brain to the total mixed irradiation field that is present.

During a recent National Academy of Sciences Institute of Medicine peer review of the canine program it was suggested by the referees that some of the tolerance experiments could be performed using dogs with the scalp and temporal muscle surgically reflected prior to irradiation in order to avoid the problems noted above. This is being seriously considered and the results of some exploratory calculations to determine the peak cNs fastneutron dose for canine subjects with and without scalp and temporal muscle reflection have been completed and are summarized here.

The calculations were performed using the recently-developed bnct edit/rtt MC BNCT analytic dosimetry software package ${ }^{3}$. This software packāge employs the Monte Carlo technique to compute three-dimensional neutron and photon flux and absorbed dose distributions within a BNCT irradiation volume that is geometrically described by non-uniform rational B-spline surface equation fits to the various regions of interest, as outlined (for this application) on a series of axial medical images of a typical Labrador Retriever head. Figure 1 shows the spline surface fits to
\end{abstract}

Advances in Neutron Capture Therapy, Edited by

R.F. Barth and A.H. Soloway, Plenum Press, New York, 1993

603 

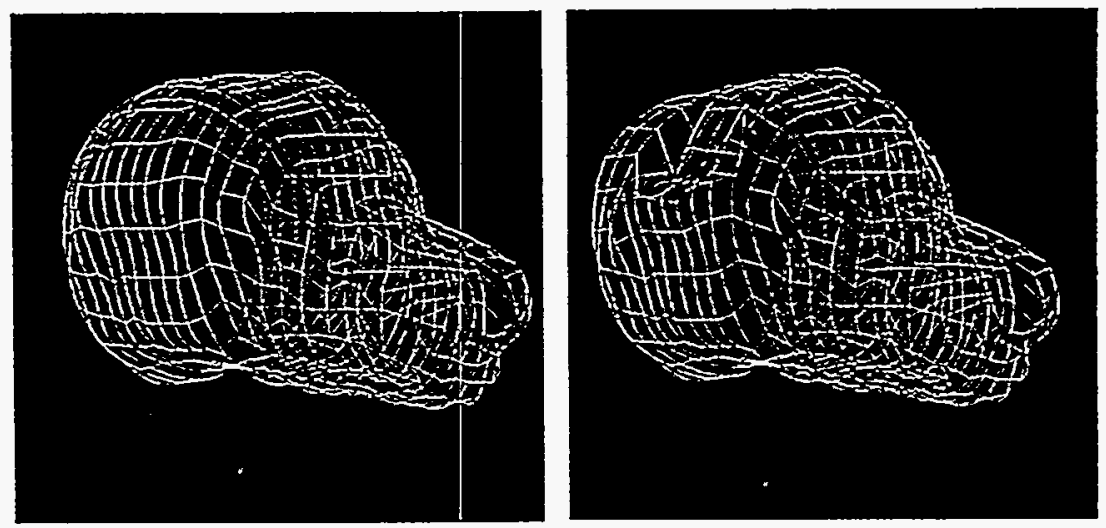

Figure 1. Reconstructed outer surfaces for the intact and surgicallymodified canine head models.

the outside surface of the canine head for both the intact dog and for a head model that has been modified to simulate the removal of the skin and temporal muscle overlying the right hemisphere of the brain. Both models also include surface reconstructions of various interior structures (brain, eyes, sinus cavities, etc.). These have been deleted from Figure 1 for clarity.

Three calculations were performed. All three assumed the presence of a $5 \mathrm{~cm}$ by $10 \mathrm{~cm}$ BMRR beam delimiter aperture over the right hemisphere of the canine brain with the long dimension of the aperture lined up parallel to the longitudinal axis of the head. In the first case the intact dog model is used and the beam impinges at an angle of incidence that is perpendicular to the top of the head. The second case employs the reflected-scalp model, again with the beam perpendicularly-incident on the top of the head. In the third case scalp reflection is assumed and the beam is incident on the head at an angle that brings the exit plane of the delimiter as close as possible to the exposed skull surface overlying the brain. At first glance, this would be expected to increase the fast neutron dose to the brain relative to the case with perpendicular beam incidence. Calculated results for the fast neutron dose in the three cases are illustrated in Figures 2-4. In all cases the fast neutron dose is normalized to a peak total physical dose in the brain (with no boron) of 10 Gy. As expected, scalp reflection increases the fast neutron component of the total dose significantly (about a factor of 2), assuming perpendicular beam incidence for both the intact (Figure 2) and reflected-scalp (Figure 3 ) cases. The relative difference in fast neutron components between these two cases has been independently confirmed using three-dimensional deterministic calculations based on the canine head model described in Reference 4. It is interesting to note, however, that with scalp reflection, a decrease in the relative fast neutron component is observed when the beam is obliquely incident (Figure 4) rather than perpendicularlyincident (Figure 3). This may be explained by the fact that the delivery rates of all dose components increase with oblique beam incidence, but the delivery rates of those components that result from thermal neutron interactions increase more than is the case for the fast-neutron component. Hence when one normalizes to a specified total peak dose, the relative fast neutron component decreases for the case with oblique incidence. That this would be the case was not immediately obvious prior to the completion of these calculations. It is a consequence of the complex nature of the neutron transport phenomena associated with BNCT. This illustrates the importance of performing detailed three-dimensional planning calculations in connection with all in-vivo BNCT irradiations. 
Peak Total Physical Dose $=10 \mathrm{~Gy}$

Reference Fast-neutron-dose $=1.8 \mathrm{~Gy}$

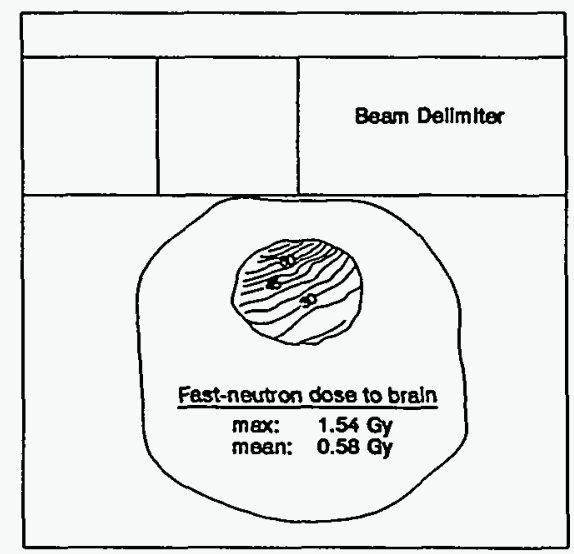

Figure 2. Canine brain fast-neutron-dose percentage contours for the intact canine head model.

Peak Physical Total Dose $=10 \mathrm{~Gy}$ Reference Fast-neutron-dose $=1.2 \mathrm{~Gy}$.

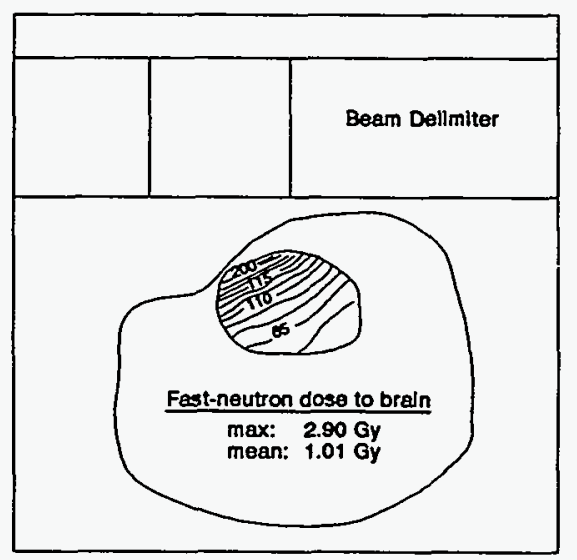

Figure 3. Canine brain fast-neutron-dose percentage contourg for the surgically-modified canine head model. 
Peak Total Physical Dose $=10 \mathrm{~Gy}$

Reference Fast-neutron-dose $=1.5 \mathrm{~Gy}$

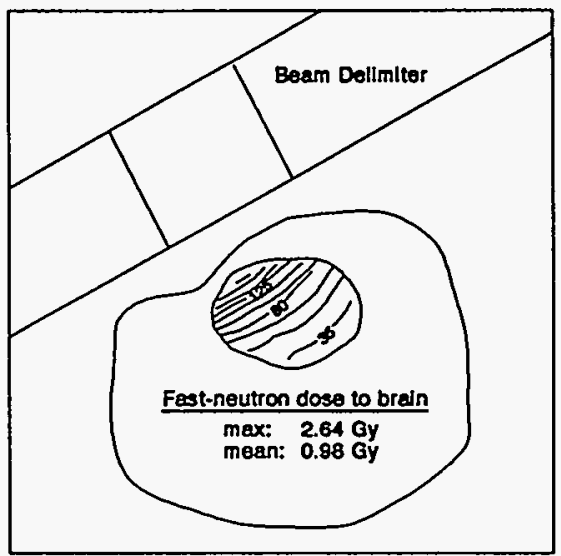

Figure 4. Canine brain fast-neutron-dose percentage contours for the surgically-modified canine head model with a rotated beam angle of incidence.

\section{ACKANOWLEDGEMENTS}

Ken Bradshaw, working in collaboration with the University of Utah, provided the sequence of canine magnetic resonance images used to construct the calculational models employed in this study.

This study was performed under the auspices of the U.S. Department of Energy, Office of Energy Regearch, DOE Field Office, Idaho, under Contract Number DE-AC07-76IDO1570.

\section{REFERENCES}

1. P.R. Gavin, et. al., Large animal model studies on the use of BNCT for the treatment of brain tumors, Proceedings of the Fifth International Symposium on Neutron Capture Therapy, OsU, September 1992, (To be published).

2. D.W. Nigg, et. al., Demonstration of three-dimensional deterministic radiation transport theory dose distribution analysis for boron neutron capture therapy, Medical Physics, 18:1, 43-53, January 1991.

3. D.W. Nigg, Analytical dogimetry and treatment planning for BNCT, Proceedings of the Fifth International Symposium on Neutron Capture Therapy, OSU, September 1992, (To be published).

4. J.M. Moran, et. al., Macroscopic geometric heterogeneity effects in radiation dose distribution analysis for BNCT, Medical Physics, 19:3, 723-732, June 1992. 


\section{Dose Optimization for Boron Neutron Capture Therapy of Spontaneous Canine Brain Tumors}

C. Atkinson and F. Wheeler

This article is a reprint from Advances in Neutron Capture Therapy, pp. 607-611, (1993). 

DOSE OPTIMIZATION FOR BORON NEUTRON CAPTURE

\title{
THERAPY OF SPONTANEOUS CANINE BRAIN TUMORS
}

\author{
Carol A. Atkinson, Floyd J. Wheeler \\ Idaho National Engineering Laboratory \\ Idaho Falls, Idaho 83415-1575
}

\section{INTRODUCTION}

The ultimate success of Boron Neutron Capture Therapy (BNCT) will depend, among other things, upon the ability to calculate, a priori, accurate and optimal radiation dose distributions in the target volume. Twenty-one dogs with spontaneously-occurring brain tumors have been treated, at the Brookhaven Medical Research Reactor (BMRR) in a collaborative effort between the Idaho National Engineering Laboratory (INEL), Washington State University (WSU), and Brookhaven National Laboratory (BNL). Because these 21 treatments were designed to demonstrate safety rather than efficacy, the dogs involved did not necessarily receive irradiations corresponding to a treatment plan designed to maximize the estimated cell kill at the target location. Four of these dogs (with intra-axial tumors above the tentorium) were chosen as test cases to determine the effects of optimizing treatment. Although the tumors present in all four dogs were of the same type, they varied significantly in size and location. Optimum treatment was found by varying boron concentration and incident neutron beam position and size. The effect of using a beam having a "cleaner," more forward-peaked angular distribution [Georgia Tech Research Reactor (GTRR)] was also examined.

\section{DISCUSSION}

A brief description of the four dogs chosen for this study and their "as irradiated" parameters is given in Table 1. A standard calculational model was developed using regular-geometry primitives ${ }^{1}$ and a calculated spectrum for BMRR. ${ }^{2}$ The following variations, representing several variables on which the irradiation would be physically dependent, were applied to this calculational model to determine an optimal treatment plan for each dog: the beam port opening was varied between a $10 \times 10 \mathrm{~cm}$ square opening and circular openings of $7.62,6.35,5.08$, and $3.81 \mathrm{~cm}$; the head was positioned as close to the beam port opening as possible while simultaneously trying to minimize the distance between the tumor and the beam port; the ${ }^{10} \mathrm{~B}$ concentration was increased to $100 \mathrm{ppm}$; the boron tumor-to-blood ratio was varied between 1 and 5 ; and selected cases were repeated using a calculated spectrum for the GTRR. ${ }^{3}$ Monte Carlo methods for neutron and gamma transport ${ }^{2}$ were used to determine flux distributions throughout each model.

Generally-accepted relative biological effectiveness (RBE) values for boron, nitrogen, gamma, and fast neutron were applied to the results of these calculations to determine an effective dose. The following RBE values were assumed: 2.3 for ${ }^{10} \mathrm{~B}$ at the target location ${ }^{4}, 2.7$ for nitrogen ${ }^{5}, 4.5$ for fast neutron ${ }^{6}$, and 0.6 for gamma. ${ }^{7}$ Due to geometric considerations, a compound 
Table 1. Results of selected calculations used in determining an optimal treatment plan for four previously irradiated dogs having spontaneously. occurring brain tumors.

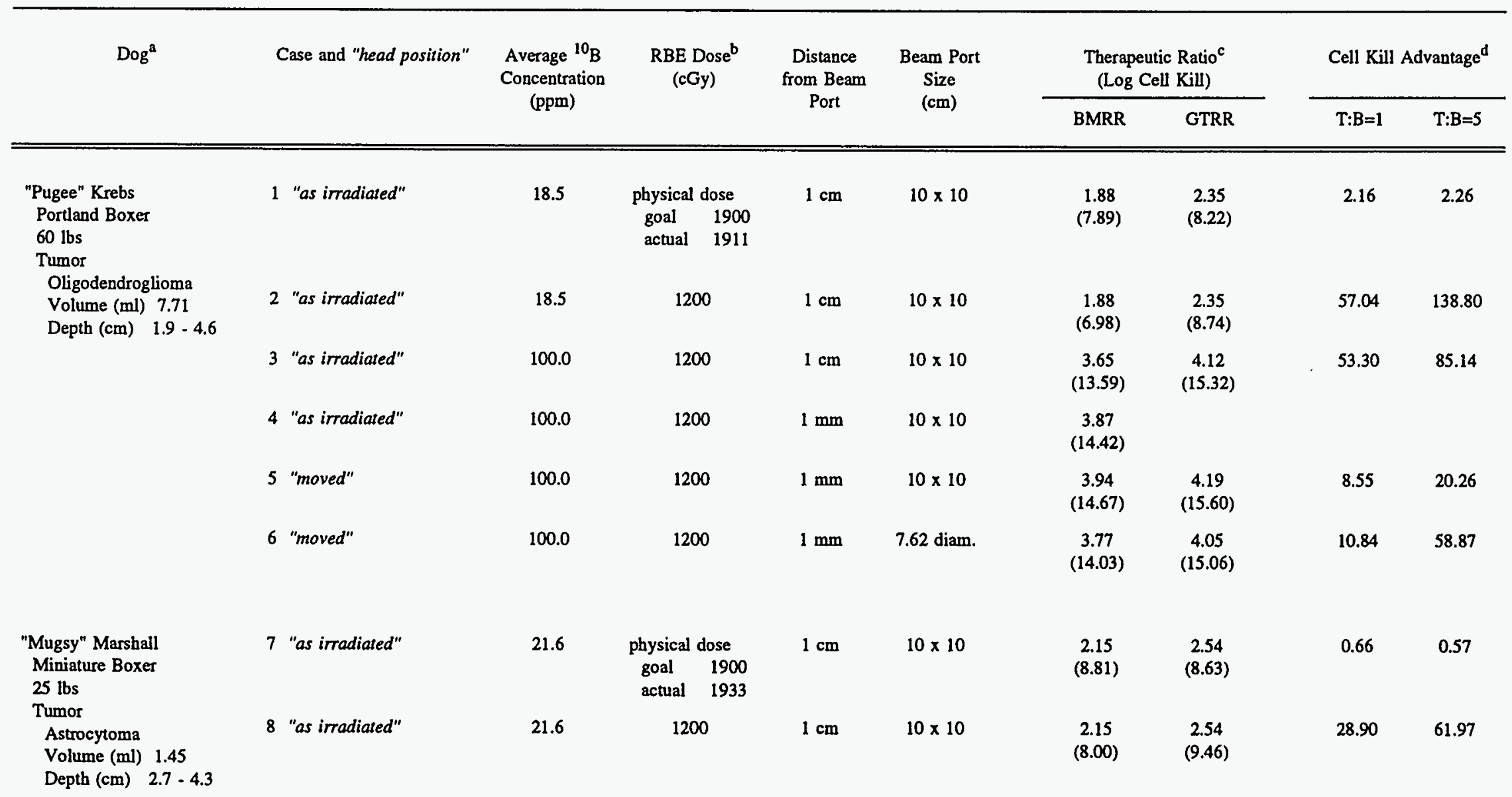




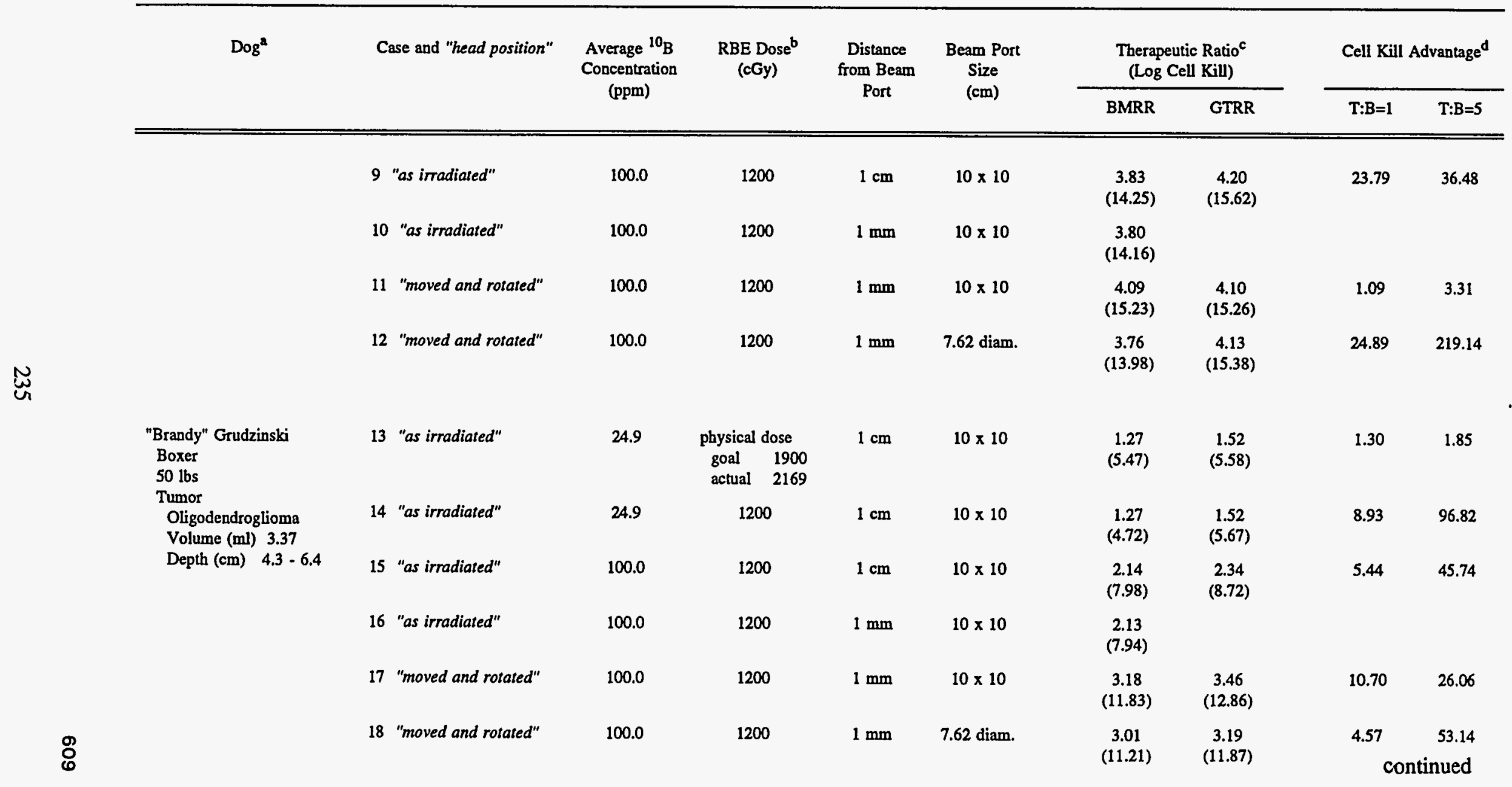


factor of 0.33 was used for ${ }^{10} \mathrm{~B}$ in healthy tissue. ${ }^{6}$ The doses were normalized to $12 \mathrm{~Gy} \mathrm{RBE}$ at the thermal peak. Therapeutic ratio and log cell kill were computed for comparison between treatment plans developed for the same neutron source; cell kill advantage was computed for comparison between the two different neutron sources.

Results of Monte Carlo calculations for these four dogs are given in Table 1. For the less forward-peaked beam, BMRR, it appears that a generalized treatment plan could be determined to produce optimal tumor cell kill: high ${ }^{10} \mathrm{~B}$ concentrations; large beam port opening; and the head positioned to minimize the distance between the tumor and the beam port. However, for irradiations planned in a more forward-peaked beam, GTRR, a generalized plan is not so obvious. This can be seen in the results for "Mugsy" (Cases 9, 11, and 12) where tumor cell kill could be improved by increasing the distance between the head and the beam port and using a smaller opening.

Under almost all circumstances, the more forward-peaked beam will produce a greater cell kill at the tumor location as illustrated by the values given for the cell kill advantage in Table 1. To illustrate another important feature of using a "cleaner", more forward-peaked beam, the boron tumor-to-blood ratio (illustrative of the ability to get higher concentrations of boron to the tumor) was increased from 1 to 5 and cell kill advantage was again computed. In almost all cases, the cell kill advantage increases, sometimes dramatically, as the tumor-to-blood ratio increases.

\section{CONCLUSIONS}

To see significant increases in estimated tumor cell kill, optimal treatment plans specific to each patient are recommended. The determination of these plans depends on several parameters: depth and location of the tumor within the brain, concentration of ${ }^{10} \mathrm{~B}$ that can be delivered to the tumor mass, and the angu. Ir distribution and energy spectrum of the neutron source. It is also seen that the importance of beam quality is magnified as ${ }^{10} \mathrm{~B}$ delivery systems improve.

\section{REFERENCES}

1. F.J. Wheeler and D.W. Nigg, "Three-Dimensional Radiation Dose Distribution Analysis for Boron Neutron Capture Therapy," Nuclear Science and Engineering, 110, January, 1992, pp. 16-31.

2. F.J. Wheeler, D.K. Parsons, B.L. Rushton, and D.W. Nigg, "Epithermal Neutron Beam Design for Neutron Capture Therapy at the Power Burst Facility and the Brookhaven Medical Research Reactor," Nuclear Technology, 92, October, 1990, pp. 106-117.

3. D.W. Nigg, G.J. Storr, and F.J. Wheeler, "Physics Parameters for an Epithermal-Neutron Beam at the Georgia Institute of Technology", Proceedings of the Fifh International Symposium on Neutron Capture Therapy, Columbus, Ohio, September 14-17, 1992, these proceedings.

4. D. Gabel, R.G. Fairchild, B. Larsson, K. Drescher, and W.R. Rowe, "The Biological Effect of the ${ }^{10} \mathrm{~B}(\mathrm{n}, \alpha)^{7} \mathrm{Li}$ Reaction and its Simulation by Monte Carlo Calculation," Proceedings of the First International Symposium on Neutron Capture Therapy, Cambridge, Massachusetts, October 1214, 1983, BNL 51730, Brookhaven National Laboratory, R.G. Fairchild and G.L. Brownell, editors, p.128.

5. J.O. Archambeau, Swine skin: A model to evaluate dose recovery from different radiations, in: "Clinical Aspects of Neutron Capture Therapy," Plenum Press, New York, 1989, pp.9-20.

6. P.R. Gavin, F.J. Wheeler, R. Huiskamp, A. Siefert, S.L. Kraft, and C.E. DeHaan, "Large Animal Model Studies of Normal Tissue Tolerance Using an Epithermal-Neutron Beam and Borocaptate Sodium," Proceedings of the International Workshop on Macro and Microdosimetry and Treatment Planning for Neutron Capture Therapy, Massachusetts Institute of Technology, Cambridge, Massachusetts, October 31 - November 1, 1991, to be published.

7. K.F. Fu, Influence of dose rate on normal tissue tolerance, in: "Radiation Injury to the Nervous System," P.H. Gutin, S.A. Leibel \& G.E. Sheline, eds., Raven Press, New York, 1991, pp.69-87.

\section{COPYRIGHT}

The submitted paper has been authorized by a contractor of the U.S. Government under DOE Contract No. DE-AC07-76ID01570. Accordingly, the U.S. Government retains a nonexclusive, royalty-free license to publish or reproduce the published form of this contribution, or allow others to do so, for U.S. Government purposes. 


\section{In Vivo and In Vitro Uptake of Boronated Compounds by B16-BL6 Murine Melanoma}

C. Elstad, K. Meinkoth, B. Mathison, G. Meadows, D. Kinder, M. F. Hawthorne, K. Shelly, D. Feakes, and W. Bauer

This article is a reprint from Advances in Neutron Capture Therapy, pp. 619-622, (1993). 



\title{
IN VIVO AND IN VITRO UPTAKE OF BORONATED COMPOUNDS BY B16-BL6 MURINE MELANOMA
}

\author{
C. A. Elstad ${ }^{1}$, K. R. Meinkoth 1 , B. A. Mathison ${ }^{1}$, G. G. Meadows 1 , D. H. \\ Kinder $^{1}$, M. F. Hawthorne ${ }^{2}$, K. Shelly ${ }^{2}$, D. A. Feakes ${ }^{2}$, and W. F. Bauer 3 \\ ${ }_{1}$ Department of Pharmaceutical Sciences, College of Pharmacy, WSU, \\ Pullman, WA 99164-6510 \\ 2Department of Chemistry and Biochemistry, UCLA, Los Angeles, CA 90024 \\ 3INEL BNCT Research Programs, EG\&G Idaho, Idaho Falls, ID 83415-2208
}

\section{INTRODUCTION}

A crucial requirement for effective boron neutron capture therapy (BNCT) of cancer is the selective localization of high concentrations of boron in tumor tissue relative to adjacent normal tissue, thus minimizing damage to normal tissues caused by the neutron beam. Several promising boron compounds are presently available as boron-delivery agents for neutron capture therapy of malignant melanoma. More are expected to be developed in the near future. Therefore, there is a need to experimentally describe these boron-delivery compounds in vitro and in vivo, to screen these agents by critically comparing their efficacies with known boronated agents, and to effectively optimize their BNCT potential.

We have developed standardized in vivo and in vitro experimental assays to evaluate boron pharmacokinetics and distribution in tumor and control (normal) cell lines after exposure to selected boron-delivery compounds. We have shown that boronated compounds accumulate both in vitro and in vivo in the highly invasive and metastatic B16-BL6 (BL6) murine melanoma. This project has emphasized the potential of various boron compounds with affinities for melanin, such as para-boronophenylalanine (BPA) which is an analogue of natural melanin precursors ${ }^{1,2}$, or of boron-delivery carriers such as liposomes which preferentially accumulate in neoplastic tissue ${ }^{3-5}$.

\section{MATERIALS AND METHODS}

\section{Tumor Cells and Culture Conditions}

The well characterized BL6 variant of the B16 murine melanoma was chosen for these studies 6.7 . BL6 is highly invasive and highly metastatic. For routine culture, cells were grown in vitro in Dulbecco's modified Eagle's complete minimal essential medium supplemented with $10 \%$ heat-inactivated fetal bovine serum, sodium pyruvate, nonessential amino acids, 2-fold vitamin solution, L-glutamine, $100 \mathrm{U} / \mathrm{ml}$ penicillin, and $100 \mu \mathrm{g} / \mathrm{ml}$ streptomycin. MEM Selectamine Kits (GIBCO Laboratories, Grand Island, NY) were used to formulate all amino acid-limited media ${ }^{8}$. These media were prepared using dialysed FBS to eliminate free amino acids in the serum. To determine uptake of BPA, tumor cells were exposed in the culture medium to BPA at concentrations ranging from $25-100 \mathrm{mg} /$. Cells were incubated in a humidified atmosphere of $5 \% \mathrm{CO}_{2}$ in air at $37^{\circ} \mathrm{C}$ and harvested using 4 
mM EGTA to avoid the membrane-altering effects of trypsin. Cell viability was determined by trypan blue exclusion.

\section{Animals and In Vivo Screening of Boron Compounds}

Specific-pathogen-free, female B6D2F1 mice were purchased from Jackson Laboratories, Bar Harbor, ME, at 6-8 weeks of age and housed singly in the WSU Wegner Hall Vivarium, which is AAALAC accredited. Mice were inoculated subcutaneously into the dorsal hip with $1 \times 10^{6}$ viable BL6 tumor cells in $200 \mu \mathrm{l}$ of $\mathrm{Ca}^{2+}$ - and $\mathrm{Mg}^{2+-}$-free saline solution, as described previously ${ }^{9}$. Approximately 1-2 weeks later, unanesthetized tumorbearing mice were inoculated with $200 \mu \mathrm{l}$ of boron compound into the dilated lateral tail vein. In other studies, BPA was also injected intraperitoneally as a slurry at a concentration of 750 $\mathrm{mg} / \mathrm{kg}$ body weight. Following injection of boron compound, mice were sacrificed through a 72-hour time period. At each time point, tissues (tumor, blood, liver, spleen, brain, muscle, kidney, skin) from six mice were collected and analyzed by ICP-AES for boron content.

\section{RESULTS AND DISCUSSION}

We have shown that boronated compounds accumulate both in vitro and in vivo in BL6 murine melanoma. In vitro uptake of boron by BL6 increases with increasing levels of BPA (25 to $100 \mathrm{mg} / \mathrm{l}$ ) (Figure 1). Levels of boron in cells exposed to $100 \mathrm{mg} \mathrm{BPA} / \mathrm{ml}$ are significantly greater $(p<0.05)$ than levels in cells cultured in $25 \mathrm{mg} \mathrm{BPA} / \mathrm{ml}$.

Figure 2 shows that this uptake is enhanced 7-fold when levels of tyrosine and phenylalanine are restricted to $4 \mu \mathrm{g} / \mathrm{ml}$ ( $22 \mu \mathrm{M}$ tyrosine, $24 \mu \mathrm{M}$ phenylalanine) which corresponds to an $88 \%$ reduction compared to levels found in normal medium 8 .

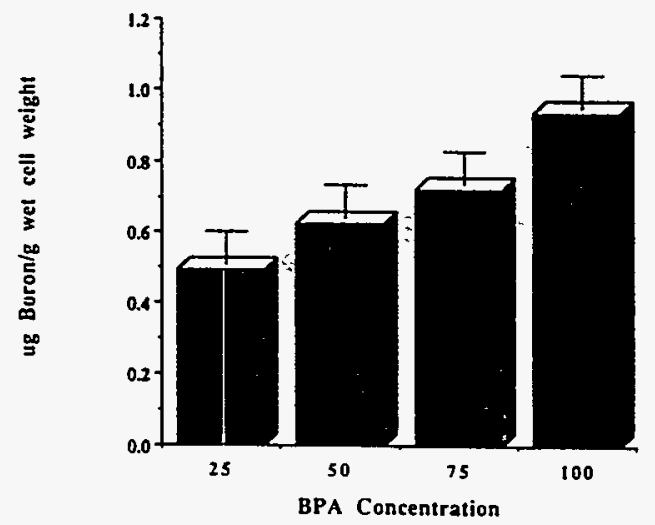

Figure 1. Cellular uptake of boron by B16-BL6 exposed in vitro for 6 hours to increasing concentrations $(25-100 \mathrm{mg} / \mathrm{ml}$ medium) of BPA in the culture medium.

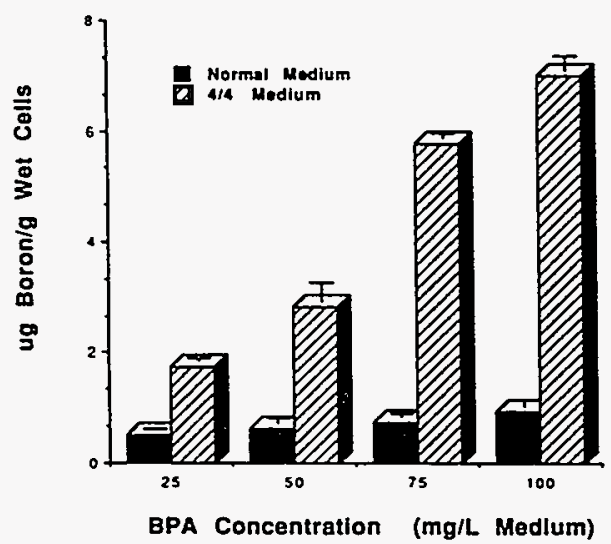

Figure 2. Cellular uptake of boron by B16-BL6 exposed in vitro for 6 hours to increasing concentrations of BPA in normal culture medium or medium restricted in tyrosine and phenylalanine to $4 \mu \mathrm{g} / \mathrm{ml}$ each. 


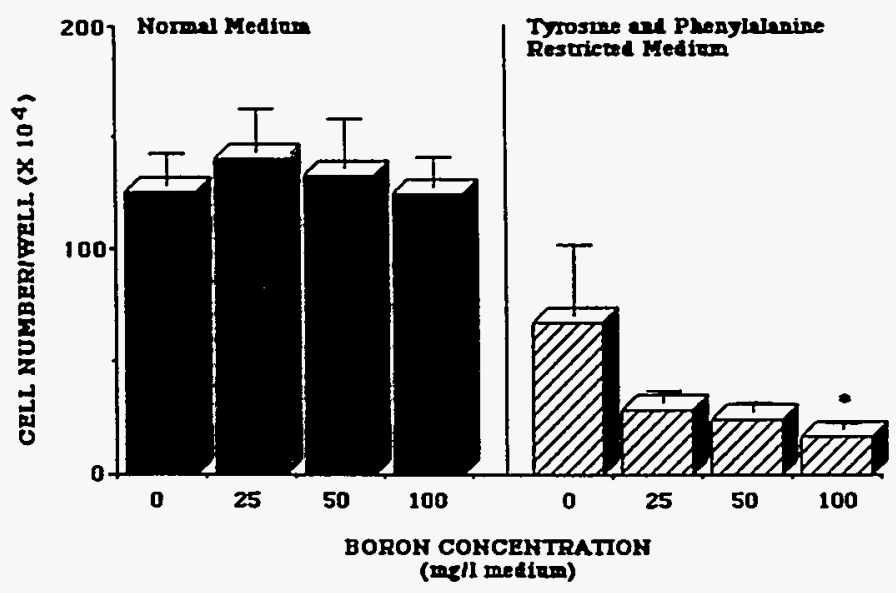

Figure 3. Growth of B16-BL6 on day 5 of culture in normal or tyrosine/phenylalanine-restricted medium and increasing concentrations of BPA.

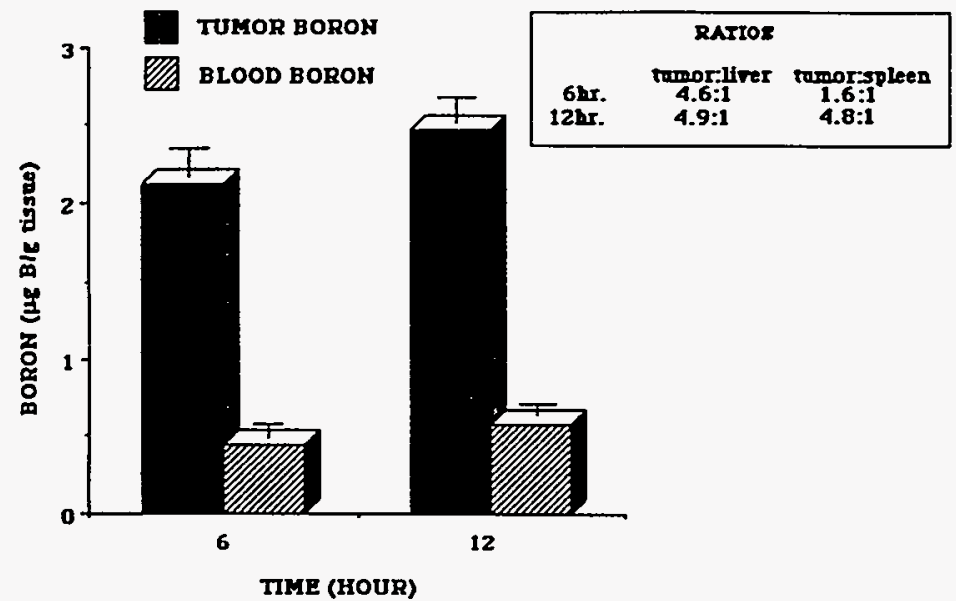

Figure 4. Distribution of boron in mouse tissues 6 and 12 hours after intraperitoneal injection of BPA as a slurry at a concentration of $750 \mathrm{mg} / \mathrm{kg}$ body weight. Tissues were collected from six mice at each time point.

This increased uptake is not due to any growth modulatory effects of BPA since in vitro growth of BL6 is not affected by exposure to exogeno:1s BPA (Figure 3).

We have also investigated the in vivo biodistribution of BPA. After intraperitoneal injection into mice of $750 \mathrm{mg} \mathrm{BPA} / \mathrm{kg}$ body weight as a slurry, respective tumor:liver and tumor:spleen ratios were 5:1 and 2:1 at 6 hours after injection and 5:1 and 5:1 at 12 hours after injection (Figure 4). Tumor boron levels at both times points were $2-2.5 \mu \mathrm{g} \mathrm{B/g}$ tissue.

The effectiveness of liposomal delivery of boronated compounds to BL6 murine melanoma tumors has also been investigated. Following intravenous injection of liposomalencapsulated boron compounds into tumor-bearing mice, boron accumulation in subcutaneous BL6 tumors typically reaches maximum levels within 24 hours postinjection with concentrations approaching subtherapeutic levels for BNCT. A representative in vivo screening of a liposomal-encapsulated compound is shown in Figure 5. Tumor:normal tissue (muscle or brain) ratios generally range from 5:1 to 11:1. Liposomal delivery of low doses of boron has resulted in therapeutic quantities $(\geq 15-20 \mathrm{ppm})$ of boron in other tumor systems, specifically the EMT6 murine mammary tumor ${ }^{10}$. These low doses minimize toxicities to the mice and may facilitate future experiments investigating alternative delivery protocols.

We are developing in vitro and in vivo assays which utilize tissue culture and small animals (mice) to determine the effectiveness of boron compounds for BNCT before more costly and time-consuming large animal studies are conducted. We are using these in vitro and in vivo techniques to enhance selective uptake of boron into tumor cells to optimize BNCT. Identification of alterable factors influencing boron uptake could have a significant impact on therapy of malignant melanoma as well as other cancers. 


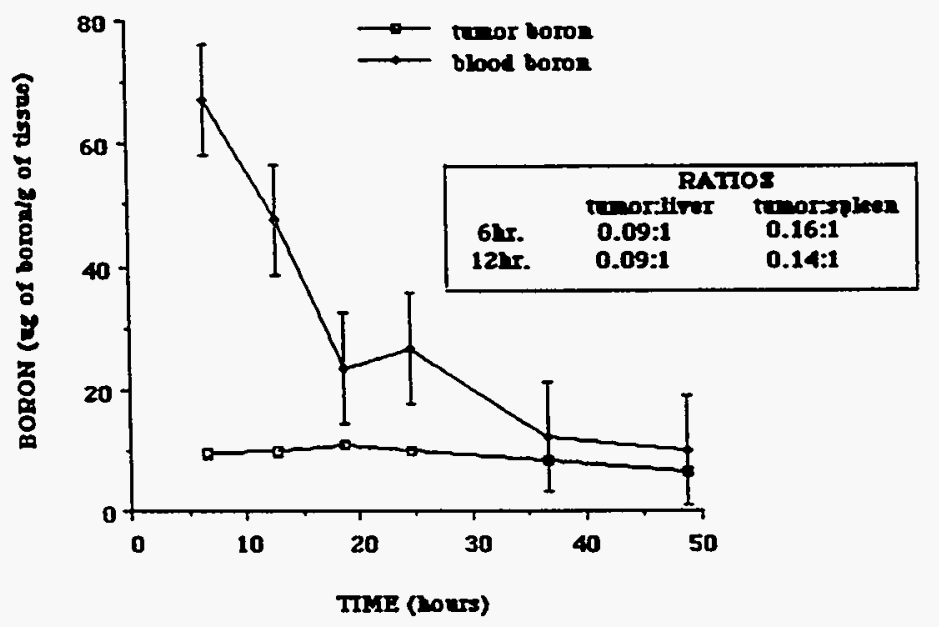

Figure 5. Accumulation of boron in tissues after intravenous injection of $200 \mu \mathrm{l}$ of liposomal-encapsulated $\mathrm{Na}_{2}{ }^{n}-\mathrm{B}_{20} \mathrm{H}_{18}$. Injected dose of boron $=212 \mu \mathrm{g}$. Tissues were collected from six mice at each time point..

\section{ACKNOWLEDGMENTS}

These investigations have been funded by the U. S. Department of Energy, Office of Energy Research, DOE Idaho Field Office, Contract number DE-AC07-D01570.

\section{REFERENCES}

1. F. Alam, B. V. Bapat, A. H. Soloway, R. F. Barth, N. Mafune, and D. M. Adams, Boronated compounds for neutron capture therapy, Strahlenther. Onkol. 165:121 (1989).

2. J. A. Coderre, J. D. Glass, R. G. Fairchild, P. Micca, and J. Kalef-Ezra, Boronophenylalanine for neutron capture therapy of melanoma, Strahlenther. Onkol. 165:215 (1989).

3. A. Gabizon and D. Papahadjopoulos, Liposome formulations with prolonged circulation time in blood and enhanced uptake by tumors, Proc. Nall. Acad. Sci. USA 85:6949 (1988).

4. D. A. Potter, C. M. Gorschboth, and P. d. Schneider, Liposome uptake by melanoma: In vitro comparison with hepatocytes, J. Surgical Res. 39:157 (1985).

5. A. Rahman and P. S. Schein, Use of liposomes in cancer chemotherapy, in: "Liposomes as Drug Carriers", G. Gregoriadis, eds, John Wiley \& Sons, New York (1988).

6. I. R. Hart. The selection and characterization of an invasive variant of the B16 melanoma, Am. J. Pathol. 97:587 (1979).

7. G. Poste, J. Doll, I. R. Hart, and I. J. Fidler, In vitro selection of murine B16 melanoma variants with enhanced tissue-invasive properties, Cancer Res. 40: 1636 (1980).

8. C. A. Elstad and G. G. Meadows, Modulation of B16-BL6 murine melanoma metastatic phenotype by tyrosine and phenylalanine restriction in the absence of host selection pressures, Anticancer Res., submitted (1992).

9. C. A. Elstad and G. G. Meadows, Phenotypic stability of B16-BL6 melanoma exposed to low levels of tyrosine and phenylalanine, Anticancer Res., 10: 1313 (1990).

10. K. Shelly, M. F. Hawthome, and P. G. Schmidt, Liposomal delivery of boron for BNCT, Proceedings of the 4th International Symposium on Neutron Capture Therapy for Cancer, 1991. 


\section{The Use of Boron Neutron Capture for the Treatment of Pituitary Tumors}

B. Albertson, M. Millan, S. Binney, G. Willeke, S. Martsolf, J. Johnson, and D. Loriaux

This article is a reprint from Advances in Neutron Capture Therapy, pp. 623-628, (1993). 



\title{
THE USE OF BORON NEUTRON CAPTURE
}

\section{FOR THE TREATMENT OF PITUITARY TUMORS}

\author{
Barry D. Albertson ${ }^{1}$, Monica A. Millan ${ }^{1}$, Stephen E. Binney ${ }^{2}$, Gail B. Willeke', \\ Steven W. Martsolf ${ }^{2}$, Jennifer E. Johnson ${ }^{2}$, and D. Lynn Loriaux ${ }^{1}$ \\ 'Division of Endocrinology, Diabetes, and Clinical Nutrition, Department of \\ Medicine, Oregon Health Sciences University, Portland, OR \\ 2Department 、f Nuclear Engineering, Oregon State University, Corvallis, OR
}

\section{INTRODUCTION}

Pituitary tumors comprise approximately $15 \%$ of all intracranial tumors ${ }^{1}$. The clinical presentation of these tumors is diverse; morbidity and mortality can be high. Current treatment modalities include conventional $x$-irradiation and/or neurosurgery, but are inadequate for the most part. Neither are uniformly successful and both are relatively non-specific. However, several features of these tumors make them ideal candidates for Boron Neutron Capture Therapy (BNCT). First, the tumors are localized in the pituitary gland, rarely metastacising to other areas of the body. Second, they are rarely larger than $2 \mathrm{~cm}$ in diameter. Third, the tumors are outside the blood brain barrier. And fourth, the pituitary tumor cells contain cellular membrane receptors that bind and internalize specific hypothalamic polypeptide releasing hormones. The ablity to target boron-10 $\left({ }^{10} \mathrm{~B}\right)$ containing compounds to these pituitary tumor cells with releasing hormones and subsequently kill these cells with BNCT is currently being evaluated in pituitary tumor cells in vitro.

\section{METHODS AND MATERIALS}

\section{CHEMICAIS AND REAGENTS}

Rodent pituitary tumor cells (AtT-20) were obtained from the American Type Culture Center, Rockville, MD. Cells are cultured in Dulbecco's Modified Eagle Medium (DMEM) (Gibco Labs, Gmad Island, NY) with antibiotics added (penicillin/streptomycin) and $10 \%$ fetal bovine serum (FBS) (Gibco). All cell washings are performed with sterile 
Dulbecco's phosphate buffered saline (D-PBF) (Gibco) in $15 \mathrm{ml}$ conicle centrifuge tubes (Corning Labs, Corning, NY). Cells are harvested from T-150 culture flasks (Coming) with a rubber policeman, or from six-well cell culture dishes (Corning) with trypsin/EDTA $(0.05 \% / 0.53 \mathrm{mM}$ ) (Versene, 1:5000) (Gibco). Cells are irradiated in sterile, $20 \mathrm{ml}$ polypropylene scintillation vials. After serveral washings cells are counted on a hemocytometer after staining with the supravital stain Trypan blue (Sigma Chemical Co, St. Louis, MO) using an Olympus CK2 inverted microscope.

\section{CELL IRRADIATION FACILITY}

Cell irradiations are conducted in the thermal column of the $1 \mathrm{MW}$ Mark II Oregon State University TRIGA Reactor. Three stringers of the graphite thermal column have been removed resulting in a $10 \mathrm{~cm} \times 30 \mathrm{~cm}$ cross sectional beam. The innermost end of this region is filled with a bismuth shielding assembly which holds the cell samples, followed by a $50 \mathrm{~cm}$ long graphite plug. The thermal neutron and gamma ray fields in the thermal column fall off exponentially with relaxation lengths of 30 and $80 \mathrm{~cm}$, respectively. For these measurements, the rear bismuth shield and graphite plug were absent.

Bare and Cd-covered gold foils were used to measure the thermal and epithermal neutron fluxes at the sample location. These values are $5.2 \times 10^{10} \mathrm{n}-\mathrm{cm}^{-2}-\mathrm{s}^{-1}$ and $9.2 \times$ $10^{8} \mathrm{n}-\mathrm{cm}^{-2}-\mathrm{s}^{-1}-\Delta \mathrm{u}$, respectively, at $1 \mathrm{MW}$. The fast neutron flux, measured in a single unshielded graphite stringer using indium threshold foils $\mathrm{s}^{2}$, is $1.5 \times 10^{8} \mathrm{n}-\mathrm{cm}^{-2}-\mathrm{s}^{-1}$. Thus, a reasonably pure thermal neutron beam has been achieved: $\phi_{\mathrm{th}} / \phi_{\mathrm{cpi}}=56 ; \phi_{\mathrm{tt}} / \phi_{\mathrm{fart}}=$ 350.

Gamma ray dose was measured with $\operatorname{TLD}-400\left(\mathrm{CaF}_{2}: \mathrm{Mn}\right)$ thermoluminescent dosimeters. The gamma dose rate at $1 \mathrm{MW}$ in the cell sample was $1.1 \mathrm{cGy} / \mathrm{s}$. The ratio of gamma dose to the thermal neutron fluence was $2.0 \times 10^{-11} \mathrm{cGy}-\mathrm{cm}^{2}$.

\section{CARBORANE CAGE SYNTHESIS AND RELEASING HORMONE CONJUGATION}

${ }^{10} \mathrm{~B}_{10}$ carborane cages have been synthesized, purified, and supplied by Professor $\mathrm{M}$. Frederick Hawthorne, Department of Chemistry and Biochemistry, UCLA. The uniqueness of the carborane cage lies in the incorporation of two carbon atoms combined as an integral part of the cage with an attached acetate reactive group and positioned so that the "cage" can be conjugated via the acetate to protein or polypeptide molecules like the releasing hormone ovine corticotrophin releasing hormone (oCRH). The conjugation of the cage is carried out with a classic peptide linkage, thereby joining the cage to the amino group on the terminal serine residue of oCRH. These synthetic steps and the final high pressure liquid chromatography purification of the conjugate are performed by Dr. Harry Chen, ERRB, NICHD, NIH, Bethesda, MD (Figure 1).

\section{EXPERIMENTAL CELL LINE (AtT-20)}

The AtT-20 rat pituitary corticotroph tumor cell line, established in 1953 by Furth et al. ${ }^{3}$, responds to $\mathrm{CRH}$ in a dose dependent fashion with the production of the intracellular second messenger, cyclic adenosine monophosphate (cAMP) (Figure 2). 


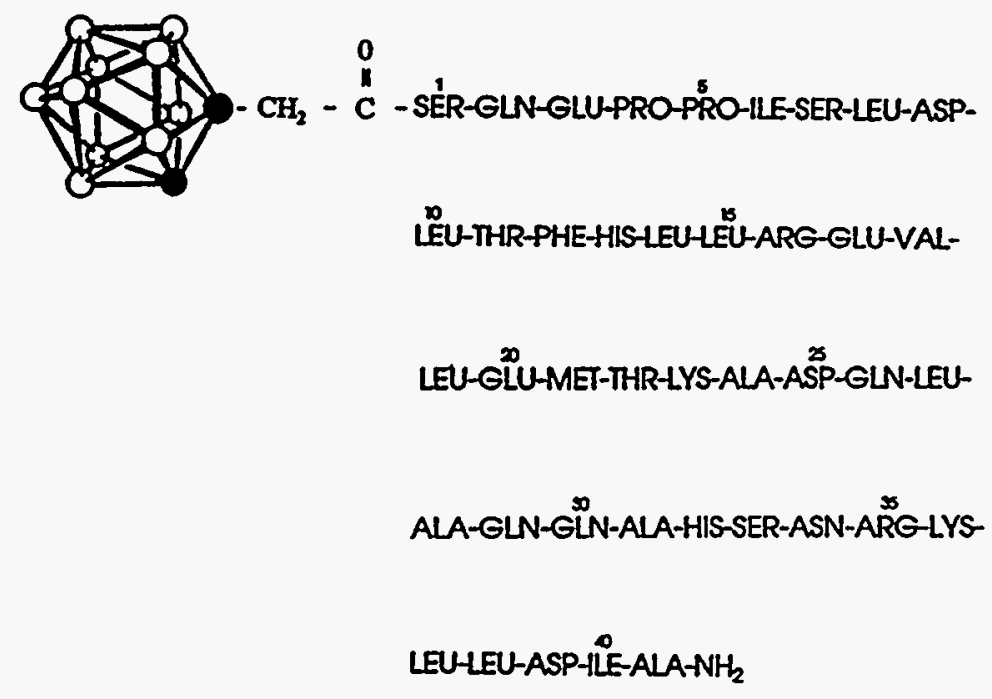

Figure 1. The amino acid sequence of $0 \mathrm{CRH}$ is shown with the conjugated carborane cage $\left({ }^{\circ} \mathrm{B}_{10}\right)$. Carborane cage boron atoms are shown as open circles, carbon atoms are shown as black circles. The acetate reactive group is shown conjugated to the number 1 serine residue of oCRH.

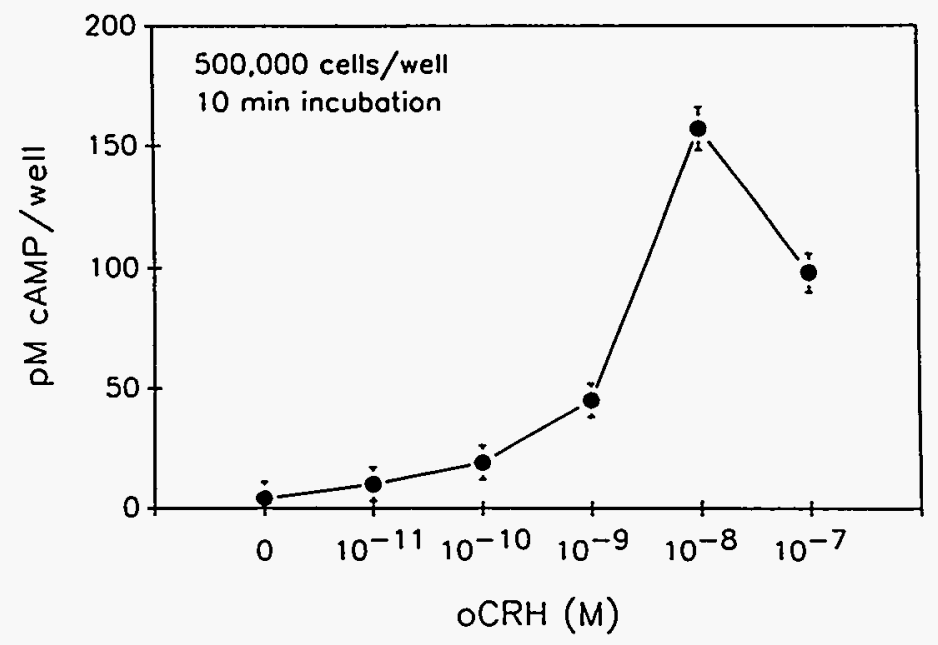

Figure 2. c-AMP dose response after a 10 minute exposure of AtT-20 cells to varying concentrations of oCRH. 500,000 AtT-20 cells were plated in 12-well cell culture dishes in D-MEM-10\% FBS. Cyclic AMP was measured using a radioimmunoassay with antisera provided by the National Hormone and Pituitary Agency, NIAMD, Baltimore, MD. Each point represents the mean \pm 1 SD of triplicate wells. 


\section{DISCUSSION AND RESULTS}

\section{RETENTION OF BIOLOGICAL ACTIVITY OF CORBORANE-CRH}

Carborane conjugated ovine $\mathrm{CRH}$ (oCRH) has been shown to retain biological activity, evaluated by the ability to stimulate the production of intracellular levels of CAMP when compared to equimolar concentrations of pure oCRH in in vitro experiments using the AtT-20 cell line (Figure 3).

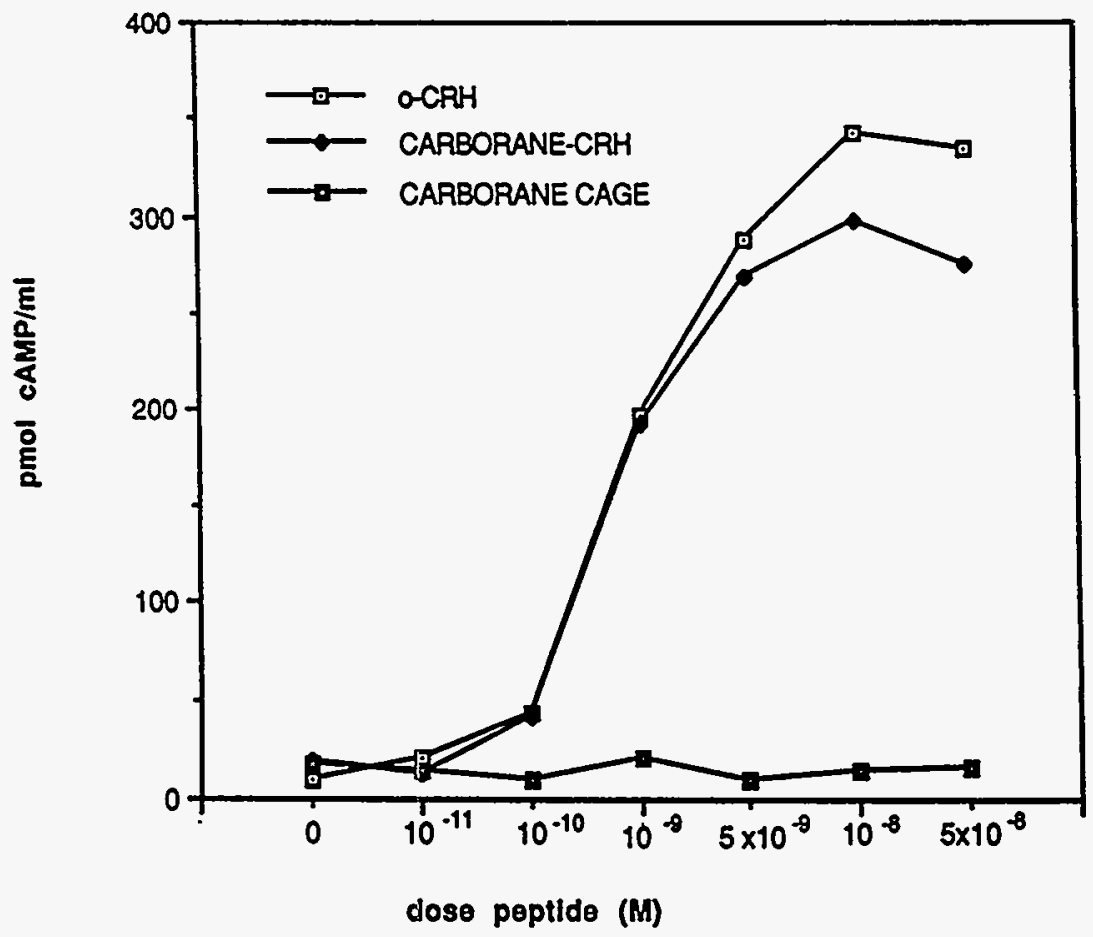

Figure 3. Cyclic AMP response of AtT-20 cells to oCRH, carborane-CRH, or carborane cage alone at various concentrations. Dose of peptide, carborane-CRH, or cage alone ranges from $10^{-11}$ to $5 \times 10^{-2} \mathrm{M}$. Picomoles of cAMP produced by 500,000 AtT-20 cells cultured in 12-well culture dishes per ml media is shown on the $\mathrm{Y}$ axis.

\section{EXPERIMENTAL PARADIGM}

AtT-20 cells are incubated in a water-jacketed $\mathrm{CO}_{2}$ incubator in the presence of oCRH, carborane conjugated oCRH or unconjugated carborane cage and oCRH. After a specified length of time the cells are washed in D-PBS, removed from the T-150 culture flasks using a rubber policeman, and placed into sterile, plastic cell irradiation vials. The vials are positioned in the thermal column as described above. The cells are exposed for up to 10 minutes at different reactor powers. The cell vials are subsequently removed, cell media aspirated after centrifugation ( $800 \mathrm{rpm}$ for 10 minutes), the cells washed in D-PBS, and replated in T-50 culture flasks in D-MEM with 10\% FBS. Cell viability is assessed over time using trypan blue. 


\section{REACTOR DOSIMETRY}

AtT-20 cells in D-MEM were placed in sterile $20 \mathrm{ml}$ scintillation vials and irradiated under different radiation dose protocols to determine the maximal gamma and/or neutron dose that AtT-20 cells in our in vitro paradigm can withstand. The cells were washed and subcultured in D-MEM for 5 days. Live AtT-20 cells (those that are attached to the plastic culture dish and exclude trypan blue) were removed from the dish using Versene and counted in a hemocytometer. The results of this pilot experiment are shown in Figure 4.

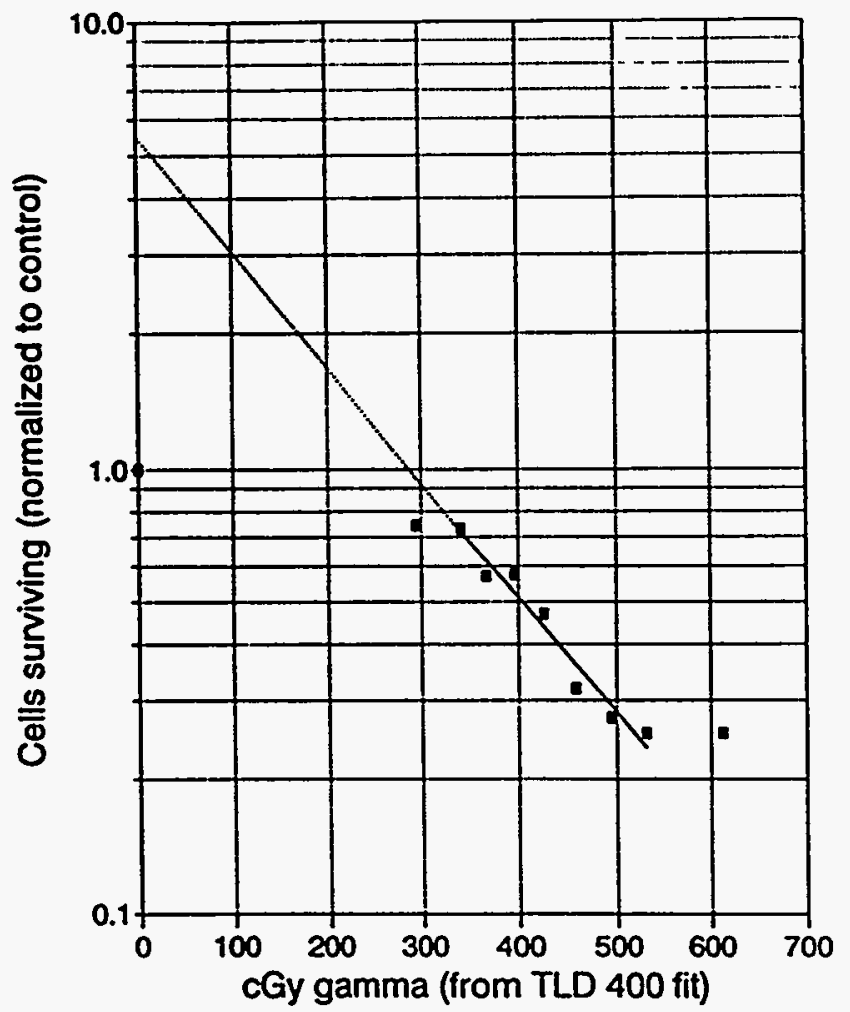

Figure 4. AtT-20 cell survival after 5 days, following exposure to varying gamma doses in the reactor thermal column. The data suggest that the $1 / \mathrm{e}$ dose for AtT-20 cells was $170 \mathrm{cGy}$ with an extrapolation number of 5.5. The initial AtT-20 cell number at day 0 was $5 \times 10^{\circ}$.

\section{BNCT EFFECT ON AtT-20 CELLS}

AtT-20 cells were preincubated with oCRH $\left(5 \times 10^{-7} \mathrm{M}\right)$ or carborane conjugated oCRH analog $\left(5 \times 10^{-7} \mathrm{M}\right)$ for 18 hours at $37 \mathrm{C}$, washed with D-PBS, and irradiated for 4 minutes at $500 \mathrm{~kW}$ (representing a gamma and neutron dose that does not appreciably harm the cells) as derived from figure 4. The cells were removed from the thermal column, washed twice with D-PBS and subcultured in T-50 flasks with D-MEM-10\% FBS for 8 days. Cell survivability was assessed at that time using trypan blue. The results are shown in Figure 5. 


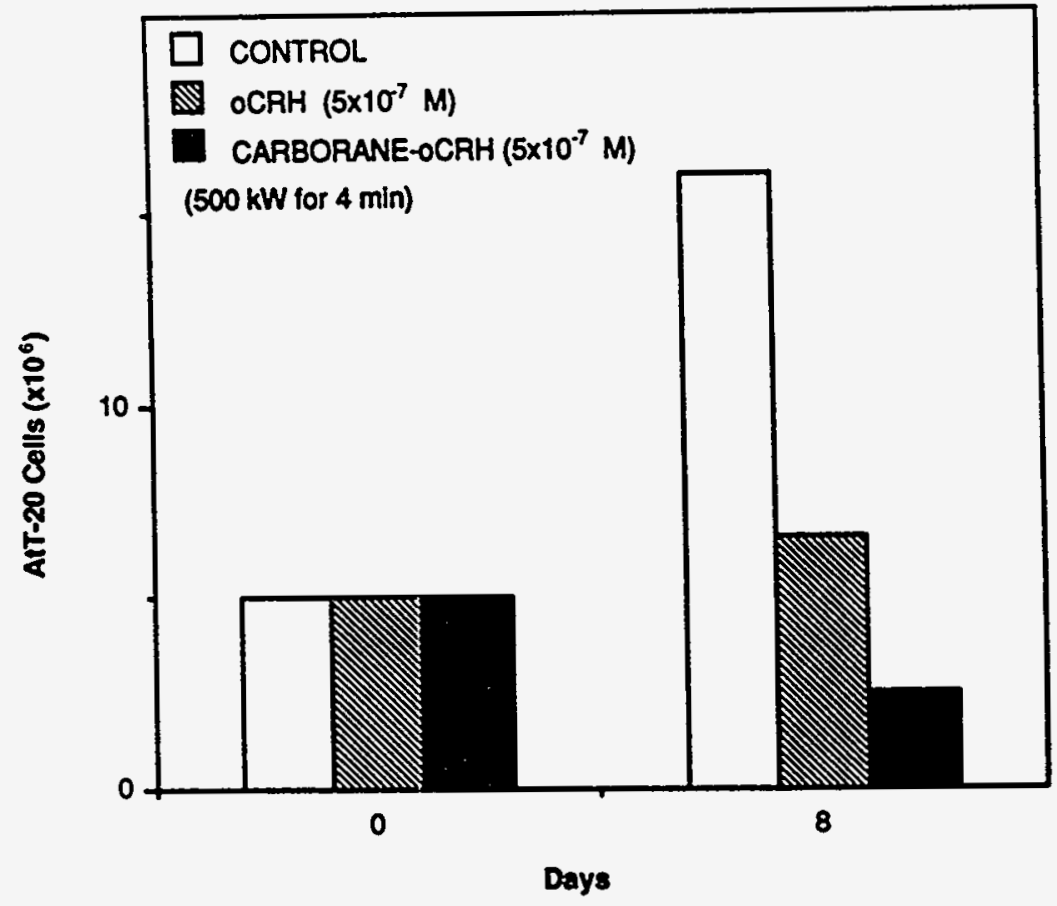

Figure 5. Control AtT-20 cells receiving no irradiation grew over an 8 day period from $5 \times 10^{6}$ cells/flask to approximately $18 \times 10^{6}$ cells/flask. Cells preincubated in the presence of $5 \times 10^{-7} \mathrm{M} \circ C R H$ grew over the same period of time from $5 \times 10^{5}$ to $6.5 \times 10^{6}$ cells. However, cells preincubated with carborane- $\alpha C R H$ decreased in number from day 0 to $2.5 \times 10^{6}$ cells/flask (day 8), suggesting a BNCT effect.

\section{CONCLUSIONS}

These preliminary data suggest that pituitary tumor cells can be killed using BNCT by linking enriched boron to hypothalamic releasing hormones. Strategies to decrease even further the contaminating gamma dose delivered to the AtT-20 cells are being tested. Cell loading experiments are currently under way (in conjunction with cellular boron quantitation using inductively coupled plasma-mass spectrometry and radio-iodine labelling of the carborane-CRH conjugate) to maximize the amount of carborane cage retained by the pituitary tumor cells so that the tumor cell to non-tumor cell ${ }^{10} \mathrm{~B}$ ratios are as high as possible. These studies will provide important insight into the design of in vivo experiments using rodents and sub-human primates to demonstrate the efficacy of BNCT for the treatment of pituitary tumors in humans.

\section{REFERENCES}

1. K. Thapar, K. Kovacs, E.R. Laws, and P.J. Muller, Pituitary Adenomas: Current Concepts in Classification, Histopathology, and Molecular Biology. The Endocrinologist 3(1): 39-57, 1993.

2. Y.D. Harker, R.A. Anderl, G.K. Becker, and L.G. Miller, Spectral Characterization of The Epithermal Neutron Beam At The Brookhaven Medical Research Reactor. Nuc Sci Eng 110: 355, 1992.

3. J. Furth, E.L. Gadsen, and A.C. Upton, ACTH Secreting Transplantable Pituitary Tumors. Proc Soc Exp Biol Med 84: 253, 1953. 